








\begin{tabular}{|c|}
\hline COUNTRY LIFE EDUCATION \\
SERIES \\
Edited by \\
CHARLES WILLIAM BURKETT \\
Kansas State Agricultural College \\
Types and Breeds of Farm Animals \\
By CHARLES S. PLUMB, Ohio State University \\
Principles of Breeding \\
By EUGENE DAVENPORT, University of Illinois \\
Other volumes in preparation
\end{tabular}




\section{PRINCIPLES OF BREEDING}

A TREATISE ON THREMMATOLOGY

OR

THE PRINCIPLES AND PRACTICES INVOLVED IN THE ECONOMIC IMPROVEMENT OF DOMESTI-

CATED ANIMALS AND PLANTS

BY

E. DAVENPORT, M.Agr., LL.D.

Professor of Thremmatology in the University of Illinots

Dean of the College of Agriculture

Director of the Agricultural Experiment Station

\section{WITH APPENDIX}

BY

H. L. RIETZ, Ph.D.

Assistant Professor of Mathematics in the University of Illinois

\section{GINN \& COMPANY}

BOSTON · NEW YORK - CHICAGO - LONDON 

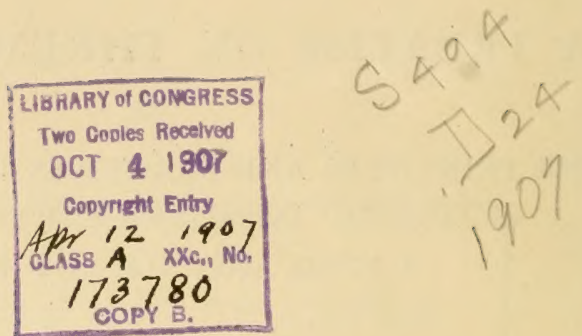

Entered at Stationers' Hall

COPYRIGHT, I9O7

BY EUGENE DAVENPORT

ALL RIGHTS RESERVED

77.9

$1-30120$

Tbe Atben xum-press

GINN \& COMPANY . PRO.

PRIETORS - BOSTON - U.S.A. 


\section{PREFACE}

Tivo classes of people have been in mind in the preparation of this text, viz. the student of agriculture in the college and experiment station and the practical breeder upon the farm. Both need to know all that evolution has to teach of methods that may be employed in still further adapting to our needs such animals and plants as have been domesticated because of their valuable natural qualities.

The general purpose has been first of all to define the problems involved in animal and plant improvement; to free the subject from the prejudice and tradition that have always befogged it; to bring to the study whatever facts are fully known to biological science; to recognize and define somewhat clearly the present limitations of knowledge, and to indicate as iwell as may be the directions from which further and much-needed light is most likely to come. Last of all and more than all, it has been the purpose to encourage, and if possible induce, more exact methods of study and of practice than have hitherto characterized this branch of agricultural science.

It is yet too early to prepare an icleal treatise upon this most intricate subject, and no one is more conscious than the author of the many deficiencies and shortcomings of this attempt. Some effort, however, is surely needed at this time to clear the atmosphere, to give the student of agriculture at least a rational point of view, and to bring him into comradeship with those who are earnestly studying biological problems and through whose efforts these vexed questions are sure sooner or later to find a solution. This, together with the pressing need of a text in his own class room, is the author's only warrant for the present volume.

No new theories of evolution are proposed. The chief object has been to distinguish what is known from what is merely traditional; to give as much as possible, within the limits of available space, of the best established facts bearing upon this subject; to 
call attention to approved methods of study, and to indicate lines of research most likely to furnish valuable information in the not distant future.

It is necessary to introduce a considerable amount of mathematical work in the later chapters. No excuse is offered for this introduction, and it is earnestly desired that the reader give special attention to this portion of the text, whether easy or difficult of following, because it is by this road that many new principles will arrive and that many of our future operations must be ordered; for nothing is clearer than that the successful brecter of the future will be a bookkcper and a statistician. For the convenience of the non-mathematical reader general formulac are placed in footnotes, and some of the more abstract matter is placed in the form of an appendix for the benefit of the more mathematically inclined.

The writer has taught this subject for fifteen years and is fully aware of the pedagogic difficulties involved as well as of the limitations of knowledge. He has tried many different outlines and many different methods of presentation, and has chosen the one here employed because in experience it seems the most favorable for the presentation of the subject-matter involved and at the same time for putting the student in a frame of mind favorable for the undertaking of economic breeding operations and for the reception of new truths as they shall be discovered.

Variation rather than heredity has been chosen for the initial and leading thought because better calculated, as experience has shown, to afford a favorable outlook and to develop such conceptions of evolution as are most useful later on.

The evolutionist who might chance to scan these pages would be struck by the absence of some of the cardinal features of evolution, as he would also note the exceeding prominence given to certain other questions of seeming minor importance. Herein exists the difference between thremmatology and evolution, and this very matter has given the author more difficulty than all others, viz. to rearrange values and to determine proper relations of old questions in a new field.

We must cliscuss the causes of variation even though we are told by the best students that such attempts are premature. A 
minor matter in evolution, curious rather than otherwise, it is a vital one in thremmatology, and we must discuss the subject the best we are able, if only to learn how little we really know about it and to point attention in the right direction.

No attempt has been made to include exhaustive references. On the other hand, they are confined for the most part to a few standard books easy of access, and to save time the references are mostly to definite pages. A general and more extended list follows the summary of nearly every chapter, enabling the student to pursue that particular subject further if desired; but there is no attempt at a complete bibliography. It was hoped that if the list of references could be kept small the student and the breeder would be the more likely to provide themselves with standard literature bearing on the subject. I have made the freest use of standard authors, giving full credit in all cases, generally in the form of reference to text and page. This course has been dictated by the desire to furnish the student with reliable facts rather than a series of academic discussions upon disputed subjects.

I desire to acknowledge the very great services of Dr. Rietz, to whom I am indebted for much assistance in the more statistical portions, and for the preparation of the appendix especially directed to the mathematical student, not as a text but as an introduction to further study in this special phase of science.

I am also indebted to many of my colaborers in the University of Illinois and elsewhere, as well as to numerous breeders in this and other states, who by their assistance have contributed much to any success which this volume may meet.

Its possible merits, therefore, I must share with others; its defects and shortcomings are my own.

E. DAVENPORT

UNIVERSITY OF ILLINOIS

URBANA 



\title{
CONTENTS
}

\section{INTRODUCTION}

PAGE

\author{
INTRHUCTION
}

CHAPTER

$$
\text { PART I - VARIATION }
$$

I. VARiation in General

I. Variation Universal among Living Beings

II. Variability the Basis for Improvement

III. Nature of Variability .

IV. Meaning of the Term "Character" . . . . . . II

V. Dominant and Latent Characters . . . . . . 13

VI. The Unit of Variability . . . . . . . 15

VII. Distinctions as to Kinds of Variations . . . . . I7 References . . . . . . . . 24

II. Morphological Variation . . . . . . . 25 References . . . . . . . . 29 29

iil. Substantive Variation . . . . . . . . 30

IV. Meristic Variation . . . . . . . . . 33

I. Symmetry ? . . . . . . . . 34

II. Meristic Variation in Linear Series . . . . . . 39

III. Meristic Variation and Bilateral Symmetry . . . . 65

IV. Symmetry in Variable Parts . . . . . . 68

V. Meristic Variation in Radial Series . . . . . . 70

VI. Importance of Meristic Variation . . . . . . 73 References . . . . . . . . 74

V. Functional Variation . . . . . . . . . 75

I. Variation in the Degree of Activity of Normal Functions between Different Individuals of the Same Species . . . 77

II. Variation in the I)egree of Activity of Normal Functions within the Same Individual . . . . . . . . $9^{\text {I }}$

III. Modification of Normal Functions by External or Other Influences . . . . . . . . . . 98

IV. Normal Functions exercised under Abnormal Conditions . 107 References . . . . . . . . Ic9

VI. Mutations . . . . . . . . . . . . . . . . . . . .

I. Distinction between Mutation and Ordinary Variation . . I

II. Examples of Mutation . . . . . . . . II I 
Cirapter

III. Experiments of De Vries . . . . . . . . II4

IV. American Experiences . . . . . . . . . 129

V. Economic Significance of Mutations . . . . . $\quad 135$

VI. Biological Significance of Mutations . . . . . 136 References . . . . . . . . 139

\section{PART II - CAUSES OF VARIATION}

INTRODUCTION . . . . . . . . I4I

VII. The Mechanism of Development and Differentiation a 142

I. Protoplasm the Physical Basis of Life . . . . . 142

II. The Cell the Unit of Structure . . . . . . 143

III. Mechanism of Cell Division (Mitosis) . . . . . 145

IV. Cell Division with and without Differentiation . . . 149

V. Physiological Units . . . . . . . 152

References . . . . . . . . . 154

ViII. Internal Causes of Variation . . . . . . . . 155

I. INTERNAL INFLUENCES AFFECTING PRIMARILY THE INDIVIDUAL

II. Bisexual Reproduction a Fundamental Cause of Variation . I60

III. Maturation and the Reduction of the Chromosomes a Cause of Variation . . . . . . . . 163

IV. Bud Variation . . . . . . . . . I 8 I

V. Influence of the Condition of the Germ upon Development . $18_{2}$

VI. Xenia, or Fertilization of the Endosperm . . . . 183

VII. Telegony . . . . . . . . . . . 185

VIII. Intra-Uterine Influences . . . . . . . . IS9

IX. Reversion and Atavism . . . . . . . . . 192

X. Individual Characters dependent upon Sex . . . . . 194

II. IVTERVIL IIFLLEVIES AFFECTTNG THE R.ICE IS I IIHOLE . . . . . . . . . . 196

XI. Relative Fertility, or Genetic Selection . . . . . . 196

XII. Physiological Selection . . . . . . . . 201

XIII. Selective Death Rate; Longevity . . . . . . 201

XIV. Bathmic Influences . . . . . . . . . 202

XV. Physiological Units . . . . . . . . . 208

XVI. Germinal Selection . . . . . . . . . . 213 References . . . . . . . . 217

IX. External Influences as Causes of Variation . . . 220

I. General Effect of Locality upon Plant and Animal Development . . . . . . . . . $22 \mathrm{I}$

II. Influence of Food upon Variability . . . . . . 225

III. Effect of Moisture upon Development . . . . . 230

IV. Effect of Contact upon Protoplasmic Activity . . . . 2 $233^{\circ}$

V. Effect of Gravity upon Living Matter; Geotropism . . 236

VI. Effect of Light upon Living Matter . . . . . . 239 
VII. Influence of Temperature upon Living Matter . . . . 254

VIII. Effect of Chemical Agents upon Protoplasmic Activity . . 264

IX. Effect of Saline Solution upon Development in Aquatic Animals

$\mathrm{X}$. Influence of Use and Disuse upon Development . . . . $2 \$ 5$

XI. External Infiuences as Causes of Variation in Type . . 290

References . . . . . . . 294

X. Relative Stability and Instability of Living Matter . . 295

I. Evidence from Stability of Type . . . . . . . 20,6

II. Evidence from Mutability of Species . . . . . 298

III. Evidence from Reversion and Atavism . . . . . 305

IV. Evidence from Disappearance of Parts . . . . . 306

V. Evidence from the Direct Action of the Environment . . 307

VI. Evidence from Acclimatization . . . . . . . 30\$

VII. Evidence from Regeneration . . . . . . . . 316

VIII. Internal Factors in Regeneration . . . . . . . 332

IX. Evidence from Grafting . . . . . . . . 335

X. Evidence from the Origin of New Cells and Tissues . . 336

XI. Evidence from Development and Differentiation . . . $33^{8}$

References . . . . . . . . 345

\section{PART III — TRANSMISSION}

Xi. Transmission of Modifications due to External. Influences

I. Introductory . . . . . . . . . 348

II. Evidence from the Nature of Variation . . . . . 356

III. Evidence from Mutilations . . . . . . . 364

IV. Evidence from Food Supply . . . . . . . 370

V. Evidence from Acclimatization . . . . . . . 374

VI. Evidence from Habit and Instinct . . . . . . . . 386

VII. Evidence from Use and Disuse . . . . . . . 404

VIII. Evidence from Disappearing Organs . . . . . 400

IX. Variations due to Causes not affecting the Germ are not

Transmitted . . . . . . . 416

References . . . . . . . . 418

XII. Type And Variahility . . . . . . . . . . . . 4ig

I. Type . . . . . . . . . . 420

II. Variability, or Deviation from Type . . . . . . +25

III. Practical Hints on the Taking and Grouping of Measurements 435

IV. Probable Error . . . . . . . . . . 437

V. Comparative Type and Variability for Different Characters in the Same Population . . . . . . . 444

VI. Effect of Selection upon Type and Variability . . . 445

VII. Indirect Effects of Selection upon Type and Variability . . 447

VIII. Studies in Type and Variability of the Same Variety of Corn raised under Different Conditions as to Fertility . . . 449

References . . . . . . . . $45^{2}$ 
I. Meaning of Correlation . . . . . . 453

II. Calculation of Coefficients of Correlation . . . . 455

III. The Correlation Table . . . . . . . . 458

IV. The Correlation Coefficient . . . . . . . . 459

V. The Regression Coefficient . . . . . . . . 466

VI. Studies in Speed Records of Trotters . . . . . . 468 References . . . . . . . 472

XIV. HEREDTY . . . . . . . . . . . . 473

I. How Characters behave in Transmission . . . . 473

II. Statistical Methods of Study of Heredity . . . . $\quad 478$

III. The Regression Table . . . . . . . . 479

IV. Like Parents beget Unlike Offspring and, conversely, Like Offspring may be begotten by Unlike Parents . . . $4 \delta 2$

V. Regression. In general, the Offspring is More Mediocre than the Parents; that is, Whatever the Parentage, the Offspring exhibits a Strong Tendency to regress toward the Mean of the Race

VI. The Measure of Heredity . . . . . . . . ${ }_{4}^{4} 86$

VII. The Mean of the Offspring not necessarily the Same as the Mean of the Parentage . . . . .

VIII. Extremes of a Race relatively Less Productive than the Means . . . . . . . . .

IX. Progression. Parents in general produce a Few Individuals More Extreme than the Race . . . . . . 492

X. The Exceptional Individual arises either from Mediocrity or from the Exceptional Parent . . . .

XI. Fraternal Variability, - Offspring of Same Parents not Identical . D Dathematical

XII. Characters tend to combine in Definite Mathematical Proportions

XIII. Mendel's Law of Hybrids

XIV. The Law of Ancestral Heredity . . . 5 ' 3

XIV. The Law of Ancestral Heredity . . . . . . 525

XV. Limit to the Reduction of Variability . . . . . 534

XVI. Power of Selection to permanently modify Types by the Establishment of Breeds

490

XVII. Breeding True, or Stability of a Character established by Selection . . . . . . . . 5 5 r

XVIII. Duration of Varieties, Breeds, and Family Strains . . 544

References . . . . . . . 547

XV. Prepotency

I. Data from the Trotting Records illustrating Prepotency . 55 I

II. Prepotency in Sex. . . . . . . . 567

III. Influence of Age on Prepotency. . . . . . . 573

IV. Influence of Constitutional Vigor upon Prepotency . $\quad 573$

References . . . . . . . 575 
XVI. Selection . . . . . . . . . . 577

I. Ideals in Selection . . . . . . . 578

II. Historical Knowledge of the Breed Necessary . . . 579

III. General Principles involved in Selection . . . . . $5^{\mathrm{SI}}$

IV. Rational Selection . . . . . . . . 592

XViI. Systems of Breeding . . . . . . . . . . 599

I. Purposes in Breeding . . . . . . . . 590

II. Grading. . . . . . . . 602

III. Crossing or Hybridizing . . . . . . 6c8

IV. Line Breeding . . . . . . . . 610

V. Inbreeding . . . . . . . . . 613

VI. Breeding from the Best . . . . . . . 626

References . . . . . . . 628

XVili. The Determination of Sex . . . . . . . . 629

I. Theories . . . . . . . . 6 6 . . . . 63

II. Influence of Nutrition . . . . . . . . . 631

III. Influence of Fertilization . . . . . . . . 632

IV. Sex in Mammals . . . . . . . . 634

V. The Accessory Chromosome and Sex Determination . . 634 References . . . . . . . 637

XiX. Plant Brefing . . . . . . . . . . . 639

I. Advantages and Limitations . . . . . . . . 639

II. Soil and Culture Conditions . . . . . . . . 64 I

III. Systems of Planting . . . . . . . . . . 643 References . . . . . . . . . 65I

XX. Animal Breeding . . . . . . . . 651

I. Advantages and Disadvantages . . . . . . 654

II. Fewer Characters for Selection . . . . . . 656

III. Fashion . . . . . . . . . 658

IV. Show-Ring Consequences . . . . . . . 660

V. Testing of Sires and Dams . . . . . . 660

VI. Weathering a Period of Depression and Preserving the IIerd $66_{5}$

VII. Records. . . . . . . . 666

VIII. Disposal of Surplus Females . . . . . . 672

IX. A Market for Sires . . . . . . . 673

X. Community Breeding . . . . . . . 674

XI. The Young Breeder . . . . . . . . 675 References . . . . . . . . 676

XXI. DeVelopMent . . . . . . . . . . 677

APPENDIX . . . . . . . . . . . 68

INDEX 



\title{
THE PRINGIPLES OF BREEDING
}

\author{
THREMMATOLOGY
}

\section{INTRODUCTION}

The main title of the present volume was chosen because selfexplanatory, though it less accurately expresses the scope of the subject than does the sub-title, which is only beginning to come into general use.

Thremmatology, from the Greek thremma, a thing bred, is a term proposed by Ray Lancaster ${ }^{1}$ to cover the principles and practices concerned in the improvement of domesticated animals and plants.

The term is broader than the "principles of breeding" because it includes development as well as reproduction. It is distinct from evolution in general, which attempts to explain the principles and forces connected with the origin and development of all forms of life but without the slightest reference to economic considerations. In evolution a protozoön is as important as a pig, a hyclra of as much significance as a horse, and the nost pestiferous weed as much an object of interest as either corn or wheat.

Thremmatology limits itself to those species and varieties whose natural qualities made them useful to man in the beginning, and it asks and seeks to answer this one question, How can they be made still more useful and better adapted to the purposes of an advancing civilization? In this study forms of life that are of no economic value are of no special concern except as their consideration may throw light upon clomesticated forms. This latter is often the case, for it is doubtless true that the same principles apply to all species, economic or otherwise, because none were

1 See Encyclopedia Britannica, ninth edition, XXIV, 84 I. 
specially created for matn any more than for any other animal. It is a seneral biological truth that covything lives moto itself, - not where it choses but where it call ; not 11 )on what it likes best but upon what it can get.

It may seem to the stuclemt that an undue amount of attention is given to variation and that a dispropertionate amount of space is devoted to that subject. In that event I have to say that vatriation is not the antithesis of heredity but rather its constant and insep)arable attendant, and that the facts of variation constitute the best portion of that stock of information with which the student must become possessed before he is reaty to stuely the principles involved in those gencralizations upon which practical oprerations may be safely based.

One is painfully aware, too, of the necessity of ranging far and wide for fates, and the student cammot fatil to fecl ofttinces that the subject-matter in hand is far removed from angriculture. When this is the case it is becaltse we are foreed to take what is available and make the most of it. Unfortumately the worliers in strictly agricultural fiedes are all too few and the reliable data deplorably meager, though some original and I trust valuable matter has been recently adeled to our stock of knowledge.

While the same principles doubtless apply in thremmatology as in evolution, yet important distinctions are to be observed. First, there is every reason to suppose that even funclamental laws apply to different species in different walys. For example, Inclian corm secms particularly sensitive to close breceling, whereas wheal is almost (xclusirely inbred and has been so inbred for unknown generations. Again, the circumstances of the case often introduce into the problem certain ecomomic considerations not resting upon sencral corolution. For example, man camnot afforel the "countless ages "and " untold generations" which are accoreled nature for accomplishing results. In practical breeding operations substantial results must follow at once and exhibit a high degree of suceess within the periof of a lifetime or they will be discarded as valueless.

Experience show's that the purposes, standarels, and methods of a successful breeder are seldom handed down from one man to another, even to his own son. Even if that could be dome, it 
would constitute no exception to the rule that a man must realize the fruit of his own labers and in his own genceration, for breceling is a business and must be marke to paly. The breceled must therefore work faster than nature, and thremmatringy tannot make use of a leisurely oprerating evolutionary prine ij le unle.. its action can be acceleraterl or its cumulative effects cxatrerematerl. Yet atrain, man cannot afford the immense numbers and the: whellesale destruction that characterize nature's mothorls of wrotinger changes. Animals and even plants cost momey; and a relatively large proportion must meet the conditions, or the conterpriac must be abandoned.

Business considerations therefore set arelurous limitations "1] ron thremmatelesy in respeet to beth tine and numbers from which crolution in general is entirely frex. The breeding business hats its own particular probleme, some of the most jmejertant of which unfortunately the known farts of corolution are least ables to answer. The profitable sturly of this subject will, howeses, lx: assisted by a clear statement of these problems.

The problems of the breeder. Cirtain ruectirn- -tanrl clearly out in the minds of practial brecter-, and throgh an attempt to answer them seriatim womlel not be: the lest mether of study, and though some of them cannot be answered with certainty in the present state of knowlerler. yet mothines is of more consequence at the rutset than that the sturlent gret a clear irlea of the problems needing solution and towards whose sollution the: stuely of thremmatrlesy is directerl. They are sub-tantially at follows :

To what extent are the charareristir iof an inclivilual at maturity cluce to its ancestry (hererlity), and to what extent are they

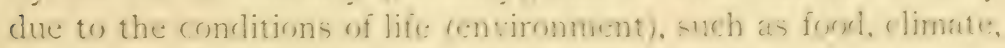
exercise, and general care during development?

Are the influences of the conditions of life limiter to the inciividual or are they in certain in-tanre- and to srme extent rarrierl wer upon the offeprines? that i-, are the effects of envirmunent inherited?

Can variations be directly controllerd to any cowtent whatow...r. or only inrlirectly through selection and by special care durine development? 


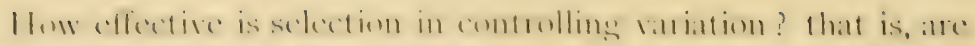

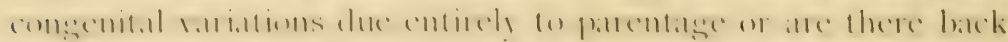

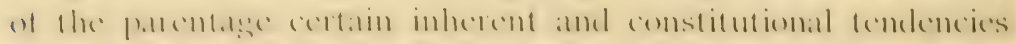

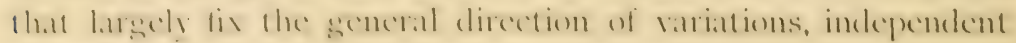
of sclection?

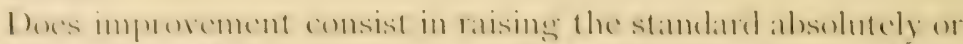

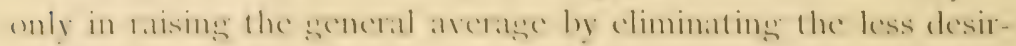
able? that is, does breeding improve upon the best or does it only bring the gencral mass nearer to the upper level?

To what extent is evolution a gradual process and to what

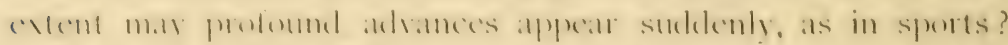

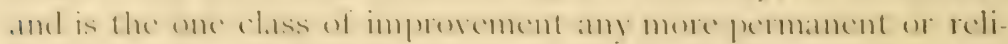
able than the other?

Do all possible values of a variable character appear or are certain values seldom or never presented? that is to say, is

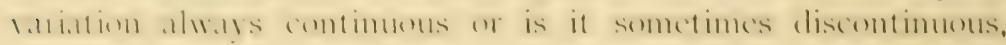
making certain things impossible because the proper variations of combinations do not appear on which sclection may te based?

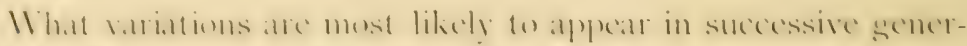
ations of any given breed, variety, or type?

Are variations correlated? that is, do they tend at all to move together, sugesesting relations of cause and effect?

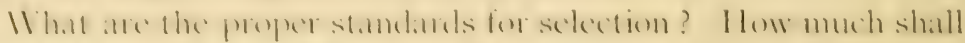
be given to utility and how much to appearance?

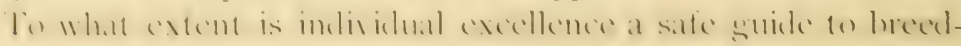
ing powers?

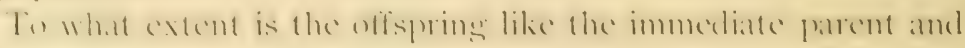
to what extent does it resemble more remote ancestors?

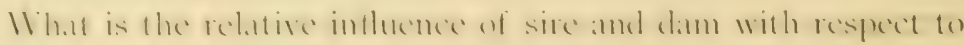
transmission of characters?

To what extent do the condition of the male at the time of

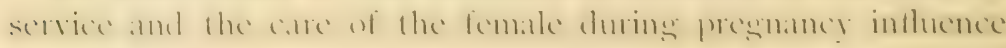
the offspring ?

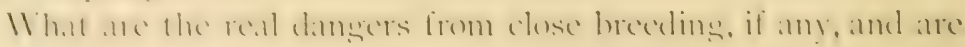
they certain or only probable?

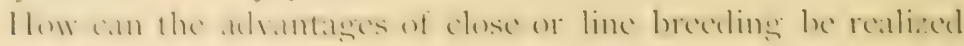
without encountering its dangers? 


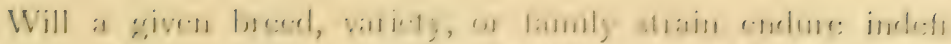

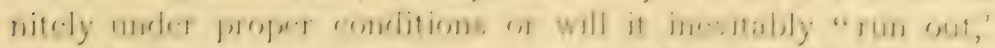

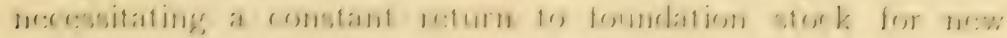
combinations as the basis of improved strains?

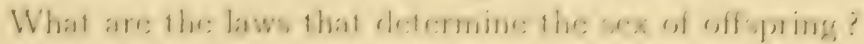

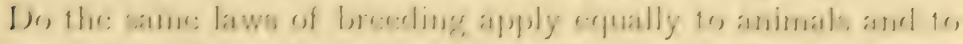

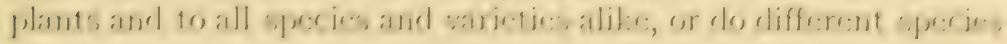
operate under somewhat different laws?

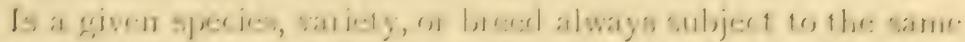

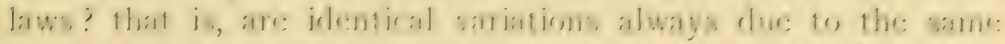

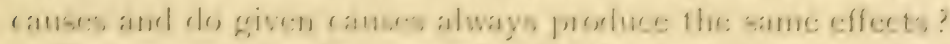

llow can results les secureal enth the least wastace: either in time or numbers?

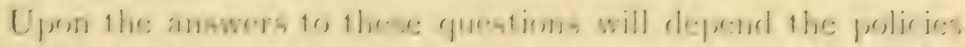

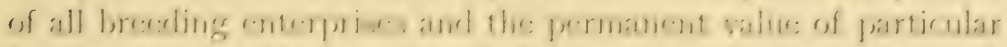

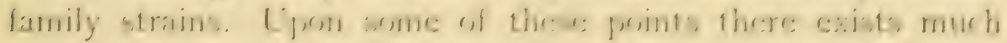
sper iffe and rediable informatien; upen othere, unfortunately, the: covidence is yet anty and uncertain. At the preent rate of

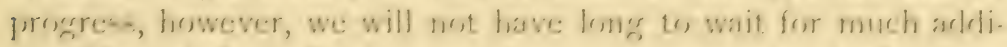
timal knowleclere. In tise meantine we mut male: the best use prossible of the information and experience at hand.

These problems an lect br: answereal not by directines antert-

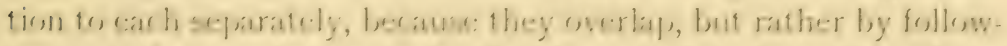
inger colt what are lenown to lor: the chararteristic lines of aturly in

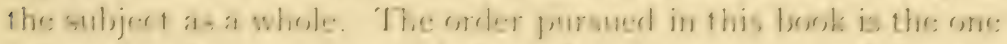
bedireverl to be ment favorable lasth for this purpore and for the:

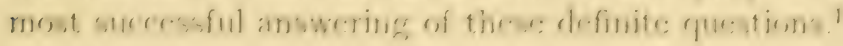

I A fair knowledge of general evolution is presumed on the part of the student and reader. If this is not in his prossession, he will do well (o) read at least

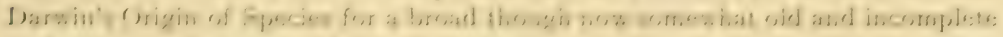
outlork upron the general field. If he desires to go further and enter the field of controversy, he can do so most directly by reading Weismann's kissays on IIeredity and his Germ J'lasm, trgether with Romanes' Examination of Weismannism and his two volumes of J barwin and After Jarwin. If this is done, it would be well to finish with IJabit and Instinct by Morgan. 



\title{
Part I-VARiation
}

\author{
CHAPTER I
}

\section{VARIATION IN GENERAL}

\section{SECTION I-CARIATION UNIVERSAL AMONG IIVING BEINGS}

The most obvious fact about living beings is their variability. Not only do species differ from each other by many and widely different characters, but individuals within the species are distinguished by differences readily discernible, at least by the trained observer. The general differences between horses and cattle, for example, are specific and distinct and therefore striking even to the casual observer; but to the trained eye all horses are not alike, and so it is that differences are detected within the species. Two individuals may be recognized as possessing the same characters and therefore related by descent, but invariably these characters differ in clegree or in their proportions one to another.

Two animals may be of the same or of different colors, but in either event the parts are differently proportioned. The leg of one is longer, larger, or more crooked than that of the other. The bones composing the two are not of equal, or even of proportional, lengths. Two cows of the same breed differ marvelously in the amount of milk they can yield in a year, and some are known to produce three times as much butter fat as others from the same amount of the same kind of feed. ${ }^{1}$ Again, some milk is rich in fat ( 6 or even 8 per cent) while other is poor (2 per cent or even less). Some horses, because of their conformation, travel more easily or more rapidly than others, and some are more intelligent or more enduring or more docile.

1 See data from Agricultural Experiment Station, University of Illinois. 
Some dogs (bloodhounds) trail marvelously well; others (greyhounds) scarcely at all. Some hens lay more eggrs than others, and of different color and size.

Some animals are hard feeders, while others lay on flesh readily. Some beef is coarse in its grain; other is fine and tender. Some is well marbled with fat; other is not. Sometimes the flavor is delicate; again it is rank, and often it is insipid.

No two trees are alike in their growth or branching habit, though similar within the same variety, and the widest difference is often found in leaves from the same tree.

Differences extend to minute particulars and include all characters. The student should early form a clear conception of the fact that differences extend to all characters however insignificant or minute. Besides, he should understand that they include function as well as structure, and that not only external anatomy and conformation are involved but internal organs and their activity as well, and no greater mistake can be made than to define evolution as "a study in morphology."

If we so define the word "variation " as to cover any change in detail of structure or function which our faculties enable us to detect, then we may say that variation extends to all characters, internal or external, structural or functional, and if the study lay in the realm of ethics, cconomics, philosophy, or religion, we should add, material or immaterial.

The individual is therefore so distinctly a unit that its identity is at once recognized and the principle is conceded that " no two are alike" and that variation is universal.

Limitation of variability. The exception to the universality of variability is in the realm of non-living matter. The specific gravity and other properties of iron, gold, sodium, or chlorin are constant, and their relations and combining powers with other chemical substances are, under identical conditions, invariable and therefore well known.

Oxygen, hydrogen, and even nitrogen and carbon, combine always in definite proportions, and though their combinations are exceedingly numerous yet when the conditions are known the exact combination can be foretold; moreover the properties of this combination will not only be definite but they will be identical with those of all other similar compounds. In this 
way sodium chlorid, for example, has always definite and wellknown properties not subject to variability.

It is to be noted of course that the properties of the conpound $\mathrm{NaCl}$ are totally different from the properties of the elements that compose it, $\mathrm{Na}$ and $\mathrm{Cl}$. They are nevertheless clistinct and invariable as well as new. This distinction between the variability of living matter and the constancy of non-living matter should be borne in mind later on when discussing some of the causes of variation.

\section{SECTION II-VARIABILITY THE BASIS FOR IMPROVEMENT}

Improvement is possible only where variability exists. The compound $\mathrm{NaCl}$ being constant, it would be impossible to produce an improved variety of sodium chlorid, because the compound is always the same and cannot be had with other than its standard and invariable properties. Improvement in this commodity is limited, therefore, to its mechanical form and cannot extend to its constitution.

Living matter, upon the other hand, while possessed of definite properties, does not exhibit these properties always in the same degree, and observation and experience have both shown that profound changes may be made in either the form or the constitution of both plants and animals by the simple method of judicious combinations of desirable deviations.

If there were no variability, and if living matter were as constant in its properties as is non-living matter, then we should be certain of what we already have, but no improvement would be possible. As it is, with variability everywhere, living organisms are both capable of improvement and liable to degeneration, for both are the logical consequence of variability. Man must therefore work for what he possesses in the way of animals and plants, and they will serve him well or ill according to his knowledge and skill in dealing with their variations.

Accordingly he cannot know too much about the variations that are likely to occur, - their nature, their extent, and the causes that control their appearance and determine their permanency. 
Ife cannot know too much about life and its vicissitudes, about living things and what they do.

An animal is born into the world. Its energies are first devoted to nutrition and growth. It builds its own machine and builds it quickly out of materials lying close at hand. In good time it is finished and all its energies are at a maximum. It seems like a stable thing that must live forever. But reproduction occurs, securing a succession of its kind. One after another of its faculties fail, and its condition is again reduced to that of bare existence, with youthful recuperative powers gone forever. By and by some vital function fails. Then life goes out; the organism breaks down and returns its elements to the inorganic world. Such is the brief history of a bit of matter temporarily endowed with life, - fleeting as a breath; any service it may render us must be caught in the passing.

\section{SECTION III - NATURE OF VARIABILITY}

The exact nature of variability is a most obscure subject, and one that cannot be fully comprehended in the present state of knowledge. Whether the distinctions between living and non-living matter will always remain as marked as they now seem to be, only future discoveries will determine. We have as yet only touched the fringe of this great subject, but enough is known to enable us to begin to penetrate some of its mysteries.

At least two general principles may be laid down in the present state of knowledge without much chance of error:

I. That all characters of plant and animal life, whether structural or functional, are exceedingly variable.

2. That ordinary variation is the result of a change in the relations between a number of associated characters through the deviation of one or more of the members, and not the introduction of an absolutely new character.

We speak loosely of "introducing new characters," but in truth improvement consists, not in the introduction of absolutely new characters, but in the intensifying of desirable old ones and the subordination of those that are undesirable. For example, 
when we undertake to improve the quality of wool we limit our attempts to the sheep, with which wool bearing is a natural character. Whether the horse or the hore could be made to grom wool is a question. If the hen could be made to produce milk or the cow to grow feathers, that would be the introduction of a new character in the strictest sense of the term.

But nothing similar to this has ever been accomplished by man. The particular group of characters that constitutes a giren species appears to be strangely fixed, and improsement seems to consist in changing the relations of these characters among themselves rather than in the introcluction of new members. How this particular grouping arose originally and how a new member (character) might be introluced are questions for the student of general evolution. They are questions, morener, upon which the present state of knowledge sheds little light, and, so far as is known, the study of the practical breeder is limited to methods of dealing with groups of characters already associated and constituting well-marked types and forms.

\section{SECTION IV - MEANIN( O OF THE TERM "CHAR.ACTER"}

This is a much-abused term, lessely useel in a variety of meanings. For example, when an inclividual differs slightly from another we say he has different characteristics. What we really mean is simply that his characters differ in their derelopment, not that he has different characters. Ilis bone is not sor round or his hock so crookecl; the crops are not so full (rr the milk so rich; the eye of the postato is not so sumken or the color of the fruit so high in one specimen as compared with another, and we say loosely that the characters are clifferent.

Now the truth is the characters are not different in kind but only in desree and proportion. Tie say of one horse that he has speed and of another that he has not speecl. The fact is that they both have some speed, but only one has enough to attract attention and be worthy of remark. This use of terms, unfortunate as it may be, is probably too common to be changed; indeed, the mere use of terms is of less importance than a clear comprehension of the facts. 
The term "character" is employed in this text to designate one ") those details of form or function which, taken together, constitute a well-marked group of animals or plants more or less closely related by descent, and this is the only sense in which the term ought to be used. Thus the color characters of the horse are black, bay, brown, gray, etc., but not red, green, or blue, although these characters are not unknown to the animal world, being common with birds.

Used in this sense, a "character" belongs primarily to the race or (moly) of which the individual is a member. It is therefore not peculiar to any particular individual and is in no sense personal property. Thus not only the color of the coat but the form of the body, the peculiar function of any of its organs, as in milk production or the secretion of poisons, any special mental attitude or intellectual function, or even a particular crook of limb or special body marking of any kind that runs commonly through the groups, is properly spoken of as a racial character. Those characters do not come and go, but on the contrary they remain with the race indefinitely. The individual horse, for example, will be marked by one or possibly more of the color characters of his kind, — black, white, bay, etc., — but he will not be marked with characters not of his kind, as red, green, etc. From this we see that the individual is not so good a unit for study as is the group to which he belongs and the racial characters that compose it.

Now the personality of the individual so strongly impresses us that we instinctively regard him as an actual unit, and we speak loosely of his characters as if they were personal property peculiar to this individual alone, whereas he possesses nothing that is not common to his race. His differences are in degree, not in kind.

What we mean to designate in the individual is the particular combination of racial characters that make up his personality, knowing perfectly well that the characters of all indivicluals within the race are racial characters and no other, and that every individual that may ever arise by descent will be limited as to his details to some combination of the characters of his race. Now the characters of any race are so many, their deviations are so 
wide, and their power to move independently of one another is so great that, according to the doctrine of probabilities, an almost infinite variety of combinations is possible. Ifence no two inclivicluals are ever likely to be identical. We thus arrive at the conclusion that the proper study of the breeder is not so much the individual as it is the normal characters of the race to which he belongs.

\section{SECTION V-DOMINANT ANI) IATENT CHARACTERS}

The race as a whole clearly possesses more characters than can ever be utilized in the visible make-up of any single indiviclual. Among all the colors of horses, but one, or at most two, can be found in any special instance. The race is therefore a kind of composite of all the individuals that compose it, or, more properly speaking for purposes of study, it affords a wide assortment of elements out of which individuals are composed.

The individual transmits the characters of the race. If the group of characters constituting a species is larger than that constituting an indiviclual, as with color among horses; and if an indiviclual may transmit a character which (apparently) he does not possess, and experience shows that he does, then it follows that the individual is in actual possession of more characters than those directly involved in his visible make-up.

For example, the offspring of two black horses will likely be black, but it may be bay, brown, or any other color characteristic of the horse kind. It is safe to say that it will not be red, green, or blue, because these colors are known not to belong to the horse kind, though all are freely found in nature.

Milk secretion is confined to the fentale sex, yet a bull whose dam is a heavy milker will transmit milking quality almost as successfully as will a cow. In this instance the male transmits a quality that he does not apparently possess and that could not become functional in his case.

From this we infer that the individual, whatever his particular make-up, transmits all the characters of the race, and none other; and that he is therefore possessed of all the racial characters of his kind in some degree visible or potential. From 
this we conclude that the apparent make-up of the individual depends upon the particular characters that happen to be strongest, that is, most highly developed in his case, but that he is in actual possession and may transmit any and all the characters of the race to which he belongs, but no other.

We now arrive at the distinction between dominant and latent characters, which is as follows: Those characters that are prominent in any individual are said to be dominant with him because well developed and plainly evident, and all other racial characters are said to be latent because not eviclent, although they are known to be present from the fact that they are transmitted to the offspring, often becoming the dominant characters in future generations.

The term "latent" should not convey the impression of hiclden or lurking characters, but rather undeveloped possibilities of the race within the individual in question. With this conception the student will be saved much mental confusion when dealing with heredity and reversion.

Elementary characters. In a biological sense the ultimate unit of variability, therefore, must be something less than the racial characters which we have been discussing, because they themselves are complex rather than simple. We speak of the leg of a horse or the quality of milk as a whole. Even if we narrow the point to the conformation of the hock or the proportion of fat, we yet have characters clearly made up of parts. The hock is an exceedingly complex structure, and seven, possibly nine or ten fats and oils are found in the fat of milk.

As almost unlimited color effects are made up by few primaries in different proportions, and as all ordinary materials are made up of a few chemical elements in different combinations, so in all probability if we could make the ultimate analysis we should find that all these characters are made up of definite living units, that we may call, for want of a better name, elementary characters.

These elementary characters have received many and various names. They are the stirp of Galton, the biophors of Weismann, and the physiological units of biologists generally. In general they are the smallest conceivable living units, comparable with 
the molecule of chemical compounds. Such elementary characters are supposed not to be variable except as they effect different combinations among themselves.

\section{SECTION VI - THE UNIT OF VARIABILITY}

The unit of variation is therefore not the individual but the racial characters that constitute the particular group, and that run down the line of descent like the strands of a rope and out of which individuals are made up, - some with one combination, others with another, after the fashion of threads in a fabric, forming patterns here and there, now of one design now of another, as they wander apparently aimlessly here and there. ${ }^{1}$

It is evident, however, that the actual basis of character deviation is sometimes exceedingly complex. Milk secretion, for example, while limited to certain animals and confined to the female sex, is properly recognized as a distinct character; yet its successful functioning depends upon a variety of considerations, - the general health of the body, the nervous temperature of the individual, the power of the stomach to provide large quantities of prepared food, the ability of the kidney's to do their work, and the power of every organ in the body to discharge its function successfully and fully under heavy strains. All these are as important to successful milk production as are large and active milk glands, and an accident at any point will cause deviation in milk yield either as to quantity or quality or both.

Deviation in color, on the other hand, may be due to presence or absence of pigment, which may be regarded as a chemical substance secreted at a single point. In this and in similar cases the actual basis of deviation is simple and readily cletected. From this it will appear that the ultimate seat of variation, whose fluctuations are responsible for character deviations, may be exceedingly difficult if not impossible to identify.

1 The unit of variability must not be confused with the unit of selection : the latter, of course, is the individual. We cannot separate his characters, but must take him as he is, for better or for worse; but we must do so fully realizing that each of his separate characters has an identity of its own, so that the unit of variability is far within the necessary unit of selection. 
It has been assumed that the ultimate unit of organized beings is the cell. This is true in a structural sense only, for there is positive evidence that the cell is itself made up of various and distinct elements capable of somewhat independent action in both cell division and growth. The content of a cell is not to be regarded as a mass of amorphous protoplasm to be halved or quartered by chance, but on the contrary it is an organized body with a distinct difference between the nucleus with its definite number of chromosomes (the supposed seat of the physiological units that give character to its activities) and its surrounding cytoplasm or food material.

Again, a whole group of similar cells may constitute a special organ (liver, kidney, or heart), discharging a highly specialized function quite different from that of any other portion of the body. This colony of many cells discharging the same function appears to move together, thus constituting a kind of functional unit larger than and quite clistinct from the ultimate physiological units that must reside within the cell.

Correlated variation. Still again, it is found in practice that occasionally whole groups of characters seem to be so correlated as to move together, so that having found one we may reasonably expect to discover the other. Familiar examples of this are found in nearly all cases of reversion. For instance, a white calf among Devon cattle will almost certainly show black or brown points (ears, nose, and legs), while a white shorthorn will not. The one is a case of reversion to the ancestral color of the breed, - the wild cattle of Britain; the other is simply the appearance of one of the normal color characters of the race. In increase of numbers of parts there is some tendency to repeat a whole group, as in cases of " double hand." 1

The same tendency for many distinct characters to move together in groups is found in cases of so-called "sports," in which we instinctively recognize something more than ordinary variation.

These instances of grouping of characters normally independent in such a way as afterward to move together is to be distinguished from such variation as is involved in extreme milk

1 See under Meristic Variation. 
production, in which results are to be ascribed rather to fortuitous variations among independent units than to anything like a linking together of the separate characters involved.

The ultimate unit. The student must maintain a clear vision as to distinctions of this sort. From the biological standpoint we accept as the unit of variability the ultimate physiological units that must reside within the cell ; for purposes of everyday use, however, we assume as a unit that detail of form or function that is important to the breeder, understanding perfectly well that, considered physiologically, it doubtless consists of a number of ultimate units which, like the elements in a chemical compound, are entirely capable of other and distinct combinations.

To repeat, we may assume either one of these conceptions as the unit for study according to the purpose in hand. In the text the word "character" will be used to denote that detail of (racial) structure or function which is important to the farmer and to this study. The character will be considered as the practical unit of variation, knowing perfectly well that the ultimate unit of variability lies much farther back in the constitution of the protoplasm itself. When this is in mind the terms "elementary character," "physiological unit," or some equivalent term will be used.

Whatever the situation, the student must not consider the individual as the unit of variability or he will come to grief both in study and in practice, nor should he become confused by those occasional and remarkable correlations of characters that suggest a unit of variability unduly large.

\section{SECTION VII-DISTINCTIONS AS TO KINDS OF VARIATIONS}

In the critical study of variation it is necessary to observe certain distinctions that are often overlooked, resulting in more or less confusion as to what is really involved in the term "variation."

Variation quantitative or qualitative. The first question that should arise in the mind of the student touching any deviation is this: Is the difference one in degree merely (quantitative) or 
is it a difference in kind (qualitative)? For example, one horse is exactly like another, only larger: the difference is quantitative. Another is no larger, but he can draw more and has greater endurance: the difference is qualitative. One cow gives more milk than another: the difference is quantitative; but a third gives better milk, and the difference is qualitative. One apple is larger than another of the same variety (quantitative variation), but another is different in texture and flavor (qualitative variation).

When, therefore, in the study of a racial character as represented in the same or in different individuals it is found to have varied, the first question to ask and answer is this: Is the deviation one in kind or merely in amount? Is it qualitative or merely quantitative? Is the change to be regarded as one in nature or only in degree? If the student will carry these distinctions always in mind, he will avoid much needless confusion.

Variation continuous and discontinuous. It is not to be assumed that variations differ from one another by infinitesimal increments. The differences may be infinitesimal (continuous variation) or they may be "cliscrete" (discontinuous variation).

Darwin supposed, and it is commonly assumed, that variation is by nature continuous, and that new forms originate by the gradual accumulation of insensible differences through the agency of long-continued selection. This means that if all the individuals that ever lived could be assembled and so assorted as to bring nearest together those that are nearest alike, it would then be found that they would grade into one another by imperceptible (lifferences, and that any gaps that might occur would be due to the effect of sclection in blotting out intermediate forms.

Now this is a hasty assumption, indicating in these days but a superficial acquaintance with the manner of variation. IVe camnot assume that all possible values in variable characters are presented; incleed, we know very well that in many cases all possible values are not presented, and that some intermediate forms never arise. For example, peaches often give rise to nectarines, but there is a gap between the two that apparently is never filled. Dirwin called an occurrence of this kind a "sport," as if it were an instance in which all ordinary laws were set aside, 
whereas it only shows that the variations of the peach are often discontinuous, with wide gaps representing spaces not filled by variation.

To get a full understanding of this ground the student must form a clear conception of the distinction between continuity and discontinuity as used in this connection.

A man grows from childhood to maturity. In doing so he passes through all possible weights and heights between those of infancy and maturity. We cannot represent all these values by any of our units of weight or measurements because all numbers are by nature discontinuous. The only measure of continuity is a line, because a line, curved or straight, represents all values sensible and insensible between its two extremes. IVe can thus plot continuity, but we cannot measure it except by cutting it into sections and measuring it at stated points as if it were discontinuous, ignoring the intervals.

Changes of temperature are continuous, as are those of humidity, illumination, and all growth in the sense of extension in size, whether plant or animal. All motion, whether regular or irregular, is continuous because all intervening spaces are included.

Discontinuity, on the other hand, implies vacant spaces not represented by values. The good singer goes abruptly from one note to the next, giving a discontinuous series of tones, while the unskilled vocalist slicles up or down the scale, giving rise to a continuous series of tones in his effort to find the proper note. The latter is not music because the ear is not pleasantly affected by this confused jumble of sound waves arising from the intermediate tones. Good music consists of a series of tones not flowing into one another but cut sharply off and cast into a discontinuous scries, striking the ear at intervals with sound waves that fit with mathematical precision.

All number is by nature discontinuous. By fractions we attempt to bridge the space between contiguous units, as between I and 2 ; but however small the fraction, there is yet a space, and a sensible and measurable one, between the fraction and the next unit. I $\frac{9999}{1000}$ is not 2 , nor will it ever become 2 this side of infinity by the addition of any number of nines to the numerator and ciphers to the denominator. It will constantly approach 2, 
but will never reach it, because definite number is discontinuous. This is why we can never accurately measure continuity except by a line, and this is why we cannot express in numbers the growth of an animal or plant, except approximately in terms of discontinuity.

All chemical compounds are made up of elements in definite proportion. They are therefore discontinuous. We have $\mathrm{H}_{2} \mathrm{O}$ and $\mathrm{I}_{2} \mathrm{O}_{3}$, but no intermediate is possible, - this again for numerical reasons. Plants and animals generally are dimorphic, the one form being male, the other female: this is discontinuity. Some species are trimorphic or even polymorphic. The ant is either male, female, worker, or soldier, and though they all belong to

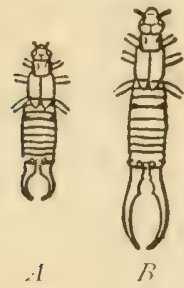

FIG. I. Dimorphism illustrated: two types of the common earwig. 13 is more common than $A$, and the type is more pronounced, but there are almost no intermediates. - After Bateson the same species there are no connecting links in this discontinuous chain.

Dimorphism without respect to sex occurs in many beetles, and is exceedingly marked in the common earwig, ${ }^{1}$ as shown in the accompanying diagram.

The shape of this curve shows clearly that here are two distinct forms of male, a large and a small one, living together and arising naturally out of the common mass, yet showing almost no intermediates.

Students of breeding familiar with the older types of Hereford will recall that the breed was almost dimorphic in that two distinct types tended to appear with singular perverseness, refusing either to blend or to undergo modification. There was the old, large, solid-bodied, thick-meated, deep-ribbed type, ideal except as to lateness of maturity; then there was also the pony-built type, - short in the barrel and lacking in depth behind, though well proportioned in front.

The shorthorns are almost polymorphic in possessing not one but a variety of types, each standing out with extreme distinctness and not readily merged.

The more the matter is examined the more it will be seen that strange and unaccountable gaps are found everywhere. Many a

1 Bateson, Materials for the Study of Variation, pp. 36-42. 
breeder has spent his life and his substance in the vain attempt to produce a desired intermediate between two forms, either one of which are easily secured. The question arises, therefore, Are some intermediates impossible? Can we briclge the space between the nectarine and the peach ? between the apricot and the plum?

With all these examples before us, we see at once that to proceed upon the theory of continuity is a gratuitous assumption not borne out by facts. Force and physical agents generally seem to be continuous in their different manifestations, shading one into another with imperceptible gradations; but organized matter, living or non-living, seems to be constructed upon the plan of discontinuity, in which case we may expect to find differences that are casily perceptible, and should not be surprised at the appearance of wide spaces between nearly related forms or at these remarkably distinct gaps that often occur between a standard form and its offset, which Darwin called a "sport" and which we in these days call a "mutant."

With this view of the case, we should not expect to find all nature united by imperceptible gradations, even providing all living beings past and present could be assembled and assorted according to nearest resemblances. Realizing the discontinuous nature of all chemical combinations, living or non-living, we should expect to find notable gaps representing spaces not taken by any possible form, and appearing quite independent of any selective process.

This distinction is exceedingly important at the outset of this study. If all variations are continuous, then all shades of difference, however minute, may be expected to occur naturally, and we may hope to secure them by breeding. If, however, some variations are cliscontinuous, then for these characters minute gradation is impossible, and we may expect descent to follow along certain lines only.

Most of the conditions of life are without doubt naturally continuous in their variations. This is certainly true of temperature, moisture, light, and food. Discontinuity must therefore arise from within, and is evidently connected with the nature of organisms. This is not difficult to appreciate when we recall the essential discontinuity of all chemical compounds or other organizations built upon the basis of distinct units. 
The student must not therefore assume the possibility of intermediate gradations and insensible differences when dealing with biological phenomena. Many of these differences are essentially and necessarily discontinuous. It remains to discover which these are and to discover the bearing of discontinuity upon the results to be accomplished by selection.

If all variations were continuous we might hope to be able theoretically to accomplish any desired result and secure any desired shade of difference by selection; but if not, then there will remain notable gaps that cannot be filled. The natural corollary of all this is that we can accomplish by selection almost any desired shade of result with those variations which are by nature continuous, but that with those variations which are by nature discontinuous, our efforts in this respect will be limited.

Distinctions arising from the nature of the characters involved. I Iaving determined whether the deviation is quantitative or qualitative, continuous or discontinuous, we next inquire into the real nature of the variation as it affects the organism. Manifestly this depends upon the character or characters involved.

Those concerned with form will, in their deviations, give rise to morphological differences. On the other hand, deviation in characters distinctly functional will give rise to differences in organic activity without regard to form.

Accordingly four distinctly different kinds of variation are recognized :

I. Morphological, relating to differences in form or size. By nature they are always quantitative, but may be either continuous or discontinuous.

2. Substantio', relating to differences in quality of the structure as distinct from mere form or size. By definition they are always qualitative and generally, if not always, continuous.

3. Meristic, relating to deviations in pattern, especially as to repeated parts, as in extra fingers and toes, doubling of petals, stooling of grain, etc. Variations of this kind are either quantitative or qualitative, generally the former, but are of necessity discontinuous.

4. Functional, relating to deviations in the normal activity of the various organs and parts of the body or the plant, such as 
muscular activity, glandular secretions, etc. They are either quantitative or qualitative, continuous or cliscontinuous, though rarely the latter.

A clear understanding of these distinctions is necessary to an intelligent study of the nature and causes of variation. Accordingly enough attention will be given to each to acquaint the student with the way in which variation behaves, partly for its own sake and partly as preparation for careful inquiry into methods of dealing with deviations in those plants and animals that we have domesticated and appropriated to our use, and which we would see still better adapted to the purposes of man.

Summary. Variability is the universal rule among living beings. Literally no two are alike. The differences extend to all characters and to the most minute particulars. Non-living compounds exist in definite proportions, and their qualities are constant, not variable. Variability is the only basis for improvment. No improvment is possible, in the strictest sense of the term, with respect to inorganic compounds, but living matter being variable is capable of change and therefore of improvement.

Variation consists not in the introduction of new characters but in different proportions or relations among the old ones. All characters are racial, and all individuals actually possess all the characters of the race and none other. This is shown by the characters that are transmitted to the offspring.

The unit of variability is in no sense the individual, though he must be accepted as the unit for selection. The real unit of deviation is the racial character, but back of that, in a biological sense, lie the elementary characters or physiological units, whose various combinations constitute racial characters.

Variation is both quantitative and qualitative, both continuous and discontinuous, and these distinctions should be clearly in mind at all times.

\section{SPECIAL ExERCISES}

Prepare a list of variations that are (1) quantitative, (2) qualitative, (3) morphological, (4) substantive, (5) meristic, (6) functional, (7) continuous, (8) discontinuous. 


\section{ADDITIONAL REFERENCES}

Antmals and Plants under Domestication. By Charles Darwin. 2 vols.

Discontinuous Variation. (An example.) By E. R. Saunders. Proceedings of the Royal Society, LXII, II-25.

Origin of Species by Means of Natural Selection. By Charles Darwin. I vol.

Theory of Organic Variation. By H. S. Williams. Science, VI, $73-84$.

TYPE, How FIXED. (On grenetic energy of organisms. Is variability and not permanency the normal law of organic life?) By H. S. Williams. Science, VII, 72I-729.

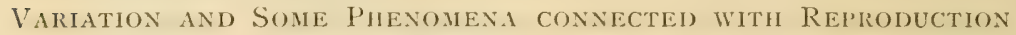
AND SEx. By Adam Sedgwick. Science, XI, 88I-894, 923-930.

VARIATION Discontinuous. (Study of a recent variety of flatfish.) By H. C. Bumpus. Science, VII, 197.

VARiation in Plants. (A study of the portions of a leaf on which chlorophyll is found.) Experiment Station Record, XIII, 423.

VARIATION IN TRILlium GRANDIflorum. (A record of the variations observed in 185 cases.) By H. W. Britcher. Maine Experiment Station Bulletin No. 86, pp. 169-196; also Experiment Station Record, XIV, 634. 


\section{CHAPTER II}

\section{MORPHOLOGICAL VARIATION}

Morphological variation has reference to differences in form. If two or more individuals possess the same structural characters and if they have all attained the same relative development, then the different individuals will differ only in size; but if the characters have not attained proportional development in the different individuals, then we shall note differences in form independent of mere size. This is the simplest of all forms of variation and is the one chiefly in the mind of older biologists, even leading to the mistake of supposing that evolution is essentially a study in morphology.

The cause of morphological differences may lie in extremely favorable or unfavorable conclitions of life, especially as regards food and climate, affecting different characters differently, or they may arise from internal and constitutional causes, as in giants and dwarfs, or in such extreme differences as in the mulberry leaves shown in Fig. 2.

Instances of morphological variation are so common and so easily noted as to scarcely require mention. Two apples are exactly alike except that one is larger than the other. It is a clear case of morphological variation. In this instance there is no difference in the characters of the two individuals except that cell division and growth have proceeded farther in one case than in the other. Aside from this they are iclentical. If two stalks of corn or if a number of pigs, sheep, cows, or horses are exactly alike except as to size, then their differences are quarititative only, and the effect is morphological merely.

Again, two horses are of the same breed, - that is, possess the same characters, - but their characters are not equally, that is, proportionately, developed. In one the leg is ionger, the hock shorter, or the face wider between the eyes. These differences 


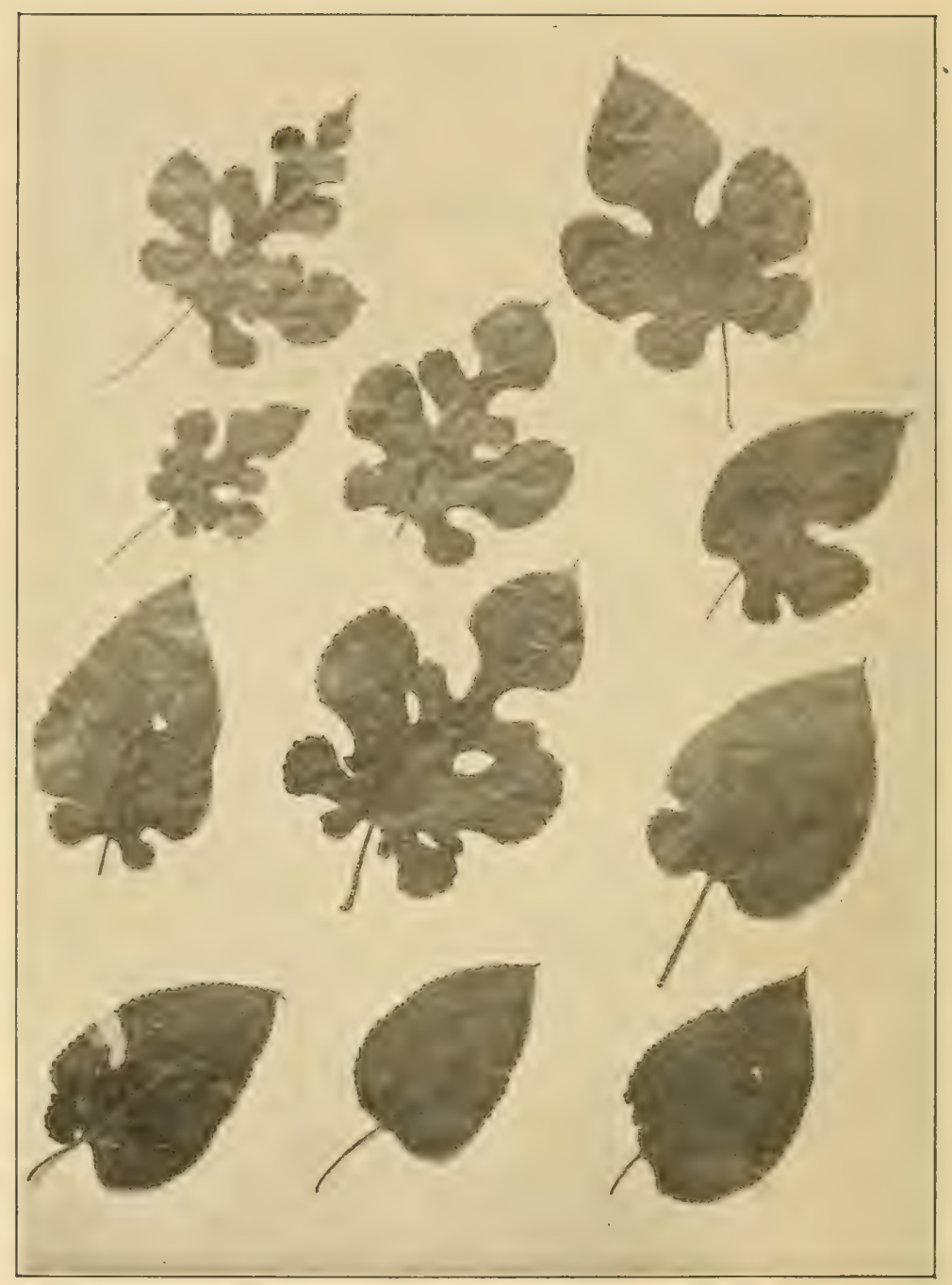

FIG. 2. Morphological variation illustrated: different forms of mulberry leaves picked from the same tree on the same day 
are all quantitative and morphological, but they influence form rather than size as a whole, because their development is not proportional one part with another.

Variation seldom simple. Instances of the above kind are, however, extremely rare. Variation is so common that other differences generally accompany those of form. The two apples may differ in color, flavor, or texture as well as in size, in which case substantive variation has also occurred. One of the horses may have an extra rib, one of the pigs a solid hoof, or one of the sheep more fibers of wool to the square inch, in which case meristic variation is present. The pulse of one of the horses may be faster than that of the other, or the milk of one cow may be richer in fat, showing functional deviation.

And so it is in practice that two or more forms of variation may be and likely will be found present in the same individual. But however that may be, all differences in form or size are regarded as morphological, no matter what other differences may be found, and it is important that the student early form the habit of distinguishing clearly between the different kinds of variation present, even in the same individual.

The limits of size. Every species has a general arerage of size to which most individuals closely approximate. A few, however (giants), greatly exceed this size, and others (dwarfs) fall far short of it. All investigators agree upon the conclusion that this difference in size is clue to the number and not the size of individual cells; in other words, size is dependent upon the energy of cell division. ${ }^{1}$ This energy is exceedingly active in youth, gradually decreasing to zero at maturity, except as to certain parts (reproductive organs, skin, and sometimes the teeth and horns). In most species accident to a part will stimulate cell division, leading to a more or less successful regeneration. For the most part, however, cell clivision does not proceed rapidly after maturity, and the limit of its activity is in general the limit of growth. Giants therefore represent excessive cell division above the normal, and dwarfs, arrested development, an abnormally early cessation of cell division.

1 Wilson, The Cell in Development, pp. 388-394. 
The causes involved in this abnormal behavior of the cell in division are exceedingly obscure. They are certainly sometimes connected with food and care in early life, and no doubt they are often constitutional. Every stockman knows about the stunted pig, calf, or colt, and that it sometimes, but rarely, recovers; that is to say, cell division once checked does not easily return to the normal rate. The trait, however, easily becomes constitutional and hereditary, for whole families (strains) become undersized and others as much above the medium.

Energy of growth not to be confused with bodily or functional activity. While the larger animal of his kind, whether it be the individual or the strain, represents the greater energy of cell division in body building, it by no means follows that the body when built will possess a greater degree of activity than will its normal or smaller neighbor; indeed, the opposite is likely to be true, because the larger body works at greater disadvantage, having greater inertia to overcome and more dead weight to carry about. This is eminently true of all animals whose service involves transporting the body from place to place, as among horses. It is manifestly impossible for a heavy horse to equal a light one in speed without the expenditure of far more power in doing it. This is not only because of the extra weight but because of mechanical disadvantages as well. In activities not involving motion this difference in size, within reasonable limits, does not exist; small men, for example, are doubtless no more and no less intellectual than are large ones.

Importance of morphological variation. Next to those of color, differences in size are the most noticeable of all variations; but they are by no means the most significant, and their importance is likely to be greatly overestimated. Except in a few instances, as with draft horses, mere size is of far less consequence than is commonly supposed.

Generally speaking, it is some quality other than bulk that determines value, and it will be fortunate for breeding when the popular notion that "the biggest is the best" shall have passed away. The largest apple is not the best for eating, nor the largest bull the best for breeding purposes. However, this does not free the student and breeder from considerations of size, 
because extremes both ways are in most cases to be avoicled, and the highest excellence and the most reliable breeders will commonly be found among the individuals of medium size.

Differences in form, arising from relative inequality in clevelopment of structural parts, are of more consequence than are differences in mere bulk, in which development has been proportional. This is especially true in horses, in which differences in relative development of structural parts may seriously affect the appearance or interfere with the action of the individual. And so it is that, while morphological differences are of far more significance to the student of general evolution than they are to the farmer, they yet constitute a phase of variation not to be overlooked by the student who is interested in the improvement of domesticated forms.

\section{ADDITIONAL REFERENCES}

DARININIANA. By Asa Gray. I vol.

DARWINism. By A. R. Wallace. I vol.

Expression of the Emotions in Man and Animals. By Charles Darwin. I vol.

From Greeks to Darwin. By H. F. Osborn. i vol.

Lamarck: His Life and Work. By A. S. Packard. I vol. 


\section{CHAPTER III}

\section{SUBSTANTIVE VARIATION}

Substantive variation has reference to differences in quality as distinct from form or size. It regards the composition or make-up of the body or its members, and refers to the constitution, or inherent nature, of the organism.

Everybody recognizes differences in muscles, whether firm and strong, or soft, flabby, and weak. We distinguish the bone of a horse as dense or as soft, porous, and spongy. The horn of the hoof is hard and tough or soft and "shelly.'

Meat is fine in grain and high in flavor or coarse in grain and lacking in quality. ${ }^{1}$ It is either juicy and rich or dry and tasteless. The gamy flavor of wild meat, both of mammals and birds, is especially tasteful to the huntsman, and whether due to breeding or to feed, it is certainly characteristic of wild life everywhere. No two cuts of meat are alike, whether wild or tame, and these differences are so pronounced as to be commonly recognized; incleed, languagre abounds in adjectives descriptive of differences in quality of food stuffs.

At one time milk was sold on the quantitative basis only, but now the per cent of fat is the basis of value. The intelligence of an animal or of a man depends less upon the size and weight of the brain than upon the quality of the brain matter and the depth of the convolutions.

One apple is sweet, another is sour, and still another is insipjicl. One fruit is highly flavored, another is tasteless. The sugar of beets, of cane, and of maple is the same; but the two former are simply sweet, while the latter is accompanied by a highly volatile ether that adds a peculiarly delicious aroma.2

1 Pigs fed heavily on cotton-seed meal make a pork strongly flavored with cotton-seed oil. See Grindley, Jonmal of American Chemical Society, Vol. XXVII.

2 Isolated by Kedzie, of the Michigan Agricultural College, from samples furnished by the author. 
The sugar content of beets varies from 4 or 5 per cent to over 20 per cent. It is also exceedingly variable in cane. Wheat is richer in protein than is corn, but both are variable, and corn has been bred with a protein content higher than that of wheat. ${ }^{1}$

Plants differ in their ability to withstand frost as do animals to resist disease. A single stalk of corn may remain fresh and green when all its neighbors have been killed. Certain individuals seem immune to particular diseases, and appear to be able to resist infection indefinitely.

Color in general is based upon definite chemical constituents or upon the character of the surfaces presented for refraction of light. In either case it is a matter of inherent quality and is substantive.

Importance of substantive variation. The significance of substantive differences depends upon the instance. Speaking generally, these differences are of high value. They are usually, though not invariably, correlated with efficiency, and in such cases they possess a utilitarian interest.

A dense bone is better than a soft and fibrous one. Everybody prefers a good apple to a poor one; we have a decided preference for certain aromas; the juicy, highly flavored steak is better than the dry, tough, and tasteless one.

Color is a utility character among flowers. ITe buy them for their color, their form, and their odor. They have no other value, and of these characters their coloring is of the most importance.

Color, however, is in general the most deceptive of all characters, - - deceptive because it is striking and because we greatly prefer certain color effects over others, even though not correlated with utility. IVe carry this preference beyond reason. A red apple will sell for more than one of any other color; yet we buy an apple not to look at but to eat; and no one has shown a correlation between color and quality in fruit.

Horses with white skin are proverbially subject to certain diseases. For this and other reasons color has no little significance in horses, but among cattle it has practically no meaning whatever; and yet how decideclly do color markings figure in

1 The lowest protein content discovered in the breeding experiments at the University of Illinois up to date (1907) is 6.13 per cent, and the highest is 17.79 . 
many score cards for pure-bred animals! Now the facts are that a cow is kept for what she can do, and there is nothing inherent in mere color that is indicative of her ability to convert feed into either milk or meat. She is therefore neither better nor worse for her color except as it is an index of blood lines when she is to be used as a breeder.

So striking are color differences, however, and so distinct are our preferences, that we instinctively follow our prejudices in this respect, quite regardless of more important consiclerations, until the whole fabric of breeding is interwoven with "fancy points" in the shape of color markings that greatly confuse the breeder in his attempts to select individuals for breeding purposes.

To-day the majority of prize-winning shorthorns are either roan or pure white. Twenty-five years ago no breeder would have dared to show a roan animal, and if a white calf had been dropped in his herd he would have destroyed it at once and kept the matter as secret as possible, so strong was the red color craze following the American worship of Bates cattle. This craze, which was always groundless, cost the breed and their admirers dearly and checked by a decade or more its progress upward.

Probably of all substantive variations color is, excepting among flowers and ornamental plants, of the least consequence to man ; yet the prejudice is with us, and the breeder who expects to sell his product must reckon with it. IIe should do it intelligently, however, realizing fully that individuals vary greatly in inherent quality quite independently of color.

In general it may be said that substantive differences, though not so easily detected, are yet of far more significance to the farmer than are those of either form or size. 


\section{CHAPTER IV}

\section{MERISTIC VARIATION}

Meristic variation has reference to a deviation in the number or arrangement of repeated parts involved in the plan or pattern upon which any particular organism is built.

A plant or an animal is not an amorphous lump of living matter. On the contrary, it is made up of parts, each of which has a kind of identity of its own, many of which are similar, and all of which are definitely related and placed in some sort of orderly arrangement.

Reflection discloses the fact that each organism is developed upon a specific plan, essentially different from that of any other, and that with most organisms the pattern is composed of a definite number of similar parts more or less repeated.

Thus the chicken has two legs, and the horse has four legs, that are more or less alike. The flower has many petals, the corn plant many leaves; the spinal column is composed of vertebra very much alike, and organisms generally possess many parts that more or less closely resemble one another.

From this it appears that the individual animal or plant is not a unit in respect to form, but rather that it is made up of many units, some of which are practical cluplicates. Thus the idea of multiple parts in orderly arrangement (morism) comes at once into the study, and variation in the number or character of these repeated parts (meristic variation) is a broad and complicated subject affording considerable insight into the nature of variation. Accordingly it is profitable to pursue it at considerable length, not so much for the material involved, which consists largely of abnormalities of no practical interest or value, but because no other phase of variation affords so much information upon the real nature of living matter and its adherence to or deviation from a definite plan. 


\section{SECTION I - SYMMETRY}

The central thought in all meristic stuclies is symmetry, by which is meant that opposite sicles of an organism possess parts that are either iclentical or at least similar. Thus the petals upon one side of a flower are in most cases like those upon the opposite side, and the blossom is made up of a number of similar parts very much alike and several times repeated. ${ }^{1}$

Among higher animals, however, opposite sides are similar but not identical, and here arises the distinction between radial symmetry and bilateral symmetry.

Radial symmetry and radial series. By this is meant that kind of pattern in which the separate parts are identical and each part is capable of replacing any other in the series. Common examples are the petals of most flowers (legruminous and the like excepted), the leares and lateral shoots of plants, the capsules of many seeds, such as the apple, orange, etc, the rows of corn upon the cob, the parts of the sea urchin, the starfish, and the Radiolaria generally.

In all cases of this sort the incliviclual parts could each replace any other part of the series. The pattern is therefore spoken of as one of radial symmetry, and the parts as members of a radial series.

Bilateral symmetry. Among higher animals a different symmetry is observable. While each sicle has its comterpart upon the other, yet there is a distinction between the right and the left sides and between the dorsal and the ventral surfaces. In such cases the parts while similar are not alike, nor could they replace each other.

1 Symmetry is well-nigh zniversal. All organisms arise through cell division in one or more planes, and some degree of symmetry is to be expected from the manner in which growth takes place.

But symmetry is not confined to multicellular structures. Appendages consisting of single cells are frequently symmetrically placed, and many organisms, which are single celled and therefore microscopic, as diatoms, secrete a skeleton with regular markings as symmetrical as hoarfrost and quite as beautiful.

All this is curious rather than valuable to the student of thremmatology who is interested in multicellular beings; yet it all throws light on the method of life, and we are able to lay down the principle that symmetry is not only the natural corollary of development by cell division, but that it is also a general principle in living matter. 
The right hand and arm are made upon the same plan as the left, but could not replace them because they would not fit; the one is the reverse of the other.

Reflected in the mirror the right hand seems to be the left, but it is an illusion, for the right side is the negative or optical image of the left with all its elements reversed. Hence a part upon the one side could not replace the corresponding part upon the other. It is its counterpart, not its cluplicate as among leaves and petals. Thus we arrive at the distinction between the complexity of bilateral symmetry and the simplicity of radial symmetry. It is also significant that bilateral symmetry is characteristic of higher animal life, and radial symmetry of lower animals and plants.

Dorsal and ventral surfaces. The fundamental fact at the bottom of bilateral symmetry is the distinction between dorsal and ventral surfaces, necessitating differences in the quadrants that are forced to work in opposition to each other. ${ }^{1}$

Indian corn and the grass family generally are as distinctly bilateral as is the horse or man, yet there is no thought of dorsal and ventral differences, and hence no distinction between right and left.

For example, let the leaves of a corn plant and the arms of a man extend east and west. Then the north and south sides of the corn plant will be alike. Not so with the man. In the one direction (we will say the south) will be his spinal column and the general framework of the body ; in the other (to the north) will be his face, his nose, his eyes, and all the active external parts. Moreover his hands are made to oppose each other and to work together with this (the ventral) sicle of the bocly. There is therefore bilateral symmetry in one direction but not in the other.

With the corn plant the case is different. It does not move from place to place, and it presents its plain sides indifferently to the world. Accordingly no distinctions similar to dorsal and ventral are possible. ${ }^{2}$

1 The word "opposition" is here used not in the sense of "antagonism" but as "placed opposite and working with"; as, The thumb is "opposed" to the other members of the hand, thereby making a working unit.

2 None of these distinctions should be confused with homologous parts or with analogous parts, nor should the ideas be confounded.

Homologous parts belong to two individuals, not one, and they are such as bear corresponding structural relations to their respective organisms, suggesting 
Bilateral symmetry not complete. Curiously enough not all the parts follow the same plan as to bilateralism and symmetry. What has been said refers to paired organs standing on opposite sides of the body, as hands, arms, legs, eyes, ears, etc.

Many organs not paired present curious facts to the evolutionist. The nose, for example, has no counterpart, but it stands on the median line and has a bilateral symmetry of its own, being made up of right and left halves. The liver and the heart, however, while consisting of right and left halves, are unpaired organs, placed not on the median line, but the one upon the right, the other upon the left. Each has a bilateral symmetry of its own, with distinct right and left sides, yet both are unsymmetrically placed.

The stomach, on the other hand, is an unpaired organ lying unsymmetrically across the body, and its own bilateralism is not between right and left but from front to back. The kidneys present the anomalous phenomena of paired organs with a bilateralism of their own but at right angles to that of the body, being also from front to back.

Longitudinal symmetry and linear series. Inasmuch as all growth is by cell division, we might expect longitudinal symmetry as well as lateral symmetry. Owing, however, to the definite relations of both animals and plants to the external world, it is not much developed, and there is but a suggestion of longitudinal symmetry to be found in either plant or animal forms.

Most plants are both geotropic and heliotropic; that is, one part goes down into the earth in response to gravity and the other upward toward the light and against gravity. This makes

common descent. Thus the leg of a man is homologous with that of a horse or a bird, because of structural resemblances. In the same sense his arm is homologous with the fore leg of the horse or the wing of a bird.

Analogous parts are such as serve the same purpose in different organisms though structurally distinct. Thus the flipper of the whale, which is a modified hand, is analogous to the fin of a fish, and the gill of a fish is analogous to the lung of a mammal, because it serves the same purpose, though there is no structural relation between the two.

The homologue or the analogue of a part is therefore to be found in another individual and of a different species. Symmetry, on the contrary, with its corollary of multiple parts, refers to individuals taken singly and to the interrelations of their parts. 
the two extremities at once very different, and forestalls the development of any very pronounced symmetry longitudinally:

Animals in their locomotion establish clifferent relations at their opposite extremities, thus preventing exact symmetry in this direction, and yet reminders of inherent tendencies toward universal symmetry are constantly encountered. Long worms, for example, though distinctly different at the extremities, are yet composed of rings very much alike throughout most of their length, even permitting locomotion backward with considerable facility.

Longitudinal division, however, with or without corresponding symmetry, is everywhere found both in plant and animal life, especially in the latter, and linear series of similar parts present as many opportunities for variation as are afforded by radial series either with or without bilateral symmetry. Thus the rings of worms, the vertebra and the ribs of the body, the joints of the fingers and of insect parts, - all these are fertile sources of meristic variation.

Homœesis in meristic variation. This is a form of variation in which one part assumes the characters or appearance of another, usually quite distinct. It is a frequent accompaniment of meristic variations. For example, an extra vertebra may be found in the dorsal series, increasing the number by one, - all normal. This is meristic variation of the simplest kind, with no homocosis.

On the other hand, it may be situated at the front of the dorsal series and partake somewhat of the character of a cervical (forvard homocosis), or it may be located at the rear of the dorsal series and in many respects resemble a lumbar (backward homoeosis).

This posterior clorsal vertebra may bear a rib that is bifurcated at the extremity, one branch effecting a union with the sacrum, the other floating, in which case there is doubt as to the real character of the additional vertebra, whether dorsal or lumbar.

In much the same way misplaced organs are often found. A leaf may be seen growing from a fruit, an antenna may spring from an injured eye, or foot appendages may develop instead of those proper to the extremity of the antenna.

All cases of this order, in which one organ through some disturbance assumes the character of another organ, are known as 
homecotic variations, and homoesis of some sort is a frequent accompaniment of meristic variation in longitudinal series. The best instance of this is found in the petals of flowers which are recognized by botanists as modified leaves, and instances are not rare in which the specimen plainly shows the various transition states from leaf to sepal, sepal to petal, and petal to stamen.

This tendency of one part to assume the character and discharge the functions of a neighboring part is an important phase of variation, throwing much light upon the general subject of development.

IVith this introduction the student is prepared for the study of meristic variation somewhat in detail, in which he will find that while each species is built upon its own somewhat peculiar and definite pattern, yet this pattern is subject to many and profound alterations, and the organism is frequently able to exist upon another and much-distorted plan, all of which goes far toward enlightening the student as to the variations that may be expected in the organic world. Studies in meristic variation are useful to the student of thremmatology, not so much for their own sake as for the light they shed upon the nature and manner of variation.

Examples of meristic variation. Examples of meristic variation are to be found at every hand. In the doubling of flowers and the stooling of grain, in increased or reduced numbers of fingers and toes, in the four-leaved clover and the branching habit of many plants, - everywhere are seen alterations in the customary plan on which nature does its work.

Fortumately an extended and valuable collection of meristic variations, mostly among animals, has been made by Bateson. ${ }^{1}$ He lists his data under 886 headings, each recording from one to several authentic cases.

Equally complete data covering plants have not been collected, though it is among plants that meristic variation is most common. Indeed, it is so common and so evident that formal collection is hardly necessary. The student is therefore referred to plant life out of doors and to Bateson's collection for a fuller study of this important subject, a bare outline of which, as a guicle, being all that is attempted here.

1 Bateson, Materials for the Study of Variation [Macmillan \& Co., I894]. 


\section{SECTION II - MERISTIC VARIATION IN IINEAR SERIES}

Vertebræ. Among fishes and snakes variation in the number of vertebra may be very great. In mammals it is smaller but yet distinct, as in the following examples, instances of which, according to Bateson, could be multiplied indefinitely.

Erinaceous Europeus (The Hedgehog) ${ }^{1}$

\begin{tabular}{|c|c|c|c|c|c|c|}
\hline No. & Cervical & Dorsal & Lumbar & Sacral & Coccygeal & Total \\
\hline I & 7 & 14 & 6 & 4 & II & 42 \\
\hline 2 & 7 & I 5 & 6 & 3 & $10+$ & \\
\hline 3 & 7 & 16 & 6 & 3 & $9+$ & \\
\hline 4 & 7 & 15 & 6 & 4 & 12 & 44 \\
\hline 5 & 7 & I 5 & 6 & 4 & II & 43 \\
\hline 6 & 7 & 14 & 6 & 3 & $9+$ & \\
\hline 7 & 7 & 15 & 6 & 3 & II or 12 & \\
\hline 8 & 7 & I 5 & 6 & 3 & I3 & 44 \\
\hline 9 & 7 & 15 & 6 & 3 & 12 or 13 & \\
\hline
\end{tabular}

It will be noticed that $\mathbf{I}$ and 5 differ only in the dorsal region, which fact, however, affects the total, but that 4 and $S$ differ both in the sacral and the coccygeal without affecting the total.

Commenting on the phenomena of an additional lumbar or sacral vertebra, Bateson says:

... There is a strong suggestion that (in cases of this kind) the number of vertebre has been increased by simple addition of a new segment behind, after the fashion of a growing worm; the variation of vertebre thus seems a simple thing. But there is evidence of other kinds, which plainly shows this view of the matter to be quite inadequate.

What this evidence is he proceeds to show by succeeding examples, a few of which are reproduced here:

In a skeleton of Python tigris ${ }^{2}$ (No. 602, Muscum of the Collegc of Surgcons) the vertebre are normal up to the 147 th inclusive. The I 48 th and I 49th are, however, abnormally short from front to back, suggesting arrested development with imperfect separation, although each vertebra bears a normal rib on either side.

1 Bateson, Materials, etc., p. I03.

2 Ibid. pp. 103-105. 
Passing backivard in the same specimen, the 166 th vertebra is seen to be normal on the left but double and bearing two ribs on the right, thus greatly crowding the ribs on that side. The i 85 th vertebra is reported in the same condition, both being doubled on the right side and single on the left (see Fig. 3).

Following, Bateson ${ }^{1}$ gives two examples of the reverse condition, namely with duplicity on the left side, and another with

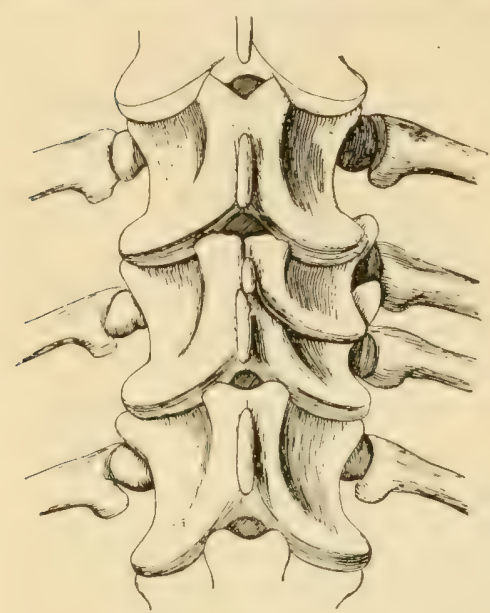

FIG. 3. Meristic variation in vertebrx: double on right side. - After Bateson duplicity on the right, showing clearly that meristic variation in one sicle of a bilateral symmetry may or may not involve the other side.

Ribs. Variation in the dorsal region necessarily involves the ribs. Aside from this, all evidence goes to show that partial division of the ribs is much more common than is variation in the number of vertebra. In man, for example, the cartilage is frequently divided for a considerable distance ( $\mathbf{I} .5$ in.) back from the sternum, often involving a real bifurcation of the rib itself.

Homœotic variation in vertebræ and ribs. ${ }^{2}$ These may be outlined as follows (all in man, except as noted) :

I. Cerical rescmbling dorsal: backacard homaosis. The distinguishing character of dorsal vertebrae is the bearing of ribs, but this character is often assumed by neighboring cervical, being common on the seventh and not unknown on the sixth. Of fifty-seven cases examined by Struthers, forty-two showed ribs on both sides and fifteen on one side only, showing a tendency to preservation of symmetry. The completeness of development ranges all the way from the merest rudiments (rare) to a perfect rib connected by cartilage to the sternum (also rare), the commonest form ending free or being joined by cartilage to the first true rib. 
2. Dorsal resembling cervical: forward homoosis. Not so common as above, but Struthers ${ }^{1}$ describes a specimen in which the first pair of ribs is defective. On the left side the rib extends but two fifths of the way around, where it articulates with a process on the second rib. On the right side it joins the second rib about one inch beyond the tubercle. As seven normal cervical vertebra are present in this specimen, it is to be regarded as a modified dersal rather than an extra cervical assuming the characters of the dorsal, as in the preceding cases.

3. Dorsal resulbling lumbar. Frequently the twelfth rib in man is rudimentary, in which case the last dorsal vertebra assumes the form and general appearance of a lumbar.

4. Lumbar rescmbling dorsal. Cases of a thirteenth rib are not unknown but are more rare than the reduction of the twelfth.

5. Homaosis betwecn lumbar, sacral, and cocygeal. The last lumbar may unite on one or both sicles with the sacral, in which case the lumbar develops processes to assist in the support of the ilium. On the contrary, the first sacral may remain detached, thus becoming practically a lumbar. Similar relations obtain between the sacral and the coccygeal.

A careful study of this whole subject develops the following facts :

I. That an increase in the number of parts in one region may or may not affect the total number in the series.

2. That consequently a change in number in one region may or may not be accompanied by changes in other regions of the same series; that is, changes in the dorsal do not imply changes in either the cervical or the lumbar.

3. That homoesis in vertebre and ribs is confined to members contiguous ; that is, if a cervical resemble a dorsal, it will be that cervical lying next to the dorsal series.

4. That the tendency is for an extra member to resemble somewhat the members of the next region; that is, an extra dorsal is likely to resemble a lumbar or a cervical, if not to entirely replace it, suggesting that it arose at the $c n d$, and not in the middle, of the dorsal series.

1 Bateson, Materials, etc., p. Iog. 
5. That forward homoessis in one region is not necessarily attended by forvard homocosis in other regions of the same series.

6. That in greneral (especially in man, where this has been most studied) forward homeosis is attended by a total increase of the series, and backward homœesis by a decrease.

7. That an abnormality on one side may or may not be attended by a like abnormality on the other, though the tendency is strongly to the preservation of bilateral symmetry.

8 . That when one part resembles another it is the member lying contiguous; that is, a dorsal vertebra will resemble a cervical or a lumbar, not a sacral, and lying between the stamen and the leaf are the petal and sepal and all intermediate gradations, either present or obliterated.

Meristic variation in spinal nerves. Branches from the spinal cord emerge between the vertebre, so that in general the system of spinal nerves is determined by the rertebrac. Aside from this, however, the emergence of the branches varies greatly both in number and in conformation, even when the vertebra are normal.

Fürbringer's ${ }^{1}$ studies in birds show that the minimum number of spinal nerves forming the brachial plexus (supplying the wings) is three, but in some species it rises as high as six. Moreover, in some instances the number varied from four to five within a single specics, and in one (the pigeon) the variation was from four to six. As might be suspected, the two sides are often differently supplied. For example, in one specimen (goose) "the plexus was formed on the right side by nerves XvI, xVII, XVIII, and XIx, while on the left side it received a strand from the xxth nerve in addition to these."

Furbringer's tables show that in some specimens of the goose the wings were supplied by the nerves $x \mathrm{~V}$ to $\mathrm{xIx}$, while in others they were supplied by the xvirth to the xxth. In the dove the brachial plexus was formed by the $x$ th branches of the spinal nerve in some specimens, by the xirth to the xvth in others, and in one case by the xith to the xIVth as an intermediary.

1 Bateson, Materials, etc., pp. I 29-1 35 . 
Herringham ${ }^{1}$ dissected to their origin the nerres forming the brachial plexus in fifty-five human subjects (thirty-two fetal and twenty-three adult). Quoting from his work, Bateson says:

The origin of the ulnar nerve was traced in thirty-two cases, fourteen being adults. It (the ulnar nerve) was found to arise in four different ways. Most commonly it arises from the vinth and $1 \mathrm{xth}$; this occurred in twentythree cases. With the virith and ixth is sometimes combined a strand from the vith, as shown in five cases (four fetal, one adult). In three fetal cases it arose from the vinth only, and in one fetal and one adult case from the virth and vinth. . . In several cases the branch from the vinth was much larger than that from the ixth, but the reverse was never met with.

Similar conditions were found elsewhere with man, the gorilia, baboon, and chimpanzee, and the following principle was set forth: "Any given fiber mary alter its position relative to the vertebral column, but will maintain its position relative to other fibcrs."

\section{Homøosis in insects and other small} animals. The replacement of one part by another, while common among plants (modified leaves and stems), is comparatively rare in animal life. It is, however, by no means unknown, and some striking examples are quoted from Bateson to show the remarkable manner in which a perfect part may arise in a most unusual place, among which are the following $:^{2}$

I. Specimens of sawfly (Cimbex axillaris) in which the left antenna ended in "a well-formed

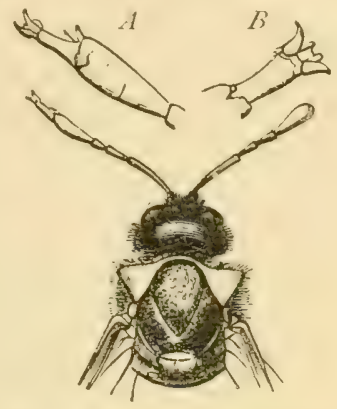

Fis. 4. IInmontic variation in sawfly: right antemna normal; left antenna bearing a foot. $A$ and $B$, enlarged.-After Bateson foot, having a pair of normal claws and the plantula between them" (Fig. 4). Right antenna normal. ${ }^{3}$

2. A male bumblebee (Bombus variabilis) taken in Munich showed the left antenna "partially developed as a foot," bearing "a pair of regularly formed claws like the claws of the foot."

3. A male specimen of $Z$ ygana filitendule "possessing a supernumerary wing arising in such a position as to suggest that it replaced a legr "(Fig. 5). The extra wing was on the left side and projected from the underside of the body after the exact fashion of the leg, which was absent. The specimen

1 Bateson, Materials, etc., pp. $135^{-1} 3^{8}$.

2 Ibid. p. 147

${ }^{3}$ Ibid, pp. 146-155. Professor Bateson vouches for the genuineness of this specimen, which he himself carefully examined, although it belonged to Dr. Kraatz. 
belongs to Mr. Richardson, and was examined by Professor Bateson as closely as was possible without removing- the hairs, to which the owner objected. It is well known that supernumerary wings may arise with the normal number of legs. In this case the closest examination failed to reveal

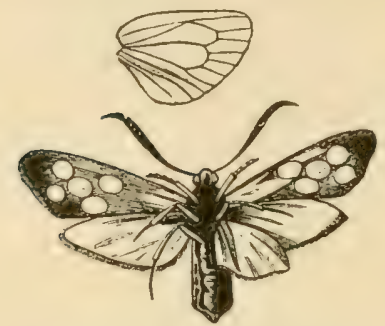

FIG. 5. Supernumerary wing on left side.-After Bateson even a rudimentary leg, and there was certainly "no empty socket or other suggestion that the rest of the leg had been lost."

4. Specimen of Palinurus penicillatus with an "antenna-like flagellum growing up from the surface of the (left) eye."

5. The female crayfish has normally a pair of oviducal openings on the bases of the antepenultimate pair of walking legs. This specimen possessed in addition a pair also on the penultimate, showing irregular segmentation.

6. Another specimen, also of the crayfish, possesses an extra pair of oviducal openings, as in the last, except that they were placed on the last pair of legs, skipping the penultimate. It is noteworthy that this is the normal position for the sexual organs of the male, except that the openings were placed in their own proper position on the leg and not "at the posterior surface of the joint as the male openings are."

7. Bateson himself examined 5 \& 6 female crayfish for abnormalities of oviducal openings. Of this number he found $56_{3}$ were normal and 23 abnormal, as follows:
I. Extra oviducal opening on left penultimate leg . . . . 7
2. Extra oviducal opening on right penultimate leg . . . . 10
3. Extra oviducal opening on both penultimate legs ... . I
4. Extra oviducal opening on both penultimate and last legs . I
5. Single oviducal opening on left side only . . . . . . . 3
6. Single oviducal opening on right side only . . . . . . $\frac{1}{23}$
Total abnormal specimens

Bateson reports but one abnormal specimen out of $7 \mathrm{I} 4$ males examined by him, and this abnormality consisted in the absence of a generative opening on the right side.

8. Among earthworms will be found many cases of imperfect segmentation, showing more rings on one side than upon the other, often suggesting a spiral rather than a series of rings. Great irregularity is also found in the position of generative openings, as to whether paired or single, ${ }^{1}$ although the male parts are always posterior to the female, whatever the number of the ring on which either is borne.

\section{Cervical fistulæ and auricular appendages in mammals.} Cervical fistulic are openings in the neck, occurring singly or in pairs and located anywhere from the median line backward as

1 Bateson, Materials, etc., pp. $15^{6-166 .}$

2 Ibid. pp. I $74^{-1 S o,}$ 
far as the angle of the jaw. The opening is sometimes slight, but often it extends completely to the pharynx. In the latter case it is possible to pass an instrument the size of a small quill, provided the opening is comparatively straight, otherwise its completeness or incompleteness may be ascertained by the injection of a liquid.

Bateson quotes Fisher ${ }^{1}$ as describing sixty-five persons with seventy-nine fistulae. Fourteen of these were bilateral (occurring

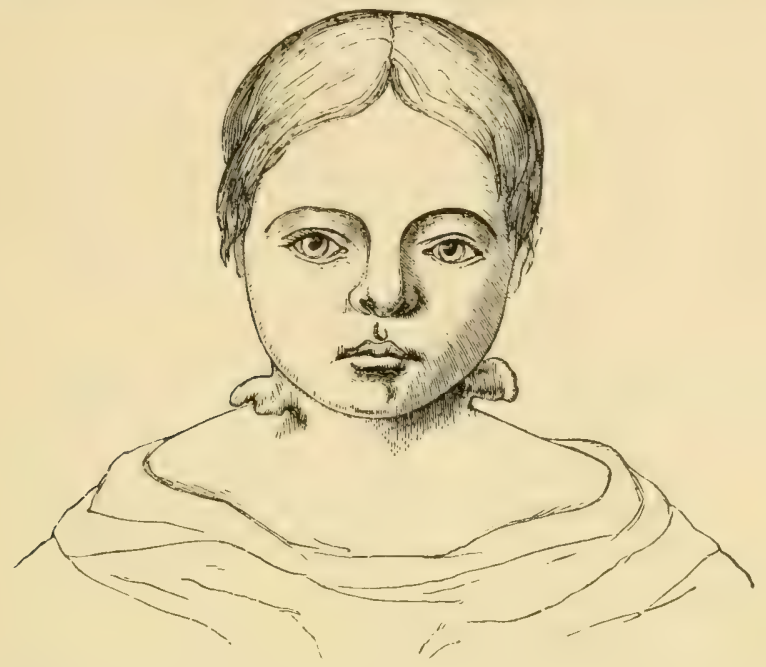

FIG. 6. Child with supernumerary auricle on each side of the neck. - After Bateson, from Birkett

on both sides), and fifty-one were unilateral, of which thirtythree were on the right side. He adds, "There was evidence of heredity in twenty-one cases."

Auricular appendages, often called supernumerary auricles, are not at all uncommon. They are non-functional growths occurring in the neighborhood of the ear but below it, and are generally accompanied by some deformity of that organ. They consist of little flaps of skin or, more commonly, of cartilaginous growths identical in texture with that of the normal external ear.

1 Bateson, Materials, etc., p. I75. Obvious errors in figures prevent further quotations that would otherwise be of interest, 
One of the most remarkable cases ever described is that of an infant brought to Guy's hospital in $185 \mathrm{I} .^{1}$ Another was of a child having a well-cleveloped supernumerary auricle on each sicle of the neck (see Fig. 6). These appendages were easily remored and proved to be entirely cutaneous, though each was served by a small artery.

Whether cervical fistula are to be regarded as remains of unclosed gill slits, or whether they are to be regarded as repetitions of the external ear, in any event their presence shows a pronounced tendency to repeat certain characteristic structures in this particular region of the body.

Growths of this character are by no means confined to man. Cervical auricles (the so-called "wattles") are common in sheep, especially merinos. They are well known in goats and are exceedingly common in many strains of unimproved swine. Strange as it may seem, these repetitions of the ear appendages are unknown in either the horse or the ox.

Meristic repetition in mammæ. ${ }^{2}$ One of the chief distinguishing features of mammals is milk secretion. Speaking generally, this occurs at some point or points on either side of the ventral surface of the body on lines rumning from the armpit to the groin. In swine and in dogs it is distributed throughout the entire extent of these mammary lines. In cattle, horses, goats, sheep, etc., it is confined to the rear extremity of the line, and in the elephant it is as decidedly forward, the udder being located at the armpit. In the human being the point of normal activity is relatively further back (down) than in the elephant, but yet above the middle.

This latter point is established by the fact that supernumerary nipples are found both above and below the normal. The fact that no less than three supernumeraries have been found above inclicates that the normal mammae are perhaps fourth in a full series. It is to be noted in this connection, however, that in most cases supernumeraries are situated below rather than above the normal. These structures vary all the way from mere nipples resembling warts and entirely unaccompanied by mammary tissue 
up to well-formed organs fully functional. Curiously enough supernumerary mamma are more common in men than in women.

IBateson ${ }^{1}$ quotes bruce as having found in 23 I I females fourteen cases (0.605 per cent), and in I 645 males forty-seven cases (2.857 per cent). In another series 3 I 5 subjects were examined, showing twenty-four cases (7.6 per cent), nineteen being male and five female. Bardeleben is also quoted as having examined 2736 recruits (all males, of course). In this series " 637 cases $(23.3$ per cent) were seen, 2 I9 being on the right sicle, 248 on the left, and 170 on both sides."

The largest number of supernumerary mammae ever recorded was in a subject described by Neugebauer." This patient had five pairs of nipples, of which the fourth, numbered from above, was the normal. When the child was being suckled milk oozed from each of the uppermost pair, but all other supernumeraries yielded milk only with pressure.

Extra teats in cows are too common to need mention except to call attention to their excessive number. The cow Rose, famous for her record at the Illinois Station," had in all eight mammx, six of which were fairly well dereloped, though only four were functional. ${ }^{4}$ It is noticeable that supernumeraries are nearly always posterior to the normal or else constitute a cloubling of one of the normals. Every milker knows by sad experience that these supernumeraries are not only common but frequently functional.

A close study of this subject shows that repetition of these parts may be by pairs or singly; that the repeated parts may be on the same or on different levels; that they may be out of line, being in some cases very near the median, and that the normal nipple may be doubled. From the latter fact we further establish the point that meristic variation may occur in two ways, - either by addition to the series or by division of a normal number. We shall find the same in teeth.

1 Bateson, Materials, etc., pp. $1 S_{2}-1 S_{3}$.

2 Ibid. p. 183.

${ }^{3}$ See Bulletin No. 66.

4 These supernumeraries were not symmetrically placed. On the right side the two extra teats were placed behind the two functional, as is commonly the case ; but on the left side only one supernumerary was so placed, while the other was between the two functional teats. 
Meristic variation in teeth. As Bateson remarks, "Tecth arise by special clifferentiation at points along the jaw, as mamma arise by special clifferentiation at points along the mammary line," and we shall see that with teeth as with mammae these points of special differentiation may frequently lie outside the normal region, that they are subject to increase or decrease in number, and that the increase may be due either to the addition of a member to the series, to the interpolation of a member, or to the division of a normal member.

Before considering special cases it is well to note that the similarity between the right and left jaws is that of ordinary bilateral symmetry, but that there is also a kind of symmetry, not very close but still marked, between the dentition of the upper and that of the lower jaw. It should be further noted that in many animals, as in the shark, alligator, etc., the dentition constitutes a series in which the separate teeth differ from one another mainly in size. But mammals for the most part are heterodont; that is, the series is broken up into groups which differ among themselves, though the members of the separate groups resemble one another closely. Thus the incisors are quite different from the canines, which in turn differ from the premolars and the molars. The different incisors, however, are very much alike, and the same is true of the canines and the various molars and premolars. Meristic variation in a heterogenous series like this is manifestly much more complicated than in a simple series like the mamma or the ribs. With this introduction attention will be called to a few special examples quoted from the 237 cases that have been collected by Bateson. ${ }^{1}$

I. One hundred and fifty-two adult skulls of anthropoid apes showed twelve cases of extra teeth. One was an incisor, one was anomalous, and the others were molars. This is nearly 8 per cent abnormal, as against 425 Old World monkeys that showed but two cases of extra teeth, -less than one half of I per cent.

2. Adult orang, with an additional posterior molar on both sides above and on the left side below. No trace of extra molar on right side was discovered, "though there is almost as much room for it as on the left side." Extra molars perfect but slightly smaller than the normal.

1 Bateson, Materials, etc., pp. 195-273. 
3. Skull (orang) No. $20+3 a$, Oxford Museum, is normal except as to the

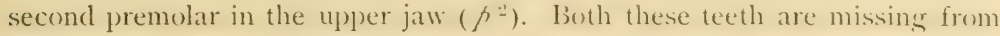
their proper place. There is plenty of space on the left side but somewhat less than the normal on the right side. The missing tooth of the right side is present in the skull, but instead of being in its proper place it stands up from the roof of the mouth within the arcade immediately in front of the right canine and almost exactly on the level of the second incisor, being in the premaxilla at some distance in front of the maxillary suture.

\section{Discussing this case, Bateson observes:}

That this tooth is actually the second premolar which has by some means been shifted into this position there can be no doubt whatever. It has the exact form of the second premolar and is of full size. It stands nearly vertically, but is a little inclined towards the outside. The canine is, by the growth of this tooth, slightly separated from the second incisor, and the first premolar is consequently pushed also somewhat further back. Hence it happens that the diastema space for the second premolar on the right side is not of full size. This should be understood, as it might otherwise be imagined that the contraction was due to a complementary increase in the size of the other teeth, of which there is no evidence.

The missing premolar on the left side was not visible, but "on the left side of the palate there was a very slight elevation at a point homologous and symmetrical with that at which the second premolar on the right side was placed. . . A small piece of bone was here cut away, with the result that a tooth of about the same size and formation as $f^{2}$ was found imberded in the bone." In this case, therefore, the upper premolars on both sides had "traveled away from their proper positions and taken up new and symmetrical positions in the palate, anterior to the canines."

As Bateson pertinently remarks (italics and parenthesis mine), "The facts of this case go to show that the germ of a tooth contains within itself all the clements necessary to its deaclopment in its oren true form [even in an abnormal position], provided of course that nutrition is unrestricted." This is a significant point of peculiar interest to students of thremmatology, not because of its bearing upon dentition but because of the light it affords upon the basis of variability and the ultimate units of variation.

4. Gorilla from the Congo, with a fifth incisor standing almost in the micldle of the lower jaw. It has the characteristic chisel shape of the incisor, but it is "turned half round so that the plane of its chisel stands obliquely." 
5. Dog with lower jaw and teeth normal, but with upper canines imperfectly divided. The division was more complete on the right side, forming practically two canines standing in line with the regular teeth.

6. I)og with first premolar in right sicle of upper jaw doubled, both teeth being normal in shape, the anterior somewhat the larger.

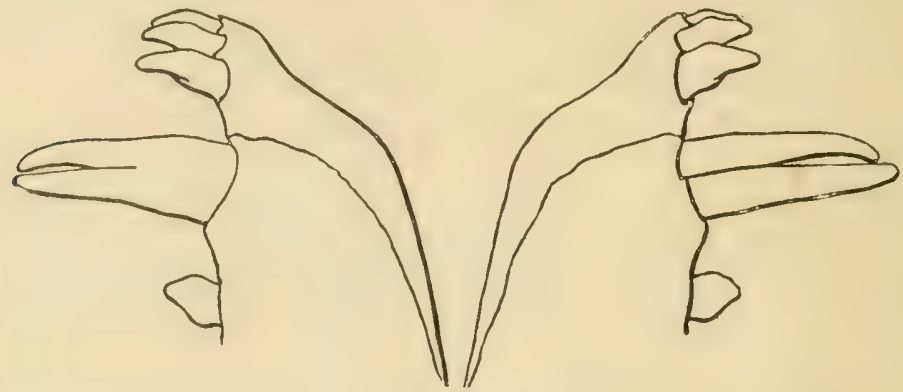

FIG. 7. Merism in teeth: canines partially divided. - After Bateson

7. Dog with an extra premolar on both sides above and below, the dentition formula being $p \frac{5-5}{5-5}$.

8. Sledge dog: "All teeth normal, except left upper $p^{2}$. This tooth normally has two roots. Here it is represented by two teeth, each having one root."

9. Absence of first premolar frequently quite common in Eskimo dogs, suggesting a breed peculiarity.

I0. Among domestic dogs supernumerary molars were found in twentyeight cases out of $3+5$ skulls examined, as follows, ${ }^{1}$ the normal dentition of the $\operatorname{dog}$ as to molars being two above and three below $\left(m \frac{2}{3}\right)$.

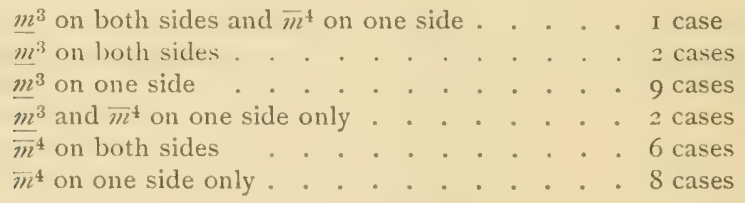

This strongly suggests the formula $m \stackrel{3}{4}$, which is that of Otocyon (Lalanules dog) and of the fossil Amphicyon, the supposed doglike progenitor of the bears. It calls to mind the further remarkable facts that Otocyon itself varies from $m \frac{3}{4}$ to $m+4$ and that it is the only mammal outside the marsupials

1 Bateson, Materials, etc., p. 220. 
that ever has four molars on both jaws, ${ }^{1}$ which goes far to inclicate a marsupial ancestry.

Remembering that the teeth are considered as one of the few most reliable bases for classification, the remarkable variation in their number, character, and position throws no little light on the manner in which variation behaves, which is the chief reason for their extended notice here.

Supernumerary eyes. The development of extra eyes seems to be confined to insects, which afford a number of excellent examples of the development of normal tissue in abnormal situations.

Bateson's Nos. 4I9 to 42 I are all cases of the development of a third eye in Coleoptera. In every case these extra eyes are quite distinct from the normal. In No. 4 I 9 the supernumerary was small and lay abutting against but distinct from the right eye. Its color was brownish yellow, while the normal eye was black. In $\mathrm{No}$. 420 the extra eye was on the left side but quite independent of the normal eyes, which were exactly alike. In No. 42 I the extra eye was on the left sicle of the head, which was rather less developed than the right. This eye is borne upon an irregular chitinous loop, having a diameter of about $2.5 \mathrm{~mm}$. This loop is attached to the substance of the head before and behind, and these two attachments are distant from each other about I mm. The diameter of the eye is about $2.5 \mathrm{~mm}$., thus occupying the full surface of the loop, and its faceting is said to be "not quite regular, and finer and slighter than that of the normal eye." It is thus a very good attempt at a functional third eye.

Supernumerary wings in insects. Bateson ${ }^{2}$ reports and describes fifteen cases of extra wings among insects, - sometimes on one side, sometimes on the other, but generally if not always smaller than the normal; sometimes plainly identified with the fore wing, more frequently with the posterior; occasionally normal in coloring and scaling, but as a rule abnormal. In one instance it took the form of a large upright scale and in another of a winglike appendage to the left anterior wing.

1 Lydekker, Library of Natural History, p. 580.

2 Bateson, Materials, etc., pp. $28 \mathrm{r}-285$. 
Meristic variation in horns. ${ }^{1}$ These appendages afford good material for studies in variation. They sometimes consist of horny matter (cattle, sheep, rhinoceros, etc.) and sometimes of true bone, as in the leer. They sometimes persist through life (cattle, sheep, goats, etc.) and sometimes are periodically shed, as with the antlers of the stag. They sometimes, as in cattle, have a bony case, which is a true outgrowth of the skull, but often, as in the rhinoceros, they have no connection whatever with the bone beneath. Again, the antlers, which are bony, separate with a clean scar from the bone of the skull, as a leaf stem parts from its twig.

The meristic variations of horns are no less remarkable than their substantive variations just mentioned. They are for the most part symmetrically placed in pairs on either sicle of the skull just above the eyes, though the horn of the rhinoceros is borne upon the nose and therefore upon the median line.

Variation in number occurs either symmetrically or asymmetrically. If the rhinoceros has an extra horn it will be just above and on the median line with the normal. Sheep may have an extra pair just external to (behind) the normal, ${ }^{2}$ or there may be three on one side and two on the other. In the latter case the third horn will be a little one lying between the normal and the more ordinary extra horn. In still other cases, according to Bateson, a double core will be found incased in a kind of "double-barreled" single horn.

Among cattle no increase in the normal number of horns is known to the writer, but their entire absence is common. Indeed, the readiness with which the polled character appears is astonishing, ${ }^{3}$ particularly as it is associated with a peculiar prominence (the poll) lying between and often slightly below the normal base of the horns. In cattle, meristic variation in horns seems to be associated neither with divided horns or extra prongs.

1 Bateson, Materials, etc., pp. $28_{5}-2 S_{7}$.

2 Four-horned breeds are not unknown. Bateson, Materials, etc., p. 285 .

${ }^{3}$ It is a well-known fact that if either parent be polled the horns are almost certain to be absent in the offspring, and Storer, in his Wild White Cattle of Great Britain, says there is evidence that these park cattle have been several times alternately polled and horned since their inclosure in the parks. 
Besides sheep Bateson gives three specific cases of increase in the number of horns, as follows: (I) a family of goats in which the four-horned character was hereditary for " many generations"; (2) chamois with two "well-formed and symmetrical extra horns" ; (3) roebuck, two specimens of which are figured. Of these one has two horns on one

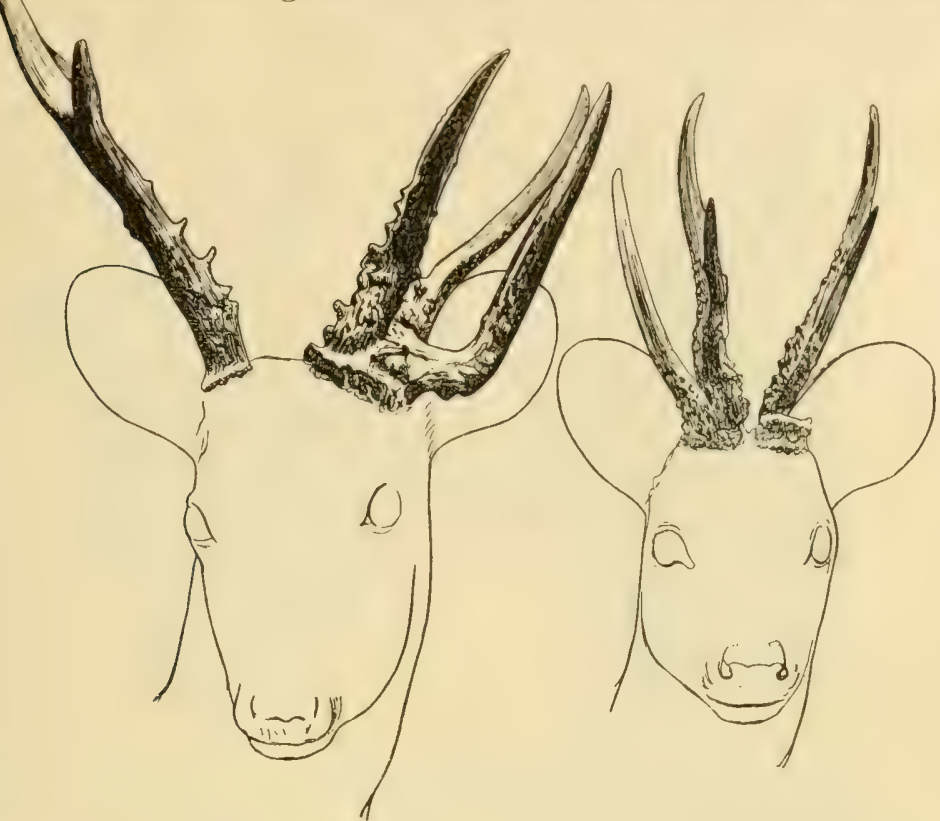

FIG. 8 . Abnormal horns in roebuck: all but one have undergone meristic variation. - After Bateson

side and three on the opposite side, while the other has three on one side, the other being normal, consisting of a single horn with one prong near the summit (see Fig. 8).

Meristic variation in digits. ${ }^{1}$ Variations in these parts are peculiarly complex. There may be an increase or a decrease not only in the total number but also in the parts or joints that compose the several members.

The best example covering both these points in the same individual is Bateson's No. $485 .^{2}$ In this case the right hand

1 Bateson, Materials, etc., pp. 3I I-4IO.

2 Ibid. p. 327. 
has the usual number of cligits, but the thumb has three instead of two phalanges, though its general shape is normal. In the left hand there is much confusion in the region of the thumb. There is an extra cligit, but its true character is not so evident. It is sharp, like a finger, but functions as a thumb. Internal to this is a thumblike supernumerary with a true nail, but from its position it is functionless (see Fig. 9).

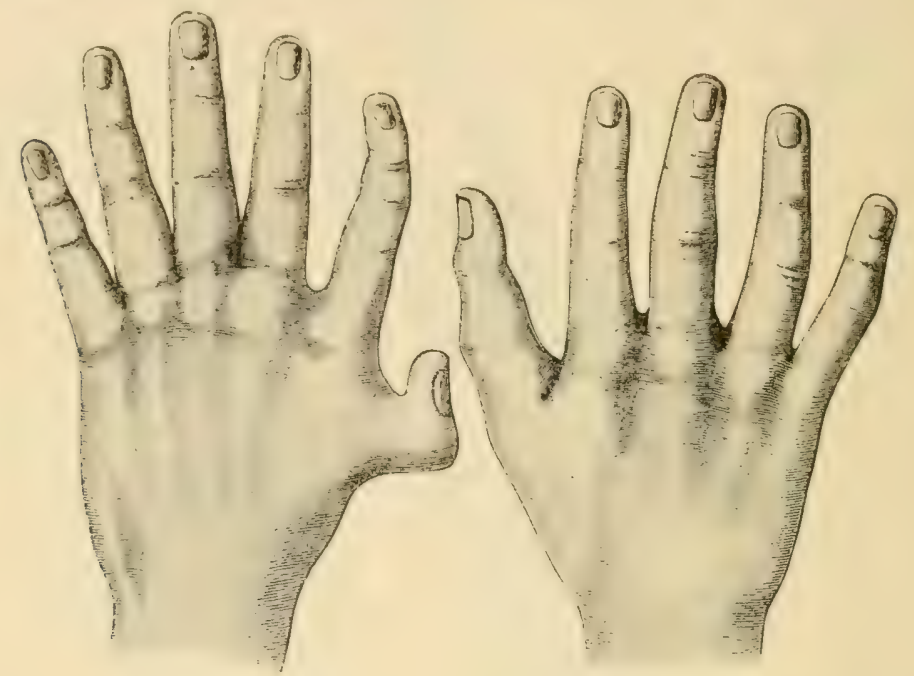

FIG. 9. Meristic variation in the hand: right and left hands of the same individual, showing on the left hand a duplication of the forefinger at the expense of the thumb, and on the right hand an extra joint in the thumb. - After Bateson

A fifth real finger, making six digits in all, is not uncommon. Its truc position, however, is by no means always easy to determine.

Speaking generally, extra digits may arise in three ways, either by addition to the series of an outside member next the thumb or the little finger, by the insertion of a member at some point within the series, or by the doubling of a member. Just which has taken place in any given case is not always easy to determine.

Reduction in the number of digits is common and takes place in three ways, - by the loss of an outside member (generally 
the thumb, when the radius is absent), by the suppression of a member within the series (ectrodactylism), or by the union of two or more members (syndactylism).

Syndactylism may occur in all degrees, from mere webbing to a real bony union, as in the case of solid-hoofed hogs. Fig. Io

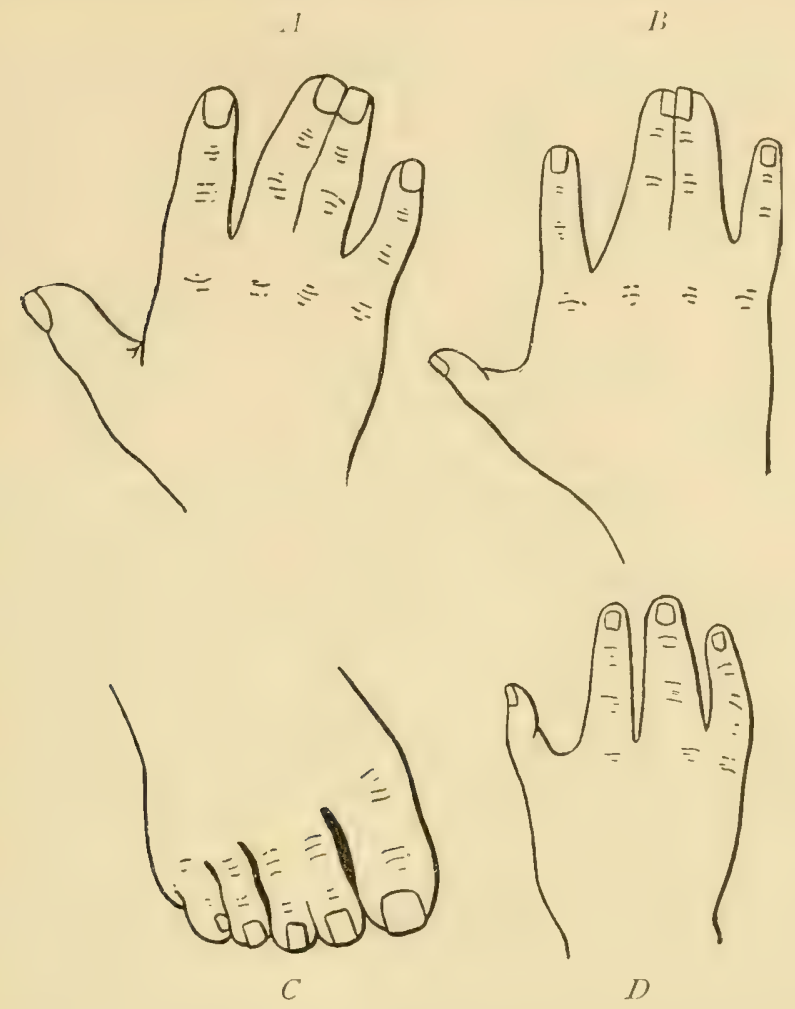

FIG. 10. Degrees of syndactylism in digits: the general shape of the member is preserved even when one digit is suppressed. - After Bateson

exhibits a case, $D$, in which the normal shape is preserved in the absence of a member (ectrodactylism), with nothing to suggest a union; that is to say, digits III and IV seem to be fully represented by a single digit, normal in character but replacing two members of the usual series. 
To fully appreciate the significance of this subject we need to remind ourselves of evolutionary history with respect to digits.

Man, for example, has normally five digits in all extremities. The same is true of the bat. The ox has only two toes and the horse but one, yet there are rudiments of others in both cases, strongly suggesting that at some remote period the number might have been greater.

All things considered, it looks as if, for some uncxplained and at present unexplainable reason, animal life in most of its higher forms had been originally constructed upon a plan of five as regarls the extremities. True, many, if not most species, have long since departed more or less widely from the original plan, and yet the numeral five is as distinctly characteristic of the digits in animals as it is of petals in the rose family among plants.

I How this number has been gradually reduced to a final form, sometimes of two, as in cattle, sometimes of one, as in horses, is a chapter in development that belongs to the ancient history of evolution. Moreover it is a chapter that, for obvious reasons, must be read backwards and reconstructed from its fragments.

It will assist in this reconstruction, and, what is of more consequence to the breeder, it will throw much light upon the manner of development and the unit of variability, if the student will consider the present condition and evident ancestry of a few characteristic species with respect to digits.

Bats have five digits in both wing and leg; though the thumb is modified into a strong claw.

Birds have three digits in the wing, namely, I, the thumb, which makes the so-called bastard wing; II and III, which make the true wing; IV and $\mathrm{r}$, missing. Radius and ulna are both present. In the leg the fibula is a mere splint, lying by the tibia.

There is but one metatarsus, but it is large and heary, ending in three pulley-like surfaces, over which play the tenclons that are attached to the three toes directed forward (II, III, and Iv). This plan suggests that the three middle metatarsals of the normal foot have here become united along the shank into one, but with three surfaces preserved for attachment of digits. Most birds have also a toe behind. This is regarded as digit I, but 
no bird has shown a trace of $\mathrm{V}$. Added together, this all means that birds have lost digit $v$ from the leg, if they ever possessed it, and $I V$ and $r$ from the wing, with $I$ in a fair way to ultimately disappear from both wing and leg, except when functional in the latter.

The cat has normally four digits (II to v) on each foot, all with three phalanges, and all furnished with claws. Besides this, I is represented on the fore foot by a pollex (thumb) of two phalanges, and a non-retractile claw, while on the hind foot the hallux (great toc) is rudimentary, consisting of a small bone articulating with the cunciform but bearing no claw. Of all animals, aside from man, the cat is the most subject to supernumerary cligits, especially on the fore foot. In the great majority of cases the doubling is in the region of digit 1 . Often the extra member is shaped, not like its neighbors, but rather as if belonging to the opposite foot, though sometimes it is indifferent. For exhaustive material on this subject, see Bateson, Materials for the Study of Variation, pp. 31 3-324.

Speaking generally, the dog tribe has five toes in front ${ }^{1}$ (digit I not touching the ground) and four behind (I absent).

The seal has five digits on all extremities, though the hand is modified into the flipper, and the foot is but slightly functional and evidently well on the road to extinction.

The whale generally has five digits in front incased in skin to form a flipper, though this number is often reduced to four, and in all cases II and III have more than the usual number of joints. The only traces of a hind limb are a few small bones beneath the sacral region and occasionally a part of a limb. ${ }^{2}$

In the manatee and the dugong the process has gone farther. Though these aquatic mammals have exceedingly serviceable flippers with five digits, yet the hind leg has been entirely lost. The vertebra in the sacral region are not united, and even the pelvis is represented only by a pair of splint bones, though some fossil forms show a rudimentary femur or thigh bone. ${ }^{3}$

1 Excepting the African hunting dog, which has four (Lydekker, Library of Natural History, p. 496).

2 This is similar to the loss of wings in the case of the New Zealand apteryx.

${ }^{3}$ Lydekker, Library of Natural History, p. II 56. 
The bear has five toes, all round, with an adclitional claw in digit II behind, which he uses for combing.

In mice and rats digit $\mathrm{I}$ in front is rudimentary. This case is unique because in most instances, where a difference is noticeable, the recluction in digits has proceeded farther behind than before.

Snakes, especially the large ones, occasionally show external vestiges of hind legs, and internally are frequently found traces not only of the pelvis but likewise of the thigh bone or femur. ${ }^{1}$ This shows clearly that the snake is a somewhat recent form developed from lizard-like ancestors with limbs, the hind pair of which must have been placed not far from the middle point of the much-elongated body. This view is strengthened by the fact that as a rule but one lung is developed, showing that the body is more slender than formerly.

Of all studies in digits the most interesting is that of the ungulates or hoofed animals. It is also the most profitable, because the majority of our valuable domesticated animals are included in this classification.

The interest arises from the fact that out of this stock have developed two very different forms of feet, viz. the two-toed (as cattle) and the one-toed (as horses), both evidently havingdescended from five-toed ancestors, each by a process of its own.

for example, cattle, sheep, deer, pigs, etc., have two toes (III and Iv) well developed into a serviceable foot, with two others (Ir and v) standing behind, not touching the ground (pigs), often rudimentary (deer), and frequently represented merely by splints (cattle). Occasionally all trace of these cligits is lost (giraffe).

On the other hand, the horse and his kind have but a single toe (III) ; but on either side is a well-developed splint, the remains of the second and fourth metacarpals in front and of the corresponding metatarsals behind. They are, however, without functional significance, being attached only above and extending downward with slender shafts and free ends not supplied with digits.

In this connection it is to be noted that the extinct protohippus of the United States and the hipparion of Europe, both decidedly 
horselike animals and regarded as ancestors of the modern horse, had each three toes that probably reached very near the ground.

Passing still further back (down) in geologic time and looking for a still more remote ancestor, we get beyond what can be called a true horse, as can the protohippus and the hipprarion. But yet there is among these long-extinct forms sufficient horselike character to suggest ancestry, as with the forest horse and desert horse of the Whitney find in Wyoming, forty inches high and three toes down. ${ }^{1}$ As we progress in this direction, however, the toes increase in number to four and even five, clearly inclicating that the modern horse has developed from a five-toed ancestor like the Eohippus, twelve to sixteen inches high and all toes down, also discovered in $\mathrm{V}_{\text {yoming whe }}$ it flourished, according to Osborn, some three million years ago or thereabouts. ${ }^{2}$

If we begin with the modern two-toed species and attempt to read their story backwards, we soon land among the same fouror five-toed primitive forms just mentioned, forcing the conclusion that the one-toed and the two-toed species of recent times have each descended from five-toed progenitors, - indeed, we may even believe from the same five-toed progenitors.

The manner of this descent is not difficult to trace by the comparison of modern species with similar extinct forms in successive downward (backward) geologic times. In almost the lowest tertiary rocks of both North America and Europe occur fossil remains of large ungulates. These "Coryphodons" were supplied with five-toed feet much like the elephant of to-day, that has survived by virtue of his teeth and in spite of his feet.

Ascending to the Miocene Tertiary, we find large ungulates still remaining, but cligit 1 is gone, while the metacarpal (or metatarsal) has become much lengthened and the third and fourth members greatly strengthened, not only in their own development

${ }^{1}$ Henry F. Osborn, Origin and History of the Horse.

2 The development of the horse from an ancestor only twelve to sixteen inches high and with five toes, all down, is the best instance of progressive evolution of which we have any knowledge. Doubtless the evolution of other species has been no less extended and fascinating, but of no other case do we possess so complete a history, thanks for which are in large measure due to the generosity of the late William C. Whitney and to the labors of Professor Henry F. Osborn. See also chap. $\mathrm{x}$, sect. ii. 
but also as regrards their articulation with the small bones above. This foot is now on the road to becoming inclifferently either a two-toed or a one-toed form, depending upon whether if and $\mathrm{v}$ reduce together or whether in takes the lead.

In this comnection certain intermediate or stranded forms are of no little interest. For example, the elephant has five toes in front, with four and sometimes three behind. The rhinoceros has three both before and behind, but the extinct form often had four. The tapir, which is also regarded as a remnant of ancient life preserved until the present, has generally three toes, though sometimes four and occasionally two. In any case, however, digit III is largest and symmetrical in itself, showing affinity with the line of descent that has developed the single-toed forms.

The camel has two toes, while the nearly related chevrotain has four, two being recluced. The hippopotamus has four short toes, all down, all hoofed and partly webbed, showing affinity with two-toed forms in that the symmetry is about a line drawn between digits III and IV. This is the same plan as that of the pig, except that in the latter the foot is more contracted, the toes being flattened on the inside and the second pair not touching the ground.

The kangaroo is anomalous, having five toes in front and in general four behind, of which $\mathrm{v}$ is much the largest; $\mathrm{V}$ is small, and II and III are much reduced and incased in a common integument.

With this brief survey of specific differences in respect to digits, certain individual deviations will have an added meaning. Bateson gives us the following: ${ }^{1}$

I. Horse having supernumerary toe on inside of right fore foot, presumably digit II. It articulated with an extra bone in the lower row of the carpus and was provided with a hoof, "convex both sides, resembling the hoof of an ass" (see Fig. I I).

2. Foal having two toes on each fore foot, otherwise normal. The carpus was in this case normal, but the extra toes were again borne on the inside and were provided with a small hoof.

3. Horse having a rudimentary digit on inside of left hind foot. This again results from a slight development of digit 11 , which is the most common cause of polydactylism among horses.

1 For variation in the feet of the horse, see Bateson, Materials, etc., pp. 360-372. 
More rare than this are: (I) the development of IV, making an extra toe on the outside; (2) development of II and IV, with III normal, making three toes in all, after the fashion of the protohippus; and (3) development of II and Ir with III aborted, resulting in an abnormal two-toed foot. All these forms are well known among horses.

4. Horse with supernumerary on outside of each fore foot, illustrating condition mentioned above (development of digit IV) (see Fig. 12).

5. Horse with both splint bones bearing digits on each foot, illustrating condition 2 (II and IV developed normal, making a three-toed horse).

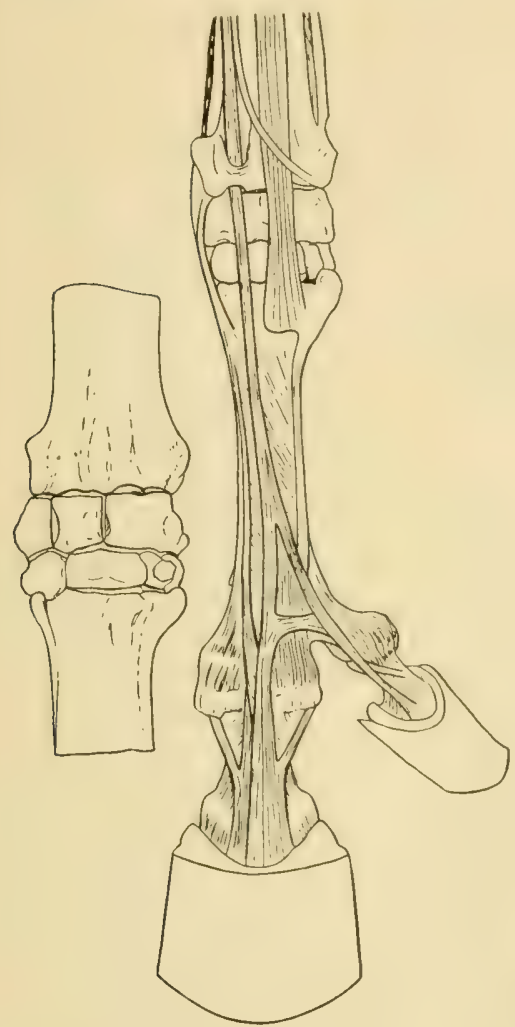

FIG. II. Right fore foot of horse (front view): as the hoof of the horse is regarded as digit III, this extra member is to be considered as digit II. - After Bateson

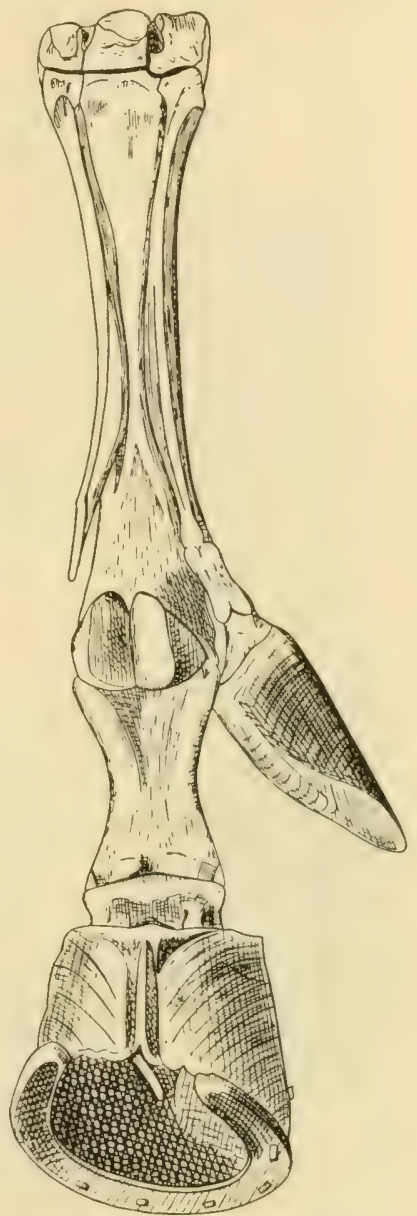

FIG. 12. Right fore foot of horse (rear view) : this extra toe is to be regarded not as digit II but as digit IV. - After Bateson 
6. Foal with right fore foot hearing two complete digits symmetrically developed, each bearing well-formed hoofs " that are flattened on the inner sides and curve toward each other like those of the artiodactyles (cattle, etc.). This illustrates condition (3) just mentioned (see Fig. I3).

Cattle, sheep, and pigs afford deviations no less interesting : 1

I. Calf having three digits on right fore foot, borne on a single

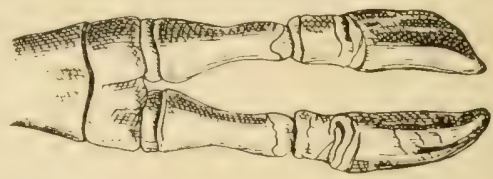

FIG. I3. Foot of horse: digit III suppressed, digits II and IV developed. After Bateson

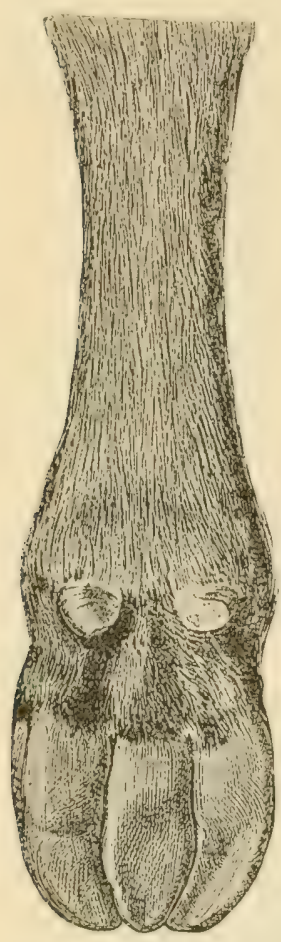

common bone after the fashion of the birds and fully symmetrical (see Fig. 14).

2. Heifer having three fully developed toes on each hind limb. In this case the supernumerary was clearly digit II.

3. Calf with "supernumerary toe placed between the digits of the right manus (fore foot). This toe

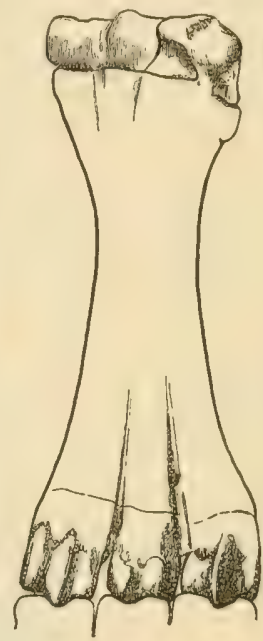

FIG. I4. Right fore foot of calf: three digits present, each supplied with both flexor and extensor tendons. - After Bateson

1 Bateson, Materials, etc., pp. 373-39o. had a hoof and seemed externally to be perfect, but on dissection it was found to contain no ossification, but was entirely composed of fibrous tissue and fat." 2

4. Cow, full-grown, right fore foot with four digits arranged in two groups of two each (see Fig. I 5).

This is clearly a case in which the increase is due, not to the reappearance of an ancient lost toe like II or $\mathrm{v}$, but rather to the doubling of the normal digits III and IV through ordinary meristic variation. ${ }^{3}$

5. Calf, left hind foot with five toes, "an inner group of two toes curving toward each other, and an outer group of three, of

2 Ibid. p. 377.

${ }^{3}$ No case is better than this to suggest caution to the student of evolution. When an extra toe appears among those forms whose ancestors were known to 
which the middle one was almost bilaterally symmetrical, while the hoofs of the other two turned toward it." 1

Bateson says of the pig that he knows of no case of polydac. tylism in the hind feet. All cases described are of the fore feet, and the extra toes are on the internal side of the digital series.

Syndactylism in cattle, sheep, and pigs. By this term is meant a real union of digits II and III into a single bone incased in a single hoof, as in the solid-hoofed hogs.

According to Rosenberg, as quoted by Bateson, ${ }^{2}$ in the normal sheep " the metacarpals II and $\mathrm{V}$ are distinct in the embryonic state, afterwards completely uniting (fusing) with III and IV." ' throws some light upon the whole question, as tending to explain not only certain cases of polydactylism but all cases of syndactylism." Again quoting Bateson:

I. A young ox having the two digits of the right fore foot completely united.

2. Calf: each foot having only one hoof, though all the bones were normal.

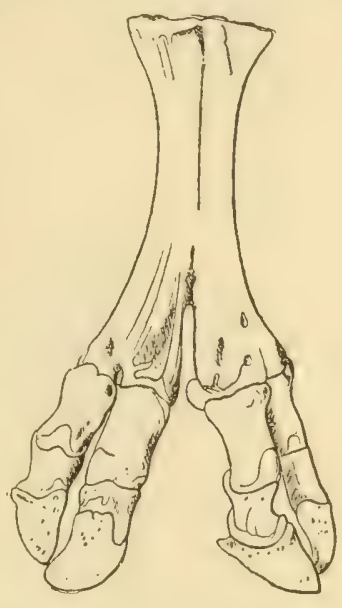

FIG. I 5. Said to be the right fore foot of cow : digits in two groups of two each. - After Bateson (from Delplanque)

3. Same as above, except that in the fore foot the normal digits (III and IV) were completely united, bearing a single hoof. The same condition was found behind, except that the hoof was more pointed.

4. A fore foot and a hind foot of the same individual (pig), in which the two chief digits were completely united, viz. represented by a single series of bones.

possess a greater number of digits, it is habitual with many to regard it as a case of atavism, - the reappearance of a long-lost character. But how is it in the case of man when a sixth or even seventh digit appears? This must be meristic variation and not atavism, because no six-toed species of any sort has ever been described or its existence suspected. Meristic variation, therefore, is not limited to lost characters or to numbers once normal, but may go far in excess of either. Here, then, is need for discrimination, for even the appearance of a character that has been once lost is not absolute evidence of atavism.

1 Bateson, Materials, etc. p. $3^{8}$ r.

${ }^{2}$ Ibid. p. $3^{8} 3$.

3 The former from failing ever to unite, the latter from a continuation of the fusing process to include III and IV.

4 That is, II unites with III, and v unites with IV during development. 
Other and similar cases are griven, though the latter is the only one described in which the syndactylism is complete in all four feet. It is, however, generally simultaneous in fore and hind feet. ${ }^{1}$

Absence of parts in a linear series. Men with hands but no arms, with feet but no legs, are not unknown. Whether the missing parts are really dropped out of the series, or whether

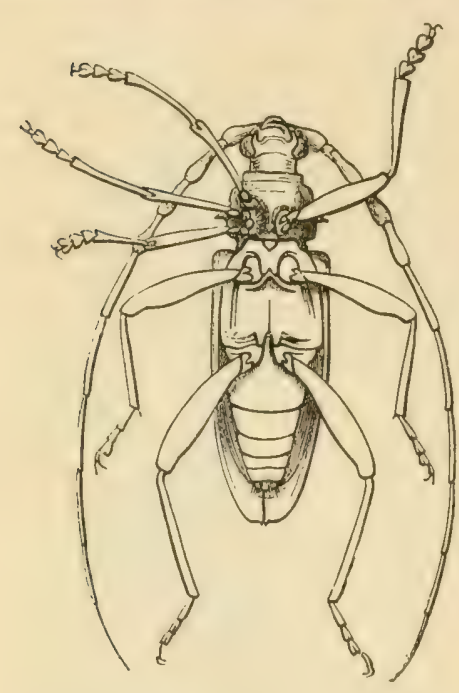

FIG. I6. Meristic repetition in leg: right leg of beetle repeated in triplicate. - After Bateson they were originally present but suffered abortion during embryonic development, being represented at maturity by rudimentary parts, is uncertain, though zoölogists would incline strongly to the latter view. The exact fact would have an important bearing upon the unit of variability, the nature of heredity, and the manner of differentiation.

Whatever the fact in this regard, variations of this order are manifestly rare as compared with the increase or decrease in strictly multiple parts. In other words, while considerable deviation in the number of similar members (as fingers) is common, it is exceedingly uncommon for an entire group (as the hand) to be omitted, - rarely from the end of the series (as the foot or hand) and still more rarely, if ever, from the middle of the series, as would be the case in a truly missing arm (humerus, radius, and ulna), but with the hand present, coming directly from the body.

Extra legs. The repetition of a member as complicated as a leg is extremely unusual but by no means unknown. The writer

1 Bateson gives many similar cases, each with some peculiarity of its own. Solid-hoofed pigs are seen so frequently and at points so widely removed both in time and space (mentioned by Aristotle and reported from many regions of the earth) (see Bateson, Materials, etc., p. 387 ) that this would seem to be a variation that has often arisen afresh. 
saw one specimen of a leglike appendage growing from the left sicle of the neck of a calf near the point of the shoulder. The legr was not more than two thirds the usual length, and was twisted and functionless, though it terminated with a hooflike growth. ${ }^{1}$

Extra legs are common in insects, sometimes throughout their entire length, sometimes doubling at the femur (see Fig. 16).

\section{SECTION III - MERISTIC VARIATION AND BILATERAL SYMMETRY}

Meristic variation among paired organs and those standing singly on the median line throws no little light upon the nature of bilateral symmetry and also incidentally upon the manner of variation.

Speaking generally, paired organs may double on either side separately or on both sicles (digits, leers, wings, etc.), or they may unite into a single organ with its axis on the median line (horseshoe kidney).

Most of the examples of meristic variation already given are of repetition in paired organs in bilateral symmetry. It remains to call attention to the opposite condition, - the fusion of a pair into a single organ standing on the median line :

I. A good example of this is that of a roebuck having the horns compounded for fully half their length into a single "bean " standing on the middle line ${ }^{2}$ (see Fig. 17).

2. A honeybee ${ }^{3}$ having the two compound eyes united into one at the top of the head with no groove or line of division between them. ${ }^{*}$

3. Posterior ends of kidney united (in man), forming a horseshoe kidney with three renal arteries on each side. This case is in sharp contrast to Bateson's No. 407 , with a single large kidney on the left and two smaller, one below the other, on the right.

1 This specimen is described from memory, as it was seen before these phenomena were matters of personal interest.

2 Bateson, Materials, etc., p. 460.

${ }^{3}$ Ibid. p. 46 r.

4 The compounding of eyes has already been mentioned. It apparently occurs only in insects, but is a good example of the development of highly differentiated tissue in abnormal situations, illustrating not only meristic variation but functional variation as well. 
Conversely, unpaired organs standing on the median line may be divided so as to form a pair of organs symmetrically placed.

It should be noted that in general a single organ standing on the median line, as the nose, is symmetrical both with reference

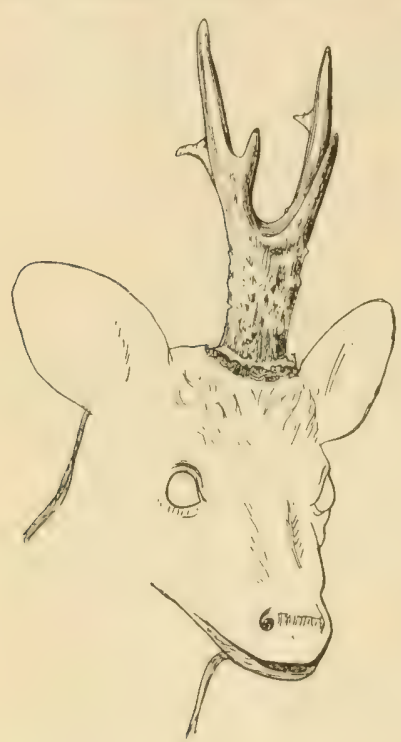

FIr. 17. Compounding of paired organs: the two homs of this roebuck are united into a single beam for a considerable distance, but afterwards they separate. - After Bateson saying that "The organs most often divided in man are the sternum, neural arches, uterus, penis, etc., and of these, specimens may be seen in any pathological museum. ${ }^{1}$ Organs more rarely divided are the tongue, epiglottis, uvula, and central neural canal." 2 These latter are in reality cases of axial cluplicity. ${ }^{3}$

1 Bateson, Materials, etc., pp. $45^{0-45^{S}}$.

2 Teratology is that branch of biology which treats of abnormalities, and it affords many cases of extreme variations. This study has been considered as curious rather than profitable, and yet, as such abnormalities are coming to be regarded as frequently due to a defective germ, it may yet prove that attention to cases of this order may furnish the key to the solution of questions involving the unit of variability.

to itself and to the median line, but that for the most part paired organs, though symmetrical with reference to the median line, are not themselies nccessarily symmetrical bodies (ears, arms, hands). In other cases of paired organs, however (eyes, kidneys), the members do have a kind of symmetry of their own.

Again, nothing is more common, especially among plants, than to find a single organ on the median line appearing as a paired organ in certain individuals or in nearly related species or varieties.

Bateson gives as examples of the last the posterior petal in Veronica, which in most related species appears as a pair of petals lying on either sicle of the middle line.

After giving numerous instances of division of median organs in fishes and in insects, he cites authority for 

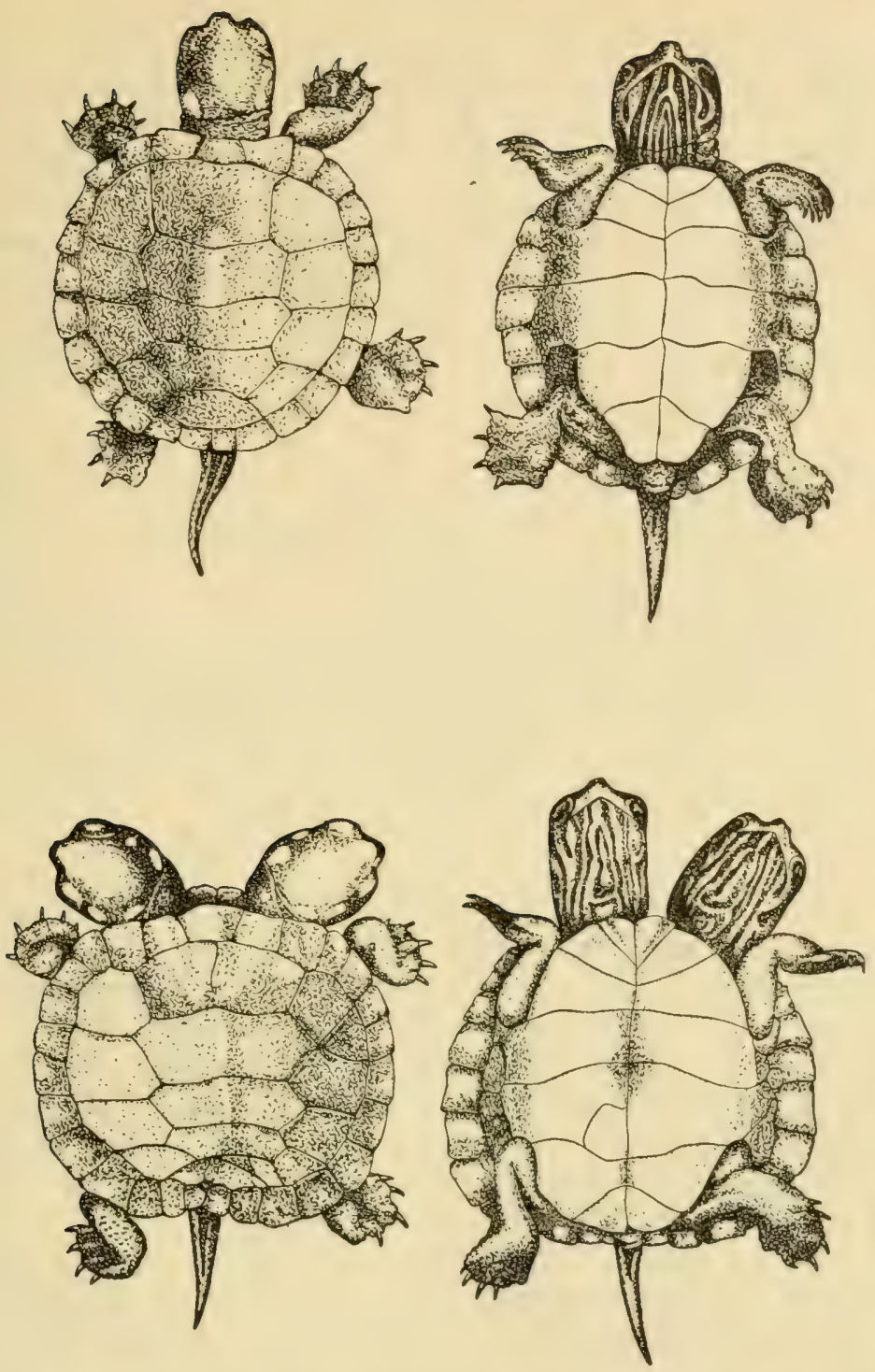

FIG. I8. Double-headed turtle compared with the usual specimens two to three days old. Note effect on shell plates. In this specimen the movements of the legs on opposite sides were not well coördinated. - After Bateson 
Fig. Is shows a case of double head in the turtle. Many similar instances have been described, but this is especially interesting because " the two heats seemed to act independently, and it is said there was no concerted action between the feet of the two sides." The same phenomena of double monsters are said to be frequently noted in fish-hatching establishments. Among snakes "some twenty cases are recorded of complete or partial duplicity, nearly always of the head. Several of these were animals of good size, and must have led an independent existence for some considerable time." 1

Similar cases of doubling are known in birds and even in mammals, but among these higher animals the practical difficulties in sustaining existence with extreme abnormality are very great, and they commonly do not long survive.

Between this division of a single organ lying on the median line and the doubling of so important a part as the head, there seems to be no clear line of clemarcation. This doubling may even further, as in the case of the Siamese twins, until the specimen is regarded as essentially two individuals united by some sort of attachment.

\section{SECTION IV - SYMMETRY IN VARIABLE PARTS}

Without a cloubt meristic variation in one organ of the body is likely, but not certain, to be accompanied by abnormality in another. For example, a variation among the cligits of the fore fort is likely to be associated with a similar variation behind, still more likely on the opposite sicle, but not positively with either.

Again, there is some suggestion of symmetry within the part itsclf in which the variation occurs. A good example of this is Bateson's No. 495 (Fig. I9).

This is a left hand, and the four extra fingers seem to represent not the thumb of that hand but the fingers of the opposite (right) hand, thus seeming to aim at a kind of secondary symmetry within the member. ${ }^{2}$

In the description of this case we are told that this double hand and arm were very muscular, so that it was not possible

1 Bateson, Materials, etc., p. 56 r.

2 Ibid. p. 335 . 
to decide in the living subject whether or not there was a doubling of the bones of the forearm. The eight fingers were in two groups of four earh, with a wide space between. The two "hands" were thus opposed to each other and could be folded upon each other. The power of independent action of these digits was limited, showing an insufficient supply of muscles. If the two index fingers, IV and $\mathrm{V}$ (really if and II), were extended, the other six could be flexed; either group of four could be flexed independently of the other, or the three fingers of either

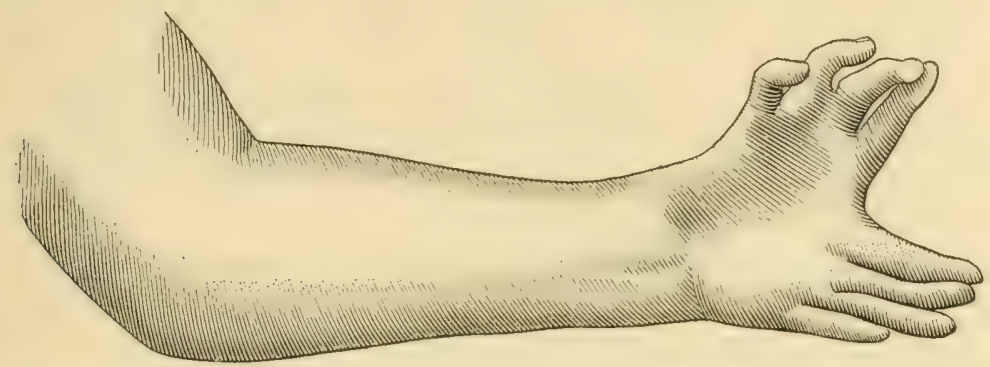

FIG. I9. Symmetry within the variable part. IIere it would seem that an attempt has been made to repeat the hand, or rather that an attempt at repetition of the thumb has resulted in a doubling of the hand.-After Bateson

hand could be flexed alone. The inder fingers alone could not be flexed while the other six were extended.

Bateson gives several other cases of "double hand" (Nos. 496-500), all giving the impression that the doubling is not simply of digits but of a hand as a arhole. I Iis No. 5 I 3 is the case of a double thumb, in which the two are symmetrically opposed to each other.

It is unfortunate for our purpose that so large a proportion of cases cited as examples of meristic variation should be among human subjects. This is only because it is here that the matter has been most studied. The idea has been advanced that domesticated species are more variable than wild ones, and man more variable than his simian congeners. The point is not well taken, because careful study shows the ape, the chimpanzee, the baboon, and the gorilla to present the same meristic deviations in respect to digits and the same abnormalities in dentition as are found 
in man. Again, while digital variation is exceedingly common in chickens, it is rare in birds generally, and is almost unknown in ducks and geese which have long been domesticated.

The fallacy above alluded to seems to have arisen from the fact that domesticated species are better known than wild ones, and that certain variations at least are more likely to be preserved. In any event they are more strongly impressed upon our attention. The truth seems to be that variability depends upon the nature of the part and the relative stability of the species in question, not upon its clomestication or its place in the scale of life.

IVe can therefore avail ourselves of any material bearing upon the general question wherever it may be found, hoping, however, for the early coming of the time when the variations within the particular field of thremmatology shall be better known and more accurately described.

Asymmetrical development in symmetrical parts. The case of the narwhal illustrates a fact in variation which, though seldom so apparent, is doubtless often potentially present, and if so, is certainly to be reckoned with by the breeder.

In the narwhal the canine tooth (in the male only) develops as a tusk, often attaining a length of seven or eight feet, or half the length of the body. The peculiarity is that normally only the lift tusk derelops, and in the few cases seen in which both are developed the right tusk is spirally twisted from lift to right, exactly like the left tusk, and not in the opposite direction, as we should expect. What is still more astonishing is that no case has iar becn describid in abich the right tusk was deacloped alone instead of the left. Either both are developed or the left one only, and in the former case they are essentially both left tusks.

\section{SLCTION V-MERISTIC VARIATION IN RADIAL SERIES}

Except in the lower forms, radial series are characteristic of plant rather than of animal life. In the branching of stems and the parts of flowers, members of radial series are everywhere about us. Their variations are always interesting (doubling of flowers) and often exceedingly valuable (stooling of grain). 
Botanists would say that what seem to us as radial series, with the members standing on the same horizontal level, are in most cases really shortened stems, bringing these parts into a relation which is apparent rather than actual, as woukl happen if we could telescope any long stem until the leaves, regularly disposed along its length, should come to occupy the same plane. ${ }^{1}$

In this view of the case the petals of flowers and the branching of stems, as in the stooling of grain, would be examples of linear series very much shortened rather than of radial series, according to the strictest definition of the term. For our purposes, however, this structural point may be waived, and all apparent cases of radial symmetry treated as actual.

Observations indicate and experiments show that members of such series may be increased in number almost indefinitely. All the members may be cloubled simultaneously (as five petals increased to ten), or any one member (original segment) may double or eren triple, or it may be entirely suppressed without reference to other members of the series.

The natural method of doubling seems to be for cell division to proceed one step beyond the normal, giving rise to two instead of one. If this occurs in all the members (petals), then the members will all be doublecl, as ten instead of five; if only in part, then only that portion will be affected, making six, seren, or even eight instead of five. Thus we have clover running all the way from the normal three up to as high as seven leaflets.

Manifestly if cell division proceeds two stages beyond the normal, each of the twin pair again divicling, it will result in

1 Leaves are arranged in regular order upon the stems of plants according to a system constituting the mathematical series, $\frac{1}{2}, \frac{2}{3}, \frac{3}{3}, \frac{5}{8}$, etc., in which the numerator indicates the number of circuits around the stem to reach a leaf directly over the one with which the count was started, and the clenominator the number of leaves that would be passed in such a circuit. It therefore represents the number of members in a whorl of a shortened stem of this character.

Corn, for example, belongs, with all other members of the grass family, to the fraction $\frac{1}{2},-$ built upon the plan of two. This number runs throughout the plant, and while the number of rows of corn on the cob may vary freely from eight to twenty-four, no case of an odd number of rowes has ever been reported. This fact tends to set some limits to even so wayward a thing as meristic variation, which seems never to have produced an ear of corn with an odd number of rows. This seems marvelous when we consider the havoc it works with digits and with even so complicated a structure as a head. 
quadrupling that member of the series. If, however, only one of this pair should clivide again, we should then have one plus two, or three, new parts in place of the one that was normal. Again, if all four should start and one abort, it would likewise result in three developed members instead of four that should have appeared.

All these various processes may take place, but whatever the final result, and whatever number ultimately develops, the method is that of doubling through cell disision, giving rise naturally to even numbers. Oeld numbers are explainable, however, by supposing that one of a pair continues the process one step farther than its twin, or else that one of the members fails to develop. In these ways an original member of a radial series may at any time (levelop into two, three, four, or more; and if all the members take part, a true doubling results.

Meristic variation and cell division. In the last analysis, therefore, variation in the number of members in a radial series is reducible to questions of cell division. Indeed, we may go further and note that all cases of meristic deviation arise in this manner ; that the preservation of the normal number of multiple parts depends upon successful cell division up to a certain (normal) point and its abrupt cessation at that point; and that all sorts of abnormalities may arise through excessive multiplication, through abortion, or through some other disturbance of the process of cell division.

This view of the case helps to explain why it is that meristic variations in radial series are among the easiest to explain of all variations which may present themselves to the breeder.

Considerations of this character make clear the futility and shortsightedness of appealing to reversion or atavism to explain what may be a mere incident in cell division, - an incident, moreover, that may never have occurred in phylogeny, may not be even common in ontogeny, and is therefore not to unduly impress the observer. ${ }^{1}$

1 These terms will be frequently used in the text. Phylogeny refers to the development of the species, ontogeny to the development of the individual. The latter is supposed in a general way to repeat the steps of the former, though with this view of the matter important gaps are of frequent occurrence. 


\section{SECTION VI - IMPORTANCE OF MERISTIC V.IRIATION}

Nothing is of more direct benefit to man than the stooling of grain, and the doubling of flowers is of prime importance to students of the beautiful. Digital variation, and indeed most of the examples among animals, are not only of no practical use but they constitute deformities that would at once be eliminated from the fields of any intelligent stockman.

Their study is, however, useful to the student in two ways: first, as showing him that freaks are by no means uncommon and therefore not to be specially prized; and second, to show the manner in which variation operates and the size of the unit involved, together with something of its relations to other and similar units in the same body. The careful student will not, therefore, waste his time in trying to establish a race of solidhoofed hogs the first time a specimen of the kind turns up in his yard, but he will utilize the information afforded in meristic variation generally to advance his understanding of the manner in which variation behaves and of the relations that obtain between the several parts of a highly differentiated body.

The purpose at this time is to secure a mass of characteristic facts on which future studies may be based. Most of the dangers of erroneous procedure in this field arise from a paucity of wellauthenticated instances and from restricted views of their real significance.

Summary. Meristic variation refers to deviations in the plan or pattern on which the organism is built. Its central thought is symmetry. Symmetry may be radial with the members identical, or it may be bilateral with opposite members, as optical images the one of the other. Distinctions of right and left arise from those of dorsal and ventral, and have reference to the relation of the individual to the outside world. Organs symmetrically placed may or may not have a symmetry of their own, but the tendency is for a part to establish some kind of symmetry within its own members.

When parts are multiplied they may be like the other members of the series in which they arise, or they may imitate those of neighboring series (homocosis). Repeated parts are especially 
subject to meristic variation. The general plan is preserved, but wide variation in the details is common, as in the nerve branches from the spinal column.

The part repeated may be simple, like a digit; or it may be an entire group, as a whole hand or an entire leg.

Meristic variation has its seat in cell division. It is of little utility in animals though highly useful in plants, but its phenomena are valuable for the insight they afford into the manner of variation, the general persistence of plan, and the unit of variability.

Exercises. I.et the student give ten separate examples of meristic variation not mentioned in the text and describe each fully, stating all that is involved of symmetry, homoeosis, etc.

\section{ADDITIONAL REFERENCES}

VARIatiox. A cock with no spurs on the leg, but with well-developed ones on either side of the comb. By E. S. Dexter. Science, VII, I36. Vegetable Teratology. By Maxwell T. Masters. 


\section{CHAPTER V}

\section{FUNCTIONAL VARIATION}

By functional variation is meant a deviation, not in form or in the number of parts lut in the functions that they perform. The living animal (or plant) not only is something; but it docs something, and plants and animals differ among themselves not only in what they are but in what they do.

Each portion of a highly differentiated organism has its own peculiar activity, which is essentially different from that of any other part of the same organism. These activities are not constant but variable; and inasmuch as many animals and not a few plants are kept not for their appearance but for what they can do, any deviations in their performance ability are of prime importance to the breeder, who is bent upon their increased efficiency and their permanent improvement for the service of man.

Now plants and animals are considered as high or low in their development according to the degree of differentiation or division of labor between their clifferent parts. In the protozoön the functions of life are few, and its relations to the environment are simple. Accordingly its activities are exerted and its obligations to life clischarged by the common mass of undifferentiated protoplasm, perhaps without so much as a stomach, reproduction being effected by a direct division of the whole mass.

In higher organisms (metazoans), however, life is more complex and the responsibilities of existence are heavier. These are met by specialized structures, such as the mouth to take food, the stomach and intestines to dissolve and prepare it for use, the liver to convert certain portions into specially usable form, the lungs to absorb air, the blood vessels to carry it and the digested food to all parts of the body, where each extracts what it needs and can use. 
Then there are organs, as the kidneys, whose function is to remove waste products that would otherwise accumulate and destroy the body. There are others, as the udder and various glands, whose function is to manufacture some particular product to be used either within or without the body. There is a system (the muscular) for moving the body as a whole or for the exercise of any of its parts, and a network of nerves forming a ready and rapid means of communication. There is a heart to drive the blood, and perhaps a bony skeleton to hold the complicated mass together.

Now the activities or functions of these various parts are by no means constant and invariable from day to day. In other words, there is probably as much deviation in function as in form, and for the purpose of the farmer it is even more important.

Evolution not a study in morphology only. The mistake is often made of defining evolution as exclusively a study in morphology. ${ }^{1}$ It means more than that. Living beings are something besides form, and their evolution something more than the development of their form; indeed, in their service to man both animals and plants are valued less for their structure than for their function, which is what they can do. And so it is that

1 "The problem of development is an acknowledged morphological problem." - C. B. Davenport, Experimental Morphology, l'art 1, Preface.

The conception here alluded to is not difficult to account for. The idea of evolution or development as opposed to the older assumption of special creation was first announced at the very close of the eighteenth century, but was not generally before the public until the appearance of the Origin of Species, after the middle of the nimeteenth century (IS59). At that time biologists were chiefly concerned in classification as based upon external structure or form. It is not strange, therefore, that the discussion should have first arisen, and the battles incident to a new, startling, and, in the popular mind, sacrilegious theory have been first fought out, in the field of morphology.

Gradually, however, biologists began to concern themselves more and more with internal structure (histology), and, quite to their surprise, they found themselves still within the field of evolution. Then came studies in function (physiology), showing conclusively that this, too, is a matter of development and subject to variation and heredity. It is therefore not only erroneous but for the breeder dangerously misleading to consider the study of evolution as confined to the field of morphology, which is not the exclusive nor to him even the primary manifestation of the great principle of evolution. There is evoluion of function as well as evolution of form. 
the breeder, intent upon enhancing their service to man, sees in the variation of the functions natural to domesticated animals and plants the greatest opportunity for improvement.

So true is all this that the successful breeder may be clistinguished from the novice at this point. The latter is likely to be attracted, first of all, by form or color, because differences of this sort are striking and easily noticed; while the former will always keep foremost in mind the question, Why is this animal (or plant) valuable to man, and what is it to do?

The student cannot, therefore, know ton much about the natural functions of domesticated animals and plants and the deviations to which they are subject. He should know this, not only as a guide to his selection but also as constituting valuable information upon the nature of evolution and the possibility of influencing the causes that control the development of living beings, functionally as well as structurally.

Instances of variation in functional activity are easily divisible into four classes.

I. Variation in the degree of activity of normal functions between different individuals of the same species.

2. Variations in the legree of activity of normal functions within the same inclividual.

3. Modification of normal functions by external or other influences.

4. Normal functions exercised under abnormal conditions.

SECTION I - VARIATION IN THE DEGREE OF ACTIVITY OF NORMAL FUNCTIONS BETWEEN DIFFERENT INDIVIDUALS OF THE SAME SPECIES

Variation in milk secretion. This is a function peculiar to one class of animals (mammals). It is the product of a highly specialized structure and is practically confined to the female sex. Moreover, it is of peculiar economic as well as physiological importance, and there is no better example to bring out much that is involved in functional variation.

The structure of mammary-gland tissue is characteristic wherever found, but the quality and flavor of its product (milk) 
are not the same for any two species (functional variation between species).

Again, no two individuals of the same species can be depended upon to give exactly the same quality of milk, for herd records show that the milk of different cows varies naturally from less than 3 per cent to more than 6 per cent fat ${ }^{1}$ (functional variation between inclividuals). Nor is this dependent upon the food supply, for all authorities agree that the proportion of fat to other solids is dependent upon the individual and not upon her feed. Moreover, differences nearly as wide as these quoted may be found within the limits of a single herd and therefore under identical conditions as to feed.

Still again, two individuals of the same breed will produce radically different amounts of milk or fat, whichever is measured, from identical amounts of the same kind of feed. This has been repeatedly and conclusively shown by Professor Fraser of the University of Illinois. ${ }^{2}$ Probably no fact in animal physiology is of more far-reaching importance than is this marked instance of functional difference between individuals.

Three experiments were conducted in the attempt to determine the limits of this difference between cows considered good enough for a place in a commercial herd. In the first ${ }^{3}$ Eva produced 48 per cent more milk and I I per cent more butter in ninety-one days than did Janet, and in doing so consumed no more grain and but 7.6 per cent more roughness. These cows were both mature, were fresh on the same day, and neither suffered accident during the experiment, yet Eva produced 1057 pounds of milk and 12 pounds of fat out of an extra feed of I 2 pounds of hay and corn stover, - a difference greater than any margin of profit the dairyman may hope to realize.

The second experiment was a comparison between Rose, a native cow nine years old, and Nora, a native cow six years old."

1 The actual range in milk is far greater than these figures. Single milkings have been known to run as low as 1.8 per cent fat, and Jersey cows near the close of lactation often give milk with 9.0 per cent fat.

2 See Bulletin No. $5 I$ and Bulletin $N_{0} .66$, Agricultural Experiment Station, University of Illinois, May, I 898 .

3 Ibid. (5I) p. 103.

4 Bulletin No. 66, University of Illinois, November, 1901. 
Rose commenced April I 3 and Nora, May 22, I 899, and both were milked for a full twelve months. Both were in grood health, and both continued in good flow until the last, Rose averaging over is pounds of milk per day and Nora nearly it pounds for the last seven days of the test. Each consumed all the feed she cared to take, the only restriction being that its composition was the same for both. Neither was in any sense beefy, but Rose gained I 8 I pounds and Nora 165 pounds from August I to April I, showing that they were evidently working at or near their limit of milk production. The grain fed was corn meal and oil meal, and the roughage consisted of clover hay, rape, green corn, and corn silage, always in combination with one or more of the following, - gluten meal, wheat bran, and ground oats. They were never on pasture during the experiment, and, as has been stated, the feed was identical in quality for both.

Rose consumed slightly the heavier ration and yielded decidedly the larger product both in milk and fat. The following table exhibits the total feed consumed and the product yielded for the entire period of twelve months: ${ }^{1}$

Comparative Milk Production on Basis of Food Consumed

\begin{tabular}{|c|c|c|c|c|c|c|c|c|c|}
\hline \multicolumn{6}{|c|}{ Cow } & \multirow{2}{*}{$\begin{array}{c}\text { FEED }^{1} \\
6477.92\end{array}$} & \multirow{2}{*}{$\underbrace{}_{\text {I I }, 329.00}$} & \multirow{2}{*}{$\begin{array}{c}\mathrm{FAT}_{\mathrm{AT}} \\
564.82\end{array}$} & \multirow{2}{*}{$\begin{array}{l}\text { BUTTER }{ }^{1} \\
658.95\end{array}$} \\
\hline Rose. & . & & . & . & - & & & & \\
\hline Nora. & . & 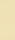 & . & . & . & 6 I 89.06 & $7,759.00$ & 298.64 & $348.4 \mathrm{I}$ \\
\hline Differe & ace & & . & - & - & 288.86 & $3,570.00$ & 266.18 & 310.54 \\
\hline Per cen & & . & . & . & . & 4.67 & $46.0 \mathrm{I}$ & S9.13 & 89.13 \\
\hline
\end{tabular}

Cast in verbal form this means that Rose was able to produce 47 per cent more milk and 89 per cent more butter than Nora, with the consumption of but 4.67 per cent more feed. Reducing both to the same basis of food consumed, it appears that with a given amount of feed for cacry 100 pounds of milk gizen by Nora, Rose gaíc 139.5 pounds; and for ciery 100 pounds of

1 Feed in pounds of digestible nutrients. Butter reckoned at 16.66 per cent water, adding one sixth to the butter fat. Per cent of difference calculated on Nora as a base. 
butter fat (or butter) produced by Nord, Rose produced ISO.7 pounds. For purposes of milk procluction, therefore, feed wats worth 39.5 per cent more when fed to Rose than when fed to Nora, and for butter production it was worth so per cent more. This, then, is the true measure of the functional difference between these two cows, and it is good and sufficient ground on which to base breeding operations. Further, it is to be noted that this is not the difference between a good cow and a poor one but between two good cows; for Nora produced 348.4 pounds of butter, which, as Professor Fraser remarks, is nearly three times the average yield ( 130 pounds) of cows in the United States, and almost one half more than the average yield (250 pounds) of what are considered profitable cows in Illinois.

It may be added at this writing (1906) that Rose, though used in many experiments and exhibited at various state fairs and at the St. Louis Exposition, is still living, hale and hearty at sixteen years of age, and is still an economical producer of milk. She has an average yearly record of $3 S_{t}$ pounds of butter fat for ten years, ${ }^{1}$ and though she has been in many tests since the one just reported she has never been beaten but once. That was in the following case, which bears further on the present point. Three cows were in this test with Rose, - Tina Clay's Queen, known to be a poor cow, and two natives, known as No. I and No. 3, supposed to be two of the four best cows bought for experimental purposes out of a herd of one hundred. Reduced to the same feed basis, and taking the yield of Queen as Ioo, that of No. 3 would be represented by I 2 I, of Rose by 304, and of No. I by 312. This is a rate of more than three to one against the poor cow, or over two and onc-half to one betwech grood cozes on the same feed basis.

This difference in the efficiency of individual cows is dependent not so much upon daily differences as upon the ability for long-time performance. Some cows will give a heavy yield for three or four months, and go dry in six or seven months; others will give a profitable yield almost continuously. Both extremes are deceptive. The herdsman will almost certainly overrate the

1 Since the above was written Rose has completed a twelve-year record of $725 \mathrm{~S}$ pounds of milk and 360 pounds of butter fat as an average. 
former and underrate the latter, so prone are we to remember striking and maximum clata.

These are not isolated and peculiar cases. Professor Fraser of the University of Illinois tested 554 cows in 36 commercial dairy herds of the state for a full period of twelve months each. He found that the best 25 per cent of the whole number tested were able to produce an arerage of 30 I pounds of butter fat per year, while the 25 per cent of lowest efficiency were able to produce an average of but 133.5 pounds, - a range of considerably more than two to one. The practical significance of this difference is pointed out by Professor Fraser as follows: If it costs thirty dollars a year to feed the poorer cows and thirtyeight dollars a year to feed the better ones, then at present prices a herd of twenty-five of the latter will produce as much nit profit as would a thousand of the former. A little calculation will show the immense saving in labor in keeping the smaller herd, and, what is equally significant, the relatively smaller investment in animals, feed, and barns, and the smaller rolume of business generally.

The faculty of producing a high yield of milk manifestly depends not only upon the activity of the mammary glands but also upon the capacity of the stomach to handle a large amount of feed, and the ability of every organ of the body to discharge its normal functions regularly and to endure the wear and tear of sustained exertion under heary pressure. This particular function of milk production is, therefore, a kind of resultant or algebraic sum of many body functions, and we should not expect to find its maximum except rarely and in few individuals. A simpler function practically independent of others would therefore be unhampered by their weaknesses, and it would reach its maximum in a higher proportion of individuals.

Variation in meat production. That the same principle is operative in meat production is abundantly shown by experiments. Steers were fed separately from calfhood to full maturity at the Michigan Experiment Station. ${ }^{1}$ The experiment was commenced as a breed test by Professor Samuel Johnson, and completed by the writer as a test of individual differences in ability to put on gain in proportion to feed consumed.

I Bulletin No.69, Agricultural Experiment Station, Michigan. 


\section{Gain in Proportion to Feed Consumed}

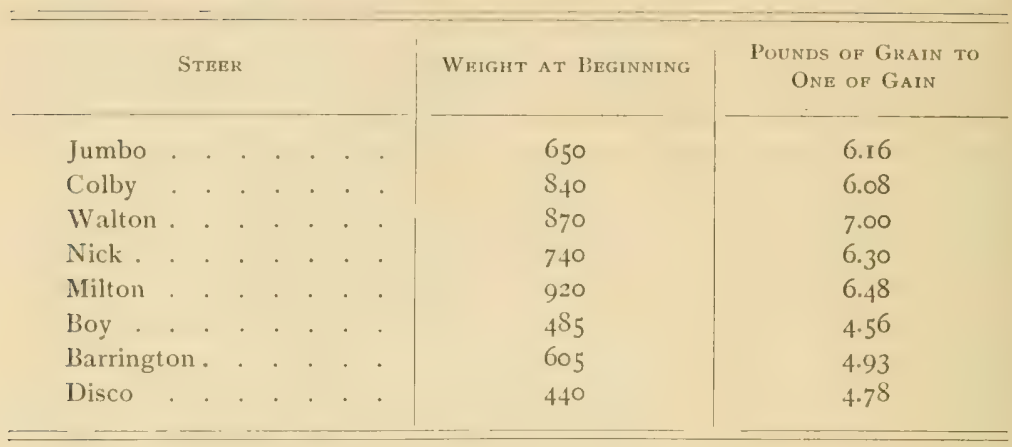

Differences of this character are further shown by Professor Mumford's experiments with the various market grades of steers. ${ }^{1}$ Feeding cattle are divided in the markets into six grades, from fancy selected down to inferior. A car load (sixteen) of each of these six grades (ninety-six animals in all) were fed on the same ration for a period of 179 days. The animals were all natives, though the better grades showed a much higher percentage of good blood than did the lower. The following table shows the relative ability of these six grades of steers to handle feed and convert it into gain :

\section{Relative Efficiency of Different Grades of Steers}

\begin{tabular}{|c|c|c|c|c|c|c|c|c|}
\hline \multicolumn{5}{|c|}{ GRADES } & \multirow{2}{*}{$\begin{array}{c}\text { Gain PER } \\
\text { SteER } \\
460\end{array}$} & \multirow{2}{*}{$\begin{array}{c}\text { Total Gain } \\
\text { i6 SteErs } \\
7362\end{array}$} & \multirow{2}{*}{$\begin{array}{l}\begin{array}{c}\text { Total Dry Mat- } \\
\text { Ter Consumed }\end{array} \\
73,267\end{array}$} & \multirow{2}{*}{$\begin{array}{c}\text { Driy Matter pek } \\
\text { Pound of Gain } \\
9.95\end{array}$} \\
\hline Fancy . & . & . & & . & & & & \\
\hline Choice. & . & & & . & 455 & 7284 & 88,093 & 12.09 \\
\hline Good . & . & - & . & . & 419 & 6705 & 81,017 & 12.08 \\
\hline Medium & . & • & . & . & $3^{8 \mathbf{r}}$ & 6095 & 79,535 & I 3.05 \\
\hline Common & . & . & . & . & 395 & 6322 & 75,875 & 12.00 \\
\hline Inferior & . & . & : & . & 350 & 5607 & 72,494 & 12.93 \\
\hline
\end{tabular}

Here is a variation of over 3 I per cent $(460-350)$ in the total gain of sixteen steers under equal opportunities, and, what is

${ }^{1}$ Bulletin No. go, Agricultural Experiment Station, University of Illinois. 
more significant, a difference of over 30 per cent in the feed required for a pound of gain. This shows the inferior feeding quality of the lower grades, due partly to age and partly to lack of breeding.

Composition of corn as influenced by functional deviation. One hundred and sixty-three good seed ears were selected from a strain of corn known as Burr's White. ${ }^{1}$ They presented to the eye no more differences than are usual with seed corn. Three rows of kernels from each were analyzed for protein and also for fat. As a result the protein in the various ears was found to range from 8.25 per cent to $\mathrm{I} 3.87$ per cent and the fat from 3.84 per cent to 6.02 per cent.

Composition of Corn from i 63 Different Ears

\begin{tabular}{|c|c|c|c|c|c|c|c|c|c|}
\hline $\begin{array}{c}\text { Corn } \\
\text { No. }\end{array}$ & AsH & ProteIN & FAT & $\begin{array}{c}\text { CARbo- } \\
\text { HYURATES }\end{array}$ & $\begin{array}{c}\text { CORN } \\
\text { No. }\end{array}$ & ASH & PROTEIN & $F_{A T}$ & $\begin{array}{l}\text { CARBO- } \\
\text { HYDRATES }\end{array}$ \\
\hline 76 & I. 70 & 10.05 & 4.77 & 83.48 & 104 & I. 54 & II .82 & 4.43 & 82.21 \\
\hline 77 & 1.45 & 10.42 & 5.24 & 82.89 & 105 & 1. 37 & 12.36 & $4.8_{4}$ & $8 \mathrm{I} .43$ \\
\hline 78 & I. 55 & 11.00 & 4.90 & 82.55 & 106 & I. 33 & II.I 5 & 5.21 & $\mathrm{~S} 2.3 \mathrm{I}$ \\
\hline 79 & 1.62 & 10.89 & 4.88 & 82.61 & 107 & 1. 33 & 9.47 & 4.97 & 84.23 \\
\hline So & 1.63 & I I. 50 & 4.58 & 82.29 & IOS & 1.30 & I 1.04 & 4.67 & 82.99 \\
\hline $8 I$ & 1.47 & I1. 49 & 4.26 & 82.78 & 109 & I. 45 & 10.82 & 5.65 & 82.08 \\
\hline 82 & I.39 & I I. 78 & 4.83 & 82.00 & I 10 & 1.60 & $\mathrm{I} 2.8 \mathrm{I}$ & $5.2 \mathrm{I}$ & So. $3^{8}$ \\
\hline $8_{3}$ & I. 17 & 9.08 & 4.05 & 85.70 & I I I & I. 3 I & 10.76 & 4.13 & 83.80 \\
\hline 84 & I. $5 \mathrm{I}$ & 12.79 & 4.25 & 8 I. 45 & 112 & 1. 26 & 10.48 & 4.54 & 83.72 \\
\hline $8_{5}$ & 1. 46 & 11.76 & 4.94 & $\mathrm{Si}_{4}$ & 113 & I.IO & $9 \cdot 30$ & $4 \cdot 3^{8}$ & $8_{5.22}$ \\
\hline 86 & I. 50 & I 2.07 & $4.6 \mathrm{I}$ & $S_{1} . S_{2}$ & 114 & I. 33 & 9.12 & 4.10 & 85.45 \\
\hline 87 & I. 59 & 12.40 & 4.74 & 81.27 & 115 & I. 29 & $10.4 \mathrm{I}$ & 4.17 & 84.13 \\
\hline 88 & I. 35 & $9 \cdot 34$ & 4.84 & 84.47 & II 6 & I.IO & $8.3^{8}$ & 4.88 & 85.64 \\
\hline 89 & I.6I & 10.71 & 4.70 & 82.98 & II 7 & I. 42 & 9.95 & 4.23 & 84.40 \\
\hline 90 & I. 55 & 9.90 & 4.97 & $83.5^{8}$ & 118 & 1.44 & II. 40 & 5.02 & 82.14 \\
\hline 9 I & 1. 56 & 10.68 & $4.9 \mathrm{I}$ & $82.8_{5}$ & II9 & I. 55 & $12.3 \mathrm{~S}$ & 4.62 & SI. 45 \\
\hline 92 & 1.46 & 12.96 & 3.97 & 81.61 & 120 & I. 39 & 9.97 & 4.42 & $8_{4} .22$ \\
\hline 93 & 1.48 & I1 80 & 4.80 & 81.92 & $12 \mathrm{I}$ & I. 36 & 10.09 & 4.82 & $S_{3.73}$ \\
\hline 94 & I. 74 & I I 89 & 4.55 & 81.82 & 122 & 1. 36 & $10.3 I$ & 5.25 & $S_{3.0 S}$ \\
\hline 95 & 1.55 & 10.49 & $5 \cdot 5 \mathrm{I}$ & 82.45 & 123 & I. 34 & 9.68 & 4.01 & S.4.97 \\
\hline 96 & I. 60 & II.IO & $4 \cdot 3^{8}$ & 82.92 & 124 & I. 44 & II 87 & $4.6 \mathbf{I}$ & $82.0 S$ \\
\hline 97 & 1.59 & I I .84 & 4.96 & 81.61 & 125 & I. 34 & 10.73 & 4.53 & $8_{3.40}$ \\
\hline 95 & I.39 & 10.23 & $5 \cdot 5 \mathrm{I}$ & $\mathrm{S}_{2} . \mathrm{S}_{7}$ & 126 & 1.49 & 13.57 & 5.72 & 75.92 \\
\hline 99 & 1.42 & 8.40 & 4.9 I & 85.27 & 127 & 1.43 & II. 53 & $4 \cdot 31$ & 82.73 \\
\hline 100 & 1.65 & I2.28 & $4 \cdot 76$ & SI.3I & I 28 & I. 33 & I I. 64 & 4.57 & 82.40 \\
\hline IOI & I. 30 & 10.08 & 4.86 & $S_{3.76}$ & I 29 & I. $3^{6}$ & I1 1.25 & 4.16 & $S_{3 \cdot 23}$ \\
\hline 102 & 1.49 & $11.8_{3}$ & $4 \cdot 5^{I}$ & 82.17 & 130 & I. 35 & I 1.86 & 5.01 & SI.7S \\
\hline 103 & I. 44 & II.25 & 4.78 & 82.53 & I3I & I. 47 & 10.49 & 4.86 & $S_{3} \cdot I S$ \\
\hline
\end{tabular}

1 This was the foundation of Dr. Hopkins' work in corn breeding at the Uni versity of Illinois, as reported in Bulletin No. 55 and Bulletin $N o .100$. It will be further discussed later on. 
Composition of Corn from i 63 Dhferent Ears - Continued

\begin{tabular}{|c|c|c|c|c|c|c|c|c|c|}
\hline $\begin{array}{l}\text { Cintex } \\
\text { No. }\end{array}$ & $A s 11$ & P'LUTLIN & Fix & $\begin{array}{c}\text { C.NRBL- } \\
\text { HYDRATES }\end{array}$ & $\begin{array}{l}\text { Cums } \\
\text { No. }\end{array}$ & $\Lambda_{S H}$ & Prutein & Fst & $\begin{array}{l}\text { C.NREO- } \\
\text { HYDRATES }\end{array}$ \\
\hline 132 & I. 55 & I I.I 3 & 4.55 & 82.77 & 186 & I. $4^{8}$ & 10.78 & 4.74 & 83.00 \\
\hline I33 & I. 39 & I I. I 3 & 4.10 & $8_{3} \cdot 3^{8}$ & 187 & I. 28 & 10.49 & $4 \cdot 44$ & 83.79 \\
\hline 134 & 1.30 & 10.85 & 4.45 & 83.40 & 188 & 1.53 & 13.10 & $5 \cdot 5 \mathrm{I}$ & 79.86 \\
\hline I 35 & $1 \cdot 37$ & I 1.29 & 4.53 & 82.81 & 189 & 1.32 & $9 \cdot 5^{8}$ & 5.63 & 83.47 \\
\hline 136 & 1.59 & I I .43 & 5.10 & SI.SS & 190 & 1.25 & I I. 50 & 4.95 & 82.30 \\
\hline I 37 & 1.47 & 11.61 & $4.4 \mathrm{I}$ & 82.51 & I9I & 1.29 & 11.19 & $4 \cdot 31$ & $83.2 \mathrm{I}$ \\
\hline${ }^{1} 3^{S}$ & 1.36 & 11.36 & 4.53 & 82.75 & 192 & 1.51 & II. 49 & 4.07 & 82.93 \\
\hline 139 & I. 57 & 9.8 I & 5.23 & 83.39 & 193 & 1.36 & $9 \cdot 47$ & $4 \cdot 5^{I}$ & 84.66 \\
\hline 140 & 1.34 & 10.53 & 4.18 & 83.95 & I 94 & 1.50 & 11.47 & 4.65 & $82.3^{8}$ \\
\hline 141 & I. 45 & 12.42 & $4 \cdot 5 \mathrm{I}$ & S1.62 & 195 & I. 54 & 11.09 & 4.37 & 83.00 \\
\hline 142 & I. 37 & $9 \cdot 3$ I & 4.82 & 84.50 & 196 & 1.30 & $9 \cdot 44$ & 3.95 & $85.3 \mathrm{I}$ \\
\hline 143 & 1.29 & I I. 33 & $4 \cdot 49$ & 82.89 & 197 & 1.26 & 11.20 & $4 \cdot 46$ & $8_{3.08}$ \\
\hline I 44 & 1.42 & II. 39 & 4.99 & 82.20 & 198 & I. 44 & 10.23 & 4.53 & 83.80 \\
\hline 145 & I. 45 & 8.25 & $4.8 I$ & 85.49 & I99 & 1.29 & 10.64 & 4.67 & 83.40 \\
\hline 146 & I. 47 & I1.29 & 4.83 & $82.4 \mathrm{I}$ & 200 & I.39 & 10.13 & 4.84 & 83.64 \\
\hline 147 & 1. 26 & I $2.2 \mathrm{I}$ & 4.49 & 82.04 & 201 & I. $3^{8}$ & 9.64 & 5.22 & 83.76 \\
\hline $14^{8}$ & I. 54 & I 1.9 .4 & 4.74 & 81.78 & 202 & I. 39 & I 1.26 & 4.96 & 82.39 \\
\hline 149 & 1.36 & I 1.29 & 4.08 & 83.27 & 203 & 1.26 & 10.48 & 4.59 & 83.67 \\
\hline I 50 & I. 44 & I I.7 I & 4.03 & 82.82 & 204 & 1.66 & I 2.57 & 4.82 & 80.95 \\
\hline 151 & I. 40 & $9 \cdot 3^{I}$ & $4.9^{6}$ & 84.33 & 205 & 1.46 & 10.71 & $5.3^{6}$ & 82.47 \\
\hline 152 & I. $4 \mathrm{I}$ & I 1.90 & 4.09 & 82.60 & 206 & I. 34 & 10.27 & 4.65 & 83.74 \\
\hline I 53 & I. 35 & $12.5^{I}$ & 5.19 & So.95 & 207 & 1.25 & I1 1.09 & 4.27 & 83.39 \\
\hline I 54 & 1.42 & I I.I 3 & 5.02 & 82.43 & 208 & 1.48 & 12.05 & 4.78 & 81.69 \\
\hline I. 55 & 1.44 & I I.05 & 4.53 & $82.9^{8}$ & 209 & I. 48 & 10.22 & $4 \cdot 30$ & 84.00 \\
\hline 156 & I.39 & I I. 74 & 4.I 4 & 82.73 & 210 & I.45 & I I.1 6 & $4 \cdot 75$ & 82.64 \\
\hline I 57 & I.46 & 10.02 & 4.88 & $\mathrm{~S}_{3} .64$ & $21 \mathrm{I}$ & I. 48 & 10.44 & $4.2 \mathrm{I}$ & 83.87 \\
\hline $15^{8}$ & I. 45 & 10.66 & 4.5 I & $\mathrm{S}_{3} \cdot 3^{\mathrm{S}}$ & 212 & I. 27 & 9.75 & 4.12 & 84.86 \\
\hline I 59 & 1.48 & 11.53 & 4.65 & 82.34 & 213 & I. 53 & 12.40 & 4.75 & $8 \mathbf{I} \cdot 3^{2}$ \\
\hline 160 & 1.43 & $11.5^{\circ}$ & $4 . S_{3}$ & 82.24 & 214 & $1.5^{8}$ & 10.22 & 4.43 & 83.77 \\
\hline I6I & 1.47 & I I. I I & 4.93 & 82.49 & 215 & I. 45 & 9.22 & 4.60 & 84.73 \\
\hline 162 & 1.48 & 12.09 & 5.61 & So. 82 & 216 & 1.42 & 10.27 & 4.35 & 83.96 \\
\hline 163 & 1.29 & 10.78 & 5.09 & $82 . S_{4}$ & 217 & 1.32 & 9.39 & 4.83 & 84.46 \\
\hline 164 & 1. 30 & $9 \cdot 3^{6}$ & 4.34 & 85.00 & 218 & I. 40 & $9 \cdot 74$ & 4.71 & 84.15 \\
\hline 165 & 1.47 & 10.50 & 4.75 & 83.28 & 219 & I. 37 & 9.92 & $4 \cdot 3^{2}$ & $84 \cdot 39$ \\
\hline 166 & 1.65 & I I.29 & 3.84 & 83.22 & 220 & I. 43 & 9.63 & 5.23 & $8_{3.71}$ \\
\hline 167 & 1. 37 & $9 \cdot 5^{8}$ & 4.72 & $8_{4.33}$ & 221 & I. 32 & 10.33 & 5.01 & 83.34 \\
\hline 168 & 1.49 & 10.94 & 4.34 & 83.23 & 222 & I. 4 I & I 2.34 & $4 \cdot 57$ & 8 r.68 \\
\hline I69 & 1.60 & II.79 & 4.22 & 82.39 & 223 & I. 49 & $10.5^{8}$ & 4.64 & 83.29 \\
\hline 170 & 1. 36 & I1 1.06 & 4.39 & 83.19 & 224 & 1.52 & 11.36 & 4.63 & 82.49 \\
\hline I 7 I & I. 44 & I I.IS & 5.75 & $\mathrm{Si}_{1} 63$ & 225 & I.33 & 9.1 5 & 4.55 & 84.97 \\
\hline 172 & I. 45 & 12.28 & 3.99 & 82.28 & 226 & I.36 & $10.3^{1}$ & 5.08 & 83.25 \\
\hline I 73 & 1.39 & 10.14 & 4.35 & $8_{4.12}$ & 227 & 1.46 & 12.63 & 5.15 & 80.76 \\
\hline 174 & I. 30 & I0.19 & 5.22 & 83.29 & $22 \mathrm{~S}$ & I. $4 \mathrm{I}$ & 12.16 & 4.12 & 82.31 \\
\hline 175 & 1.40 & I 2.68 & 5.29 & So.63 & 229 & I. 36 & II.04 & 4.52 & 83.08 \\
\hline 176 & I. 37 & 9.86 & 4.73 & 84.04 & 230 & I. 43 & 12.10 & 4.29 & 82.18 \\
\hline 177 & I. $4 \mathrm{~S}$ & 13.06 & 4.93 & 80.53 & 231 & I. 33 & I0.95 & 4.60 & 83.12 \\
\hline I 78 & I. 37 & 10.93 & 4.76 & 82.94 & 232 & I. 52 & 12.76 & 4.10 & 81.62 \\
\hline 179 & 1.32 & 11.87 & 5.03 & 81.78 & 233 & I. 40 & $9 \cdot 75$ & 4.14 & 84.71 \\
\hline 180 & I. 39 & 11.27 & 4.55 & 82.79 & 234 & I. 39 & 10.78 & $4 \cdot 76$ & 83.07 \\
\hline I 81 & 1.47 & 9.66 & 4.21 & $8_{4.66}$ & 235 & 1. $5^{8}$ & 9.97 & 5.27 & $8_{3.18}$ \\
\hline I82 & I. 37 & 10.97 & 3.94 & 83.72 & 236 & I. 40 & I0.1S & 6.02 & 82.40 \\
\hline 183 & I. 54 & 10.32 & $5 \cdot 46$ & 82.68 & 237 & I. 47 & II.16 & 5.13 & 82.24 \\
\hline 184 & I. 44 & 10.68 & 4.89 & 82.99 & ${ }^{2} 3^{S}$ & 1.60 & I 1.42 & 5.20 & $8 \mathrm{I} .78$ \\
\hline 185 & 1.42 & 9.33 & 4.49 & $8_{4.76}$ & & & & & \\
\hline
\end{tabular}


The wicle variation in all constituents, particularly in protein and fat, indicates that profound differences existed in the functional activities of the plants that procluced these ears. In order to determine whether these differences are constitutional and therefore hereditary, the twenty-four ears highest in protein were planted (separately) for the "high protein plot" and the twelve lowest for the "low protein plot." In the same way the twenty-four highest and the twelve lowest in fat were planted for "high fat" and "low fat," respectively. This has been continued from its beginning in $1896-1 \$ 97$ until the present (1907), and is still in progress, the practice being each year to plant separately the ears that show the highest and the lowest values in respect to these particular constituents. The following table shows the average composition of the seed corn and the crop for the first year of the experiment:

\section{Protein}

Highest protein ear out of $\mathrm{I}_{3}$ analyzed Lowest protein ear out of 163 analyzed 13.87 Difference.

Average of 24 high protein ears planted I 2.54 Average of 12 low protein ears planted Difference .

9.03

$3 \cdot 51$

Average of crop from high protein seed Average of crop from low protein seed

Difference.
I I.IO

10.55

.55

\section{FAT}

Highest fat ear out of 163 analyzed . . . . . . . 6.02

Lowest fat ear out of 163 analyzed . . . . . . . . 3.84

Difference .

Average of 24 high fat ears planted . . . . . . 5.33

Average of 12 low fat ears planted . . . . . . . 4.04

Difference. . . . . . . . . . . . 1.29

Average of crop from high fat seed . . . . . . . 4.73

Average of crop from low fat seed . . . . . . . . . $\frac{4.06}{67}$
Difference 
It is sufficient for the present purpose to note that there was a difference of 5.62 per cent $(13.87-8.25)$ in the protein content of the highest and the lowest ears of the 163 analyzed; of $3.5 \mathrm{I}$ per cent (12.54-9.03) in the seed planted; and of 0.55 per cent (I I.IS-IO.55) in the crop harvested the first year:

It is not the intent to pursue this subject further at this point. It will be fully discussed under heredity and under plant breeding, but enough has been quoted to show that these functional differences are both cistinctive and hereditary, and that in it all the ear is the unit to such an extent that it is entirely practicable to permanently infiuence functional differences by selection. Indeed this has been done already to such an extent that corn has been produced with a higher protein content than wheat.

Variation in sugar production. Sugars of various kinds are produced by many plant and animal activities. Certain plants excel in this particular function, and among these wide differences have been found, leading to marked and permanent increase in the amount of sugrar produced. The beet, for example, though originally producing but from 4 to 6 per cent of sugar, has been so improved and its sugar-producing activities have been so increased as to yield specimens containing as high as 25 per cent of sugar and whole crops averaging 14 per cent.

Cane is also variable, and every one familiar with the maple knows that certain trees will yield a large amount of exceedingly sweet sap, while others yield but little, which little may be either sweet or tasteless, — indeed, even bitter.

Variation in speed, scent, and organic activities generally. One horse is faster or more enduring than another, not so much from conformation as from inherent activity and power of endurance. Some dogs are especially keen in scent, others are defective, and the hearing instinct is much better dereloped in some individuals (dogs, cats, horses, cattle, bircls, etc.) than in others. ${ }^{1}$

Mental qualities, personal tastes, and intellectual ability in general are conditioned not upon conformation but upon the

I It is more than likely that some of these differences are connected with the degree of development of certain portions of the nervous system. They are none the less functional, however. 
peculiar action of certain parts possessed by the race in common, but whose special functioning in each particular case determines the place of the individual in the scale of life.

Variation in vital functions. For present purposes the animal body may be regarded as a colony of organs, each endowed with its own peculiar function, the life of the whole and of every member being dependent upon the degree of success with which each portion does its work. The whole is, therefore, as strong as its weakest member, and when the whole is put to work in service for man, that service will depend not only upon the functional activity of the special organ involved, as the udder or the muscular system, but also upon the successful discharge of all vital functions when subjected to the unnatural strain involved in working under pressure. The point at which the machine will break down or fail to do successful work is, therefore, a matter of relative strength of parts, and in the last analysis the limiting element in performance is not infrequently one or more vital functions, which experience shows are as variable as are other and, from the biological standpoint, less important characters.

The beat of the heart, in man for example, though steadily decreasing in rapidity from infancy to old age, yet varies between different individuals at maturity all the way from less than fifty to more than eighty beats per minute. Athletes tell us that the slow beat is characteristic of long-distance running and sustained effort generally, but that individuals of this order are ill adlapted to short-clistance rumning or other work requiring quick response to stimulus.

There is a marked difference in the digestive powers of different animals, and some individuals starve because the stomach and intestines are unable to dissolve sufficient food to meet the demands of the body, - and there are all degrees of starvation. ${ }^{1}$ Others with excellent digestion but with limited powers of assimilation fail to make use of the full supply that

1 Wide study of men, animals, and plants will reveal many cases in which the individual has accustomed itself to an abnormally small food supply. The effect is not necessarily fatal, but it is shown in reduced output either of labor, body product, or other form of organic activity. 
is put into the circulation. In the case of Rose and Nora, previously cited, what did Nora do with the excess of food consumed? Digestion experiments with individuals indicate no such differences in digestion among healthy animals as the differences in milk yield that are known to exist between cows. The only conclusion is that in a case like this the surplus food passed on out of the body, laying excessive labor mpon the excreting organs as well as incurring loss upon the one who provided the feed. ${ }^{1}$

From this it will be seen that the excreting organs themselves act as a kind of safety valve, and that much depends upon their relative ability to discharge their functions well. This they are better adapted to do in some individuals than in others, but that every effort is made to keep up with demands is evidenced by the fact that if one kidney is lost the other acts for both, usually increasing in size. Speaking generally, a cow will give as much milk from three teats as from four. Whether this is from compensation, as with the lost kidney, or whether it is true only because cows are seldom worked up to their limits, the data at hand do not determine.

The tremendous increase in the activity of the salivary glands on the part of tobacco chewers, the increase in size of muscles through use, and the marvelous development of skill in eye and hand by long-continued practice, as in the playing of musical instruments, reading on the part of the blind, etc., - these are all familiar examples of functional development through practice. That this development is or may be greater in some individuals than in others is too well known to need more than passing mention in this connection.

Resistance to disease and invasions of the animal economy generally differ greatly in different individuals. Some are absoIutely immune to certain cliseases, others peculiarly susceptible.

It is a matter of common observation that in fields of corn killed by frost an occasional stalk remains green and unaffected, showing unusual powers of resistance, due to some constitutional difference. Without a doubt these are the differences

I This is conclusive proof of the fact that appetite is not a safe guide to the amount of food that can be profitably consumed. The most that can be said is that it is a good indication of body use among animals whose efficiency is known. 
that go far toward constituting the essential distinction between annuals and biennials in temperate regions.

And so it is that the value of an animal or a plant depends not only upon what it can do but also upon its powers to enclure sustained exertion; and this is indirectly dependent upon the vital functions, which are therefore of prime consequence to the farmer. The horse that died in a Michigan coal mine at fiftyfour years of age after having worn out more than five generations of harness mates; Old Granny, the Galloway cow that died at nearly thirty-six years of age, having raised no fewer than twenty-five calves; men who live to be a hundred or thereabouts, - these are examples not of individuals that have been shielded from hardships, but rather of those splendid pieces of animal machinery whose every part easily performs its proper function to any limit laid upon it by the exigencies of life. ${ }^{1}$ That these functional differences exist and that they are in a measure hereditary are facts that challenge the most careful attention of the thoughtful breeder.

1 Benjamin Franklin IIarris died of pneumonia in Champaign, Illinois, May 7 , 1905, at the age of ninety-three years, four months, twenty-two days. He was personally known to the writer as remarkable not so much for his advanced age as for the fact that he was in full possession of all his powers, and actively engaged in business until within a week of his death. He organized the First National Bank, which at his death was operated by three generations of the Harris family; but he was president to the last in fact as well as in name, and the management deferred to his judgment even in matters of detail.

He was the owner and operator of over five hundred acres of prairie land, and was one of the earliest and largest cattle men in the Middle West. He was a noted feeder before a market was established in ('hicago, and was both a buyer and a seller on that market every year of his life afterward. He was always a believer in heavy cattle, and he finished and sold the one hundred heaviest cattle ever marketed in this country and, so far as is known, in the world, - an achievement of which he was always especially proud.

This instance of longevity is given not as an extreme in respect to years but in respect to retention for so long a time of all the powers of body and mind. Mr. Harris never had a second childhood, and was a good example of what a splendid machine is the human organism, which ordinarily breaks at the weakest point but does not wear out. How full of weak points animal organisms really are and how weak these points must be are considerations forced upon the mind by the fact that Mr. Harris' span of life was more than three times that of the average man. These three instances of extreme longevity in animals and man, showing what is possible in animal life, afford to the breeder ample food for reflection. 
Variation in fertility. Certain bircls regularly produce two egres, others three, and still others four, before incubation. The average hen, following her natural habit, lays a "setting" (ten to fourteen) and then suspends for incubation. The "crop" of ova has been laid, and time is required for another to come forward. The Maine station, however, has succeeded in greatly increasing the production of eggs, and has produced one hen that has laid two hundred and fifty-one eggs during a single year.

Most cows produce only five or six calves, many only one or two, and some not any, yet Old Granny (No. I in the Galloway Herd Book) produced twenty-five, the last one in her twentyninth year. The difference between regular and shy breeders is the difference in the functional activity of the reproductive organs, and next to performance ability it is the most important character in the eyes of the breeder. Even longevity itself is not its equal from the standpoint of the improver, because quality cannot be said to exist in the race unless the individuals that possess it are sufficiently fertile to insure its easy and certain perpetuation.

Accumulation of functional variations. Having shown the marvelous differences in functional activity between different individuals, and having shown that these differences are hereditary, as in corn, it follows necessarily that functional variations may be accumulated into true breed distinctions, and that strains of animals and plants may be permanently established with exceedingly high efficiency in desired lines; indeed, this has been already accomplished, though we are still far short of what is possible.

For example, the beef breeds are more economical producers of meat than are the dairy breeds, and the converse is true as to milk production. These two functions have, therefore, been largely separated along breed lines. But it cannot be said that one beef breed is more efficient than another in meat production, or that any one dairy breed stands out preëminently as the most economical producer of milk. This is partly because breed differences have been largely built up along lines other than those of efficiency, and partly because all breeds contain many individuals of low efficiency in their own breed characters and high efficiency in those of other breeds. Some Jerseys, 
therefore, are better feeders than certain shorthorns, and there are to be found among the latter breed individuals that are more economical producers of milk than are many of the Jersey breed.

It has been said that no race was ever taken by a part-bred horse over one that was racing bred. Whether literally true or not, it is substantially correct, so intensely have the racing ability and instinct been developed in certain breeds.

These facts, together with what is known as to corn and beets, show clearly that much yet remains to be clone in the way of developing functional activity, thereby increasing efficiency.

\section{SECTION II - VARIATION IN THE DEGREE OF ACTIVITY}

OF NORMAL FUNCTIONS WITHIN THE SAME INDIVIIUUA,

Of no less scientific or economic interest than the data given in the last section is the fact that functional variation is by no means confined to differences between individuals. The principle applies, though to a less extent, to the inclividual itself, whose activities are not constant but variable from day to day and throughout its life.

Daily fluctuation in normal functions. It will be found upon investigation that the ordinary functions of the body are unexpectedly irregular. Even the heart action is not absolutely constant; it is slower when the body is at rest than when it is in action and is subject to great acceleration in certain diseases.

All organs of the body work better some days than others; indeed, there is a distinct periodicity with each, - a period of increase, followed by one of maximum activity, and that again by one of subsidence. This is the way organs rest. These periods are evidently of different lengths, and it is therefore only occasionally that the body as a whole is at its maximum. A good example of this periodicity with functional deviation from day to day is, again, that of milk secretion, which, as has been remarked, is a kind of resultant of all the activities of the body. The following table gives the variations in the quantity and character of milk from a single cow for a period of one month. ${ }^{1}$

1 Bulletin No.5I, Agricultural Experiment Station, University of Illinois. 


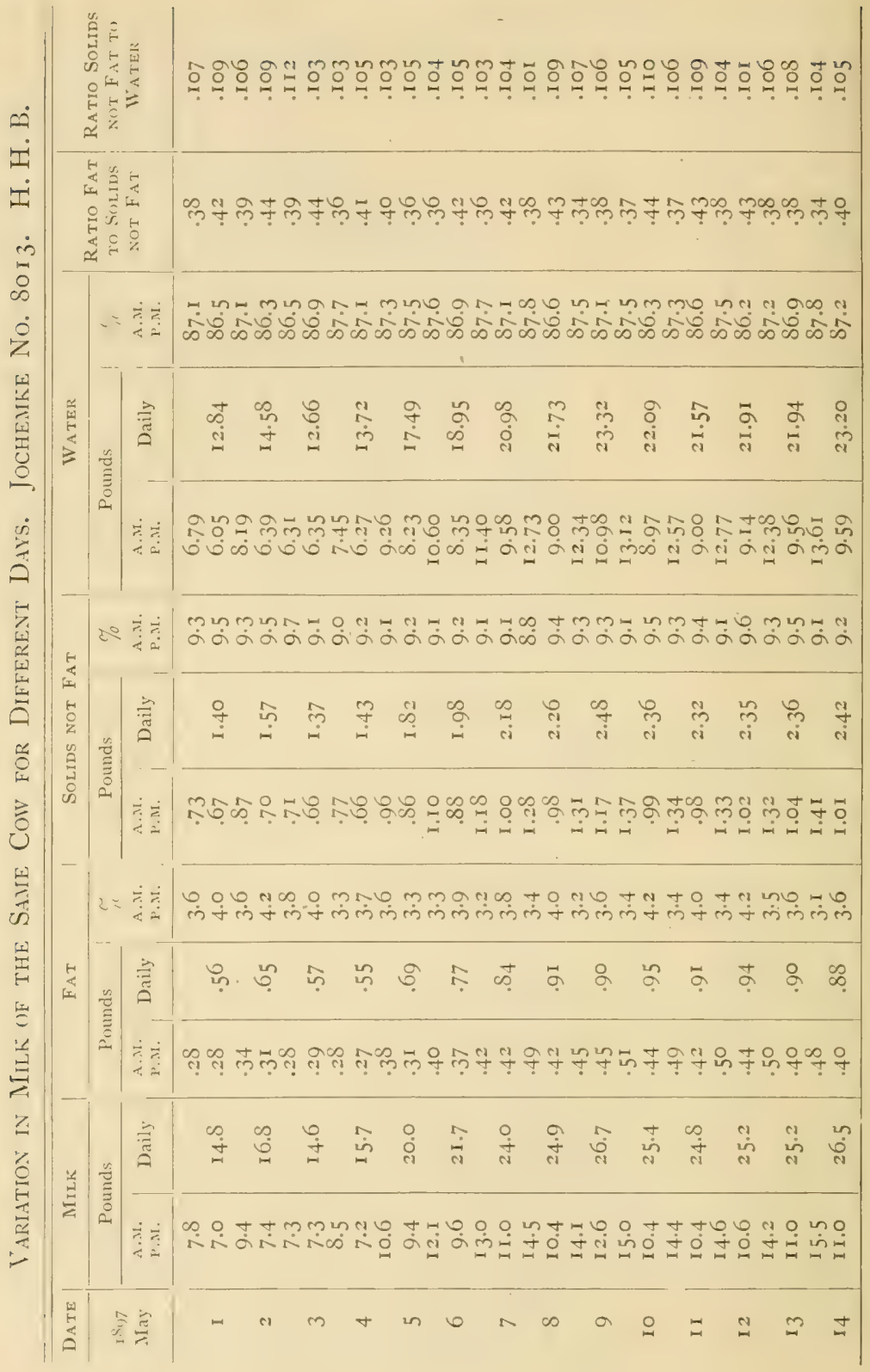




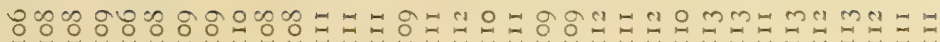

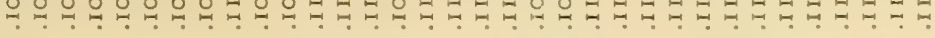

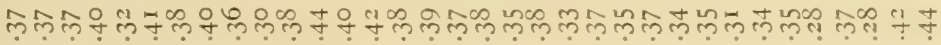

40 - N किळ人

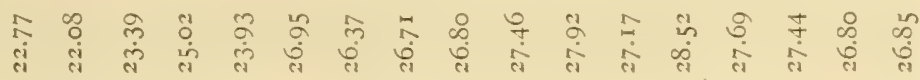

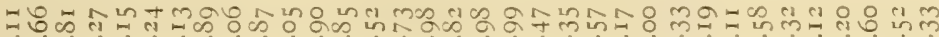
ஊव்

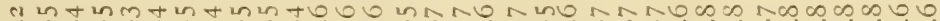

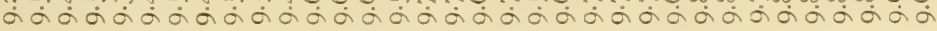

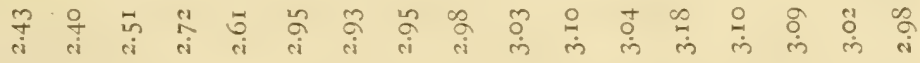

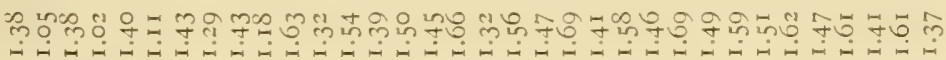

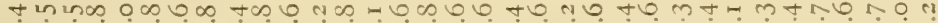

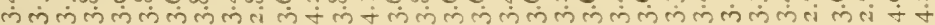

용

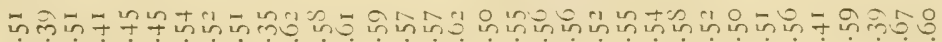

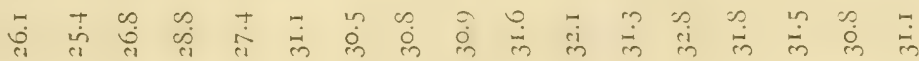

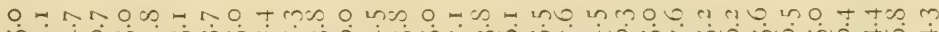
ம் $\dot{0} \dot{0}=$ in்

\# 
Comment is hardly necessary to show the irregular working of the animal machine, which in this case was a mature, strong, and healthy cow. The per cent of fat varied all the way from 2.7 to 4.2 within the space of twenty-four hours.

Influence of age upon functional activity. At birth the vital functions and those of growth are at their maximum. At this time growth seems to be proceeding with the "energy of embryonic development." It continues at a maximum for a time, ${ }^{1}$ gradually declining in rate until maturity, when growth of the general body ceases, although for certain parts (hair, teeth, horn, hoof, reproductive cells, etc.) it continues nearly or quite through life. In case of accident many parts not commonly indulging in growth after maturity are able to regenerate with more or less success (bone, skin, blood vessels, nerves), but among the higher organisms generally growth practically ceases at maturity. ${ }^{2}$ It is for this reason that feeding enterprises are most profitable with young animals that are still growing. At this age functional activity is greater and general bodily efficiency higher.

One reason why the lower grades of feeders are less economical producers than the better grades is that the animals are older, the difference in age between fancy selected and inferior steers being one to two years.

Strength and endurance are evidently at their maximum somewhat after maturity, although with the passing of that age is also lost something of the power of rapid recuperation and repair.

The reproductive functions, undeveloped until a considerable period after birth, attain a high degree of energy, if not their maximum, somewhat before full maturity is reached. They then decline, and fail entirely in old age. Their duration is therefore considerably less than the life of the individual, often dropping below 50 per cent of the full life period.

1 The curve showing rate of growth at different ages has not been sufficiently worked out, but enough has been done to indicate that the maximum rate of growth is attained a few days after birth and that this maximum is never again reached.

2 Trees continue to increase in size, to some extent at least, during life, illustrating a marked difference between the higher plants and animals. 
At what period the reproductive functions are most successful in producing young of a high order of excellence, whether in youth, middle age, or old age, is not determined, though it is a point on which light is badly needed. It is certain that the practice of using young and immature sires is almost universal, especially among cattle. That there is clanger in continued reproduction from immature animals, even though they are sexually vigorous, there is grave reason to fear, and yet, in general, reproduction antedates maturity.

The duration of profitable service depends, of course, upon the nature of the function involved. The life service of a racing horse is manifestly less than that of a work horse, and the life of a meat animal is less than that of one kept for milk.

Influence of exercise: use and disuse. The beneficial effect of use in developing and perfecting the functions of the body has been recognized from the most ancient times. Athletes train for this purpose. Musicians practice for many hours every day; indeed their chief labors arise from the need of constant and severe exercise of the musical faculties in order to achieve any considerable degree of perfection and to hold it after it has been acquired.

Horses designed for racing are worked almost from the first in order to make the most of any natural ability to trot or run. Cows are believed to be more efficient producers of milk if they begin at two years of age and are kept, so to speak, in constant practice, and barrenness is believed to be less likely if heifers are bred early than if left to attain maturity without producing young.

Darwin discovered that the wing bones of wild duclis as compared with their leg bones were relatively heavier than those of tame ducks, corresponding to their respective habits of life. The arm of the blacksmith and the wing of the ostrich; the remarkable leg of the kangaroo and the remains of that of the whale; the brain power of the busy man and that of the sluggard, - these and scores of examples that might be cited show not only that exercise develops, quickens, and perfects the body functions, but they show, too, that their very retention or loss depends in the long run upon their constant and rational use. 
Blind people acquire a quicliened and an educated sense of hearing and a touch that amounts almost to a separate sense. In the same way the developing of the bodily functions and activities generally depends upon judicious use (exercise); and the skill of the trainer and the results he is able to achieve depend not only upon his knowledge of methods that will most certainly insure the exercise of the desired parts but also upon his judgment as to how severe and protracted that exercise should be in order to secure the maximum effects of use and not incur the destructive consequences of overuse. This is as true in the feeding yard as upon the race track, and applies as well to the raising of good and profitable feeders as to the developing of racing horses, the education of drivers and saddlers, the training of hunting dogs, the "trying out" of homing pigeons, or the teaching of canaries to sing by never allowing the young birds to hear a false note.

Influence of feed upon functional activity. The relation of the amount of feed to its economical consumption is a subject needing careful investigation. Enough is known to warrant the assertion that animals can and do learn to take amounts far larger than can be really used. When a steer consumes over a bushel of corn a day he has simply formed the eating habit as the result of a morbicl appetite, nor is this appetite an inclication of body needs or a guaranty of its powers to economically convert the feed into meat, milk, or labor.

It is significant that steers very gradually brought into full feed will never take these enormous amounts. Professor H. W. Mumford of the University of Illinois finds that under such circumstances twenty pounds of grain per clay is all the animal will take.

Consumption of extreme amounts is, therefore, evidence only of the quantities of feed the digestive tract can carry and discharge without calamity, of its power to secrete gastric and other digestive juices, and of the ability of the excreting organs to eliminate unused and unusable surplus from the body

In the case of Rose and Nora the latter consumed the same feed as the former but returned but little more than half as much. She was undoubtedly, from the standpoint of economy, 
overfed, but whether the same individual would make cheaper returns on less feed is not so well known as it should be. In the mechanical world the highest return of energy per unit of consumption is realized when the machine is working full but somewhat below its maximum capacity. Doubtless the same principle holds with living machines, but on this point we are sadly in need of accurate information.

On one point we are certain. The animal (or the plant) is able to adjust itself to a wide range of food supply, providing the change be gradually made. Not only individuals but whole families for generations live in a condition of semi-starvation, of ten quite ignorant of their real condition, if indeed they are not so indifferent as to prefer to continue in the old way rather than to disturb their tranquillity by increased exertion. Such a state may easily become chronic in man or animal, but it is unprofitable because all other functions are suspended or reduced to a minimum in order that the vital functions may be clischarged at all and the animal not die outright. There is no nicer problem for the stockman and the feeder than this: How much shall I put into this animal machine in order to realize the highest net efficiency, after first providing for those activities which are necessary to the life of the machine, - the vital functions?

Influence of hard conditions. Under hard conditions the functions of life may be disturbed but not destroyed. Under these conditions valuable activities are carried forward upon a reduced scale, and they often give rise to losses that are no less serious because invisible. The most common example of this is in the case of ill-fed or much-abused animals and of badly nourished crops or trees: some milk is secreted, but it is insufficient and its quality is poor; the plant is weak, with little resisting power against insects or clisease, and with little ability to mature its crop; the apples are there, but they suffer for the means of development.

Every one who has had experience with unthrifty animals or plants knows how difficult and how slow is the process of restoration of normal activity after it has once been seriously checked by neglect or disease. This is because the condition readily becomes constitutional, tending to continue through life. 


\section{SECTION III - MODIFICITION OF NORMAI, FUNCTIONS BY EXTERNAL, OR O'THER INFLUENCES}

To what extent are normal functions dependent upon favorable environment and how do they respond to changed conditions? A few notable facts will throw some light upon this all-important question.

Galls. An insect stings a plant. Under the influence of the poison a gall is formed. If this gall be shown to a biologist he will at once state with certainty the plant which produced the gall and the insect that made the injury, so definite in form and appearance is the resulting growth and so distinct is it from any normal growth of the uninjured plant.

In other words, specialized plant tissues subjected to a certain injury produce a new kind of growth almost as specific as when operating under the laws of heredity. IIere the functional activities of the plant at the affected point have been not destroyed but permanently alfered in such a manner as to give rise to new structures of definite form and often with specific chemical properties, as in the case of nutgalls ${ }^{1}$ containing 30 to $S 0$ per cent of tamnin. In cases of this kind a new function has been developed suddenly out of old materials, and it at once gives rise to new and distinct products, both substantive and morphological.

Abnormal overgrowth of disordered animal tissues. We have just noted that vegetable tissues subjected to specific injuries often suffer such a derangement of their functions as to cause the production of an abnormal but characteristic overgrowth of the injured part. Quite similar is the result of the invasion of the animal economy by specific germs from without, as in the case of Bacillus tubcrulosis. Here the growths (tubercles) resulting from the apparent attempt to encyst the foreign material are sufficiently characteristic to serve as a name for the disease (tuberculosis).

Tumors generally, whether malignant or benign, are perverse overgrowths of normal tissues of the body, whose ordinary

1 All formulas for good black writing ink include gallnuts (or nutgalls) as the characteristic ingredient. Those commonly used are produced on the oak by the sting of the gallfly (Cymips galla-tinctoria). 
functions have been deranged by some cause, external or internal, and whose activities have been diverted to the production of abnormal but characteristic tissue with " no typical termination to its growth."

Nearly all tissues of the body ${ }^{1}$ are subjected to this derangement of their ordinary functions, resulting in the suspension of all activities except that of growth, which proceeds with the "energy of embryonic development" and continues indefinitely.

Though often supplied with special blood vessels and a system of nerves, these growths are entirely functionless and therefore useless to the body. Being parasitic they are always a drain upon its resources, and often from their nature or position they constitute a real menace to its existence.

A tumor represents a bit of differentiated body tissue that for some reason or other has abandoned its characteristic functions, cut loose from all restraints of heredity, set up an independent existence of its own at the expense of the colony of which it has been a respectable and dependable member, and has now devoted all its resources to growth, which, as has been said, proceeds with the energy of embryonic development, resulting in nothing but functionless masses of living matter, strongly suggesting a reversion to primitive undifferentiated tissue.

Under conditions not well understood all sorts of abnormal growths may appear. In this way an antenna may appear where an eye ought to be, ${ }^{2}$ or it may end in a foot instead of a feeler. ${ }^{3}$ The writer knew a young lady of culture and of no little natural beauty except for the fact that, growing from one cheek, was a tuft of coarse black hair three or four inches long. Her normal hair was brown and her complexion clear. What functional disturbance could have given rise to such a growth is as mysterious as it was unfortunate.

Ossification is a natural process, but under the influence of excessive strain it may proceed to an abnormal extent, as in spavin, where the entire hock joint becomes solicl through the ossification of the fluid thrown out as the result of injury.

1 Muscles, fatty tissue, connective tissue, bone. cartilage, nerves, glands, blood vessels, the covering of the brain, etc.

2 Bateson, Materials, etc., p. I51. $3 \mathrm{nt}^{3}$ Ibid. pp. 146-147. 
Derangements of a more fundamental nature often arise during embryonic development, resulting in monsters of all degrees of abnormality. Teratology has little interest to the biologist generally, because these abnormal caricatures of life constitute nothing but sporaclic offshoots of the species. Developing from defective grerms and having no connection with the line of descent, they are of little interest to the evolutionist. Their interest to the thremmatologist lies in their bearing upon functional actirity and the desrie of cortainty with which specialiacd tissues may bi depended upon to discharge their hereditary and proper functions.

Variation due to the suppression or failure of the reproductive functions. The abdomen of the crab Carcinus manus normally has seven segments. In the female these are distinct. In the male the abdomen is much narrower, and the divisions between the third, fourth, and fifth segments are obliterated. Males, however, inhabited by the parasite Saculina do not develop sexual characters, and in them the segmentation is complete, as in the female. ${ }^{1}$

A young male is castrated. The parts removed are in no sense vital, and they seemingly have no connection with other organs of the body. All the bodily functions except those of reproduction proceed, but not as bifore. In general the development of the shoulders and neck will be arrested, and they will remain lighter and finer. The voice, the nervous temperament, the disposition, and the general activity of the body are all affected. The mane of horses will be thinner, finer, and shorter. The hair of face and neck in cattle will be finer and less curly. In hogs the tusks and shoulder plates do not develop. The growth of the horns is stopped in sheep, but in cattle the only effect is to make them slightly longer and a little more slender, approaching the female type. The hincler parts of the body as a whole clevelop rather more in castrated than in entire animals, and there is a general approach to the form of the female. It is noteworthy in this connection that the same general effect follows the failure of the sexual powers with advancing age, except that the body development has already taken place.

\footnotetext{
1 Bateson, Materials, etc., p. 95.
} 
Females deprived of their ovaries develop to some extent the characters of the male. Spayed heifers are not at all like bulls, but they do resemble steers. Unsexing animals seems, therefore, to induce a kind of mediocre development, although it gives rise to four distinct types instead of two for each species.

Many females in later life assume certain characters of the male. Cows bellow and paw dirt like bulls; hens grow spurs and try to crow; women sometimes grow a straggling beard and acquire a heary roice. These changes do not by any means appear in all cases, but when they do appear they may be regarded as symptoms of loss of the sexual function and of cessation of breeding powers. ${ }^{1}$

This influence over the functions of the body by organs apparently having no connection with the parts affected is akin only to that of certain glands like the thyroid, whose function is entirely unknown, but in whose absence children grow up defective both physically and mentally.2 We are at this point very near to the forces that determine the activities of living matter, but the mysteries involved are in no sense cleared up; they rather deepen instead as they are studied. It is as if our vision were obstructed, not by a curtain that can be clrawn aside affording a view beyond, but rather by a solid wall fixing the limits not only of vision but of progress as well.

\section{Functional variation due to the modifying influence of the} conditions of life. The conclitions of life are most active in stimulating or depressing normal activities, but they are not without effect upon their character as well. Plants having a fixed abode are more dependent upon their environment and therefore less resistant than animals, though species living in confined waters are little better off than plants in this regard.

1 Though bearing but indirectly upon the present question, it is worthy of remark at this juncture that many individuals of each sex seem to be naturally endowed with more than the usual proportion of the characters of the opposite sex and to be correspondingly short in those of their own. Thus we have our "mannish" women and our "effeminate" men, distinguished not only for their tastes and their mental characteristics generally but for their body conformation as well. These abnormal unions of male and female traits are often strange mixtures indeed, and may well be avoided in the breeding yard.

2 Loeb, Physiology of the Brain, p. 207. 
Plants, and animals too for that matter, growing in cold climates or under hard conditions suffer profound changes, to which they become accustomed (acclimated) and which are ever afterward constitutional. IVe become accustomed to cold or to heat and are thereafter less affected by extremes. Recent calorimeter tests show that the temperature of the human body is lowest from three to five o'clock in the morning and highest from one to three in the afternoon, thus following fairly close the minimum and maximum of outside temperatures. These conditions continue even if the subject works at night and sleeps in the daytime.

Two conditions tend to produce hard and spiny growth in vegetation. These are intense light and extreme dryness. Both are found in tropical regions, and when they occur together their maximum results follow as to harshness and spines. These conditions can be verified in the laboratory, showing conclusively that the character of growth is, in a measure at least, dependent upon surroundings.

Speaking generally, plant lice reproduce parthenogenetically during the growing season of the summer, and during this time only wingless females are produced. IVith the approach of cold weather, however, a winged bisexual brood is produced that lives over winter.

These conditions can be produced artificially in the greenhouse at any time by lowering the temperature and allowing the plants on which the lice feed to clry up. Thus we may say that wings and sex may be developed at will by the manipulation of the conditions of life.

The so-called conversion of one species into another by influencing its environment has been largely overstated, and yet the facts are that when Schmankewitsch ${ }^{1}$ grew Artcmia salina in water whose saline content was gradually increased, the caudal fins and their bristles "progressively degenerated " until, in many cases, these appendages had disappeared, the animal thus assuming the character of $A$. milhansenii, which normally lives in waters of extreme density. These experiments were undertaken because he secmed to have observed this transformation taking place naturally in a lake crossed by a dam, and which was inhabited

1 Bateson, Materials, etc., p. 96. 
by both species, the one above the other. This dam broke, mixing the two species, but in three years original conditions were practically restored. The experiments were undertaken to learn whether real transformation had taken place or whether the result had been brought about by selection.

The experiment seems convincing; and the point is further strengthened by the facts that Schmankewitsch restored the caudal fins by reducing the solution, and that when the reduction was carried below the normal of $A$. salina, within three generations the last segment of the body divided after the fashion of A. branchipus, another related species. The facts are the more remarkable as $A$. salina reproduces parthenogenetically, while A. branchipus is not known to do so.

Biologists are extremely careful not to assume the absolute conversion of one species into another by any such direct methods as have here been noted, the question being, rather, whether they are all goorl species; but that single characters are profoundly influenced by changed conditions, and come to resemble the same character in another and related species, is too well established to be longer questioned. What light this may finally throw upon the origin of species is problematical, but it serves the present purpose in showing the power of environment to profoundly modify the functional activities of living beings.

If a cutting of willow, currant, or other suitable growth be planted in the earth, roots will start from the part below the ground, and leaves and branches from the part above. If, now, it be cut off at the surface of the ground and the top portion be planted again, it will again take root at the new point of severance which had before borne leaves. The process may be continued indefinitely, or until the piece is used up, showing that roots or leaves may be developed at will at any point along the cutting, according as it is placed below or above ground.

If a cutting be planted, roots will develop only at the lower end. If, however, before planting it be cut into two pieces, each will develop roots on the part below ground, and in many species this will occur even if the pieces be inverted and planted top down. ${ }^{1}$

1 This is most likely to take place in young wood, less likely in old wood. See Morgan, Regeneration, pp. 7 I-9I. 
A maple tree growing in Urbana had forked into two nearly eculal parts about six feet from the ground. One part was split down and torn off in a heavy storm, when it was seen that roots hard developed in the crotch and were evidently at work upon the soil that had blown from the street and the moisture that had accumulated from rains.

The writer was excavating for a basement. A black cherry tree stood some ten feet from the line of the wall. In taking this out many of the roots were severed, their cut ends being left in the bank of undisturbed earth. In a few days these cut ends were clothed with a growth of green leaves. IIere was tissue that, under normal conditions, functions only as roots, yet upon occasion readily gives rise to both leaf and stem.

All plants and most animals maintain definite relations to light, and if free to move, orient ${ }^{1}$ themselves with reference to the clirection of the light rays striking the surface of their bodies. A plant bends toward the window because of the contracting effect of the light upon the protoplasm of one side of the stem. Many larve are negatively heliotropic; that is, the lighted side of the body is more irritable and they move away from the light, coming to rest only in dark places, where they feed and mature. Others are positively heliotropic when hungry and negatively heliotropic when fecl. Such larva will climb trees and feed upon the leaves or buds until filled, when, becoming sensitive to light, they descend and hide in the ground, under rubbish, or in any other place shielded from the sun. This action has been erroneously attributed to a semi-intelligent instinct. It is nothing but functional dependence upon external stimuli. This is not the place to pursue the subject in detail, - which will be done when discussing "Instinct and Reflex Action," - but it is the place to note the wonderful dependence of certain normal functions upon external influences.

An animal is invaded by a foreign germ and suffers from clisease. It is in most cases ever after immune to that clisease. What change has been worked in the animal economy? IVe

1 By orientation is meant the direction in which the long axis of the body is brought to rest with reference to surrounding bodies or influences, such as grav. ity or light. 
know that as long as the white corpuscles are able to discharge their proper function the resistance is complete. Why do they weary of their work, and what condition is left behind which assures absolute resistance to future invasions?

The phenomenon of acclimatization in general represents a condition in which an organism has undergone a permanent change in its vital functions, foreed upom it by the exigencies of life. In the future studies, however, it will be seen that the clisturbing effect of adverse conditions, if not ton severe, may be gradually overcome, and the animal or plant resume its functions, either modified or ummodified; and it will be seen further that if the changes be gradual, the immunization will extend to a point that would have been fatal at the outset. Thus organisms may be reared in a gradually intensified poisonous solution, or in a liquid whose temperature is slowly raised, and in this way a point may be reached many degrees above the power of normal organisms to withstand. The subject cannot be pursued further in this connection, for it is a large one, with many other bearings; but the student should bear it in mind throughout the study.

Irregular functioning. An interesting phase of irregular functioning is found in the so-called "instinctive acts," more properly reflex actions, which by peptular conception are supposed to proceed with unerring accuracy. This assumption is natural in view of the complex nature of many of these acts, all of which have the appearance of being under the control of reason. For example, note the complicated nature of the process necessary to the successful deposition of the egg of the yucca moth (Promula yuccasclla). We are told ${ }^{1}$ that these moths emerge simultaneously with the flowers of the yucca, which open but for a single night and are practically dependent upon this particular moth for fertilization. When ready to oviposit, the female gathers a bundle of pollen from one flower, flies with it to another, pierces the tissues of the pistil of the latter, and lays her egg; after which she ascends to the stigma of the same pistil and "stuffs the fertilizing pollen pellet into its funnel-shaped opening."

Now this process is necessary not only to the fertilization of the yucca, but also to the grub that hatches from the egg, which

1 Morgan, Habit and Instinct, pp. I $3^{-1} 5$. 
otherwise would be left without food. There is, therefore, a particular sequence in this complicated performance that must be observed or failure results; and failure is fatal to the existence of both species.

Moreover, this act is performed but once in the lifetime of the moth, who has no knowledge of the acts of her predecessors, and is therefore not proceeding from simulation; nor has she opportunity to learn the fate of her offspring and profit by the experience.

Now the truth is that, unerring as is this performance, a good many orules escape, from failure of the egg to hatch or from other causes, and thus the yucca is able to mature some seed. That complicated processes of this kind are not always carried out in proper sequence and full detail is shown by careful study of different individuals, as pointed out especially by Professor C. S. Crandall in his studies of the apple and plum curculio. ${ }^{1}$

Careful study of these complicated acts and of the body functions in general must convince the student not only of their nice adjustment but (what is of equal consequence) of their exceeding variability and irregularity within limits that certainly are by no means narrow.

Cases of "double personality," in which the individual behaves for a time as another and distinctly different person, are too well known to require more than a passing notice. These are instances in which an entirely new set of functions is brought into play, - distinct from the normal, yet working: together to the accomplishment of definite ends.

But a few of the many modifications of normal functions have been mentioned. This is not the place to exhaust the subject. Only enough has been given to show the student that even the highly specialized functions are subject to the laws of variation. The matter will be more completely covered under "Causes of Variation."

1 Bulletin No. 98, Agricultural Experiment Station, University of Illinois, pp. 500-502. The account of the different ways in which three different females performed their work is given in full under " Transmission of Modifications," section on "Habit and Instinct." 


\section{SECTION IV - NORMAL FUNCTIONS EXERCISED UNDER ABNORMAL CONDITIONS}

The mammary gland, normally confined to females, is commonly functionless until after pregnancy; but by manipulation of the udder, heifers and other females may be made to yield milk without bearing young. Again, rudimentary mammæ, present generally in males, are occasionally accompanied by considerable development of mammary tissue, nearly always but not necessarily functionless. ${ }^{1}$

Most remarkable of all, mammary-gland tissue has been known to develop in extremely unusual places upon the body. Mammary tumors in the axilla (armpits) are described as of "common occurrence in lying-in women." 2 These tumors have no duct, but in squeezing they yield "both colostrum and milk," following in the same order as from normal mammx, and oozing through the skin "at the situations of the sebaceous follicles."

Besides these there is "indisputable evidence of the presence of a mammary gland on the thigh . . ., on the cheek . . ., on the acromion (shoulder point) . . , and in the labium majus. . . In the two last cases the mammary nature of the gland was proved by microscopic examination." 3

Similar conditions may be produced artificially by grafting, and all sorts of abnormalities may testify to the persistence with which highly specialized tissue continues to discharge its functions, often under the most discouraging circumstances. ${ }^{4}$

For example, Hunter and Duhamel grafted the spur of a young cock into his comb, "where it continued to grow to its normal size." 5 "Bert transplanted the tail of a white rat to the body of Mius decumanus (the common brown rat), where it continued alive." 5 The same experimenter bent over the tail

1 Dr. Hottes, a personal friend of the writer, knew a young man in Germany who was suckling an infant.

2 Bateson, Materials, etc., p. I $\$_{5}$.

${ }^{3}$ Ibid. p. I 87 .

4 As when a piece of mammary gland was grafted into the ear of a guinea pig; when the pig became pregnant the gland commenced to secrete.

${ }^{5}$ Morgan, Regeneration, pp. 178-179. 
of a rat and grafted it back into its own body. After it had united he severed it at the normal base and thus provided the animal with a "reversed tail." Ile found, however, that the tail of the mouse did not grow as well in the body of the rat and woukd not unite at all with the body of either the dog or the cat. ${ }^{1}$

Born succeeded in uniting the anterior and posterior parts of the tadpoles of two different genera of frogs (Rena csculcuter for anterior and Bombinator igncus for posterior). The combination lived for ten days, when it was killed because of pathological changes. ${ }^{2}$

In the same way Harrison made up an individual of two species (Rana rirescens and Rance palustris). This he kept alive until after its transformation into a frog, "each half retaining the characteristic features of the species to which it belongs." " This being true, it is not surprising that many varieties of apple can be grafted into the same tree top. Examples of this sort might be muliplied indefinitely, as in the making up of worms by grafting together pieces of two different species, in which each piece preserves its specific characters; but enough has been given to show the persistence with which specialized tissue continues to discharge its natural function even uncler the hardest of conditions. ${ }^{3}$

The circumstances under which living matter can discharge its normal functions unaltered, either in character or in degree, and the limits beyond which these functions must cease or undergo alteration, - all this is not only a question of deep biologrical interest but it is one of special significance in breeding, because it throws no little light upon the real nature and causes of variability, a subject upon which we sorely need information if variations are ever to be controlled either in their development or in their transmission.

Summary. We are to regard variations in function as well as in form, of activities as well as of structure, of what an animal or plant does, as well as what it is.

The body functions are not constant, but variable. They are variable as between different individuals and also with the same

1 Morgan, Regeneration, pp. $178-179$.

${ }^{3}$ Ibid. chap. ix, pp. I 59-189, "Grafting and Regeneration."

2 Ibid. pp. $18_{3}-18_{5}$ 
individuals at different times. They are variable not only in degree but also in kind, and normal functions may be disturbed, even altered, by external infuences. Conversely, usual functions may be discharged under most unusual conditions.

All variation is either continuous or discontinuous, and continuity must not be assumed. Many of the fundamental qualities of living matter, such as definite composition, argue for discontinuity.

\section{ADDITIONAL REFERENCES}

Immunity and Adaptation. Biological Bulletin, IX, I4I-I5I.

Geldings more Susceptible to Disease than Mares. Experiment Station Record, XI, 896.

Variation in Immunity to Axthrax (among sheep). By Martinet.

Experiment Station Record, XIII, I86.

Insectivorous Plants. By Charles Darwin.

For good evidence on functional variation consult the speed records of trotting and running horses and the Advanced Registry of Jersey and Holstein-Friesian Cattle. 


\section{CHAPTER VI}

\section{MUTATIONS}

\section{SECTION I - DISTINCTION BETWEEN MUTATION AND ORDINARY VARIATION}

The deviations from type heretofore considered are those of individuals rather than of groups. Whether quantitative, substantive, meristic, or functional, they represent the fluctuations of individual members of a species or a variety about the normal type of the race, not necessarily exhibiting any tendency to depart permanently from that type.

The study of these deviations shows that, while no two individuals are alike, yet the departures of certain individuals in one direction are compensated by departures of other individuals in the opposite direction. In other words, the members of a race cluster closely about what may be called a center of fluctuation, which is, in most cases, comparatively stationary. Because of this fact we may have a relatively fixed type, indicating a practically stationary race, even in the midst of considerable individual deviation. ${ }^{1}$

Mutations, on the other hand, mark sudden and distinct departures from type. The pendulum swings, but does not return. A new center of fluctuation is established, from which individuals deviate in all directions as before. It is not that the old center is abandoned, - for the mass of individuals still cluster about it as before, - but it is that a new center is established, about which a new group clusters, and all close observers recognize at once that a new type has been born into the world

1 This fact is extremely confusing, especially to animal breeders. In the midst of wide variations and with but few individuals living at any one time, the breeder is often unable to tell whether his general average (or type) is improving, retrograding, or standing still. This matter will be alluded to again under "Type and Variability." 
and a new race is established on the earth. This new group and its center constitute a mutation, and the individuals are spoken of as mutants. Variation has become discontinuous as well as continuous.

These sudden offshoots from established species were noted by Darwin, who called them sports. He considered, however, that new species are formed only by the slow but continuous action of selection working with ordinary fluctuations (continuous variations), building up new types a little at a time through the gradual accumulation of slight but favorable deviations.

His so-called "sports" were therefore mysterious, and from the fact that under natural conditions they generally disappear rapidly by crossing, he was led to attach little importance to these sudden departures from the established type. ${ }^{1}$ Later researches, however, have given them unexpected significance.

The distinguishing feature of a mutation is that there are no intermediates between the old type and the new, which was therefore attained not by slow degrees but by a sudden leap; that there is but a slight tendency to revert to the old form, but that if reversion takes place at all it is complete at once and the return is to the old type and not to an intermediate form. The mutant is distinctly a case of discontinuity.

\section{SECTION II - EXAMPLES OF MUTATION}

The classic examples of mutation are the weeping willow and the nectarine. They are to be regarded, however, as familiar illustrations of general principles widely operative and giving rise not to few but to many distinct types.

When a seed germinates it puts out two sprouts. One is positively geotropic; that is, it responds to the force of gravity and grows downward into the soil, developing the root system. The other is negatively geotropic; that is, it grows upward

1 The student of evolution should gain the conception that the type of a race is not a fixed point from which deviations radiate; it is rather the center of gravity of all the individuals of the race, its exact location depending upon the extent and direction of individual deviations, shifting slightly from time to time with the causes that influence variability. 
against gravity, and with an energy sufficient to maintain it in a fairly upright position, developing stems, branches, and leaves.

Occasionally this latter geotropism fails, and the branches hang downward, forming a "weeping" variety. This is especially common in the willow and the birch, though by no means unknown in other trees, notably the elm, maple, and beech.

"Cut-leaved" varieties and "fan tops" 1 occasionally arise suddenly, all of which may be preserved by grafting or by budding, so that with proper attention we may have weeping, cutleaved, and fan-top varieties, not of a few but of many species of trees and shrubs, although the readiness with which a particular mutation may appear in one species is no guaranty of its appearance in another.

A tree which has always before borne peaches may suddenly bear nectarines, or more likely a single branch may make the departure, the remainder of the tree continuing to bear peaches as before. In any event the mutation may be propagated by bud or possibly by seed, - in which latter case a nectarine-bearing tree results. This tree may bear nectarines all its life, or it may occasionally bear peaches on the whole or a portion of its top. The significant fact is that there is no intermediate between the peach and the nectarine, and yet the one may arise at any time from the other. ${ }^{2}$ The mutation from peach to nectarine is clearcut and distinct, and the reversion from nectarine to peach when it occurs is equally complete.

The apricot appears to be related to the plum much as the nectarine is to the peach. In both cases the main stocks (peaches and plums) exist in many varieties, and the mutations (nectarines and apricots) in but few. In the case of the former (the peach) the main stock is downy, while the mutant is glabrous, or destitute of downy covering. In the latter, however, the conditions are reversed, for the main stock (the plum) is glabrous, while it is the mutant that is downy.

1 A fan-top tree is one in which the branches are borne on opposite sides, after the fashion of corn. In a small forest plantation belonging to the writer is a fantop linden, now grown to considerable proportions.

2 Inasmuch as the peach is considered as the main race, the nectarine is said to arise from the peach by mutation. Therefore when peaches are bome upon nectarine trees the case is considered to be one of reversion. 
Because the apricot has never been observed to arise direct from the plum as the nectarine has repeatedly been known to arise from the peach, and because the apricot trees have never been known to bear plums as the nectarine trees occasionally bear peaches, - because of these facts botanists have quite generally ceased to regard the apricot as a sport from the plum, and are agreed, I believe, in considering it as a distinct species.

However, it behaves precisely like a mutant, and in considering the means by which new types originate the presumptive evidence is strong that the apricot originally sprang from the plum stock. Though it is true that some mutations are frequently repeated, it is also true that others arise but rarely. The nectarine is unusual in the frequency with which it reappears, and the readiness with which the peach and its mutant exchange places has perhaps no parallel.

In many respects the apricot appears like an intermediate between the peach and the plum. The external appearance of the fruit is that of the peach. The pit is smooth, resembling that of the plum. The bark of the tree is like that of the peach, but the leaf is like that of the plum. There is nothing to suggest a hybrid origin, though everything to suggrest that this strange plant and its fruit are in some way composed of the elements of both the peach and the plum.

Nor would a hybrid origin be at all necessary to this fact. Certain characters are general, rumning through many species quite independent of consanguinity. Thus the weeping or the cut-leaved habit is common to a great variety of species only remotely related. The downy character is common with both fruit and leaf, and almost every downy or pubescent species has its glabrous or smooth variety, - its mutant in all probability, and one that easily and frequently arises. So also, without doubt, the reverse is true by which smooth species occasionally throw off downy or pubescent varieties. Now this particular character of pubescence, while simple enough in itself, is yet exceedingly noticeable, and serves to insure a specific name, unless indeed the direct origin happens to be extremely well known, in which case the mutant is likely to get off with a varietal distinction. 
In the same general manner, color is likely to fail, and nothing is more common in nature than albino varieties. Thus we have our white currant, strawberry, raspberry, and even the blackberry, - almost every thicket affording its examples and speaking eloquently of the freedom with which nature creates new forms, and if we will only open our eyes to see what is going on about us, we shall learn much of how it is done.

Albinism among animals is even more common than among plants. Men, dogs, cats, horses, cattle, sheep, bears, rabbits, rats, mice, and many other species are distinguished by albino varieties.

These distinctions, marked though they are, arise doubtless from the simplest causes. For example, if an animal for any reason fails to secrete pigment in the normal manner it is from necessity an albino, and if the failure is hereditary an albino race is likely to be established, although unrestricted breeding greatly reduces its probability through crossing with other forms.

\section{SECTION III - EXPERIMENTS OF DE VRIES 1}

Hugo De Vries, professor of botany in the University of Amsterdam, long ago became convinced that Darwin's theory of the origin of species through the gradual accumulation of fortuitous variations is not the only means of creating new types. Darwin taught not only that existing types had been preserved by selection because they in some way fitted the conditions of life, but that the intervening spaces between species and varieties represent extinctions through the agency of natural selection.

De Vries came to believe that, in many cases at least, the new type springs suddenly from the old, without gradation and without intervening forms, and that while selection may shape up the new type and perhaps the better fit it for existence, yet the selective process is in no way responsible for its origin. Indeed, one of the earliest evidences, to his mind, that new types often arise without the agency of selection, was the notable fact that new forms arising spontaneously in nature are for the most

1 Hugo De Vries, Species and Varieties, their Origin by Mutation [Open Court Publishing Company, Chicago]. 
part promptly exterminated by the rigors of natural selection, which therefore could not have been the chief agency in their creation.

Accordingly he conceived the idea of cultivating a few unstable forms under conditions such as would protect and preserve any mutations that might arise, hoping in this way to throw some light on the origin of new types and to determine whether in the origin of species natural selection works principally upon individuals or upon types.

Experiments with toadflax ${ }^{1}$ (Linaria vulgaris). These experiments were designed to test the origin of the peloric form. ${ }^{2}$ The toadflax was chosen, first because the peloric form is known to have arisen repeatedly, and second because the change involved is slight, structurally speaking. These two considerations gave reason for the hope that if the species were put under careful observation and control, he (De Vries) "might be present at the time when nature produces another of these rare changes."

The experiments commenced in I $\$ \$ 6$ with normal plants bearing "one or two peloric flowers," as is common with most individuals of this genus. The roots were planted in the garden, and flowered and seeded in IS87. This second generation was grown for three years, producing in $1889^{3} \mathrm{one}$, and in I 890 two, peloric structures. The seeds of these were saved and produced the third generation in r890-189r. Among some thousands of blossoms in this generation there was one five-spurred flower. This was pollinated by hand and luckily produced "abundant fruit, with enough seeds for the entire culture of I892, and they only were sown." 4

1 De Vries, Species and Varieties, etc., pp. 464-487.

2 The normal flowers of the toadflax are exceedingly unsymmetrical. Aside from bearing a short spur, they are described as consisting of a "two-lipped corolla, the lower lips spreading and three-lobed, with a base so enlarged as to nearly close the throat." Plants bearing such unsymmetrical flowers as do toadflax, snapdragon, etc., are known occasionally to produce peloric, that is, symmetrical, flowers. Not only that, but peloric varieties are not unknown, and these experiments were designed to solve the manner of their origin.

3 The toadflax is a biennial.

${ }^{4}$ Peloric flowers of this species are commonly sterile, but in any case are dependent upon artificial fertilization. They are by nature ill adapted to preserve themselves. 
Up to this point in the experiment each generation required two years, as the toadflax is a biennial, not blooming until the second year. After this, however, the seedlings were started under glass and transplanted to the garden in June. By this means the new plants were made to produce flowers and seeds the first year.

About twenty plants of this (fourth) generation were secured, and under this treatment most of them produced seed the first year. Only one peloric flower was observed, however, in the entire lot. The plant bearing this flower and one other were preserved, and all others were destroyed. These two fertilized each other freely and produced Io cc. of seed, but no more peloric flowers appeared. It is from this pair of plants, however, that a peloric race finally sprung. ${ }^{1}$

In IS94 about fifty plants were in flower. There was no reason for considering these plants any more promising than previous sowings, except that "stray peloric flowers were observed in somewhat larger numbers than in previous generations, eleven plants bearing one or two, or even three, such abnormalities." De Vries wisely remarks that this "could not be considered as a real advance, since such plants may occur in varying though ordinarily small numbers in every generation."

However, besides these eleren individuals, each bearing one or two abnormal flowers, there airs a single plant bearing only' peloric floacrs. The mutation had arisen and De Vries "was present at the time."

This plant was carefully kept, all others being destroyed, and the next year it bloomed again, bearing only peloric flowers. It was true to its type. In this connection De Vries says :

Here we have the first experimental mutation of a normal into a peloric race. Two facts were clear and simple: [first] the ancestry was known

${ }^{1}$ It has been said that the flowers of one plant are sterile to pollen from the same plant. De Vries ascertained by careful experiment that this is true in about 50 per cent of the cases, so that, though a much higher degree of fertility exists between individuals than within the individual, absolute barremness between all flowers of the same plant cannot be asserted. The point is not significant in the present connection, but it is important as demonstrating that fertility and sterility are not always in direct proportion to consanguinity, and that, though close breeding may be commonly infertile in certain strains, it by no means follows that it is always infertile even in the same strain. 
for over a period of four generations. . . T This ancestry was quite constant as to the peloric peculiarity, remaining true to the wild type as it occurs everywhere in any country, and showing in no respect any tendency to the production of a new variety.

[Second] the mutation took place at once. It was a sudden leap from the normal plants with very rare peloric flowers to a type exclusively peloric. The parents themselves had borne thousands of flowers during two summers, and these were inspected nearly every day in the hope of finding some pelorics and of saving their seed separately. Only one such flower was seen. . . There was simply no visible preparation for this sudden leap.

This leap on the other hand was full and complete. No reminiscence of the former condition remained. Not a single flower on the mutated plant reverted to the previous type. . . The whole plant departed absolutely from the old type of its progenitors.

The next object was to seek for other mutants from the same lot of seed ${ }^{1}$ and to compare their proportion with the proportion coming true from the seed of the first mutant.

Accordingly De Vries planted his entire remaining stock of seed, which, it will be remembered, was grown from the pair of plants one of which bore a single peloric flower, but both of which were immediately descended from the single five-spurred flower of the third generation.

From this seed he grew about two thousand plants in wellmanured soil. About I 750 of these bore flowers, and among these sixteen, or about I per cent, were wholly peloric. As these seeds were of the same generation that produced the first mutant, he concludes that the chance of a peloric mutant is not orer one in a hundred.

De Vries next undertook to determine whether the mutation would be repeated in another generation, for up to this point all the mutants had arisen from the same lot of seed. For this purpose he saved seeds from normal plants so isolated as to prevent crossing with peloric strains. In one instance he "obtained two and in another one peloric plant with exclusively manyspurred flowers," showing conclusively that mutations are iterative, and that the same conditions that produce one mutant will from time to time produce others altogether similar.

1 It will be remembered that the original stock of seed of this generation was Io cc., but that only enough had been grown to produce fifty plants, leaving a quantity still on hand. 
New type persistent. Next he undertook to determine to what extent these mutant pelorics would "breed true," in order to compare the proportion with the previously ascertained I per cent. In this he encountered difficulty because of the high degree of sterility of peloric flowers. He had in all some twenty plants, and pollinated artificially more than a thousand flowers. Of these he says :

Not a single one gave a normal fruit, but some small and rudimentary capsules were produced bearing a few seeds. From these I had ing flowering plants, out of which 106 were peloric and the remainder ( 13 ) one-spurred. The great majority (some go per cent) were thus shown to be true to their new type. Whether the 10 per cent reverting ones were truly atavists caused by stray pollen grains from another culture cannot of course be determined with sufficient certitude.

This experiment determines not only the distinctness of the new type and the suddenness of its formation, but its essential purity as well; for it bred true in 90 per cent of the cases, while the probability of original mutation was slight, certainly not over I per cent.

The total lack of intermediate steps in the control experiments is significant. Their absence in nature is not less so (for if they were present as transition steps toward the formation of peloric races, they would certainly be discovered, particularly when we remember that the species is a perennial), and the conclusion seems inevitable that the transition is abrupt, and the new type, repeatedly re-formed, is without doubt to be regarded as a true mutation.

The common snapdragon, whose flowers are exceedingly unsymmetrical, also has a peloric race. "But the snapdragon is self-fertile, and so is its peloric variety," observes De Vries. These mutations are therefore much more easily preserved, and are, as we should expect, more common than in the toadflax, so common and so distinct as, without a cloubt, to give rise to real hybrids with the old form.

What is true of toadflax and snapdragon is held to be true of unsymmetrical flowers generally; namely, a strong tendency to give rise from time to time to peloric varieties, not by gradual change of the parent stock but by sudden offset, or mutation. 
Experiments in the production of double flowers. ${ }^{1}$ After remarking that mutations occur as often among cultivated as among wild plants, De Vries drops the caution that in all experimentation of this order hybridism nuust be carefully guardecl against." He observes, too, that white varieties seem comparatively old, as they are common in the wild state, while double flowers are rare in the wild state and correspondingly recent, indicating their origin under cultivation, and thus making the matter of doubling a farorable character with which to conduct investigations upon mutation.

In the experiments upon peloric toadflax nothing new was attempted. The object was to repeat what nature was known of ten to have done, but so to control conditions as to "be there" when it happened next time.

In this experiment, however, De Vries determined to attempt a new mutation, - that is, to try to secure double flowers where they had never been observed in nature. He accordingly chose the corn marigold (Chrysanthomum sigctum), common in the grain fields of central Europe, and its cultivated variety, grandiflomm. The number of ray florets is variable in both, but is, on an average, thirteen in the wild and twenty-one in the cultivated. This indicated the latter as the more favorable for the experiment, and it was therefore chosen; but it is far from pure, for many of its heads have as few as thirteen rays. Only six out of the first lot of three hundred plants reached an average of twenty-one, and these were selected as the foundation.

The seeds of each of these were sown separately. Five gave proof of being still mixtures with the wild form and were rejected. The offspring of the sixth plant averaged twenty-one ray florets, and after counting some fifteen hundred heads the two plants were selected whose secondary heads made the best showing. The progeny of these plants also averaged twenty-one,

1 De Vries, Species and Varieties, etc., pp. 489-515.

2 If a new form is a mutant it will "breed true " to itself in the great majority of cases and perforce hybridize with the original stock. If, on the other hand, it is an ordinary hybrid, it will not breed true, but will observe the principle of Mendel's law (to be discussed later), by which a certain definite percentage is of the original types. Thus it is comparatively easy to ascertain whether a new type is the product of a single race by mutation or of two races by hybridization. 
and De Vries considered that the strain was now pure and that "no further selection could be of any avail."

One of these two plants was distinguished by producing two secondary heads with twonty-two rays, whereas generally only the terminal head reached so many as trucnty-one, the other retrograding often as low as to thirteen. This exceptional plant was distinguished only by these two secondary heads. Its terminal head had but twenty-one rays, and the average of all its heads was not exceptionally high; but no other plant out of hundreds had ever produced secondary heads with more than twenty-one rays, and it was from this plant that the doubleflowering line developed three years later.

This plant appeared in I896. Its seed was sown in I897. The largest number of rays in the terminal head suddenly increased from twenty-one to thirty-four; next year ( $\$ 98$ ), to forty-eight; next (I899), to sixty-six; and during this time the general average for all the heads increased remarkably. No indication of doubling had, however, yet appeared. The improvement was such as follows selective breeding with fluctuating variability, - improvement by gradual change and without mutations.

Late in the season (September) of this year (1899), however, three secondary heads appeared on one plant with a few ray florets scattered over the disk. The mutation anxiously awaited for seven years had suddenly appeared in this small, belated way toward the close of the growing season, and in a manner that would have escaped the attention of any but the most painstaking investigator, ${ }^{1}$ and that would have invited extermination in nature.

This was in I 899 . The heads were of course pollinated with other and inferior flowers, but in 1900 the highest number of rays rose to one hundred, and in $190 \mathrm{I}$ reached two hundred. He remarks, "Such heads are as completely double as are

1 The student will note that every flower of thousands of plants was carefully examined, and that in every case the foundation of the mutation was in an inconspicuous plant, certain to be overlooked by casual observers. The obvious lesson is that only the most careful and systematic examination will detect the foundation stock, so easily does it escape notice in the general mass and so readily is it lost unless isolated and protected. 
the brightest heads of the most beautiful double commercial varieties of composites." He adds :

The race has at once become permanent and constant. Real atavists or real reversionists were seen no more after the first purification of the race. It has of course a wide range of fluctuating variability (considering all the heads), but the lower limit has been worked up to about thirty-four rays, a figure never reached by the gramdiflurum parent, from which my new variety is sharply separated.

Unfortunately, the best heads now produced are sterile, so that seeds must be secured from inferior stock and the variety must be propagated from slightly inferior parentage. Selection has, therefore, reached its limit, unless a fertile strain arises, which is entirely possible.

This mutation is decidedly new. It had never been known, nor had anything approaching it ever been discovered in this species. The only hope that it might appear was belief in the principle, and the fact that doubling had taken place in other composita. Right royally was De Vries's prophecy fulfilled, and again was he "present" when it happened; not only that, but in this case nature evidently would not have produced this mutation without assistance. Here nature has accomplished with help a work which she was powerless to accomplish alone, but abundantly able to achieve with a little assistance.

Experiments in the production of new species. ${ }^{1}$ De Vries was not content with the simple production of varieties. He desired to show that the principle of mutation produces species as well. ${ }^{2}$ He cultivated many species of wild plants in his garden, choosing wild in preference to cultivated, because he regarded the latter as evidence of what had recently taken place and, therefore, not the best stock for further mutation in the near future. In other words, he desired to be present before the mutation

1 De Vries, Species and Varieties, etc., pp. 516-546.

$2 \mathrm{Ile}$ distinguishes sharply between varieties and species. The variety differs from the main stock in but a single character, progressive or retrogressive, while the species differs in all characters, some of which are perhaps progressive and others retrogressive. He likes to distinguish elementary species from all other types, as these are in his estimation the most stable forms in nature; and when any race assumes the "mutative state" it is likely to throw off, if conditions are favorable, a large number of new elementary species, each with its new center of variability. 
happened, rather than to enter just afterward and only in time to note results with no evidence as to methods.

Of all the hundreds of plants cultivated he found the evening primrose most fertile in distinct strains, both in the wilcl and in the cultivated state. Other species gave rise to varieties freely, but no other appeared to be sufficiently mutable to give rise freely to what he regarded as elementary species.

Of all the primroses, (Enothera Lamarckiana, commonly called Lamarck's evening primrose, was the most prolific in distinct forms, and accordingly this was chosen by De Vries for special attention in his experiments. It is described as "a stately plant with a stout stem, attaining often a height of $1.6 \mathrm{~m}$. or more. When not crowded, the main stem is surrounded by a large circle of smaller branches growing upward from its base so as to form a dense bush. These branches in their turn have numerous lateral branches. . . Contrary to their congeners, they are dependent on visiting insects for pollination."

Orclinarily this primrose is a biennial, producing rosettes in the first year and stems in the second year. Both the rosettes and the stems are highly variable in nature, producing a number of clistinct races, some of which show a marked ability to hold their own under natural surroundings, while others are too weak to endure.

Many of these De Vries regarded as new species. Experiments to determine this point were commenced with stock discovered near Hilversum, and three plans were followed: first, to transplant the apparent new species into the garden whenever the new race was sufficiently strong; second, to reproduce weak races by sowing seeds from "indifferent" plants growing wild; third, to sow the seeds from the introduced plants. "These various methods," he adds, "have led to the discovery of over a dozen new types never previously observed or described."

These new plants are divisible, according to De Vries, into five different heads or "groups" : (I) those that " are evidently to be considered as varieties in the narrower sense of the word," representing retrograde development; (2) "progressive elementary species" which are "as strong as the parent species "; (3) "progressive elementary species," but weaker than 
the parent, and "apparently not clestined to be successful" ; (4) certain forms that are "organically incomplete" ; (5) "inconstant forms."

Group (I), retrograde varieties. Of this class the following three forms were discovered, all produced in nature as well as in the garden:

$O$. leififolia, the smooth-leaved variety, constant from seed and never reverting except from crossing. As strong and fertile as the parent.

$O$. breistylis, the short-styled form. In this the ovary is so placed that it is reached by very few pollen tubes. Thus while the plant is vigorous it is but indifferently productive of seeds, and as De Vries says "many [capsules] contained no seeds at all; from others I have succeeded in saving only a hundred seeds from thousands of capsules." These seeds, however, reproduce the variety without reversions to Lamarckiana.

O. nanella, the dwarf, "a most attractive little plant,... very short of stature, reaching of ten a height of only $20-30 \mathrm{~cm}$., or less than one fourth of that of the parent." The flowers are as large as those of the parent; the leaves are much smaller and with no reversion in seedlings, even in repeated and successive generations.

Group (2), progressive elementary species, and vigorous ; two forms discovered :

O. grigas, the giant, deserving its name not from being higher than its parent, but because it is "so much stouter in all respects." The stems are often twice as thick as in the parent (Lamarkiana), and the "internodes are shorter and the leaves more numerous, covering the stems with a denser foliage." The flowers are larger, and the seed capsules are smaller and filled with fewer but larger seeds than in the parent plant. It has a strong tendency to remain a biennial.

O. mbrincris, the red-veined form. In this the veins of the leaves are distinctly tinged with red and the fruits are streaked with red. The plants are in many ways a counterpart of the giant, except for the red tinge and distinctly lighter foliage. This latter probably accounts for the marked tendency on the part of this form to become an annual. Like the giant, this form 
is true to type when grown from the seed, and its recurrence is far more common than is that of grigas, which is extremely rare.

Group (3), progressive elementary species, but weakly. Two forms :

$O$. albida, the albino, with whitish, narrow leaves, "apparently incapable of producing sufficient quantities of organic food." The scedlings are exceedingly delicate, and if left to themselves will be speedily overgrown by their more vigorous neighbors; but if transplanted and given the best of care, they make fairly vigorous plants the second year, comparing fairly well with the parent stock but bearing fewer seeds. They come true even to the third generation and the type remains distinct.

O. oblonga, the narrow-leaved form. It " may be grown either as an annual or a biennial. In the first case it is very slender and weak, bearing only small fruits and few seeds. In the alternative case, however (biennial), it becomes densely branched, bearing flowers on quite a number of racemes and yielding a full harvest of seeds."

The investigator says :

We have now given the description of seven new forms which diverge in different ways from the parent type. All were absolutely constant from seed. Hundreds or thousands of seedlings may have arisen, but they always come true and never revert to the original $O$. Lamarckiana.

He adds the remark that they have inherited the condition of mutability to some extent and are evidently themselves able to produce new forms, but that they do so but rarely.

Two other forms belong to this group, - O. scmilata and O. Icptocarpa, - but their characters do not merit special description.

Group (4), forms organically incomplete :

$O$. lata is a pistillate variety, wholly dependent for fertilization upon other forms, and it had therefore no opportunity to establish its type, which, however, freely appeared. It is a "low plant," but with "dense foliage and luxuriant growth." Its presence can be detected in the seedling by the "broad, sinuate leaves with rounded tips." Being pistillate, it produces seed only when cross pollinated, in which case its characters are 
transmitted to a portion only of its offspring, thus behaving like hybrids. Indeed, he specifies that "on the average one fourth of the offspring are lata," the others assuming the character of the pollen parent," - a strict example of hybridism between a weaker and a stronger form, according to Mendel's law.

Group (5), inconstant forms :

$O$. scintillans is a perfectly fertile form, bearing smooth, darkgreen leaves with glistening surfaces. It is a natural dwarf, easily cultivated as an annual. When fertilized with its own pollen to produce a "pure" strain, it is found that the seedlings all resemble the parent, but that soon afterward they diverge into various types. Some of these resemble the original parent stock (Lamarckiana) and others remain pure, but the proportion is very variable. These might be regarded as simple reversions, except that occasionally other types appear, especially oblonga, lata, and nanella, the first often constituting io per cent of the sowings. It thus shows a disposition to give rise to the same distinct forms as does its own parent, and is thus regarded by De Vries as being itself in a "highly mutable state."

O. clliptica is a narrow-leaved, inconstant type, exceedingly "clifficult of cultivation." Though fertile to its own pollen, it "repeats its type only in a very small proportion of its seeds."

There are thus "a dozen new types springing from an original form in one restricted locality and seen to grow there, or arising in the garden from seeds collected from the original locality.' Most of these types behave with a constancy that ranks them, for breeding purposes at least, as clistinct forms, good elementary species, - new things in the earth that may be held constant or that may be slightly modified by the exercise of selection among the fluctuations to which all types both old and new are subject. The experimenter observes :

It is most striking that the various mutations of the evening primrose display a great degree of regularity. There is no chaos of forms, no indefinite varying in all degrees and in all directions. On the contrary, it is at once evident that very simple rules govern the whole phenomena.

History of the experiment. In all De Vries made four different series of pedigree cultures of the evening primrose, extending from five to nine generations and including thousands of 
plants. The types that arose at different times have already been described, but considerable interest and no little profit attaches to the details of the experiment, especially in regard to the order and manner of the appearance of the new types. The following is abstracted from the experimenter's account of one of these four experiments, running through eight generations. ${ }^{1}$

Beginning in the fall of 1886 he took nine large rosettes of $O$. Lamarkiana from the field and planted them in the garden. The second generation was sown in I8S8 and flowered in I889. The seed produced fifteen thousand seedlings, of which ten were divergent at once, - five lata and five manella. No intermediates appeared. "They came into existence at once," says De Vries, "fully equipped, without preparation or intermediate steps. No series of generations, no selection, no struggle for selection was needed. It was a sudden leap into another type, - a sport in the best acceptation of the word." 2

The third generation of ten thousand plants showed three lata and three nanella, besides one mbrinervis.

Growing expert in detecting mutants at an early stage, he discovered 334 young plants out of 14,000 of the fourth generation (1895). This is about 2.5 per cent. Of these I 76 were oblonga, 73 lata, 60 nanella, I 5 albida, 8 mbrincris, I scintillans, and I gigas.

The larger number and wider range of mutants discovered this year are to be ascribed to growing skill in detecting them at an early age. Manifestly such immense numbers must be greatly reduced at the earliest possible date, and without doubt some good forms were overlooked in the earlier generations. After this (fourth) generation the number of seedlings was greatly reduced, with the effect of reducing the number of mutants and also the chances of the rarer forms appearing at all ; indeed, gigas never appeared again, and sintillans not after

1 De Vries, Species and Varieties, etc., pp. 549-556.

${ }^{2}$ It may occur to the student to object to the conclusion on the ground that the parent stock taken from the field may not itself have been pure. If, however, the stock had been in any sense hybrid, the departures should have been, according to Mendel's law, more than ten; but not in this or in later generations did either parent stock or mutant behave like a hybrid in this respect. 
the sixth generation. The entire results of the eight generations of breeding are given in the following table.

Eight Generations of a Mutating Strain of Evening Primrose (O. Lamarckiana)

\begin{tabular}{|c|c|c|c|c|c|c|c|c|}
\hline $\begin{array}{c}\text { GENEIRA- } \\
\text { TIONS }\end{array}$ & $\begin{array}{c}0 . \\
\text { gigras }\end{array}$ & Albida & Oblongra & $\begin{array}{l}\text { Rubri- } \\
\text { nervis }\end{array}$ & $\begin{array}{l}\text { Lamurik- } \\
\text { iana }\end{array}$ & Ninetha & Lata & $\begin{array}{l}\text { Sintil- } \\
\text { lans }\end{array}$ \\
\hline I & & & & & 9 & & & \\
\hline II & & & & & I 5,000 & 5 & 5 & \\
\hline III & & & & I & 10,000 & 3 & 3 & \\
\hline IV & I & I 5 & 176 & 8 & 14,000 & 60 & 73 & I \\
\hline $\mathrm{V}$ & & 25 & I35 & 20 & 8,000 & 49 & 142 & 6 \\
\hline VI & & II & 29 & 3 & I , 800 & 9 & 5 & I \\
\hline VII & & & 9 & & 3,000 & I I & & \\
\hline VIII & & 5 & I & & 1,700 & $2 I$ & I & \\
\hline
\end{tabular}

In the opinion of the experimenter here are numbers enough and types sufficiently distinct to warrant the enumeration of certain laws or principles that appear to govern the appearance of mutants, especially in the species under observation. This De Vries attempts to do, but without presuming to say how closely they may apply to other strains of plants or animals.

Laws of mutability for evening primroses. De Vries' experiments. On the basis of his experiments with the evening primrose the investigator announces the following laws of mutability as applying to that species : ${ }^{1}$

I. "That new elementary species appear suddenly, without intermediate steps." As proof he points out that no intermediate forms appeared to fill the gaps, and that no selection was necessary to establish the type.

2. "New forms spring laterally from the main stem." "The current conception concerning the origin of species (or new forms generally) assumes that species are slowly converted into others. The conversion is assumed to affect all the individuals in the same direction and in the same degree. . . The birth

1 De Vries, Species and Varieties, etc., pp. 558-575. These laws, while announced for the evening primrose, are without doubt of wide if not general application. 
of a new species necessarily seemed to involve the death of the old one," at least the old merged into the new.

The experimenter points out, however, that through all the process of originating a dozen or more distinct forms, the parent stock continued unchanged, and still constituted the principal strain of all the primroses, ${ }^{1}$ and from this he deduces the law that mutants are laterals.

3. "New elementary species attain their full constancy at once." "Constancy is not the result of selection or of improvement. It is a quality of its own. It can neither be constrained by selection if it is absent from the beginning, nor does it need any natural or artificial aid if it is present."

De Vries remarks that scintillans repeats its characters in but part of its offspring, and that he has "tried to deliver it from this incompleteness of heredity but in vain. . . The instability seems to be here as permanent a quality as the stability in other instances. Even here no selection has been adequate to change the original form." He regards it as itself in a state of instability.

4. "Some of the new strains are evidently elementary species, while others are to be considered as varieties."

Elementary species are regarded as possessed of progressive characters, but varieties as differing from their parent stock in but a single character, and that in the way either of an assumption or of a loss. The elementary species is, therefore, a new agrgregation of characters, while the variety is simply the old form minus a single character. Whether this distinction holds, remains to be cletermined. Much of the argument turns upon what is to be considered as a character and when it is lost. For

1 A natural corollary to this observation is to remark upon the erroneous popular assuniption that of similar and contemporaneous forms the more primitive are necessarily the progenitors of the more nearly perfect. For example, it is hastily assumed that if evolution is true then man must be the direct descendant of the ape. But the ape, though very old, is still an ape, and he is not descending into anything but apes. Though evidently developed from the same original stock at some time and in some way, whether by one or by many mutations nobody knows, yet the gap between us is evidently fixed and not growing less or being bridged at any point. Good evolution regards related forms as connected by ties of consanguinity, but whether direct, or, what is more likely, indirect, running to some extinct common ancestor, only a novice will attempt to say. 
example, is the smooth leaf or stem considered as having lost a character as compared with its downy relative?

5. "The same new species are repeatedly produced," that is to say, the same new forms arise again and again, showing that the tendency to their production is inherent and persistent. "This is a very curious fact," remarks De Vries. "It embraces two minor points, - the multitude of similar mutants in the same year, and the repetition thereof in succeeding generations. Obviously there must be some common cause. This cause must be assumed to lie dormant in the Lamarckians of my strain, etc. . . . The germs of the oblongar, lata, and nanella are very irritable and are ready to spring into existence at the least summons, while those of gigas, mbrincris, and scintillans are far more difficult to arouse." May not the same be true in nature generally, and may not the same strain arise again and again, commonly failing to persist because as a rule all conditions are against it?

6. Mutability is distinct from fluctuating variability. Darwin regarded the new type as built up by the operation of selection upon fluctuating variability, establishing a new type by the gradual accumulation of farorable variation, all others (intermediates) being exterminated. De Vries regards mutability as distinct from fluctuating variability, and considers that he has presented experimental evidence to show that it is entirely competent to give rise to new forms suddenly, without intermediates and without the aid of selection. I Ie of course believes that all types, both old and new, are subject to fluctuating variability, and that through selection some improvement is possible, but that this is not the sole or principal method of securing new types.

7. "The mutations take place in nearly all directions." Some are larger, some are smaller than the parent; some stronger, others weaker; some plainer, others more brilliant. The species is not, therefore, drifting; it is sending out new types from all sides.

\section{SECTION IV - AMERICAN EXPERIENCES}

The experiments of De Vries are strongly confirmed by the experience of breeders, especially in the production of new varieties of fruits and regetables. Many of these have been so long 
uncler cultivation that nothing is known of their origin. Of others, on the contrary, the life history is well known.

When Europeans peopled America they naturally brought with them their fruits, their vegetables, their grains, their grasses, and their clomestic animals. The new country was rich in native species, both plant and animal, but the European species had the advantage of being better known and better adapted to the special needs of man. Accordingly, wherever the introduced varieties succeeded, the corresponding native types were neglected; but when the European varieties failed, then the natives were developed. It is from this latter class that some important observations may be made. ${ }^{1}$

The gooseberry. ${ }^{2}$ The large English gooseberry was too tender for the American climate, and withal was exceedingly liable to milclew. Native varieties flourished widely in the forests. Unfortunately the varieties bearing the largest berries were exceedingly thorny, both on bush and fruit. Side by side, however, with these prickly sorts were smooth varieties, free from "prickers" both on fruit and bush. These were freely transplanted to the gardens of the pioneers and furnished an acceptable fruit. ${ }^{3}$ In good time they developed improved sorts, — first the Houghton, a seedling originated by Abel Houghton of Lynn, Massachusetts, sometime in the early forties. Then came the Downing, a seedling of the Houghton, first described in 1853 , the fruit of which is said to be "the largest yet known, being about twice the size of the I Ioughton's seedling, its parent; it is pale or light green, without any blush, and smooth. The skin is very thin and the fruit as delicate and tender as any European gooseberry on its native soil. The flavor and aroma are perfect."

Bailey observes in this connection, "This berry, now known as the Downing, is the standard of excellence in American gooseberries, and is probably grown more extensively than all other varieties combined; and yet it is only two remores from the wild species."

${ }^{1}$ L. H. Bailey, Evolution of our Native Fruits [The Macmillan Company, New York].

2 Ibid. pp. 389-399.

3 The writer remembers very well as a boy searching the woods, and especially the swamps, of Michigan for these smooth varieties for transplanting. 
Is not this a natural mutation in the truest sense of the term ? If not, then it is merely a question of teminology and definition. The fact remains that it arose sucklenly, a distinct type, and remains true with no characteristics of a hybric. We need a term for this sort of thing which is occasionally occurring everywhere in nature, in our gardens and in our herds, and I know of none better then the one already in use - mutation.

The strawberry." The wild strawberry grew everywhere in northern North America. There were not only many distinct types of the red, but, like the native raspberry and the blackberry, it had everywhere its albino race. Good progress had been made in the cultivation of the native strawberries, and without doubt good varieties would in time have developed; but the intreduction of the Chilean berry (the parent of most present varieties) seems to have put a stop to this. The most promising of all native strains was the Fragaria Chilocnsis, a native to Oregon and the Pacific coast; but, as Bailey observes, "the garden progeny of its South American branch is already so good that there is little reason for returning to the wild for a new start." Here is a curious instance of the successive supplanting of varieties. European sorts were vanquished by developments of New England natives. Then the wild type of Oregon came into the struggrle and threatened to supplant them both, for it was full of promise. But its prosperity was its own defeat, for its own Chilean brother has now supplanted everything in that it is the stock which is furnishing our improved varieties. Any student of this subject will recognize the comparative readiness with which these new types spring up.

The blackberry.2 The blackberry grows wild both in America and in Europe, but is said to be cultivated only in North America. It is not more than fifty years since improved varieties were introduced, and its real cultivation dates only from about i 875 .

There are two principal types of the wild blackberry growing in the northern United States: (I) the "high bush," long and luscious, loving the shade, represented in its cultivated types, according to Bailey, by the Taylor and the Ancient Briton;

1 L. H. Bailey, Evolution of our Native Fruits, pp. 424-432.

2 Ibid. pp. 298-330. 
(2) a smaller variety growing in sumny, open places and bearing small, round, losse-grained fruits, ripening late and exceedingly sour. This type is represented in cultivation by Lawton, Kittatimny, Snycler, Agawam, Erie, and others. Neither of these yielded readily to cultivation and restraint, and this fact served in an early clay to earn an evil reputation for what Professor Card calls this "gypsy of the fruits." Nevertheless, they yiclded to persistent efforts, and have given rise, as Bailey puts it, "to a lost of varieties. . very many of them wildings, or chance bushes found in fence rows."

The first-named variety was the Dorchester, introduced about I $S_{4}$. Its exact origin is unknown, though its originator (probably Captain Lovett) is known to have transplanted wild plants for many successive years. Whether this first civilized gypsy was a sport or simply a strain improved by selection is not now capable of proof, and yet its constancy is good presumptive evidence.

Wilson's Early was known in $S_{54}$, the Holcomb in 1855 , and in I 857 the Lawton (first called New Rochelle) was introduced, being at once declared superior to the Dorchester. Of these the Wilson was "discovered in the wild about I $\$ 54$ by John Wilson of Burlington, New Jersey" ; and the Lawton, formerly New Rochelle, "was found in the town of New Rochelle, New York, by Lewis A. Secor." These two strains have given rise to numerous distinct modern varieties. The "loose-cluster" strains are regarded by horticulturists as the descendants of the IVilson. The origin of certain other varieties seems to be as follows:

In I870 Mr. William Parry, of New Jersey, "selected a healthy young I)orchester and planted it in the same hill with a strong, healthy WVilson's Early (for breeclers), located far away from any other blackberries." 1 In IS75 the seed from some of the largest berries growing on the Wilson were planted. One plant only was regarded as valuable, and all others were destroyed. This new strain was named VVilson Junior. The fruit was "large, early, and very fine," and so prolific that in I 884 "one acre yielded I IO., bushels of fruit, by the side of five acres of IVilson's Early in the same field, with the same culture, which areraged

1 Bailey, Evolution of our Cultivated Fruits, p. 3 I6. 
but 53 bushels." The Eureka was produced in exactly the same way in I $\$ 77$. In I 879 Rioter and Farmer's Glory were also produced from berries growing on the Wilson, and Gold Dust and Primordian from berries growing on the Dorchester. The Gold Dust was remarkable for the fact that its entire crop ripened within a period of four clays. It was thus distinct from all other blackberries in at least one important character.

The Sterling Thornless arose as a chance seedling of the IVilson on the farm of John Sterling at Benton Harbor, Michigan. It is, as its name indicates, destitute of thorns, and is a distinct mutation, to be carefully distinguished from other strains of thornless blackberries, which, according to Bailey, are "specifically distinct from the common bush blackberry."

Plums. ${ }^{1}$ According to Bailey not a single commercial variety of plum has ever originated from the native stock of New England, New York, Pennsylvania, or Michigan. This is partly because the European sorts thrive well and partly because the natives of this region "are less prolific of large-fruited forms than those farther west."

Some excellent varieties have arisen, however, from native stock elsewhere. The Miner was produced from seed of native stock planted in I $8 \mathrm{I} 4$ by William Dodd in Knox County, Tennessee. The Robinson was a seedling from North Carolina stock. Wayland "came up in a plum thicket in the garden of Professor II. B. Wayland of Cadiz, Kentucky," and was introduced about 1876 . The Missouri apricot was found wild in Missouri. The Golden Beauty was found in the same way in Texas, the Pottawattamic in Tennessee, and the Newman in Kentucky.

The Wolf originated from seed gathered from wild trees in Iowa. The Pottingstone was found on the bank of Pottingstone Creek, Minnesota, and the Quaker was found wild in Iowa. Literally scores of well-clefined varieties have arisen from native stock. It would be too much to say that none of these are hybrids. Undoubtedly many of them are the product of crossing, but this origin cannot be consistently claimed from chance seedlings found in a thicket of ordinary wild stock. Mutation, whatever

1 Bailey, Evolution of our Native Fruits, pp. I70-226. 
it is, must be credited with having produced many new forms spontaneously.

Grapes. ${ }^{1}$ " North America is a natural vineyard," says Bailey, and yet with the most skillful and persistent attempts to cultivate the European varieties for wine making, they have not succeeded. Under these circumstances nothing is more natural than that valuable native varieties should arise, providing the capacity was inherent in the species.

John Adlum wrote, about 1 \$23, "The way is to drop most kinds of foreign vines at once and seek for the best kinds of our largest native grapes." He is to be remembered for the introduction of the famous Catawba, which was "found wild in the woods of Buncombe County in extreme western North Carolina in $\mathrm{I} 8 \mathrm{O} 2 . "$

The Catawba is, therefore, almost certainly a sport of the wild grape growing in profusion in that region. In I 843 came the Diana, a seedling of the Catawba.

In I $S_{40} \mathrm{Mr}$. E. W. Bull bought a house in Concord. Some seedlings of wild grapes sprang up about it, one of which fruited in I843. It was so excellent in quality that all others were destroyed and the new variety was named the Concord. This seedling has since given us the Worden, Moore Early, Pocklington, Eaton, and Rockland, of which the first has long been famous. The Concorl, itself a mutant, seems to have been peculiarly rich in possibilities for still other races.

"In the year I 82 I I Ionorable Hugh White, then in the junior class in Hamilton College, New York, planted a seedling vine in the grounds of Professor Noyes, on College Hill, which still remains, and is the original Clinton."

These are only a few of the many varieties of grape of American origin, tracing directly to wild native stock.

Lost possibilities. Had other domesticated plants and animals brought from Europe succeeded less admirably, what enrichment might have come through the native flora and fauna of America!

The prairie chicken would have been improved if the domestic hen had not succeeded. The turkey was a new thing and was therefore seized upon. The buffalo would not now be extinct

${ }^{1}$ Bailey, Evolution of our Native Fruits, pp. I-126. 
if cattle had acclimated less successfully. Native grains other than maize would have been dereloped had it not been for this competition, and native grasses have not lived up to their possibilities. This is through no fault of theirs, though we still lack " the best American grass."

\section{SECTION V - ECONOMIC SIGNIFICANCE OF MUTATIONS}

Because of the waywardness of sports, - the impossibility of predicting their appearance, the readiness with which they disappear when interbred with the parent stock, and their very frequent inability to reproduce at all, - because of all these considerations it has become fashionable to declare sports in general to be of slight economic importance and umworthy the breeder's serious attention. The only course left open for improvement is, therefore, the slow one of gradual accumulation through selection of minute but favorable variations, according to the theory of Darwin.

The best of evidence exists, however, for believing that this is a hasty and unwarranted conclusion, and that many, if not indeed most, of our really valuable new types have arisen suddenly as mutations and not gradually through infinitesimal differences, as is commonly supposed. The experiments of De Vries and the American varieties of fruits both come near enough to the origin of types to more than warrant this view of the situation and to afford ground for the greatest hope that unsuspected possibilities still exist in many if not most donesticated species, - possibilities of spontaneously giving off varieties representing essentially new combinations of the characters of the species and consequently possessed of different and perhaps enhanced economic value. The work of Luther Burbank ${ }^{1}$ and of our commercial seedsmen add confirmation to this hope, which, if well founded, promises new methods in breeding and vastly increased possibilities for improvement.

The small numbers involved in animal breeding reduce enormously the chances of mutations appearing; and yet nearly every

1 W. S. Harwood, in The Century Magazine, March and April, 1905; also New Creations in Plant Life [The Macmillan Company, I906]. 
species has thrown off its albino variety, which in most cases is easily propagated. Hornless cattle occasionally appear in nearly all breeds, and the type is comparatively easy of preservation. It is more than likely that the different types in the larger breeds, which breeders find so difficult to break up, are in reality quite distinct.

In future chapters dealing with the measurements of variation and the statistics of heredity in general, it will appear that even fixed types afford sufficient deviation to keep a breeder busy with selection; in other words, that the animal breeder dealing, as he is, with small numbers will always find sufficient variation to lead him to suppose that he is getting results of his selection even when he has not shifted the center of variation the slightest. Much that passes for breeding is nothing more than this ineffectual multiplication, and it is not too much to say that hundreds of breeders and thousands of animals have lived and died without affecting the breed in the slightest.

The writer is strongly of the opinion that while selection is a powerful agent for "shaping up " and "finishing off " a fairly acceptable type, and while it is the only means of deciding what shall live and what shall clisappear, yet much of the real advance in both animal and plant breeding is likely to come through distinct offsets which are now called mutations, and which in Darwin's time and until recently were erroneously, if not reproachfully, denominated "sports."

\section{SECTION VI - BIOLOGICAL SIGNIFICANCE OF MUTATIONS}

Too much mystery has surrounded this matter of sports, and there has been a too ready tendency to evoke the aid of latent characters to explain this and almost every other unusual phase of evolution.

In truth, there is no more mystery about mutations than about heredity in general, which is a complication of mysteries. It is not a question of latency but of relative prominence of characters, of the possible loss of a racial peculiarity, or, what 
is more likely, a new combination of the elements out of which characters are made up.

Every new being is the result of a new combination of racial faculties transmitted from two family lines, possibly differing in essential particulars. This new combination is certain to throw some characters into prominence and others into the background, and results occasionally in strikingly new effects. This is usually the case in hybridization, but it follow's in less degree in ordinary reproduction, which cliffers from hybriclization more in degree than in kind.

Again, many characters, though exceedingly noticeable, rest after all upon a comparatively simple basis. Such, for example, is pubescence in plants, which depends upon the activity or non-activity of a few cells in developing a hairy growth. Nearly all species present both forms, - the one in which the character is present, and its opposite in which it fails to develop. Similarly, almost any character may fail, giving rise to a distinctly new creation. If the failure is not at a vital point it may be transmitted, in which case a new type has arisen.

The origin of a new type by the addition of a character is, biologically considered, much more complicated and difficult of understanding; yet even this is not beyond some degree of comprehension. The probability is that what we call racial characters are less complicated than we may at first suppose. The unlearned savage could scarcely beliere that the almost infinite variety of colors of natural objects are due to different combinations of very few primaries. The effects produced by three-color printing are almost beyond belief, yet we are fully advised as to the real basis for all these variations; while the effects are striking, the means are simple.

So it is, we may imagine, in the ultimate make-up of what we call racial characters : their elements are doubtless fewer than we have supposed, and the possibilities of their combinations and recombinations are greater than we have hitherto imagined. Whether all possible combinations of these elements actually take place we do not know, but all facts go to show that they occur in great variety, the most striking and permanent of which we call mutants. 
If a new race is produced by hybriclization, then a new combination of characters has been effected, and it is fair to assume that the combination is richer in possibilities and possesses a larger number of characters than did either parent. Mutation teaches that new assortments of characters may take place, in some cases at least, without hybridizing.

If a racial character, as color or hairiness, is lost, we recognize the new type and name it as a new creation. It may be more valuable to us than its parent, but it must be recognized biologrically as having lost something to which it was racially entitlecl.

Again, if all normal characters acquire an unusual development, relatively or absolutely, as in giants, or if their development is abnormally arrested, as in clvarfs, we again recognize the new departure, and it is a good mutation.

Still again, if certain characters only undergo change in development, while others remain normal, then relative values are changed, the effect is altered, and we recognize a different type. This, too, is a good mutation, provided the new relation persists. All these changes can be worked with the normal characters of the race, without the introduction of new characters or even the supposititious aid of latent characters. Soberly considered, these changes are none other than the student of biology would expect, unless indeed racial characters are bound together much more rigidly than present evidence would lead us to suspect.

Summary. Not all variations are continuous and connected with the type by insensible differences. Some deviations are discontinuous, with a tendency for future variations not reverting to the main type but clustering about a new center of variability, thus setting up a new type. Such a deviation is called a mutant, and new strains may arise in this manner, as well as by the slower Darwinian method of heterogeneous variation, out of which new types are established by the slow process of selection.

Both the experience of breeders - especially with new varieties in America - and numerous instances of experimental evidence show conclusively that new strains not only may, but in actual practice do, orisinate in this manner, suddenly and completely, without any apparent preparation and with little tendency to revert to the original or main type, which continues as before 
Mutants, like their parent types, are subject to fluctuating variability, which is a necessary law of reproduction, and they may be improved - that is, shaped up - by judicious selection, but their principal characters and main trend were fixed when the type arose.

\section{ADDITIONAL REFERENCES}

Arbisisu. (A critical study of its causes.) By E. Pantanelli. Experiment Station Record, XV, 55 .

Atavic Mutation of the Tomato. By C. A. White. Science, XVII, $76-78,234-235$.

Atavism in the Potato. By S. Rhodin. Experiment Station Record, XI, 7 IO.

Determinate Mutations. (De Vries and others quoted.) By M. M. Metcalf. Science, XXI, 355-356.

Evolution and Adaptation. By T. H. Morgan. Science, Xix, $221-225$.

Evolution without Mitation. By C. B. Davenport. Science, XIX, 215. Inheritance of Monstrosities. (Experiments of twelve years.) By Hugo De Vries. Experiment Station Record, XI, 546.

Mutation and Selection. (What causes mutations? Are they all in one direction?) By M. M. Metcalf. Science, XIX, 75-76.

Mutation in the Tomato. By C. A. White. Science, XIV, S4I-S44. Mutation Theory. (A review of Species and Varieties.) By (. B. I)avenport. Science, XXII, 369-372.

Mutation TheOry of DEVRIFs. (Twenty-eight lectures by the author at the University of California, 1904.) Experiment Station Record, $\mathrm{XVI}, 745$.

Mutation Theory of DeVries. By D. T. McDougal. Experiment Station Record, XIII, 324-619; XIV, 226, 526 .

Mutation Theory of Organic Evolution. (A brief but pointed survey of the subject.) By W.E. Castle of Harvard University. Science, XXI, 52I-543; from standpoint of animal breeding, 52I-524; from standpoint of cytology, 525-528.

Mutations in Plants. By D. T. McDougal. American Naturalist, XXXVII, 737-770; also in Experiment Station Record, XVI, 23.

Origin of Species. By Hugo De Vries. Science, XV, 72I-729.

Origin of Species through Selection contrasted with their Origin through Appearance of Definite Varieties. By T. H. Morgan. Popular Science Monthly, LXVII, 54-66.

Prepotency of Individuals with Abnormal Variation or MutaTION. (A study of cats with extra toes.) By H. B. Torrey. Science, XVI, 55t-555. 
Some Causes of Saltatory Variation. By C. H. Eigenmann. Proceedings of the American Assaciation for the Advancement of Science, 1900, XLIX, 207.

SPorTs. (Author concludes there are other laws than Mendel's and Galton's.) By C. B. Davenport. Science, XIX, I5I; also in Experiment Station Record, XV, 753.

SPoRTS on Grapevines. By J. C, Talback, Experiment Station Record $\mathrm{XV}, 478$.

SPORTS; THE PEACH-Nectarine. Journal of the Royal Horticultural Society, XXYI, 596-59S; also in Experiment Station Record, XIV, 45.

The Mutation of Lycopersicum. By C. A. White. Popular Science Monthly, LXVII, I 51-162.

The Mutition Thlory. (A defense.) By Thomas L. Casey. Science, XXI1, 307-309.

The Origin of a White Blackberry. By Luther Burbank. Experiment Station Record, XIV, IO7I.

Theory of Mutations. By A. A. W. Hubrecht. Popular Science Monthly, LXV, 205-223. 


\section{Part I I - Causes of Variation}

\section{INTRODUCTION}

Variation is at once the most promising agent for improvement and the most powerful and subtle force for undermining and destroying what has already been attained. Because of this and with a view to their possible control, the breeder is especially interested in the cause's that lead to deviation in plant or animal characters.

It is said that it is yet too early to inquire into the causes of variation, because our stock of accurate knowledge is too limited to permit a settlement of this most complicated question. That the matter cannot be fully settled in the present state of knowledge is beyond question, but the writer does not share the opinion that discussion at this stage of proceedings is unprofitable.

The student of general evolution may well assume the rôle of a curious but disinterested observer, note what passes before his eyes, and take his choice as to the questions that shall engage his attention. Not so with the farmer and breeder. His funds are tied up in his animals and his plants. He is breeding them not for amusement but for profit, and he is interested in results not thousands of years hence but in those that may be confidently expected within the limits of a lifetime.

Ile above all men, therefore, is interested in variation and the causes that induce it; and we are bound in his interest to study the question assiduously, to determine what is known and what is not known on this most important point, and to indicate as well as we are able the direction from which further light may be expected. To this end everything is important that is connected with variability in a causative way, whether its effect is upon either the form or the function of living matter. 


\section{CHAPTER VII}

\section{THE MECHANISM OF DEVELOPMENT AND DIFFERENTIATION}

Before specific inquiries can be profitably made into the causes of variation it is necessary to become fairly familiar with what is known of the essential comstitution of living matter and of its manner of growth and differentiation.

After attention has been bestowed for a time upon these consiclerations, it will be evident to the student that here, in the inner workings of living matter, are fundamental causes of profound variations, even in protoplasm seemingly the most stable.

\section{SECTION I - PROTOPLASM THE PHYSICAI, BASIS OF LIFE}

Protoplasm is a greneral name for all matter that is endowed with life, but the student must never forget that the biologist, like the chemist, is dealing with matter composed of well-known chemical elements united in definite proportions. The known differences between living and non-living matter are, for our purposes, the following :

I. Living matter is endowed with a mysterious force called life.

2. Matter so endowed has a much more complicated chemical composition than has non-living matter, or than can be maintained after the life principle has departed. Living matter at death, therefore, breaks up (or down) into ordinary chemical compounds. The true constitution of living matter cannot, therefore, be determined by any known methods of analysis, which reveal only the elements involved but not their exact relations during life.

3. Matter enclowed with life is able to appropriate to itself other outlying matter and to increase its bulk through growth. 
4. This growth is not of bulk merely, but it is attended by differentiation, so that one part is distinctly different from another.

5. As growth proceeds bits of this bulk are thrown off, each of which constitutes a new individual capable of independent existence, - reproduction.

6. The new individual is substantially, but never exactly, like the one from which it arose, and here lie the chief mysteries of breeding. In reproduction there are no duplicates.

Nothing approaches this in the inorganic world save crystallization. Crystals add matter to their bulk and thus may be said to grow. Moreover, the matter is added in an orderly manner, resulting in a kind of definite structure with exact angles always the same, but nothing like differentiation exists. One part of the crystal is like another; it has no power of reproduction and is possessed of no force comparable with life.

The student should early learn that the field of biology is distinct, but he should also fully realize that it lies within and not outside the range of chemistry, and that living matter is not freed from its ordinary affinities by reason of its association with life, but on the contrary it continues as before to be subject to the ordinary physical and chemical relations of matter generally. If he can do this, he will simplify many of his difficulties.

\section{SECTION II - THE CELL THE UNIT OF STRUCTURE}

If a bit of liver, bone, wood, or any other form of plant or animal tissue be examined under the microscope, it will be found to possess a definite structure, and to consist of a large number of separate divisions, each filled with a gelatinous mass called protoplasm. These separate divisions or cells are apparently alike throughout the substance of any particular tissue, - as the liver, - but they differ greatly in different tissues of the same body (bone, muscle, brain). Biologists have been unwilling to consicler the individual as the unit, because he is too large and his structure and activities are too complicated. They have, therefore, chosen to regard the individual as a colony of many and variously differentiated cells. 
In assuminer 1lue cell as a unit many structural clifficultices

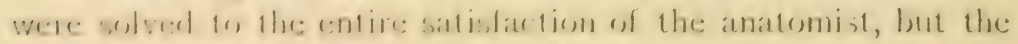

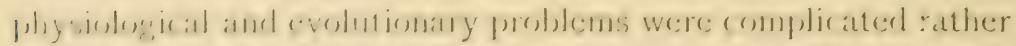

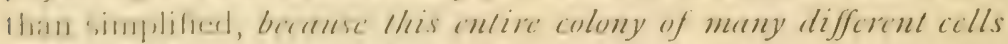

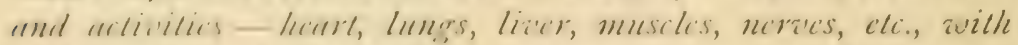

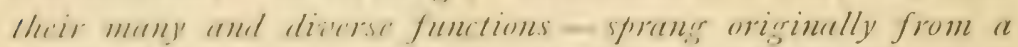
sinele wh: merenver, this colemy will throw off a succession of

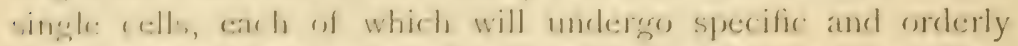

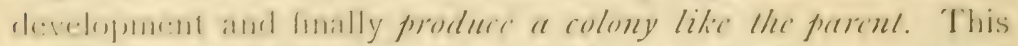

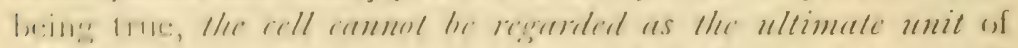
living naller, moleses we atsome: some: kind of unity between all 1hu: r. Ils ; some: lamel of intercellubar forre: to insure that differcondiation shall talie: place at the: proper perints and stages, wherwise the original cell would develop inter a lump of protoplasm or into a colony of cells all alike.

The simberell fornt which a new individual is an revelop

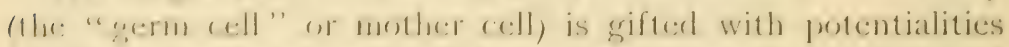
for Hhe: entire lxeing, with all its complications of structure and with all its variety of function. Biologists at one time were

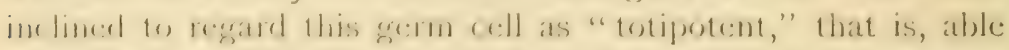

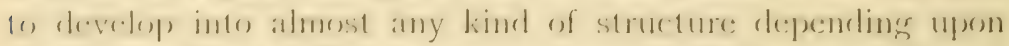

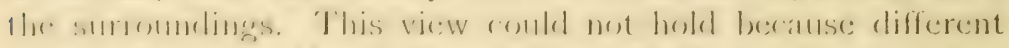

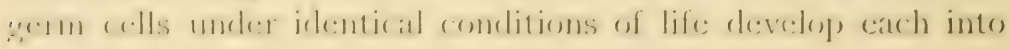
its own species.

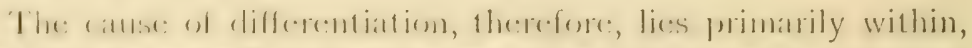

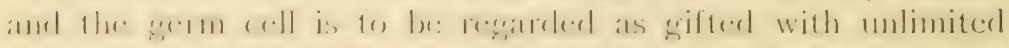

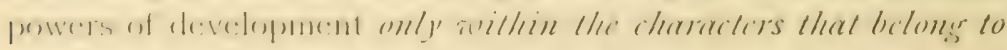
llec species.

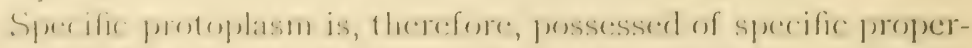

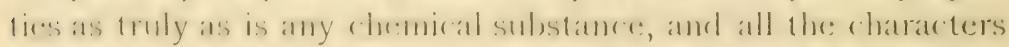

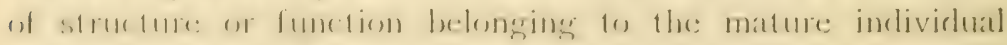
are to be regarded as in some way "inherent in the germ." The cell is, therefore, like the individual, too large and too

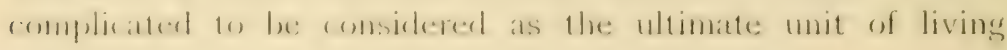
matter.

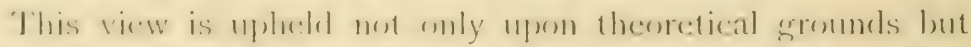
alse by the known facts of its complleated struefure and its rematrable: behaver doring all division and groweth, a subject 
which it were well to consides before proceceling further with the search after the "ultimate unit of living matter," and therefore of groweshe of differentiation, and of varialsility.

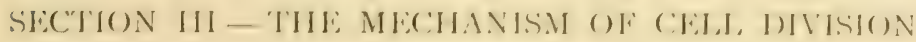 (MITOOSIS)}

Growth in the sense of increase of size is the direct result of cell division. I atre bodies do not have liaree alls than smatl ones, but they have more of them. Growth is, therefore, in proportion a cell division, - the mechanism of which is esceedingly suggestive of the methoeds by which lines of descent are: preserved and the proper development assured.

When the protoplasm of an ordinary growing cell, plant or animal, has absorbed material until it has rearthed at certain maximum size, it then prepares for division. This is mot at lump) division in which the new cells cach get one half of the: bulk of the parent cell, but it is qualitative as well as quantitatioe, and is based on an exceedingly orderly procedure, which insures not only that each datughter cell shall receive its shate of the mass but also that this share shall be irlentical in glatity with that inherited by the sister cell of the same division.

Those portions of the cell contents most intimately concerned in the process of clivision, and therefore of chief interest here, may be briefly described as follows:

Fleating in the gencrat protrolasmic mass (the cytoplasm) is a small body (the nucleas) of greater density thats its strmomel. ing matter and the evirlent seat and initial proint of all construr tive processes.

Scattered through the mass of the nucleas and semerally, but not alwalys, in the from of minute cranules is the sor alleal "chromatin matter," natued from its intense reation to statinum agents.

These granules of which the chromatin natter is (alp parently) cromposed are the "chromatin gramules" of some anthros, the

1 The word "apparently" is inserted because the granular character of chromatin matter has not in every case been made out, and because its granular character is less pronounced at some times than at others. 
"chromomeres" of others, the "microsomes" of still others, and the "ids" of Weismann.

Lying generally in the cytoplasm just outside the nucleus will commonly, but not always, be found an extremely minute highly staining body, the centrosome, about which, when division is about to occur, the near-by matter is thrown into radiating lines like iron filings about the poles of a magnet, giving the whole a kind of starlike appearance.

These are the portions of the cell most concerned in cell divisions, and their special characters are most pronounced and the differences most distinct just previous to the act of division, and least well marked in the cell during its "resting stage" between divisions.

The actual process of cell division whereby one cell gives rise to two and by which growth is attained is essentially as follows :

When division is about to take place the chromatin matter (granules) assumes the appearance of a fine network rumning through the mass of the nucleus, the granules looking like beads strung upon a thread. This network commonly, though not always, condenses into a ribbon or thread (the spireme), which, however, speedily breaks up transversely into a definite number of segments, generally in the form of short rods, straight or curved (the chromosomes). Whether or not the reticular or network form passes through the spireme stage, the result is always the same; namely, the chromatin matter becomes clivided into a definite number of chromosomes. Here are two remarkable and significant facts; first, the number of cluromosomes is constint in all indivituals of the some species; and second, "in all species arising by sexual reproduction the number is even." 1

While the chromatin matter has been engaged in breaking up (or down) to form the chromosomes, another significant process

1 Wilson, The Cell, p. 67. The author here gives the number of chromosomes characteristic of certain species as follows: some of the sharks, 36 ; mouse, salamander, trout, and lily, 24; ox, guinea pig, and onion, 16; grasshopper, 12; Ascaris, 4 or 2 ; the crustacean Artemia, 168; man, 16 or possibly 32 . In this connection it is worthy of note that varieties of the same species often differ in the number of their chromosomes, the significance of which variation has not yet been determined. 
has been going on. The centrosome has divided and the two new bodies derived from it have separated and migrated to opposite sides of the nucleus, each surrounded by its radiating lines, in which condition they are known as "asters" (stars).

During this migration the asters are generally (not always) visibly connected by lines, but in either case by the time they have reached opposite sides of the nucleus they will be seen to lie at opposite ends of a spindle-shaped body (the amphiaster) consisting of lines, among which lie the chromosomes.

Matters are now ready for the final and significant acts of cell division. The chromosomes arrange themselves end to end along the equator of the spindle, and at right angles to its axis ; whereupon each chromosome splits lengthavisi, one group of halves migrating to one aster (centrosome), the other to the other, where each clusters about its own center, forming a new nucleus with its centrosome. The cell wall now becomes constricted, clividing the cytoplasm approximately equally (sometimes very unequally) between the two new cells, and the division is complete. The resting stage ensues, during which preparation is made for another division ; indeed, the centrosome occasionally divicles, in anticipation of the next division, even before all the details of the first division are complete. For a graphic outline of the complete process of mitosis see Figs. 20 and $2 \mathrm{I}$.

This, in general, is the process of cell division which, with more or less variation, attends all growth. The significant facts brought to light in this complicated process are: (1) that the number of chromosomes is constant for all individuals within the species; (2) that for all forms arising by sexual reproduction the number is eren; (3) that however its details may vary, cell division consists essentially in a splitting of the chromosomes, by which each daughter cell secures (apparently) an exact equivalent of what is received by the other daughter cell of the same division. Cell division is therefore not a lump division of the cell mass, but it is meristic, insuring a strictly qualitative division in which one half of each chromosome descends to either daughter cell. ${ }^{1}$

1 These same facts have added significance when considered in connection with the germ cells, reproduction, and the problems of heredity. 

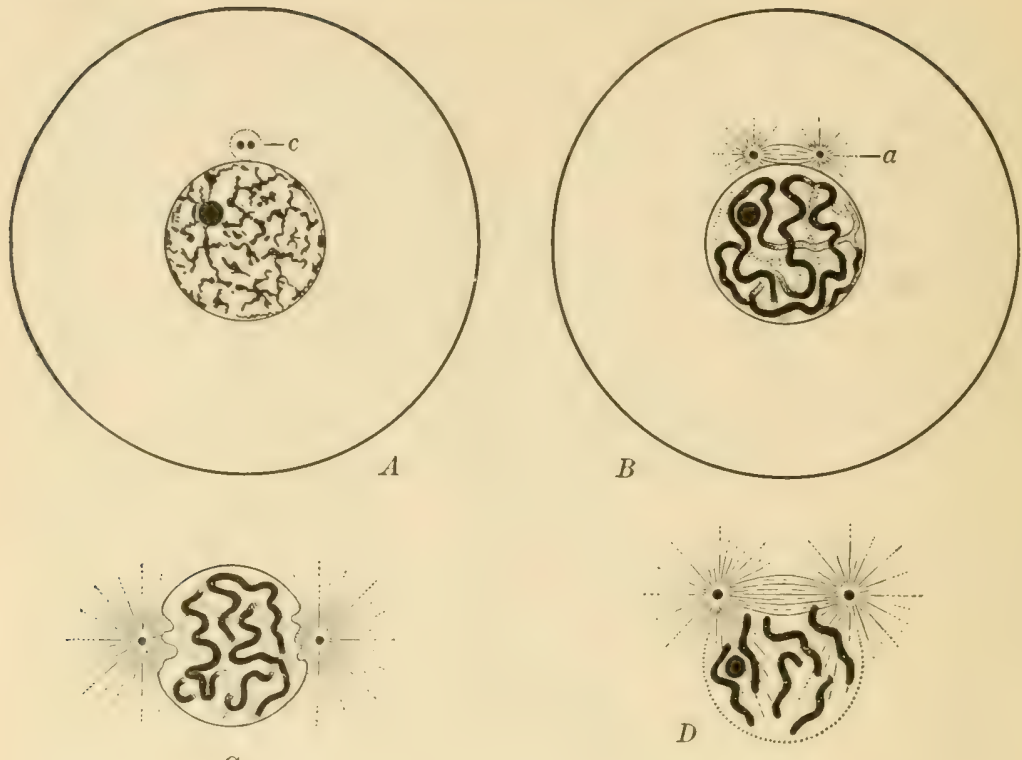

$C$

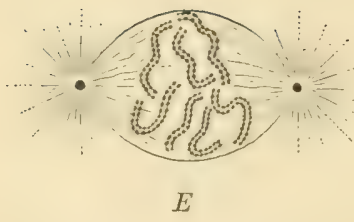

Fig. 20. Diagrams showing the prophases of mitosis

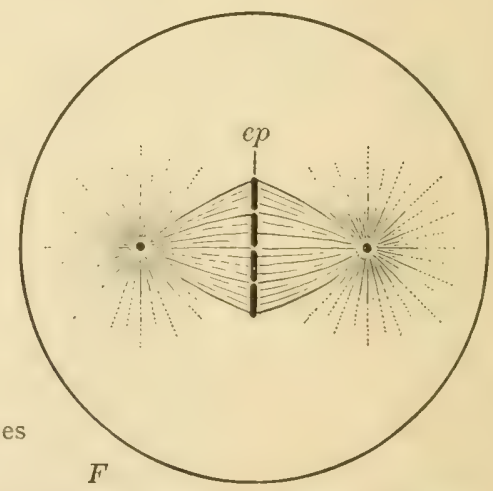

A. rmating cell with reticular mucleus and true nucleolus: at $i$ the attraction splhere containing two centromoses. $B$, early prophase: the chromatin forming a continuous spircme, nucleolus still present; above, the amphiaster $(a)$. $C, D$, two different types of later prophases: $C$, disappearance of the primary spindle, divergence of the centrosomes to opposite poles of the nucleus (examples, some plant cells, cleavage stages of many eggs); $D$, persistence of the primary spindle (to form in some cases the "central spindle "), fading of the nuclear membrane, ingrowth of the astral rays, segmentation of the spireme thread to form the chromosomes (examples, epidermal cells of salamander, formation of the polar bodies). $E$, later prophase of type $C$ : fading of the nuclear membrane at the poles, formation of a new spindle inside the nucleus; precocious splitting of the chromosomes (the latter not characteristic of this type alone). $F$, the mitotic figure established; ep, the equatorial plate of chromosomes. - After IVilson 


\section{SECTION IV - CELL IOIVISION WITH ANI) WITHOUT DIFFERENTIA'TION}

The accomplishment of this minutely accurate division of certain portions of the mother cell between the daughter cells at division suggests two points: (I) that the matter thus carefully
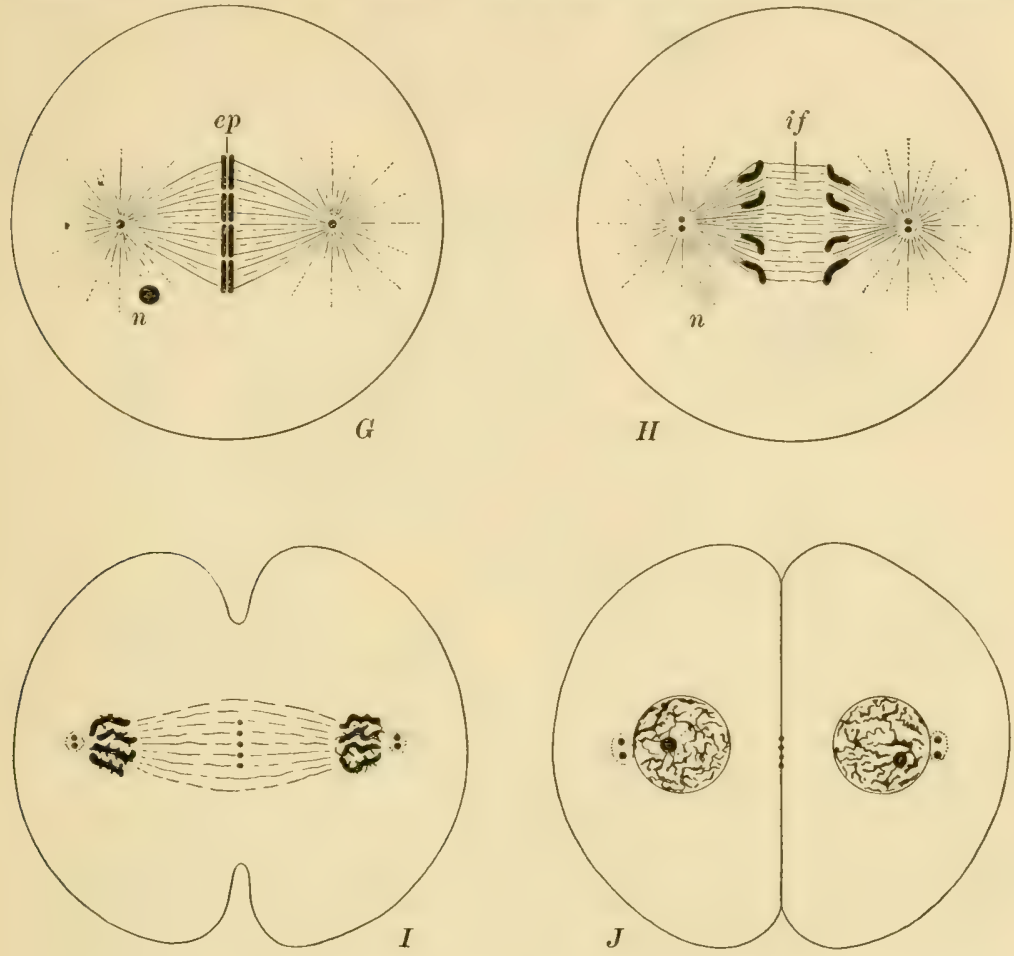

Fig. 21. Diagrams of the later phases of mitosis

$G$, metaphase: splitting of the chromosomes $(e p) ; n$, the cast-off nucleolus. $H$, anaphase: the daughter chromosomes diverging, and between them the interzonal fibers (if), or central spindle; centrosomes already doubled in anticipation of the ensuing division. $I$, late anaphase or telophase, showing division of the cell body, midbody at the equator of the spindle, and beginning of reconstruction of the daughter nuclei. $J$, division completed. - After Wilson

divided is of special importance in shaping the activities of future cells; (2) that daughter cells so provided should be identical, and their after growth should not only be alike but should 
also be similar to that of the mother cell from which they are descended.

For all cell division within the same tissue this latter is true; that is to say, in the growth of liver tissue, bone tissue, or any other specific structure the cells appear to be identical and their resulting growths alike. But it must not be forgotten that these different tissues all arose originally from a single cell; in other words, that some cell divisions are attended by differentiation. When? How? Here lies the chief mystery of variation. The mechanism of cell division would seem to be specially designed to frei'cut deviation and to insure absolute transmission

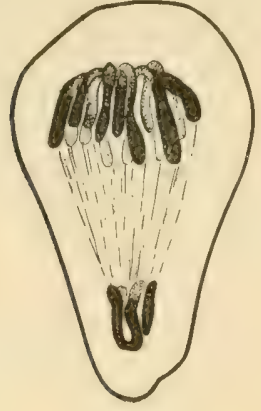

$A$

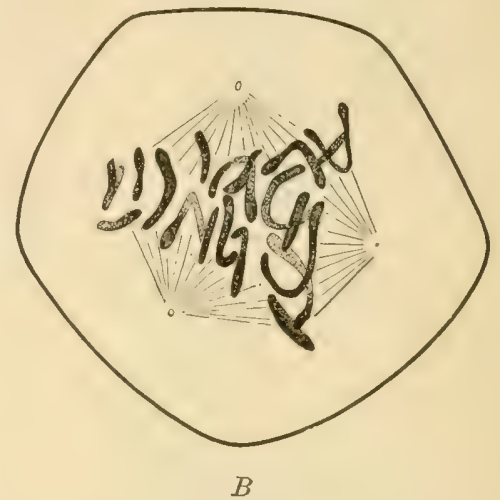

$B$

Fig. 22. Pathological mitosis in epidermal cells of salamander caused hy poisons $A$, asymmetrical mitosis after treatment with $0.05 \%$ antipyrin solution; $B$, tripolar mitosis after treatment with $0.5 \%$ potassic iodid solution. - After Wilson, from Galeotti

from mother cell to daughter cell. It does not account for or indeed appear to admit of differentiation of tissues or variation in growth. But differentiation does take place and variation is a fact to be in some way explained.

Irregularities in cell division. Not all cell clivision, it is true, proceeds with the regularity and perfection of plan indicated in the description, which is the common method in higher animals and plants and may therefore be regarded as fairly typical. The known abnormal cases are of several distinct kinds :

I. Asymmitrical mitosis, in which the chromosomes are not equally distributed to the daughter cells, most of them massing 
at one pole, some of them perhaps being lost altogether in the mass of the cytoplasm.

2. Multipolar mitosis, in which the number of centrosomes is more than two and the resulting daughter cells three or more.

Both these abnormal processes, however, are characteristic of abnormal growths, such as cancers and tumors, and are therefore considered as pathological. It is a suggestive fact that such irregularities may be artificially produced by poisons and other
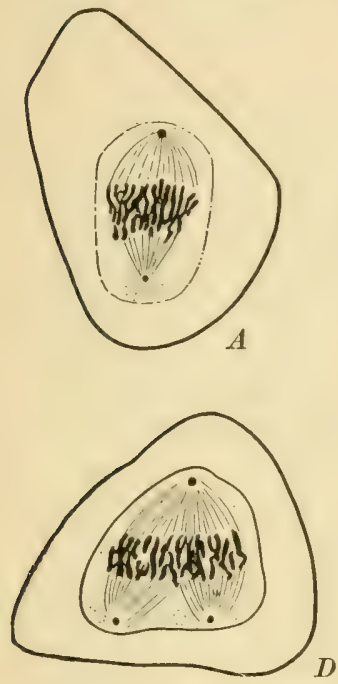
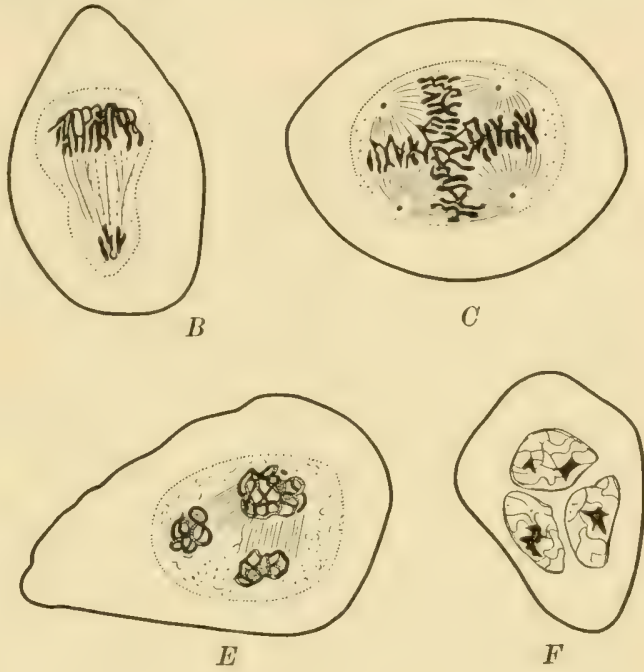

Fig. 23. Pathological mitoses in human cancer cells

$A$, asymmetrical mitosis with unequal centrosomes; $B$, later stage, showing unequal distribution of the chromosomes; $C$, quadripolar mitosis; $D$, tripolar mitosis ; $E$, later stage; $F$, trinucleate cell resulting. - After Wilson, from Galeotti

chemical substances such as chloral, quinin, nicotin, antipyrin, cocain, etc. ${ }^{1}$ (See Figs. 22 and 23.)

3. Amitotic division, ${ }^{2}$ - that is, division without the formation of the amphiaster or the splitting of the chromosomes. This form of cell division is effected by constriction, resulting simply in a lump division of the mass of the nucleus, without reference to qualitative considerations. In this case the claughter cells would not, presumably, be alike. This form of cell division 
is of rare occurrence, is never known in embryonic tissues, and is characteristic of tissues "on the way towards degeneration." 1

4. Quite generally uniccllular organisms display extreme irregularities in mitosis, some species omitting one and others another of the processes typical in higher species. Prominent among these deviations is the failure of chromatin granules to unite to form definite chromosomes. In place of this the individual granules themselves divide, suggesting that fission of the sranules is the clementary and cssential feature of nucliar division. ${ }^{2}$

Other minor deviations are known, though much of the field is yet unworked. These may account to some extent for differentiation during cell multiplication, and yet so far as is at present known all processes that do not accomplish an equitable division of the chromatin granules through the splitting process are looked upon as distinctly pathological. Normal cell dirision, therefore, secms to be in the interest of constancy, not differentiation, and what power it is that produces one sort of tissuc from another, as must happon in the developing cmbryo, is still a mystory.

\section{SEC'TION V - PHYSIOLOGICAL UNITS}

This difficulty has led to the assumption of some sort of physiological units, some of which are active at certain stages of development, others at other stages; and the chromatin granules whose qualitative division is in most cases carefully insured are quite generally regarded as the repository of these units and the common vehicle of hereditary transmission. Such were the gemmules of Darwin," the stirp of Galton, the idioplasm of Nägeli, and the determinants of Weismann. ${ }^{4}$

1 Wilson, The Cell, pp. 116-12 I.

2 Ibid. p. 90.

${ }^{3}$ See Darwin, Animals and Plants, chap. xxvii.

4 Weismann's elaborate theory of heredity regarded the germ plasm as the original substance, of which the body is the natural expansion. This "ancestral germ plasm " is unchanging, unchangeable, and, so long as the species endures, is immortal. Ile regards this germ plasm as comprised ultimately of "biophors" (life bearers), which may be spoken of as living molecules. These biophors, or ultimate units, are combined in an orderly manner into "determinants," whose activity at development determines what the particular part shall be. These 
Whether or not any of these theories finally hold, this significant point remains, - that an adequate theory of heredity must account for the following facts :

I. A single cell thrown off from the sexual parts of a mature individual will, under proper conditions, produce an entirely new individual in all essential respects like the parent, but in minor respects different.

2. Commonly a cell or a number of cells taken from any other part of the body will wither and die, or, if growth follows, only one kind of tissue develops; but in some instances (the begonia and others) the smallest bit of leaf, under favorable conditions, is able to grow and produce a new plant capable of bearing blossoms and seeds. ${ }^{1}$

3. The mechanism of cell division seems admirably adapted to insuring growth without differentiation.

4. But differentiation does take place, and in process of development a great variety of different cells arise from the single original germ cell.

5. These "differentiations" take place at different but proper stages, insuring orderly arrangement and, for the most part, uniform results.

6. There is always more or less variation between individuals, showing that the problem of development and inheritance is something else than absolute descent without change.

We still seek, therefore, physical units with sufficiently exact properties to insure the general character of development, with such mutual relations as shall provide for orderly, not simultaneous development, sufficiently elastic in their constitution (or combining powers) to admit of certain deviation, and each withal gifted with the power of nutrition, growth, and multiplication by division. Such in general are the properties of the physiological

determinants are united into "ids," which are held to be identical with chromatin granules, and these in turn are assembled into "idants," which correspond with chromosomes. For a full explanation of Weismann's theory, see his Essays on Heredity, chap. iv, and his Germ Plasm, chap. i.

1 It is a significant fact that if two begonia leaves be placed on sand simultaneously, one taken from a plant just about to blossom, the other from one just past the blossoming period, the plant from the former will flower first. For Weismann's views, see his Essays on Heredity, chap. iv. 
units required to explain the function and achievements of the germ plasm, - the bit of vitalized matter that holds within its substance all the potentialities of its particular kind of life.

Summary. The causes of variation are closely connected with the mechanism of growth and differentiation. The cell is the unit of structure and all growth is by cell division; but it is not the unit of differentiation of different parts of the body, because all parts arise from one original cell, the germ cell.

Cell division seems admirably adapted to insure absolute transmission without variability of any kind. But both differentiation and variability are facts. We seek, therefore, a "physiological unit" more minute than the cell, whose activities and possibly whose combinations with other physiological units of different properties are able to bring forth first differentiation within the body and later differences between different individuals.

\section{ADDITIONAL REFERENCES}

Chromosome Tesicles in Maturation. By W. M. Smallwood. Science, $\mathrm{XXI}, 386$.

Cytological Features of Fertilization. By W. H. Blackman. Proceedings of the Royal Society, London, LXIII, 400-40I.

Fertility of Eggs after Removal of Cock. By L. G. Jarvis. Experiment Station Record, XI, 67I.

Laws of Embryonic Development: the Law of Von Baer. By Otto Glaser. Science, XV, 976-982.

Mechanism of Development. By William Turner, F.R.S. Popular Science Monthly, LVII, 56I-575.

Ontogenetic and Phylogenetic Variation. By H. F. Osborn. Science, IV, 786-789.

Problem of Development. By E. B. Wilson. Science, XXI, 28i-293. Protoplasmic Structure. By E. B. Wilson. Science, II, 893-899; $\mathrm{X}, 33-45$.

Some Orservations and Consinerations upon Maturation Phenomena of Germ Cells. By T. H. Montgomery. Biological Bulletin, VI, 1904.

Structure and Formation of Pus Cells. Experiment Station Record, XIV, I016.

Vitality of Pollen. (Roses, twenty-two days; clivias, three months.) Experiment Station Record, XIII, 620; (Bear, thirty days) XV, 872 . 


\section{CHAPTER VIII}

\section{INTERNAL CAUSES OF VARIATION}

While the causes of variation are both internal and external to the organism, the facts of the last chapter must satisfy the student of breeding problems that many of the processes attendant upon growth and reproduction are fruitful sources of variability. It is the purpose of the present chapter to discuss these internal influences somewhat at length. They are of two kinds, - (I) those affecting the individual only, and (2) those affecting the race as a whole. It is expedient to distinguish between these two classes, and the chapter will be divicled into two parts, corresponding to these distinctions, as follows: (I) internal influences affecting primarily the individual ; (2) internal influences affecting the race as a whole.

I-INTERNAL INFLUENCES AFFECTIN'G PRIMARILY THE INDIVIDUAL

\section{SECTION I - CELL DIVISION}

Growth is the result of cell division. Manifestly, therefore, all differences in size or in pattern are intimately dependent upon the extent and regularity of this process.

Morphological variation due to cell division. Whatever influences underlic the phenomena of mitosis, all questions of form or size are absolutely dependent upon the extent to which cell division and its attendant growth proceed. The individual cells in giants are not larger than those of normal specimens, but they are more numerous; and in dwarfs they are not smaller, but fewer in number. ${ }^{1}$ What energies decide how far cell division shall proceed and when it shall stop in the case of each separate

1 Wilson, The Cell, pp. $3^{88}-389$. 
organ we do not know. Food and climate undoubtedly exert a general influence, as we shall see, but altogether aside from this there must be profound internal forces or interrelationships, upon the normal exercise of which all typical results depend.

Consider the development of a normal individual from the fertilized ovum to maturity. The circumstances require not only that arm, leg, and bone, heart, liver, and brain, arise at the proper time and place, but also that the attendant coll divisions in cach proced to the requisite number and then stop. If the number be ton few, a dwarf is the result; if too large, a giant; and if too few in some parts (arrested development) or too large in others (hypertrophy), the individual is thrown out of proportion and is recognized as more or less of a monstrosity according to the degree of disproportion. To be sure, all these things occasionally happen, and yet, in the majority of cases, the process of cell clivision is adjusted with a nicety that is nothing short of marvelous; in any event, the results secured, though varying somewhat in total development, are yet almost absolutely proportional (?). ${ }^{1}$

Whatever may be the controlling force to decide at what point cell division in each case shall stop and when the individual as a whole shall cease to grow, the plain physiological fact is that all considerations of size (quantitative variation) are fundamentally those of cell division.

The cessation of growth at maturity cloes not imply the loss of power of cell division, because most forms of life, plant or animal, have more or less powers of regeneration if a part is lost or injured. If a leg of a salamander be cut away, it will speedily be restored, bones and all, as good as new. A tail of a lizard is readily broken off, separating not between two vertebra but at the midlle of a vertebra (in some species generally the seventh caudal)." When the tail regenerates, however, the vertebra do

1 At this point the author questions his own statement. As a matter of fact, the data involved have not been submitted to absolute mathematical determination. We do not know whether the normal deviation in size due to variation in cell division is the same for all species; nor do we know whether in giants and dwarfs all parts bear the same relative proportions as in normal specimens; indeed, there is ground for believing that they do not. In the most general sense, however, the statement is true.

2 Morgan, Regeneration, p. 198. 
not regenerate, and in their place there is only a "cartilaginous tube attached to the broken vertebra." 1

In the first case (that of the salamander) cell division, which would normally remain suspended through life, was able upon occasion not only to resume activity but also to begin back at the proper point in ontogeny ${ }^{2}$ and repeat its normal processes from that point onward. Moreover, in this particular instance it can do this not once but many times. ${ }^{3}$ In the lizard, on the other hand, regeneration is not complete, as no true vertebra are formed. Higher animals generally have but slight powers of regeneration, but all have enough to repair ordinary injuries to the skin, bone, nerves, etc., showing that the power of cell division is not entirely lost at maturity; in other words, that cessation of growth when the normal size is reached is due to some cause other than the failure of the power of cell clivision. There are many cases of abnormal size of certain parts due to a failure of this process to arrest itself at or near the proper point. "Big heads," "giant kidneys," and similar pathological cases are instances in point, but whether the division is mitotic or amitotic has not, so far as the writer is informed, been determined.

While the limitation of cell division can certainly be influenced, especially by the food supply and by exercise, it is manifest that its absolute control is, and doubtless always will be, largely beyond our power. All animals get feed enough to more than build their bodies, and the point at which growth ceases seems to be mainly constitutional. If we could regulate size directly, it would rastly simplify the process of breeding, but as it is now, we are obliged to "breed for size" and feed accordingly.

1 Morgan, Regeneration, p. 198.

2 Ontogeny refers to the development of the individual, as phylogeny refers to that of the race.

3 The absolute limits of regeneration are not known. Speaking generally, they are high in plants and low in animals. The salamander has been known, however, to restore tail and all four legs six successive times (Morgan, Regeneration, p. 5). The deer grows a new set of antlers every year. This is hardly a case of regeneration, however, because successive growths are each more complicated than the former, each adding its characteristic prong; but it is a good instance to show the persistence of the power of cell division. 
Meristic variation in general due to cell division. All differentiation involving numbers of cluplicate parts manifestly has its seat in cell division. An additional division at the point of origin of the series cloubles the nomber, but an extra division at the point of origin of a member adds a pair, if both daughter cells develop, or a single member if but one develops.

When the number in a meristic series is even the series is easily conceirable as having arisen from a corresponding number of cell divisions. For example:

2 in the series, 1 division

4 in the series, 2 divisions

6 in the series, 2 divisions, with one pair dividing again

8 in the series, 3 divisions

10 in the series, 3 divisions, with one pair dividing again

If the number of members is odd, it is only necessary to assume that one of the even numbers failed to develop, or, what is more likely, that one of a pair indulges in additional division, - its sister member remaining single; thus :

3 members, I division, one member dividing again

5 members, 2 divisions, one member dividing again

7 members, 2 divisions, two members dividing again

9 members, 3 divisions, one member dividing again

The frequent recurrence of five as a digital number is one of the mysteries in creation, and its singular persistence is another. It is, however, subject to many deviations, as was seen in the chapter on "Meristic Variation"; even in the rose family there is an occasional loss of one of the members.

The frequent presence of six digits is not to be explained by reversion, as nobody supposes that number ever to have been characteristic in any species, - a fact that should be noted by some of our friends who are always ready to invoke the aid of atavism to explain every abnormality.

Meristic variation, like other deviations arising from external causes, is to some extent hereditary, and capable of being influenced, if not absolutely controlled, through selection. No other method is known, aside from the fact that external injury 
to many plants and certain animals results in budding and multiplication of parts. We cut the main stem of a small tree or shrub in order to increase the number of side branches. Somewhat similarly, injured parts are of ten doubled in regeneration. ${ }^{1}$ In this way lizards may be made to produce an increased number of toes and even double feet, legs, and tail. It is supposed that double feet, sometimes seen even in mammals, may be produced by a "fold of the ammion constricting the middle of the beginning of the young leg " 2 in the embryo. This, however, is curious rather than valuable to us, as it tends to explain abnormalities rather than to point a way to practical improvement.

Irregularities in cell division a cause of variation. ${ }^{3}$ The characteristic act in cell division seems to be the splitting of the chromosomes (or chromatin granules) and the migration of exact equivalents to each new daughter cell, strongly suggesting that the assortment of "physiological units" (whatever they may be) received by one daughter cell is an exact duplicate of that received by the other, thus insuring an orderly and systematic development through a strictly qualitative division of hereditary substance at each and every stage of growth.

The whole mechanism of mitosis seems adjusted to this end, and if the assumption is true its significance can hardly be overestimated. If this careful adjustment of the mechanism of cell division is necessary to orderly development, it is manifest that any substantial deviation is likely, if not certain, to result in variation more or less profound. Such deviation is characteristic of amitotic division generally, and it is more than conceivable that the ordinary process is subject to occasional "slips." Some chromatin granule may fail to clivide at the proper moment and may pass over to one daughter cell entire, ${ }^{4}$ or, conversely, it may indulge in an extra division. Substantial deviations in the process are known to occur not rarely but frequently. For example, the splitting sometimes takes place in the spireme stage, sometimes after the formation of the chromosomes; sometimes

1 Morgan, Regeneration, pp. 137-139.

2 Ibid. p. 139.

3 See previous chapter.

4 This is known to occur in certain instances in maturation. 
the centrosome divides before the resting stage, more commonly afterwarl. Taking it all in all, here is an exceedingly complicated procedure, only semi-mechanical and therefore subject to deviations. Absolute constancy demands no failure in the final (b)joct of exact qualitative division, but the student sees many possibilities for unequal division and therefore for deviation in growth. Is this the fundamental cause of mutations? One thing is certain, - living forms are made up of elements, and these elements are subject to strange combinations throughout the entire range of plant and animal life, and the facts seem to teach that from time to time combinations may arise that are entirely new. Moreover, whole units seem occasionally to be "lost out," as when horned cattle suddenly give rise to polled strains, hairy species to smooth varieties, colored to albino, etc.

Conversely, do vital elements like chemical radicles assume new combinations from time to time, giving rise to new characters and new types which we call "mutants"? We do not know, and yet we feel the conviction that at this point we are very close to the "origin of characters," the cause of mutations and of variation in general.

Manifestly, in so far as irregularities in cell division may be a cause of variation, the matter lies absolutely beyond our control except that lines in which it is believed to occur may be avoided in selection. Here is a field, however, too far beyond our present knowledge to admit of anything more than the merest mention. We confidently believe that the future will shed more light on this obscure subject.

SECIION II-BISEXLAL REPRODUCTION A FUNDAMENTAL CAUSE OF VARIATION

Among higher animals and plants the new individual is the clirect product of two others, - the male and the female parent, - and is of necessity different from either, being a product of both. In bisexual reproduction, therefore, biologists recognize a fundamental cause of variation, - slight if the parents are of like blood lines, extreme if of radically different, as in hybriclism. 
This view of the case is borne out by the facts of fecundation or fertilization of the orum, which may be briefly clescribed as follows: 1

The ovum. This is the finished product of the sexual cells of the mother parent, and consists of a mucleus with its characteristic chromatin granules surrounded by a comparatively large mass of cytoplasm.

The sperm cell. This is the finished product of the sexual cells of the male parent. It is called a spermatozoön in animals, spermatozoid in lower plants, and pollen grain in higher plants. It is in all cases vastly smaller than the corresponding orum, being almost clestitute of cytoplasm. The characteristic elements of the ovum are its nucleus and the cytoplasm, while the characteristic elements of the spermatozoön are its nucleus, borne in the "head," and a centrosome, generally carried in the "middle piece." The tail, formed from the small amount of cytoplasm, seems to have no function beyond providing motile power, and is absent in the pollen of higher plants.

Fertilization. Both the orum and the sperm cell have arisen in their respective organs by the method of cell division, displaying in the process the ordinary phenomena of mitosis." But both have reached the end of their powers of self-division, and if left alone they will be thrown off from their respective points of origin to wither and die.

If, however, they are brought near together, mutual attraction ensues, the spermatozoön (or other sperm cell) enters the ovum, the nuclei approach each other and fuse, the centrosome divides, an amphiaster is formed, and cell division ensues. The orum is now fertilized, segmentation proceeds, and a new individual is established in an independent existence.

The new individual is thus the possessor of actual living matter (physiological units) derived from both parents, and thus inherits literally the substance of both, having come into direct possession of material identical with the living matter of both parents.

1 For a fuller discussion of this subject, see Wilson, The Cell, pp. I78-23r.

${ }^{2}$ For a brief statement of what is involved in maturation, see the next section. 
All that is involved in fertilization is not well unclerstood, but its essential feature is the mion or fusion of the maclear matter (chromosomes) of the germ cells from ta'o parents to form the clearage or segmentation mucleus whose subscquent growth and dirisions "grize rise to all the muclei of the body'." This fertilized orum becomes, therefore, the first cell of the new being, which inherits directly and equally a portion of the nuclear matter from both parents, so that "crery nucleus of the child may contain nuclear substance derived from both parents." 1 Here, then, is the arenue of all inheritance, and, as the new individual is a kind of blend of both parents, we see in fertilization an initial and primary cause of variation.

This is the only form of variation recognized by Weismann in his earlier writings as in any sense hereditary. All deviations in development due to external causes were conceived to affect the body (soma plasm ${ }^{2}$ ) only, exerting no influence upon the ancestral germ plasm." True, he later announced the theory of germinal selection, in which a kind of struggle for existence is conceived as taking place between the "biophors" (physiological units), by which some prosper and multiply exceedingly while others are crowded out entirely. ${ }^{4}$ This would give another cause of variation within the germ plasm of each individual.

Biologists generally recognize internal causes of variation other than these, and yet this union of the chromosomes from different indivicluals taking place at each new generation must be regarded as a very effective means of introducing variability. Even if the offspring of a single parent, as in parthenogenesis, should be an exact duplicate of the parent, - which it is not, every one would recognize the fact that the blending of hereditary substance from two parents must of necessity produce an individual with a new combination of faculties.

It is a variation, however, confined not only to the characters of the race but also to the family possessions of the particular parents. Bisexual reproduction cannot be looked upon as a means

1 Wilson, The Cell, p. 182.

2 "Soma plasm" is a term used to represent the protoplasms of the body in general as distinct from the output of the sexual cells (germ plasm).

${ }^{3}$ Weismann, The Germ Plasm, chap. ix.

${ }^{4}$ Weismann, Germinal Selection (pamphlet). 
of introducing new characters into the race, and while it is manifestly a fruitful source of never-ending combinations of racial characters in new individuals, yet variations so introduced are comparatively slight except when the two parents belong to separate lines.

Fertilization of the ovum is something more than a stimulus to growth. It is a real union of material bodies, physiological units, or whaterer they may be called, representing the hereditary substance of both parents. Biscxual reproduction is therefore not only a gruaranty of transmission of racial characters but also an assurance of inheritance with some variation.

Control. Here is a fundamental cause of variation practically under the control of the breeder through selection. True, his knowledge and his judgment are insufficient to insure him against mistakes in mating, and it is also true that there are many other influences at work to produce variations, but this is the field in which the breeler can exert the largest influence, and it is by selection that the greatest results in improvement have been attained up to date.

Sexual selection, ${ }^{1}$ preferential mating, ${ }^{2}$ and assortative mating." Powerful as are these influences in clirecting the trend of variability, they yet belong to general evolution because they are elements in natural selection, and they have no place in the present discussion.

\section{SECTION III - MATURATION AND THE REDLCTION OF} THE CHROMOSOMES A CAUSE OF VARIATION

Fertilization is a process whose inevitable consequence would seem to be the "piling up" of melear mattor indefinitcly" for if, with each new generation, the chromosomes (or physiological units) of the one parent are added to those of the other, it would seem that in time the resulting nuclear matter would speedily become "unmanageably large" and inconceivably complex, - an event certain to follow except for a series of very remarkable

1 Darwin, Origin of Species, see Index.

2 Pearson, Grammar of Science, pp. 425-428.

${ }^{3}$ Ibid. pp. 429-437. 
facts occurring just previous to fertilization and by which the number of chromosome's in both the male and fimale germ cells is reduced to one half the usual or somatic mumber, so that their union at fertilization restores the true number of chromosomes typical of the race. Thus, if the somatic number of chromosomes is sixteen, the number in the germ cells at fertilization will be eight each, or sixteen after fusion of the nuclei. This process by which the number of chromosones is halved in the germ cell is known as reduction, and is supposed to be the significant feature of the maturation process by which the male and female germ cells are prepared for union.

Parallelism in the sexes. Maturation and its attendant phenomena of reduction in the number of chromosomes is a subject that must be considered separately in the male and the female, and yet there exists a strange parallelism worthy of notice. To quote Wilson ${ }^{1}$ :

Recent research has shown that maturation conforms to the same type in both sexes. . . Stated in the most general terms this parallel is as follows: In both sexes the final reduction in the number of chromosomes is effected in the course of the last tato cell divisions, or maturation dirisions [as they are called], by which the definitive germ cells arise, each of the four cells thus formed having but half the usual number of chromosomes. In the female but one of the four cells [resulting from the two maturation divisions] forms the ovum proper, while the other three, known as the foldar bodics," are minute, rudimentary, and incapable of development. In the male, on the other hand, all four of the cells become functional spermatozoa. This difference between the two sexes is probably due to the physiological division of labor lietween the germ cells, the spermatozoa being motile and very small, while the egg contains a large amount of protoplasm and yolk, out of which the main mass of the embryonic body is formed. In the male, therefore, all of the four cells may ${ }^{3}$ become functional; in the female the functions of development have become restricted to but one of the four, while the others have become rudimentary.

1 Wilson, The Cell, p. 234 .

2 The author is here speaking specifically of reproduction in animals, as plants do not form polar bodies. The difference in plant and animal reproduction is, however, more in form than in significance.

3 The author says "may" become functional. He means by this that each of the four cells (spermatozoz) arising from the last two divisions is capable of fertilizing an ovum, while of the four cells arising from the last two divisions in the female only one is capable of being fertilized. 
Maturation and reduction in animals and in plants radically different. This process is far better understood in animals than in plants, and in many respects it is radically different in the two. It is simpler in animals and more direct. In them the last two cell divisions always (apparently) give rise in the male to four functional spermatozoa, but in the female to one functional cell, retaining nearly all the cytoplasm, and to three polar bodies incapable of fertilization and destined to wither away and disappear. The same general facts seem to hold for animals of all species, and Wilson remarks ${ }^{1}$ :

The evidence is steadily accumulating that reduction is accomplished by two maturation divisions throughout the animal kingdom, even in the unicellular forms; though in certain Infusoria an additional division occurs, while in some other Protozoa only one maturation division has thus far been made out.

Among plants, also, two maturation divisions occur in all the higher forms, and in some at least of the lower ones. Here, however, the phenomena are complicated by the fact that the two divisions do not, as a rule, give rise directly to the four sexual germ cells, but to asexual spores which undergo additional divisions before the definitive germ cells are produced." [The end product, however, shows the same reduction in the number of chromosomes.]

A brief description of reduction in animals is worth considering somewhat in cletail, as it is fairly well known and cannot fail to impress the student with its fundamental significance and the nicety of adjustment of the mechanism of living processes.

Reduction in the female. ${ }^{3}$ Among animals the production of the female germ cell (the ovum) is the special function of the ovaries. In the tissues of these organs cell division proceeds under the usual mitotic plan, giving rise to a series of cells known as ögronia. At a certain point mitotic division halts, and each cell prepares for the final (maturation) changes. Food material is absorbed, the cytoplasm increases in bulk, the nucleus greatly enlarges, and the cell, now known as an öryte, is ready

1 Wilson, The Cell, p. 235 .

2 Ibid. pp. 235-236. Note that, in general, polar bodies are not formed in plants.

${ }^{3}$ Ibid. pp. $236-240$. This description applies to the animal. The details are distinctly different in plants, to be discussed later. 
for the last two, or maturation, divisions. In this condition the egrg cell remains until near the time of fertilization, when the process of maturation proper takes place.

The significant details of this interesting series of changes are concerned with the nucleus and are substantially as follows: During the long resting stage preparatory to these final divisions the nucleus increases in bulk and the chromatin matter assumes the reticular form characteristic of the resting stage of dividing cells in general. In this condition the nucleus is known as the "germinal vesicle." Up to this point the number of chromosomes is the same as that of the body cells in general. Their identity is, of course, now lost, but as the time for the first maturation division arrives, instead of the spireme of ordinary mitosis breaking up into the usual number of chromosomes, there appear more or less spontaneously a number of "primary chromatin masses " in the form of rods, rings, or V-shaped bodies, each of which ultimately breaks up into four smaller bodies. These groups of four are always one half the usual or somatic number of chromosomes.

Whether the chromatin masses appear in the form of rods, rings, or otherwise, the final result seems to be always the same; namely, the breaking up of each into four smaller bodies, either by tae longitudinal divisions or by onc (the first) longitudinal and onc transierse. The details differ in different species and have been worked out in but few cases. It is not important here to trace the bewildering differences, but rather to describe typical behavior. ${ }^{1}$

Having assumed this condition the nucleus now migrates to the margin of the cell, each of the groups of four (tetrads in rodshaped cases) splitting into two smaller groups of two each (dyads). ${ }^{2}$ The mass now divides, one pair from each group

1 For a full discussion of the different forms of reduction, see IVilson, The Cell, V, 233-287.

"The terms "tetrad" and "dyad" of course apply only in the case of rodshaped masses. In the case of rings (common in animals) and V-shaped masses (common in plants) the parting into four takes place gradually as the work proceeds, while in the case of rods the division into four takes place early and the parts are distinct from the first. In this formation the two divisions take place much more rapidly than in the case of rings, which split and divide slowly. The 
remaining in the cell, the other passing outside, forming the first polar body, which may or may not undergo further division.

The portion now remaining within the cell consists of groups of two each, instead of four, and their number is of course the same as before, namely, one half the somatic number of chromosomes. Immediately now, without assuming the resting stage, the dyads, or groups of twos, turn one fourth around, taking a position at right angles to the margin of the cell, and at once diaide again, one member of each pair remaining behind in the egre cell, the other passing ont, forming the sicond polar body'.

The first polar body carried away one half the nuclear matter, and the remaining half has now been divided equally between the second polar body and the main cell, which is now ready for fertilization and is from this time on spoken of as the orum.

Neither polar body carries any appreciable quantity of cytoplasm, and both are destined to degenerate and disappear. The first one, however, containing half of the total nuclear matter, commonly divides once, so that the first polar body represents not one, but two cells, - the first and the third polar bodies.

The total result, then, of this complicated process seems to be the equal division of the chromatin matter between the ovum, capable of fertilization, and three polar bodies, destined to extinction.

A group of four cells thus arises, - namely, the mature egg (ovum), which after fertilization gives rise to the embryo, and three small cells or polar bodies (incapable of fertilization), which take no part in the further development, are discarded, and soon die without further change. The egg nucleus (of the ovum proper) is now ready for union with the sperm nucleus," which process is known as fertilization.

"In some cases - for example in the sea urchin - the polar bodies are formed before fertilization, while the egg is still in

tetrad form is always chosen for description because the details are capable of more definite statement. Whatever the form of the masses, however, the final result seems always the same; namely, a reduction to one half the usual number of chromosomes, and this by the method of division and extrusion.

1 In rare instances the polar bodies have commenced to segment, but they never proceed far in development.

2 Wilson, The Cell, pp. 236-237. 

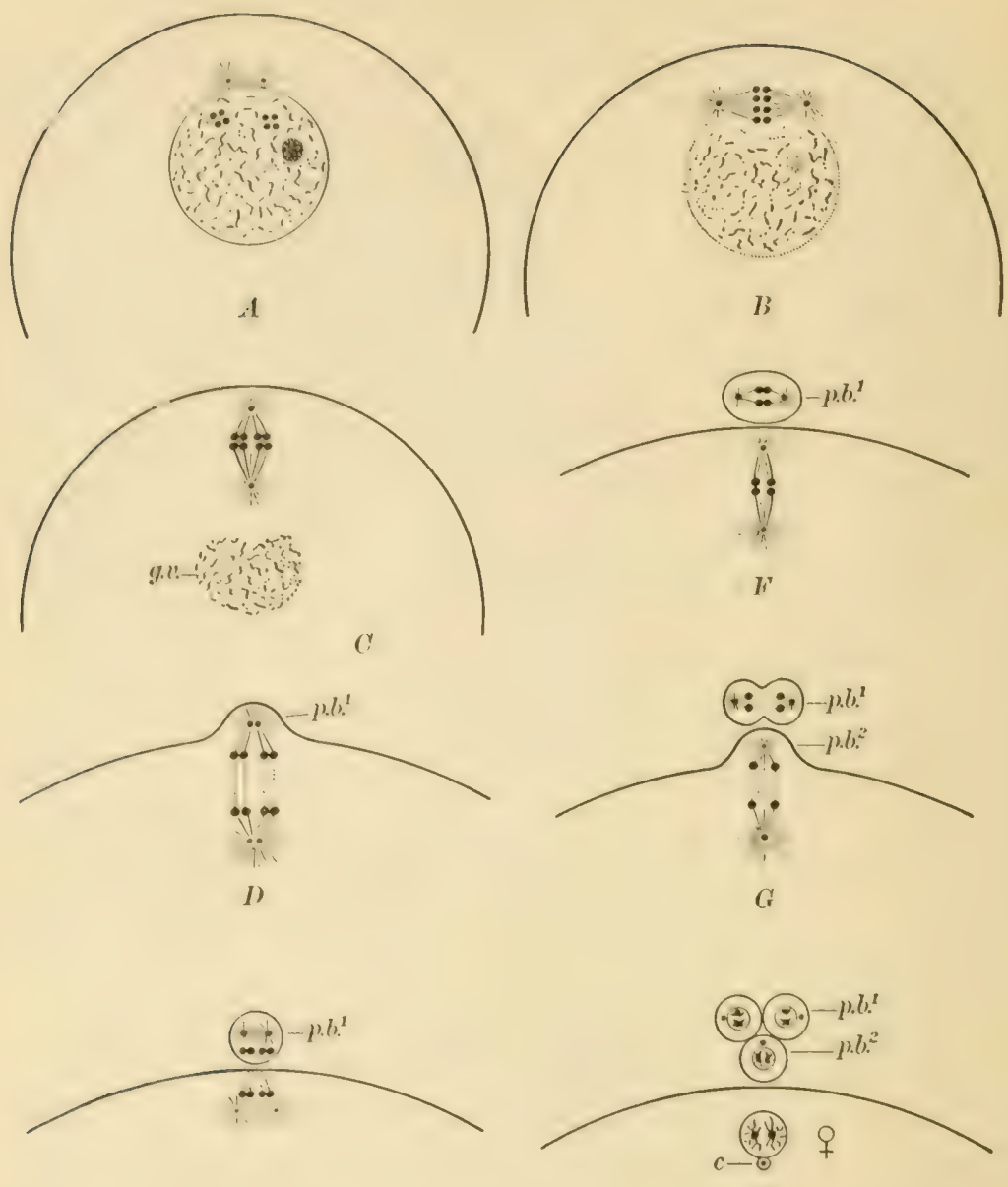

E

II

Fig. 24. Diagrams showing the essential-facts in the maturation of the egg. 'The somatic number of chromosomes is supposed to be four

$A$, initial phase: two tetrads have been formed in the germinal vescicle. $B$, the two tetrads have been drawn up about the spindle to form the equatorial plate of the first polar mitotic figure. $C$, the mitotic figure has rotated into position, leaving the remains of the germinal vesicle at g.z'. D, formation of the first polar body: each tetrad divides into two dyads. E, first polar body formed: two dyads in it and also in the egg $\left(f, 6 .{ }^{x}\right)$. $F$, preparation for the second division. $G$, second polar body forming and the first divicling: each dyad divides into two single chromosomes. $H$, final result: three polar bodies and the mature ovum, each containing two single chromosomes, or half the somatic number; $c$, the egg centrosome, which now degenerates and is lost. - After Wilson 
the ovary. More commonly, as in annelids, gasteropods, and nematodes, they are not formed until after the spermatozoin has made its entrance; while in a few cases one polar body may be formed before fertilization and one afterwarel, as in the lamprey eel, the frog, and in Amplioxus. In all these cases the essential phenomena are the same. Two minute cells are formed, one after the other, in rapid succession and near the upper or animal pole of the ovom; and in many cases the first of these divides into two as the second is formed."

To what extent this division is qualitative is unknown. Of one thing we are certain : somerallere in the process the number of chromosomes has bien reduced to chatly one half the number characteristic of the spccies.

It was formerly supposed by Van Beneden, Weismann, and Boveri that reduction consists in the casting out and degeneration of half of the chromosomes. "Litter researches conclusively showed, however, that this view camnot be sustained, and that reduction is affected by a rearranscrment and redistribution of the nuclear sulstanci, without loss of any of its essential constituents." 1 This is said because the groups - tetrads, rods, rings, etc. -- arise spontanconsly in the nucleus in the retuced number. The loss occurs later in the extrusion of the polar bodies, but no corresponding loss takes place on the male sicle because all four cells are functional, though not all alike.

Reduction in the male." The maturation processes in the male and female are practically iclentical in their results, with two exceptions; namely, first, in the male the four cells resulting from the maturation divisions are all alike and all functional, and second, they are exceedingly small in size as compared with the ovum, being almost destitute of cytoplasm.

The spermatogonia, corresponding to the orgenia of the female, arise in the testes by mitotic division, with the full somatic number of chromosomes. As in the female, they reach a stage where division ceases for a time and enlargement ensues, in which condition the cells are known as spermatocytes (corresponding to oöcytes in the female).

1 Wilson, The Cell, p. 233.

2 Ibid. pp. $241-242$. 
At the proper stage each spermatocyte undergoes two divisions (maturation divisions) into four cells, called spermatids, each of which develops a tail and becomes functional, in which finished condition it is known as a spermatozoön, when it is ready to enter and fertilize the ripened ovum.

The history and distribution of the chromatin matter in the male is identical with that in the female, so that each spermatozoön inherits one fourth the chromatin matter and one half the cluromosomes of the original cell. In plants the process differs but the general results are the same.

Significance of reduction. On the female side three fourths of the chromatin matter has been extruded in the polar bodies, and therefore lost to the line of descent. Whether reduction takes place by extrusion or by rearrangement, one thing is certain: when the second division is transverse, and possibly when it is longitudinal, it results in an uncqual division of pluysiological units, if the identity of the chromosomes and the chromatin granules has any meaning. If this division be anything else than strictly qualitative, then the extrusion of the polar bodies means a loss of something qualitative on the female sicle.

On the male side the loss is not absolute, because all four cells are functional, but if reduction has the meaning we attach to it, these four spermatozoa are not identical but different in the hereditary substance with which they are provided.

Significance of fertilization. Ifere, then, are two sexual cells ready for union. Each has lost large portions of its chromatin matter, the evident vehicle of transmission, and each brings to the union but one half the number of chromosomes characteristic of its species, strongly suggesting a loss of certain chromatin granules and the hereditary qualities they represented.

When fusion of the nuclei of these two germ cells takes place at fertilization, however, the act of union again restores the full and proper number of chromosomes, which will remain characteristic of the new individual throughout its life, and which it will hand down to posterity, always through the same complicated method we have attempted to describe. The number of chromosomes is evidently kept constant by the complicated process of reduction during maturation and by fertilization 
afterward; but what about their character? In what condition have they emerged from this seemingly incomprehensible tangle? Is nature as careful to preserve their quality as it is their number?

What opportunities for profound variation! Certainly if chromatin matter has any fundamental meaning, and if chromosomes are in any way representative of physiological units, and if they in their turn are in any way representative of racial characters, these processes must have some meaning in variation. Certainly we have been very near in all this to the material basis of transmission of racial characters, and to fundamental and initial causes of variation.

Something has been lost in the two peculiar divisions attending maturation. Some definite groupings of hereditary substance have disappeared from the line of descent. They could not have represented, ordinarily, definite portions of a body, but they must represent something. What chances for accident! And, in the light of these marvelous phenomena, do we wonder that individuals are sometimes born minus a leg, an arm, or some other part? Do we wonder that vital parts are so of ten affected, and that one third of our children die in infancy? How many die before birth, and how many more die at some stage in embryo!

Evidently all that is required to make a living being is a fairly perfect development of the s'ital parts, quite regardless of the presence or absence of the many other racial characters that should be present in the perfect individual. Is it surprising that perfect individuals are so few, and that defectives are frequently so far from the type? Iere is material for study on the part of criminologists and courts of justice, as well as students of methods of economic improvement.

Reduction and fertilization in plants. It may be said in general that in animals the evidence tends to the assumption that reduction takes place at the extrusion of the second polar body ; that each group (rod, ring, or V-shaped body) is in reality a doubled (bivalent) chromosome, and that the first polar body remores one half of each (split) chromosome, while the next removes every alternate chromosome.

While the facts of reduction in plants are yet in a hopeless tangle, it is safe to say that the evidence tends to show that no 
true polar bodies are formed, ${ }^{1}$ but that the chromosomes suddenly appear in reduced number at the first division, as if it were effected directly by the segmentation of the spireme thread (of the maturing germ cell) into half the somatic number of chromosomes.

While details vary greatly, botanists recognize two stages in the development of the female germ cell of the plant, neither of which is iclentical with maturation in animals, though the first is fairly comparable thereto. The first stage, or sporogenesis, follows after that active massing of food material which marks in both plant and animal the preparation for reduction. At this time the nucleus divides quickly into four daughter nuclei, each of which is supplied with half the number of chromosomes that characterizes the species. The precise methods followed out are matters of much dispute among botanists, but the significant fact is that reduction is accomplished at this stage.

Of these four daughter nuclei none are extruded, but three of them degenerate in the cytoplasm, while the fourth increases in size to form the embryo sac, which, without waiting for fertilization as among animals, continues to divide, - commonly twice, - griving rise to eight sub-nuclei, which arrange themselves in definite prositions. Two of these sub-nuclei remain near the center of the embryo sac and give rise to the enclosperm; three migrate to the extremity nearest the point of attachment with the pistil, and one of these (and one only) the so-called egg nucleus - unites with the nucleus of the pollen grain to form the fertilized germ; the three remaining migrate to the other extremity of the embryo sac and concern themselves with establishing a food supply with the parent plant.

On the male sicle the process is simpler. The pollen nucleus divides, one half forming the pollen tube, along which the other half travels, dividing again at some point before uniting with the egrg nucleus of the embryo sac. These divisions are evidently reducing divisions, as the pollen-grain nucleus brings to the union a reduced number of chromosomes.

1 Disputed by Chamberlain, who believes that "the egg with its three polar bodies constitutes a generation directly comparable with the gametophytic generation in plants." See Botanical Gazette, XXXIX, I39; see also under "Xenia," in this text. 
Thus, while the plan is different in plants and in animals, the first stage, sporogenesis, in plants seems fully comparable with maturation in animals, and the same general end is accomplished. ${ }^{1}$

After all, the manner of division is primarily of interest to the physiologist and does not concern us. Our interest is in the fact that maturation in sencral inatiols an actual loss of chromatin matter (hereditary substance) and ar reluction in thi munber of chromosomes, and consequently of physiologianl anits. In the present state of knowledge it seems safe to assume that both these results follow, whatever the mechanism of maturation in each particular instance. If this be true, here is a fertile and initial cause of profound variation, an excellent opportunity for losing important elements of the physical make-up, but, so far as we can see, no chance for positive gain, unless it be by new combinations, because nothing is introduced.

Phenomena such as these are remarkable for what they suggest rather than for conclusions that can be positively drawn. The suggestion is that of substantial deviation in the very fundamental process of transmission of the hereditary substance, a deviation that cannot but be fruitful of variation in resulting individuals.

Weismann's prediction. It is noteworthy that reduction was predicted by Weismann on purely theoretical grounds some years before it was known as a fact. ${ }^{2}$ Ie argued for its recurrence as a physiological necessity to prevent the piling up of "ancestral idioplasm," - the physiological units to which he afterward gave the name of "ancestral units," " - and later developed the intricate sysiem of biophors, ${ }^{4}$ determinants, ${ }^{5}$ ids, and idants, ${ }^{6}$ by virtue of which he explained the constitution of the germ plasm, ${ }^{7}$ and which he used as the basis for his famous theories of heredity. ${ }^{8}$

1 For full discussion, see articles by B. M. Davis, American Naturalist, XXXIX, Nos. 460 and 463 .

2 Weismann, Essays on Heredity, I, 357, 363-396; II, II4-150; also Weismann, The Germ Plasm, chap. viii. $\quad 3$ Weismann, Essays on Heredity, II, I 6.

4 Weismann, The Germ Plasm, pp. 40-53. $\quad{ }^{5}$ Ibid. pp. 53-60.

${ }^{6}$ Weismann, Essays on Heredity, II, I $3^{6-1} 3^{S}$; Weismann, The Germ P'lasm, pp. 6o-75.

7 IVeismann, The Germ Plasm, chap. i, pp. 37-85.

${ }^{8}$ Ibid. chap. ix, pp. 253-293. 
The later discovery of the mechanism of maturation and of the extrusion of the polar bodies was a startling confirmation of Weismann's prediction, and went far to fix his theories of heredity in the minds of many biologists. In this connection Wilson very pertinently remarks : ${ }^{1}$

The fulfillment of Weismann's prediction is one of the most interesting results of recent cytological research. It has been demonstrated in a manner which seems to be incontrovertible that the reducing divisions postulated by Weismann actually occur, though not precisely in the manner conceived by him, . . but it remains quite an open question whether they have the significance attributed to them by Weismann.

Just when the reduction occurs is not known. It was at first assumed that it occurs in connection with the extrusion of the second polar body, - an assumption based upon the development of parthenogenetic eggs. But plants do not form polar bodies, and again there is great uncertainty as to whether the rods (tetrads), rings, or V-shaped bodies (whose number is half the usual number of chromosomes) are to be regarded as representing the usual number of chromosomes split and arranged in pairs, - in which case the second polar body would accomplish the reduction; or whether the chromosomes as formerly known never emerge from the nucleus of the oöcyte, so that the identity of the chromosomes is in some way lost and the reduction is effected at this carly stage by some sort of internal fusing, or perhaps by an entire rearrangement of chromatin granules. On this point the evidence is confusing, but on two significant points there is no doubt, - the loss of chromatin matter out of the line of descent, and a reduction of the chromosomes in the germ cells to one half the somatic number. ${ }^{2}$

Composition of the chromosomes. ${ }^{3}$ In view of the important office of the chromosomes and the many theories of heredity based upon their nature and constitution, in view also of their evident importance in all studies on inheritance and variation,

1 Wilson, The Cell, p. 246.

${ }^{2}$ By "somatic" number is meant the number characteristic of the soma, the body in general as distinct from the germinal matter whose function is not growth but reproduction.

3 Wilson, The Cell, pp. 294-304. 
it may be well to note the substance of what is really known about their actual constitution.

That they are not masses of homogeneous matter is certain, and that they consist of numbers of small granules capable of multiplication and division seems equally certain. To quote Wilson :

The facts are now well established (I) that in a large number of cases the chromatin thread consists of a series of granules (chromosomes) imbedded in and held together by the linin substance: (2) that the splitting of the chromosomes is caused by the division of these more elementary bodies; (3) that the chromatin grains may divide at the time when the spireme is only just beginning to emerge from the reticulum or resting stage.

Because of these facts there arises the strongest tendency to attach individuality to the chromatin granules and to conceive them as built up of definite, though often diverse, physiological units, thus constituting a semi-mechanical basis for heredity, and incidentally for variation as well. This assumption Weismann and others have made. Whether the facts should be pushed to this extreme interpretation is, in the opinion of the author, as yet uncertain. The facts are extremely suggestive, to say the least, and it is certainly not too much to believe that at this point we have touched the physical basis of life and in some fashion the very root of inheritance and variation; indeed we may proceed upon the conviction that transmission is a function of the chromatin granules.

Reduction as a cause of variation. The most remarkable and suggestive fact about living beings is the numerical constancy of chromatin units (chromosomes) for each species, and the most remarkable and suggestive of all the vital processes is their reduction before fertilization. If, as we suppose, the chromosomes are the physical basis of inheritance, then in the loss of chromatin matter at maturation lies a fundamontal canse of variation, and one quite independent of the effects of fertilization afterward.

Reduction would seem to be a process calculated to insure that no two germ cells, even from the same inclividual, should ever be alike, and this is the most evident reason for the 
essential differences in children of the same parents, even in the case of twins. ${ }^{1}$

It is true, of (course, that mo two inclividuals, even twins, can be developed under conditions of life exactly iclentical ; and yet the differences of conclition cannot account for the fact that, while one brother resembles his father, another may resemble

1 Twins are considered as arising from separate ova, as in the case of multiple hiulis (joigs, dogs, etc.), and, of course, ats exhibiting the deviations to be expected from different germs and distinct fertilization, as in litters generally.

Some twins, however, are so nearly alike (identical twins) as to suggest the posilsility of the having arisen from a single ovum in some way separated into halves at its first cleavage, each half developing an individual. 'This view is evidently favored by Geddes and Thomson (see The Evolution of Sex, p. 41).

If twins should be developed in this manner, they would evidently be of the nearest possible similarity, for they represent but one ovum and but a single fertilization.

This possibility is supported by the experiments of Roux, Endres, and Walter, in which eath blastomere of the two-cell stage of the frog sometimes (not always) is capable of cleveloping into a perfect individual. I riesch, working with echinoderms, established the same facts, which are also well known in the case of Amphioxus (see Wilson, The Cell, p. 4rig).

Conversely, when two fertilized ova of sea urchin, or Ascaris, adhere accidentally, they may develop into an embryo of unusual dimensions (see Loeb, Studies in General I'hysiology, Part II, p. 676).

When, however, the blastomeres are not separated, but one of them is killed by a heated needle (Roux), the uninjured half alone develops, but it produces at the best a kind of half larva (right or left half), "containing one medullary fold, one auditory pit" (Wilson, The Cell, p. 399). Chun, Driesch, Morgan, and Fischel, working with etemophore eggs, howerer, found that isolated blastomeres of the two-, four-, or eight-cell stages developed "defective larva, having only four, two, or one row of swimming plates." Also "Crampton found that in the case of the marine gasteropod llyancrsa isolated blastomeres of two-cell or fourcell stages semented exactly as if forming part of an entire embryo, and gave rise to fragments of a larva, not to complete dwarfs as in the echinoderm" (Wilson, The Cell, p. 419). This attempt to form entire individuals from a portion only of a fertilized egg, resulting as it often does in dwarfs, seems to the writer a proces closely akin to regeneration (which see in chapter on "Relative Stability and Instability of I iving Matter"), and would seem to raise doubts as to its successful occurrence in the higher animals.

Though not bearing especially upon the point in question, the matter of twins in cattle is unique and worthy of mention. Three kinds of twins are known in cattle: "(1) the twins may be both female and both normal; or (2) the sexes may be different and normal; or (3) both may be males, in which case one always exhibits the peculiar abnormality known as a 'free-martin,' - the internal organs are male, but the extemal accessory organs are female, and there are also rudimentary female ducts" (Geddes and Thomson, The Evolution of Sex, p. 4 ). This is a kind of hemaphroditism, and not, as is commonly supposed, "a heifer twin with a bull." 
his mother or one of her male ancestors. Differences such as these must arise from strictly internal causes, which seem to set a natural and incritable limit to what may be accomplished through selection. Here would seem to be an irreducible minimum in variation, arising directly through reduction. ${ }^{1}$

Control. In so far as variations arise through changes in hereditary matter during the processes of maturation and recluction, they are, and must doubtless always remain, entirely beyond the influence of the breeder. It is quite evident that here is a degree of cleviation and an element in breeding that must be left to nature and subjert to the lara's of chance atithin the range of chararters meturat to the raci. That this will alwalys be a bar to absolute success is evident, but that it constitutes the strongest known argument for purity of blood is, in the opinion of the writer, beyond question, becallse the dhances of unfortunate dealiations are reduced in proportion to the furty of blood and the absence of undesirable characters.

Variation in parthenogenetic reproduction." IIad IVcismann's original assumption been correct to the effect that sextlal uniom is the only constitutional cause of variation, then individuals arising from parthenogenetic reprefuction should, barring the influence of surrounding conditions, be alike, because only the

1 Endeavoring to determine the function of the cytoplasm and nucleus, Boveri removed the nuclei from the eggs of sea urchins and afterward admitted spermatozoa to these enucleated ova. Development followed in a few cases, but the nuclei were smaller than in larva normally fertilized, contained lut half the mumber of chromosomes, and the resulting larvx possessed the "pure parental characters."

It is not supposable that anything like this occurs in nature, and yet it raises the question whether, after reduction, every remaining clement of the mucleus of both parents always plays its part in development. Should it fail to do so for any reason, herein would lie a sufficient cause for the occasional remarkable resemblance of offspring to one and not the other parent.

2 While in all higher animals and plants a union of a male with the female cells is necessary to each fertilization and to the production of young, it is by no means true among other organisms, especially in rotifers, crustaceans, and insects with which "parthenogenesis has become a fixed physological habit," thromgh which the unfertilized female cell develops a perfect individual.

It is now well known that the queen of the honeybee, if prevented from mat. ing, will yet lay eggs capable of development, lut they will all be dromes (males). After mating she can lay either fertilized or unfertilised egss, the fertilized developing into workers (undeveloped females), — or, if properly fed, into queens, the unfertilized into drones as before. Ifter the male element is eshatusted (she 
female and her germ cell are involveel. But individuals thus arising through unisexual reproduction aray widely, a fact easily credited when the phenomena of reduction are remembered.

Parthenogenesis being limited to lower animals, the range and character of variations are for the most part difficult of detection and measurement. It is known, however, that great differences in size occur among individuals parthenogenetically produced, and characters generally are so variable in such individuals as to lead to the statement that the variability of offspring

never mates but once) she is, of course, capable of laying only unfertilized or "drone eggs." In this way, in crossing, the drones and the workers may actually be of different breeds.

I'lant lice reproduce parthenogenetically during the summer season, producing only females; but as the temperature lowers with approaching autumn a mixed brood of both males and females appears, which, upon mating, produces the longlived, winter-enduring eggs. It is noteworthy that the parthenogenetic eggs of bees develop males only, while those of plant lice develop females during the summer, both sexes appearing as autumn approaches.

It is also noteworthy that under the artificial heat of greenhouses, approximating perpetual summer conditions, parthenogenesis continues indefinitely, and males are not produced unless the plants become badly dried up.

Parthenogenesis differs greatly in degree. It is supposed to be complete in certain minute crustaceans and in many rotifers among which " no males have ever been found." It is "seasonal" in the aphis (plant lice), and "partial" in the honeybee and "in some of the lower animals which are not themselves normally parthenogenetic, but have relatives which are." Occasional parthenogenesis has been frequently observed. An example is the silk moth, in which Nussbaum found that out of 1102 unfertilized eggs . . 22 developed ... up to a certain point. It is supposed that in all cases of parthenogenesis many eggs fail to develop.

In this connection it is noteworthy and extremely suggestive that among higher animals - frogs, hens, and even mammals - the unfertilized ovum occasionally begins segmentation, never proceeding far, however, on its parthenogenetic course.

The student should understand that in all probability a large number of eggs fail to develop into complete individuals even in the most successful parthenogenesis, just as do many fertilized ova fail along the way (see Weismann, Essays on Heredity, I, 175). There are all degrees of parthenogenesis, from the perfectly successful down to zero.

Bearing upon the general subject are the interesting experiments of Loeb in artificial parthenogenesis, especially of the sea urchin, normally bisexual, but which, after immersion in a saline solution of high density and subsequent return to normal sea water, commenced segmentation and afterward developed living larra. Magnesium and potassium salts proved most effective, though in general any treatment avails that serves to zeithdrane a portion of the zuater from the unfertilized egg (see Loeb in American Journal of Physiology, III, 434; also Loeb, Studies in General Physiology, Part II, pp. 576-626, 638-692; Methods, pp. 766-772 ; Geddes and Thomson, Evolution of Sex, pp. $\left.1 \delta_{3}-198\right)$. 
asexually reproduced is not "immensely reduced below the variability of the race." 1

In the honeybee only the male sex is produced parthenogenetically. In plant lice it is commonly the female alone under high temperature, and both sexes under lower. Weismann bred separately two varieties of Cypris reptans for some seven years, covering more than forty generations and " many thousand individuals." One variety, A, was light in color; the other, B, was dark. No males were ever discovered in either, and it is supposed that this species produces only parthenogenetically. While, for the most part, the descendants of each were extremely alike, yet "minute differences invariably existed."

Not only was this true, but in $I 887$, three years after the experiment commenced, "some individuals of the dark green variety, $B$, appeared in the aquarium with the light variety:" The same variation appeared a second and a third time, and in the last instance intermediate forms could be made out. In IS9I another case occurred, and in the same year its converse appeared, - a few typical light individuals among the dark colony that had "bred true many years." 2 Does this experiment also throw light on the origin of varieties, and were these mutations? However this may be, it clearly shows that variability is not entirely dependent upon sexual union, and that even distinct varieties may arise without the intervention of sex.

The first significant fact in maturation of parthenogenetic eggs is that they produce but one polar body. ${ }^{3}$

From this point on two alternatives seem possible. In the first place, a second polar body appears to be forming in the usual manner and the separation of the nuclear matter takes place, but instead of passing out of the egg it remains bchind, fusingagain with the meleus of the egr proper, which straightway undergoes development, with its chromosomes increased to

1 Pearson, Grammar of Science, pp. 472-473.

2 Weismann, Essays on Heredity, I, I6I-164.

3 Often this polar body divides, giving the appearance of two, but a second one is not formed. It is quite remarkable, though entirely consistent, in the case of aphis, honeybees, and certain other forms that produce both sexually and asexu-

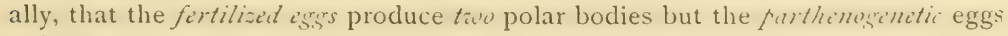
only one. 
the proper number. In this case the second polar body appears in the rôle of a male element, so we may speak of this as a kind of "fertilization by the second polar body."

In the other form of parthenogenesis, however, there is little suggestion of a second polar body; certainly no formal separation and later fusing of nuclear matter takes place. On the contrary, development takes place directly upon the extrusion of the first polar body, and it is significant that individuals arising in this way posscss but half the number of chromosomes as compared with those arising by senual reproduction or by the method just described. Some species (as in Artemia) ${ }^{1}$ reproduce parthenogenetically by both methods, giving rise to two distinct varieties, one with half the number of chromosomes characteristic of the other. ${ }^{2}$

Mutation as related to reduction and fertilization. Mutants scem to be departures characterized by a sudden loss of some racial character or by its possession in some unusual degree. They do not appear to be endowed with characters new to the race, except when artificially produced by hybridization.

If the process of reduction means the loss of hereditary material, and if fertilization means its restoration, and if either means in any sense new combinations, then we can see in the two phenomena taken together, or even singly, abundant opportunity for the most profound variations; indeed, admitting their possibility through these causes, the wonder is that they are not yet more common and infinitely more remarkable.

If there is in any sense, however slight, a qualitative loss through reduction, then by the law of chance the time is certain to come when something unusual will appear. Is it not more than likely that here lies a fruitful source of sweeping changes, as well as of the more obscure differences that are everywhere about us? And is it not likely that still greater and more frequent changres would present themselves were it not that fertilization is for the most part restricted to comparatively narrow lines?

1 Wilson, The Cell, pp. $2 S_{2}-2 S_{3}$.

2 Artemia thus varies from 84 to 168 , according to the particular method observed. 


\section{SECTION IV - BUD VARIATION ${ }^{1}$}

Variation is not necessarily connected with reproduction in the ordinary sense of the term. One limb of a peach may produce nectarines. A single branch of a tree may assume the weeping habit or the cut-leaved form. Not only are these wide deviations between buds of the same tree well established, but also all shades of differences exist, showing that one part of a plant may vary independently of another, quite after the manner of meristic variation among animals.

Bailey ${ }^{2}$ calls attention to the fact that the plant is not an individual with a simple anatomy like an animal, but that "its parts are virtually independent in respect to (I) propagation, . . (2) struggle for existence among themselves, (3) variation, (4) transmission of their characters by means of either seeds or buds."

Each bud, therefore, has a kind of individuality of its own. All but the first are developed asexually, yet all shades of differences will be found among these different members of what we call a plant or tree; hence each branch or phyton is a bud variety, and one which can be propagated by cuttings or by seeds or by both, and in either case can doubtless be improved by selection. ${ }^{3}$

Bailey makes the statement 4 that "the seeds of bud varieties are quite as likely to reproduce the variety as the seeds of seed varieties are to reproduce their parents." 5 He quotes Darwin in saying that "moss roses (which are bud varieties) generally reproduce themselves by seed, and the mossy character has been transferred by crossing from one species to another." If this be true, - if bud variations are transmitted by the seed, even to the slightest degree, - then the changes wrought in bud variation must be profound, extending as they do to the constitution of the germ, a fact which argues much for the ever-present

1 Bailey, Survival of the Unlike, pp. So-1o6.

2 Ibid. p. 105.

${ }^{3}$ Ibid. pp. 90-92.

${ }^{4}$ Ibid. p. 94.

5 Professor Bailey does not intend to say that seeds of bud varieties are certain to come true, but rather that no seed exactly reproduces the parent plant. 
liability to internal change and not at all, as is erroneously supposed, for the inheritance of acquired characters, because the characters in question were not "acquired" in the ordinary acceptance of the term, - they were the result of internal, not external, impulses.

\section{SECTION V-INFLUENCE OF THE CONDITION OF THE GERM UPON DEVELOPMENT}

Staleness. Both the male and the female grerm cells are capable of living for a considerable time after maturation, so that fertilization may be somewhat delayed; how long is not known, and what the effect of delay may be is not fully understood.

Experiments by Vernon upon the ova and spermatozoa of the sea urchin of different ages, from nine to forty-five hours, indicate that the size of the larva is in some degree dependent upon the freshness of the germ at fertilization. ${ }^{1}$ The results of a number of trials were as follows:

I. With stale ova and stale sperm the resulting larva differed but slightly from the normal (in which both were fresh).

2. With fresh ova and stale sperm the larva were distinctly larger (5.8 per cent).

3. With stale ova and fresh sperm the larva were distinctly smaller than when both were fresh (4.9 per cent).

It is certain that the above combinations as to staleness are possible in the fertilization of mammals by mating and of plants by pollination. Whether the results are the same and whether the differences persist through life are, of course, unknown. The facts recorded are suggestive, but whether they will ever be useful remains to be determined.

Individuality of the germ. That successive germ cells from the same individual may be substantially different, even aside from considerations of maturation, is a fact beyond question. The ear of corn, likie its tassel, matures from the base upward. The tip kernels are not only younger but decidedly smaller than their half-sisters at the base. The different peas in a pod are

1 Vernon, Variation in Animals and Plants, pp. 105-108. 
not equally developed. One of the twin pair of oats is more or less undeveloped. Is this difference in size due to season, food supply, room, or to some peculiarity in the germ? It may be lack of room in pod-bearing plants, but it cannot be that in the case of corn. The strong presumption is, in the opinion of the writer, that these differences in size are partly due to differences in food supply but more largely to inherent differences in the germs.

\section{SECTION VI - XENIA, OR FERTILIZATION OF THE ENDOSPERM, - DOUBLE FERTILIZATION}

If one kind of corn be fertilized by another, the mixture will show the first year. For example, if white and yellow corn be planted side by sicle, the white ears will have many yellow grains, showing at once the effects of cross fertilization. These " off " kernels are the mixed seeds, but, reasoning from analogy, we should not expect the mixture to appear until the grains are planted and the generation of mixed breeding is at hand. The visible part of the kernel is not the germ; it is the "endosperm," or surrounding portion, which serves as food for the sprout until the young plant has established itself. It is related to the germ much as the white of egg and its shell are related to the yolk. Fertilization is of the germ. How, then, do these outside parts become affected?

It will be remembered that in the animal the female germ gives rise to one mature functional cell, the orum, and three non-functional, the polar bodies; that the male cell gives rise also to four mature cells, the spermatozoa, all functional, and that the nucleus of the one unites directly with that of the other without intervening muclear divisions.

In plants, however, it is found to be substantially different. The mature female cell, corresponding to the ovum, instead of awaiting fertilization, continues its activity, undergoing generally two (sometimes more) divisions of the nucleus, giving rise to eight, or some other corresponding number of "sub-nuclei," which remain floating within the cytoplasm. It will be remembered that of these eight sub-nuclei only one is capable of functioning as an egg nucleus; also that two others remain near 
the center of the embryo sac to form the endosperm. It will be remembered, also, that the pollen nucleus undergoes a second division during its progress down the pollen tube and before uniting with the egg nucleus.

Of this divided nucleus one portion unites with the single functional member of the female group, making the germ, and in cases such as are now under consicleration the other joins with the minor members concerned with the development of the endosperm. In this way, by means of this kind of double fertilization, the endosperm is itself affected and the crossing is evident the first year.

Of course this visible effect upon the endosperm is of itself purely transitory, having no influence upon the line of descent. The real effect of pollination is manifestly, as in all other fertilization, confined to the germ.

Whether the two fertilizations are similar as to comparative influence of the two parents no one knows, nor cloes it greatly matter. The effect upon the endosperm enables us to detect the cross, if it is capable of detection, and to remove the contaminated seeds if we desire to retain purity. If the object be to secure crossing, we shall of course subsequently deal with the products of the cross-bred germ, which only are significant from the breeder's standpoint.

Just what species indulge in this double fertilization is not well known. It is, however, well established in a large number, and the process is supposed to be common rather than unusual.

Effect of crossing upon fruit in general. What the layman calls fruit is commonly not the endosperm that has been under discussion but the thickened and much developed fleshy receptacle on which the seeds are borne. It has been claimed that these parts are directly influenced the first year by crossing, so that the character of strawberries, apples, pears, melons, squashes, etc., depends much upon the source of the pollen used in fertilization.

This claim has never been well substantiated by direct experiment. Dr. Burrill, of the University of Illinois, tells me that he crossed Crescent strawberries both with the Sharpless and with a wild berry especially selected for its insignificant, worthless fruit. Nobody was able to detect the difference in the resulting 
crops. So far as is known to the writer, the same principle holds in other fruits. It is the endosperm and not the receptacle that is directly affected by fertilization, and any influence upon the latter must be indirect and comparatively slight.

Possible indirect effect of pollination upon the development of fruit. Though the receptacle is not itself fertilized, its development is conditioned upon that of its superincumbent seeds, which are themselves directly dependent upon fertilization for their development.

This fleshy growth of the receptacle is, therefore, the result of a kind of stimulus from the growing germ, and it is conceivable that this stimulus may differ somewhat in degree, depending upon the source of the pollen. In this way the siac of the fruit might be indirectly influenced by the pollen; and in fruits like the pear, which are not concentric about the seeds, even the shape might be influenced in the manner noted.

All this is quite independent of certain markings of fruit which may arise by those dispositions of color which are everywhere responsible for stripes and spots, and whose causes are not as yet understood.

\section{SECTION VII - TELEGONY}

The term "telegony" is synonymous with "infection of the germ " and the "influence of previous impregnation."

By this is meant the supposed influence of the male upon the female in such a way as to affect future offspring by other sires.

Breeders of animals quite generally believe that the influence of one impregnation, especially the first, is permanent and will affect all future offspring; indeed, some go so far as to say that a female once mated to a male of a different breed is ever afterwards, for breeding purposes, herself a cross-bred animal. ${ }^{1}$

This supposedly permanent effect of the male upon the female has been especially claimed for horses, dogs, and men.

Telegony in horses. The classic example among horses, and the one that is everywhere cited as proof of the theory, is the

1 This theory seems to be limited to animals. The writer is not aware that it has ever been claimed for plants. 
instance of Lord Morton's mare mentioned by Darwin. ${ }^{1}$ This mare bore a colt by a quagga, which was of course striped after the manncr of his sire. She afterwards bore two colts by a stallion, both of which were said to have been marked with bars on shoulders and legs supposedly showing the effects of the quagga upon the offspring of the stallion.

Professor Ewart, of Edinburgh, has recently repeated this experiment on an extended scale, with results showing no trace of the quagga beyond his own offspring. ${ }^{2}$ Recent investigations in contemporary literature throw grave doubt upon the essential accuracy of the data at Darwin's hand. They seem to show that the supposed resemblance of the stallion colts to the quagga was exceedingly fanciful, probably being nothing beyond what appears frequently in young horses of the purest parentage, dun-colored horses as a rule showing more or less tendency to stripes and bars.

It is one of the best evidences of the power of tradition that this single instance, happening more than a hundred years ago, has done duty ever since to prove (?) an exceedingly doubtful theory and an almost unaccountable belief. It is remarkable that so uncertain a circumstance, and one so easy of repetition, with universal experience tending constantly to throw light upon the subject, should have been so excessively overworked. It shows, as no other instance has ever shown, the persistence of tradition, the extent of credulity in the presence of the phenomenal, and the willingness of men to repeat an assertion, or even an opinion, until by mere repetition it comes to have all the force of authority. The thanks of the world are due to Professor Ewart for his excellent work in disposing, by direct experiment, of a citation that has done damage long enough. It is to be hoped that the question may at least be held open until some sort of positive evidence is brought forward that is worthy the credence of careful students.

Telegony in dogs. Dog fanciers are pretty generally credited with believing in telegony, especially in case of first matings.

1 See Darwin, Animals and Plants under Domestication, chap. xiii, p. 17, of second edition by Appleton. (Quoted from Philosophical Transactions, I82I, p. 25).

2 Breeders' Gazette, XLI, roog. 
The best students, however, insist that very little real evidence has been produced on the subject, and none at all tending to prove the existence of this influence. ${ }^{1}$

With a view to testing somewhat the real extent of this belief, the author addressed letters to the best-known dog fanciers of the United States. Of thirty-seven answers received, one writer is a believer in telegony; six somewhat mildly express uncertainty; two are non-committal; and twenty-eight are outspoken against the theory. The most outspoken of them all is a well-known fancier of long experience. Judging from this small number, it would seem that this belief among dog fanciers has been overrated.

Proof by the method of instance. Without a reasonable doubt belief in telegonic influence rests upon stray instances, difficult of understanding by those who happened to be the observers, and hastily accepted as evidence. Now nobody should be more careful than the breeder to judge accurately the nature and value of evidence. A single instance may be good negative testimony, but it is seldom worth much as positive evidence. The products of breeding are so many and so various, and the causes of variation are so numerous and so complicated, that a particular result can seldom be assigned to the operation of any single cause. It is more likely the mixed or composite result of many influences, both internal and external; and in order to know the effect of a single cause it is necessary to isolate the case if possible, or, if not, to resort to the examination of large numbers of cases, subject to varying degrees of influence, and thus indirectly to estimate the effect of any special cause of variation. For example, stripes and bars were once common color markings of horses, as they are now of asses, especially zebras and quaggas. Consequently a certain proportion of colts, whatever the parentage, will be born with traces of shoulder and leg. markings. Now, under the laws of chance, a certain portion of these will be the direct offspring of striped or barred sires, and will attract no attention, the markings being considered hereditary. By the same law of chance a certain (smaller) portion will be the offspring of parents not barred, and a still smaller number

\footnotetext{
1 Proceelings of the Royal Society, LX, 273.
} 
will be the progeny of unbarred sires and out of dams onee mated with barrel sires for other offspring. This smallest portion, getting its bars not by direct descent but by reacrsion, will most likely be erroneously considered to have derived them from the barred male not their sire. The same is true of other markings, and on such evidence as this the theory of telegrony has been built up), and, so far as proof goes, it rests on no better foundation as yet. In order to secure evidence amounting to proof, it is necessary to examine large numbers involving both positive and negative evidence, in order to secure trustworthy averages. Whenever this has been done the theory of telegony fails of support.

Telegony in man. The statistical method has been applied by Pearson ${ }^{1}$ in the case of man. He, together with Galton, possesses data covering hundreds of individuals in Engrish families. Ile reasoned that if the sire exerts a permanent influence upon the dam, tencling to assert itself in all future offspring, then this influence must be in a sense (cumulative, so that the younger sons in the family will tend to resemble the father slightly more than will the older sons, conceived before such influences have become established.

His study covered 385 brother brothers and 450 sister sisters, taken two and two. In some instances there was considerable difference in ages, and in others they were successive children. I is data covered both height and arm length, and after making the usual allowances for sex and age Pearson concludes that, so far as these characters are concerned, "no steady telegonic influence exists."

Again, the many successive marriages of both colored and white women to men of opposite color should afford numerous examples of telegony were it a consequential force in heredity.

Scientific objections to the theory of telegony. If telegony exists, its influence over hereditary characters must be explained, so far as present knowledge goes, in one of three ways: (I) some effect upon the tissues of the female such as will influence future ova in their maturation or the embyro in its development; (2) something like a partial fertilization of immature and uncleveloped ova, in such a way as to influence their character at

1 procedings of the Royal Society, LX, 273 . 
maturation; (3) the retention of the spermatozoa from the first mating, and their action in successive fertilizations.

As to the first, there is no scientific ground for assuming the slightest effect of the spermatozoa upon the tissues of the female. It is the ovum that is fertilized, not the female, as was at one time supposed when fertilization was regarded solely as a stimulus.

As to the second, there is no ground for believing that the nuclei of growing immature ouggonia are in condition to unite, or that they are capable of uniting, with the nuclei of other cells in any capacity whatever.

As to the third, there is every ground for believing that the spermatozoa are not retained for any considerable time, else successive births would occur from a single mating. Moreover, as but one spermatozoön takes part in fertilization, the blended effect of two sires is impossible. It is even impossible in multiple births when two services are close together. If a litter of pigs is the result of two matings by different sires, some may resemble one sire and some the other, but none will resemble both.

\section{SECTION VIII - INTRA-UTERINE INFLUENCES}

It is a widespread tradition that distinct characters, especially abnormalities, may be impressed upon the individual while in utcro through the imagination or other strong mental impression of the mother. It has even been held in the case of hens, which would necessitate the exertion of the influence upon the orum itself.

The usual argument is that the intimate contact between the mother and the fetus renders the latter peculiarly susceptible to influences affecting the former. Thus marks and deformities of all sorts are popularly attributed to unfortunate sights and experiences of the mother before the birth of the young. Peculiarly marked calves are said to owe their markings to the strong mental impressions created by a steer or by other cows, and colts are believed by many to owe their color not so much to the sire as to the gelding mate that worked beside the dam while she was carrying her young. Persons with whom the tradition is strong often display a blanket of a pleasing color before the eyes of the 
mare at the time of service, and of course are extremely careful to protect her from umpleasant colors of any sort. ${ }^{1}$ The hold of this theory upon the popular mind is the best example afforded by breeding of the strength of tradition. The supposed reason on which it rests has slight basis in fact. The contact between the mother and the fetus is not so intimate as is popularly supposed. The fetus is absolutely dependent upon the mother for nourishment, it is true, and it lies floating in its fleshy incasement, which is in intimate contact with the tissues of the uterus; but there is no organic connection, no nervous interrelation whatever.

Anything which would curtail or shut off nourishment would of course injure or destroy the fetus. It is also subject to other accidents, as becoming entangled in its own cord, which may thus divide a limb or cause strangulation, - all of which, however, is quite aside from the matter in point.

The real question is whether, and to what extent, the fetus is influenced by peculiarities of nourishment during its development. It would of course be injured by poisons, and the clanger from administering anasthetics is great, but this discussion is limited to the direct effect of mental impressions.

The indifference of the fetus to its source of nourishment is shown by an experiment of Heape, ${ }^{2}$ performed for another purpose, but throwing light upon these questions. In this experiment "two segmenting ova were obtained from an Angora doe rabbit which had been fertilized by an Angora buck thirty-two hours previously, and were immediately transferred to the upper end of the Fallopian tube of a Belgian hare rabbit which had been fertilized three hours before by a buck of the same breed as herself. In due course this Belgian hare doe gave birth to six young. Four of these resembled herself and her mate, but the other two were unduubted Angoras." . . B Both of the Angoras were born bigger and stronger than any of the other young, and

${ }^{1}$ For a good collection of alleged instances, see Miles, Stock Breeding, pp. $28 \mathrm{I}-295$, or consult any neighborhood oracle.

2 Vernon, Variation in Animals and Plants, pp. I19-120; also Procedings of the Royal Society', XLVIII, 457.

${ }^{3}$ The Angoras were characterized and easily distinguished by their long, silky hair and their habit of swaying the head from side to side. 
they all along maintained their supremacy in this direction." Whatever this experiment proves or does not prove, it shows conclusively that a fertilized Angora germ preserves and develops its inherent characters perfectly in an exceedingly foreign environment, on which it evidently depends only for nourishment.

Mental impressions and nervous conditions are commonly invoked to explain birth marks and other natural abnormalities, such as the loss of a finger. In this connection two facts are to be carefully considered : first, there is certain to occur a large number of marks ("strawberry," "cucumber," and others) and many malformations of one kind or another. Scarcely an individual is absolutely free from something of the kincl. Again, mothers are subjected to all sorts of sights, sounds, and experiences during the many weeks of embryonic development, and it would be strange indeed if out of the thousands of cases some correspondence between marks and experience could not be figured out, especially by one whose belief is fixed and who, having the case at hand, needs only to find the proper "corresponding experience." The law of chance alone will insure an occasional correspondence between the two, - entirely enough to start the tradition and to maintain it afterward. As in theories concerning the control of sex, any theory stated will be verified half the time because there is but one alternative, so here, while the alternatives are more, the correspondence is certain sometimes to appear under the law of chance alone.

Another fact to be reckoned with is that if the fetus were so sensitive to mental impressions as to require the display of properly colored blankets, - if females were so susceptible as this to surrounding sights, — what a jumble of colors our domestic animals would speedily display. In the opinion of the writer this tradition has neither a scientific basis nor well-established instances, and it is time it no longer occupied the minds of breeders to the exclusion of far more important matters. In this connection it is worthy of remark that if the average breeder were half as familiar with important facts, and half as attentive to their bearing upon his operations, as he is familiar with and attentive to floating traditions, we should have a far smaller proportion of worthless animals. 


\section{SECTION IX - REVERSION AND ATAVISM}

These two terms are used to designate characters appearing in the offspring but not visible in the parents. "Reversion" is used to indicate resemblance to a comparatively near-by ancestor, not the parent, while "atavism" refers to exceedingly remote ancestors, sometimes of other and foundation species.

Thus, if a dash of impurity of blood enters a herd, it will appear occasionally for many generations. This would be spoken of as a "reversion to the strange blood." If a sire or dam has some peculiar character, like white stockings in horses, a peculiar horn in cattle, or a habit in man, it is likely to appear from time to time in future generations, even after its real origin is forgotten. This is a reversion. English breeds of cattle are developed from the ancient wild white cattle of Britain, and the occasional appearance in all these breeds of a white calf with red or brown ears, lower legs, and tail brush is to be expected. It is a reversion, not a proof of mixed blood. Of course the animal so marked is useless for breeding purposes, but no reproach to the herd, and none necessarily to the dam that produced it, for reversions for the most part seen to come singly.

Atavism, on the other hand, goes farther back. For example, mammals during their carly embryonic development still show traces of the gill slits, thus betraying their undoubted one-time connection with the same stock which gave rise to the aquatic animals. These gill slits occasionally persist, failing to close, and grive rise to the abnomality known as "cervical fistula." It is an undoubted atavistic abnormality, - an extreme case of course, because of its antiquity.

Cases of this kind are to be carefully distinguished from mere meristic variations. For example, the sudden appearance of a three-toed horse would be regarded as atavistic, for all horses once had three toes; but a sixth digit in man is certainly not ataristic, for we have no evidence that man ever possessed normally more than five digits. The criminal instinct in certain men is undoubtedly atavistic, showing not so much a delight in evil doing as an entire absence of the higher sense of right doing. 
There are certain intermediate cases difficult to name. An occasional cow gives no more milk than her wild progenitor; a hog resembles not his immediate kind, but his striped and longnosed ancestor, the wild boar of the bush; the horse or the sheep paws snow the first time he sees it, though cattle do not; the dog turns many times around in lying down, as if making his nest; the occasional horse has bars on his legs and stripes on his shoulders. Which term shall be applied?

These are undoubtedly line cases, and all would not agree as to whether they should be regarded as reversions or instances of atavism. Because of our very frequent need for a term to cover experiences met almost every day by the breeder, and due to more near-by causes, the writer is of the opinion that it is better for our purposes to extend the meaning of the word "atavism " well forward, making it cover cases of remote characters, even within the species (like those just given), leaving the term "reversion," which will be much more frequently needed, to cover the more near-by cases that occur every day in our herds, and that would be traceable, could we know all the facts, to an old but not remote ancestor.

Inheritance is from the race. It is evident that inheritance is not limited to the visible characters of the immediate parent. We constantly forget that every individual is possessed of and capable of transmitting all the characters of the race to which he belongs. We forget that his visible characters are not his total possession, but only those which are relatively most prominent in his case. Other combinations are easily possible out of the same elements in slightly different proportions, and it is not so strange as we think that a character once in possession of a race tends to persist indefinitely and, perforce, occasionally to become visibly apparent. It is as bound to appear, under the law of chance, as is the one black ball in the box of a thousand or a million, if only throws enough are made.

The work of Galton, while mostly confined to man, yet shows clearly that inheritance is not in strict line with the visible parental characters, but is in large measure independent of the immediate parents. ${ }^{1}$

1 See the Regression Table, sect. iii, cliap. xiv. 
Galton has endeavored to assess mathematically the fraction of direct inheritance, or, more accurately, the similarity between the child and its various ancestors. From his studies he concludes that the visible or dominant characters of the child are, on the arerage, inherited (that is, correspond with those of the various ancestors), roughly, as follows ${ }^{1}$ :

From the immediate parents

50 per cent

From the grandparents

25 per cent

From the great-grandparents

I 2.5 per cent

From the great, great-grandparents . . 6.25 per cent

Earlier ancestors in proportion

Pearson, working with larger numbers and diverse characters, concludes that Galton's fraction of direct inheritance $(0.50)$ is too high, and is inclined to believe it not above 0.40 for blended characters. The subject will be pursued farther under "The Law of Ancestral Heredity," but this glimpse into the nature of inheritance is the best method known to the author to dissolve the almost supernatural mystery that has been thrown around reversion and its natural corollary, latent characters. The study can be pursued no farther at this point, but what has been said will serve to show that reversion (regression) is a fertile cause of variation as calculated from the type of the parent. It will serve also as an introduction, preparing the student for the more serious study of inheritance later on, when we shall learn that the real type, from which all departures should be reckoned, is the type of the race, and not the special type of the parent, or even of the mid-parent.

\section{SECTION $\mathrm{X}$-INDIVIDUAL CHARACTERS DEPENIOENT UPON SEX}

That both sexes possess and transmit all the characters of the race is a well-established fact in evolution. It is also true that the particular characters to undergo development, and the extent

1 Vernon, Variation in Animals and Plants, p. 123; Proceedings of the Royal Society, LXI, 40I, 1897; Galton, Natural Inheritance, p. I91. This does not mean that every individual will inherit in this proportion, but that the fractions express averages. 
to which they will develop depends very much upon the sex of the individual. How much allowance to make on account of sex in comparing one inclividual with another of a different sex we are in most cases unable to say, - not from the impossibility of knowing, but from the fact that in respect to most characters the matter has not yet been worked out. It is easily possible, however.

For example, in respect to stature, men are 8 per cent taller than women, so that when the heights of the latter are multiplied by $1.08^{1}$ the two are strictly comparable, and not before. When this is done the difference duc to sex has been eliminated, and the statures of men and women may be directly compared.

In general, males and females exhibit the same characters, but in varying degrees. For example, the woman as well as the man has hair on the face, but in less amount; the male as well as the female has nipples, but they are rudimentary. Among mammals and the domestic animals generally the male is heavier in front, generally of a more robust build, and considerably larger than the female, - a distinction that by no means holds in animal life generally.

In the present state of knowledge we simply know that the general appearance of the individual and its character development are largely dependent upon its sex; but to what exact extent remains in most cases to be determined, and the determination must be made before we can compare individuals of different sexes with any degree of accuracy. Without a doubt distinctions in sex have been greatly overworked, the differences being mostly of degree rather than of kind, ${ }^{2}$ and far less consequential than has been supposed.

Individuals deprived of their sexual organs by castration or by spaying do not develop their primary sexual characters. The castrated male is not a female, as is sometimes erroneously believed, but a male arrested in his development ; and the spayed female is an undeveloped female. As would be expected, both

1 These data are the result of Galton's study of the stature of English people. See Galton, Natural Inheritance.

2 It is idle to attempt to prove that certain characters, aside from those of reproduction, are especially identified with one sex. 
take on the secondary sexual characters (those dominant in the other sex) prematurely young. ${ }^{1}$

Many entire individuals never develop strongly the primary characters of their own sex. There are effeminate males and masculine females, - those in which the characters of the opposite sex are unusually dereloped. It is needless to say that such individuals are not the best parents.

\section{II-IVTERNAL INFLUENCES AFFECTING THE RACE AS A WHOLE}

Over against those causes that may operate in the case of each individual to warp its development are to be considered those that influence the race as a whole, turning the line of descent more or less permanently aside from former channels. Some of these influences are clearly defined and easily recognized; others are problematical, the discussion not having yet passed beyond the stage of a plausible theory.

The student of thremmatology and the breeder should be always mindful that the purpose of all good breding is not simply to hold what w'e already have but to produce now types better adapted than the old to the purposes of man. Accordingly any and all lines that promise any hope of success should be assiduously investigated.

\section{SECTION XI-RELATIVE FERTILITY, OR GENETIC SELECTION 2}

The assumption that all members of a race are equally fertile in se and inter se (of themselves and between each other in all (irections) is not only hasty but dangerously incorrect. To quote Pearson, "Fertility is not equally distributed among als individuals."

1 The entire animal with increasing age, its own sexual characters abating, begins to take on those of the other sex. Thus the hen grows spurs, the cow bellows and paws the dirt, women grow scanty beards, and old men's voices grow light.

2 Pearson, Grammar of Science, pp. 376, 414, 437-449, 462. 
If this be true, and practical breeders know that it is true, then an interesting and important question at once arises; namely, What characters are correleted with the highest fortility? This is important, because these are the ones that will become the dominant characters of the race, certainly unless opposed by the most rigid selection or by other powerful influences. This is genetic selection, - an ever-present influence over the line of descent, tending to establish what might be called a natural type.

Unfortunately we possess no accurate data on this point among domestic animals, but Pearson's work ${ }^{1}$ among men and plants is sufficient to settle the principle that such a definite influence exists.

He finds, for example, that daughters are not taller than their mothers, but that they are taller than wives in general. Now not all wives are mothers, and these data mean simply that taller women are on the average more fertile. There is thus some correlation between fertility and stature. This is genetic selection, and under it the stature of women (English) may be expected to gradually increase until such correlation is satisfied, unless held back by other influences.

Mothers are less variable, but daughters more so, than wives in general; that is, progressive selection exists, for not all daughters marry, and not all who marry produce young. If the standerat diation from the race were the serme for cach, the'n no scliction would bi involied, but it is progressively less from daughter to wife and on to mother. The difference bitaten danghter and wife is due to preferential mating, the espocially' usly indiaiduals being less likely to find a mate; but the differcuce between wife and mother is due to relatio' fertility.

The fact that in general the mother is nearer the average than is the wife shows that the race is fairly stable; but the fact that wives are shorter than mothers has but one meaning, that in respect to stature the race is yet unstable.

Extensive studies in eye color indicate that dark-eyed individuals, both men and women, are slightly more fertile than are the lighter-eyed. This means that the dark-eyed will progress (increase) upon the light-eyed and the race will grow darker-eyed,

1 Pearson, Grammar of Science, pp. 44I-445. 
unless the tendency shall be held in check by the greater attractia'cuess of lighter cyes, - prefirential mating. This would be a long and slow process, but it would avail much to reduce, though it could never overcome, the effects of the higher fertility of the darker-eyed individuals.

Pearson collected 4443 capsules of wild poppy. ${ }^{1}$ They showed the following distribution arranged according to the number of stigmatic bands :

\begin{tabular}{|l|r|r|r|r|r|r|r|r|r|r|r|r|r|r|r|r}
\hline Bands .... & 5 & 6 & 7 & 8 & 9 & 10 & II & 12 & 13 & 14 & 15 & 16 & 17 & 18 & 19 \\
Frequency... & I & I I & 32 & 56 & 148 & 363 & 628 & 925 & 954 & 709 & 397 & 155 & 51 & 12 & 1 \\
\hline
\end{tabular}

The largest number of capsules (954) had I 3 bands and the next largest number had I 2. Very few had so many as i 8 or I9, or so few as 5,6 , or 7 . The type number of bands is then 13 .

He provided receptacles and kept the scals of each group separate. He says :

To my great surprise, however, my receptacles for 12 and 13 were filled up with the contents of very few capsules, those for I I and i 4 more tardily, those for 10 and 15 only with emptying a great number of capsules, while I could hardly get any seed at all from those capsules with very many or very few bands; they were practically sterile. The type capsules were enormously fertile, [while] those with even a moderate deviation from it [were] relatively or even absolutely infertile. ${ }^{2}$

This being true, the poppy has become about as stable as is possible, for its highest fertility is with its most numerous population. This plant was growing wild in nature. Obviously the great bulk of seeds clistributed would be of the type number, I 3 or near it, and the mass of descendants would arise from seeds close to the type. What chance now would there be in nature for a large colony of six-or seven-banded strains to arise? Very little, unless they happened to possess some decided advantage in the struggle for existence, in which case the type would speedily shift in that direction; but as long as the highest fertility remained with the higher number of bands, the race would be unstable.

\footnotetext{
1 Pearson, Grammar of Science, pp. 443-444.

2 Ibid. p. 444.
} 
Suppose it were the purpose of man to develop a poppy with fewer, or with more, than the natural number of bands, - say seven or seventeen. Under what disadvantage he would work as long as the fertility remained relatively low! and he would never succeed unless he separated the plantings from the more prolific type. This is genetic selection.

Breeders are constantly operating against the drag of infertility without knowing it, and are as often wondering why better results do not follow, especially when only approved mating is practiced. Consider the mathematics involved in, say, three lines of descent of different degrees of prolificacy. For the salie of simplicity in illustration let us suppose three cows were living in a herd together. One of these cows raises two calves and becomes barren; another raises four before she ceases to breed, and another six. For the sake of further simplicity let us suppose that one half the calves are females, and that each daughter descendant exactly repeats the performance of her dam and then becomes barren. How will the account stand in a few generations ? 1

Cunulative Effects of Fertility as shown by the Relative Number of Female Dfscendants of Cows of Various Degrees of Fertility

\begin{tabular}{|c|c|c|c|c|c|c|}
\hline \multirow{2}{*}{ Cows } & \multirow{2}{*}{ Calves } & \multicolumn{5}{|c|}{ Generations } \\
\hline & & I & 2 & 3 & 4 & 5 \\
\hline No. I & 2 & I & I & I & I & I \\
\hline No. 2 & 4 & 2 & 4 & 8 & I 6 & 32 \\
\hline No. 3 & 6 & 3 & 9 & 27 & $8 I$ & 243 \\
\hline
\end{tabular}

This tabular presentation shows that after five generations of this kind of breeding there would be but one fortile cow of the first order in the herd, ${ }^{2}$ while if all had been kept there would be 32 of the second order and 243 of the third. What an

1 There is no longer any doubt that fertility is an inheritable character.

2 There might be any number of living barren ones if the strain happens to be a favorite and is long-lived. 
opportunity for selection in the latter instance, with none whatever in the former! The first untimely death would render the line extinct, which is perhaps the best fate that could overtake a race which at best is able only to hold its initial number good.

Of course artificial conditions have been assumed in order to bring out the principle. It does not work out in this regular and evident manner in our herds, but the principle of genetic selection is at work, nevertheless. It would be fortunate if it were more evident, for the herds are few that do not contain a large proportion of females that contribute nothing to the real line of descent, though they now and then give birth to exceptional individuals. The quality is good, but the rate of reproduction is too low.

How many a breeder has spent fruitless years in ineffectual attempts to build up a strain excellent in itself but essentially infertile! Witness the fate of that remarkable family of shorthorns, the Duchess. This famous family, in its glory, was never surpassed, yet it was genetic selection that exterminated the line. Fortunate indeed is the breeder who knows this principle and realizes its full power whenever he finds himself opposed by it.

The student must not get the impression that genetic selection is an enemy only. A prolific line tends as strongly to establish and maintain itself as does a barren one to rush headlong to extinction. Genetic selection is therefore a friend powerful for good, as well as an enemy powerful for evil; but it is as quiet and unobtrusive in the one relation as it is insidious in the other. The breeder has only to be eternally conscious of the fact that if he is to succeed he must have numbers, not occasional births, but regular and generous. Then he may be sure that he is not trying to do a thing on which nature has set the seal of her clisapproval through non-production. However worthy and however valuable intrinsically the strain may be, it is worthless unless he can produce it with certainty and in any desired numbers. "Beware of the shy breeder, and treasure the old female that breeds regularly and true." This doctrine establishes a coöperation with nature that insures results, and without it genetic selection will work against us, not for us. 


\section{SECTION XII - PHYSIOLOGICAL SELECTION}

The term "physiological selection" refers to the fact that certain individuals, fertile enough of themselves, will yet absolutely fail to breed with a particular indiridual of the opposite sex. ${ }^{1}$

This principle is now well established and is recognized as a large cause of fruitless marriages. Individuals are frequently barren in one marriage and perfectly fertile in another. Physiological selection is a phase of genetic selection, and while of extreme importance in the marriage relation it constitutes no special menace to our herds. In general it has little bearing upon the development of a breed, but is often exceedingly troublesome when it is clesired to effect a particular combination of blood lines.

\section{SECTION XIII-SELECTIVE DEATH RATE; LONGEVITY}

The total population depends as much upon longevity as upon fertility and the prevailing type at any moment depends as much upon the individuals that die out of the world as it does upon those that are brought into it.

If the draft by death is equal, or rather proportional, from all types of the race or breed, then the existing type will be the same as that born into the world; if not, it will be different.

As there is little use in attempting to breed a strain, however clesirable, that is not at least fairly prolific, so there is little use in spending time and expense upon short-lived strains, especially of milch cows and horses, which are valuable largely in proportion to age.

For reproductive purposes the "age" of an animal is the age at which he stops breeding, but for other purposes it is the age at which he can no longer render valuable service in the clesired direction, such as labor.

1 This principle was first announced by Romanes ("Physiological Selection," Journal of the Linnann Society", XIX, 337-4II), though what he had in mind evidently included what is now known as "genetic selection." It was proposed as showing that other principles are at work to fix types, aside from the struggle for existence. 


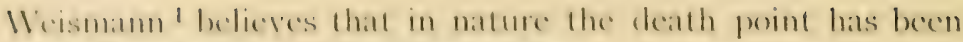
fivel all an ane most profitable lo the race as a whole. That is

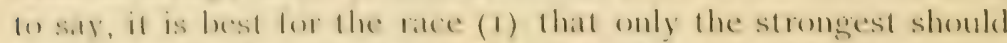

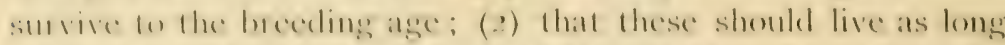

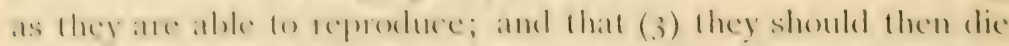
.mol rease for acouply form and consume food which would other-

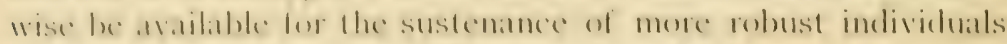

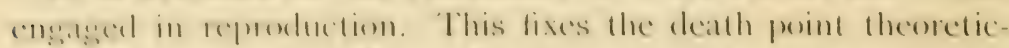

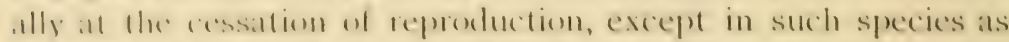
llose in which the youme need the care or the colucative assist-

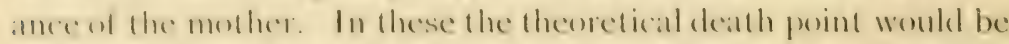
.11 the mallutey of the last fomme. This of course is in reference lo wihl speries, and Wismamn believes that mature has estab-

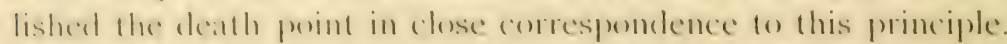

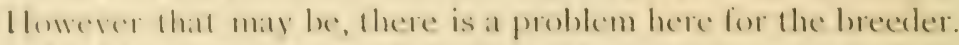
It is tor him to tis the death limit well beyomel the periogl of the

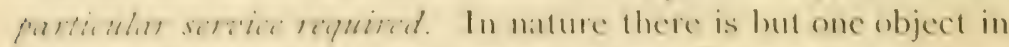
lite, sell posestaltion and reproduction. On ont farms there .16 whe whjerts. The horse is for laber, and his servicable

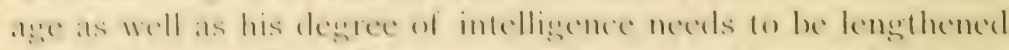

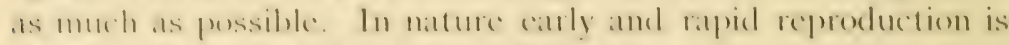

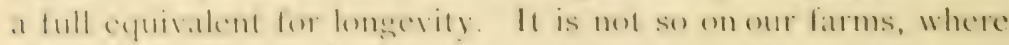

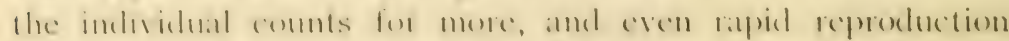
cammot take the place of long life and fathful service.

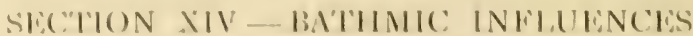

Do species possess inherent tendencies to vary? If a race

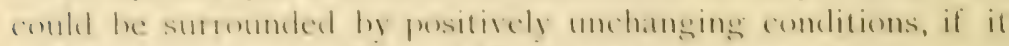
combl promlace asevually, and if all lypes were equally vigorous

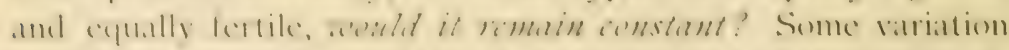

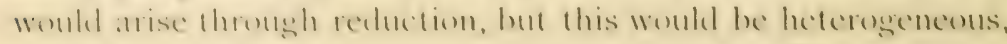
- that is, now in one direction, now in another. The real queslions the bathmic evolutionist asks are these: Is there a tend-

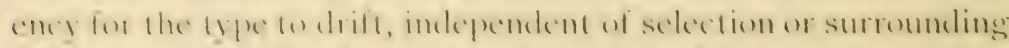

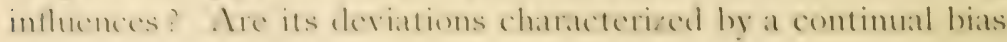

1 Weismann, lissays on Ileredity, 1, 11-163; see also Pearson, Chances of De:ulh, pl). 1-.12. 
in favorite directions? Does it vary progressively because: impelled in these direstions by "swowth force" or other inheremt encrey? Are species hedel to their present stamelards by outside: influences? or, if not "helel," are they drifting in spite of us? Is the life principle constant or perioglic in its atctivities; and are these internal energies that vitalize matter and that determine development and differentiation,- are they imbliferent as to the frenel of the type, of do they run more casily in some channels than in others? Is variat ion in some sense subjeet to and direeted by a natural bias? 'This is the ficlel of bathmis evolution, and these are the questions involved. No one is more interested in their discussion than is the breeder of domesticated forms.

Two principal theories covering the field of bathmie evolution have been proposed, both incaprable of absolute promf, as all such theories must be, but both of interest to the breeder.

Acceleration or retardation of growth force. This principle is annemeerl by Cope as an internal and ereepresent cause of prongessive covolution, rumming through all foms of life and

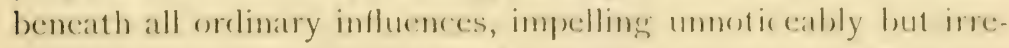
sistibly in rertain directions. It is, after all, alcererling to this atubor, the most suldte and most potent callse of depatture fom

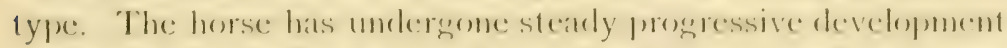
from an animal of the size of a jack rablsit mp (1) his present proportions and perfection. This is dee, areorlinge tor cope,

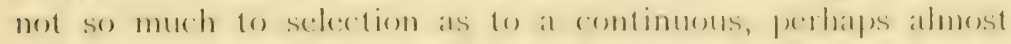
unprecedented, acciloration of groweth force.

This theory attempts an explatin much of evolution through the energy of growth, thus throwing into the discussion it dynamic elenent commonly megrlected by evolutionists. In this connection Pearson pertinently remarks:

There is nothing more (or less) unscientific in using an inherent growth

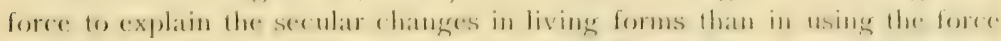
of eravitation inlerent in matter on explain the devedepment of planetary

1 Pearson, Crammar of Science, pp. 375-377. The term "bathmic" as here used does not include genetic selection or any other selective agent, internal or external, because the effects of all such influences tend to come to a rest and not to constitute a "continual bias."

2 Cope, Origin of the littest, pp. 18-30, 190-192, 396-398; P'rimary lactors of Organic Evolution, pp. 473-49\%. 
systems from nebulic. The ultimate action of vital units in each other's prescnce would be no more, nor less, of a mystery than the ultimate action of material units. . . . The real objection to bathmic evolution lies not in any a priori reason against an inherent growth force, but to the obvious historical fact that such a force has been used to cover all sorts of obscure reasoning and even sheer foolishness. Science would welcome above all things a description of the action between vital units as simple as the law of gravitation, provided it gave a causal account of variation; and the welcome would be none the less sincere if the action showed that variation was biased and that evolution would be irreversible even with a reversed sequence of physical environments. ${ }^{1}$

Cope's theory of acceleration or retardation of growth force is of course merely quantitutiz'c, and would explain any differences that might arise through either size or proportions of parts, or faculties dependent upon such proportions. It does not attempt to explain the introduction of characters, and if it can be in any way controlled no method has yet been pointed out.

We all allude to the same general thought when we use the words "vitality" and "constitution" to denote not so much tenacity of life as vigor of growth, and we all recognize that some individuals and some lines possess this faculty in much higher degree than others. Some individuals never survive the embryonic stage; others dic in infancy ; still others reach full maturity, and a few persist to an advanced age. As death comes only with the failure of some vital function, the individual may persist long after he is stripped of everything that makes life enjoyable.

It is so with races. Some seem endowed with phenomenal vigor, while others are preserved from extinction only with the greatest difficulty and by the narrowest margin, not only because of low fertility but also by reason of inherent lack of vigor. The existence of these internal forces is not a matter of cloubt, and their office in directing variation is an interesting and valuable problem which the present strate of knowledge is insufficient to solve.

Orthogenesis. Closely akin to Cope's conception is Eimer's theory of orthogenesis ${ }^{2}$ (straight, creation), or, as he calls it, "definitely directed evolution."

1 Pearson, Grammar of Science, pp. 375-376.

E Eimer, On Orthogenesis and the Importance of Natural Selection in SpeciesFormation (pamphlets, $5^{6}$ pages) [Open Court Publishing Company]. 
This theory of Eimer's is put forth as a protest and a counter proposition to the theory of Darwin, - afterward very much elaborated and extended by Wreismann and others, - which was to the effect that all evolution is the result of heterogeneous growth trimmed down and shaped up by the extinction of individuals possessing unfavorable characters. The natural assumption of the extreme selectionists is that utility is the basis of all sclection, and that only useful characters will be preserved, the inevitable corollary of which is that all cristing chavacters are useful.

Now the necessary consequence of selection is that after a time all existing forms and characters will come to "fit" or agree with the conditions of life, which are the natural agents of selection. This "fit" is so accurate as to deceive many observers and lead them to declare selection to be a fundamental cause of variation.

Eimer points out two facts ${ }^{1}$ : first, that there can be no selection until a choice is presented, - therefore that the selective process follows and does not precede the origin of a deviation; that selection may and does cause the race to vary, but that it has nothing $t$ o do with the prescutation of the variation in the first individual, - a position in which he is certainly correct.

He argues, sccond, that it is not truc that all characters are useful, but that many species endure those that are inconvenient and unfortunate, yet not sufficiently detrimental to be fatal, else the line would become extinct and no such instances would erer be seen.

His position is that, first of all, "organisms develop in definite directions without the least regard for utility, through purely physiological causes, and as the result of orgrmic growth." 2

Then, after all the characters hare developed together, they are passed upon by natural selection in the struggle for existence, this process blotting out only those sufficiently detrimental to unfit the individuals so afflicted for continuing the struggle in competition with more favored forms. Selection does not remove a handicap, or relieve a race from all undesirable characters. It eliminates only the worst, and down to a level sufficient to establish a kind of "equilibrium of life."

1 Eimer, Organic Evolution, sects. ii and iii.

2 Eimer, On Orthogenesis, p. 2. 
In this view of the case characters bad and good develop together. The a'orst ones are eliminated, but many undesirable or indifferent ones are left behind as not beingr sufficient to turn the scale against the individual or the race. Thus many undesirable characters linger in all races, and, what is more to the point, utility is not the canse of cither the origin or the persistence of charactiss, but only of thit obliteration avhen sufficiontly detrimental to destroy the indiadual and therefore cut off descent in that particular line..

The writer shares the opinion that this is the true limit of the selection process under nature, and that the presence of unfavorable characters argues for their having arisen from causes quite independent of selection.

In our yards and fields we control selection according to whatever standards we may please to establish, but if unfavorable characters develop in nature, where selection aims directly at life, will they not be likely to develop also, unnoticed, under our own selection, especially when we do all within our power to preserve life ? ${ }^{1}$

The presence or absence of a principle aside from selection, yet responsible for the presence of characters, turns very largely upon the (question as to whether, after all, there are well-established instances of characters independent of utility, and therefore of selection. The presence of such characters is easily shown. For example, what is the utility of the scrotum among mammals? Would it not have been better with sheep, for instance, if the testicles had remained within the abdominal cavity, where they develop, and where they would be safe, instead of descending into an external sack exposed to frequent injury? Undoubtedly it would have been better for indiziduals, for many have not only lost these organs, but their lives as well, from this unfortunate position; but the mumber lost is not sufficiont to scriously affect the race. ${ }^{2}$ In other words, selection has aimed at this vulnerable

${ }^{1}$ It is noticeable that nature allows reproduction to go on unrestricted, and then slays by the millions. Man cannot afford this wholesale destruction of numbers. He seeks to prevent undesirable births, - a kind of advance selection that has both its advantages and its disadvantages.

2 This shows that what is bad for the individual is not necessarily bad for the race ; conversely, what is best for the race is often hard upon or even fatal to the individual. This is the very essence of selection. 
point many times, and in numerous cases with effect, but mammals as a race have been able to enclure the handicap, else they would long since have become extinct.

This shows that some causes other than utility are responsible for the appearance and continuance of racial characters; that teleology ${ }^{1}$ is not a universal principle, and that the function of selection is restrictive, not creative.

Other characters not teleological may easily be mentioned :

I. The peculiar minute markings on diatoms and on other inconspicuous organisms.

2. The green color of leaves, due simply to the fact that chlorophyll is green. This is no more of an inherent necessity than that coal should be black or gold yellow.

3. The digital number five which runs generally through vertebrates, which has often been modified and often left intact. Certainly the original number five could not have been teleological. It is not enough to assume that changed conditions might have rendered an organ detrimental which was once useful. There are too many obviously useless characters.

4. The phosphorescence of pelagic animals, ${ }^{2}$ and the pearl of the oyster, which is due to injury. Is this beauty useful or is it accidental ? ${ }^{3}$

5. The bright color of deep-sea fishes. Is it any more significant than the (acciclental) color of chlorophyll-bearing leaves ? ${ }^{4}$

6. The horns of stags, - useful (?) in battle, but weapons as dangerous to the possessor as to his enemy.

The list might be extended indefinitely. Eimer's argument is that characters such as these have been produced not through selection but in spite of it, and through the agency of organic growth in definite directions, which is orthogenesis. It would be difficult to be always certain that no basis of utility exists or ever has existed simply because it is not now erident, yet no

1 The doctrine that development is in line with utility and that everything is useful is known as "teleology."

2 Eimer, Organic Evolution, p. xiii.

${ }^{3}$ Eimer, Orthogenesis, p. 10.

4 In certain leaves of bright color the chlorophyll is unable to dominate the stronger shades of other chemical substances, and the leaf is not green but some other color. 
careful student of evolution doubts any longer that there are many misfits in mature. Whether they have arisen, as Eimer asserts, by reason of organic growoth, and whether they are evidences of difinitily dircted deaiation, is quite another matter.

There is no doubt of the persistence of a character once started, even in the face of selection, but whether it be necessary to invole the aid of an internal directive force to explain it is a question upon which more evidence is sorely needed. It is exceedingly important for the breeder to know and recognize all the inherent tendencies with which he must finally reckon, and it may be necessary to go beyond physiological units, corresponding to chemical atoms or molecules, and invoke some form of "growth force" corresponding to chemical energy to explain the mysteries of development. Any theory, however, that will even reasonably account for these mysteries must be, in the present state of knowledge, largely an assumption, and let the assumption be as simple as possible until we can defend its complexity by a mass of well-established facts. In the opinion of the writer the existence of such a principle as orthogenesis is more than problematical, except as it is an expression of the relations that naturally obtain between physiological units, whatever they may be.

\section{SECTION XV-PHYSIOLOGICAL UNITS}

The "gemmules" of Darwin, the "stirp" of Galton, the "idioplasm" of Nägeli, the "biophors," "determinants," and "idls" of Weismann, and the "physiological units" of other writers are all attempts to explain inheritance of definite qualities by assuming that the germ cell which passes over from parent to offspring at the time of procreation is composed of definite units of living matter, each with its specific properties, among which are nutrition and multiplication, which together constitute growth, and - considering the separate properties of the different units of which a given individual is composed growth in defuite directions.

In support of this general theory it may be urged that the individual is what he is very largely because of intrmal qualities. Corn and wheat grow side by side, drawing their nourishment 
from the same soil and the same atmosphere. The most nourishing food and the most deadly poison are produced side by side under identical crtemal conditions. A man divides his dinner with his dog: one portion simply nourishes the dog and provides energy to watch sheep or perchance to kill them; the other results in strength to bless the ages, or perhaps in crime to shock the world, - each according not to the nature of the food but to that of the animal that consumes it, and to the support of whose peculiar energies it contributes.

This kind of difference in living organisms is traceable to the endowments of a single cell, - the only material that passes over from parent to offspring, - and, regard it as we may, we must see in this single bit of living matter all the potential qualitics of the race, all the differences between the corn and the wheat, between the man and his dog. They are all there, represented in some material way in the constitution of the germ cell. There is thus a material basis to heredity.

We must accept one horn or the other of the dilemma: either conceive this single cell as directly endowed with all the qualities of the race, defining its development, or else endow it with the capacity to develop in this fashion or that according to stimuli. But whence come the stimuli? Certainly not altogether from without, or the man and his dog would become alike, if consuming the same kind of food; and to assume that the influences are internal is only to push the puzzle one step farther away and to assume possibly an immaterial in place of a material basis.

The most simple and direct explanation of the phenomena of inheritance and definite development is to consider the germinal matter as consisting of units of some sort endowed with life and the power of growth. This assumption of the physiological unit is not so violent nor so different from other accepted scientific assumptions as it at first may seem. In the non-living world we assume the existence of the atom, whatever its ultimate constitution, as a minute, indivisible, and indestructible unit of matter. The association of some millions of like atoms makes a measurable quantity of an element like gold, silver, iron, chlorin, or sodium. 
Something over eighty distinct kinds of matter are known; therefore some eighty kinds of atoms are assumed, and this exhausts the possibilities so far as unlike like atoms are concerned (unless other atoms are subsequently discovered or created).

But this does not exhaust the possibilities of matter, for these atoms combine together, forming new units (called molecules) with clistinct properties. Thus $\mathrm{NaCl}$ (sodium chlorid) is different in every way from either the sodium or the chlorin atoms that have united to produce it. In this way these (eighty) various atoms effect all sorts of combinations, many of them exceedingly complex, ${ }^{1}$ each constituting a new material unit, a sufficiently large number of which constitutes a measurable quantity of a substance whose real composition could rarely be predicted by any of its visible properties. The following table of chemical formula is presented for two purposes: (I) to show the exceeding complexity of ordinary materials; (2) to show how certain groups of atoms (as $\mathrm{CH}_{2}$ or $\mathrm{CO}_{2} \mathrm{H}$ ) ${ }^{2}$ behave as units, effecting profound changes in the properties of their compounds. It is evident that the possible combinations, even with the few most common atoms, - as C, H, O, N, Fe, Na, K, P, S, - are practically infinite when they are able to organize themselves into larger units, giving rise to complex series like the table on the following page. ${ }^{3}$

In this table the radical $\mathrm{CO}_{2} \mathrm{H}$ runs through the entire series, giving a kind of genetic quality to the compounds, while specific differences accompany the varying numbers of $\mathrm{C}$ and $\mathrm{H}$ atoms present with the radical. It is to be noted, however, that these $\mathrm{C}$ and $\mathrm{I}$ atoms are in clefinite proportion to each other, namely, $\mathrm{C}_{11} \mathrm{I}_{231+1}$; that is, for every atom of $\mathrm{C}$ there will be one more than twice as many atoms of $\mathrm{H},+\mathrm{CO}_{2} \mathrm{H}$, - all of which is extremely suggestive as early steps in the world of organized matter.

1 The composition of strychnine, $\mathrm{C}_{21} \mathrm{H}_{22} \mathrm{~N}_{2} \mathrm{O}_{2}$, and that of grape sugar, $\mathrm{C}_{12} \mathrm{H}_{22} \mathrm{O}_{11}$, are both exceedingly simple as compared with many known substances.

2 Such groups of atoms that move together are known as "radicals." They are in every sense units and are capable of replacing or of displacing other atoms in their constructions.

${ }^{3}$ We are told by the chemists that more than one hundred thousand separate compounds are now known. 
Monobasic Acids of the Acetic Series, $\mathrm{C}_{n} \mathrm{H}_{2 n+1} \mathrm{CO}_{2} \mathrm{H}$

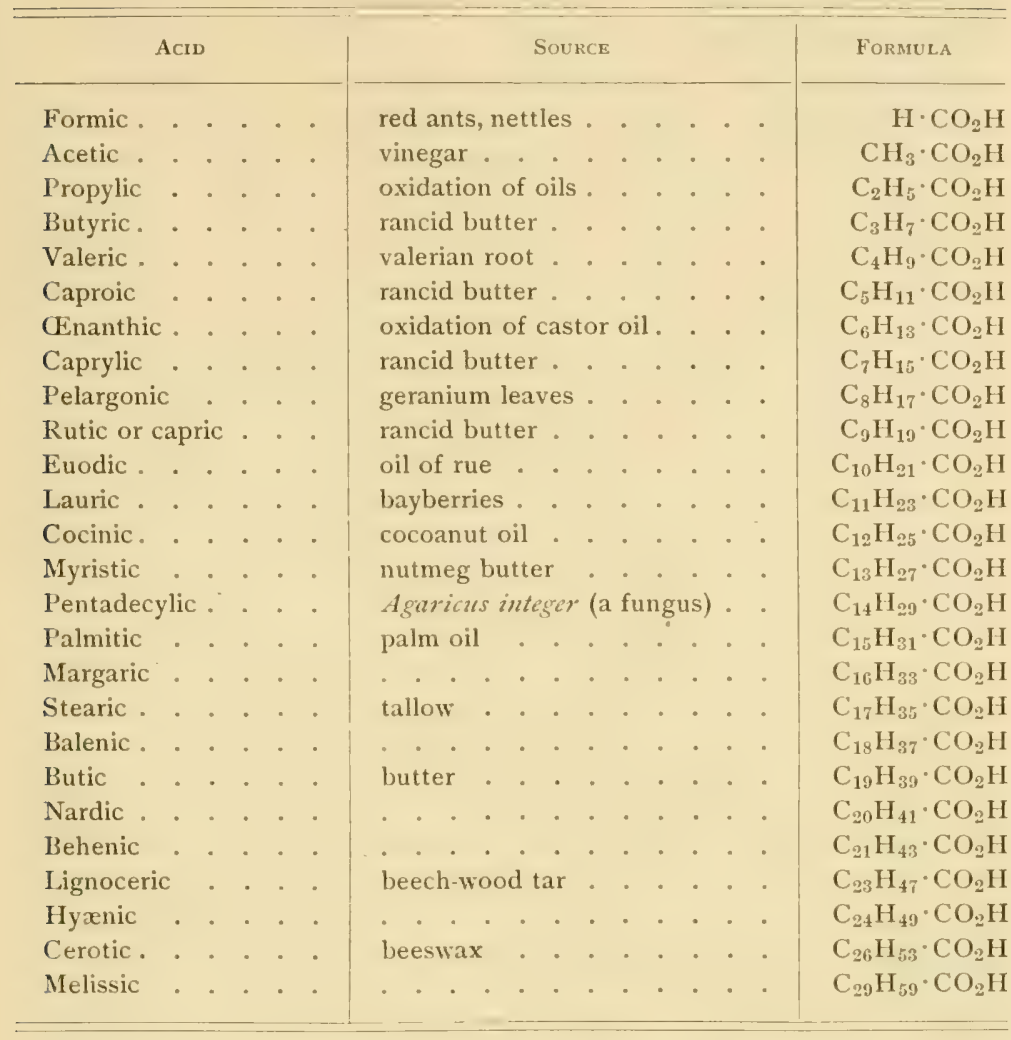

Observe the exceeding complexity of these compounds, and notice that they stand in definite series with uniform differences; there are no breaks in the series and no missing members until we reach $\mathrm{C}_{21} \mathrm{H}_{43}$. Note too that these compounds may themsclves combine with others; thus stearin, the characteristic fat of tallow, is $\mathrm{C}_{3} \mathrm{H}_{5}\left(\mathrm{C}_{18} \mathrm{H}_{35} \mathrm{O}_{2}\right)_{3}$, in which case $3 \mathrm{H}$ from the acid radical and $3(\mathrm{HO})$ from the glycerin radical have united to form $3\left(\mathrm{H}_{2} \mathrm{O}\right)$, or water.

Above everything else in this series note the will difference in plysical properties arising from a slight difference in chemical 
constitution. For example, it is significant that in this series both the solubility in water and the acid strength diminish as the proportion of carbon increases.

In addition to the properties of non-liaing nnits the theory of plyysiological units as the basis for specific characters in living matter requires one distinctive and additional quality, namely life, with its attendant phenomena, - the power of nutrition and growth. But what are nutrition and growth? Considered in general terms, nutrition is simply the power of one chemical compound (the living) to enter into the composition of another (the food) and break it up and readjust its elements on a basis like its own, leaving the residues to take care of themselves.

This readjustment of the non-living food to the composition of the living plant or animal we call nutrition, and it means essentially an increase of the living at the expense of the nonliving; in other words, growth through the numerical increase of living units.

There is often readjustment in the non-living world when two compounds are brought together, -- the weaker giving way to the stronger affinity, — but there is no such wholesale "carrying over" of matter from one to another as in the phenomena we call nutrition and growth. This is a true invasion of the non-living by the living world, transferring matter almost indefinitely, unto itself, not only preserving its own identity in the meantime but impressing it upon the appropriated materials as well.

These physiologrical or vital units are therefore conceived to be the smallest living units, like molecules in non-living matter, except that they are far more complex in constitution and are endowed with the power of self-multiplication through nutrition. This requires growth and division after the manner which has been noted in chromatin granules, except on a scale infinitely more minute.

This conception of the action of living units has its similitude in the non-living. Crystallization is a growth, in the sense of increase of size, but it is not attended by transformations equal to those in living matter. Furthermore, crystallization is growth without differentiation, except as to geometric form. Either 
the physiological units are capable of a cycle of differentiated energies, or else the race is possessed of many kinds of units, each inactive until its turn, then playing its rôle in suitable order. What then establishes the order of activity and calls out each unit at the proper time? Herein lies the mystery, and while the physiological unit seems a biological fact, it after all does not solve the mystery of differentiation; it only pushes the problem one step farther away.

Unsatisfactory though it is to attempt to solve the mystery of inheritance and differentiation by means even of vital units, still nothing else that has yet been proposed comes nearer satisfying the needs of the case, and we cannot fight off the following convictions, namely :

I. That there is a material basis not only of life but of racial characters as well, and this material passes to the individual by means of the germ cell.

2. That the processes of life are essentially chemical.

3. That if the whole truth could be known, the physiological units of vital activity may not be fundamentally different from atoms, molecules, and radicals actuated by chemical affinity. Broadly speaking, there are suggestive similarities between the chemical behavior of living matter and that of laboratory material generally, and these similarities are constantly turning up, even where least expected.

Whatever the truth may be as to the unit of vital activity, of two things we are sure: first, there is a unit of some kind, -- a center of activity; and second, it is a chemical material possessed of life. Finally, to be useful, these units must be conceived as capable of absorbing nourishment, and of selfmultiplication indefinitely.

\section{SECTION XVI — GERMINAL SELECTION}

The difficulty in seeking causes for inheritance and variation is that we are likely to prove too much. For example, if sufficient plasticity is assumed to fully account for the high degree of variation that often occurs, then variation is sufficiently provided for, but this view makes a thing like inheritance a matter 
of extreme improbability. ${ }^{1}$ On the other hand, if influences are discovered which are really efficient in setting bounds to variability, then they make the transfer of characters from parent to offspring so absolutely certain, regular, and fixed, as to seem to leave little or no possibility of variation.

This latter is the case with the hypothesis of physiological units. Weismann recognized its limitations and proposed the theory of germinal selection ${ }^{2}$ to account for variation as well as inheritance.

This theory assumes that the "biophors," or the physiological units by whatever name they may be called, are engaged in a kind of struggle among themselves within the germ, much as are plants and animals in the larger world outside.

Any theory of physiological units must include their absorption of food and their power of self-multiplication. If these activities proceed at a uniform rate for each unit involved, then no variation would result from this multiplication; but if proportions change, or if the vitality varies, then variation would necessarily result from these causes alone. Now these activities must be either constant or variable. Weismann assumes that they are variable; that these units of various relative numbers and strengths are competitors among themselves, one with another, for food; and that those most energetic in food absorption and capable of the most rapid multiplication will not only be the most vigrorous but they will also exist in relatively the largest numbers. They will therefore tend the more to impress their characters upon the later development of the individual.

Under this view of the case the "balance of power" is constantly shifting, always in favor of the most vigorous and rapidly multiplying units. Believers in physiological units must either follow Weismann in this conception or else assume on the part of the units absolutely equal powers of nutrition and multiplication, for multiplication there must be if such units avail anything in the rôle of inheritance.

1 All things considered, inheritance and not variation is the mystery. The wonder is, not that individuals vary, but that they follow as closely as they do the type of the race to which they belong.

2 Weismann, On Germinal Selection as a Source of Definite Variation (pamphlet, second edition) [Open Court Publishing Company]. 
Weismann reminds us that "by far the largest part of qualitative modifications . . rest on quantitative changes. A determinant," says he, "must be composed of heterogeneous biophors, and on their numerical proportion reposes, according to our hypothesis, their specific nature. If this proportion is altered, so also is the character of the determinant;" and further: "for fluctuations of nutriment and the struggle for nutriment, with its sequent preference of the strongest, must take place between the various species of biophors as well as between the species of determinants. But changes in the quantitative ratios of the biophors appear to us qualitative changes in the corresponding determinants." 1 And again : " $B y$ " a selection of the kind referred to the germ is progressively modified in a manner corresponding with the production of a difinitely directed progressia'c aration of the part." 2 In this way Weismann would "explain" Eimer's orthogenesis; but it is noteworthy that none of the thcorics yet proposed awill account for the original introduction of a new character in the race, whether represented and transmitted by a physiological unit or not. Germinal selection would provide for changes in relative proportions of characters, and even for their utter extinction, but not for their introduction, unless, indeed, characters may originate by new combinations of old elements.

One is almost forced to the conclusion that in nature loss or modification of characters is far more common than their origin and introduction. It looks as though most of the changes arise in this way, yet it is conceivable that an entirely new quality might arise through a relatively slight modification of the chemical or physiological make-up of the vital units. It is seen and recognized that in the non-living world a slight change in the radical is followed by a profound alteration in physical and chemical properties, and that this sweeping change may be induced by comparatively slight and even external causes. May not the same be true of vital radicals or units, and may not new characters arise more readily than we suppose, all perhaps out of elements fewer, and transformations simpler, than we have hitherto imagined?

1 Weissman, On Germinal Selection (second edition), pp. 46-47. ${ }^{2}$ Ibid. p. 35. 
Control of internal causes affecting the race as a whole. Whatever causes of this nature may be at work in our fields and yards, - and they are to be reckoned with, - our control over them is secondary and indirect. Their effects, if present, are at once insidious and sweeping.

We can be mindful of the effects of genetic selection and the selective death rate, and provide against them, at least to a large degree. If growth force and orthogenesis are also forces, they can be assisted or held in check by selection, but they can never be absolutely controlled ; and if germinal selection is a fact, it is going on entirely independent of any control which present knowledge enables us to exercise except through selection.

Summary. All that is involved in heredity is contained in a minute bit of living matter passed from parent to offspring, and whose development will constitute the new individual. The impulse to development, therefore, and its fundamental possibilities are forces internal to the germ and to the living organism.

It is not difficult to see many causes of variation in the internal processes known to be involved in the activities of living protoplasm. Growth is the result of cell division, which seems to proceed upon plans calculated to insure qualitative as well as quantitative equality as between the daughter cells. Any deviation from the plan, however, - and deviations are known to occur, - must result in variation. This is especially true in the reduction process which is characteristic of maturation in both sexes, and which probably lies at the basis of bud variation and of many mutations.

Fertilization and sexual union are processes calculated to effect new combinations out of the elements involved, though the possibilities in this direction would be rapidly reduced by close breeding or by any other circumstance which simplifies the ancestry.

Doubtless the condition of the germ has some influence, but it is not well understood. The phenomenon of xenia, or double fertilization in certain plants, causes the seed coats to vary the first year in the same direction as the germ. Telegony is a myth, and intra-uterine influences are doubtless limited to those of nutrition, except in cases of disaster. 
Reversion and atavism are but special instances under the law of ancestral heredity to be discussed later, serving to show that inheritance is partly from ancestors back of the parent.

Certain internal factors are of such nature as to affect the race as a whole. Genetic selection is based upon the fact that all individuals are not equally fertile, and that the type tends strongly to assume that of the most prolific. Bathmic influences, such as "growth force" and orthogenetic bias, have been advanced as explanations of those inherent tendencies that appear to characterize most species and that give an underlying trend to their direction in descent.

The basis of all vital activities is conceived as being some kind of living unit, comparable with the atom and the molecule in the non-living world, whose activities constitute growth and differentiation, and whose reactions with outside matter and with each other are fruitful causes of variation.

\section{ADDITIONAL REFERENCES}

Bud Variation and its Bearing upon Weismannism. By L. H. Bailey. Science, I, 28I-29I.

Bud Variation, Causes of, and Illustration. By R. M. Kellog. Proceedings of the Michigan Horticultural Society, 1897, pp. I2I134; also in Experiment Station Record, XI, 424-425.

Bud VARlation of Concord Grape. By W. Paddock. Garden and Forest, No. 456, pp. 464-466; also in Experiment Station Record, VIII, 290. Color Effects in Crossing Sweet and Fuixt Corss. Bulletin Illinois Experiment Station, No. 21; also in Experiment Station Record, XIII, 740.

I)termate Variation and Orgaxic Selection. By J. M. Baldwin. Science, VI, 770-773.

The Development of the Hybrids between Fundulus HeteroClitus and Menidia Notata, with Especial Reference to the Behavior of tile Maternal and Paternal Chromatin. By W. J. Moenkhaus. American Journal of Anatomy, III, 29.

EFFect of Fertilization upon Fragrance. Experiment Station Record, VIII, 55.

EfFect of Spaying upon the Quality of Milk. Experiment Station Record, XIV, I82.

Essays on Heredity. By A. Weismann. 2 vols.

Evolution in a Determinate Line. By Bashford Dean. Biological Bulletin, VII, I05-112. 
Eyolution on Pliedetermined Lines. By T. D. A. Cockerell. Science, XIII, 3II-3I2.

Experimental Study of Variation. By J. C. Ewart. Report of the British Association for the Advancement of Science, LXXI, 666680.

Experments in Crossing Corn and Watermelons. By F. C. Card and G. E. Adams. Experiment Station Record, XIII, 740.

Experiments upon the Influence of the Sexual Cells upon the Somatic. By G. W. Field. Biological Bulletin, II, 346-347.

Heterotypical Division in Maturation. By T. H. Bryce. Report of the British Association for the Advancement of Science, I90I, pp. 685-687.

Hytridzation of Corn and Watermelons. By F. C. Card. Experiment Station Record, XI, 928 .

Hyiridizing Melons: Effect in Sugar Content. Experiment Station Record, XVI, 229.

Hyiridizing Zeiba ANi Horse. (Experiments of Baron de Parana in Brazil.) Experiment Station Record, XI, 972.

Inimiduality OF Ciromosomes. By H. Metcalf. Proceedings of the Nebraska Academy of Science, I90I.

Inimeritance in Partienogenesis. By Ernest Warren. Proceedings of the Royal Society, LXV, 154-158.

Organic Selection. By J. M. Baldwin (Science, IV, 724-725; V, $634-636)$, E. D. Cope (Science, II, 124), and H. F. Osborn (Science, VI, 583-587).

Orthogenetic Variation. By H. F. Gadow, Cambridge. Science, XXII, 637-640.

Orthogenetic Variation in Certain Mexican Species of Lizards. By H. F. Gadow. Proceedings of the Royal Society, LXXII, 109-126. Physiological Selection. By G. J. Romanes. Science, Vil, 6o6-6o8. Premature Fertilization injurious to Fruit. Experiment Station Record, XIV, 634.

Problem of Develoiment. By E. B. Wilson. Science, XXI, 28I-293. Secondary Sexual. Characters. (A study of the effects of castration.) By S. G. Shattuck and C. G. Seligmann. Proceedings of the Royal Society, LXXIII, 49-58.

Sexual Repronuction; Distinction from Asexual. By Richard Hertwig (translated by W. C. Curtis). Science, XII, 940-946.

SoNg of Birds Kept from their own Species. By William E. D. Scott. Science, XIX, I 54.

Sterile Frut Blossoms. By S. A. Beach. New York Station Bulletin, CLXIX, 33I-37I.

Stfrility of Cattle, Causes of. Experiment Station Record, XI, 289. Telegony. By J. C. Ewart. Proceedings of the Royal Society, LXV, 243-25I. 
Telegony (Disproved). By J. C. Ewart. Popular Science Monthly, LVII, 126-133.

Telegony (DISPROVED): AcCount of EWART'S Experinent With Zebroids. By J. C. Ewart. Breeders' Gazette, XLI, I009; also in Transactions of the Highland Agricultural Society, 190I, pp. 8I-I34; and in Experiment Station Record, XI, I077; XIII, 275; and XIV, 76 .

Telegony (Disproved): Experiment with Guinea Pigs. By C. S. Minot. Report of the British Association for the Advancement of Science, 1906, p. 606.

The Chromosomes in Heredity. By W. S. Sutton (1903). Biological Bulletin, IV.

The Germ Plasm. By A. Weismann. I vol.

The Physical Basis of Heredity. By K. H. Eigenmann. Popular Science Monthly, LXI, 32-44.

Theory of Heredity and Telegony. By J. C. Ewart. Nature, LX, 330-333.

Xenia, Example in Apple. By L. H. Bailey. Science, IV, 498-499.

Xenia, or Double Fertilization. (Review of work of De Vries, Correns, and Webber.) Experiment Station Record, XII, 42I, 7I7; also XIII, 620 .

Xenia in Maize. By Hugo De Vries. Experiment Station Record, XI, IOI6. 


\section{CHAPTER IX}

\section{EXTERNAL INFLUENCES AS CAUSES OF VARIATION}

It was long ago noted, and the most casual observer cannot fail to discover, that individuals of the same species vary greatly according to their environment, - meaning by that term all the external conditions of life, such as climate, food, friends, enemies, and all those outside influences, favorable or unfavorable, among which the individual finds itself born, and with which it must live upon the best terms possible if it would live at all. That these extermal agencies exert a direct effect upon living matter is beyond question, and it remains to give attention to the nature and extent of this influence as a partial answer to the question we would solve, - the dependence of organized living matter upon the external world for the nature and range of its activities. Anything we may learn upon this point will be a contribution to the stock of knowledge out of which we shall one day determine all the causes of variation.

Without a doubt the great bulk of variability is due to causes internal to the organism, mainly in the form of inherited tendencies. Pearson, after exhaustive statistical investigations, remarks, "The individual contains within itself, owing to a bathmic law of growth, a variability which is itself quite sensible, being 80 or 90 per cent of the variability of the race." 1

Even then, however, these internal influences are dependent upon outside conditions for their opportunity. A born giant must have food in abundance, but no amount of food would make a giant out of a dwarf. Nor will it avail to awaken, late in life, forces that once might have been active. Some divarfs are therefore born and others are produced by insufficient food.

The external conditions of life affect variability in four distinctly different ways : (1) through natural selection, influencing

1 Pearson, Grammar of Science, p. 473. 
the type; (2) by affording or withholding the offortunity for the proper development of the characters born into the individual and therefore representing internal forces; (3) by exerting, directly upon the organism, a stimulating or a depressing effect upon its normal activities; (4) in extreme cases by temporarily or permanently modifying the character of normal functions.

The first manifestly affects the type and the race as a whole, while the second, third, and fourth primarily affect the individual. It is with these latter that we are now concerned.

The hasty student credits to external conditions all that aiould hafpen if these conditions a'ere aithdrawn. This is erroneous. A very large part of all that happens is due primarily to internal causes, because different races are differently affected by the same conditions. The fundamental cause of variability is therefore to be sought in the form of inhiritid characters, eren though these are dependent upon external conditions for their development, which may themselves seem to be direct causes of variation.

It is therefore proper enough to speak of external conditions of life as causes of variability, providing we know what we mean thereby and are careful to distinguish between their indirect effect, on the one hand, in affording or withholding the conditions of derelopment in which their influence is secondary, and their direct influence, on the other hand, in stimulating, depressing, or altering the activities of the organism.

With these distinctions in mind we may study the effects of outside conditions upon variability without danger of attributing to them what properly belongs to inherited faculties.

SECTION I - GEXERAL EFFECT OF LOCALITY LPON PLANT

\section{AND ANIMAL DEVELOPMENT}

It is a matter of common knowledge that the texture and quality of garden vegetables depend very much upon the conditions under which they are grown, and that the highest flavor of the orange, peach, pineapple, and edible fruits generally is found only in specimens from certain favored localities.

Thus cantaloupes of extremely high quality developed first at Rockyford, Colorado, and afterward in a few other sections. 
American seed growers generally seem to have settled upon Kansas as the spot most favorable to the development of the highest quality in the watermelon, and it is accordingly the favorite seed-producing locality. Darwin tells us that "the seed of the Persian melon yields near Paris a fruit inferior to the poorest marliet kinds, but at Bordeaux yields delicious fruit." 1

European varieties of grapes failed so utterly in eastern North America as to necessitate the developing of varieties from the native vine.

Indian corn develops local varieties with extreme readiness, but they seldom succeed when transferred even short distances, at least until time enough for acclimatization has elapsed. The writer sent a standard white Illinois corn, ripening in about a hundred and twenty days and capable of maximum yields (seventy-five bushels per acre), to be grown in Michigan, Wisconsin, Maine, and Mississippi. ${ }^{2}$ In Maine it failed to ripen, but at all other points it ripened in about a hundred days, producing small, inferior ears, altogether worthless as a commercial crop. That it should hurry through its period of growth at the north was not surprising, but that it should do the same at the south, where it had even more time at its clisposal than at home, is unaccountable.

Wheat, on the other hand, is a cosmopolitan crop, and while varieties succeed better in some localities than in others, yet a new variety seldom fails, and sometimes succeeds even better than in the locality whence it came. However, it is altogether likely that no known wheat-growing region equals England in natural advantages for maximum yiclds. This is supposed to be due to the humid atmosphere and cloudy skies during the later stages of growth, in sharp contrast to the bright skies and hot dry air of America at this season. If this be the true partial explanation of the occasional phenomenal and always high yields in Great Britain, ${ }^{3}$ we may hope for equal results some day in the similar climate of Oregon.

1 Darwin, Animals and Plants, II, 264.

$2 \mathrm{Wisconsin,} 200$ miles north; Michigan, 200 miles north and 100 miles east; Maine, 300 miles north and 900 miles east; Mississippi, 450 miles south.

3 The average wheat yield of the United States is between $I_{2}$ and I 3 bushels per acre, while that of Great Britain is almost exactly 30 , and a maximum of 90 bushels has been reported. 
The experiments of Bonnier upon this general subject are classic. ${ }^{1}$ He divided dandelion, helianthus, and many other plants growing in the valley, and planted one half of each individual plant on the mountain at an elevation of 2300 to 2400 meters, leaving the other half in the valley (how much below he does not say). As the division was macle by a vertical cut through the fleshy root, the two halves must have been practically alike.

He found that the portions on the mountain developed plants much smaller than those in the valleys. The difference was mainly in the length of the internodes, not in their number. The leaves of the dandelion were less than one fifth as long when drawn to scale, and the flower stalks were not one tenth as long. The mountain plants in general developed higher colors.

Darwin tells us that the medicinal qualities of digitalis are " easily affected by culture," and that "the wood of the American locust tree (Robinia) when grown in England is nearly worthless, as is that of the oak tree when grown at the Cape of Good Hope." "2 The same author quotes Sir J. E. Tennent as saying that "in the Botanic Gardens of Ceylon the apple tree 'sends out numerous runners underground, which continually rise into small stems, and form a growth around the parent tree.'" 3 If this be true, its naturally slight tendency to sprout has in this locality developed into a pronounced habit.

Sheep, especially the merinos, are cosmopolitan, and yet they succeed nowhere else as in New Zealand. On the other hand, according to Darwin they seem not to succeed in the West Indies or on the west coast of Africa, where "the wool disappears from the whole body except over the loins." $f$ The writer has seen the same thing in Brazil, except that the best-clothed portion of the body was the back of the neck, the same spot on which the vicuna bears its fur.

The statement is frequently made that fat-tailed sheep rapidly lose this character when removed from their native saline pastures, but the assertion needs confirmation, for the writer has

1 C. Bonnier, Recherches expérimentales sur l'adaptation des plantes au climat

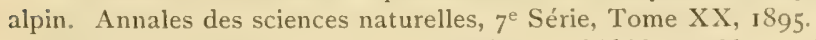
2 Darwin, Animals and Plants, II, 264.
3 Ibid. p. 266.
4 Ibid. I, IO2. 
seen a respectable development of fat when the sheep were kept under ordinary conditions.

Onc of the most remarkable and seemingly best authenticated instances of the evil influence of locality upon character development is the almost uniform failure to maintain the quality of certain English breeds of dogs when bred in India. We are inclebted again to Darwin ${ }^{1}$ for the remarkable statement that in that country the bulldog rapidly loses his ferocity, and of all dogs the hounds decline most rapidly.

Instances might be multiplied indefinitely, but two things must be borne in mind by the student when dealing with this class of facts : first, there is the greatest opportunity for error or exaggeration from inexact observation and report; second, the plant or animal is exposed to a multitude of new conditions when transplanted to a new locality, only a portion of which are inherent in the conditions of life. Commonly the breeders or attendants are not familiar with the new form and do not afford proper conditions, as in not giving suitable food to animals or in failing to afford sufficient room to large-growing varieties of plants. ${ }^{2}$

After making due allowance, however, for all these considerations, the fact remains that the conditions of life evidently do exert a strongly modifying influence upon development.

Locality a comprehensive term. There is little use in attempting to determine the exact influence of each separate locality. The term is an exceedingly comprehensive one, including many things, - climate, by which we mean not only temperature, moisture, and light, but their comparative proportions in that particular spot; season, by which we mean the succession of climatic conditions; food (both as to quantity and quality), on which the creature absolutely depends, not only for life but also for growth; habits of life, radical changes in which may be forced upon the animal by its habitat.

This is all too complex for profitable study and discussion. We must separate locality into its elements and determine, if we

1 Darwin, Animals and Plants, I, 39.

2 It will be noted in this connection that most of the instances cited are those of deterioration. 
can, the particular modifying influence of each of the conditions of life with which animals and plants are surrounded. In this way we may get important information upon this most difficult and unsatisfactory subject. Accordingly we undertake to ascertain the effects due specifically to food, temperature, light, etc., - the elements that, taken together, constitute the conditions of life.

\section{SECTION II-THE INFLUENCE OF FOOD UPON VARIABILITY}

The best evidence goes to show that food affects development both quantitatively and qualitatively. It is expedient to consider the two separately.

Quantitative effects of food. In general, as every stockman knows, full feed means increased size, provided always there has been no check during development. This is not only the experience in the yards everywhere, but the world over the largest animals are found on the best feeding grounds. Doubtless other external influences affect size, but certainly no other equals the food supply, and if maximum derelopment is expected food must not be withheld, especially during the early stages of growth. No amount of later feeding, after the individual has accustomed itself to a reduced supply, can make amends for early shortage. This is itself a deriation which easily becomes permanent and follows the individual through life.

Development, however, bears no direct ratio to food consumed; that is to say, the greater portion of all food is consumed in supporting the vital processes, altogether without reference to increase of weight or to labor performed. Under the best of feeding we rarely recover Io per cent of the food consumed in the form of growth or increase of weight, and seldom realize as much as one sixth in the form of labor or other output of the body. The great mass of the food is either not digested at all or goes to support the internal activities of the body, or else is digested and passed out of the body without serving any useful purpose whatever.

It is a significant fact that stunted animals (and plants as well) seldom recover from the evil effects of arrested development, 
and that under-development due to insufficient food is quite distinct from divarfing, especially among animals, in that the body does not develop proportionately. In underfed calves, for example, the head outgrows the rest of the body, the legs are long, and the joints are large.

In general, full feed means not only increased size but early maturity as well, which is of even greater consequence. Because of the large proportion of food never recovered in gain, it is manifest that any shortening in the period of development results not only in improved quality but also in the saving of feed; in other words, early gains are economical gains and they tend to higher quality.

Effect upon fertility. The amount and character of food often exert profound physiological influences. For example, the fertility of the female honeybee is mainly due to food, the sterile workers and the fertile queens developing from the same eggs. If, even after the worker eggs are hatched and the larvæe well developed, they be taken from the worker cells, put into queen cells, and fed "queen's food," they will develop into queens, a fact often taken advantage of by the bees themselves when by accident all the prospective queens have been lost. Here fertility is largely a matter of food, although an occasional worker is known to produce eggs. This general difference between worker and queen must therefore be regarded as one of development dependent mainly on the food supply.

Speaking generally, excessive food supply leads to infertility among both plants and animals. The former vegetate luxuriously, but they do not blossom and fruit so abundantly as under a full but moderate supply of plant food.

Whether the effect in question is due to overfeeding or to some one or two elements in particular is not well established. Enough is known, however, to justify the assertion that extreme proportions of nitrogen produce luxuriance in stem and leaf at the expense of flower and fruit, but there is exceeding doubt whether this effect would follow a well-balanced food supply with plenty of phosphorus.

Excessive feeding of animals, especially females, tends to fatty degeneration of the essential sexual organs, and consequently to 
sterility, and this result is hastened if the food contains unusual proportions of carbohydrates, especially sugar.

Effect of feed upon variability. It is a common belief that plants and animals are more variable when well fed than otherwise. Doubtless this is true, so far at least as appearances go, for only under such favorable conditions are all the faculties that are born into the exceptional individual able to develop and become visible. When a race is living under mediocre conditions there is a dead level in development. The mediocre individuals have relatively the best chance, and few will rise above the condition of mediocrity.

Whether full feed is a direct stimulant to variability or only brings potential differences to the surface is therefore an open question. The procedure indicated is, however, in either case the same; namely, to provide maximum conditions if the breeder expects to realize the utmost from his best individuals or hopes ever to find variations worth preserving.

Herein lies the fact that well-bred animals often require more feed than their scrub relatives. It was upon that point that they departed from their kind - not that they contracted to exist on less feed, but that they were able to handle more feed and put it to good use. If the purpose of the breeder were to develop races with a minimum maintenance ration, it could be done; but we keep domestic animals not for their society but for what they can do, - for what they can manufacture out of corn, oats, and hay. We improve crops, not to see upon how poor land they may live, but rather to increase their ability to construct valuable food materials from the mineral elements of the soil and the inorganic constituents of the atmosphere. Not minimum of consumption but cconomic consumption is therefore the virtue sought.

Evil effects of overfeeding. The plant will seldom suffer from abundance of food, although it is not impossible. Excess of nitrogen causes rank growth of stem, but phosphorus is needed for seed. Something akin to a balanced ration is doubtless best for plant as well as animal, yet the former has more of selective ability than the latter.

The animal is easily overfed, and if so the injury is likely to be permanent. It results (I) in disorders of the digestive tract; 
(2) in disorders of the excretory organs; (3) in excessive fat; (4) in sterility, especially in females, through fatty degeneration of the essential sexual organs.

Qualitative effects from the nature of the food. The color of plants and Howers, and even of animals, is said often to be directly influenced by their food, but it is exceedingly difficult always to separate fact from tradition in this particular field, because the average person has an exaggerated conception of the influence of food upon the constitution of the organism, often believing that a meat diet, for example, inclines to ferocity and uncontrollable temper generally. Darwin ${ }^{1}$ mentions the practice of feeding hemp seed to bullfinches to darken their color; the fat of a certain fish to parrots, causing them to "become beautifully variegated with red and yellow feathers." He also states that the shells of mollusks are largely influenced by the amount of lime in the water in which they grow, and Beal succeeded in growing blue hydrangeas from pink stock by occasional application of alum water to the roots.

That the flavor of milk and eggs is largely dependent upon food has long been known, as has the specific effect of certain foods upon the texture and flavor of meat. Mumford found at Illinois that the fat of pork fed upon large amounts of cotton seed proved, on chemical examination, to contain a proportion of true cotton-seed oil, showing that a portion at least of the food had been carried over and stored unchanged in the tissues.

That this is the exception, or perhaps more correctly the lesser result, is proved by the fact that in general the identity of the food is lost in the nature of the organism, showing that the organism and not its food dominates results. The same meat becomes $\log$ or man indifferently, indicating that food compounds are not as a rule carried over but rather "suffer profound disruption," being reduced, if not to their elements, at least to comparatively simple compounds, the energy set free in the disruption being "utilized in the subsequent work of construction." 2

The higher organisms are comparatively independent of their food so far as qualitative changes are concerned. They seem

1 Darwin, Animals and Plants, II (second edition), 269-270.

2 Encyclopædia Britannica, XIX (1885), article "Physiology," 2 I. 
able to extract the materials for their activities often from the most forbidding sources and under the most discouraging circumstances. Indeed, many forms of animal activity are dependent for support not so much upon the materials of the food as upon its cncrgy. This applies more especially to mature animals and their functional activity, but we also remember that in the business of body building, materials are required in large excess of the actual amounts retained in the body.

Lower forms of life, however, seem often greatly dependent upon their culture medium. Thus many bacteria grow quite differently upon the potato and in agar or in beef broth, and a germ disease often presents in one species symptoms substantially different from those it presents in another.

The animal and the plant (among the higher forms) are nourished upon fundamentally distinct plans. Both require that food be in the soluble form before it can be useful, and each makes use only of such available materials as it may need. But the animal is provided with an elaborate excretory system by which it frees itself of all residues, both of undigested food and of broken-down tissues, and also of digested food in excess of requirements. By this means the animal is promptly freed from all redundant food material.

It is not so with the plant. It takes in food by absorption at its roots, and the water carries with it anything and everything that may happen to be dissolved. If nothing poisonous enters, the plant will live, but it will be loaded with residues, because it has no excretory system. The water passes off by evaporation at the leaf surface, leaving at that point large quantities of whatever was dissolved in the juices of the plant. We are not surprised, therefore, that vegetation of the same species differs very widely in composition in different localities, especially with respect to mineral content, depending upon the character of the soil in which it was grown. This difference may be, as in the case just cited, quite independent of vital processes, and due to nothing more than accident.

Plants, and the simpler organisms generally, are of necessity far more dependent upon their environment, and especially their food, than are the higher animals, that have to a large extent 
freed themselves from the bondage due to the accident of birthplace, being able to move about and therefore to establish in the widest sense an independent existence.

Notwithstanding all this, and after making allowance for the grosser influences over lower organisms, the fact yet remains that, to a slight extent, and to a slight extent only, the animal is influenced by the character of his food. That this influence is larger upon the products of the body than upon the body itself is certain; and upon the subtler qualities, like flavor, rather than the more essentially biological characters such as structure.

\section{SECIION III - THE EFFECT OF MOISTURE UPON DEVELOPMENT}

Animals as a rule are quite independent of moisture, providing their direct needs for water are satisfied in the way of body consumption in addition to that needed to reduce the effects of internal heat by evaporation. ${ }^{1}$

Plants on the other hand do not have the circulatory system of animals, and they depend upon water, taken in by the roots and evaporated by the leaves, to actually carry food to all parts of the structure. Their need for water is therefore far above the amounts necessary for actual composition. For example, it requires the evaporation of something like the equivalent of eight inches of standing water over the entire field to mature an average corn crop.

1 It is often erroneously taught that animals consume carbonaceous foods to sustain the body temperature, while the truth is that all food actually utilized is broken down and its energy set free. This energy is disposed of in three ways: first, to a slight extent in effecting the recombination of the elements of the food into the exceedingly complex protoplasm of the body or its products; second, by the body or some of its parts in the form of internal or of external work; or, third, it is radiated in the form of heat of low intensity. In this way the body is a factory that is constantly producing heat, which must be disposed of or the structure will be destroyed. There are but two ways of doing this, - by radiation and by evaporation. The body is thus constantly producing and is as constantly losing heat. Its actual temperature is certain to be something above that of the surrounding medium, - how much will depend upon the relation between the rapidity of production and the facilities for radiation and evaporation. The body temperature is thus a kind of algebraic sum, and it depends upon a great variety of conditions. Small animals radiate rapidly, large ones less rapidly, for radiation is a surface action, and their surface is less in proportion to the bulk. Hogs radiate slowly, because of their blanket of fat. They do not sweat, and thus are easily overheated. 
Water, or the lack of it, is therefore in many countries and in many seasons the limiting clemcut; that is to say, the yield is limited not by the available fertility or the ability of the crop but by the moisture present.

In excessively wet seasons crops are notoriously " soft," that is, lacking in substance. Just what the difference is has not been well established, but size has been attained at the expense of quality. There is good reason to assume that it is the result of abundance of water, leading to full cellular development, but deficiency of evaporation and transfusion of food due to cloudy skies, resulting in a lack of actual dry matter.

Effect of moisture in the atmosphere. It is saicl that moist atmospheres produce fineness of hair or fur in animals and delicate foliage in plants, and that a dry atmosphere inclines to a harsh, dry coat and to spiny growth in plants. Under natural conditions, however, moisture is often associated with coolness and shade, and dryness with great heat and intense light. Certain it is that fur-bearing animals are found in cool climates and that vegetation is delicate in the temperate region but harsh, dry, and spiny in the arid sections. These facts are well known and universally recognized, but how much is due to moisture alone cannot well be determined in nature.

Resorting to direct experiment, however, we find that the same plant may be grown with or without spines according to the degree of moisture in the surrounding atmosphere. Spines are undereloped leaves, as thorns are abortive stems, and anything that checks growth tends to their production. That this is mainly the result of a dry atmosphere, however, is easily shown in the laboratory.

"Lothelier has made numerous observations in which inclividuals of the same species were placed side by side, some exposed freely to the air and others kept moist under a glass shade." Under conditions such as this "Berberis anlgaris bore nonspinescent leaves in a moist atmosphere, but spines alone in a perfectly dry one. Again, the shoots which in Lycium barbarmm, Elcr Europieus, etc., would normally have formed thorns, by arrested development and sclerosis (hardening), in a very damp atmosphere continued to grow, and elongated into leafy branches." 
Microscopical examination showed that in the dry-air specimens "the palisade cells were well developed and there was a special consolidation of fibrous tissues." 1 The same author continues :

Again, the common water reed Pharmitis communis, when growing in the unirrigated areas of the Nile valley, forms a stunted growth with very short and sharp-pointed leaves. Close to the Nile, however, . . . it grows nine or ten feet high, with long leaves almost exactly like the plants in English rivers.

All observations go to show that the number of vessels in the fibro-vascular system is greater in the aërial than in the aquatic forms of the same species, ${ }^{2}$ and the evidence in general seems conclusive that the notorious abundance of spines in tropical vegetation is due primarily to a dry atmosphere, assisted to some extent, no doubt, by the retarding effect of intense light upon growth.

A significant fact in this connection, possibly attributable to the scarcity of water, possibly to the lack of heat, is the well-known phenomenom that plant lice, producing females only during the summer, begin with approaching autumn to produce males, and that under the perpetual heat of the greenhouse the insects observe summer habits indefinitely unless the flants on which they are fecding are allowed to become dry.

As is well known, seeds may be kept for long periods if thoroughly dried. In this case the vital activities are reduced to a minimum, but probably not entirely suspended, because seeds will not last indefinitely. Certain lower forms of plant and animal life have a marked power of apparently suspending life through desiccation and resuming its activities again with sufficient moisture. ${ }^{3}$

The actual influence of water upon development is not yet well understood, except that it is one of the absolute conditions of life, and, being a fluctuating element, often limits development.

The student should fully appreciate the bearing of all this upon the matter in hand: The degrec of deaclopment of an individual at maturity is not a complete inder to his inherited charactors.

1 Vernon, Variation in Animals and Plants, p. 26.4; see also Henslow, Origin of Plant Structures, p. 40.

2 Vernon, Variation in Animals and Plants, p. 265.

${ }^{3}$ C. B. Davenport, Experimental Morphology, Part I, pp. 59-65. 
It is both something liss and something morc. It is less by so much as the individual has failed to develop because of unfavorable external conditions; it is mor by whaterer development is due to the direct infucnce of external conditions.

This is the principal reason why breeders have difficulty in knowing how much to credit to inheritance, and it is on this account that more knowledge is needed of the nature and extent of the development due directly to external causes, and therefore independent of inheritance. After all, however, it will be found that the total development from all causes is well within hereditary limits except, perhaps, in rare cases where the normal functions may have been altered by unusual conditions.

\section{SECTION IV - EFFECT OF CONTACT LPON PROTOPIASMIC AC'TIVITY $^{1}$}

Unstable chemical compounds are exceedingly sensitive to mechanical contact either from solid bodies or from liquids or gases in rapid motion. Davenport says : ${ }^{2}$

Mechanical disturbance can induce in certain lifeless compounds violent chemical changes. Compounds which are so affected are preëminently unstable. This instability, however, varies greatly in degree. In some cases the blow of a hammer is required to upset the molecules, the result being often a violent explosion. In other cases (e.g. chloricle or iodide of nitrogen ${ }^{3}$ ) the slightest touch of a feather suffices to produce an explosion. Now, most of the substances which explode upon impact [altering their chemical arrangement and properties] ... are organic compounds, fulminate, nitroglycerin, gun cotton, and picric-acid derivatives, - and therefore it is not surprising that we find the notoriously unstable protoplasm violently affected by contact.

"Of special interest in this connection" is the fact that "piriodic disturbances produce very important molecular changes in [certain] chemical compounds. Certain substances have a

1 C. B. Davenport, Experimental Morphology, Part I, pp. 97-110, and Part II, pp. $370-388$, from which most of the data on this subject are taken. N. B. Contact agents are technically known as molar agents.

2 Ibid. Part I, pp. 97-98.

3 When chemical terms of this kind are used outside of quotations the new form will be used, as nitrogen chlorid, omitting the $c$. 
specific rate of vilbration, so that when this is reproduced by a vibrating cord or plate, explosion of the substance may occur. Iodid of nitrogen is one of these substances which is exploded by a high note." 1 Living protoplasm is no exception to the general rule that specially unstable compounds are sensitive to contact.

Effect of contact upon the metabolism of protoplasm. ${ }^{2}$ It is a well-known fact that phosphorescence is increased by mechanical irritation. So true is this that the water thrown from the propeller wheels of a steamer in tropical regions looks like liquid fire, and a brisk breeze moving over the surface suggests at a distance the white foam of the surf.

Contact also exerts a strongly stimulating influence upon secretions, not only with lower organisms which seck attachments, and the glands of insectivorous plants, but with higher animals as well. ${ }^{3}$

Effect of contact upon movement. The first effect of mechanical disturbance in protoplasm is to check all movement. Minute organisms, tradescantia hairs, etc., cease their protoplasmic motion by the irritation of mounting under the cover glass. In higher plants a sudden jar causes cessation of movement and often a retreat of the protoplasm to one side of the cell so characteristic as to be spoken of as "fright."

If an amoba with pseudopodia out is touched or irritated, it immediately assumes the spherical form, and in general the effect of contact is to cause protoplasm to cease motion and assume approximately the spherical form, or, in other words, to occupy the least space possible. But this is to all intents and purposes contraction, and the general principle may be laid down that external contact causes contraction, especially noticeable in muscle, which is par cxcellence the contractile tissue.

This contraction most commonly, and of necessity, operates at first to draw the organism away from the irritating body (negative thigmotaxis), but if the body be long or large, so that locomotion continues, then the side next the foreign body will

1 This suggests, as the author observes, the phenomena of hearing.

2 C. B. Davenport, Experimental Morphology, Part I, pp. 98-99.

${ }^{3} \mathrm{As}$ is well known, the heifer that has never produced a calf may be made to give milk merely by persistent manipulation of the udder. 
be shortened and the organism will move in a curve that will speedily bring it into actual contact. It is noticeable, too, that real contact, being once established, is broken with difficulty. Many lower animals, as in aquaria, coming in contact with a plain surface, move along that surface until they reach a point where side and bottom or where two sides join, and where they can place their bodies in contact with two surfaces. They are likely now to move along the groove formed by the two surfaces until a corner is reached where contact on three sicles is possible. Here, if anywhere, the organism will come to rest. It is only that it is "more comfortable" ; that it moved under the molar impulse until it reached a point where further movement and more complete contact were alike impossible. Even higher animals come to the highest state of rest when in contact with foreign bodies on as many sides as possible.

Effect of contact upon direction of movement, - thigmotaxis, or stereotropism. ${ }^{1}$ It is a well-known fact that roots growing in running water grow upstram, not downstream, and that many fish at the breeding season are possessed of an irresistible impulse to move against the current (rheotaxis). ${ }^{2}$ They therefore ascend the strongest currents, leap waterfalls, and surmount every possible obstacle in upstream movements, - a passion which ultimately carries them to their breeding grounds in shallow water. It is at these times that salmon pile themselves up even above the water level and that they will follow any decoy that leads against the current, even into hopeless traps.

Thus may external agents exert a strongly modifying influence upon such essential activities of living matter as the contractility of protoplasm, resulting in definitely directed movements through their control of muscular contraction. As we shall see, contact is not the only influence capable of stimulating contractility of protoplasm and controlling the direction of movement; on the other hand, muscular tissue is exposed to the exciting influence of a great variety of circumstances both internal and external to the organism, any one of which will induce the characteristic reaction of this sort of tissue, which is contraction.

1 C. B. Davenport, Experimental Morphology, Part I, p. 105.

2 Ibid. p. Iog. 


\section{SECTION V-EFFECT OF GRAVITY UPON LIVING MATTER $^{1}$; GEOTROPISM ${ }^{2}$}

A germinating seed sends out two sprouts. From whatever position they emerge, one grows downward in response to grav.* ity, the other upward in opposition. In other words, the root is positively and the stem is negatively geotropic; that is to say,

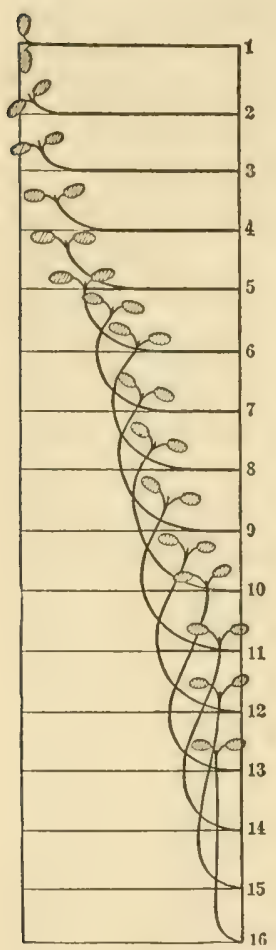

FIG. 25. Influence of geotropism : behavior of a growing plumule in righting itself after being placed in a horizontal position, as at 1 . After C. B. Davenport, from Strasburger each contains within itself some quality that puts it into definite relation with the center of the earth, but in opposite directions.

That this tendency is something considerable is shown by the fact that it is capable of exertion against pronounced resistance, as in burrowing through the soil or persisting against mechanical obstructions.

This definite relation to gravity seems to exert itself in the manner of inward forces responding to outsicle conditions; for if a piece of stem be altered in its position, future growth readjusts itself as promptly as possible in

1 C. B. Davenport, Experimental Morphology, Part I, pp. I I2-I24; also Part II, pp. 391-402, from which most. of the facts here cited are taken.

2 Two series of terms are in use, of substantially the same meaning: one (see "geotropism" and "geotropic"), with endings derived from the Greek meaning to tum; the other (see "geotaxis" and "geotactic"), with Greek endings signifying to arrange. We thus have also "chemotropism," with its adjective "chemotropic," over against "chemotaxis," "chemotactic," "heliotropism," and "heliotactic," etc. The latter endings were supplied when the effect of chemicals, gravity, and light upon free-moving organisms, as bacteria, swarm spores, etc, was first noted, which effects led to definite "arrangements"; but when similar effects were noted upon larger organisms not free to move, like the fixed plants, but which manifested the effect by turning, the term "tropism" came into use, signifying a turning. This term and its derivatives seem to express more accurately what really happens in most cases, and they are coming to be preferred in all generalized considerations. Ience in the text the term "geotropism" is preferred to "geotaxis" and similarly in most places for all corresponding terms. 
accordance with this inward polarity and the new conditions (see Figs. 25 and 26).

Vöchting's experiments as here figured, and as described by Morgan, ${ }^{1}$ indicate that the formation of stem or of root is a

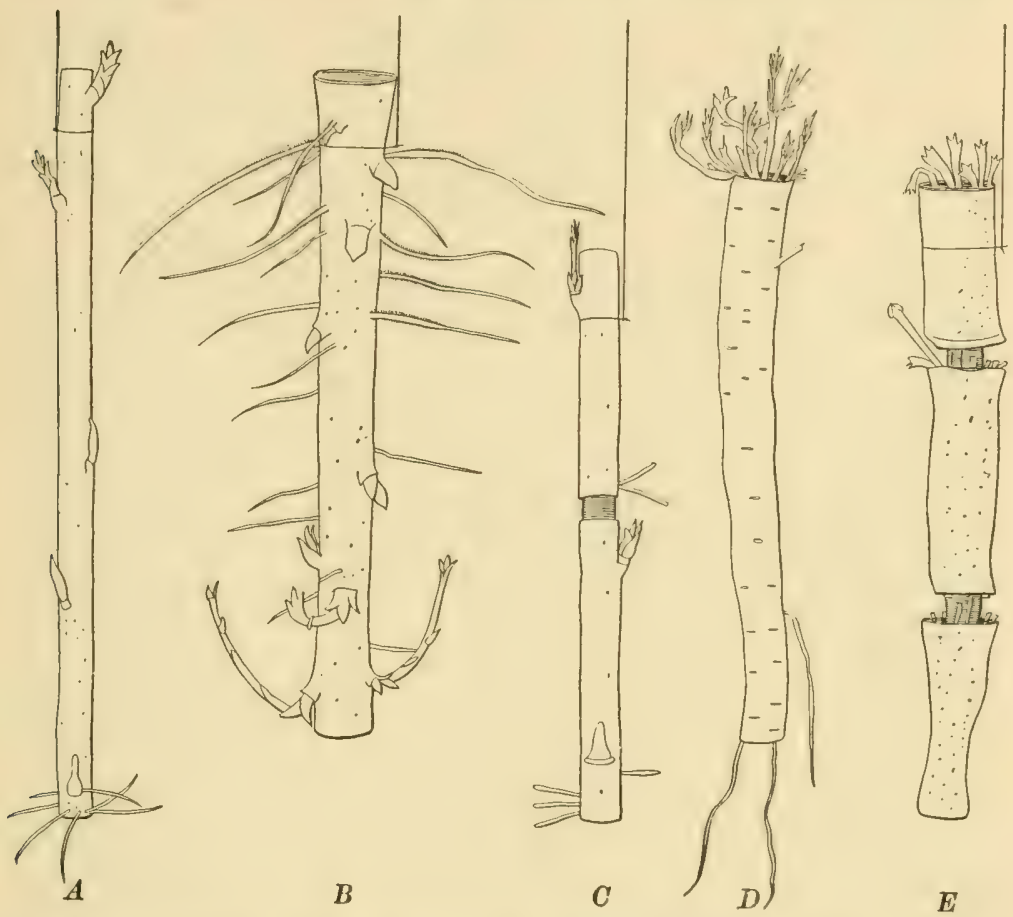

FIG. 26. Influence of polarity and of gravity upon the character and direction of growth. - After Morgan, from Vöchting

$A$, piece of willow (cut off in July) suspended in moist atmosphere, with apex upward; $B$, older piece of willow (cut off in March) suspended in moist atmosphere, with apex downward; $C$, piece of willow with a ring removed from the middle, apex upward; $D$, piece of root of Populus dilatata, with basal end upward; shoots from basal callus; $E$, piece of root of same with two rings removed; new shoots develop from basal callus and from basal end of each ring

question of both internal causes (polarity) and external influences (gravity), that is to say, the character of growth is a function both of internal and external conditions.

1 Morgan, Regeneration, pp. $72-\delta_{3}$. 
A stem of willow severed from its parent plant and suspended, apex upward, in a moist atmosphere, will of course send out shoots from its apex and roots from its base. If a ring of bark be removed from the micldle of the stem, then sprouts will issue from the apical extremities of the sections and roots from the basal encl. Neither of these experiments determines whether gravity or polarity is chiefly instrumental in the production of stem and root, but if the piece be inverted and suspended apex downward (in a moist atmosphere), we shall get some light on the two forces, external and internal.

Under these conditions the apical end, now downward, will yet produce stims, but they will change their direction with reference to the axis and point upreard, while the basal end will produce roots, but they will extend downward. In this case each end has produced its characteristic growth, and each has responded to gravity in the usual way (see Fig. 26), except that, if the piece be of the older wood, roots will appear throughout the entire length. The force that fixes the character of growth appears to be internal; that which fixes its direction appears to be mainly external.

If a begonia leaf be planted in the ground or suspended in moist air, whatever its position roots will start from the basal end of the stem at its point of scicrance, and afterward shoots will arise just above the point of origin of the roots, the bocly of the leaf withering away. (By "above" is meant between the origin of the root and the apex of the leaf, whatever its position.)

By this we see that the stem has a distinct polarity, producing sprout and root, each at the proper end; that the leaf has no true polarity, producing primarily only roots; but that wherever and however produced, a distinct geotropism characterizes both stem and root.

There is little geotropism in leaves, or in the horizontal stems of many plants running along or just below the surface of the ground. However, the stems and roots produced at the nodes of these underground stems are both geotropic.

Geotropism in animals. Geotropism is much less marked with animals than with plants. IVe may say that only fixed organisms 1 Morgan, Regeneration, pp. 74-76. 
would be likely to develop decided geotropism; or, conversely, we may say that organisms with marked geotropism would be likely to become fixed. In either event less geotropism would be expected among animals, and either assumption would square with the facts. Many lower organisms truly animal are distinctly geotropic, however, and most animals show a decided preference as to position with reference to gravity. Both with land animals, high or low, and with fish as well, the ventral side is carried downward, and the anterior portion in general upward.

Effect of geotropism upon protoplasm. The protoplasm of cells, plant or animal, is not homogeneous. The nucleus is heavier than the cytoplasm, and together with chlorophyll granules and starch grains tends to settle to the lower side of the cell, giving it a kind of polarity due to gravity. "In many ova the yolk sinks to the lower pole and the cytoplasm floats on top, in whatever position the egg may be held," - a fact which " undoubtedly has an important effect upon development." 1

General effect of gravity upon development. There is no room for doubt as to the profound effect of gravity upon development. However, this influence of gravity has been continual and constant on all existing species for untold generations, and it may be looked upon as having already exerted the maximum of its influence upon all forms of life.

The effect of gravity upon development has, therefore, long ago reached the position of a constant force to be reckoned with, and is now to be regarded as a fixed factor in development rather than as a present cause of individual deviation, — to be studied more for the sake of learning the degree of dependence of living matter upon outside forces rather than as a direct means of further change.

\section{SECTION VI - EFFECT OF LIGHT UPON LIVING MATTER}

In all chlorophyllaceous plants the amount of carbon fixed, and therefore the total of growth in the sense of increase in dry matter, is in almost direct proportion to the expanse of leaf surface and the amount of light that falls upon it.

${ }^{1}$ C. B. Davenport, Experimental Morphology, Part I, p. I I4. 
Light has other influences, however, than those exerted through the fixation of carbon. For example, strong sunlight tends to check growth in the sense of increase in bulk, and when these two effects of light are combined, as they are in the tropics, they give us naturally the slow-growing, generally small, and extremely dense wood of the lower latitudes.

Briefly, light, like gravity, exerts specific effect upon matter. Many of the effects of gravity (positive geotropism) may be regarded as arising from the elementary properties of matter, for naturally all matter is attracted by, and approaches as nearly as possible to, the surface of the earth; that is, matter in general may be said to be positively geotropic. Sensitiveness to light, however, should be regarded as due to the special componnds that constitute living matter, rather than as a property of matter in general, for matter in general is indifferent to light.

Light exerts influence upon living matter, especially plants, in three distinctly different ways: (I) through its heat rays, affecting temperatures; (2) through the so-called chemical (actinic) rays, causing definite chemical reactions in the protoplasm; (3) through the luminous rays, influencing especially the direction of growth in those parts that are so fixed as to be incapable of free movement. Certain of these influences are worthy of somewhat extended consideration.

Chemical effects of light. ${ }^{1}$ While matter in general in its simpler compounds is quite indifferent to light, yet certain compounds are notoriously dependent upon its influence; that is to say, many combinations are effected more readily and others only in the presence of light (photosynthesis).

Oxidation of vegetable oils is much more rapid in daylight than in darkness. Hydrogen and chlorin unite explosively in the prescnee of light. Chlorin passed through alcohol in strong sunlight unites with it, forming chloral hydrate, and chlorin compounds generally are sensitive to light.

The whole field of photography is dependent upon the action of light upon the halogen salts of silver, gold, platinum, and other metals, clue to the so-called "chemical rays" extending

1 C. B. Iavenport, Experimental Morphology, Part I, pp. I6I-165, from which most of the instances under this heading are taken. 
from the blue upward and most pronounced in the invisible " ultraviolet" portions of the spectrum. These particular wave lengths seem closely akin to chemical energy, and their effect, invisible and subtle as it is, should not be overlooked.

Certain organic compounds are readily formed only by the aid of light; thus the reaction $\mathrm{C}_{14} \mathrm{H}_{8} \mathrm{O}_{2}+\mathrm{C}_{6} \mathrm{H}_{5} \mathrm{CHO}=\mathrm{C}_{14} \mathrm{H}_{3}$ $(\mathrm{O} . \mathrm{H})\left(\mathrm{O} . \mathrm{CO} \cdot \mathrm{C}_{6} \mathrm{H}_{5}\right)$ takes place in sunlight, but in clarkness the substances are indifferent to each other. ${ }^{1}$ It is uncler this same principle that the vegetable substance chlorophyll is able to break up the $\mathrm{CO}_{2}$ of the atmosphere and fix the carbon in the form of starch, setting free the oxygen. This is the most distinctive act of plant life, and yet it takes place only in the presence of light. The student is therefore prepared to realize that light is one of the controlling forces, not only in effecting chemical compounds in the non-living world but in the activities of living matter as well; that in many respects its action is fundamental (as in the fixing of carbon), in others incidental, and in still others even accidental (as in the color of chlorophyll or of gold or silver). In any event it is an influence to be taken account of when one is engaged in the study of the circumstances that control the activities of living matter.

Effect of light upon functional activity.: The effects of sunlight upon growth are of three kinds, - one due to the heat rays of the lower spectrum, the others to the luminous and the so-called chemical or "actinic" rays of the upper spectrum, from the blue to a considerable distance beyond the violet. Strange as it may seem, the influence of light upon the fixation of carbon is greatest in the thermic rather than in the actinic region of the spectrum. Timiriazeff ${ }^{3}$ kept a plant in the clarkness until the starch in the leaves had been absorbed. "Then in a dark room a prismatic spectrum was thrown upon the leaf and the position of Fraunhofer's lines indicated on the leaf. After three to six hours starch had formed under the influence of the light, only in the region of the absorption bands of chlorophyll lying between $B$, and $D$," as determined by treating first with

1 C. B. Davenport, Experimental Morphology, Part 1, p. I63.

2 Ibid. Part I, pp. I66-I8o; Part II, pp. 416-436.

${ }^{3}$ Ibid. Part I, pp. $169-170$. The italics are mine. 
dewht th (Swlories, and then with indin, which forms its chat-

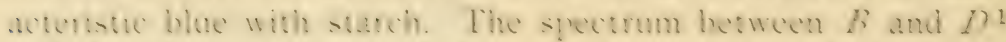
includes the unper pare of the red, the ormper and the bower parts of the yellow, - the thermic rather than the actinic portion of the spectrum.

Aupong both planes and animals light has an importane indu-

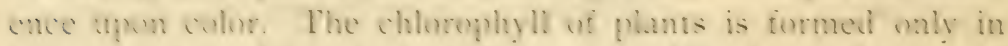

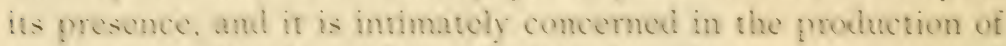
pigments in the skin. Not mly that, but the arrangement and 1) situm of pugmenary matter, whether bing mat the surface and well diftused - thus giving color to the animal - or lying collerted in massis leoper in the skin and having little eftect uphen the whtor, are due fargely an the dirces eftert of light calling upon the skun ot the animal. In the way cortain animals.

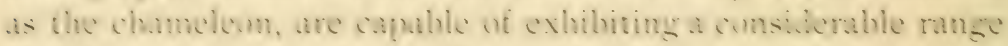
af covers, giving vise to the fietum that they are able to imitute any color near which they may be situated."

It has been customary to cite the fact that cave animals are ficyuenty less highily colore i chan thein congeners of che lamd, as eviderne that colon is tumbmentally dependent upon light.

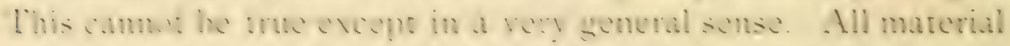
substumes hase sume whation to light and therefore have some color: 11 hat the color of a boly may be is theretore dopendent primarly upme its chmpestium, and in this sense its colur may be sand on be weritental. - a remark shat is as tree of chlorophyll as it is of gold or silver, or of red, white, or yellow brick.

but when the particular compound happens on be one like

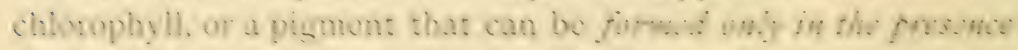
$\because \because \frac{1}{2}$ then and then only can colut be sald to depend upun

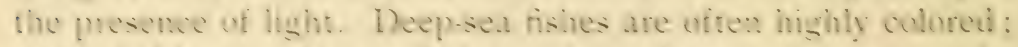
rocks htaden on the earth have their chameteristio tint: the

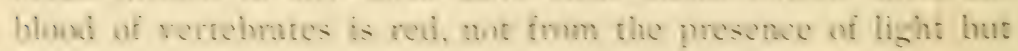
from the presence of compounds of iron. In all these cases the color artses from a substance in no sense dependent upon loghe tue ut fimmation and evistomee, and the cuss is distimet

1 The vibrations at this point are approximately $525 \times 10^{12}$ per second $1525,-$ cecosececo.cool.

2 C. B. Davenport, Experimental Morphology, Part 1. pp. 10=-104. 
from one in which the color is due to a substance formed only by the aid of light. It is only in this latter eatse that light maty truly be said to be a direct extemal canse of aratiation.

Examples of this are found in the coloring of louit, either under normal conditions or in "frut photography," a process by which pictures may be made to appear on highly colored fruit by shading with a sereen deriverl like a negattive from the picture to be transferred. ${ }^{1}$

The sum is supposed to exert a direct effect upon the skin, ranging from the tan of the white man to the dark color of the tropical races. This secms an ill adaptation, and so it is as regards the heat, for black objects are warmer than white ones; but the adaptation is not to the heat rays but to the chemical, for black pigment is almost totally non-actinic. Ilence we may say that dark-skimned people have lost something in heat resistance, but they have gained what is of more conseguence, - a screen against the actinic rays. ${ }^{2}$

Light exhibits its most charateristic effect upon the eye of higher animals. It here gives rise to two remarkable actions, muscular contraction of the iris, by which the amount of light admitted is regulated, and a nerve stimulus, which forms a definite image on the retina, as upon a mirror, and which is perfectly comprehended by the mind. Whether the colors and images seen by all eyes are absolutely ielentical is obviously a matter that can never be determined. It is of course safe to assume that the images, in so far as form is concerned, are identical, because the outlines are due to the mechanical laws of refraction, but the colors as comprohended may be due in part, if not entircly, to physiological peculiarities. That is, the color which 10 one is red may look to him as yellow does to another, - a supposition entirely plausible when we remember that with some individuals sound always sugrests color as well, so that the name Jones immediatcly suggests black, or red, or some other color, differing with different individuals. What relation or coordination between the auditory and the optic nerves can be responsible for this sort of mixed impression we do not know.

1 Literary Digest, September I6, 1905, D. 38x.

2 Ibid. October 7,1905, p. 485 . 
Vital limits. Chlorophyllaceous plants are absolutely dependent upon light for their very existence, but parasitic plants, and animals in general, are not dependent upon light in any vital sense, because they, like animals, subsist upon highly organized materials in which the fixation of carbon has been already accomplished by other organisms. ${ }^{1}$

All known facts indicate that animal life in general is essentially successful in total darkness. Mules have been kept in mines for twenty years, and beyond temporary sensitiveness of the eyes no effect was perceptible. Prisoners have spent their lives in dungeons. All embryonic development in mammals takes place in the total darkness of the mother's body. It is doubtless not too much to say that light has no effect whatever upon the rital functions of the higher animals; that it is as unessential in this respect to animals as it is indispensable to plants.

Bacteria, as a rule, not only do not need the light, and flourish best in darkness, but strong sunlight is almost uniformly and quickly fatal; indeed, direct sunlight is recognized as one of the most successful germicides (which is of itself the principal reason why plenty of light should be provided wherever clomestic animals are kept). This fact is illustrated by inoculating a gelatin plate uniformly with bacteria, as Bacillus anthracis, covering the plate with a piece of black paper out of which some pattern is cut, as the letter $\mathrm{E}$, and exposing it all to strong sunlight for a few hours. If the plate is then put into an incubator the bacteria will grow, except over the area exposed to the light, in which area they have been killed. ${ }^{2}$ From the well-known fact that bacteria in a r'acumm are not affected by light we conclude that death is due to oxidation in the presence of light, a phenomenon common in organized compounds.

Light rigor. The movements of protoplasm in general are retarded, or even stopped, in the presence of intense light, so that rigor preceles death. There is therefore (for plants) an optimum, minimum, and maximum intensity, and between

1 This is written in general terms, and regardless of the fact that certain lower organisms, whose nearest relatives are distinctly animal, themselves bear chlorophyll.

2 C. B. Davenport, Experimental Morphology, Part I, pp. I71-I 72. 
these limits protoplasm is stimulated by sudden changes, rapidly becoming accustomed, however, to alterations within a narrow range (phototonus), and soon resuming its normal activity, except that as the intensity approaches the point of rigor activity appears to be permanently checked.

Retarding effect of light upon the rate of growth. To the higher organisms generally, sunlight, howerer intense, is not fatal, but it not infrequently retards the rate of growth, especially among plants. This accounts for the relatively slower growth of tropical vegetation as compared with that of higher latitudes, and for the fact that growth in the sense of increase in bulk is more rapid at night than in the daytime. Sachs found ${ }^{1}$ that the curve of growth reached its greatest height at daylight, then commenced to decline, reaching its minimum a little before sunset. Davenport points out that this fluctuation is opposed to the effects of temperature, which is more favorable in the day than at night, so that the final results are somewhat less than the total influence due to light.

This fact is well illustrated in experiments upon seedlings, grown both in darkness and in light, - investigations that are entirely feasible, because at this stage the young plant depends upon the old seed for its nourishment. It is invariably found that seedlings grown in the clarkness have grown at the faster rate.

That different rays have different effects upon the growth of plants is easily shown. Flammarion cultivated sensitive plants for three and a half months (July 4 to October 22) in red, green, white, and blue light. At the close of the experiment the plants had attained heights as follows: in the red light, $420 \mathrm{~mm}$.; in the green, $152 \mathrm{~mm}$.; in the white, $100 \mathrm{~mm}$.; and in the blue, $27 \mathrm{~mm}$., with general appearances shown in Fig. 27.

In this experiment the greatest heat rays were of course transmitted with the red light, but the general temperature was regulated by currents of air passing through the various chambers."

It has been roughly stated that light has no effect upon germination. In general this is true, though careful experiments indicate that most seeds germinate slightly earlier in darkness

1 C. B. Davenport, Experimental Morphology, Part II, p. $42 \mathbf{r}$.

2 Ibid. pp. 427-429. 
than in light, and a few, prominent among which are the poas, germinate more readily in the light, as do also the spores of ferns and the seeds of the mistletoe. ${ }^{1}$

Evidence as to whether sunlight influences the growth of animals is inconclusive. Experiments have been conducted with tadpoles, snails, the eggs of certain fish, and with the young of higher animals, but while slightly better growth is reported

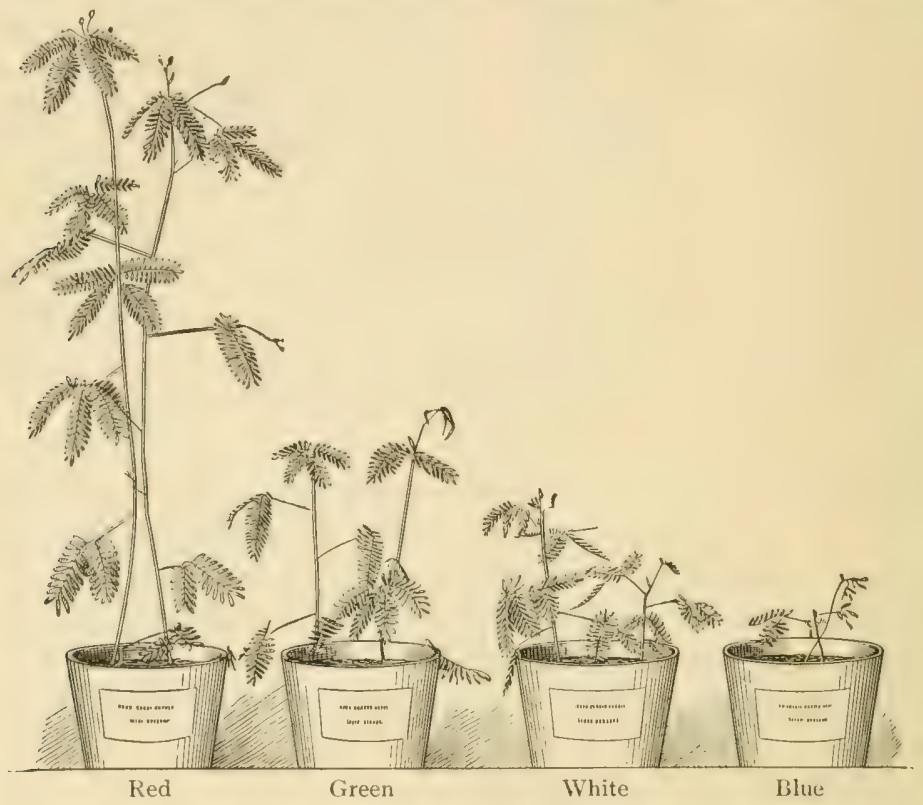

FIG. 27. Effect of light upon rate of growth: sensitive plants grown for three and a half months in red, green, white, and blue light. - After C. B. Davenport, from Flammarion

during daylight, it is not certain that other conditions did not contribute to the results. ${ }^{2}$ Experiments in feeding fattening animals in darkness and in light fail to establish special differences.

All considerations point to the conclusion that light exerts a strongly modifying influence upon living matter in general, but that the higher animals are substantially free from its direct effect except to some extent in the matter of color.

1 C. B. Davenport, Experimental Morphology, Part II, pp. 4 I 9 and 424.

2 Ibid. pp. 425-426. 
Influence of light upon the direction of locomotion or of growth ; heliotropism. Irritability to light is one of the properties of protoplasm. This reaction is generally in the form of contraction, as with muscle fiber ${ }^{1}$ resulting in a shortening of the side next the source of light. With free-moving plants and animals this gives direction to locomotion, and they gradually swing about until both sides are equally lighted, when, if motion continues, the creatures will of necessity progress toward the light. This is positive heliotropism. Quick-moving forms are often carried into the source of light by their very impetus, before the repellent effect of great heat has time to act. In this way moths fly into the candle and are killed, while slowermoving forms are checked by the heat in time to save themselves. In this latter case the future movements will be a resultant of the positive heliotropism and negative thermotropism, by which the insects are held at a certain radius circling about the source of both light and heat, as if not able to leave it, as indeed they are not.

Negatively heliotropic forms, like earthworms, are those whose protoplasm does not contract in the presence of light, but, on the contrary, expands. These are carried away from the light, and if, in their wanderings, a lighted area is approached, they are unable to enter it.

When the organism is not free to move, as in the case of stems of higher plants, the effect will be manifested in the dircttion of grouth, which is all the response to heliotropism possible under the circumstances. In cases of this kind the stem will bend toward, or away from, the source of light, according as the plant is negatively or positively heliotropic, until all sides are equally lighted, in which position it will remain during growth, as do other forms during locomotion.

This placing of the body (or stem) with reference to the various "tropisms" is technically known as "orientation," and

1 As most of the examples to follow are confined to the lower animals, and to plants in which protoplasm is comparatively exposed, it is well to remind the student that the higher animals are not destitute of the same properties, and that they have one exposed region peculiarly sensitive to light, namely the iris of the eye, whose muscles contract promptly under its influence. 
this particular orientation with reference to the rays of light, whether parallel to their direction or transverse, has received the special name of "phototaxis." 1

Effective rays. All experiments indicate that the blue rays are the effective ones in producing heliotropic effects. ${ }^{2}$ Some organisms seem sensitive to the yellow, but both red and ultraviolet are alike inoperative. Heliotropism is therefore an effect decidedly due to the luminoils rays.

Contributary conditions." Heliotropism is dependent upon a variety of conditions both external and internal to the organism.

1 There is great uncertainty as to terms. The one just quoted ("phototaxis") is in its root a protest against the old term "heliotropism" in that it recognizes light as the active agent rather than the sun (helios), which is a source not only of light but of heat and chemical energy as well; and it recognizes, too, that these influences are exerted as light, quite independent of the sun or any other particular source.

Of late there has been a strong disposition to substitute this root for the older term, giving us phototropism in place of "heliotropism." Those disposed to this view would go farther and discriminate between those movements that appear to occur with reference to the direction of the rays, which is "phototaxis," and those which are made with reference to the intensity of illumination, which is "photopathy." These students atso recognize the fact that irritalility and the consequent movements, whether positive or negative, depend very much upon the intensity, so that organisms that are negatively heliotropic at high intensity are positively heliotropic at low intensity, suggesting a middle point at which the organism will be held, as with deep-sea animals that come near the surface at night but sink to considerable depths in the bright light of day, the water acting as a screen. This neutral point or satisfied condition is described as "phototonus."

It may be necessary to recognize all these distinctions when the subject is better understood, but it is more than likely that these different behaviors are only different manifestations of the same natural irritability to light on the part of protoplasm in general, and that the so-called "phototaxis" or even "phototonus" is only the condition of the organism after it has brought itself into such position that one irritability is balanced by another (which is always easy with bilateral symmetry), or that it is a kind of acclimatization acquired by the protoplasm to the particular intensity at hand.

The theoretical objection to direct reference to the $s u n$ is certainly sound. The attraction (or repulsion) is with reference to light as light, and not to the sun as its source. But the sun is preeminently the source of light, and for this reason, and with a proper understanding of the facts, the author prefers for our purposes to adhere to the single old term, at least under the present state of knowledge, for if properly understood it seems substantially correct and serves all practical purposes fairly well.

2 C. B. Davenport, Experimental Morphology, Part I, pp. 201-203; Loeb, Studies in General Physiology, pp. 29-31.

3 Ibid. pp. I96-201. 
It is first of all dependent upon intensity of light, feeble illumination being ineffective, even if the characteristic rays be present. Not only that, but some organisms are positively heliotropic in moderate light and negatively heliotropic in strong light. In this way many fishes are held in a nearly constant illumination, rising or falling in the water according to the intensity of sunlight, and coming completely to the surface at nightfall.

Another element in heliotropism is temperature, its influence being most active at the maximum or normal, and lessening or disappearing as it falls.

Still another controlling influence is the condition of the animal. Many caterpillars are positively heliotropic only when unfed. They are thus led to ascend trees when hungry and to descend when filled. ' Still again, certain animals are heliotropic only under peculiar circumstances ; for example, Loeb found that winged ants exhibited no reaction to light except at the time of their nuptial flight, when they were decidedly helistropic."

Influence of light upon the direction of growth. ${ }^{3}$ Instances of this influence upon the stems of plants are almost too common to need mention. The leaning of plants toward the window, or of trees over a stream, can be seen almost any day.

It is noticeable that most leaves appear to be destitute of heliotropism, and yet it is not impossible to detect traces of its influence. On careful observation it will be noted that some leaves tend to present their upper surface at right angles to light rays, while others tend to present the edge.

It is a general principle of orientation that organisms with radial symmetry, like most plants, present as nearly as possible the cnd of the long axis to the source of light, with the lateral parts equally lighted; while organisms of bilateral symmetry, like most animals, tend to present the dorsal surface at right angles to the light, with the oral (head) end nearest its source, the right and the left halves equally lighted, and the ventral surface shaded.

1 Loeb, Physiology of the Brain, p. I89.

2 Loeb, Studies in General Physiology, pp. 52-53.

3 C. B. Davenport, Experimental Morphology, Part II, pp. 437-445. 
In the very highest animals little but the eye is sensitive to light, most parts being protected by a heavy epidermis or other covering, so that heliotropism in its strictest sense is in them limited to the visual parts. In lower animals, however, with bodies less protected, light exerts a controlling influence upon movements.

Influence of light upon the direction of locomotion. ${ }^{1}$ It has already been explained that some animals exhibit heliotropism only at certain periods of their lives, or only in certain conditions, as when hungry. Others, however, are constantly and uniformly sensitive. The common house fly is positively heliotropic, while the larvae of the same, hatched in the dark, soon become strongly negative, and so continue while in the larval condition. $^{2}$

This difference between the larval and adult stage is common, and led Loeb at first to suppose it to be a general principle, a conclusion invalidated by the fact that caterpillars and their imagoes behave alike. ${ }^{2}$

Both moths and butterflies are positively heliotropic, but moths are "attuned" to a lower intensity. This, with their more rapid flight, is responsible for their wholesale destruction by the naked flame, which the slower-flying butterflies avoid as a source of heat.

The tendency of many small animals to creep into crevices as if to hicle must not be understood as evidence of negative heliotropism, much less as evidence of timidity. It is often due simply to contact irritability (stereotropism), for it is a well-established fact that living matter is sensitive to contact with other solid substances. This is the principle discussed in Section IV and the one that generally lies at the basis of the huddling together of individuals, or of their crowding into corners or crevices. Loeb brought out this principle very nicely with some negatively heliotropic butterflies which wedged themselves closely between two plates of glass in the presence of light, showing how one tropic influence - in this case contact irritability - is competent

1 C. B. Davenport, Experimental Morphology, Part I, pp. I80-210; Loeb, Studies in General Physiology (I)05), pp. I-I14, from which most of the examples are cited.

2 Loeb, Studies in General Physiology, p. 20. 
to overcome an opposite but weaker one, - in this case negative heliotropism.

It is a noteworthy fact that irritability to light, while a property of protoplasm in general, is more pronounced in some cases than in others, even within the same organisms. This is true not only in the cy'es of animals, but, in general, the oral (anterior) end of eyeless animals is much more sensitive to light than is the aboral; ${ }^{1}$ as also is the dorsal surface more sensitive than the ventral. Light therefore operates strongly to influence not only the position but the locomotion of animals as well.

The following conclusions from Loeb upon the influence of light are valuable. ${ }^{2}$ In substance they are:

I. "The dependence of animal movements on light is in every point the same as the dependence of plant movements on the same source of stimulation."

I. "The direction of the median plane or the direction of the progressive movements of an animal coincicles with the direction of the rays of light."

2. "The more refrangible rays of the visible spectrum are more effective than the less refrangible rays."

3. "Light of a constant intensity acts as a constant stimulant."

4. Heliotropism is in a large measure dependent upon the intensity of light, differing for different animals.

5. "Heliotropic movements occur only between certain limits of temperature."

II. "The orientation of an animal toward a source of light depends on the form of the body, just as the orientation of a plant to light depends on the form of the plant."

I. "Symmetrical points on the surface of dorsiventral animals possess equal irritabilities."

2. The "irritability of the oral pole of an animal is different from the irritability of the dorsal pole," and is generally greater.

3. "The irritability of the ventral surface is different from the irritability of the dorsal surface."

"These three conditions taken together cause dorsiventral animals to place their median planes in the direction of the

1 Loeb, Studies in General Physiology, pp. 76-78.

2 Ibid. pp. 8I-84. 
rays and to move toward or away from the source of light in this direction."

4. "Eyeless animals behave in this respect like animals having eyes."

III. "Heliotropic irritability of an animal manifests itself frequently only at certain epochs of its existence."

I-4. In winged ants this epoch is at the nuptial flight; in plant lice it is when the wings are present; in the larva of Musca vomitoria negative heliotropism is most prominent when the larva is fully grown; and in a large number of animals the irritability is opposite in the larval and in the adult stages.

5. "Both night and day Lepidoptera are positively heliotropic, and their heliotropism is similar to that of every other positively heliotropic animal. The period of sleep of the night Lepicloptera, however, falls in the daytime, and only for this reason is their heliotropism manifested exclusively at night." 1

IV. "In many animals heliotropic irritability is connected with sexuality." Ants are sensitive only at the time of the nuptial flight, and in both ants and Lepidoptera the males are more sensitive than the females.

V. "The behavior of an animal clepends on the sum total of its different forms of irritability."

VI. Many animals are "compelled to orient their bodies against the surfaces of other solid bodies," or to bring their bodies "in contact with other solid bodies on as many sides as possible (stereotropism)."

VII. Animals " may be forced by light to move from diffused light into sunlight and to remain exposed to the high temperature of the sunlight, even though it may cause their death."

Considering all the "irritabilities" and "tropisms" to which animals and plants are exposed and to which they react, it is not necessary to appeal to instinct, or even to the nerrous systcm, to explain all movements of animals, nor is it well to ascribe to them such anthropomorphic qualities as love of light, distaste for darkness, preference, avoidance, curiosity, reason, or other such bases of higher intelligent action.

1 The question naturally arises, Why is the daytime the period for sleep in a positively heliotropic animal? The answer has not yet been given. 
To these conclusions two observations may well be added for present purposes :

I. Heliotropism, like many other reactions of protoplasm, arises from the nature of the organism, and not necessarily from

$f^{11: 22}$

f1:18

f1:12

P11:02

f10:44

$\int 10: 55$

(2). 14:00 to $14: 20$

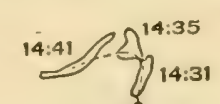

(4), 14:34 to $14: 42$

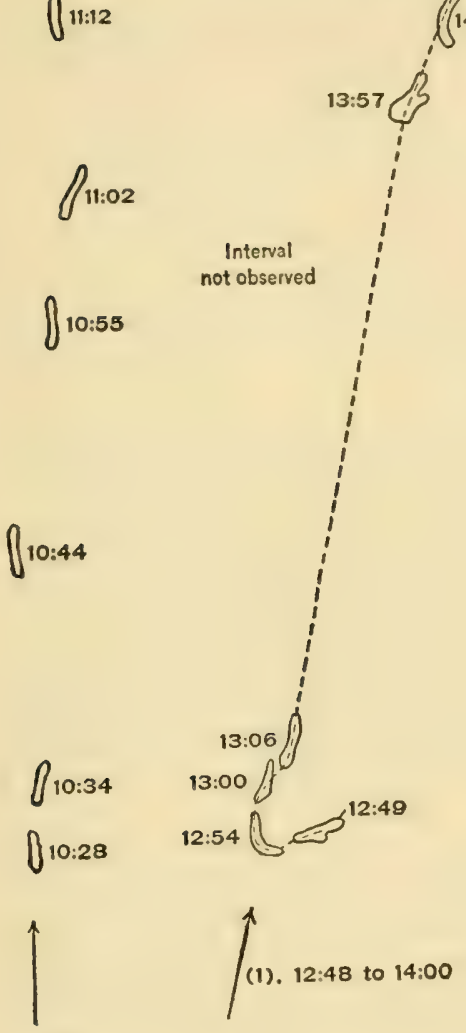

FIG. 28. Effect of light upon locomotion: movements of an amœba in response to changing directions of light. Arrows show the direction of the light rays, and figures show the hour $(13=1$ o'clock, etc.). - After Davenport

a basis of utility. For example, roots are in general positively heliotropic, - a quality that is not of the slightest usefulness, and which must be regarded as entirely accidental. Again, this 
quality is often fatal, as in the case of moths. In general, however, matters have long since become adjusted to these reactions as to other necessities governing the behavior of living matter.

2. All experiments indicate a high degree of variability among individuals, not only as regards the degree of response to heliotropism, but also as regards the effect of all other outside influences, even that of poisons.

Among examples furnished by the extended investigations into this subject, we have space to note but two.

The amoba, which represents about the simplest form of animal life, is an excellent medium for illustrating sensitiveness to light. Fig. 28 exhibits the movements of one of these bits of living matter under the influence of light, whose direction, as shown by the arrows, was occasionally changed, the figures indicating the hour, - all of which is strongly sugrgestive of the process of driving sheep.

The other example to be noted is the effect of light upon the position of the chlorophyll granules in the leaf cells, under different degrees of illumination, whether on the exterior walls, the partition, or the interior walls.

Conditions that determine heliotropism. Some organisms are positively heliotropic in one intensity and negatively so in another; some are positive at one temperature and negative at another; some are positive in a certain concentration (of sea water) and negative in another, and the general principle may be stated that decreasing the concentration has the same effect as increasing the temperature. ${ }^{1}$ It thus appears that the same organism can often be made positively or negatively heliotropic at will by altering the surrounding conditions of life.

\section{SECIION VII - INFLUENCE OF TEMPERATURE UPON LIVING MATTER}

The relation of heat to living matter is mainly, but not exclusively, quantitative; that is to say, the effect of heat is principally upon the rate of growth and activity. In general, each species has its maximum, above which protoplasm becomes

${ }^{1}$ Loeb, Studies in General Physiology, pp. 265-294. 
inactive (heat rigor); its minimum, below which all activity ceases; and its optimum, - that point at which growth is most rapid. Certain facts in this connection are noteworthy:

I. The maxima, minima, and optima are not the same for different species.

2. Protoplasm is killed if carried much above the maximum, - the organism decomposes and is destroyed.

3. Temperatures below the minimum are not fatal except in the presence of moisture, which, on conversion into ice, destroys the structure of protoplasm by the act of expansion.

4. The optimum at which growth is most rapicl is nearer the upper than the lower limit.

5. Both the optimum and the maximum may be raised by careful methods involving gradual acclimatization.

Specific effect of heat upon protoplasm. ${ }^{1}$ Beginning at the optimum and decreasing both ways to the limits, it may be said in general that protoplasmic activity is in proportion to the temperature. This is true of the amount of oxygen absorbed, of carbon dioxid evolved, of chlorophyll formed, and of carbon fixed, - in other words, of metabolism. The same is true as to movements of protoplasm and its irritability to light, contact, or other stimuli. The following table exhibits the number of electric shocks per second required at different temperatures to produce tetanus in the neck muscles of a tortoise. ${ }^{2}$

Effect of Temperatures upon Animal Activities

\begin{tabular}{|c|c|c|c|}
\hline Temperatures & $\begin{array}{c}\text { SHOCKS TER SECOND RE- } \\
\text { QUIRED TO PRODUCE } \\
\text { TETANUS }\end{array}$ & Temperatures & $\begin{array}{c}\text { SHOCKS PER SECONd RE- } \\
\text { QUiRed to PRONUCE } \\
\text { Tetanus }\end{array}$ \\
\hline $4^{\circ} \mathrm{C}$ & I & $2 \mathrm{I}^{\circ} \mathrm{C}$. & 25 \\
\hline $9^{\circ} \mathrm{C}$ & 5 & $2 S^{\circ} \mathrm{C}$ & 34 \\
\hline
\end{tabular}

Effect of heat upon the rate of growth in plants." The relation of temperature to plant growth is well shown in the tables on the following page. ${ }^{4}$

1 C. B. Davenport, Experimental Morphology, Part I, pp. 222-23I.

2 Ibid. p. $230 . \quad{ }^{3}$ Ibid. Part II, pp. 450-460. ${ }^{4}$ Ibid. p. 45 I. 
(ikowth in Millimeters of the Plumules and the Raidcres of Sefi)IINGS (IROWN UNIER I)IFFERENT TEMIERATURES FOR 4 S HOURS (Sachs)

\begin{tabular}{|c|c|c|c|c|c|c|c|}
\hline \multicolumn{4}{|c|}{ Piumeles } & \multicolumn{4}{|c|}{ Ranicles } \\
\hline Temperature C. & Maize & Bean & Pea & Temperature C. & Maize & Bean & Pea \\
\hline $\begin{array}{l}14-16^{\circ} \\
16-18 \\
18-20 \\
20-22 \\
22-24 \\
24-26 \\
26-28 \\
28-30 \\
30-32 \\
32-34 \\
34-36 \\
36-38 \\
3^{8}-40 \\
42.5\end{array}$ & $\begin{array}{r}5.6 \\
\\
11.0 \\
13.0 \\
\\
9.1 \\
4.6\end{array}$ & $\begin{array}{l}\text { I1.0 } \\
10.5 \\
\text { I } 5.0 \\
10.2 \\
7.5\end{array}$ & $\begin{array}{l}10.0 \\
5.7 \\
5.0 \\
5.5\end{array}$ & $\begin{array}{l}17.0^{\circ} \\
25.7 \\
26.3 \\
28.5 \\
33.2 \\
34.0 \\
38.2 \\
42.5\end{array}$ & $\begin{array}{r}2.5^{1} \\
24.5 \\
39.0 \\
55.0 \\
25.2 \\
5.9\end{array}$ & $\begin{array}{r}39 \\
47 \\
34 \\
30 \\
28 \\
22 \\
7\end{array}$ & $\begin{array}{l}4.0^{1} \\
41.0 \\
17.0 \\
\text { I } 2.2\end{array}$ \\
\hline
\end{tabular}

Maxima, Minima, and Optima for Various Species arranged ACCORDING TO THE OPTIMA ${ }^{2}$

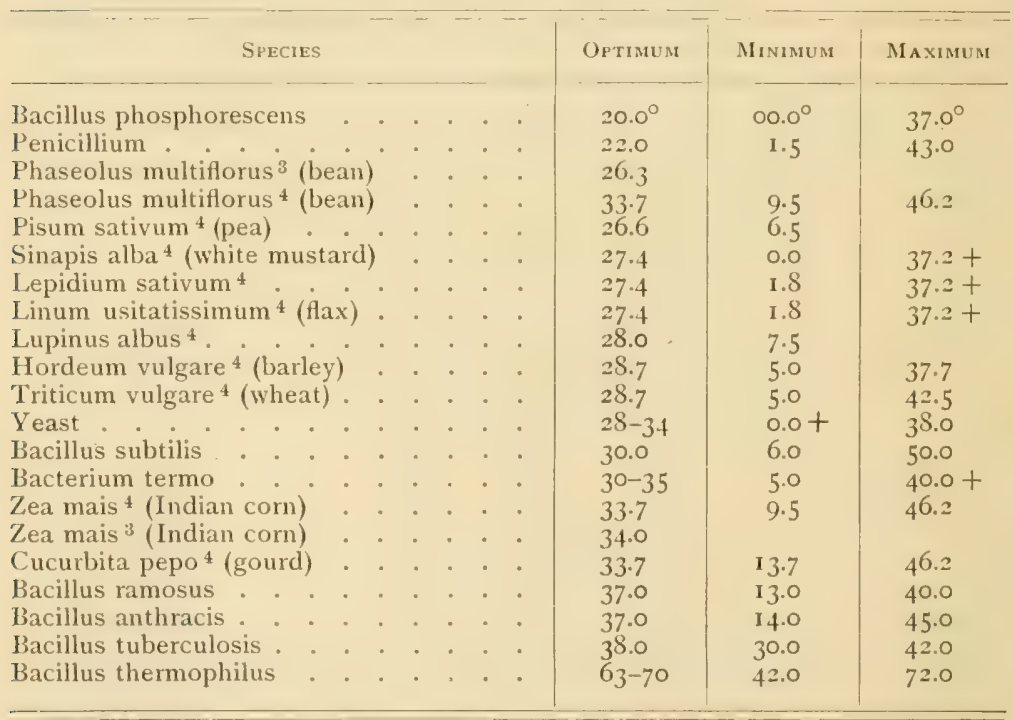

1 Growth during 96 hours.

2 C. B. Davenport, Experimental Morphology, Part II, p. 454.

${ }^{3}$ Radicle. 4 Plumule. 
Commenting on this table, its author observes in substance:

I. That in general the optima, the minima, and the maxima rise and fall together; that is, a species with a high optimum will also have a relatively high maximum and minimum.

2. That species vary greatly; so much so that the maximum of one (B. phosphorescens) may be below the minimum of another (B. thermophilus).

3. That the optimum for the radicle and the plumule may be widely apart, as in the bean.

4. That, in general, the optimum is in close relation to the natural habitat of the species, as in $B$. phosphorescens that lives in the moderate temperature of the sea, and in B. thermophilus that lives in the high temperature of decaying manure. From collateral evidence this must be ascribed to acclimatization.

5. That of all the species noted, the bacteria have the greatest range in optimum, showing that they are, as yet at least, less fixed in their organization.

6. That the minimum never falls below $\mathrm{O}^{\circ} \mathrm{C}$., the freezing point of water, which is the minimum for vital activities.

7. That the maximum temperature tends to be rather constant with related species, and among flowering plants the range is but $9^{\circ}\left(37^{\circ}-46^{\circ}\right)$. But $46^{\circ}$ is a fatal temperature for most protoplasm, and $50^{\circ}$ is the limit, showing how near the limit some species have been pushed. The extraordinarily high temperatures of $P$. thermoplizhs must be regarded as an instance of acclimatization, of which other striking examples are found in hot springs. ${ }^{1}$

8. That the range from minimum to maximum varies with the species. In this table the range is least for $B$. tuberulosis $\left(12^{\circ}\right)$ and greatest for $B$. phosphorescens $\left(37^{\circ}\right)$.

9. That the "wonderful adjustment" of critical temperatures to the environment of the species is not to be regarded as evidence of selection, but, as is elsewhere shown under "Acclimatization," it is due to the modification arought in the protoplasm by the temperature itself.

1 To the above may be added the observation that the optimum lies nearer the upper limit; that is, the difference between the optimum and the maximum is less than the difference between the optimum and the minimum. 
Effect of heat upon growth in animals. ${ }^{1}$ All larger land animals have accuired facilities for maintaining practically a constant temperature. This is not true for all animals, many of which, like marine species, are notably clependent for their temperature upon the accident of environment, in which respect they differ but little from plants. It remains to note, therefore, what influence heat may exert upon the growth of animal life so conditioned as to be dependent upon the surroundings for its temperatures.

Incklase in Lincith (Millineters) of Young; Tadpoles (of Frog and of Toad, under Different Temperatures, from the $24 \mathrm{TH}$ TO THE 48 Th Hour after Hatching ${ }^{2}$

\begin{tabular}{|c|c|c|c|c|c|}
\hline \multirow{2}{*}{$\begin{array}{c}\text { Temperatures } \\
\text { C. }\end{array}$} & \multicolumn{2}{|c|}{ Average Growth } & \multirow{2}{*}{$\begin{array}{c}\text { Temperatures } \\
\text { C. }\end{array}$} & \multicolumn{2}{|c|}{ Averagr Growth } \\
\hline & Frog & Toad & & Frog & Toad \\
\hline $9-1 I^{\circ}$ & $4 \cdot 5$ & 3.0 & $23^{-25^{\circ}}$ & & $41 \cdot 3$ \\
\hline $11-13$ & $5 \cdot 3$ & $5 \cdot 3$ & $25^{-27}$ & $31 \cdot 5$ & 39.0 \\
\hline${ }^{1} 3^{-1} 5$ & $4 \cdot 3(?)$ & I 5.5 & $27-29$ & 40.0 & \\
\hline $15^{-17}$ & & 16.3 & $29-3 I$ & $47 \cdot 5$ & 56.8 \\
\hline $17-19$ & $9 \cdot 5$ & & $3^{I-33}$ & 40.2 & $55 \cdot 3$ \\
\hline I9-2I & I 9.8 & 21.2 & $33-35$ & $43 \cdot 5$ & \\
\hline $2 I-23$ & & & & & \\
\hline
\end{tabular}

Twenty-nine to thirty-one degrees seems to be about the optimum temperature for both, from which the table shows that the land-living toad prospered rather better under the higher temperatures than did the frog, as he certainly suffered more uncler the lower, but that in both the rate of growth was substantially in proportion to the temperature.

The author of the table reports that the interval between fertilization and hatching in cod varies from thirty days at a temperature of $-2^{\circ}$, to thirteen days at a temperature of $6^{\circ}-8^{\circ}$; that herring vary from forty days at $2^{\circ}-4^{\circ}$, to eleven days at $10^{\circ}-12^{\circ}$; and that the time required for the frog to attain a development at which the head and tail are sharply defined is, at I $5^{\circ}$, six days; at $33^{\circ}$, one day.

1 C. B. Davenport, Experimental Morphology, Part II, pp. 457-460.

2 Ibid. p. 457. 
Birds develop only at high temperatures. The normal temperature for the chick is $38^{\circ}$. Féré2 incubated at temperatures varying from $34^{\circ}$ to $41^{\circ}$. The individuals were all examined at the same absolute time, and the following figures express the percentages of derelopment attained, taking that of $38^{\circ}$ as the standard : ${ }^{1}$

\begin{tabular}{lll|c|c|c|c|c|c|c|c}
\hline Temperature . . . . . & $34^{\circ}$ & $35^{\circ}$ & $36^{\circ}$ & $37^{\circ}$ & $38^{\circ}$ & $39^{\circ}$ & $40^{\circ}$ & $41^{\circ}$ \\
Index of development &. & 0.65 & 0.80 & 0.72 & $?$ & $\mathbf{1 . 0 0}$ & $\mathbf{1 . 0 6}$ & $\mathbf{1 . 2 5}$ & $\mathbf{1 . 5 1}$ \\
\hline
\end{tabular}

The author remarks that some doubt attaches to the figures under $35^{\circ}, 36^{\circ}$, and $37^{\circ}$, and calls our attention to the fact that somewhere not far above $4 \mathrm{I}^{\circ}$ the series would become zero. But for the range given, the development is in proportion to the temperature, although the highest given $\left(4 \mathrm{I}^{\circ}\right)$ is considerably above that attained under natural conditions. The growing click therefore does not, in nature, achicue its optimum.

Effect of heat upon the direction of growth, - thermotropism.2 Without going into the methods of investigation, it appears that, independently of the influence of light or other "tropisms," plants are often positively or negatively thermotropic largely according to temperatures. The plumule of seedling maize, for example, is known to be positively thermotropic at ordinary temperatures, while the radicle is positive between $15^{\circ}$ and $35^{\circ}$, indifferent at $37.5^{\circ}$, and negative above. The indifferent point with the bean (radicle) is given at $22.5^{\circ}$.

The subject is little understood, and though the impulse of thermotropism is weak as compared with that of heliotropism or geotropism, it is supposed to be one which inclines the organism to align itself in accord with the direction of heat rays, although it is true that thermotropic plants are sensitive to conducted as well as to radiant heat.

Temperature limits of life. ${ }^{3}$ All experiments indicate that as the temperature rises above the maximum the first effect is heat

1 C. B. Davenport, Experimental Morphology, Part II, p. 459.

2 Ibid. pp. 463-467.

${ }^{3}$ For extended discussion, and for tables of temperature limits, see (. . B. I)avenport, Experimental Morphology, Part I, pp. 23 I-249. 
rigor, which soon passes into death. The more rapid the rise the lower the death point, and the more gradual the rise the greater the resistance. Again, if the temperature does not rise too high, the heat rigor may gradually pass off, and activity may be resumed, even at temperatures which at first were followed by entire suspension of activity, and even by rigor. This is the first stage in the process of acclimatization.

Sachs found that a sensitive plant kept at " $40^{\circ} \mathrm{C}$. for one hour suffered loss of sensibility during twenty minutes after removal. Raised slow'ly to $50^{\circ}$, sensibility was only temporarily lost, but $52^{\circ}$ proved fatal. Immersed in water, heat rigor occurred at a temperature $5^{\circ}$ to $10^{\circ}$ lower." 1 Hofmeister found that hairs from the stem and leaf of Echallium agreste, showing lively movement, when gradually raised from $16^{\circ}-17^{\circ} \mathrm{C}$. to $40^{\circ} \mathrm{C}$. "became motionless," but that "after one or two hours movement returned and was very violent. Cooled and raised again to $45^{\circ} \mathrm{C}$, the protoplasm was motionless at first, but after seventeen minutes movements recurred but were not rapid." 2

The vital limit varies greatly with the species. Thus, roughly speaking, for bacteria it is $45^{\circ} \mathrm{C}$.; for cryptogams, generally $45^{\circ}-50^{\circ}$, with an occasional one at $60^{\circ}$; flowering plants, $45^{\circ}-50^{\circ}$; protozoa, $40^{\circ}-45^{\circ}$, with a few as high as $60^{\circ}$; mollusks, $30^{\circ}-40^{\circ}$; worms, $45^{\circ}-50^{\circ}$ (tardigrades, dried, $98^{\circ}$ ); crustaceans, $26^{\circ}-43^{\circ}$; insects, $27^{\circ}-43.7^{\circ}$; fish, $27^{\circ}-40^{\circ}$; salamander, $44^{\circ}$; frog, $40^{\circ}-42^{\circ}$; $\log$, rabbit, and man, $44^{\circ}-45^{\circ}$; vertebrate muscle, $40^{\circ}-50^{\circ} .^{2}$ This series exhibits a wide range of resistance to excessive heat, yet few organisms can endure much above $50^{\circ}$.

All experiments and observations indicate that death from high temperatures is caused by coagulation of the albumen of the protoplasm, a circumstance showing that albumen carries into its vital relations its ordinary property of coagulation by heat. That living matter contains many easily coagulable proteids no longer admits of doubt, and that their coagulation causes death is evidenced by the fact that once in this condition they do not return to their normal state.

1 C. B. Davenport, Experimental Morphology, Part I, p. 232.

2 For full tables from which these abstracts are made, see C. B. Davenport, Experimental Morphology, Part I, pp. 234-237. 
Death from low temperatures appears to result from entirely different causes. Protoplasm seems to contain no substance but water that undergoes either chemical or serious physical change by low temperatures. Many yeast cells endure - I I $3.7^{\circ} \mathrm{C}$. (Schumacher).

De Candolle subjected "various $d r y$ ' seeds and spores of bacteria to a temperature of nearly $-200^{\circ}$, at which temperature the atmosphere becomes liquefied, but without fatal effects." "Cilia from the mouth of the frog were cooled to $-90^{\circ}$, and recovered their movement upon raising the temperature." "Eggs of the frog, lowered slowly to $-60^{\circ}$, can revive." From facts such as these Davenport concludes that "there is no fotal temperature for dry protoplasm. ${ }^{1}$

The first effect of lowering temperature is a slowing of activity, followed, finally, by complete cessation. As is pertinently remarked by the author just quoted, "The fact that cold rigor usually occurs close to the zero point (C.) inclicates that the activities of protoplasms are closely determined by the fluid state of water," and "the critical point for vital activity has been adjusted to this critical point of water." 2

There is much lack of information upon the exact cause of death from excessive cold. Among the higher animals the immediate cause is without doubt asphyxia from the cessation of the blood flow; but among the simpler organisms the rinatter is not so clear. What evidence we have seems to indicate that the primary cause of death is in all probability the mechanical rupture of protoplasm and cell wall by freezing water expanding as it solidifies.

In any event experience and experiment agree in indicating that protoplasm is resistant to excessive cold in the absence of moisture, and in all study of this matter we are to remember two facts: first, that the freezing point of all protoplasm is lower than that of water only; and, second, that as long as the slightest activity is present heat is being produced. From these two facts the protoplasm is able to resist actual solidifying much

1 C. B. Davenport, Experimental Morphology, Part I, pp. 240-242. Though not so stated in the text quoted these temperatures are $C$.

2 Ibid. p. 242. 
longer and under much lower temperatures than we should at first suppose.

Substantive variation due to temperature; color markings. Early in the section it was remarked that the effects of temperature are qualitative as well as quantitative. IVithout doubt temperature exerts a controlling influence upon the color of butterflies, as has been determined by a number of direct experiments.

For example, I'anessa liana and $V$.prorsa were long regarded as distinct species. Lewanu is "characterized by a yellow-andblack pattern on the upper side of the wings," while prorsa "has black wings with a broad white transverse band and delicate yellow lines rumning parallel to the margins." 1 Later this was recognized as a case of "scasonal dimorthism," the yellowand-black lezana being the spring brood and the darker prorsa being the summer brood; that is to say, liatua, emerging in the spring, breeds immediately, producing a summer brood (prorsa), and this brood in the same way gives rise to a generation which passes the winter in the chrysalis form, emerging in the spring as lerana. Thus these two "species" are produced from the same stock, the difference being that one passes the chrysalis stage in the summer, the other in the winter.

That this difference is one of temperature was proved by direct experiment. Dorfmeister ${ }^{2}$ succeeded in producing prorsa directly from prorsa by the application of warmth to the pupa, and "by the application of cold he obtained from lea ana not the pure lcarna form, but one intermediate between it and prorsa," - an intermediate occasionally observed in nature and known as $V$. porima. ${ }^{2}$

Weismann, repeating the experiment, found that by using lower temperatures liana could be produced directly from levana, and he adds, "The converse experiment was also occasionally successful, the pupe of the winter generation being forced to assume the summer form by the influence of a higher temperature during, or shortly after, pupation." 3

1 Weismann, Germ Plasm, p. 379.

2 Vernon, Variation in Animals and Plants, p. 233.

3 Ibid. ; also Weismann, Germ Plasm, p. 379. 
Here temperature seems to exert a controlling influence upon pigment formation, although Weismann is careful to inform us ${ }^{1}$ that the two patterns "do not correspond" ; that if we were to "superpose" one upon the other, "it would seem that the black parts in prorsa do not correspond to the yellow ones in leana, and that the white band in the former does not correspond to [either] a yellow or [a] black part in the latter. This band is, on the contrary, cutively' awanting in levana, and is represented by both black and yellow regions." 1

Again, Weismann experimented with Polyommatus fhlaras, ${ }^{2}$ a species "distributed over the whole of the temperate and colder parts of Europe and Asia." Toward the north (in Germany) the upper surface of the wings is of a "beautiful reddish-gold color," hence its popular name, "fire butterfly." But he says, "Farther south the reddish-gold color is more or less thickly dusted with black, and specimens from Sicily, Greece, or Japan often display only a few reddish-gold scales, the general appearance being almost black."

"In Germany this butterfly is double-brooded, and the two generations are similar, but in certain districts of southern Europe . . the first generation is reddish-gold, - the second, which flies in midsummer and is known as the variety cleus, having the wings well dusted with black." "As in Germany during exceptionally hot summers individuals with a blackish tint have repeatedly been caught together with the ordinary form ...." and Veismann observes that it would seem "the butterfly becomes red when exposed to a moderate temperature and black when the heat is greater.'

Attempts to produce these forms at will, however, by regulation of temperature only partially succeeded. But the conditions were severe. There was no common ancestor. Weismann undertook to produce the southern form from the northern stock and vice versa. Insects reared from German butterflies but kept in high temperatures were in many instances "dusted with black, but none of them resembled the darkest forms of the southern clcus." Conversely, butterflies raised in cool temperatures from

1 Weismann, Germ Plasm, p. 379.

2 Ibid. pp. 399-4co. 
Neapolitan stock were lighter in color than in their native habitat, but "none were so light-colored as the ordinary German form." 1 This difference he ascribes to the cumulative influence of the natural seasonal temperatures, and is quick to protest against its interpretation as inclicating an inheritance of acquired characters. He calls it a case of internal selection as between "winter and summer determinants."

However, that is of no consequence in the present connection. The facts here given show beyond a cloubt that outside temperatures exert a clirect effect upon so important a character as color. Whether this occurs by chemical clisturbance in pigment formation, by intemal selection, or by other means does not greatly matter here. There is some evidence tending to show that the light color of polar animals is due to the direct action of cold. ${ }^{2}$ This, if true, argues for chemical action upon pigment as the cause of color charges due to temperature.

Temperature an all-pervading influence. Temperature differs from most other external forces in being, for many species, at least, an all-perading influence. Higher animals and plants are themselves centers of heat production, and in general their temperatures are the algebraic sum of their own heat production and the heat of their surroundings. Lower organisms, however, are very largely dependent upon their environment for their temperatures, and in cases of this kind the entire protoplasm of the body is affected.

\section{SECTION VIII - EFFECT OF CHEMICAL AGENTS UPON PROTOPLASMIC AC'TIVITY}

All development, all differentiation, and all functional activity of living organisms are the result of protoplasmic activity; but protoplasmic activity is, in the last analysis, chemical activity, and it is certainly subject to many of the laws controlling ordinary chemical reactions. It is noticeable in the study of vital

1 Weismann, Germ Plasm, pp. 399-400.

2 The writer has somewhere read that animals on shipboard become rapidly lighter in color as the coat becomes exposed to intense cold, but he is unable to verify the report. 
processes from the chemical standpoint that some substances exert no influence upon protoplasm, while others kill it outright; that some accelerate and others retard its normal action; and that some suspend activities more or less completely, while others dir'ort them into cntively now channcls. Here is variation due to chemical disturbance of the material basis of life, and it is well to study somewhat in cletail this "modification of vital actions" from chemical causes. ${ }^{1}$ In studying this class of phenomena it is necessary, of course, to make use of simple organisms of one or of few cells, and while we cannot reason directly from these to the higher animals and plants, still all evidence goes to show that the differences are not so much in kind as in complexity.

Oxygen. All experiments indicate that no protoplasm can long survive in the absence of oxygen. In most cases it is taken directly from the air, but in others, as in anaerobic bacteria, it is probably extracted from surrounding compounds containing oxygen. Diminished oxygen retards and increased oxygen and ozone greatly accelerate the vital processes, ${ }^{2}$ all without changing their character.

A number of oxygen-containing substances greatly retard or even destroy vital activities, probably through "oxidation of the protoplasm." "3 If this be the case, and the material basis of life is subject to the ordinary chemical process of oxiclation, it shows that vital processes in the last analysis rest upon a strong chemical basis.

Hydrogen peroxicle $\left(\mathrm{H}_{2} \mathrm{O}_{2}\right)$, only slightly different from water $\left(\mathrm{H}_{2} \mathrm{O}\right)$, is a powerful oxidizing agent. One part in ten thousand (O.O I per cent) in hay infusion killed all ciliata in from fifteen to thirty minutes. Algæ survived a O.I per cent solution but ten or twelve hours, and died in a ro per cent solution in a few minutes. "Salts of chromic, manganic, permanganic, and hypochlorous acids act as intense poisons, apparently by directly yielding oxygen atoms to the plasma proteins." Chlorin, iodin, and

${ }^{1}$ C. B. Davenport, Experimental Morphology, Part I, chap. i, from which the data in this section are largely taken.

2 Small animals confined in an atmosphere of pure oxygen exhibit greatly increased activity and "live themselves to death" in a few hours.

${ }^{3}$ C. B. Davenport, Experimental Morphology, Part I, p. 3 . 
bromin in the presence of water act "fatally upon all organisms by splitting [the] water, forming hydro-halogen compounds, and leaving the oxygen to unite with the living protoplasm." 1

Hydrogen. Amobie subjected to an atmosphere of hydrogen for twenty-four minutes became motionless, some having assumed the spherical form. The same general result followed in tradescantia hairs, but from the fact that normal activity was restored by admitting air it was assumed that the results arose not from any injurious effect of hydrogen but from the exclusion of oxygen. ${ }^{2}$

Oxids of carbon, $-\mathrm{CO}_{2}$ and $\mathrm{CO}$. These two oxids of carbon have very different effects upon protoplasm. The former, like hydrogen, seems to act only by excluding oxygen, death, when it results, being due mainly to asphyxia, while the latter kills by attacking the protoplasm directly. ${ }^{3}$

Catalytic poisons.t A large number of unstable carbon compounds, neither acid nor basic and therefore not characterized by intense chemical action, are yet violent poisons. Here belong the anæesthetics, as chloroform, chloral, ethyl, ether, alcohols, etc.

These unstable compounds are characterized by a "lively condition of molecular movement" (Nägeli), which is considered to disturb the normal movements of the protoplasm, or "to lead to chemical transformations in the unstable albumen of the protoplasm" (Loew).

Catalytic substances are supposed to exert their action not by entering into and effecting new combinations but by disturbing, through their mere presence, the usual behavior of bodies. It is in this way that protoplasm suffers in their presence. Thus hydrochloric and prussic acids unite only at high temperatures, except in the presence of various ethers, when they will unite even at $-15^{\circ}$. The vigor of this catalytic action is in proportion to the molecular composition. ${ }^{5}$ Thus in the methan series with $\mathrm{CH}_{3}$ as the base we have $\mathrm{CH}_{4}, \mathrm{C}_{2} \mathrm{H}_{6}, \mathrm{C}_{3} \mathrm{H}_{5}$, etc., in which

1 C. B. Davenport, Experimental Morphology, Part I, p. 4.

2 Ibid. p. 5 .

3 Ibid. 1) 6.

4 A free extract from C. B. Davenport, Experimental Morphology, Part I, pp. 7,8 .

5 The facts here stated are taken almost literally from C. B. Davenport, Experimental Morphology, Part I, pp. 7-8. 
"the poisonous action increases up to a certain limit in proportion to the number of $C$ atoms," while "above this limit the compounds are more stable and are more indifferent, as for example paraffin $\left(\mathrm{C}_{21} \mathrm{H}_{44}\right.$ to $\left.\mathrm{C}_{27} \mathrm{H}_{56}\right)$."

Again, in such a series, if the $H$ atoms become replaced by one of the halogens, the poisonous properties correspondingly increase; thus : ${ }^{1}$

$\mathrm{CH}_{4}$, marsh gas, innocuous.

$\mathrm{CH}_{3} \mathrm{Cl}$, slightly anæesthetic.

$\mathrm{CHCl}_{3}$, chloroform, powerful anæsthetic.

$\mathrm{CCl}_{4}$, very dangerous, stupefying involuntary muscles.

Chloroform and ether affect all protoplasm, both plant and animal, higher as well as lower. They seem to produce at first (two to five minutes in a 25 per cent water solution) a "very intense excitement in the morement of the protoplasm," followed by "strong vacuolization, and then the cytoplasm gradually becomes immobile" and dies, if the influence is continued. In a similar way the various alcohols exert stupefying effects in proportion to the number of $\mathrm{CH}_{2}$ radicals present, and carbon disulphid $\left(\mathrm{CS}_{22}\right)$ is one of the most powerful catalytic poisons.

Protoplasm is therefore subject to catalytic disturbances, in which it is not different from other and more ordinary chemical materials, - a fact in itself exceedingly significant to the student looking for fundamental causes of variation.

Poisons which form salts." These are acids and bases which Loew believes, as stated by Davenport, " unite [directly] with the protein substances of the protoplasm, producing salts," disturbances that of course soon lead to death. Thus "formic acid, even in small per cents, -- 0.05 per cent to 0.006 per cent, - prevents the development of bacteria. On the other hand, some protoplasm has acquired a resistance to organic acids, the vinegar eel living in 4 per cent acetic acid," and the gland cells of some marine Gastropoda secrete from 2 per cent to 3 per cent of $\mathrm{H}_{2} \mathrm{SO}_{4}$, a strength which is fatal to most protoplasm.

1 The halogens - fluorin, chlorin, bromin, and iodin-form a group of substances of very similar chemical properties, but form, in the order named, a decreasing series as to chemical energy.

2 C. B. Davenport, Experimental Morphology, Part I, pp. I2-14. 
Nageli's experiments ${ }^{1}$ indicate that water distilled in copper ressels, or standing four days in other vessels with twelve clean copper coins per each liter of water, was fatal to Spirogyra, though the proportion of copper to water was but i to $77,000,000$.

These reactions, resulting in death rather than in modified action, are important, not as showing primary causes of variability but as proving again that living protoplasm is still subject to many of the chemical affinities that controlled its elements before they became organized into living matter. It must be remembered in this connection that many of these substances attack only living protoplasm, having no action upon dead protoplasm, showing that at death the highly complex materials have, to some extent at least, broken down.

The action of some poisons, like nicotin, is proportional to the "differentiation of nervous substance" ; others, like cocain and atropin, first excite and then paralyze the central nervous system of vertebrates, but act as violent poisons upon undifferentiated protoplasm (Protozoa). ${ }^{2}$

Toxic poisons. It is not the germ that lills, but rather its specific toxin that deranges some of the vital functions beyond endurance. The dire effects of germ diseases are therefore due not so much to the organisms themselves as to their constant manufacture within the body of a chemical poison which the protoplasm cannot enclure and preserve its normal functions. It may die in the attempt, or it may succeed and become acclimated, but while the struggle is on, the body functions will be considerably disturbed. It is significant that compounds similar to those of discase-producing bacteria have been " extracted from the seeds of some phanerogams; for example, ricin from the seeds of the castor-oil bean, etc." In this class may come the poisons secreted by certain animals, as the rattlesnake and cobra, fatal to vertebrates but innocuous to Infusoria and Flagellata.

It is also noteworthy that the blood serum of one species rapidly dissolves the corpuscles of another (red and white), and is therefore injurious or fatal according to the amounts present. ${ }^{3}$

1 C. B. Davenport, Experimental Morphology, Part I, p. I4.

2 Ibid. pp. 23 and 24 .

3 Ibid. p. 22. 
Specific secretions and glandular activity. The fact just mentioned introduces a subject full of interest. It appears that each species, and perhaps each individual, is engaged in the production of chemical substances (whether the result of metabolic or liatabolic activity is uncertain) which exert specific action upon living matter.

It is significant that some of the lower organisms producing definite substances die from the injurious effects of their own product, ${ }^{1}$ unless this is removed as formed, and that higher animals are supplied with elaborate excreting apparatus, strongly suggesting that certain of their products are deleterious to the organisms that produced them, while in other cases they are clearly beneficial. In this connection Loeb remarks : ${ }^{2}$

It is perhaps not impossible that those mental diseases that are hereditary are, in reality, chemical diseases caused by poisons that are formed in the body, just as special substances - for instance, alcohol, hashish, and other intoxicating substances - produce temporary mental diseases. The delirium of fever, as well as certain other mental diseases, may owe their origin to poisons which are formed in the body. It is quite possible that these poisons are also formed in the normal body. It is only necessary that they be formed in somewhat larger quantities or destroyed in somewhat smaller quantities in the body of the insane than in the normal man."

It is further not at all necessary that the hypothetical poisons which cause mental diseases be formed in the central nervous system. They may be formed in any organ of the body. It is only necessary that they affect the central nervous system; in other words, that they be nerve poisons.

Nothing is better qualified to make this view clear than the result which the destruction of the thyroid gland has on the mental and physical development of children. We know that in case of degeneration of the thyroid gland the growth and mental development of the child are retarded. Idiocy may result from the destruction of the thyroid gland. It has been found that an improvement, or even a cure, can be attained by feeding patients afflicted with this trouble with the thyroid substance of animals. ${ }^{4}$ Baumann found that the thyroid gland contains an element which is contained in no other organ of the body, - namely, iodin.

1 The yeast plant that forms alcohol dies when the solution has reached a strength of about 20 per cent.

${ }^{2}$ Loeb, Physiology of the Brain, p. 207.

${ }^{3}$ It is said by Lombroso, and others agree, that the criminal is characterized by excessive amounts of urea.

4 Medicinal preparations from various glands are now regularly supplied by the large slaughterhouses. 
Insect poisons. The poison of bees - formic acid - is fatal to insects and small animals, and in sufficient quantity to the larger vertebrates, including man, though frequent stings of a moderate number lead rapidly to acclimatization. It is noteworthy, too, in this connection that the sting of the insect (mud wasp, for example) does not always kill but often merely paralyzes, so that the creature stored with the egg will remain alive to furnish food to the larvæ some weeks later.

Galls. Galls are the direct result of the sting of an insect, leaving a specific poison to act upon a particular form of protoplasm. The result is not death but a diverting of the activities into entirely new channels. Darwin states that "no less than fifty-eight kinds of gall are produced on the several species of oaks by Cynips with its sub-genera, and Mr. B. D. Walsh states that he can add many more." 1

Darwin further remarks that many gall insects are exceedingly small, and that consequently the drop of poison they inject must be exceedingly minute; moreover, it is never injected but once. The growth that follows, however, is specific and continuous. He quotes Walsh as saying, "Galls afford good, constant, and definite characters, each kind keeping as true to form as does any independent organic being," ${ }^{2}$ and he calls our attention to the fact that seven of the ten distinctly different galls produced on the willow are by insects which, "though essentially distinct species, yet resemble one another so closely that in almost all cases it is difficult and in most cases impossible to distinguish the full-grown insects one from another." The difference in the quality of the poison secreted by insects so nearly alike cannot be great, yet it is sufficient to give rise to galls widely different. Last, and not least, he mentions that "Cymips fecundatrix has been known to produce in the Turkish oak, to which it is not properly attached, exactly the same kind of gall as on the European oak. These latter facts apparently prove that the mature of the poison is a more poacrful agent in detcrmining the form of the gall than [is] the specific character of the tree which is acted on." 3

1 Darwin, Animals and Plants, II, 272.

${ }^{3}$ Ibid. (second edition), 273. The italics are mine.

2 Ibid. p. 273. 
Tumors. Those overgrowths of various parts of the body, called tumors, arising from causes not well understood, have their specific characters as truly as if derived from inheritance. Whether the character of the tumor is derived primarily from the tissues affected or from some outside specific cause is not known, but it is a significant fact that the protoplasm, which derized its characters originally by inheritance, has undergone permanent and definitc alteration through the operation of causes absolutely distinct from inheritance, whether internal or extcrmal to the organism, thus showing the possibility of diverting the cuergies of inherited material into absolutely nia channels through apparently slight canses.

At this point it is well to call our attention to the profound changes (permanent variations) wrought upon the constitution of the individual by such internal-external circumstances as vaccination or the injection of antitoxin, as well as to the immunity acquired through a single attack of an infectious disease.

Germination of seeds. It is a well-known fact that certain chemicals accelerate and others retard the process of germination. Just why this is true is not clear on any other ground than that of the ordinary susceptibility of growing protoplasm to stimulants and sedatives. The process of germ development requires, in addition to what is contained within the seed, only oxygen, water, and a farorable temperature; the influence of other chemicals must be indirect.

Chemotaxis and chemotropism. ${ }^{1}$ The influence of chemical substances upon the locomotion of free-moving organisms is technically known as chemotaxis, and their influence upon the direction of growth (in plants) is known as chemotropism. ${ }^{2}$ The distinction is hardly worth observing for our purposes, because both phenomena arise from a clirect influence of chemical substances upon living protoplasm, either attractive or repellent. If attractive, it (the protoplasm) will move toward the material in

1 C. B. Davenport, Experimental Morphology, Part I, 32-45; Part II, pp. 335-342; Loeb, Physiology of the Brain, pp. 50, 88-90, I I $186-188$.

2 As has already been noted, the same distinction is often observed between geotaxis, the influence of gravity upon locomotion, and geotropism, its influence upon the direction of growth; also between heliotaxis and heliotropism as covering corresponding influences of the sun (light). 
question, providing it is free to do so (as in the case of the lower plants and all animals), or its growth will be directed toward it if (as in the case of higher plants) it is fixed and unable to move. The former is, strictly speaking, chemotaxis; the latter is chemotropism. For our purposes it is a distinction without a difference, because in the latter case the plant is unable to indulge in locomotion, and performs the nearest possible act in changing the direction of growth. Both are locomotion under the circumstances; both are due to the same cause, - a chemical affinity or attraction, - and they differ because of differences in the organisms affected in respect to the power of locomotion, not because of differences in the nature of the forees in action. The two terms are, therefore, for our purposes, synonymous as denoting a power of attraction between protoplasm and certain ordinary chemical compounds such as to cause the protoplasm to approach if it be free to move, or, if not, to grow in that direction, - which is all that can be done under the circumstances to satisfy the affinity.

Chemotaxis, or chemotropism as the writer prefers to call it, ${ }^{1}$ has but a slender hold upon higher animals. It appears to be localized in the nostrils and to manifest itself only in the sense of smell, agreeable or otherwise; but in lower organisms, even in many insects, it is apparently not confined to a minute fraction of the surface, but pervades the whole organism with an influence that is all but overpowering. It may of course be aided or opposed by other tropisms, as gravity or light, in which case the total result is the algebraic sum of all the energies operative, but that chemotropism is a force to be reckoned with in development is a fact not to be doubted.

Examples of chemotropism." Englemann noticed that bacteria uncler a cover glass will gather along the margin, or if green algre be introduced they will cluster about them so long as they are producing oxygen, but in the darkness they will not

1 The ending "taxis" is from the Greek, meaning assortment or arrangement; the ending "tropism" is from "tropic," to turn. "Chemotropism" is pronounced k:è-mŏt'rứ-p̌̀m.

2 Until otherwise noted what follows is a free though not exact transcript from data given in C. 13. Davenport's Experimental Morphology, Part I, pp. 32-39. 
be affected. In the same way nearly all kinds of motile organisms are now known to be influenced by a variety of chemical substances.

Lubbock has shown that ants retreat from essence of clove, lavender water, etc., ${ }^{1}$ placed within one fourth inch of their path, and Loeb found that the larvæ of flies creep towards a piece of flesh brought nearer than $1.5 \mathrm{~cm}$. Not only flesh and decaying meat, but meat juice in a glass, will allure, while fat has no effect. Every farmer knows how quickly flies are attracted by a dressed animal, and carrion birds by a carcass.

According to Pfeffer's experiments the inorganic salts of potassium, sodium, calcium, ammonium, magnesium, and many other metals in 0.5 per cent solution act attractively upon Bactcrium temo. "Inorganic acids . . in general act repulsively," but phosphoric acid and the phosphates are strongly attractive. Dewitz states that mammalian spermatozoa are attracted by $\mathrm{KOH}$.

"Alcohol in grades between Io per cent and I per cent acts repulsively towards bacteria," but "glycerin is neutral." Malic acid, which is of wide distribution among plants, is strongly attractive to spermatozoids, even in a 0.00 I per cent solution, a fact which is highly significant.

This principle of chemotropism acting on higher organisms gives rise to characteristic movements. In Loeb's experiments on actinians ${ }^{2}$ a piece of meat laid upon the tentacles so affected them as to cause a bending which carried the meat into the mouth, while a wad of water-soaked paper had no effect, but lay there until removed. If, however, the paper was soaked in meat juice it was received the same as a piece of real meat. (See Fig. 29.)

Now the actinian, consisting simply of a sac with a row of tentacles around the edge, without brain or nerve centers of

1 Experimenting upon means of preventing the ravages of the corn-root aphis, Forbes of Illinois found that a small amount of oil of lemon on the seed corn, before planting (costing but ten cents per acre), is able by its strong odor to repel ants from the neighborhood of the corn hill for no less than six rueeks after planting. As the young aphis is absolutely dependent upon the attentions of the ant, this treatment is effective.

2 Loeb, Physiology of the Brain, pp. 49-50. 
any kind, is certainly incapable of exercising anything like intelligent choice. The characteristic movement must have been due to the specific chemical action of the juices of the meat upon the protoplasm of the muscle fibers of the tentacle, while the paper had no such action and hence no movement followed. This movement and non-movement look like intelligence or

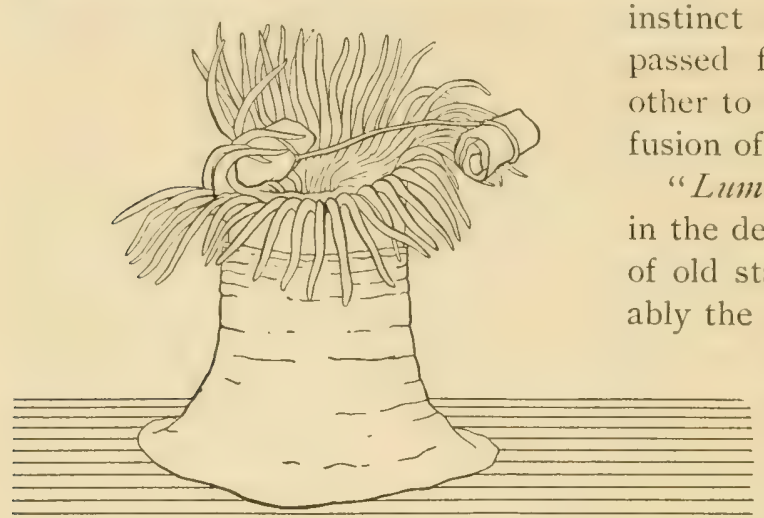

FIG. 29. Stimulating effect of certain chemicals upon muscular action: a piece of meat laid upon the tentacles of the actinian stimulates their action and it is drawn into the mouth. A piece of paper similarly placed is inoperative of certain substances contained in the compost holds them there," for "when one half of the bottom of the box is covered

with moist blotting paper and the other half with a thin layer of compost, all the worms will gather on the compost side." Decapitated worms behave in the same way, ${ }^{2}$ so that the effect is due to a general influence, not to "nerve centers." Loeb says," "I have often placed pieces of lean meat and pieces of fat from the same animal sicte by side on the window sill, but the fly never failed to lay its eggs on the meat and not on the fat." (He tried, of course without success, to raise larvæ in the fat.) "It can casily be shown that larre of the fly are positively chemotropic lowards certain chemical substances which are formed, for instance, in decaying meat or cheese, but which are not formed in fat. The substances in question are probably volatile nitrogenous compounds," and "the chemical effects of

1 This subject will be pursued further under "Instinct and Reflex Action."

2 Loeb, Physiology of the Brain, p. 90.

${ }^{3}$ Ibid. pp. 1 $86-187$. 
the diffusing molecules on certain elements of the skin influence the tension of the muscles," causing motion.

The female fly is attracted by meat, the same as are larve, and "as soon as the fly is seated on the meat chemical stimuli seem to throw into activity the muscles of the sexual organs, and eggs are deposited on the meat." This chemical stimulus is about all there is of the wonderful "instinct" by which insects are led "always to deposit their eggs in exactly the right places."

These and similar examples show the effect of certain chemicals upon free-moving organisms. It remains to illustrate their effect upon the direction of growith among plants, which are not free to move.

First of all, we are to note the effect of certain chemicals upon the tentacles of insectivorous plants. Darwin noticed that "when drops of water or solutions of non-nitrogenous compounds are placed upon the leaves of the sundew, Drosera, the tentacles remain uninflected; but when a drop of a nitrogenous fluid, such as milk, wine, albumen, infusion of raw meat, saliva, or isinglass is placed on the leaf, the tentacles quickly bend inwards over the drop." 1 Darwin found that of " nine salts of ammonia tried, all caused inflection, and of these the phosphate was the most powerful," and that "sodium salts in general caused inflection with extreme quickness." This action of nitrogenous, phosphatic, and other chemicals common to animal life, was the same upon the tentacles of insectivorous plants as upon the tentacles of lower animals subsisting upon the same kind of food." Thus plant and animal tissues appear to be subject to the same general laws in this regard.

From this point of departure, common to both plant and animal, we note that the animal, free to move, cloes so in response to this class of stimuli. What does the plant do that cannot move, even as tentacles move? In other words, how does chemotropism affect the direction of growth among higher plants?

Roots in general are supposed to grow toward oxygen, and pollen tubes will certainly turn toward the stigma of the flower,

${ }^{1}$ C. B. Davenport, Experimental Morphology, pp. 335-336.

- See the experiment on actinians previously quoted from Loeb, Physiology of the Brain, pp. 49-50. 
the supposition being that sugar is the special attracting substance in the latter case. It is noteworthy that the pollen tube is attracted not simply to its own stigma but also to the pistil and the ovule of other species, even of a different genus with which it is unable to unite. ${ }^{1}$ Davenport says : ${ }^{2}$

The results of experimentation upon chemotropism show that various substances may direct the growth of such elongated organs as tendrils, roots, and hyphæ of plants.... In many instances it can be shown that the direction of growth is on the whole advantageous to the organism. . . In other cases, however, the response seems to have no relation to adaptation.

The immediate cause of change of direction is "excessive growth on one side, due to excessive imbibition or to excessive or restricted assimilative activity.'

The evidence seems conclusive that the chemical elements constituting living matter have not entirely lost their ordinary affinities and properties; that protoplasm is in many respects subject to the laws of other chemical compounds; that its activities may be accelerated or retarded; that they may be temporarily or even permanently modified in character by chemical alteration, or even entirely destroyed by entering into new and strange combinations with surrounding substances. Here is a real cause, at least of occasional variation, - possibly of those sudden changes we call mutation.

\section{Rhythmical contraction of muscle instituted by certain chem-} ical substances. The irritability and consequent contraction of muscles clue to influences of a chemical nature have already been noticed, as in the case of the movement of the tentacles of the actinian, the attractive or repulsive effect of certain odors, and, to some extent, in the placing of insect egrs in "exactly the right spot."

It is now well known that certain salts exert a specific action upon muscle, exciting eren rhythmic contraction. As long ago as I 88 I Biedermann discovered that if the muscle of a frog be carefully excised at a low temperature $\left(0^{\circ}-10^{\circ} \mathrm{C}\right.$.), then weighted and dipped into a 0.6 per cent solution of sodium chlorid containing also small amounts of sodium phosphate and sodium

${ }^{1}$ C. B. Davenport, Experimental Morphology, p. $339 . \quad 2$ Ibid. p. 343. 
carbonate "one observes as a rule, aiter a longer or shorter period of rest, that the immersed muscle begins to beat rhythmically."

On this point Loeb remarks that sometimes only a mere tremor is noticeable, at others violent contractions; that sometimes only individual fibers are active, at others the whole muscle is involved; and that "at low temperatures these phenomena may continue for days." 1

These facts, together with Ringer's and Howell's statements that calcium and potassium salts exert a direct action upon the heart, led Loeb to extend the Biedermann investigations. He subjected the gastrocnemius muscle of a frog (unweighted) to a series of solutions of chemically pure materials in twice-clistilled water."

These experiments not only confirmed Biedermann's findings that the salts of sodium were able to excite rhythmic muscular contraction but they also added lithium, cresium, and rubidium to the list of bases, and the salts of bromin, iodin, and iron to those of carbon, chlorin, and phosphorus. These movements are periodic and continue into the second day, even at room temperatures.

Loeb determined that if a muscle be immersed in a 0.7 per cent solution of sodium chloricl, contractions will begin in from sixty to ninety minutes, but that "if a trace of alliali is added, contractions begin much sooner." This acceleration he attributes to the hydroxyl $(\mathrm{OH})$ in the alkali added. Not only that, but he ascribes the effect to the $\mathrm{H}$ involved in the hydroxyl, because the same action follows the addition even of inorganic acids, as $\mathrm{HNO}_{3}$, "if the same number of hydrogen ions are contained in the unit volume" ${ }^{3}$ but Loeb hastens to assure us that neither the hydrogen ions nor the hydroxyl ions "belong to those which are capable of liberating rhythmic contractions." They only accelerate the action of those which of themselves possess this power.

The action of sodium and other chemicals in exciting contraction is, in the opinion of Loeb, to be ascribed to their entering

1 Loeb, Studies in General Physiology, Part II, p. 5 I8.

2 Ibid. pp. 519-538.

${ }^{3}$ Ibid. p. 527. 
the muscle and there forming with its substance definite compounds, and he believes the accelerating effect of $\mathrm{H}$ or $\mathrm{OH}$ is due to their catalytic action in facilitating the formation of these compounds. In this connection it is to be remembered that the serum of the body which bathes the muscles is always, in health, strongly saline and slightly alkaline.

Further experiments clearly showed that the salts of potassium and those of calcium, magnesium, strontium, manganese, and cobalt tend strongly to pricut contraction, this being especially true in the case of potassium and calcium, forcing the conclusion that certain definite substances are necessary to contraction; that certain others tend to acclerate and still others to retard this characteristic activity of muscular tissue.

Artificial parthenogenesis through changes in the surrounding solution. ${ }^{1}$ The indefatigable labors of Jacques Loeb upon this subject have not only thrown much light upon the essential features of fecundation, but incidentally they have afforded results of high value in determining the nature and range of external influences upon the characteristic activities of living matter. ${ }^{2}$

It had long been known that many of the eggs of sea urchins, arthropods, and marine worms, cien when unfertilised, would, if left for a comparatively long time in sea water, begin to segment, reaching the two- and sometimes the four-celled stage. Loeb, and later Morgan, found "that if the concentration of the sea water be raised sufficiently by the addition of certain salts, a segmentation of the nucleus takes place without any segmentation of protoplasm [cytoplasm]. Such eggs, however, when brought back into normal sea water divide into as many cells as there were preformed nuclei." "3 In none of these experiments did the cell division "lead to the formation of a blastula. A heap of cells, at the best about sixty, were formed, and then everything stopped." As in the case of tumors

1 Loeb, Studies in General Physiology, Part II, pp. 539-69r.

2 These investigations have been published from time to time, especially in the American Journal of Physiology, and later (1905) in book form under the title, Studies in General Physiology.

${ }^{3}$ Loeb, Studies in General Physiology, Part II, pp. 540-541. 
and galls, here was growth without systematic differentiation; cell division without the formation of an embryo.

Encouraged by this degree of segmentation and by his experiments upon irritability of muscle, Loeb tried a great variety of solutions, in various degrees of concentration, in the hope of carrying the segmentation far enough to produce real embryos and live, free-moving larvæ.

He was greatly hampered by the fact that unfertilized eggs do not form membranes as do fertilized, so that growth tended to be formless, and even when assuming definite form in the blastula ${ }^{1}$ stage there were often formless masses of dividing matter lying to one side.

Briefly stated, the following facts developed during the progress of this systematic experiment :

I. In a solution of sodium chlorid the eggs were unable to reach even the blastula stage.

2. With the addition of $\mathrm{MgCl}_{2}$, however, blastula were formed, but they did not move. When afterward placed in normal sea water movement soon appeared.

3. With three chlorids ( $\mathrm{Na}, \mathrm{K}$, and $\mathrm{Ca}$ ) "the eggs not only reached the blastula stage and swam around in the most lively way, but they reacherl the gastrula and even the pluteus stage, with the exception, however, that practically no skeleton was formed." 2 Such larvæ lived about ten days.

4. The addition of a trace of $\mathrm{Na}_{2} \mathrm{CO}_{3}$ resulted in the formation of a slieleton, but it was not quite normal. It was made normal by adding a trace of $\mathrm{MgCl}_{2}$.

1 Three early stages are characteristic of the early development of all embryos: (r) the morula, or "mulberry" stage, in which cell division gives rise to a globular mass of rounded cells, each more or less distinct, like the grapes on a bunch or the seeds of a mulberry; (2) the blastalla stage, in which the outer cells become condensed, showing a distinct outer layer, - the blastoderm; and (3) the sastrula stage, in which one side becomes pushed in (invaginated), as one would push in a hollow rubber ball with his thumb, forming a kind of mouth and stomach. A few forms never get beyond this stage, but most pass quickly through it, differentiation proceeding rapidly. In higher animals the outer layer (ectoderm) gives rise to the skin and its appendages, the inner (endoderm) to the internal organs. Among sea urchins, which were here under experiment, the next stage is known as the pluteus, - the stage of free-moving larvæ. It was this stage the experimenter desired to produce.

2 Loeb, Studies in General Physiology, Part II, pp. 585-586. 
5. All experiments indicated that it is impossible to secure more than the beginning of segmentation from an unfertilized egg without raising the concentration of the sea water.

6. But for this purpose $\mathrm{MgCl}_{2}$ was peculiarly effective and normal plutei (free-swimming larva) developed from unfertilized eggs lying in normal sea water after having lain for two hours in a solution of $\mathrm{MgCl}_{2}$ of proper strength. ${ }^{1}$

7. These effects seemed to be due to the increased concentration in the sea water, bringing about increased osmotic pressure and resulting in a loss of water on the part of the egg: This loss of water seems to be the active cause of rapid segmentation, and a variety of substances were discovered which were able to bring it about.

8. The principal difference noticeable in the plutei was that those developed from fertilized eggs swam freely at the top of the water, while those developed from unfertilized eggs "were all at the bottom of the dish and unable to rise."

9. Experiments upon the marine annelid Chrtopterus ${ }^{2}$ indicated that artificial development is easier than with the sea urchin, but that it is achieved by a different solution. In the words of the experimenter, "IVe may say that Chxtopterus possesses a higher degree of parthenogenetic tendency than the Arbacia [sea urchin] eggs," 3 and "if the sea water contained only a slightly greater proportion of $\mathrm{K}$, we should find that Chætopterus was normally parthenogenetic." 4

IO. If certain forms are prevented from becoming parthenogenetic by the constitution of the sea water, we may infer that those which are naturally parthenogenetic are so by the constitution of the blood or the sea water enabling the egg to develop. ${ }^{5}$

II. "The bridge between the phenomena of natural and artificial parthenogenesis is formed by those animals in which

1 Loeb, Studies in General Physiology, Part II, p. 624.

2 "Mead had already found that if 0.5 per cent $\mathrm{KCl}$ is added to sea water the unfertilized eggs of Chatopterus throw out their polar bodies, while the addition of 0.5 per cent $\mathrm{NaCl}$ produced no such effect." - Loeb, Studies in General I'hysiology, Part II, pp. 656-657.

${ }^{3}$ Loeb, Studies in General Physiology, Part II, pp. 654-655.

4 Ibid. p. 665 .

5 Ibid. p. 683 . 
physical factors decide whether or not their eggs develop parthenogenetically." 1 The consideration seems to be largely one of change in osmotic pressure, some organisms requiring increase and some decrease. Plant lice are parthenogenetic only at high temperatures and when the host plant has plenty of water. "If we lower the temperature or let the plant dry out, sexual reproduction occurs." 1 It seems to be decided that Artcmia salina living in brackish waters is parthenogenetic, while its nearest fresh-water relative, Branchipus, is not. ${ }^{2}$

I2. From the fact that the beginning of segmentation is common in unfertilized and untreated eggs of many forms, it seems to follow that the effect of fertilization or of treatment is largely to accelcrate a process which is able to begin alone but which proceeds so slowly as to be overtaken by destructive processes and the death of the egg before an embryo can form.

I3. The introduction of a small amount of a catalytic substance at the critically proper time (at maturity) seems in most cases necessary to a cell division sufficiently rapid to insure the continuation of life.

I4. The function of the spermatozoon would seem therefore to be twofold, - first, to introduce such a catalytic substance, and second, to convey hereditary material.

No student can consider these fundamental matters and fail to realize the profound effect of external influences upon internal activities, nor can he avoid the conclusion that we must revise our ideas as to the relation even of inorganic chemistry and physical forces to the processes of life. Much that we have considered as morphological and peculiarly vital is, after all, evidently due to the operations of ordinary chemical and physical laws. This does not make the facts of variability less significant, but it does show the extent to which living organisms have become accustomed to their ordinary surroundings.

1 Loeb, Studies in General Physiology, Part II, p. $68_{3}$.

2 "Janósik has found segmentation in the unfertilized eggs of mammalians." - Loeb, Studies in General Physiology, Part 11, p. 543. Loeb expresses the conviction that possibly "only the ions of the blood prevent the parthenogenetic origin of embryos in mammalians," and that a change in their blood might be followed by parthenogenetic development. 


\section{SECTION IX - EFFECT OF SALINE SOLUTION UPON DEVELOPMENT IN AQUATIC ANIMALS}

Sea water cliffers from fresh water in two particulars, salinity and density, both of which exert marked influence upon animal life and between which it is often difficult to discriminate. A goldfish plunged into sea water at first shows "violent incoördinated movements," but shortly "becomes immobile and rises to the surface by virtue of its lower specific gravity." 1

"The effect of fresh water upon marine organisms is equally striking. They go immediately to the bottom and move with difficulty. Swimming animals swim badly if at all, and small fishes have to make much exertion to rise to the surface." 1

On many marine animals, as mollusks and fish, fresh water acts as an anresthetic, the mollusks soon yielding to paralysis, the fish appearing to suffer from lack of air. "The respiratory movements become deep and rapid. . . . The tissues become swollen so that soft-bodied animals are visibly cleformed, - in fishes the eyes are forced out, the foot of gastropods sivells, the blood corpuscles swell up and burst, and muscular tissue may increase as much as six times in volume." 2

Many of these effects are clearly due to differences in pressure which may amount to many atmospheres, but it remains to separate, so far as may be, the effects of salinity from those of specific gravity.

Rather startling claims have been made from time to time as to the conicrsion of one specic's into another by altering the degree of salinity. Further investigation seems to show that in all such cases intermediate forms are known to occur, which argues that the two forms which had been recognized as distinct were not both good species; that is to say, it is a case of one species with wide variability as to certain characters, not that of two distinct and well-defined species. If, however, differences in salinity are effective in bringing about alterations in even a single character, the fact is of interest here, no matter what specific lines should be drawn by the biologist.

1 C. B. Davenport, Experimental Morphology, Part I, p. 79.

2 Ibid. pp. 79, 8 o. 
The small crustaceans Artomia salina and A. milhansinii have been recognized as distinct, the former living in brackish and the latter in still more concentrated waters, the two differing mainly in the number and length of bristles borne at the extremity of the caudal fins.

Early in the seventies Schmankewitsch published an account of the mutual conversion of each form into the other, but the facts as given by him have been greatly overstated, as frequently happens in repetition. They are sufficiently significant as first reported, and it seems well to give the original statement as recorded by Bateson, ${ }^{1}$ which is as follows:

The salt lagoon, Kuyalnik, was divided by a dam into an upper and a lower part; the waters in the latter being saturated with salt, while the waters of the upper part were less salt. By a spring flood in the year 1871 the waters of the upper part of the lake swept over the dam and reduced the density of the lower waters to $8^{\circ}$ Baumé (= about sp. g. 1.05I), and in this water great numbers of 1 . salina then appeared, presumably having been washed in from the upper part of the lake or from the neighboring salt pools. After this the dam was made good and the waters of the lower lake, by evaporation, became more and more concentrated, being, in the summer of $1872, \mathrm{I}^{\circ} \mathrm{B}$ (about sp. g. $\mathrm{I} .103$ ); in $\mathrm{I} 873, \mathrm{I} 8^{\circ} \mathrm{B}$ (about sp. $\mathrm{g}$. 1.135); in August, $1874,23.5^{\circ} \mathrm{B}$ (about sp. g. I.I 77), and later in that year the salt began to crystallize out. In $187 \mathrm{I}$ the Artemia [as first carried over] had caudal fins of good size, bearing eight to twelve, rarely fifteen bristles, but with the progressive concentration of the water the generations of Artemia progressively degenerated, until at the end of the summer of 1874 a large part of them had no caudal fins, thus presenting the character of $A$. milhausenii. - Frscher and Milne-Edwards.

\section{Bateson adds :}

A similar series was produced experimentally by gradual concentration of water, leading to the extreme form resembling - 1 . milhunsenii. It was found also that if the animals without caudal fins were kept in water which was gradually diluted, after some weeks a pair of conical prominences, each bearing a single bristle, appeared at the end of the abdomen.

The experimenter also relates that by breeding salina in still more diluted water he attained a form resembling Schaffer's genus Branchipus. But the principal difference between the genera is that in Artemia the last segment is about twice as

1 Bateson, Materials, etc., p. 96. 
long as each of the others, while in Branchipus it is divided. It is extremely significant that this division should be produced in Artemia by culture in comparatively fresh water, but the fact is no warrant for the assertion that one genus can be converted into another by altering the environment. It rather casts doubt upon the wisclom of a classification which establishes generic clistinctions upon differences so slight and so easily brought about.

The same experimenter studied species of the genus Daphnia, and found "in their case also considerable structural and physiological changes, the fresh- and salt-water forms differing, in his opinion, by characters usually held to be specific." 1

Bateson studied the common cockle, a mollusk, everywhere present in the Aral Sea and its outlying waters of different degrees of salinity. One of the lakes (Shumish Kul) on its western shore exhibited no less than seven distinct terraces, held to represent successive stages of the water levels during its long period of drying up, with corresponding increase in salinity. The most noticeable differences in the shells taken from these successive terraces, and presumably due to increasing salinity, are outlined as follows : ${ }^{2}$

1. A diminution in the thickness of the shells, first apparent in the third terrace. In the seventh terrace this change was so marked that the shells were almost horny, and their weight was not a third of that of the shells from the first two terraces.

2. Diminution of the size of the beak [with the lowering of the level].

3. High coloration. [The author does not state which way the changes ran, whether up or down the terraces, but he remarks that all the shells of a given terrace were "very nearly alike in texture, thickness, and degree of coloration." ]

4. Gronves between the ribs appearing on the inside of the shell as ridges with rectangular faces.

5. A great diminution in absolute size of the shells on the lowest terrace.

6. Alteration in proportion of length to breadth, ranging from I to 0.80 in the shells of the first terrace to I to 0.725 in those of the serenth and I to 0.66 on the shores of neighboring

1 Vernon, Variation in Animals and Plants, p. 275. $\quad 2$ Ibid. pp. 275, 276. 
lakes. In view of these facts Bateson remarks, as quoted by Vernon, "It seems almost certain that these conditions are in some way the cause of the variations."

Biological literature is full of similar examples of the characteristic effects of varying degrees of salinity. The limits of space forbid the further pursuit of a subject which might be extended almost indefinitely. It may be sufficient to say that the specific influence of salinity upon certain characters is, beyond a doubt, well established.

\section{SECTION $\mathrm{X}$-INILUENCE OF USE ANI) DISUSE UPON DEVELOPMENT}

No fact is better or more generally known than that use stimulates and disuse dwarfs the development of many organs. To say that development is in proportion to use would doubtless be true, roughly speaking, of certain parts, as the muscular system, secreting glands, etc. It certainly would not be true of many others, as hair, feathers, bony skeleton, etc., which develop independently of use, and some of which, as hair and feathers, involve no activity in the sense in which the term is here understood.

This discussion should be limited to the distinctively active parts, and the infuence of exercise or the lack of exercise upon their development. Of these parts it may fairly be said that perfect development is dependent upon, if not proportional to, the degree of their use, especially during the earlier stages of development.

The classic illustration from Darwin, showing the leg bones of the tame duck and the wing bones of the wild duck to be relatively heavier; the arm of the artisan and the body of the athlete; the training of the track horse; the marvelous coördination of complicated nervous impulse and muscular response in the violinist and the pianist, - all these and a multitude of similar facts teach clearly that individual development of usable parts depends very much upon their early and continuous exercise.

1 Vernon, Variation in Animals and Plants, p. 277. 
Upper limits of development. In what sense is development conditioned upon use? Does use simply enable the part to attain its nomal and proper deaclopment, to which it is entitled under the laws of heredity, or does it stimulate development beyond the nomal?

Some biologists at once assume the latter to be impossible and that any unusual appearance is a case of atavism. It is true that in times long past there may have existed ducks that walked and others that flew more and better than those which Darwin examined; but when did nature produce a running or a trotting horse as good as the one of to-day? To what remote ancestor do our violinists and our pianists owe their skill, and what was the instrument on which they acquired it ?

The accompanying cut is a facsimile of a properly attested letter written with the fect by a young woman twenty-three years old who lost both arms at the age of ten. Among her other acplishments she numbers cutting, sewing (threading her own needle), drawing, sweeping, and a great variety of housework. ${ }^{1}$

Here is a case of putting parts to an entirely new use, demanding a nicety of adjustment that was never acquired even in one out of a million of the ancestors. Could there be better evidence of the fact that few individuals ever use, and therefore few ever develop, more than a fraction of the capacities born in them; that the possibilities of life are seldom realized, and that never are all faculties developed to their utmost in any single individual ?

All this is clear but it is not so easy to determine where to draw a line and say, "All development below this is due to inheritance and all above to use." The truth would seem to be that development depends upon both inherent tendencies and external conditions affording opportunities for their exercise, and that the marimum of derclopment is reachad only awhen both are at their optimum. There is much force in the word "optimum." Too much exercise, too much food, too much temperature, or too much of any of the conditions of life is as unfortunate as too little.

I The author saw one man who wrote with his feet, but they were attached directly to the body, with no legs whatever. 


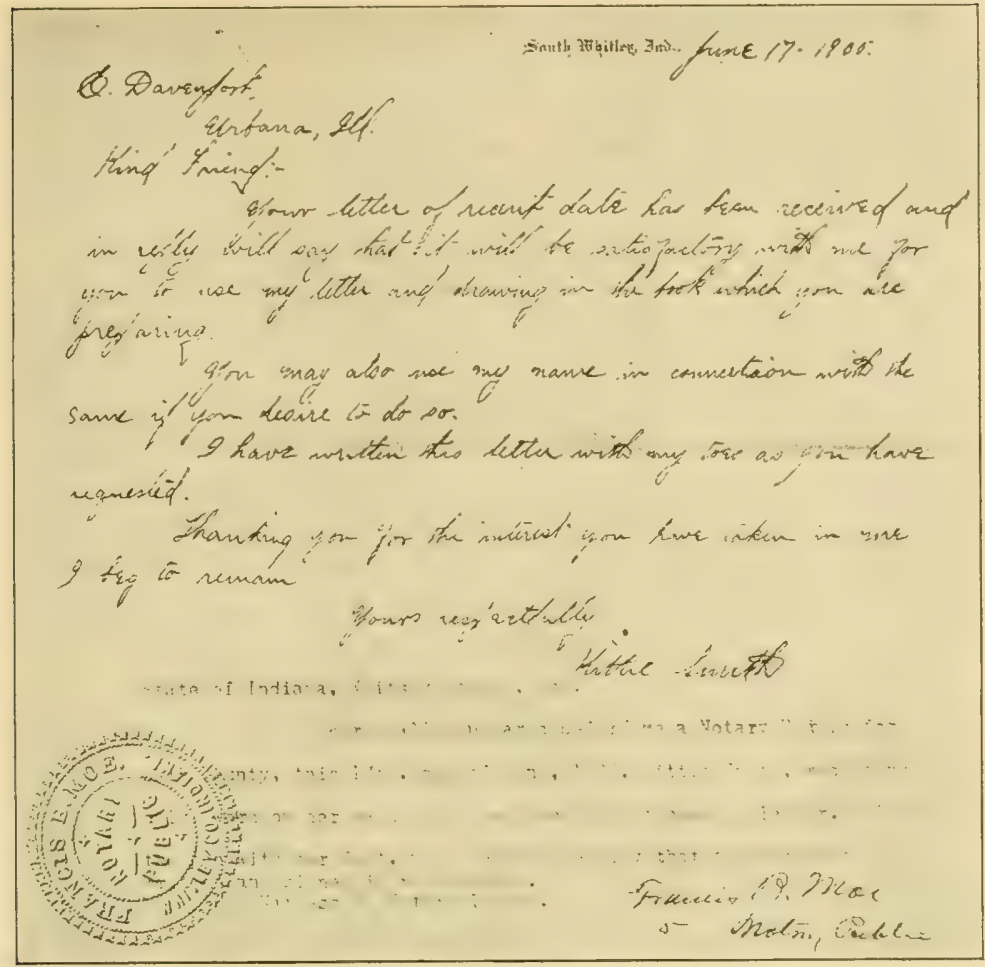

Fig. 30 illustrates the ability of a highly developed part to perform an extremely unusual service. The above letter was written with the feet instead of with the hands

It is as futile to attempt to decide whether internal or external circumstances are more helpful in development as it is to attempt to say whether food or heat is more essential to life. Both are absolutely necessary, and it suffices present purposes if the student understands that external conditions, even to the matter of exercise, are fundamentally essential to full development, and that the "limiting element" may be found external to the organism as well as internal. We shall not be able to assert how much is due to each separately, nor can we determine the coefficient to be assigned to use and to clisuse, but we are safe in resting assured that for many parts deiclopment is fairly proportional to excreise, at least within the limits of inheritance. 
Disappearing organs. The disappearance of legs from snakes and from whales, the lessening of the fore arms of the kangaroo, and of the wing of certain birds, the loss of toes from many mammals, and rudimentary conditions generally, argue for the gradual disappearance of a part that is no longer useful and no longer used.

The first stages of this disappearance can be understood as arising through the cessation of selection and the resulting panmixia, ${ }^{1}$ by which inheritance is from the grneral arerage of the whole race, instead of from a selected lot, as heretofore, resulting necessarily in degeneration as compared with a standard sustained by rigid selection. Later stages may be explained by "reversal of selection," when the hitherto useful organ has not only become useless but in some way detrimental. This would account for still further degeneracy, and is as far as the principle of use and disuse applies. ${ }^{2}$

Space cannot be taken here for the enumeration of instances showing the effects of use and disuse. They may be found on every hand, and they abound in books on general evolution. ${ }^{3}$

Hypertrophy. Unusual enlargement of a part is technically known as hypertrophy. Two kinds are recognized, - functional hypertrophy, when a part is enlarged through use; and compensating hypertrophy, which takes place when, one organ being removed or becoming functionless, another enlarges. ${ }^{4}$

The voluntary muscles of the hand and arm grow large through heavy use, but the muscles of the fingers of a musician do not undergo hypertrophy, though the total amount of work may be very large. It is only when muscular work is done against great resistance that enlargement of the muscles takes place. ${ }^{5}$

1 A term coined by Weismann and denoting "universal crossing," literally "all mixed." See Weismann, Essay on Heredity, I, 9I, I4I ; also Romanes, Darwin and After Darwin, Part II, pp. 29I-306.

2 For fuller discussion of disappearing organs, see next chapter.

${ }^{3}$ Darwin, Origin of Species (sixth edition), pp. IOS-I12 [D. Appleton \& Company]; also Animals and Plants under Domestication (second edition) [D. Appleton \& Company] : in general, II, 285-293, 345, 346; in rabbits, I, I29I34; in ducks, I, 299-301.

4 Morgan, Regeneration, pp. Ir 5-118.

5 Of course the effects of use are not limited to increase of size; they are fully as noticeable in nicety of adjustment. 
When one kidney is removed from either man, rabbit, or dog, the other becomes enlarged and the total amount of urea excreted is unchanged, and this is true even if the removal is made at maturity, after the parts have reached their probable full development.

This is allied to the fact that the full amount of urea is excreted at once upon the removal of one kidney, proving that its fellow is able to increase its labor even before hypertrophy, and showing that under normal conditions the kidney is not fully worked. There is first an increased flow of blood, then increased excretion, then increased size. The same is true of the salivary glands, the mammx of the female, the testes of the male, and quite likely of paired organs generally.

When the spleen is removed the "lymphatic glands of other parts of the body become enlarged." 1 Another kind of compensating development is the well-known increase of one faculty when another is extinct, as the hearing and the touch of the blind. In this instance, as in learning to write with the feet, the part is not only developed and trained to its utmost, but the undivided attention is fixed upon the matter in hand.

The student who bestows careful study upon the relation of the individual to his environment will arrive at three definite conclusions :

I. The impulse to development and its chief directive forces are within.

2. But the possibilities of that development, in kind as well as in degree, lie very largely in surrounding conditions and entirely external to the organism.

3. These surrounding conditions, therefore, while not logically causes of adriation, since they cannot bring about a development whose tendency does not already exist, are yet the limiting elements to all development, and many of these conditions are chemical and physical forces able to exert strongly directive influences upon growth capable of differentiation in more than one direction. On this point see also the chapter on "Relative Stability and Instability of Living Matter." 


\section{VARIATION IN TYPE:}

The student must distinguish clearly between the influence of external comditions upon an occasional individual and their celfert upon the type of the race. There are three possible ways in which the enviromment may result in a modification of type: (1) by affecting all inclividuals in the same way; (2) by selection; (3) by the inheritance of the modifications due to conditions of life. It remains to examine each somewhat carefully.

All individuals affected in the same manner, thus influencing the type directly. The molifying effects of the conditions of life hate been quite fully noted. If but few individuals are affected, it is manifest that the type will not be seriously changed; but if, on the oflere hand, crery inclividual is affected, and in the sime waty, then the type is to that extent due to the conditions of life.

For example, size is directly influenced by the food supply, and increase of size in a race, contemporaneous with a better fool supply, may farly be attributed to the farorable influence of full fecd acting upon all individuals alike.

Size is also inherited, so that the limits of development are due (1) two inflences acting together, - inheritance and food supply. It is often exceelingly difficult to determine how much for attribute to the one influence and how much to the other.

What is true of size in this respect is true of every other character that is in the slightest degree dependent upon enviromment for its development. Accordingly much uncertainty prevails as to the comparative influence of inheritance and enviromment. The racial lype can be determined only by the study of the individuals constituting the mature population; but their development is the result of two sets of causes, - the one of heredity, the other of enviromment, - both contributing to the same effect and both continuous through life.

Under the okl view every individual was regarded as the result not only of what was born into it but also of the direct influence of its enviromment. Inclividuals constitute the type, and so it is that when the conditions of life affect all individuals 
alike they necessarily influence the type of the race, but to what extent cannot be told without some method of subtracting the normal results of simple inheritance. No method of doing this has ever been discovered, and much uncertainty has always prevailed as to what proportion of existing types should be credited to the conditions of life.

The puzzling problem is greatly simplified if we enlarge the meaning of the word "inheritance" to cover not only the lines of possible development but the full capacity as well. In this view of the case the individual and the race are understood as possessing hereditary capacities for development far beyond that for which opportunities are likely ever to be afforded by environment. This largely removes the conditions of life from among the fundamental coruses of variability and relegates them to the realm of passive and permissive, though necessary, requisites for the full display of hereditary power. It tends strongly, too, to regard most individuals as something less than would be indicated by their hereditary possibilities, and to consider environment in general as the limiting, not the stimulating, element in development. With this view the writer is inclined to agree, though it necessarily reduces the extent of direct influence of the enviromment. The student is never to forget that, whatever may be the influence of surrounding conditions upon one form of life, the same influences affect other species differently, thus showing that their characteristic effect is conditioned upon the power of the organism to react, - a condition that is eminently internal and inheritable.

Selection as a cause of variation in type. Many inclividuals are unable to meet the conditions of life with which they find themselves surrounded, and in the attempt become extinct. This is selection, and it is manifest that the prevailing type of the race as it exists at any moment, being made up of selected inclividuals, is something different from that which was born into the race. It is also manifest that only a limited portion of adults will reproduce, so that selection through the external conditions of life exerts a strong influence upon type.

Selection is therefore one of the most powerful - if not the most powerful-influences known in the modification of species, 
and it may be fairly spoken of as a primary cause of variation in type. This does not make it, however, like temperature or food supply, a fundamental cause of variation in living matter. There is no basis for selection until differences have appeared from other causes. When the selection is made, based on these differences, it is certain to affect the type, and it is therefore a cause of type variation; but it was no cause of the original differences on which its action was predicated, and it is in no sense an original cause of variability. The confusion of mind which causes selection to be regarded as a cause of variation has arisen from a failure to distinguish clearly between the inclividual and the type, - between the material on which we can work through selection on the one hand, and the finished product on the other.

Variation in type through the inheritance of modifications due to environment. Whether individual modifications due to environment (acquired characters) are inherited will be discussed in a following chapter. It is the purpose here only to show the nature of the effect of such inheritance in case it does occur.

If the modifications due to external influences should perchance be inherited, even in the slightest degree, then the effects of modification would, like those of selection, become cumulative, and the type would the more rapidly conform to the environment and the more rapidly establish a "fit" with the conditions of life.

It is evident that this is a difficult field. Merely through the exigrencies of maintenance those characters will develop best that are most favored by the conditions of life, thus bringing about a kind of mass response to the exactions of the environment. Again, by the principle of selection, only those in fair accord with the environment will live, and this brings about a still closer fit. If, now, modifications due to enviromment were fully and completely inherited, the adjustment to constant conditions would speedily become so exact and complete as to leave no room for selection.

Few biologists are bold enough to claim this extreme degree of inheritance of modifications due to environment. Many deny it in toto. That neither extreme is right is comparatively easy of as good proof as we are generally able to bring in affairs biological, but where between lies the truth it is most difficult to 
determine. The first two causes mentioned, both of which are certainly at work, sufficiently explain most phenomena, but whether there is an additional fraction due to inheritance, it is most important for us to know.

It can be but a fraction at best, and being in exact line with selection and with the direct action of the conditions of life, it is exceedingly difficult of identification and of separation from the larger causes. If inheritance is to be included, however minute the fraction in a single generation, its effect is cumulative, and in time it would become the most powerful and irresistible of all causes influencing type. Its further consideration must be cleferred to a later chapter, but the result of its activity, if it has any, is in its influence upon type.

Summary. Though the impulse to development lies within, the opportunities for that development and the forces controlling subsequent activities are to be found in the conditions of life surrounding the organism.

These are generally insufficient to afford full development of all the possibilities with which the organism is endowed by heredity. Accordingly the individual does not express in its own personality the full extent of its heritage, and individuals generally are to be regarded as having realized something less, rather than something more, than their birthright.

Living matter, like non-living matter, sustains definite relations to external materials and forces, and the chemical elements of which it is composed are not freed from their ordinary reactions to other elements or to chemical or physical energies. And so it is that living matter is subject to both constructive and destructive combinations, and to definite reactions toward gravity, light, temperature, electricity, and to chemical and physical forces generally.

Herein lies the modifying effect of surrounding conditions upon the development and activities of living matter. Endowed from within with definite propertics and capacities, their realization depends very much upon outside materials and forces which provide the conditions under which the definitely organized matter is compelled to discharge its activities, and we do well to become somewhat familiar with the nature and extent of the limitations thus imposed. 


\section{ADDITIONAL REFERENCES}

Certain Habits of Animals traced to the Arrangement of THEIr Harr. By Walter Kidd. Proceedings of the Zoölogical Society of London, II, 145-158.

EFFect of Climate on Sugar Content of Beets. Experiment Station Record, XIII, 736.

FHFECT OF DARKNESS UPON VEgetaton. Experiment Station Record, $\mathrm{XIII}, 651$.

Effect of Electricity upon Growth of Plants. Experiment Station Record, XIV, 346, 352, 548; (Acetylene Gas Light), XIV, $42 \mathrm{I}, 437$; XVI, 137.

EFfect of Humiti on Growtr. Experiment Station Record, XII, 1014.

Effect of Presence or Absence of Certain Salts upon the Composition of THE CROP. Experiment Station Record, XIV, $561-563$.

EFFECT OF STARVATION ON PLANT GROWTH (first, shortage of nitrogen ; second, shortage of potassium). Experiment Station Record, XIV, I $19,347$.

Effect of Water Content upon Development of Wheat, Oats, Clover, Etc. Experiment Station Record, XIII, I25-I26, 44I-63I. Experimental Zoölogy. By J. H. Morgan. Chapters II and III, pp. $12-4 \mathrm{I}, 1$

Injurious Effect of Freezing on Development of the EMbryo IN THE EgG OF THE HEN. Experiment Station Record, XI, 577.

VARIATION OF ANTHRAX BACILIUS WHEN BRED UNDER DIFFERENT Conditions. Experiment Station Record, XIV, 293, 916.

VAriation in Nitrogen Content of Wheat. Experiment Station Record, XIII, 45 I.

VARiation in Tubercle Bacilli when found in Different EnviRONMENT. Experiment Station Record, XIV, II2I; XV, I88.

VARiations CaUsed by Fertilization. Experiment Station Record, $\mathrm{XIV}, 3+7$.

1 This excellent volume was not yet off the press when this copy was prepared. It is especially recommended. 


\section{CHAPTER X}

\section{RELATIVE STABILITY AND INSTABILITY OF LIVING MATTER}

In order to guide the student of breeding in forming his conceptions as to what may and what may not be accomplished in the way of modifying the form or function of domesticated animals and plants, everything is valuable which throws light upon the degree of froctuess in living matter; that is to say, in the relations that happen to have become established between the essential characters of existing species.

When the student for a time bestows careful study upon variation and comes to realize how radical are some of the departures from type and how sweeping are some of the cleviations from the normal, he is led to feel instinctively that living matter exists in a state of extreme instability as regards both form and function, and that almost anything is likely to happen.

When, however, he considers that, through it all, distinct types are preserved; and when he notes the singular persistence of certain characters through all the vicissitudes of time and evolution, reappearing generation after generation when they were supposed to have been long lost, and in many cases lingering after their usefulness is past and associated characters have been blotted out, - when he considers all this, the careful student will realize that stability and instability are relative terms, and he will begin seriously to inquire into the degree of stability of the various plans upon which matter has been organized and vitalized. It is therefore profitable to inquire somewhat fully into the relative stability or instability of those compounds that are endowed with life, and into their relative ability to maintain their integrity and clischarge their functions under conditions both normal and abnormal. The utility of this inquiry rests in the light it may throw upon the extent to which characters that have become typical are fixed and unchangeable, and the extent to which they may be modified. 


\section{SECTION I - EVIDENCE FROM STABILITY OF TYPE}

Although no two individuals are alike, and although a given character differs greatly in different members of the race, yet there is a specific type that is always and everywhere present; though the elements are exceedingly variable, yet the resultant composite is remarkably constant as compared with other types.

In other words, when we compare horses with horses we are impressed with the fact of variability, but when we compare horses with cattle, or even with asses, then we are led to marvel at the fixity and persistence of type. In all its variations, the horse is still clearly a horse. IVide and profound as is variability, it is yet well within limits, and certain types continue with singular persistence.

The Hubbard squash and the Morgan horse are good examples of persistence of type. With all the variability of the $\mathrm{Cu}$ curbitacex, the Hubbard squash persists, distinct in type and quality. It mixes freely with other types, but its characters are evident even then, and it possesses a singular ability to free itself from such admixtures and return again to the original.

The Morgan horse is a breed established by a single animal, and yet, a hundred years after the death of Justin Morgan, when the per cent of his blood is of necessity slight, the Morgan characters still stand out clearly, constituting a type almost as distinct as that of any existing breed. This is the solitary known instance of the founding of a breed by a single ancestor. It illustrates in a peculiar way the occasional extreme persistence of a type once formed, and is in marked contrast to the readiness with which other types break up and disappear.

The old-time persistence of the sloping rump in the Berkshire, of the narrow chest in the Poland-China, of lack of depth behind in the pony-built Hereford, of deficient crops in the Shorthorn, - these and similar defects that might be mentioned illustrate the strength with which certain characters continue, even in the face of the most powerful opposition, and argue strongly for stability of type.

It is also a general fact that species hold their types with essential success under a great variety of conditions, both 
favorable and adverse, yielding but slowly, and sometimes not at all, to modifying influences, and often suffering extinction when a slight modification would have resulted in preservation. Thus the oaks and the tulip tree have come down to us from remote ages practically unchanged, and the elephant is with us yet, substantially the same as he has been for probably thousands of years.

And yet there is constant variation, in these as in more flexible species. They have not freed themselves from variability, even though the species as a whole has come to be remarkably constant. Indeed, the more the question is studied, the more evident it becomes that a great deal that passes for variability is merely individual fluctuation around a practically stationary point, not necessarily involving actual change in type. That is to say, few indiriduals exactly reproduce the type of the species, however fixed it may have become; most of them depart slightly this way or that, making a great show of variation, so that we seem to be in the midst of bewildering differences, even though the type is practically unchanged. In cases of this sort deviations represent not so much departures from type as individual approximations to a general average.

Here is ground very deceptive to the breeder. Generally speaking, variation denotes flexibility of organization, and therefore possibility of improvement, but the breeder must not assume that great variation clenotes large possibility for improvement. Fundamentally it denotes quite as much an inherent failure to assume a distinct type; and often a lesser deviation, representing a true departure from type, affords a far more favorable basis for improvement than do those deviations that after all are merely fluctuations about a center that has a strong tendency to remain fixed. ${ }^{1}$

1 Pearson believes that the extent of variability cannot be reduced more than about II per cent, however rigid the selection. He does not claim that the type cannot be shifted more than that amount, but that, however much it may be shifted, there is still variability about the new center, and that this variability is at least $S_{9}$ per cent of the original variability of the race. See Pearson, Grammar of Science, pp. $4 S_{I-4} S_{5}$; also chapter on "Selection." This subject will be fully studied in a succeeding chapter entitled "Type and Variability." 


\section{SECTION II - EVIDENCE FROM MUTABILITY OF SPECIES}

Species do not, however, always remain unchanged. On the contrary; they frequently exhibit a progressive development truly marvelous. Horses, for example, are traceable backward by easy stages and well-clefined connecting links to a time far beyond the appearance of man upon the earth, the line ending

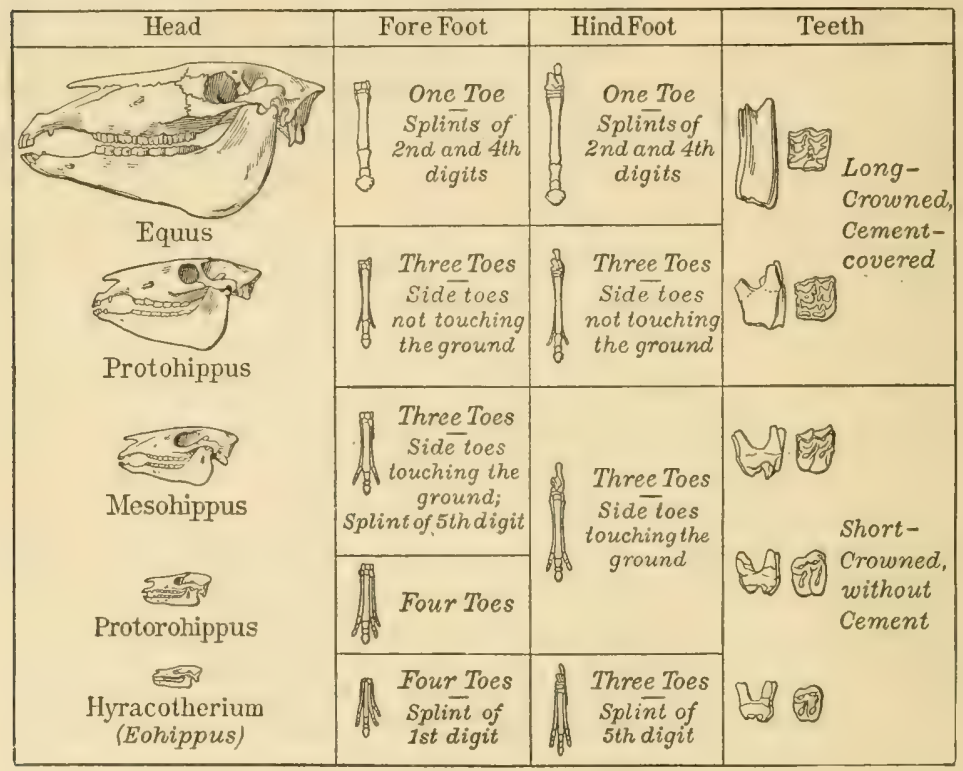

FIG. 3I. Comparative drawings of skulls, feet, and teeth of prehistoric horses, showing evolutionary development. Reproduced by permission from Origin and History of the Horse by H. F. Osborn

in one of the carliest mammals, a little five-toed creature not much larger than the domestic cat.

No less than twelve stages in this evolution are well known, and represented by specimens more or less complete: ${ }^{1}$

1 William D. Matthew, of the American Museum of Natural History, article "Horse, the Evolution of," in Encyclopxdia Americana. This is one of the best and one of the newest accounts of the development of the horse, and is chosen because of its accessibility and reliability. The outline given, while not marked by quotations, is practically an abstract of the reference. 
I. Hyracothcrimm. Skull only. Found in London clay of the Lower Eocene (earliest mammals). Specimen in British Museum.

2. Eolitpus. Much better known, coming from the Lower Eocene of Wyoming and New Mexico. Teeth like the former; four toes on the front foot, with a splint of the fifth; three toes behind, with a splint of another, showing that considerable departure had already taken place from its evident five-toed ancestry. Height of animal i 2 to 16 inches.

3. Protorohippus (Wyoming, I880). Four complete toes in front and three behind; no splints; skeleton of the size of a small dog. Described by Cope as "the four-toed horse."

4. Orohippus. Only parts of jaws and teeth, but these show some advance. Specimen at Yale University.

5. Epihippus (Upper Eocene, New World). Only incomplete specimens found, but much time has elapsed and considerable development is noted, especially in the teeth. The toes are still four and three, but the central toe is "becoming much larger than the side toes."

Collateral branches of the same period in the Old World (Paleotherium and Paloplotherium) had three toes of nearly equal size on each foot. They were very abundant at this time but seem to have become extinct, - a fate that overtook most of the branches of this fertile and progressive stem.

6. Mcsolitpus (White River Formation). Three toes on each foot, the middle much the largest, the side toes bearing little weight. By this time the animal ranges in size from the coyote to the sheep, and the molar teeth are well developed. All parts of the skeleton known. Fifth toe represented by a splint.

7. Anchithcrium (Lower Miocene). Found both in Europe and in America. Much like its predecessor, but larger, with better-developed teeth. May be one of the "side branches" rather than in the direct line of the modern horse.

8. Hypohippus and Parahifpus. A complete skeleton of Hypohippus was found, I90I, by the Whitney Expedition near Pawnee Butte, Colorado. In the forefoot small rudiments still represent the first and fifth toes, but the splints are gone; the second and fourth digits still touch the ground, though 


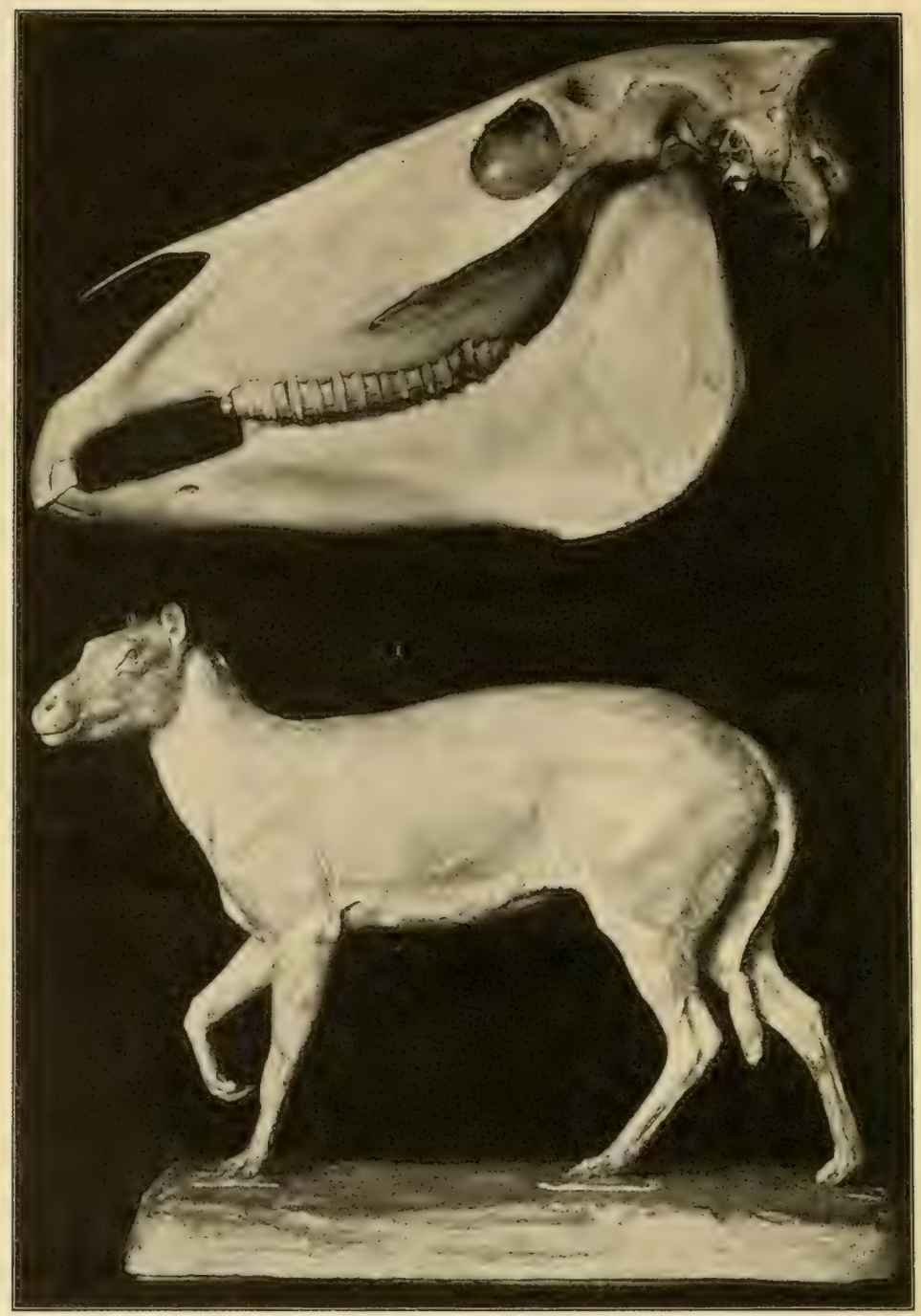

FIG. 32. Progressive evolution in the horse : the lower figure is a full-sized model of the Eohippus in comparison with the skull of the modern horse, showing that the skull of the latter horse is larger than the entire body of its ancestor. - From specimen in American Museum of Natural History, New York. Courtesy of Director H. F. Osborn 
lightly. The animal was of the size of a Shetland pony, but is regarded as being "off the direct line of descent." See Fig. 33. The companion form, Parahippus, is regarded as nearer the line, having better teeth and smaller side toes.

9 and Io. Protohippus and Pliohippus (Middle and Upper Miocene). Teeth much improved as grinders, - the valleys being filled with cement, - all showing the appearance of harder vegetation. The side toes (II and IV) are still complete, but do not touch the ground. In some species of Pliohippus they have almost disappeared.

II. Hipparion (Pliocene). Much like Protohippus; but larger, with more complicated teeth. Found both in Europe and in America, but is probably one of the "side branches."

12. Equus (Pleistocene and Recent). The modern horse, in which digits $\mathrm{I}$ and $\mathrm{V}$ have entirely disappeared and II and IV are represented by splints. This single remaining branch of the horse family (including the asses) has developed one of the most specialized of animals. It has left behind many unsuccessful relatives, representing departures that proved either unprofitable or unfortunate and coming thus to a more or less

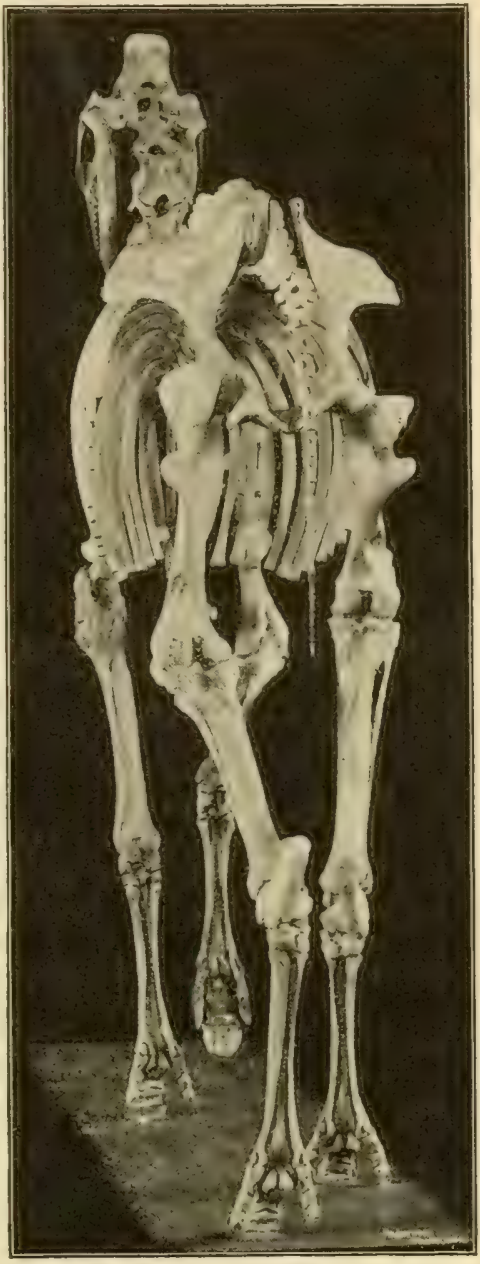

FIG. 33. Three-toed ancestor of the horse,-the Hypohippus : a complete skeleton found in the Middle Miocene, Colorado, showing the second and fourth toes touching the ground lightly. - From specimens in the American Museum of Natural History, New York. Courtesy of Director H. F. Osborn 
abrupt end. The modern horse, however, after his long and tortuous evolution, seems destined for a prolonged and notable existence. During the progressive changes in the feet the leg has been greatly lengthened, the joints modified from the loose ball and socket to the firmer hinge joint, and the teeth have become exceedingly serviceable. It is a noticeable fact that the power of a species to withstand the vicissitudes of extreme lapses of time depends very largely upon the ability of its feet, its legs, and its teeth to undergo modification. It has been said that the elephant has succeeded in maintaining himself to the present in spite of his feet, and by virtue of his excellent teeth and his remarkable proboscis.

It is noticeable that the larger part of this evolutionary history of the horse has been worked out from specimens found in western America, ${ }^{1}$ but no one believes that the modern horse is an American animal. This evolution seems to have proceeded upon substantially parallel lines in the eastern and the western continents, which were, during its progress, united by a broad strip of land in the region of Alaska; but something seems to have happened to the American branch, and it is believed that we are indebted to the European and Asiatic branch for the horse of the present.

Indeed, South America is represented by a fossil form (Hippidium) whose feet resembled Equus, except that they were short and stout. Its teeth resembled those of Pliohippus (Museo Nacional, Buenos Ayres). This form had evidently advanced nearly to that of the present, but perished in the general disaster, whatever it was, that overtook the American horse, for it left no descendants that persisted until historic times.

Causes of the evolution of the horse. As is remarked by Matthew, "the evolution of the horse, adapting it to live on the dry plains, probably went hand in hand with the evolution of the plains themselves." At the commencement of mammalian

1 Our knowledge of the evolution of the horse is largely due to the indefatigable labors of Professor IIenry F. Osborn, Director of the American Museum of Natural History, and to the magnificent generosity of the late William C. Whitney, through which extensive explorations and significant discoveries were made in Wyoming and other regions of western America. The student of this subject will eagerly await Professor Osborn's forthcoming full report. 


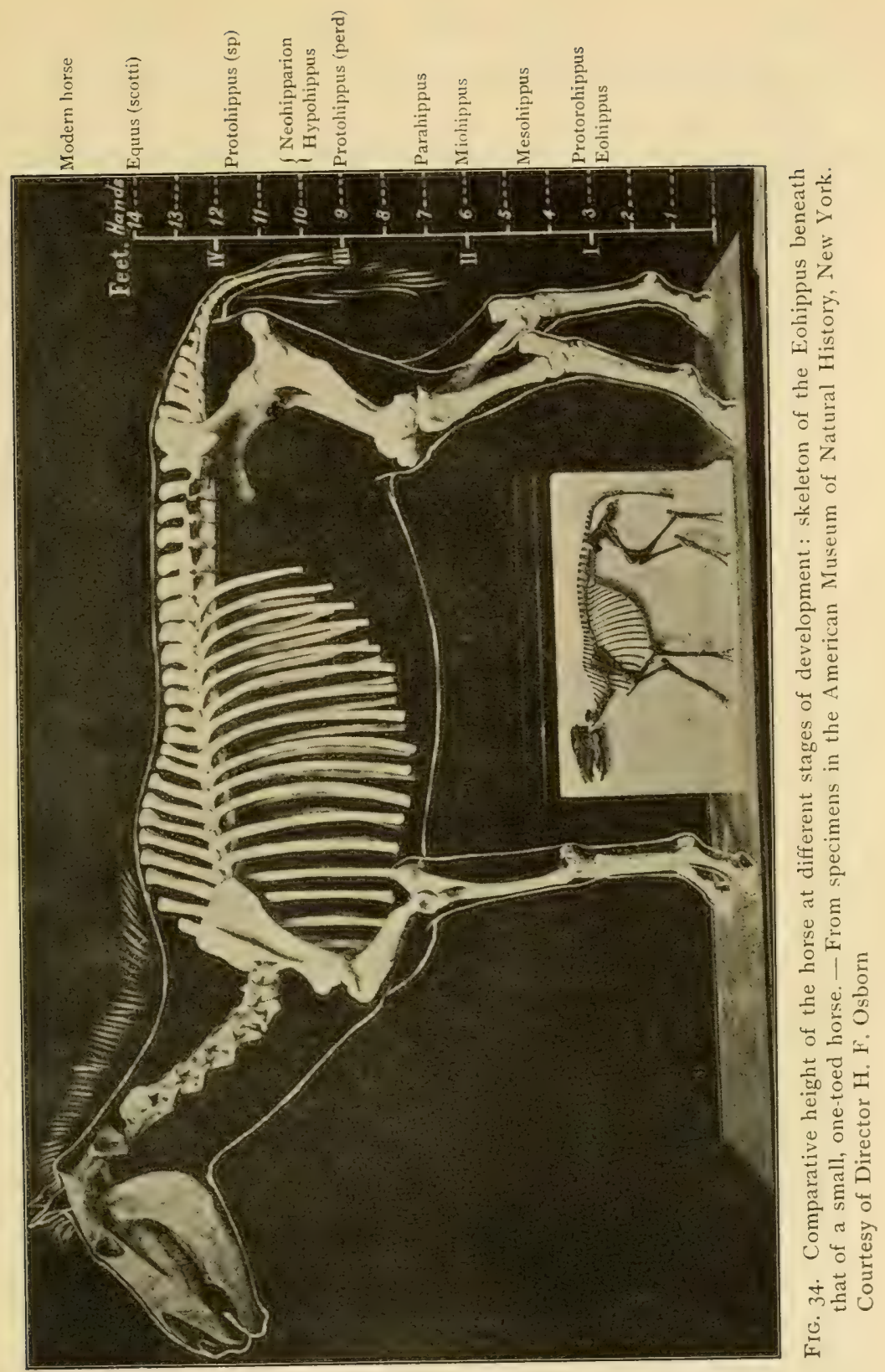


life the Mississippi valley was just emerging from the Gulf of Mexico, and the plains of westem Europe and Asia were low and wet. The climate was moist and tropical, stimulating dense and luxuriant growth of giant vegetation even as far north as Greenland. With the Tertiary came a general elevation, ushering in a comparatively cold, dry climate, favorable to grasses and the harder vegetation generally. With this came grassy plains and the evolution of races with good teeth and excellent feet and legs, fitting them to a life in the open.

With these profound changes in nature other forms underwent a development similar to that of the ancestors and other relatives of the horse. Many of these, as our cattle, sheep, swine, etc., developed a two-toed foot, and some, as the rhinoceros, stopped at the three-toed stage, but none of them became so highly specialized as the horse.

Here was a great line of descent, continuing almost for ages, and terminating in many highly specialized species that are still flexible. But it gave rise on the way down (or up) to many known, and doubtless to many unknown, branches that became extinct through some general disaster, or, more likely, because of their inherent inability to develop all the characteristics necessary to meet changing conditions. For example, as the teeth developed into molars fitted for grinding the ever-hardening forage, some species secreted cement in the valleys thus supporting the hard and grinding ridges; others did not, and it is significant that in the latter case no species endured. ${ }^{1}$ The elephant alone, of his kind, has persisted to the present, and if this is because of his teeth, and in spite of body and feet, which are ill adapted to modern conditions, it serves to show on how slender a thread the life of a species often hangs.

Present existing land species are to be regarded as representing lines of descent naturally endowed with an unusually high degree of flexibility; all the more stable and less adaptable forms having perished off the earth in the long struggle to keep up with the

1 It is worthy of remark that the central plains of South America seem to have developed a horse-like animal (Litoptirnct), losing its lateral toes and developing the hinge joint and lengthened limb; but it never developed cement in its grinders, which remained inferior, and we are not surprised that its line became extinct. 
evolution of the world as a whole. Existing species therefore represent the choicest material of the organic world. Having arrived at a high state of differentiation, they have doubtless lost something of the flexibility that marked their early and more generalized forms, yet they are to be regarded as " highly selected material," ready to the breeder's hand for still greater adaptation, not only to their own needs but to those of man.

Even when dealing with specially flexible forms, the breeder is never to forget that they are constantly giving rise to branches that are incapable of adaptation. Unhappy is the breeder who devotes his life to a branch of this kind, whether it be among horses, cattle, or any of the more slowly multiplying forms, animal or plant; for no amount of apparent variability will make up for inherent defects, nor will it, seemingly, avert the eril day of extinction.

\section{SECTION III - EVIDENCE FROM REVERSION AND ATAVISM 1}

The sudden reappearance of a long-lost character serves to remind us that combinations once effected tend strongly to return. The English breeds of cattle are supposed to have descended from the ancient wild white cattle that roamed freely over the island until the year I 200 or later, when private ownership interfered. With the inclosing of the larger estates as hunting parks, herds of these cattle were included with other game animals, and for over six hundred years they have lingered in this semi-wild condition at Chillingham, Chartley, and other parks.

${ }^{1}$ It is important that the student observe the modern distinction between "reversion" and "atavism." They both refer to the reappearance of characters once typical but now extinct. "Reversion" is the term to use when the character belonged to a near-by ancestor clearly of the same species but sereral generations removed, while "atavism " is used to denote the appearance of characters belonging to exceedingly remote ancestors, perhaps even of different species. An example of reversion is the occasional appearance of the white color in the red breeds of English cattle, and a good example of atavism is the occasional persistence of gill slits in mammals, which generally disappear during embryological development but occasionally remain as permanent openings. Canine teeth in man, normally present, are regarded as a heritage from some primitive ancestor. They may some day become ataristic. The term "reversion " covers most of the breeder's needs. It is the biologist who deals with remote species. 
During this time there has been (supposedly) no admixture with domesticated herds, and yet there appears occasionally, even in a Devon herd, a white calf whose ears, lower legs, and brush of tail are marked with the tawny red or brown of the wild ancestor, and whose matted, curly hair, upstanding horns, and peculiar facial expression bespeak his reversion to the early type.

This singular persistence of characters once typical argues strongly for stability if considered from the standpoint of the ancient character, but it speaks not less plainly for instability if considered from the standpoint of the new (present) type.

The vermiform appendix, the persistence of the tail in most mammals, - these and scores of similar instances attest the stubborn resistance of a structural part to the extinction that is inevitable; and the case of the "beard" of the turkey cock illustrates the fact that a combination of whatever order, once started, tends strongly to continue, even though useless and unmeaning.

\section{SECTION IV - EVIDENCE FROM DISAPPEARANCE OF PARTS}

The organic world is full of instances of structural parts lingering long after their usefulness has largely or quite disappeared, and after entirely new relations have been established among associated characters. ${ }^{1}$

The hind legs of the python and the whale, already rudimentary and represented only by bones internal to the surface, and those of the sea lion and the seal, evidently disappearing in the same manner; the wing of the apteryx, reduced to the merest trace hidden in the plumage, and that of the ostrich, plainly following along to the same fate; the fetal hair of the whale, and

1 This is entirely independent of the question of the influence of utility in the origin and development of a new character. It has been the fashion to assume that none but useful characters will originate. The writer, on the contrary, inclines to the belief that any character will arise whose elements are present in the organism, quite irrespective of its usefulness, and that it will continue unless prevented by selection, although manifestly it will never attain maximum prominence except through the cumulative effect of the selective process. 
but from sheer inability to support life. Manifestly all regeneration is a struggle, and a prerequisite to its accomplishment is an assured base of supplies and uninjured vital organs.

Regeneration by transformation. ${ }^{1}$ Regeneration in some of the lower animals is accomplished through rearrangement of old substance as well as through the addition of new material.
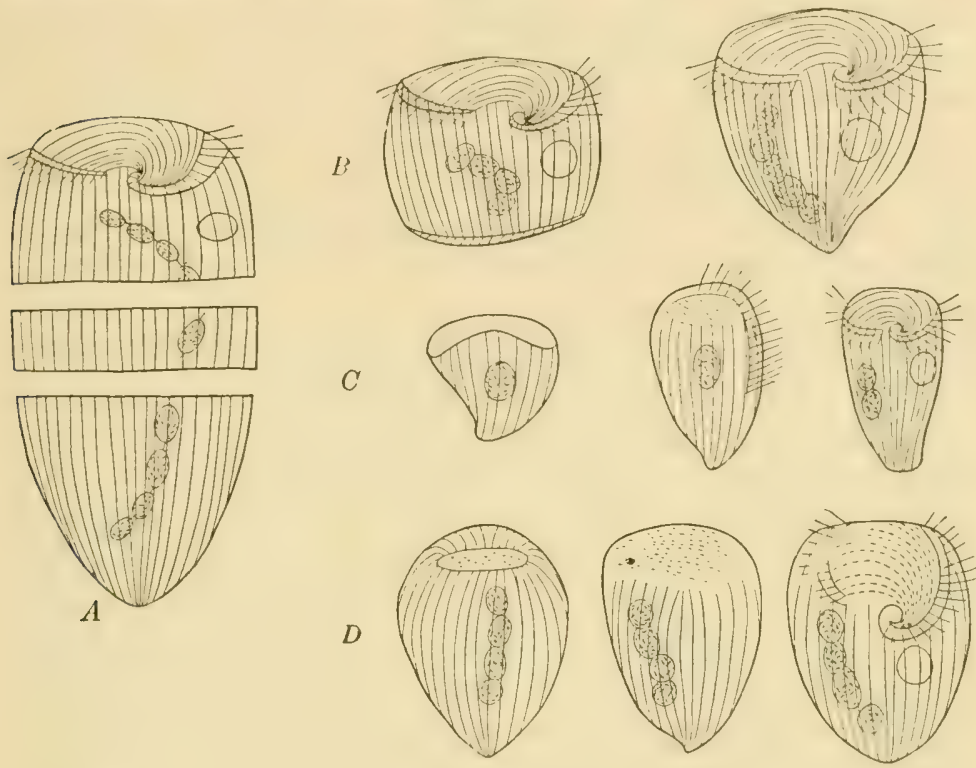

FIG. 39. Regeneration of Stentor cut into three pieces, as at $A$

$B$ : this row shows regeneration of anterior piece. $C$ : this row shows regeneration of middle piece. $D:$ this row shows regeneration of posterior piece. This regeneration is effected first of all by rearrangement of material, - each piece having been supplied with a portion of the nucleus.-After Morgan, from Gruber

If a short piece be cut from the stem of a hydra the first step in the formation of a new inclividual from the piece is the closing of the ends and a shrinking of cliameter, thus making a closed cylinder, but much smaller than the stem from which it was cut. In a day or two four tentacles appear at one end, and shortly the piece has assumed the characteristic form and proportions of a complete hydra, after which it may increase in size. The same is true of a piece of a planarian or of a Stentor (see Fig. 39).

1 Morgan, Regeneration, pp. 13-15. 
The first process seems to be 10 assume the characteristic form, afterward to increase in size; not only that, but regeneration is possible in the entire absence of food, as will be seen later, - all of which indicates a more or less extensive transformation of material.

Regeneration in embryos and eggs. ${ }^{1}$ There is much reason to believe that regeneration, especially by rearrangement, is more pronounced in the embryonic than in the adult state. The frog does not regenerate the leg, but the tadpole does. If the blastula of the sea urchin be cut into two pieces, each will develop into a perfect, but abnomally small, embryo. If the parts be separated at the two- or the four-celled stage, each is capable of dereloping into a perfect embryo, but at the eight-celled stage they are not capable of such development.

If each cell of the two-celled stage is capable of developing into a complete inclividual, then the material at this stage must be indifferent, that is undifferentiated. In other words, if the first cleavage be considered as dividing into right and left halves, and each half is capable of developing into a whole, the case is exactly like that of regeneration in the split planarian; if, however, the first segmentation be considered as dividing into anterior and posterior regions, and if each may develop an individual, it is exactly similar to the case of the regeneration of a worm that has been cut in two crosswise into anterior and posterior halves. In all cases some readjustment of material is involved.

This separation is easily effected in the sea urchin by shaking, in which case each part, below the eight-celled stage, develops an individual. If in the frog the parts, even at the two-celled stage, be separated, each collapses; if instead one half be killed by a needle, the uninjured part develops at first a laalf embryo, afterwards making more or less successful "post generation" of a whole embryo." It is therefore a perfectly well-established fact that, in certain species at least, a part of an egrg or embryo is capable of developing into a perfect individual.

To what extent this separation of segmenting eggs at the two-celled stage may take place in nature is of course unknown,

1 Morgan, Regeneration, p. I8; also chap. xi, pp. 216-24I.

2 Ibid. pp. 216-22I. 
but it has been assumed to be the cause of the production of such twins as are exceedingly similar. Hypothetical cases of this sort are known as "identical twins," supposedly arising from a single ovum instead of from two.

Experiments upon a variety of species show different powers of development from part embryos. IVilson found, and Morgan verified the fact, that in Amphioxus each of the first two or four cells could develop an entire embryo, and that the one to eight blastomeres would develop to the blastula stage but no farther. Zoja showed that the isolated blastomeres in a number of jellyfish developed each a whole embryo but of small size. ${ }^{1}$ Driesch studied the matter in ascidians and found the cleavage of isolated blastomeres to be neither like that of the whole embryo nor like the development they would each have undergone had they remained in place. They produce symmetrical gastrulæe and larvæ of small size, but lacking in certain parts. ${ }^{2}$

No one can aroid the conclusion that the phenomena of regeneration generally show an extreme stability of living matter; but they also betray, especially in lower organisms and in the developing embryo, an unexpected elasticity. To assign absolute stability or extreme instability to living matter would be to state but half the truth. A fair interpretation of the facts of regeneration leads to the conviction that living matter has the power of extreme readjustment in its effort to discharge its normal functions, and that it will discharge those functions as nearly as may be, even under dire distress, and even though important details of structure are by force omitted.

Regeneration in plants. This is different from regeneration in animals in that "the piece does not complete itself at the cut end, nor does it change its form into that of a new plant, but the leaf buds that are present on the piece begin to develop, especially those near the distal end of the piece." 3 The processes are similar in the two cases in that a piece may give rise to a whole individual, as when the begonia leaf throws out first roots and then stems, which develop into perfect plants.

Regeneration in higher animals. It is a somewhat singular fact that the lower animals possess larger powers of regeneration of lost
1 Morgan, Regeneration, p. 237.
2 Ibid. p. 236.
${ }^{3}$ Ibid. p. 15. 
parts than do those which are more highly differentiated. Still the latter are not destitute of this faculty of replacement. The teeth of many vertebrates are shed once and replaced; rarely a second replacement occurs. If the ox loses the horn, the loss is permanent; but the stag sheds his annually, each successive pair arising from the same scar or bud, but each provided with an additional prong. By what inherent quality of this particular spot are we to explain this annual change in the character of the part restored?

Birds shed their plumage, and many animals their hair, annually, as trees shed their leaves, and often the new growth differs materially from the old. The "milk teeth " are simpler than the permanent set; the color of the foal and the fawn changes with maturity; and the shape of the cotyledon gives little indication of what the real leaf will be. Nobody seeing the umbrellalike first leaf of the basswood would suspect what the later leaves will be, nor would one suspect the clover until the "third leaf " appears.

Repair of injury among higher animals seems to be exceedingly limited. However, wounded muscles can "fill up)" to some extent; cut nerves mend slowly; severed blood vessels repair themselves and restore circulation to the part; liver, kidney, glands, and tissues generally have sufficient power of regeneration to close wounds and to replace lost portions more or less perfectly, but nearly always with a scar; broken bones will knit or a small piece removed will be restored, but an entire bone cut off will not be replaced. Of all parts the skin possesses the highest power of restoration, probably because it is normally in active growth from beneath to replace the parts worn off from above.

The character of the regenerated part. The regenerated part may compare with the original in any one of four different ways :

I. It may be exactly like the original, as in the leg of a salamander (holomorphosis).

2. It may be like the original except smaller in size (meromorphosis).

3. It may be different from the original but like some other part of the body, as when an antenna replaces an eye (heteromorphosis). 
4. It may be unlike any normal structure of the body, as when a new leg is "unlike any other leg on the body" (neomorphosis).

With respect to the tissues from which. regenerated parts arise two distinct cases are to be noted :

I. Where the part regenerated springs from tissue of the same kind, requiring only an extension of growth, as when an injured muscle is repaired.

2. Where the regenerated part springs from tissue of a totally different order, as where a severed leg is restored from the cut outward, or where the lens of an eye arises from the iris, requiring differentiation as well as growth." This subject will be pursued further under the section on "Origin of New Cells and Tissues."

Effect of temperature upon regeneration. ${ }^{3}$ Planarians were cut in two transversely at the pharynx. No regeneration took place below $3^{\circ} \mathrm{C}$. Of six specimens kept at this temperature only one regenerated, and that incompletely, the eyes and brain being incomplete after six months. The temperature at which regeneration took place most rapidly was $29.7^{\circ}$, at which a new head formed in four and six-tenths days. At $31.5^{\circ}$ it required eight and a half days to complete the head; at $32^{\circ}$ regeneration commenced, but death occurred in about six days; at $33^{\circ}$ regeneration was slight, and at $34^{\circ}$ none took place, death occurring within three days. Other species showed a similar range for optimum, minimum, and maximum. ${ }^{4}$

Influence of food upon regeneration." While regeneration takes place more rapidly with a full food supply, it nevertheless proceeds without it. In this case the new growth appears to be derived not from surplus fond material but from the protoplasm itself, resulting in reduction in size.

If a planarian be kept for several months without food, in this starred condition it gradually shrinks in size, even to one thirteenth of the normal (see Fig. 40).

If a starved worm be cut in two pieces, each will regenerate, though more slowly than if fed, the new part increasing in size at the expense of the old.

1 Morgan, Regeneration, p. 24.

2 Ibid. p. 205.
3 Ibid. pp. 26-27.

4 Ibid. pp. 26-27.
5 Ibid. pp. 27-29. 
As Morgan remarks, "The growth of the new part at the expense of the old tissues is a phenomenon of the greatest importance, an explanation of which will involve, I think, the most funclamental questions pertaining to growth." To this remark it may be added that the phenomenon is also of the greatest importance in its bearing upon the relative stability of living matter. In so far as protoplasm is workd ozer to serve new purposes, it

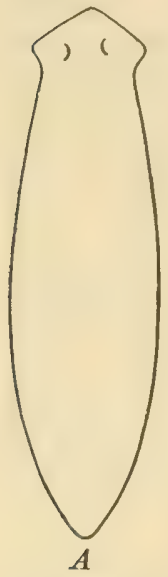

FIG. 40. Effect of star. vation upon the planarian

$\therefore$, well-fed worm; $B$, the same individual after being kept without food for four months and thirteen days. - After Morgan shows wonderful elasticity; but the fact that the organism preserves or completes its plan at almost any expense, even to itself, betrays a wonderful persistence on the part of the original plan.

It is known that the starving cat or dog will replace a large share of the dry matter of the body with water, and sacrifice all other activities to the vital processes. Plants growing with no nitrogen supply save what is contained in the seeds will soon reach the maximum of possible development, but will continue to produce new leaves at the expense of the old ones, ${ }^{2}$ as the rapidly growing stalk of the century plant feeds on its thickened leaves, or the beet and carrot feed on their thickened roots.

Effects of light upon regeneration." In the case of plants only ${ }^{4}$ does light seem to affect regeneration, and in them the influence appears to be confined to the blue rays. "Herbst observed that when the eye of certain Crustacea is cut off, sometimes an eye and sometimes an antenna is regenerated." Experiments were conducted to see whether light might be the factor which determined whether the

1 Morgan, Regeneration, pp. 27-29.

2 The writer saw the original clover plants in the first Rothamsted series for testing the nitrogen-gathering powers of root tubercles. One of these plants had no source of nitrogen but the seed. It was two years old, still producing new leaves as the old ones died down, but it had never blossomed, nor was it able to produce more than four leaves at any time.

${ }^{3}$ Morgan, Regeneration, pp. 29-30.

4 Ibid. p. 78 . 
eye or the antenna should appear, but as many eyes were regenerated in the dark as in the light. It was found, however, by both Herbst and Morgan independently, that "when the end only of the eyestalk is cut off an eye regenerates, but when the eyestalk is cut off at the base an antenna regenerates." 1

Effect of gravity upon regeneration. The effect of gravity upon regeneration in plants is pronounced, ${ }^{2}$ but only one case is known of its influence upon regeneration in animals. This is the case of the hydroid Antcnunlaria antennina. ${ }^{3}$

This animal, however, has many of the characteristics of the plant, for it lives attached by a kind of root to the bottom of the sea, and its general form is that of a branching stem, like a typical plant. All experiments show that regeneration in this form is always with reference to gravity, much as in the case of plants. Whatever the position of the piece, the new growth is upward from the most clevated part, whther basal or apical, and downward from the lower extremity and from the base of the new growth (see Fig. 4I).

The effect of gravity upon regeneration in plants may be briefly summarized as follows : ${ }^{4}$

I. If a piece of stem of the willow be suspended with its apex upward, in three or four clays roots will spring from small swellings at the basal ${ }^{5}$ end, and three or four buds will arise at the apical ${ }^{5} \mathrm{end}$, the one at the extreme tip coming first and growing fastest, others in regular decreasing order (see Fig. 26, A).

2. If the piece is long the lower buds will not start, but if it should be cut in two pieces, or if a ring of bark should be cut from the middle, each would behare as already described, showing that any point on the stem may throw out cither shoots or roots, according to its position with reference to the cut and to gravity.

3. These new growths generally arise from preëxisting buds if the piece is young, but they may arise from regions entirely destitute of preexisting buds. The writer knew a red maple tree

1 Morgan, Regeneration, p. 30.

2 Ibid. pp. 7 I-So.

3 Ibid. pp. 30-32.

4 The pieces experimented upon were suspended in moist atmosphere.

5 In all these explanations "basal" means the end that was down when the plant was in its normal position, whatever its position during the experiment. "Apical" refers to the end farthest from the base in nature. 
in Urbana, Illinois, eighteen inches or more in diameter. It forked about six feet from the ground. A heavy storm split one of the limbs away, when it was found that a dense network of roots had formed in the moist soil that had collected in the fork.
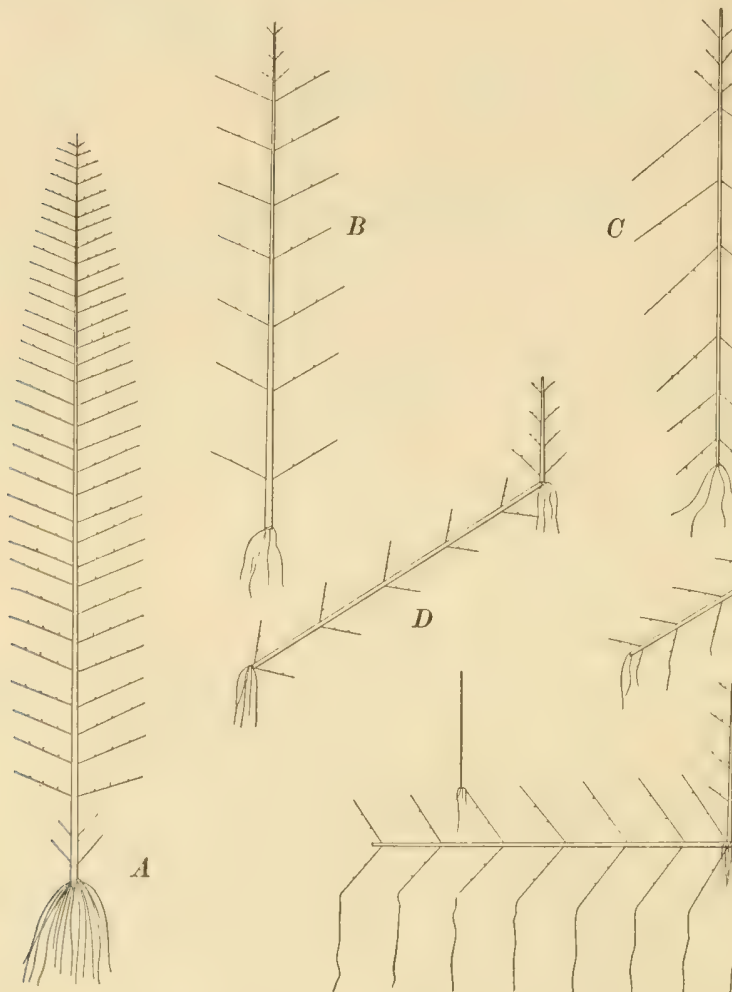

$B$
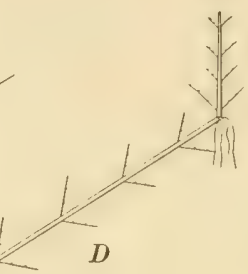

C

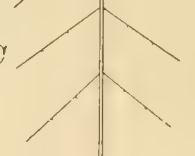

1

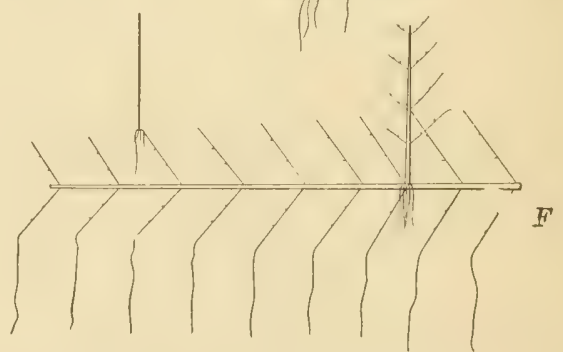

FIG. 4I. Regeneration in response to gravity in animal organisms: this creature (Antennularia antennina) resembles plants in its habit of growth, being attached; it also resembles them in its response to gravity in regeneration. - After Morgan, from Loeb

4. If the piece be suspended apex down, the shoots will still start from the apical end, bending upward, and roots will start not only from the basal end, now uppermost, but they will start from the whole length of the stem and bend downward, showing that the force determining whether shoot or root shall be put out 
is largely intomal, but that the influence determining the direction of the growth is extemal, - gravity (see Fig. 26, $B$ ).

5. This "polar difference" is most energetic in young stems, gradually lessening in older growth, though the tendency remains in quite old pieces.

6. If internodes only are used, some plants will regenerate and others will not; but if they do regenerate, the tendency is for roots to appear from the basal end and leaves from the apical, whatever the position. ${ }^{1}$

7. If a piece of the root of a poplar be suspended rertically in a moist chamber, afix downiard, leaves and shoots will appear from the basal," now the upper, end; if suspended basal end downward, the shoots will still arise from this (basal) end."

8. Certain plants, as the begonia, are able to produce new plants from even a single leaf if set in moist sand. In all cases, so far as known, roots first arise at the base of the leaf stem, or midrib section, and later shoots arise on the apical side of the roots, whatever the position.

9. Curiously, if the leaf be taken from a begonia just ready to flower, the new plant will flower very quickly after becoming established, but with few leaves and little growth; but if it be taken from a plant just out of flower, the growth will be greater and the period longer before flowering follows.

10. When pieces of stem are suspended vertically, apex upward, polarity and gravity act together; when suspended apex downward they act oppositely. The two forces may, to some extent, be separated by employing different positions. For example, if the piece be held obliquely, apical end the higher, the buds along the upper side will develop more than those on the lower side; and if it be placed horizontally, all the buds on what is now the upper side of the stem will start, but those at the apical end will grow more rapidly.

If held in an oblique position with basal end higher, different results follow; but in general it is shown that where polarity

1 Morgan, Regeneration, p. 74.

2 It should be remembered that the basal end of the root is the end nearest the stem.

3 Morgan, Regeneration, p. 75 . 
and gravity are opposed to each other the former is by far the stronger force. ${ }^{1}$

I I. If a long piece of stem be suspended by both ends, roots will start freely along the curied loa'r surface of the bent stem. If now the bent stem be reversed, roots will not only form less freely, but they will mostly be along the lower or under side of the arch, the concave instead of the convex side of the curve, and clustered closer to the basal end. ${ }^{2}$

\section{SECTION VIII - INTERNAL FACTORS IN REGENERATION}

Even among plants it is noticeable that internal influences are strongly involved in the regenerative processes. Indeed, one point of difference between plants and animals is that the latter regenerate directly from the cut surface, while the former regenerate by means of buds thrown out at the sidc and just behind the point of severance.

Again, it is to be observed that those organisms whose regeneration is influenced by gravity are the attached species, that maintain habitual and constant relations to gravity, but that those species which, like most animals, move about freely, regenerate according to internal influcnces, except in so far as vital processes are involved through food, temperature, or chemical conditions. In other words, the study of regeneration is essentially a study of internal forces, and even among plants subject to the influence of gravity the internal factors are yet the dominant ones. They are well worth study, therefore, as bearing upon the subject of this chapter and upon the inherent forces of organized and living matter.

Polarity and heteromorphosis. If a short piece be cut from the anterior end of an earthworm the main piece regenerates promptly, the small piece with difficulty or not at all; and the same is true as to the posterior end. Again, if the short piece regenerate at all, it cloes not complete itself by growing a new worm, but by growing a reacrsed hacd (or tail) like itself, so that we may have an individual with two heads and no tail, or vice versa. In other words, the polarity is reversed; for if the

${ }^{1}$ Morgan, Regeneration, p. 78 .

2 Ibid. pp. 79-8o. 
worm had been cut in the middli, both halves would have produced an entire worm, minus, perhaps, certain parts like the reproductive organs, which do not seem to be reproduced. However, the posterior half of a planarian will regenerate, producing an entire worm with typical eyes.

What it is that determines the character of the restored part is a mystery. A worm is cut at a certain point. The tissue of one piece will regenerate head with all its parts, and the tissue of the other, at the same point, will regenerate posterior parts, - unless the cut be well forward, when both pieces will regenerate antcrior parts, or well back, when both will regenerate posterior parts.

These facts cannot be explained on the theory of "formative stuffs," because head tissue may arise from posterior positions and tail tissue from anterior. For example, if an oblique cut be made into the side of a planarian, head tissue will arise if the cut be directed backward, even though made well to the rear, while tail tissue will arise from a cut directed forward, even though made at a point so far ahead that if carried entirely across the body it would sever a piece so small that it would give rise to hcad tissue only (see Fig. 42).

The determining cause of these opposite differentiations is yet a mystery.

Lateral regeneration. "Since the most familiar cases of regeneration are those that take place at the anterior and posterior

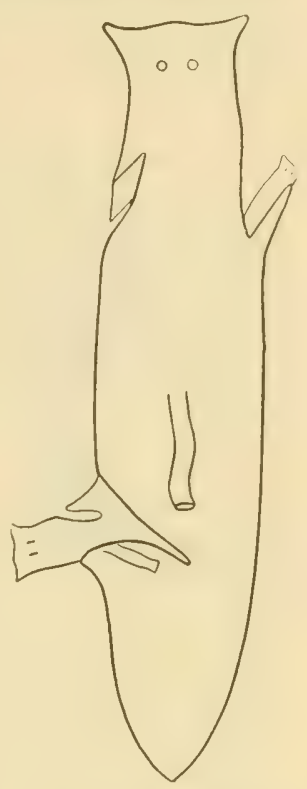

FIG. 42. P'olarity in regeneration : regeneration from the two oblique cuts opening forward will produce "head stuff," though one cut is far posterior; on the other hand, an anterior cleft opening backward produces tail matter. - After Morgan ends, we not unnaturally come to think of polarity as a phenomenon connected only with the long axis of the animal, but there are also many cases of lateral regeneration in which a similar relation can be shown." 1

1 Morgan, Regeneration, p. 43. 
If an incision be made in the side of an actinian, tentacles will arise at that point, and they will seize pieces of meat and press them against the stem, whether a mouth was formed or not $^{1}$ (see Fig. 43).

If a planarian be split lengthwise into right and left halves, regeneration takes place whether the halves be equal or unequal. The completed worm will be at first both nerrozere and shorter

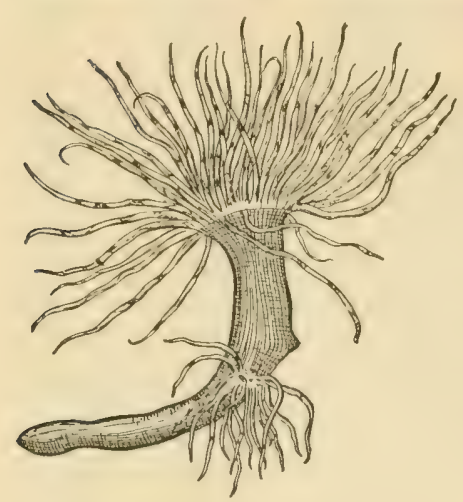

FIG. 43. Lateral regeneration: a cut in the side of an actinian produced a clump of tentacles which would seize a piece of meat and press it against the side of the body, even though no mouth were present. After Loeb than the normal, but with time and feed it will equal or even exceed the original. How the nerve cords and genital ducts are formed anew, especially from the piece so far to one side as to include none of their substance, is a mystery closely akin to that of original differentiation from the fertilized ovum. If the slice be taken well to one side, no head matter is included. In such a case the head appears first at the side of the piece, but later it assumes its proper position.

Regeneration from an oblique surface. If the tail of a tadpole be removed by an oblique cut, the regenerated tail will at first stand at right anseles to the cut and obliquely to the axis of the tail. As growth proceeds, however, the tail gradually swings into line with the axis of the body.

If the head of a planarian be removed by an oblique cut, it will begin to regenerate at the forward part, making the head at first stand at right angles to the cut, and not in line with the body.

If a piece be taken from the middle of a planarian by two parallel but oblique cuts (for example, running backward from left to right), the head will begin to regenerate from the anterior end at its forward (left) side, while the tail will begin at the posterior extremity but on the ofposite side, the final result being complete restoration of the normal shape.

\footnotetext{
1 Loeb, Physiology of the Brain, p. 52.
} 
That the new material produced in regeneration is at first totipotent - that is, capable of more than one differentiation is easily shown. If a short piece be cut from the middle of a planarian, and if, after the new material has begun to form, the whole mass be split lengthwise, both halves will derelop heads directly; but if the split is not made "until just before the formation of a head, then each half piece produces at first a half head, that completes itself later at the cut side." 1

Again, if the head be cut from a planarian and the body be split for a clistance, the split will heal and a single head will regenerate; but if a slice be taken out of the middle line of the body, or if otherwise the two pieces be prevented from fusing, then two hacals will regenerate, one on each piece. ${ }^{2}$ These two heads may later pull apart with sufficient force to tear the body:"

All these phenomena reveal great capability of readjustment as to more or less differentiated tissue. The more the matter is studied the more we discover that the line between regeneration and development is one of degree rather than of kind, and that differentiation in both cases, whether normal or abnormal, rests upon causes very imperfectly understood but closely akin to those of differentiation in general.

\section{SECTION IX - EVIDENCE FROM GRAF'TING}

When tissue of one kind, plant or animal, is removed from its connections and set into tissue of the same or of a different kind, and a union takes place so that growth ensues, the union is called a graft. From our standpoint no little interest attaches to the variety of conditions under which grafts may be effected, and to the fact that the growth upon the graft is like the part sit in and not like the tissue that supports it, - the host acts only as affording standing room and food supply to the graft. Again, if two dissimilar pieces are joined, each preserves its identity.

Grafting is comparatively easy among plants, though the species that may be joined are limited. Among animals it is more difficult, but by no means impossible.

1 Morgan, Regeneration, p. 49.

2 Ibid. p. 50.

${ }^{3}$ Loeb, Physiology of the Brain, p. 82. 
Born succeeded in uniting tadpoles of two different species of frogst, Rana esculinta for the anterior part and $R$. arrealis for the posterior. This "made-up, animal lived seventeen days. Harrison succeeded in keeping an individual made up of $R$. airescens and $R$. palustris until its transformation from a tadpole into a frog. ${ }^{1}$

In the same way earthworms may be built up of two or more individuals of the same or of different species, and the pieces used in this building up may even be reversed, so that the middle part of the made-up) worm may have its posterior part placed anteriorly, or the reverse. IVorms may be made with two heads, with or without a tail, or with two tails, with or without a head, though the latter, for obvious reasons, can live but a short time. ${ }^{2}$

Ribbert grafted a portion of mammary gland into the ear of the guinea pig, where it grew, and when the pig became pregnant the grafted tissue secreted milk. ${ }^{3}$

The whole subject of grafting, even more than that of regeneration, shows the wonderfully persistent nature of differentiated tissue, though it shows also the variety of conditions under which its activities may be discharged.

\section{SECTION X-EVIIUENCE FRON THE ORIGIN OF NEIV CELLS AND TISSUES}

New tissue may arise in regeneration in three distinct ways:

I. It may arise from tissue of its own kind; that is, the tissue produced in regeneration may arise from the same kind of tissue in the organism.

2. It may arise, not from like tissue, - of which none may be present, - but from the same point of origin and in the same manner as during embryonic development.

3. It may arise from a source entirely different from that of embryonic development, and in this case may be either normal or heteromorphic.

Examples of the first class are everywhere at hand; indeed, this is the most ordinary form of regeneration, and many have erroneously supposed it to be the only form. Ordinarily, muscle

1 Morgan, Regeneration, pp. I84-185. 2 Ibid. pp. I 7 I-173.

${ }^{3}$ Loeb, Physiology of the Brain, p. 206. 
gives rise to muscle, nerve to nerve, bone to bone, and each part to its own kind by the simple method of growth extension.

This, however, is the simplest method of regeneration, and more complex methods are necessary in extreme regeneration, where the entire supply of certain tissues is cut away, and the new growth must arise from different tissue or not at all.

Examples of the second class are less common, but not rare. Where an entire limb is gone regeneration must proceed upon a plan different from the one it would follow were only a single tissue involved, like a wounded muscle. The first new growth that arises must in some way be endowed with the power of producing not one, but many, differcut linds of tissucs. Götte, as quoted by Morgan, has studied both "the embryonic development and the regeneration of the limb of triton, especially in regard to the origin of the new bones. I Ie found that the slieleton develops in much the same way in the embryonic limb and in the regenerating limb, and the process in the latter may be saicl to repeat that in the former." If the larva is young; the new growth differs but little from the old, but if the bones had become ossified, the difference between the two is much more marked. ${ }^{1}$ Curiously enough the salamander regenerates the tail completely; bones and all, but the regenerated tail of a lizard contains not a new series of bones, but a cartilaginous tube which is attached to the broken seventh caudal vertebra. ${ }^{2}$

An example of the third case, in which tissue is regencrated from a source differont from that of cmbryonic derclopment, is shown in the reproduction of the lens of the eye of the salamander or of triton. In this case, if the lens be removed a new one is regenerated from the ufper cdge of the iris, a part of the body from which the lens of the eye nicer derclops nomally in the embryo of this or of any other vertebrate. In the embryo the lens develops from the ectoderm at the sicle of the head, having no connection with the iris.

The regeneration from like tissue is no more difficult of comprehension than is ordinary growth, and that which repeats the

I Morgan, Regeneration, pp. 200-20r.

2 Ibid, p. I98. The lizard's tail does not break between the two vertebræ, which are strongly joined, but in the middle of the vertebra, which is relatively weak. 
course of embryonic development is involved in the mystery of ordinary differentiation from totipotent or indifferent tissue; but regenerated matter which arises from tissue in no a'isc incoliced in embryonic derelopment would seem to be outsicle of the influence of heredity. In the latter case it may result in normal tissue, as in the lens of the eye, or in heteromorphic growth, as in the production of an antenna instead of an eye, or of a foot instead of an antenna.

\section{SECTION XI - EVIDENCE FROM DEVELOPMENT AND DIFFERENTIATION ${ }^{1}$}

From the fertilized ovum to the fully differentiated adult individual of the highest species is a long step. Nothing is more evident than that all this differcutiation is the result of forces resident within the single cell of the o-'um. Whatever office is discharged by outside agencies, and whatever deviations or modifications are produced thereby, the impulse to differentiate and the dirction of differentiation arise from forces internal to the organism. Moreover, this differentiation, great as it is in the finished product, when traced backward merges by imperceptible shades each into the next preceding, until the undifferentiated ovum is reached at the end (or beginning) of the series.

What is the nature of these internal forces, and what are the agencies that set them in motion, are the chief mysteries in animal and plant development. That a single germ cell, similar in its essential nature to any one of the tissue cells of which the body is composed, - that such a cell "can carry with it the sum total of the heritage of the species, that it can in the course of a few days or weeks give rise to a mollusk or a man, is the greatest marvel of biological science." 2

"In attempting $t()$ analyze the problems that it involves," continues Wilson, "we must from the outset hold fast to the fact on which Huxley insisted, that the wonderful formative energy of the germ is not impressed upon it from without, but is inherent in the egg as a heritage from the parental life of which it was originally a part. The development of the embryo is nothing new.

1 Wilson, The Cell, p. 430.

2 Ibid. p. 396. 
It involves no breach of continuity, and is but a continuation of the vital processes going on in the parental body. What gives development its marvelous character is the rapiclity with which it proceeds, and the cliversity of the results attained in a span so brief."

IVe can define the chief mystery of development as lying in the facts of differentiotion and definite tcrmination to growth, but if we ask how the impulses governing such complicated results lie latent in a single cell, and how they operate in orderly sequence beginning and ending at the proper moment, we have asked the " final question," and " in approaching it," says Wilson, "we may as well make a frank confession of ignorance."

About all we can hope to do in the present state of knowledge is to throw light upon the question of comparative stability of organized matter and note the conditions under which one kind of cell gives rise to another of a different order. On this point certain facts of development have an important bearing.

Cell division. All differentiation during development is by cleavage of the germ cell. The phenomena of clivision and mitosis are in general the same in the cleavage of the germ cell and the development of the embryo as in the division of ordinary tissue cells, except that the body cells for the most part give rise only to others like themsclic's, while those of the germ and the embryo give rise not only to others like themselves but to many different kinds as well.

This distinction is relative rather than absolute, for we remember that in certain species a small part, even a leaf, is able by regeneration to give rise to a new individual, involving differentiation similar if not equal to that of embryonic development. However, in many species and with most tissues the power of typical differentiation appears to be lost in the first development. In this connection it is well to remind ourselves that in many species the amount of chromatin matter in the somatic cells is different from that in the germ cells. ${ }^{1}$

Geometrical character of cleavage. The earliest cleavage of the germ cell is governed by two definite geometric principles announced by Sachs: (I) the cell typically tends to clivide into

1 Wilson, The Cell, p. 426 (concerning Boveri's investigations on Ascaris). 
equal parts; (2) each new plane of division tends to intersect the preceding one at a right angle. ${ }^{1}$

A typical cleavage of a spherical egg would be, first, vertical, dividing into right and left halves; second, also vertical, but at right angles to the first, dividing into dorsal and ventral portions; third, horizontal, dividing into anterior and posterior parts; after which all sorts of irregularities might be expected, including cleavages parallel with the surface, cutting in two the long cells that formerly extended to the center. The egg of the holothurian, like those of Synapta, proceeds regularly until as many as 5 I 2 cells are reached, ${ }^{2}$ while others become irregular almost at once, some quadrants dividing much more rapidly than others.

Variation in the rate of division. If clivision proceeds with perfect regularity, the number of cells will of course form an increasing geometrical series whose ratio is two, $-2,4,8,16$, 32,64, I $28,256,512,1024$, etc. Such a series has been mentioned, extending to $5 \mathrm{I} 2$.

This uniform series is rarely realized, however, owing to irregularities in the rate of division; for example, Nereis regularly grives rise to the series $2,4,8,16,20,23,29,32,37,38,41,42$, "after which the order is more or less variable." 3

In some portions of the dividing cell the cleavage proceeds therefore with much greater rapidity than in others, nor is the plan uniform in all cases, though the results achieved may be substantially identical. In general the rate of division is most rapid in the upper hemisphere of the ovum, and in some instances it proceeds very slowly, with long pauses, in the lower, giving great irregularity in the size of cells.

Temporary effect of outside influences upon cleavage. Driesch placed egros of sea urchins under pressure sufficient to flatten the spheres to disks. In this position "the amphiasters all assume the position of least resistance, i.e. parallel to the flattened sides, and the egg segments as a flat plate of eight, sixteen, or thirtytwo cells. This is totally different from the normal form of cleavage; yet such eggs when released from pressure are capable of daclopment and gire rise to normal cmbryos." "4 Without

1 Wilson, The Cell, p. 362.

2 Ibid. p. 364.
3 Ibid. p. 366.

4 Ibid. p. 375 . Italics are mine. 
doubt in nature similar temporary effects are caused by internal stress or pressure of the parts of the egg itself.

After noting other causes of irregularity, Wilson fittingly observes :

All these considerations drive us to the view that the simpler mechanical factors, such as pressure, form, and the like, are subordinate to far more subtle and complex operations involved in the general development of the

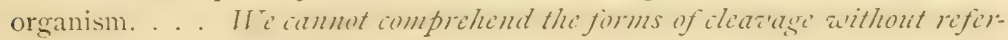
ence to the end results. ${ }^{1}$

Promorphology of the ovum. Is there something in the original shape or character of the egg that corresponds to the finished individual? Is there a polarity of the egg that is in any way related to the order of cleavage and the axis of the body?

Speaking of the eggs of insects, Wilson says $:^{2}$

In a large number of cases the egg is elongated and bilaterally symmetrical, and, according to Blochmann and Wheeler, may even show a bilateral distribution of the yolk corresponding with the bilaterality of the ovum.

Hallez is here quoted as asserting, after a study of the cockroach, water beetle, and the locust, that "the egg cell possesses the same orientation as the maternal organism that produces it ; it has a cephalic pole and a caudal pole; a right side and a left ; a dorsal aspect and a ventral; and these different aspects of the egg coincide with the corresponding aspects of the embryo." Wheeler, after studying some thirty different species of insects, reached the same result, and concluded that even when the eggr approaches the spherical form the symmetry still exists, though obscured. ${ }^{3}$

In species other than insects the egg often has a bilateral symmetry of its own, "sometimes so clearly marked that the exact position of the embryo may be predicted in the unfertilized egrr." "3

Polarity of the ovum. It is now well known that in the segmenting eggs of the frog the first two cleavage planes are i'crtical, the first corresponding to the median plane of the body and setting off right and left halves (which develop into corresponding

1 Wilson, The Cell, pp. 376-377.

2 Ibid. pp. $383-384$.

${ }^{3}$ Ibid. p. $3^{8} 4$. 
parts of the body), the second setting off dorsal and ventral areas; while the third, which is horizontal, divides into anterior and posterior portions.

The process is the same in many species, and "wherever the egg axis can be determined by the accumulation of the deutoplasm in the low'r lemisplure the egre muclus sooner or later lies eccentrically in the upper homisphur, and the polar bodies are formed at the upper pole."

In such cases of distinct polarity the cleavage planes are practically predetermined, and the products of division have each their definite rôle in development. Thus the upper hemisphere (so-called animal pole) is the seat of most active division, with smaller cells, which develop into cerebral, dorsal, and anterior portions of the body; while the lower hemisphere (regetable or nutritive pole) divides more slowly, its cells are larger, and they develop into the alimentary organs and the posterior and ventral parts generally. ${ }^{1}$

While this rule is not absolute in all species, it yet indicates a broad general principle that lies at the very threshold of development; namely, that the original impulsi to direction of groath lies in the polarity of the ooum.

Cause of polarity in the ovum. Gravity would seem to be the controlling cause in establishing a kind of polarity in the orum. The nucleus being of low specific gravity, tends to lie eccentrically nearer the upper side of the ovum, and the heavier deutoplasm settles to the lower side, the parts arranging themselves according to relative weight, like starch granules or other cell contents. Moreover, Born has shown that "if frogs' eggs be fastened in an abnormal position, - e.g. upside down or on the side, rearrangement of the egg material takes place" and "a niw aris is established in the cgrr." " Schultze discovered that "if the egg be turned upside down when in the two-celled stage a whole embryo (or half of a double embryo) may arise from each blastomere, instead of a half embryo as in the normal development, and that the axes of these embryos show no constant relation to one another." "2 Morgan learned that "either a half embryo

1 Wilson, The Cell, pp. 37S, 379.

2 Ibid. p. 422. 
or a whole half-sized dwarf might be formed, according to the position of the blastomcre." 1

Causes of differentiation. These facts show:

I. That a primary cause of differentiation lies in the polarity of the ovum.

2. That this polarity is clue primarily to gravity separating its heavier from its lighter parts.

3. That the egg is at first, in many cases at least, indifferent, and that its polarity may even be changed after having once been well established.

4. That if the segmenting orum may be separated into its blastomeres, and each may produce a complete individual (Amphioxus, etc.)," then the egg as a whole is not only totipotent but its earlier segments as well are each capable of producing all the parts of the body.

5. The polarity and the development of a part are influenced largely by its position with reference to other and cspicially larger parts.

Roux found that when, in the two-celled stage of the frog's egg, one of the blastomeres was killed by a hot needle, the remaining one developed a half embryo, ${ }^{3}$ whereas in many cases a single blastomere is known to be entirely capable of producing a whole embryo if freed from its neighbors.

Again, a section from an earthworm tencls to regenerate head matter at its anterior end and tail matter at its posterior end; but if a smail piece be grafted, cien in arerersed position, on the anterior end of a larger piece, it will regenerate head matter from its postcrior crtromity, showing how the polarity of the smaller piece is, so to speak, overcome by that of the larger."

A consideration of these facts leads Wilson to quote Hertwig as follows: The relatiac position of a blastomere in the whole [asscmblarge] determines in gencral what develops from it; if its position be changed, it gives risc to something difforent; in other words, its prospective value is a function of its position. ${ }^{5}$

1 The different cells of the earlier cleavages (the 2-, 4-, $\$$-, I6-celled stages, etc.) are known as blastomeres.

2 Wilson, The Cell, p. 423.

${ }^{3}$ Ibid. p. 380.

${ }^{4}$ Morgan, Regeneration, p. 8.

5 Wilson, The Cell, p. 4 I 5. 
6. Whether the above statement is absolutely or only relatively true, the fact is clear that living matter will often go through its changes and complete its cycle of development under extreme hardship. For example, in some species a blastomere from the two- or from the four-celled stage may develop into a whole (dwarf) embryo or into a half embryo, while in others (ctenophore) each one of the first eight may develop into a symmetrical and therefore complete individual, but lexcking the full nomal number of suimming plates. ${ }^{1}$

7. The conclusion is unavoidable that living matter is endowed with wonderful elasticity and persistence of plan, or an approximation to it, even uncler most adverse conditions, providing only these conditions do not destroy life.

And so we return to the original questions: How much of what the individual is to be is due to inheritance? How flexible is the plan? To what extent are modifications possible, and to what extent are variations inherited?

Summary. In general the facts of differentiation and of regeneration, together with the persistence of an established type in carrying forward inherited qualities even under most adverse conditions, indicate extreme stability of living matter.

On the other hand, the facts of acclimatization argue for the alterability of protoplasm, both as to constitution and as to function, inclining one strongly to the belief that such alteration may become more or less permanent with the organism.

Altogether the conviction is forced upon us that the activities of living matter proceed upon a plan inspired from within and arising from the nature of the organization, but that this plan has accommodated itself to surrounding conditions in the past and is entirely competent to do so again whenever sufficient occasion arises, provided only that the new demands be not too sweeping or too suddenly imposed.

These facts need to be constantly in the mind of the farmer, who must be prepared for comparatively sweeping changes from apparently slight causes.

We pass now to a discussion of the question as to whether or not individual modifications may be transmitted.

1 Wilson, The Cell, p. 4 is. 


\section{ADDITIONAL REFERENCES}

A Natural Hybrid. Experiment Station Record, XV, 677.

EfFect of Stock upox Sciox. Experiment Station Record, $\mathrm{XY}, 363$. EXperimental Zö̈ngy. By T. H. Morgan. Chapters XV-NXil, pp. 239-346.

Grafting Berts. (Colors that do not blend.) Experiment Station Record, XIV, 353.

Grafting Experiments with Tamples. By R. G. Harrison. Science, VII, 198-199.

INFluexce of Stock Upox Scion. By T. J. Burrill, Transactions of the Illinois Horticultural Society, 1898, pp. 6z-72. Experiment Station Record, XI, $51,152,250$.

INFLUExce of Stock cPox Sciox. Experiment Station Record, ViI, 309.

Monffications in Parasitizen Cells. Experiment Station Record, XIV, III.

Regeneration of Begoxia. Experiment Station Record, XIT, 528.

Resemblance between Cells of Malignant Growths in Man and Normal Reproductive Tissue. Proceedings of the Royal Society, LXXII, 499-504.

Variability of Organisms and of therr Elements. Science, $\mathrm{XV}$, $1-5$. 



\section{Part III - Transmission}

Up to this point the study has been confined to the nature and kinds of variations that may arise, together with the conse's that lead to their appearance. We come now to inquire a'hithir, and to wohat cxtent, these variations are transmissible.

This is the all-important question, because variations that are not transmitted are manifestly of no consequence except to the individual. Whether desirable or undesirable, they have no opportunity to affect the race as a whole either favorably or unfavorably. We may therefore disregard, in all questions of race improvement, any and all deviations that are not transmissible.

Those that are transmitted, however, are by that fact destined to exert a more or less permanent influence for good or evil. Accordingly we cannot know too much about this class of variations and the circumstances under which they descend from generation to generation and ultimately come to characterize, if not to dominate, the type of the race.

The student, and the breeder as well, is likely to become confused by the ceaseless panorama of characters and variations presented to his view as generations come and go, involving sometimes literally multitudes of individuals of all shades of difference. He must learn to pass this procession before his mental vision with the full realization that a large portion of all that he sees will have no permanent influence upon the race. He must become acute in detecting the significant factors, which are those only that are connected with transmission.

The study of race improvement is, therefore, essentially a study of the laws that govern transmission, - all that has gone before being preparatory to that study: Upon two questions the breeder must fix his attention, - What is transmitted, and how is the transmission accomplished? 


\section{CHAPTER XI}

\section{TRANSMISSION OF MODIFICATIONS DUE TO EXTERNAL INFLUENCES}

\section{SECTION I - INTRODUCTORY}

In the discussion of the causes of variation a clear distinction was made between causes internal and causes external to the organism. This distinction should still be borne in mind, though the principal discussion and all of the controversy arise in connection with external causes.

Variations due to causes internal to the germ are transmitted. So far as the writer is aware, no one has ever disputed, or even questioned, the correctness of this proposition. It is fundamental, if not axiomatic, that any change taking place in the structure of the germinal matter, which is passed on from generation to generation, which is the bearer of all characters and the exclusive basis of all transmission, - that any such alteration will of necessity make itself manifest at once, and indefinitely afterward; indeed, as long as the change in the constitution of the germ continues. This is the purpose of all selective mating, - to secure a germ endowed with as many as possible of what are considered to be desirable variations, tending to maximum development, and as few as possible that are undesirable, and these with a minimum development. Such a germ should develop an embryo, and finally an adult individual, with the highest obtainable degree of excellence.

To control the germ is the purpose of all selection. It is the object of all breeding. We practice line breeding, and even in-breeding, to give intensity along certain lines; we resort to mixed breeding, even to cross breeding, to introduce new variations and to endow the race with greater flexibility.

It is in the germ that mutations arise. The causes are obscure, but it is here, in the constitution of the germinal 
matter, that they are at work, and it is here that races are enriched, almost miraculously, with new and valuable endowments, - not frequently and freely, but occasionally and sparingly. These are the free gift of nature, arising, so far as we can now see, spontaneously, but due, as we firmly believe, to definite influences at work within the germ, which is the avenue of all transmissible variations.

Remembering, then, that all influences that affect the germ will give rise to variations, we come now to the direct question of the chapter, - Are the modifying effects of external influences transmitted? or does each generation begin anew, unhampered and unhelped by the direct effects of previous environment, whether favorable or unfavorable?

The main question. This is an exceedingly important matter ; incleed, no other question in all evolution is of such immediate and far-reaching consequence in thremmatology. This is because the influences of environment are insidious and at the same time well-nigh irresistible. If their effects are also transmissible, they will become, like compound interest or any other geometrical progression, strongly and rapidly cumulative, and therefore tremendously powerful either for good or for evil.

This is the old and hotly contested question of "inheritance of acquired characters," better stated for our purposes in the form, Are the effects of environment transmitted? It is but fair to warn the student that this is at once the most difficult and the most complicated of all the questions of evolution, and that not only must its study be critically conducted but the student must be constantly mindful of certain fundamental considerations, a few of which it will be well to note before proceeding to the discussion of the main question.

What is a deviation? What we call a variation, a deviation, or a modification, is not, like a character, an entity that can be transmitted. It is rather the desece to which a character attains, as measured from the general mean or arerage of the race as a whole. When we speak, therefore, of the transmission of a variation or a modification, we mean literally the transmission of the character as modificd, either by internal or external causes. 
Strictly speaking, therefore, it is the character, and not its molification, which is transmitted, and what we desire to know, is, whether the modilication of a character in a particular individual tends to become permanent; that is 10 saty, whether a charatede that has undergone modification will be transmitted in its modifice or in its ummodified form.

In this connection the student must be reminded that we have no waty of judging what characters or what modifications are born inte an individual except by the develepment they attain in his own persomality after reaching the adoll stage. ${ }^{1}$

for example, when noting an average inclividual we camnot saty whether he is one that was exceptionally well born, with fair (1)pertunities for development; whether he was only farrly well borm, but with exceptional opportunities; or whether he is an average both as to birth and oppertunity. All three combinations would produce about an average individual.

On the contrary, if he be an execptional incliviclual, we are fairly safe in assuming that he was both well born and well conditioned: but if he be one of the lowest inclivicluals, that he was batly born and has lived under hard conditions, -... both of which, operating together, make exen an arerage development impossible.

(O) a mature individual we may say, first, that all the evident characters of which he stands possessed were born into him, but that the derelopment they hate attained is partly a matter of birth and partly a matter of the external conditions of life. I Is difference in development as compared with the mean of the race taken at the atult stage is his deviation or variation (for we use the ferms interchangeably), and the (puestion we are now asking is, Are these individual deriations transmitted, or are all (hatacters transmitted acoording to a deat level of inheritance, learing all deviations to be accounted for as matters of inclividual attainment through more or less sucessful development?

Adaptations. The molifying effect of the conclitions of life is so profoumd that in nature exerywhere both animals and plants hamenize with their enviromment almost perfectly, thus

1 When the individual becomes a breeder his inlerited yualities will be fairly well indicated by his powers of transmission. In this way an animal truly may "breed better than he is himself." 
giving the appearance of having heen specially created for their particular surroundings. We know enough of the causes of variation, howerer, to realize that this " lit "between the amimal or the plant and its enviromment has been brought about by the direct action of outside conditions upon matrisms somewhat plastic and capable of becoming adapted to a wide ramge of circumstances, - all individuals not pessessing this addatability having long since disappeared by natural selection.

Nothing is more simple and natural than to assume that when the individual has aceuired some molification though the inflesence of the enviromment it will tramsmit this modificattion to its descendints, and that what wats at first impressed from without gradually becomes hereditary, exhibiting all the cumulative effect of transmissible qualities. All appearances are in favor of such an assumption, and it is the simplest and most direct explanation of the phenomena of adaptation and of the well-known hamomy that alway's exists between a species and its environment.

But there are at least tade ofler methoels by which the enviromment impresses itself stromgly upom the species, - both of which are always at work, both of which achieve large results of an exactly similar charater and appeatrance, and which must therefore be either subtrated or fully accounted for before we are waranted in assuming results dee to direct transmission.

The first of these is the direet effect of the conditions of life acting separatcly $u p(x)$ all the individuals of each generation and all in the same direction. This has all the apperrerme of transmission, but it is not comolative, and cam bring about no better adaptation in the species, and no greater total change, than can be wrought for each indiviclual during its lifetime. The other is dece to the selective effect of the enviromment, which is cumblative, and, so far ats we can see, abundantly able to account for all the phenomena of adaptation and for the full effects of environment.

The environment always selective. Some individuals of every generation fail utterly to condure the conditions of life and, thus fating, they leave no descenclants. The next generation, therefore, being descended from the more adaptable individuals, is 
more nearly in harmony with the environment, and this cumulative influence, constantly exerted, generation after generation, slowly but surely modifies the race in the dirction of the chirommont, giving again the appearance of inherited modifications. Now in nature this selective influence is always at work, sometimes by actually destroying the less adaptive individuals, more often by affecting not life, but fertility or longevity, or both, through little-noticed but insidious agencies.

This is, moreover, the most powerful of all the means by which enviromment influences species. It entirely outstrips the results of direct influences upon individuals, and is fully able to account for all ordinary cases of environmental influence. ${ }^{1}$

The source of the difficulty. Here is an ever-present, allpowerful influence, bending species into harmony with their environment, and its effects must be fully eliminated or accounted for before we can determine whether a residue remains to be attributed to direct transmission. This is the source of the greatest difficulty in attempting to learn whether modifications are directly transmitted.

This selective process of the environment results in the indirect transmission of such modifications as increase the chances of the individual in the struggle for existence; but what farmers want to know is whether modifications produced by the conditions of life are divetly transmitted as such, - independently of the question whether they increase or decrease the chances of life in the individual, and independently of all questions of selection.

I The individual and the type. The student must be clear as to what constitutes type. Every individual must be considered with reference to at least two generations, the one to which it belongs and the next. The type of a race at any particular moment is fixed by the personal qualities of all its adult members, and for this purpose all individuals are of equal weight, and one character or difference is as good as another. Everything counts in fixing the type of an existing generation.

But when the next generation is considered, it is not so. Only those individuals count which succeed in reproducing, and only those differences count that are transmissible. The initial or mutural type, therefore, as secured by transmission, is somewhat different in succeeding generations, nor is it ever fully expressed in adult members. The existing and visible type of any race is, therefore, at best but an incomplete expression of its possibilities. 
In nature, where selection is unlimited, it does not greatly matter whether modifications thus induced are directly or indirectly transmitted, - the only difference is a slight one in the amount of time required; but among domesticated species, where selection is largely controlled, where it is to be employed for as few purposes as possible, and where at most, for economic reasons, its use must be sparing as compared with that of nature, - here it is important to know, if possible, whether there exists, side by side with our selection, this other influence, in some cases assisting, in others opposing, our aims.

The influence of the environment upon transmission is certainly slight at any moment, but if it exists at all it is cumulative, and, being independent of selection, it is a friend to the breeder for fixing clesirable characters, as it is also an insidious enemy, to be greatly dreaded, when an undesirable character is involved.

As the cliscussion proceeds the student must realize that we are looking for that which is at best but an infinitesimal increment as compared with the larger results due to selection, the presence and influence of which he must grow skillful in detecting and assessing.

He must also be mpon his guard against evidence that is not evidence. For example, a cat learns to open a door, or a mare to hold up her foot for her feed. If the young develop the same habit as the mother, the hasty observer calls it a case of the inheritance of an acquired character; whereas the truth is that in all probability the young creatures simply learned it by observation; incleed the readiness of the young to learn by imitation is vastly underrated. In the case of the horse it must also be remembered that most individuals that hold up the foot when begging have defective roices. This defect is extremely likely to be transmitted to the young, which, finding themselves voiceless, would, if left to themselves, even entirely without example, resort to the next most convenient and natural method of begging, which is holding up the foot. It must not be forgotten that, in the horse, holding up the foot in begging is a kind of fundamental instinct, second only to vocal effort, and it may be said in general that if a horse cannot call for his feed he will hold up his foot. Against such instances as these, urged 
as proof of the inheritance of acquired characters, the stuclent must be on his constant gruard, and he must be extremely careful in the generalizations he allows himself to make in this particular field of study.

This subject has been greatly complicated by a mass of tradition that has grown up around it. The average man assumes that the effects of environment are transmitted. He does not stop to inquire seriously into the subject. He considers all doubt on this point as foolishness; but that which he considers as good proof is, in many cases at least, anything but proof.

As if this were not enough, the naturalist on his part has still further muddled the matter by a most unfortunate and confusing, not to say inaccurate, use of terms. Traditions may be ignored for purposes of study, but not so with terminology, which should be exact and accurate. The two most unfortunate terms that ever crept into cvolutionary studies are "congenital" and "acquired."

Characters congenital and acquired. It is a custom with evolutionists to assume that every adult individual is in possession of two kinds of characters: first, those which were born into it (the congenital); second, those which were forced upon it by the conditions of life or picked up as the result of experience (the acquired). They then proceed to the very natural assumption that the congenital characters, having been clerived by inheritance, will in turn be transmitted; after which they raise what would seem to be the final question, Are the acquired characters transmitted? Thus is the field of discussion staked out, and no such battle royal (of words) was ever fought out in modern times - except over questions theological - as has been waged over the question of the inheritance (or transmission) of acquired characters. ${ }^{1}$

1 This is the question that has divided the neo-Lamarckians and the neoDarwinians, and almost the entire host of evolutionists for the last twenty years have ranged themselves on one side or the other of this dispute and have allowed themselves to be identified with one or the other of the hostile camps.

As the special opponent of the theory of the transmission of acquired characters, the student should read Weismann, particularly his Essays on Heredity, his Germ Plasm, and his Germinal Selection.

The most vigorous and accessible, if not the most able, champion of the transmissionists is perhaps Romanes in his Examination of Weismannism and 
The writer does not propose to enter the disputed territory at this point, for the reason that he does not believe in accepting for these purposes the fundamental distinction between "congenital" and "acquired" characters. The use of the term "congenital" as clistinct from "acquired" is most unfortunate. Any character present at birth is, by clefinition, congenital. The list would include not only the characters typical of the race but also malformations and deformities due to conditions in utcro or to unknown causes. Thus men have been born without feet, yet the chances of transmitting the deformity are extremely remote. An arm or a leg may be misplaced during embryonic development and malformations result without essentially bearing upon the conditions that are supposed to control inheritance.

Manifestly the most profitable distinction to observe regarding the inheritance of variations is a strict discrimination between those that have arisen through causes affecting the germ plasm directly, on the one hand (blastogenic), and those that affect the body during its development, upon the other (somatogenic). Now an accident to the embryo in ntero is as much external to the germ plasm - the inherited substance on which development depends - as is an accident after birth, and therefore distinctions between congenital and acquired characters are extremely misleading, especially among mammals; less so, of course, among bircls, in which no such thing as birth exists.

his Darwin and After Darwin. Cope, in his Primary Factors of Organic Evolution, may be read with profit, as may Eimer in Organic Evolution.

Both factions claim to be disciples and exponents of Darwin, but the opponents of transmission, from their extreme appeal to selection, have come to be known as neo-Darwinians, and the advocates of transmission have accepted the name of neo-Lamarckians, from their belief in the formative influence of the environment, which was the distinguishing feature of Lamarck's view of evolution.

In general it may be said that, while there are many notable exceptions, the zoölogists tend to side with the neo-Darwinians against the idea of transmission, while the botanists, dealing with fixed and of necessity much more plastic forms, tend to go with the neo-Lamarckians.

In the opinion of the writer, Lloyd Morgan is by far the most satisfactory investigator and writer on this vexed question, especially in his Habit and Instinct, which should be read by every careful student of this subject. The inquiry is conducted to find out the truth, not to prove or to disprove the inheritance of acquired characters, and his conclusions as stated on pages 307-322 of that volume may well engage the attention of the thoughtful reader, whatever his personal views on the points in dispute. 
In every case, bird or mammal, plant or animal, the new individual begins with the fertilized germ, not at some later period called birth; and for our purposes the distinction should not be whether the variation was implanted before or after birth, but whether its cause was internal or external to the germ.

Attention should be fixed upon the germ plasm, the physical basis of life and the only known avenue of transmission from one generation to the next, and the distinction should be clearly made between variations due to changes in its structure from internal or other causes, and those changes of the organism due to influences exerted directly upon the organism during its development. As this discussion proceeds it should be clearly borne in mind that no character can be transmittid, no matter how strongly present, unless the germinal matter is in some way preziously affected. Nothing else passes over from parent to offspring, and no other medium of transmission is possible. The study is, therefore, clearly defined. Do modifications, as such, affect the germ directly, and so become transmitted; or, if not, do the same influences that affect the dereloping individual also affect the geminal matter in the same direction, giving all descendants an initial trend or modification similar to the one impressed upon the parent?

\section{SECTION II - EVIDENCE, FROM THE NATURE OF VARIATION}

In the opinion of the writer it is fundamentally wrong, both logically and biologically, to conceive of the individual as made up of two sets of faculties, - one inherited and the other acquired. The distinction not only does not rest upon good ground, but in its application to the facts of life it leads to most unfortunate conceptions and to most erroneous conclusions. It is this fundamental misconception of the function of the environment that is responsible for most of the foggy thinking which marks the contention over the question of inheritance of acquired characters, and which nowhere else bears such unfortunate fruit as in the field of practical affairs. In general evolution it does not matter greatly whether acquired (?) characters are inherited 
much or little, or not at all; the discussion is there largely an academic question. But in the fields and yards of the farmer it is the largest of all questions, and we are led to inquire sharply whether these distinctions are real; whether the differences between germinal (blastogenic) and acquired (somatogenic) characters are differences in kind or in degree; whether, in short, acquired characters, in the common acceptation of the term and in any true sense, exist at all.

The characters of the individual are the characters of the race. Careful observation will disclose the fact that e'cry' quality inherited or acquired by an adult indiaidual is posscssed in some degree by eacry other normal adult indiridual of the same race. All horses can trot some; all cows give some milk; all sheep bear wool of some color, length, or clegree of fineness; all hens have feathers; all men not idiotic can learn and speak a language; all men have some little (perhaps very little) musical ability, and all can learn to play the piano or the violin. The point here is not whether it is skillfully done, but whether it can be done at all.

It is a habit of speech to designate a low degree of quality by negative terms, and we say of the horse that trots but slowly or awkwarlly that he "cannot trot." We mean by that that he cannot trot well enough to make him valuable for this purpose. In the same way the man who "cannot sing" is the one whose singing we do not care to hear; the man who "cannot speak" is the one on whom we would not depend for the presentation of a difficult case. We do not mean of him that, like the oyster, he cannot convey information and is dumb because of the utter absence of the organs and powers of speech.

So these are relative terms, like "heat" and "cold," but our use of them in the absolute sense has built up in the popular mind an assumption that they stand for realities, not relative values of the same thing; just as the unlearned man supposes cold to be as real as heat, and black (which is the absence of all color) to be as real as white (which is the presence of all). Thus have we by our rerbiage elevated relative values to absolute distinctions in kind, creating misconceptions which we must first undo if we are to proceed safely in this study. All indiatumals 
of the serme race possess the seme charactirs; herein do racial valucs exist and horeby are racial distinctions established.

Variation practically confined to racial characters. In all the examples of variation that have been cited, - and they have purposely been many, - and in all the cases that occur in our fields and yards, idriation is confined to racial claracters. This is the experience of breeders everywhere. When dealing with cattle breeding all variation is of cattle characters. We do not expect and we do not find among cattle the appearance of characters belonging to horses, sheep, pigs, dogrs, or chickens, except as they are possessed in common. The same is true of other species, and when characters are possessed in common the variation in each case is well within the range of the species in question.

That is to say, variations in cattle all appear among wellknown and long-established characters that distinctly belong to cattle, such as the head, horns, legs, color, udder, quantity or quality of milk, etc. Among chickens the variations are among chicken characters, such as the shape and color of feathers; size, color, and quality of the egg; quality of meat, etc.

IVe find neither chicken characters appearing among cattle nor cattle characters appearing among chickens. The hen cannot give milk, nor can the cow bear feathers. There is no interchange of characters between species, either by birth or by acquisition afterward. Not only that, but even when the same character is possessed by two distinct species its arriations in cach are acll within the range of the particular spicies. For example, the legs of cattle and chickens are built upon the same general plan, but they have drifted far apart, and do not overlap even in their variations. The leg of a horse and the leg of a cow are on nearly the same plan, and yet no one would mistake the one for the other, no matter what the range of variation. Even color deviations are always within certain definite limits.

No such thing as an acquired character. Variation is, therefore, a contition, not a thing. It is the state of a racialcharacter, not the result of the introduction of a new one ; indeed, variation by the introduction of a positively new character is, if not unknown among us, a matter that belongs to general evolution 
and to the origin of races. It is not a matter that practically concerns the thremmatologist, who is working with established races and characters whose variations are fairly well circumscribed. The appearance of a positively new character among any of these races would be cause for profound astonishment. Under the present state of knowledge, and for our purposes, we may say that there are no such things as acquired characters, in any proper sense of the term. It is a figure of speech at best, and a most unfortunate one, at that.

By " acquired character," as the term is commonly employed, is always meant one of two things, - (I) differences in the degree of development of ordinary racial characters, or (2) the peculiar use to which the individual has put his natural endowments under his special conditions of life.

These are differences in degrec, not in kind. To speak of them as characters is to dignify them with a term whose meaning is eminently qualitatio' $c$, not quantitative, and this it is that has built up the false conception that individuals of the same breed or race differ from each other in something that is real; that individual differences are all qualitative, - whereas, within the race, they are quantitative merely.

To speak of these differences, which are only differences in degree of development of ordinary racial characters, or at most only differences in behavior of organs and parts known to be able to respond to various stimuli and to function somewhat differently under different conditions, - to speak of differences such as these personal acquisitions as acquired characters, is to use the term "character" in an unfortunate and singularly misleading sense.

Now a difference which is nothing more than a degree of development of a well-known character is not in itself a n'a' character. It is not in that sense an acquisition. It is more in the nature of a realization of what was before a potential possibility.

Neither is a habit entitled to the term "new," or "acquired," character. IIabit refers only to the customary use of natural faculties. Some characters, like those associated with the pro. duction of the gastric juice, for example, have but a narrow 
functional range and are quite out of control of the individual ; others, like the brain and the hand, are capable of functioning in many directions, - so many that no lifetime is long enough, or its needs and experiences varied enough, to exhaust their possibilities.

If the student hopes to follow the mazes of racial characters as they traverse the generations, like threads through the patterns of a fabric, he must not confuse either his terminology or his icleas by attaching so important a conception as "character" to what is nothing more than an indiridual manifestation of character development, or the particular personal use to which many racial characters may be put.

The faculties of the individual are limited in kind to the faculties of the race, and in degree to the intensity of their inheritance and the conditions of life. There is no case in which an individual of one race has picked up or otherwise acquired a character that belongs to another race and not to his own. All his achievements, all his capacities, are within the limits of his racial characters and the conditions controlling their development.

The individual is in actual possession of all the characters of the race. That this is true is shown by breeding experiences cverywhere, for in all cases the individual transmits to some degree all the characters of his race. Milk secretion is a character limited to mammals and functional only in the female sex, yet every dairyman knows that the bull will transmit milking qualities as successfully as will the cow.

Our experience with reversions - those "long-lost characters" that return to plague us - is convincing proof of the fact that every character of the race is potintially present in every individual, whether the degree of development be much or little. In no other manner could they be so long and so persistently preserved in the race. Their repeated appearance, long after they have ceased to be typical, only shows that they were never truly lost.

The individual is, therefore, born with all the possibilities of the race to which he belongs. Those which shall develop and fix the type will depend upon two considerations, - first, the 
relative intcusity of their inheritance, and second, the opportunities for their development.

The achievements of a race under one environment, therefore, cannot be considered as limiting, or even very closely indicating, its possibilities under another, and we nerer know the possibilities of a race until we have seen it bred and reared under a great variety of conclitions, - all of which is but another way of saying that rastly more is present and transmitted than even keen observers are aware of. Everything that belongs to the race is always present and is always transmitted in some decree. It is sheer business folly, as well as bad science, to conceive of characters as being lost for generations, then appearing again.

Whatever the individual comes to be, therefore, in his adult state, he is to be regarded as the repository of all the characters of his race, only a few of which have reached anything like their highest possible development in his particular personality, and many of which remain so undereloped as to be unnoticed perhaps throughout his entire lifetime. That they are potentially present, howerer, is attested by his descendants.

Highly differentiated races are so rich in possibilities and so great in their range of characters, that the lifetime of any individual is too short, and his environment too circumscribed, to realize more than a fraction of his possibilities; but he transmits the remainder as an undeveloped heritage to his descendants. Ithat now', if any', is the offect upon transmission, of the particular dericlopment that he has realiacd in his ow'n personerlity? IVill the spicial characters that he hers strongrly deacloped be transmitted with increased intensity becanse of their recent cutreme derelopment, or will this derelopment hat'e no cffect upon thcir initial poacrs in the ncit gencrations? This is the one question we repeatedly ask ourselies, for it is the one we most desire to answer.

Degree of development depends upon both germinal and environmental influences. The erolution of a mature and adult individual from a fertilized germ is to be regarded as essentially a process of diclopment. It has been shown that all differences between adult individuals of the same race are due to the degree of development which the racial characters have been able to attain. 
This, and not the introduction of new characters, is the basis of variation between individuals of the same race. Differences between races may be either qualitative or quantitative, or both; but differences between individuals of the same race are essentially quantitative.

Quantitatively, that which is transmitted from parent to offspring is a certain capacity for development. But possession of the capacity for development is no guaranty that development will follow. Whether or not it will follow depends upon the nature of the conditions of life, and whether they will afford the opportunity for development.

The limits of development of any racial character are fixed, therefore, by two factors : first, the initial impulse born into the individual, the intensity of which is a matter of breeding; and second, the attitude of the environment, whether favorable or unfavorable.

Manifestly with any individual the highest development will be in those characters whose inherited intensity is strongest and for whose development the environment is most favorable. Next in order will come those with high intensity but which are forced to struggle against an unfavorable environment, as well as those whose inherited intensity is less. Weakest of all will be the development of those characters whose inherited intensity is low and for which the environment is especially unfavorable. As we have seen, no matter what may be the environment, no development will take place except along lines that are clearly recognized as within racial possibilities and therefore due to transmitted impulses. These contingencies cover all cases of variation between individuals, and the real question before the student is not whether acquired characters are inherited, but it is this: Will the extreme development of a racial character under unusually favorable conditions of life augment even to the slightesi degree the transmitted tendency for dewelopment in the ncrt goncration? or are intensities the product only of changes internal to the germ plasm?

Stated more broadly the question is this: Does the dcrclopment attained by the individual influence his powers of transmission? This question should stand out clear-cut in the student's 
mind. It is not whether an individual with strong tendencies is a better breeder than one with weaker tendencies, - that is conceded; it is not whether a race living under a favorable environment flourishes better than one living under hard conditions, - that is conceded, too, for natural selection is inevitable; but the question is, whether the indiaduat will be the better or the worse as a breeder because of the special development he has acquired.

The answer to this will decide the question whether we shall keep our meat-breeding animals in high or in moderate flesh; whether we must develop the speed of our racing stallions and mares; whether a given sire or dam is a better breeder after speed is developed than was the same individual when green. It will determine the whole matter of the importance of deicloping breeding stock, not only as a means of increasing natural capacity, but as a means of intensifying the powers of transmission.

Fortunately we are not without facts bearing upon this vexed question; but the whole field is exceedingly difficult, and reliable evidence is eagerly sought. It is the more difficult to secure because of the ever-present and alway's powerful influence of selection.

The particular modifications (acquired characters) that have been most discussed and whose transmissibility has been advocated on the one hand or denied upon the other are of four distinct kinds :

I. Mutilations due to injury or destruction of racial characters after they have reached full development.

2. Habits of life arising out of the exigencies of existence.

3. Structural peculiarities due to use and disuse.

4. Adaptations to climatic conditions.

These are commonly all considered as acquisitions in the sense of additions to racial characteristics. In the view advocated by the writer they are all reducible either to different degrees of derclopment of racial characters, or to the uses to which these are put under the conditions and exigencies of life. They will be considered in the order named, always with the question uppermost in mind, Are they transmissible? 


\section{SECTION III - EVIDENCE FROM MUTILATIONS}

Mutilation is the forcible removal of a part after it has developed, or at least the destruction of those parts which are fully endowed with the power of complete development. Unfortunately, in this field the most absurd stories have gained credence, and their popular acceptance has done much to obscure the whole subject. Some one owned a cat whose tail was pinched off in a door, and straightway all her kittens were tailless. A few semi-traditional stories like this are made the foundation for believing in the transmission of mutilations, the facts being forgotten that for generations it has been the custom to remove the tails from lambs, with no sign yet of tailless sheep as a result, and that circumcision has been practiced by many tribes from the remotest times, and by the Jews certainly for four thousand years, apparently without effect upon the natural development of parts. Certainly, if any effect has been produced, it is not evident, and is so small as to be classed among negligible quantities, falling entirely outside the field of practical results.

The tail, being a portion of the vertebral column, might be expected to long resist all influences toward its suppression; but the prepuce is an unimportant and recent structural addition, yet still it lingers, despite persistent and systematic removal by force.

The question lies deeper than the surface. A mutilation, like any other difference, in order to be transmitted, must first effect the germ plasm, which is the only material carried over. If Darwin's theory of gemmules were true, then it might be conceivable that a defective part would no longer produce its share of the germ plasm, and that it would certainly disappear from the race, and that at once. The fact that persistently mutilated parts do not disappear is good proof not only that mutilations are not transmitted, but that the theory of gemmules is incorrect.

For the most part, belief in the inheritance of mutilation has rested, not upon experimental evidence, but upon instances in which natural deformities in the offspring correspond to mutilations in the parents. Such correspondence is assumed to be 
proof of a causative relation, so quick are we to accept for fact that which is not only plausible but startling. In this way a mass of evidence (?) has accumulated on this subject second in amount only to that bearing upon birthmarks and upon the "control of sex."

The law of chance. Before subjects of this character can be properly studied, the operations of the mathematical law of chance must be comprehended and their effects deducted.

If we toss a coin the odds are even that "heads "will be up; they are also even for " tails up." There being but one alternative, either heads or tails is certain to appear. When the next toss is made the odds are again even, but there is no cansatize relition between the first and second events. They may agree or they may differ; that is, both may be heads, both may be tails, or one may be heads and the other tails.

Successive tosses will give rise to an extremely irregular series, as may be shown by trial. However, if the series be continued indefinitely and tally be kept, it will be found that in the long run the heads and the tails will be equal. When the equality will first occur is entirely uncertain. It may be at the second throw or it may be at the hundredth, or even later, but it is certain to come.

The roulette wheel, as commonly used, is made up of thirtyseven color spaces, eighteen red and nineteen black, or the reverse. The wager is laid upon the number or the color on which the wheel will rest after a supposcdly impartial spin. It is evident that the probability of its resting upon a particular number is but one in thirty-seven if the wheel is mechanically perfect, and that the chances of its resting upon a particular color are not quite even. This difference of nineteen to eighteen constitutes the "advantage" of the owner over the player, and shows the hopelessness of attempting to "break the bank." This margin of one out of every thirty-seren bets is over 2.5 per cent of the business and constitutes the assured profit in a game of chance conducted honestly on this plan, - which is the one in use at Monte Carlo, the greatest gambling house in the world. ${ }^{1}$

1 See Pearson, Chances of Death, pp. 42-62. 
The successions of red and black representing gain and loss are so irregular and so confusing that the player fails to detect the ratio of more than 5 per cent that is against him, and consequently does not realize that the longer he plays the more certain are his losses. Nor does he realize that in the long run nothing is more absolutely certain than the law of chance. The deviation is not great at any point after the first few throws, and herein lies the first leceptive quality of all games of chance.

If the letters of the word "incomprehensibility" be tossed into the air in such a manner that they must fall into a line, the chances of their falling in the proper order to spell the word correctly are exceedingly remote, y'ct it is bound to happin if the trials are long enough continued.

These simple facts teach us not to attach too much importance to occasional occurrences, however strange or apparently improbable. They teach us, too, that there may be no special canse at the bottom of the occurrence beyond the mathematical law of probability. The tossing of coins shows why it is, for example, that every theory for the control of sex that ever has been or ever can be invented has been repeatedly verified. There is but one alternative, and every assumption of cause, however absurd, is certain to come true (?) half the time, which is sufficient proof for most people who depend upon memory impressions rather than upon absolute records.

Proof by the method of instance is therefore extremely hazardous. Something more than the mere fact of coincidence is necessary in order to establish a causative relation with any very high degree of certainty.

When, therefore, a deformity in a child corresponds to a mutilation in a parent we are not warranted in at once assuming a causative relation. We are to remember that deformities of all kinds are comparatively common; that mutilations are exceedingly so; that frequently a mutilation will resemble a natural deformity or an injury; and that occasionally, under the laws of probability, the mutilation of the parent will resemble the deformity in the offspring, thus suggesting direct transmission. We are to remember, too, that the law of chance must first be satisfied before we can assume causation. 
The most direct way of procedure is, however, not to endeavor to eliminate the law of chance, but, by direct experiment, to learn whether a sufficient number of occurrences can be established to clearly exceed in number any possible coincidence.

Experimental evidence on inheritance of mutilations. The facts just given show conclusively the hazard of framing theories on chance occurrences, and demonstrate the practical worthlessness of all but experimental evidence in the study of inheritance.

Unfortunately but little evidence of this kind is at hand, and, so far as is known to the writer, that which is at hand is confined to artificial injuries to the nerve, with exception of that already cited in such practices as docking and circumcision.

Romanes outlines seven classes of abnormalities that appeared in the offspring of guinea pigs corresponding to those artificially produced in the parents by Brown-Séquard and his assistants. They are in brief as follows: ${ }^{1}$

I. Appearance of epilepsy, when parents have been rendered epileptic by an injury to the spinal cord.

2. Same, when the injury had been to the sciatic nerve.

3. Change in the shape of ear in animals born of parents in which such a change was the effect of a division of the cervical sympathetic nerve.

4. Partial closure of the eyelids in young born of parents in which that state of the eyelicls had been induced by section of the cervical sympathetic nerve or the removal of the superior cervical ganglion.

5. Exophthalmia in young born of parents in which a similar protrusion of the eyeball had been produced by injury to the restiform body.

6. Gangrene of the ears in animals whose parents' ears had been affected by injury to the restiform body.

7. Absence of toes in young whose parents had eaten off their toes, which had become "anæsthetic" by reason of the section of the sciatic nerve alone or of that nerve and the crural.

8. Various morbid states of the skin and hair corresponding to a similar condition of the parents which had been brought on by an injury to the sciatic nerve.

1 Romanes, Darwin and After Darwin, II, I03-122. 
It is notable that all these experiments are based upon injury to the n're' and are of a degree of severity likely to affect the entire organism seriously. If, however, it is true that injury of any kind in the parent leads, as Brown-Séquard and Romanes evidently suppose, to corresponding deformities in the offspring; the fact is exceedingly significant. This is a field, however, quite different from that of ordinary injuries - such as the removal of a horn or a tail, producing no constitutional disturbance and leading to no organic changes. Further experiments are greatly needed to confirm, deny, or modify the results of Brown-Séquard. In the meantime it seems almost incredible that so much erroneous tradition should have grown up surrounding this matter of inherited mutilations, especially when the world for unknown generations has almost inrariably seen perfect children born from one-armed, one-legged, and otherwise mutilated parents. Indeed, if offspring inherited the ordinary mutilations of their parents, the world would have become long since a collection of monstrosities which would put to shame the rare specimens now collected in dime museums.

Inheritance of disease. The old tradition of inheritance of disease is long since disproved, and those diseases once thought to be inherited are now known to arise not from inheritance but from infection after birth, which for obvious reasons is extremely easy between parent and offspring.

The weakening effect of wasting diseases upon the parents, and the influence of this weakening upon the constitution of the offspring, inducing predisposition to cliscase, is, however, quite another matter. That many of the effects of such a disease of the parents will work injury and weakness to the offspring will be readily admitted; that such offspring will be the more susceptible to attack from diseases of all kinds will hardly be denied; but whether it will be peculiarly suscoptible to the special discase that arought havoc aith the parcut is a question on which we need much more evidence.

$\mathrm{Up}$ to date this point has not, in the opinion of the writer, been established. Although it is true that certain family lines are specially susceptible to tuberculosis, it is not yet shown whether this susceptibility is the result of inroads of this special 
disease or whether it is caused by weakness in family lines destined to disappear, and for whose extinction tuberculosis is the special agent of natural selection. The weight of evidence inclines the writer to a belief in progressive immunity from diseases of this class, - a matter touched upon under the subject of acclimatization. That the sparined mare will not transmit her spavin is as fortunate as it is true, but the question lying back of this fact is, Why was she spavined? Is the injury an evidence of weakness, or is it only the result of an accident, such as might have happened to any horse? If it is the former, then the weakness, not the sparin, will be transmitted; if it is the latter, there is in all probability not the slightest danger. If injuries of this sort were transmissible, our horses would long since have acquired a collection of sparins, ringbones, splints, sidebones, and curbs such that no leg could hold them. It is far from the purpose of the writer to advocate the use of defectives as breeders, but we cannot close our eyes to the fact that a mare which has seen hard service and bears the marks of it is in all likelihood a better breeder than another that has never been put to the test, no matter how clean and free from blemishes the limbs of the latter may be. The stubborn fact is that the risk of accident is so great that a horse put to hard service is certain to be blemished sometime ; and so far as present knowledge goes, she is as good a breeder after the accident as she was bifor, which is far from saying that every blemished horse is fit for breeding purposes.

The writer is clearly of the opinion that, even with the experiments of Brown-Séquard in mind, the evidence warrants the conclusion that ordinary injuries to the body are not transmitted to the offspring. Whether different results follow those profound injuries that reach the nerve centers and work constitutional changes in the organism is a matter on which we must await further evidence.

Mutilations have reference to characters already fully cleveloped, and therefore fully provided for in the germinal matter. If violent removal is to lead to their suppression, it must lead to it through some sort of retroactive influence affecting the germ in exactly the proper particular and no other, - a presumption 
that is inconceivable under any law of physiology that is known or that can be imagined.

The non-development of parts is another and quite a different matter. If the non-development be due to a defective germ, it of course does not come under the present inquiry. If, however, it be due to an injury at an early stage, resulting in arrested development, it may or may not be equivalent to a mutilation. If the non-development be due to the destruction of cells, as in chemical dehorning, it is to all intents and purposes a mutilation, as conditions were present for full development. If the non-development be due to malnutrition, the case is different, and belongs among cases to be considered later.

The essential weakness of the whole theory that mutilations may be transmitted lies in the fact that the characters in question are present, fully developed and functional, until removed by violence, all of which is conclusive evidence of a natural capacity for complete development. In view of our inability to conceive how the removal of a part can possibly affect the corresponding portion of an undeveloped and even unfertilized germ; in the absence of reliable experimental data and with the certainty that, were the injuries due to the multitude of accidents occurring to all forms of life transmitted all species would soon be disfigured by an overwhelming mass of inherited mutilations, - in view of all these facts we are certainly warranted in feeling assured that injuries to the fully developed body are not transmitted.

\section{SECTION IV - EVIDENCE FROM FOOD SUPPLY}

This is a very different matter from mutilation. Of all the conditions of life this is, par crecllcuce, the limiting element, not only in body building and in functional activity, but in constitutional vigor as well.

That the amount of food available is a controlling factor in the development of size is a matter too well known to require discussion. In the presence of abundant food, animals and plants of all species attain their maximum size and their maximum development in all respects. If the supply be limited, the effect is invariably seen in under-development, even though the total 
amount consumed is many times greater than that actually used in body building.

In acclimating to a shortened food supply the animal or plant is forced, not so much to make more economical use of what it can obtain as to reduce the scale of living and actually to accomplish less in the way of growth and functional activity generally. A starving animal or plant will make the most of all available food, but in addition the animal will replace a large share of the dry matter of the body with water and reduce its activity to a minimum before it succumbs, and a starving plant will still put forth new leaves, using the substance of the old to nourish the' new.

If the shortage in food comes before development is complete its effect is seen in under-development, or possibly in arrested development, - recognized by the farmer under the term "stunted." Sometimes the condition is only temporary, but more often it is permanent, when no amount of food later in life will avail to repair the damage done by shortage during development. Farmers accordingly recognize the period of growth as a "critical period," and uniformly say that if any live stock is to be short of feed let it be the older ones. Sad experience has taught the irreparable evil of shortage in food during development.

Under-nourishment strikes at the very root of life as well as at development. What is true of individuals seems true of races. Under-nourishment is followed by a lowering of tone and a lessened rate of living, while full feed and maximum conditions of life generally induce great protoplasmic activity and rapid cell division, resulting in maximum size and maximum functional activity in all parts of the structure.

All experience goes to show that weakened parents, plant or animal, give rise to young that are low in vigor and slow of growth. Seed corn that is below the normal in vitality, though it may germinate, will give rise to weak and slow-growing plants.

There are all degrees of vigor and intensity of the vital processes, from zero up, and nothing seems more potent than the food supply in influencing this matter that lies at the basis of all development and all functional activity. 
As temperature is an all-pervading influence with smaller organisms, so is food an all-pervading influence with all organisms, large or small. Without doubt it exerts a controlling effect upon the quality of germinal matter produced, as it does upon its quantity, and upon the maximum or minimum development of the body.

Constitutional vigor, which is the most valuable asset of any plant or animal, is a heritage whose seat is in the germ from which it was developed. Such a germ could be produced only by a vigrorous, healthy, well-nourished parent. Anything which weakens this parent constitutionally, which lowers its tone, reduces its vital powers, and lowers its rate of living, must of necessity affect the quality of any germinal matter it may produce and the constitutional vigor of its descendants.

We do not permit this condition to any large extent in our domesticated species, plant or animal, for we realize too well the consequences, but we have only to hol among the underfed classes and races of humans to see the evil effects of malnutrition in weakened constitutions, low vitality, predisposition to the ravages of disease, and general inefficiency wherever any great functional activity, physical or mental, is required. That this condition is transmitted does not admit of a reasonable cloubt.

On the other hand, races that are well nourished for many generations undergo maximum derelopment. This has been the experience with all domesticated animals and plants. For the most part they have been provided with all the food they needed, and they have responded with a development such as never came to them under natural conditions.

Nature never produced such specimens as our modern beef or milk breeds or our draft horses. Our achierement as breeders is due, therefore, to something besides selection, and we are forced to one of three conclusions :

I. That nature never produced a perfect specimen; that is, that natural conditions were never sufficiently farorable to allow the individual to realize the full development to which his natural endowments entitled him.

2. That with each increment of gain through selection, establishing a higher general average, a new "center of variation" 
was also established, which was bound in time to produce better specimens than ever before.

3. That there has been direct transmission of the increased vigor and powers of nutrition and growth that come from full feed.

The first conclusion is unthinkable. Nature must certainly have produced, occasionally at least, perfect specimens of their kind, and the belief that this is so is favored by the fact that wild things do not respond generously to full feed.

The second is without doubt a real fact in evolution, difficult as it is to comprehend. Later, in statistical studies, it will be found to our satisfaction that as the average is raised by selection ncu ialucs appear at the top, - a fact on which depends, without doubt, a large share of our improvement of all species.

And yet we cannot fight off the conviction that here, at this point, lying so close to the very springs of life, the absolute condition of life - nutrition - exerts a controlling influence upon that mysterious force which we call the vital principle, and whose relative strength we measure by such terms as "constitution" and "vigor." That vigor, or the lack of it, is a transmissible character no one will deny, or even doubt; and it is the firm conviction of the writer that when this vigor, or scale of living, has been strengthened or weakened from any' cause, the power of the individual to transmit a vigorous constitution to its offspring will be enhanced or lessened accordingly, and that when the last word shall have been spoken upon the disputed question of inheritance or non-inheritance of acquired characters it will be found to square with this fact.

The writer desires, above all things, not to dogmatize. Facts, not opinions, are needed in these uncertain fields; and yet, until the partisan adrocates of the opposite sides of this question will divide the question and discuss separately the three or four distinctly different issues involved, - until that time, practical breeders must not be deceived or lulled into carelessness by the dictum that "acquired characters are not transmitted."

Increased development above the natural in one form or another is the principal object in all improvement, and a large share of the possibility of such increased development lies in 
this matter of comstitutional vigor, which so largely depends upon nutrition that the breeder can afford to make no mistake at this point.

Inflences that strike at the root of the vital principle, whatever that may be, are far reaching in their consequences. To maintain the vital powers at a maximum is one of the prime objects in all breeding, and that this is to a large extent a matter of nutrition is a fact that should be fully appreciated by him who hopes to maintain unimpaired the valuable racial characters for which he breeds his animals and his plants.

There is no better maxim for the breeder than this: the results of good feed are transmitted to the offspring in the form of a vigorous constitution and large powers of assimilation and of service.

\section{SECTION V - EVIIDENCE FROM ACCLIMATIZATION}

It is a well-known fact that the inclividual acquires by experience a high degree of resistance to temperature, poisons, or other adverse conditions of life; that this modification is more or less permanent with the individual and that in grood time the race as a ahole becomes acclimatized to changed but persistent conditions. ${ }^{1}$ Is this race acclimatization in any way the result of transmission of the acclimatization of the individual ?

1 It should be clearly understood that "acclimatization" is not confined to adverse conditions; it may relate as well to adaptation to improved conditions, such as increased food supply. Indeed, the term is intended to cover accommodation to any change in external conditions of life, whether favorable or unfavorable, gradual or sudden.

As is well known, acclimatization is more successful if the subjection be gradual; but, in any event, one of two results will follow if the individual is not killed in the process: (I) it will become permanently altered, and will therefore discharge its functions in a modified manner; or (2) it will acquire by experience so high a degree of resistance as to be able to resume its usual activities after the first disturbance and afterward to discharge its normal functions in spite of adverse conditions.

There are thus two kinds of acclimatization: one in which the functions or activities are modified, the other in which the individual succeeds in resisting the changed conditions, and therefore in preserving its normal functions. Of the two, the latter is perhaps the more common. 'The former betrays the more constitutional change, the latter the greater elasticity in organization. 
To determine whether in the acclimatization of a race agencies are involved other than selection operating upon individuals, it is necessary either to eliminate the results due to selection or else to discover cases in which it does not occur. While the first is all but impossible of accomplishment with any feeling of assurance, the second is, in the opinion of the writer, entirely feasible, especially in certain lines.

The importance of the whole question and the difficulty of securing reliable data are sufficient excuse for introlucing a somewhat full discussion of certain topics which afford evidence upon the question at hand.

Extent of acclimatization. The power of the individual to adapt itself to changed conclitions is something marrelous, as has been seen under the subject of causes of variation and in the discussion of relative stability. This same clasticity of organization is characteristic of races as a whole. By means of this adaptability many species of both animals and plants have totally changed their habitat, and with this change have undergone the most sweeping alterations. The whale is dereloped from a land mammal and is suffering degeneracy as a consequence. Swine have been adapted from a diet of roots and flesh to one mainly of grain. Horses and cattle in their wild state subsisted entirely on pasture, but with us their diet is from 25 to 75 per cent grain. Sheep are mountain animals, but they have been adapted to the richest pastures and the lowest plains. The turkey, native to North America, is making his way over all the earth, as chickens have scattered broadcast from their native habitat in southeastern Asia.

The potato, native to the mountains of Peru, is now grown everywhere in temperate regions, though it never succeeded in acclimating in the tropics except in high altitudes. There are evident limits, or else the possibilities are not yet exhausted.

Corn (maize) will endure but slight change in locality without suffering seriously, yet after a few years it appears to recover tone and succeed. In this way the culture of this crop has been gradually moving northward in the United States, until now it is fully acclimated in regions in which a quarter of a century ago its culture was impossible. So sensitive is the corn plant to 
climatic change, but so readily does it adjust itself, that new varieties can be introduced successfully, provided they are given consiclerable time to acclimate on a small scale before their production under field conditions is attempted.

Wheat has extended almost over the earth, except in extreme latitudes. The varieties have become so well fixed in the various regions that when brought from long distances they seldom thrive at first. Some strains never succeed, but others acclimate perfectly in new localities. Varieties may be changed readily from spring to winter sorts, and the hibernating habit become fixed, as in other biennials.

Imported animals are seldom fertile until acclimated. It is said, however, that certain breeds of the dog never acclimate in India sufficiently well to preserve their distinctive racial characters. On the other hand, species occasionally prosper better in new localities than in old ones. Generally speaking, distance makes less difference than altitude, temperature, sunlight, and food supply. The evident principle involved is the influence, favorable or otherwise, of certain elements of climate upon the development of racial characters.

Acclimatization to temperatures. It is a well-known fact that if we bring together and put under the same conditions plants or cuttings of the same species but grown in different latitudes or altitudes, and therefore habitually exposed to widely different temperatures, those from the more northern localities and the higher altitudes will be the first to put out bud and leaf.

De Candolle of Switzerland, and Bailey of New York, have both conducted extensive experiments in this direction, and with the same results. ${ }^{1}$

The former took, among others, cuttings of the poplar, the tulip tree, and the catalpa, both from Montpellier and from Genera, and planted them at the latter place in glasses of water with sand at the bottom. In every case those taken from Geneva, the colder locality, leafed out first. The difference in the case of the poplar was about tiventy-three days, in the case of the tulip tree about eighteen days, and in the case of the catalpa twenty days.

1 Bailey, Survival of the Unlike, pp. 296-301. 
Montpellier is situated in southern France on the Mediterranean, within a few miles of the coast; Geneva is at the head of Lake Geneva in Switzerland. The two points are, therefore, separated by about two and a half degrees of latitude, with a difference of about twelve huncled feet in altitude, and with some possible differences in humidity. The main difference, however, is one of temperature; and although both the tulip tree and the catalpa had been originally introduced from America, they had evidently become so physiologically modified by their new surroundings as to exhibit substantial diversity in their reactions to the same temperatures.

Bailey found that cuttings of Lombardy poplar from northern Maine unfolded their buds two days earlier than similar cuttings taken at Ithaca. He placed under the same conclitions cuttings of the Concord grape taken from Maine, New York, and southern Louisiana, and found that they leafed out in the order of their locality, beginning with the most northerly. He reports similar results from potatoes.

These were cuttings, and the experiments show that the individual plants from which they were taken had become thoroughly acclimated. Whether any selection had been involved in this acclimatization we do not know, and we cannot tell, or even infer, from these experiments whether or not the sceds grown from these plants would have behared in the same way. What is shown is that the individual plants were so thoroughly acclimated as not to respond in the same degree to identical temperature conditions.

The same behavior has been shown, however, with all seeds that have been tried. Bailey found that corn (maize) grown in New York germinated more readily than that grown in South Carolina or (as shown by the following table) than that grown in Alabama :

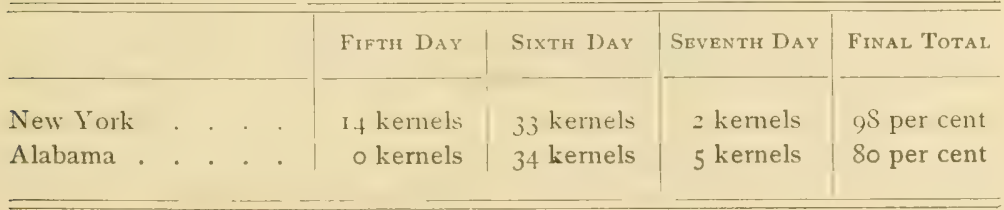


The corn from New York was evidently the better seed, because its final percentage of germination was higher.

This fact might account for some portion of the difference in promptness of germination, but we are informed that during the entire month of the experiment the plants from the northerngrown seed were the "largest and most vigorous of any." They were evidently ahead in their development. Bailey remarks that not only "corn gave the most marked results in favor of the northern samples, but there was generally a similar difference in the watermelons and beans, with not one contrary result."

Bonnier ${ }^{-1}$ made observations with Tcucrimm Scorodonia (wood sage) for eight years. When grown on the high altitudes of the Pyrenees it produced shorter stems, darker-green and more hairy leaves, and more compact inflorescence than when grown on lower land. Seeds gathered from these plants and sown in Paris, after thre years in the new habitat "produced elongated stems, with less hairy and brighter-green leaves, or plants very similar to those from seeds obtained in the neighborhood of Paris."

The same experimenter collected specimens of other species, both in alpine and in arctic regions, and found that those of the latter region had "more rounded cells" and larger intercellular spaces. $^{2}$

That races as well as individuals acclimate to temperature is easily shown and well known, but as the process is generally accompanied by selection there is difficulty in finding instances free from its influence, and it is practically impossible to assess and deduct its results. It is not impossible, however, to find cases fairly satisfactory.

The origin and history of the Shetland pony is not known, and yet it is practically certain that its small size is partly the result of a cold climate and scanty feed. Under these adverse conditions there must have been vigorous natural selection. We shall learn in the chapter on "Heredity" that this small size could doubtless be accounted for by progressive selection, but the question here is whether progressive acclimatization is not also involved.

1 Vernon, Variation in Animals and Plants, p. 312, from which the account is taken.

2 Ibid. p. $3^{1} 3$. 
All things considered, the conviction is forced upon us that the Shetlands suffered a progressive diminution because of low temperatures or short feed, or both, or else that these northern forms, living under hard conditions, lagged behind their more fortunate neighbors in the general increase in size that has attended the evolution of the horse kind generally.

The temperature of hot springs varies all the way from $50^{\circ}$ to $98^{\circ}$ C. ${ }^{1}$ So far as known they are all inhabited by living organisms. The protoplasm of ordinary plants and animals cannot endure a temperature above $45^{\circ}$. Death quickly follows the attempt to raise it much above this point, and the nearest relatives of these hot-springs species are no exception to the general rule.

Yet the fact remains that the hot springs are inhabited, and by creatures so small that their temperature must be the same as that of the waters in which they live. How have these waters become peopled with organisms living in temperatures ten to fifty degrees higher than could be enclured by the stock from which they must have descended ? ${ }^{2}$

No amount of selection could account for the fact, for there are no other known species living in an environment approaching these temperatures. There must have been progressize dcvelopment of some fashion and from some cause.

Upon this point the experiment of Dallinger is both significant and valuable. ${ }^{3}$ He reared Flagellata in an oven where control of temperature was absolute. Beginning at $15.6^{\circ} \mathrm{C}$, he took four months in which to raise the temperature through $5 \cdot 5^{\circ}$, a precaution now known to be unnecessary, as Flagellata will endure a quick rise to $2 \mathrm{I}^{\circ}$.

When the temperature reached $23^{\circ}$ the organisms "began dying, but soon ceased, and after two months the temperature was raised half a degree more." After a time it reached $25.5^{\circ}$, when they again began to die, and for eight months the temperature could not be raised even a quarter of a degree above this

${ }^{1}$ C. B. Davenport, Experimental Morphology, Part I, pp. 250-251.

2 The temperature varies slightly in different parts of hot springs, being lower near the edge.

${ }^{3}$ C. B. Darenport, Experimental Morphology, Part I, pp. 252-254: Vernon, Variation in Animals and Plants, pp. 379-3So; Journal of the Royal . Microscupical Society, VII, I9I. 
point. It seemed at this time that a "stationary point" had been reached, but ultimately Dallinger was able to make slight additions to the temperature, and, "proceeding by slow stages" and for "several years," he succeeded at last in reaching $70^{\circ}$, when the experiment was terminated by an accident.

The exact length of time employed in this experiment is not stated, but it is supposed by Vernon to be approximately six years. Thus these organisms were bred for many generations during the experiment, and it is really a case of race acclimatization. The organisms were monads, it is true, which multiply by fission, so that, as Davenport states, "the high temperatures acted upon the same protoplasm at the end of the experiment as at the beginning." Is there any reasonable cloubt that this is the process by which organisms of this character have gained access to our hot springs even under natural conditions?

In this experiment three points are noteworthy:

I. There were certain "sticking points," so to speak, that were difficult to get over, but after these were passed, additional increase of heat was easily endured. The temperature of $25.5^{\circ}$ was one of these sticking points.

2. It was found that the process of acclimatization did not become gradually slower and more difficult with the higher temperatures, for $25.5^{\circ}$ was the most difficult temperature encountered, requiring eight months to surmount, while the rise from $41.7^{\circ}$ to $58.3^{\circ}$ was made in seven months, and that from $6 \mathrm{I} . \mathrm{I}^{\circ}$ to $70^{\circ}$ in a few months (number not stated), ${ }^{1}$ showing that the limits of variability were not reached, and suggesting that the experiment might have been continued much longer and the increase pushed much farther.

3. Organisms acclimated to $70^{\circ}$ died off when returned to the original temperature of $15.6^{\circ}$, showing that the modification of the protoplasm was not only profound but also permanent. In other words, here is a species whose temperature has been raised through so many degrees, and its protoplasm so altered, that it can no longer endure its original normal temperature. It has been taken entirely out of its field and placed in another so far removed as to have no connection with its former state.

1 Vernon, Variation in Animals and Plants, p. 380. 
It is true there were deaths during this process, but the scliction was insignificant, and the conviction is absolute that the result was in no large sense due to the selective process.

In the opinion of the writer this is proof absolute of one of three things:

I. Either the direct transmission of modifications, - a thing not difficult to imagine considering the mild sort of transmission involved in reproduction by fission;

2. The direct action of the temperature upon the constitution of the protoplasmic basis of life, - a contingency not specially applicable in this case, where the germ undergoes but slight development and there is no practical distinction between germ plasm and body plasm;

3. Or, if of neither of these, then it is proof of what may be called progressive variation, in which, with a species living under changing conditions, new centers of variability are being constantly established.

The significant point is that in this instance the deviations are due not to selection but to the direct action of the environment, and we are left to explain cases of this kind by assuming either that the modifications are themselves directly transmitted, or else that the external conditions have influcuced the gcrm as well as the body.

This is not difficult to believe of such all-pervasive influences as temperature, and it may well be that certain outside influences can make themselves felt in this way when others which, from their nature, may affect the development but cannot reach the germ, will not make themselves permanently felt except through individual adaptation and selection.

Acclimatization to poisons. That individuals acquire a high resistance to poisons has already been shown. Speaking generally, living protoplasm will soon adjust itself to any chemical influence not fatal or so extremely injurious as to overcome its powers of adaptability. Physicians change remedies frequently for the reason that they soon lose their characteristic action.

The acquired resistance of man to arsenic and other poisons, of mice to ricin, of the horse to the filtrate of the diphtheria bacillus, of the rabbit to that of hydrophobia, and of animals 
in general to poisons of all sorts gradually administered, is well known. ${ }^{1}$

So far as we are able to judge, immunity to infectious diseases is produced in the same way. One attack of certain diseases serves to render the inclividual immune through life. The question that interests us at this point is this: Is this immunity in any sense, or to any degree, transmitted to the descendants?

It is claimed by some that if a sow recovers from a case of hog cholera suffered while carrying young, the pigs will be born with a high legree of resistance, if not absolute immunity; the idea being that they acquired in utro from the blood serum of the mother the same kind of immunity that could be produced by inoculation.

There is too little experimental evidence as yet, and we know too little of the real nature of acclimatization, to warrant positive conclusions. What is known, however, is sufficient to raise some interesting and exceedingly suggestive questions.

If immunity can be produced in the mother by the repeated injection of the chemical products of disease, and if such immunity be permanent, then why should not the young, whose blood serum is derived from the mother, be also of the same character? Whence come our "natural immunes"? are they mutants, or are they the products of immunizing influences from the parentage? Recent investigations seem to indicate specific qualities in blood serum, ${ }^{2}$ and it may very well be that "blood relationship" means more than we have hitherto supposed.

To what extent immunity is purely a chemical question, and to what extent it is connected with the power of the white corpuscles to attack and digest invading organisms, we do not know. In so far as it depends upon or affects the serum of the blood it may well be a transmissible quality from the fomalc mammal if not from other parents.

1 C. B. Davenport, Experimental Morphology, Part I, pp. 28-32; Vernon, Variation in Animals and Plants, pp. 386-387.

2 When the blood serum of one species is injected into the veins of another, the most injurious effects are said often to follow, and the so-called precipitin test seems to establish the fact that differences in blood serum of different species are profound. See Blood Immunity and Blood Relationship, by Nuttall, reviewed in Science, October 28, 1904 . 
Chemical action of normal secretions. In the opinion of the writer, those who discuss the subject of the transmission of modifications (acquired characters) as if it were a single issue, and dismiss it in toto as impossible upon the theoretical ground that no such modifications could by any manner of means affect the germ plasm - those who discuss and dismiss the matter in this manner commit a fundamental error in overlooking the fact that, as a whole, this is a broad question, or rather a series of questions, and that the living organisms, exposed as they are for generations to outside conditions absolutely essential to their existence, present many points of contact in which they are exceedingly susceptible to influence. They make the fatal error, too, of failing to distinguish between those circumstances that affect only the externals of the body and those all-pervading influences that affect the very constitution of the organism.

For example, it is now well known that the perfect working of the body as a whole depends upon the presence of specific secretions of certain organs, many of which were once thought to be functionless. Destruction of the thyroid gland at once arrests not only the physical but the mental derelopment. In children its degeneration results in retarded mental development and even in idiocy, a calamity that can be ameliorated, and even averted, by the injection of thyroid substance of animals. Baumann found that the secretions of this gland are characterized by the presence of iodin, which is found nowhere else in the body. ${ }^{1}$

On this same general question Vernon, after speaking of the frequently fatal effect of removing the thyroid gland, unless the animal be fed thyroid substance, remarks as follows : ${ }^{2}$

Extirpation of the suprarenal glands results in much more speedy death, and here again the injection of extracts may delay the fatal issue. Extirpation of the pancreas causes the production of severe diabetes, and ultimately death, but such an effect may be avoided by the grafting of a portion of excised gland in the peritoneal cavity or the tissues... A Again, extirpation of the total kidney substance of the dog leads, not to a diminished secretion of urine, but to a largely increased secretion, accompanied by a rapid wasting away, which soon ends fatally. Hence the kidneys may possess an influence on the metabolism of the whole body, as well as their

1 I oeb, Physiology of the Brain, pp, 207-20S. Extracts of this and other glands are now regularly prepared at our larger slaughterhouses.

2 Vernon, Variation in Animals and Plants, pp. 358-360. 
obvious secretory function. The spleen appears to have an internal secre. tion which is of influence in setting free the pancreatic ferment. Finally, extracts of various nervous tissues - brain, spinal cord, and sciatic nerve have been found, when intravenously injected, to produce a distinct fall of blood pressure, ${ }^{1}$ whilst those of the pituitary body produce a marked rise.

Here is a basis for possible transmission of such diseases as might be connected with normal secretions, as it certainly is for any external influence that could permanently affect either their character or their quantity. This opens a wide field for the possible and permanent influence of causes of variation lying originally outside the germ, but whose effects are of such a nature as to make themselves felt throughout the entire organism, and to influence not only its development and activity but its power of transmission as well.

Akin to this is the possible effect of such chemicals as alcohol, which has specific relations to protoplasm and is one of those influences that apparently are capable of penetrating to the uttermost limits of the organism. Without cloubt other material elements of food and drink exert fundamental infuences of a chemical nature, whose effects may reach the germinal matter and thus of necessity descend from generation to generation, to the distinct modification of the race.

How races acclimate. How do races become acclimated? There are at least five methods competent to explain the process :

I. The acclimatization of all thi individuals of a race, each one in the successive generations separately;

2. Silction, obliterating such inclividuals as are unable to acclimate successfully, thus restricting descent to the fittest;

3. The direct transmission of indiadual modifications (acquired characters), at least in some slight degree, the accumulation of which ultimately produces complete acclimatization in the race;

4. It is possible that the same causes which induce modifications in the individuals may also crirt influcuces so decp-seated as to affect the germ plasm directly and in this way produce all the appearances of inheritance of modification ;

1 Due probably to specific action on the heart. 
5. The process may be explained by the old principle of progressive variation.

Of these, the first two are certainly always at work. Manifestly all the indiaiduals of succeeding generations, or most of them at least, will spontaneously acclimate to the changed condition. This of itself would give an appearance of race acclimatisation, even though no change had been wrought in its inherited nature. If, now, to this is added the effect of selection, we at once recognize a powerful cause of real race acclimatization. Nor does this necessitate the destruction of any very large numbers. If only their life period be shortened or their fertility decreased, their relative importance in the race would be greatly lessened thereby and the effect of selection felt.

Either one of these two processes alone is entirely competent to account for the full apparance of acclimatization. Doubtless both are always present and at work jointly, but this does not preclude the possibility of other agencies also. The fifth possibility depends upon selection for its efficiency.

The chief objection to relying upon selection to fully account for race acclimatization is that the destruction is frequently too slight, and the race response too prompt; yet it is not sufficiently prompt and instantly complete to account for the phenomena by the successive acclimatization of all individuals separately.

There is a rapidly cumulative element somewhere. The whole movement is too rapid for selection, especially with the exceedingly moderate destruction of individuals that sometimes takes place. Plants do not acclimate by reason of most of them being killed off, yet there is a strongly progressive element involved. The inevitable conclusion is, in the opinion of the writer, that the chief effects of acclimatization are transmitted.

Whether this transmission be direct or indirect; whether it be due to the peculiar development of the individual impressing itsclf upon the germ, or to climatic influences, like temperature, food, etc., which being all-porading, affect the general state of life and influence the germ direct, is another and more important matter.

That the simple removal of a part does not affect transmission is significant. The old contention that the protoplasm of 
the germ and the protoplasm of the developed body are essentially different has long since been disproved. Both are susceptible to any influence that can reach them, and in climatic conditions generally we have abundant influences of this kind.

May we not consider as established the possibility that the germ itself, and therefore descent, may be directly modified by external influences? How far this may go, and what influences are included, is another subject, and one calling for the most careful study and requiring the most reliable data as a basis for an intelligent opinion.

The present state of knowledge is insufficient to entirely solve the problem, but there is additional evidence worth consideration.

\section{SECTION VI-EVIDENCE FROM HABIT AND INSTINCT. IS INSTINCT INHERITED HABIT?}

Habit and instinct both refer to the use which individuals make of those racial characters that are capable of action. It is a matter of common knowledge that an oft-repeated act speedily becomes a habit with the individual, and, as such, repeats itself almost mechanically; so that what was at first a nice adaptation of means to end shortly becomes little more than reflex action.

Building upon this fact, it is a plausible assumption that what is habit with one generation becomes instinct in the next. It is a sweeping but easy generalization that " no distinct line can be drawn between instinct and reason " ; 1 that instinct is inherited habit, and reason inherited instinct modified by individual experience.

This is the position taken by many of the older naturalists, especially Romanes, who defined instinct as "reflex action into which there is imported the element of consciousness," 2 and reason as "the faculty which is concerned with the intentional adaptation of means to end." 2

1 Romanes, Animal Intelligence, p. I 5.

2 Ibid. p. I7. This volume is perhaps the most extreme exponent of the idea of inherited habit, and of intelligence as lying at the basis of all animal activity. 
Thus the whole question is up again in this connection. Will the habit of the individual be transmitted to its offspring ? Is the habit of one generation the instinct of the next? If so, then we have a case of transmission of modifications (inheritance of acquired characters) of the most direct and certain kind.

The answer to the question is important for its own sake, but more especially for the light it may throw upon the main question now in hand. To arrive at a safe answer it is necessary to give more than a passing notice to the nature of instinctia' acts, and to critically determine whether instinct is built upon habit or habit upon instinct.

Nature of instinctive acts. Most acts of intelligent beings are performed for a particular purpose and for definite ends. Most such acts are controlled by a greater or less degree of purposeful adaptation of means to end that involves knowledge of results, based on experience and merging naturally into habit. Habit is then the customary use to which the inclividual puts the parts with which it is endowed by nature, after they have reached full development. Its chief interest to us at this point arises from the fact that another individual might put the same parts to a different use, while in instinctive acts use is a function of structure, and but one set of actions is possible except under greatly changed conditions.

The term "instinctive" is applied to those acts which are performed without preatous cxperience and perhaps under circumstances that preclude all linowledge of what the result will be; so that, as far as the indiatud is concerned, there is, and can be, no consciousness of purposeful action or of adaptation of means to end, and yet the action, often complicated in the extreme, may be eminently adaptive and exhibit every appearance of being the act of a most intelligent being. Young mammals suck with the lips; young waterfowls swim and dive, but land birds do not; young squirrels hicle objects, and even in a room go through the motions of digging and burying; young chicks have their own peculiar cry, and peck at shining objects; birds build their nests without instruction or assistance from older or more experienced individuals. These are instinctive acts of the simplest order; many others, however, are extremely complicated. For example, 
an insect burrows a cavity and lays its egrg. It then attacks another insect, stings it so as to paralyze but not to kill it, drags it to the burrow, tucks it in next to the egrer where it will serve as food to the larva, seals all up, and goes away.

The yucca moth emerges from the cocoon just as the yucca opens its flowers, each for a single night. The female collects pollen from one flower and kneads it into a bundle. She then flies to another, makes a puncture, and lays her egg among the ovules, after which she darts to the stigma and "stuffs the pollen pellet into the funnel-shaped opening." 1 It is supposed that this is the only way in which the insect reproduces, and the chief way in which the yucca is fertilized.

Here definite and important ends are dependent not only upon complicated acts but also upon the scrial onder of thcir performance, - all without previous knowledge or experience on the part of the agent, for the female in most cases is performing this act for the first time, and in many cases will not live till the eggs hatch. Given any amount of intelligence, therefore, she could not know the final result of her own industry, although the entire process has every affearance of intelligent, even deliberate, action. It is noticeable at once that instincts of this sort are concerned with those acts which, like reproduction, are fundamental to race preservation.

Is instinct founded on habit? The outcome of most instinctive acts is so clearly the preservation of life and the good of the species, the acts themselves are often so extremely complicated, their separate steps are so nicely adjusted to the final end, their proper serial order is so accurately observed, the appearance of deliberate, purposeful action is so genuine, the need of intelligent direction at some period of the organization of the series is so apparent, the importance of the ends achieved is so obvious, and the similarity between the instinct of a race and the habit of an individual is so close that many naturalists have leaped to the conclusion that instinct is inherited habit; in other words, that what has been found beneficial in the experience of individuals has become habitual with them, and through their descendants it has become the habit of the race. This position

1 A free transcript from Morgan, Habit and Instinct, p. I4. 
was quite generally taken by the older naturalists. As Romanes puts it, " Instinctive actions are actions which, owing to their frequent repetition, become so habitual in the course of generations that all the inclividuals of the same species automatically perform the same actions under the stimulus applied by the same appropriate circumstances." He adds : "Rational actions, on the other hand, are actions which are required to meet circumstances of comparatively rare occurrence in the life history of the species, and which, therefore, can be performed only by an intentional effort of adaptation."

If, now, instinct be inherited habit, and reason only modified instinct, then we have an unbroken chain, from the simplest adaptive act up to the highest mental generalization, - all the product of inherited experience. This is inheritance of individual modifications (acquired characters) of the most pronounced type, and if true, it affords the most important evidence upon the question now under consideration.

A critical analysis of the matter makes clear the fact that this conclusion involves the following extreme assumptions, whose correctness must be carefully considered and not accepted without question, as is too often done ${ }^{2}$

I. That instinctive acts are performed perfectly at the first attempt, - the traditional " unerring instinct."

2. That they are carried out substantially in the same way by all individuals of the race and by the same individual in successive performances. ${ }^{3}$

3. That instinctive acts are always adaptive, thus showing their ultimate origin in purposeful acts. ${ }^{4}$

4. That habit precedes instinct, and that instinct finds its directive force in inherited experience. ${ }^{5}$

It is well to consider these points somewhat carefully.

Instinct not unerring. The earliest instinct of the young mammal is to suck. Moreover, it is an instinct connected with the preservation of life; yet the calf will be entirely satisfied

1 Romanes, Animal Intelligence, pp. 16-17.

2 Read, in this general connection, Habit and Instinct, by Lloyd Morgan, especially pp. 29-1 27.

${ }^{3}$ Romanes, Animal Intelligence, p. I7.

4 Ibid. p. I5.

5 Ibid. pp. 16-17. 
with its neighbor's ear, the baby with its own fist, or the young lamb with a lock of wool, which, if the mother be young and inexperienced, it may suck until it starves. Young chicks will pick up and swallow at first whatever attracts their attention, - bits of colored yarn, or " nasty" caterpillars, as readily as "good" worms; but they rapidly learn by experience. ${ }^{1}$

The instinct of the young chick is to strike at any small object that attracts its attention either by its color or its movements; yet the first attempts are cxtremely awkward and seldom result in a catch, the bill going some distance to one side of the object. Gradually, however, it learns by experience to strike accurately. ${ }^{2}$

Walking, flying, swimming, and talking are instinctive acts with species possessing the requisite mechanism; yet the first attempts are exceedingly crude, and much experience and practice are required before effective proficiency is developed.

A fair study of the subject can but convince the student that instinct is at first but little more than an impulse to action in general, which, however, rapidly shapes up into well-ordered performance under the corrective influence of experience.

Instinctive acts are not performed in the same way either by all individuals or by the same individual at successive performances. To watch the complicated acts of the egg-laying instinct in many insects is at first to become convinced that this marvelous sequence of events is assured only by the highest intelligence or by an instinct that is unerring in its directive power; yet a little further study will convince the student that these complicated instinctive acts are not always carried forward on the typical plan, nor are they always successfully executed. On the contrary, important, even significant, steps are often omitted from the series, and different individuals differ greatly in the degree of thoroughness and the rapidity with which the work is carried forward.

For example, Crandall endeavored to note accurately all the steps in the process of making the puncture and laying the egg of the plum curculio working upon the apple. ${ }^{3}$

1 Morgan, Habit and Instinct, pp. 40-44, 50.

${ }^{3}$ Bulletin No. 99, Illinois Experiment Station, pp. 500-504.

2 Ibid. pp. 37, 47 
Of many attempts to watch and record the process from first to last, only three were successful in covering the entire period. The rest were fragmentary, covering only portions of the process. This was owing to the difficulty of keeping the insect under focus for the fifteen to twenty-five minutes required for the complete operation without disturbing its work. The record of the observer is as follows :

In the first observation the female moved about the apple for several seconds, keeping the end of her beak in contact with the surface, as if seeking a favorable spot. When the exact spot was decided upon, the minute jaws at the end of the snout began a rapid movement which quickly made an opening through the skin. This opening was no larger than necessary for admission of the tip of the beak. No skin was removed; it was simply torn and thrust aside to give access to the pulp below. Later, as the excavation proceeded, the broken skin was seen as a sort of fringe around the beak at the surface of the fruit. As soon as excavation in the pulp was commenced, the beak was deflected backward so that the work was carried on under the insect, just beneath the skin and nearly parallel with the surface. As the work advanced, the opening through the skin became slightly enlarged by lateral motions of the beak. The pulp was all eaten as excavated. During the process the beak was not once withdrawn, nor was there any cessation of motion. When the excavation of the cavity was completed the beak was withdrawn by a quick motion, the insect turned about, adjusted the tip of the abdomen to the opening and deposited an egg, which was forced to the extremity of the excavation by the ovipositor. The insect now rested without motion for two minutes; then, turning again, proceeded to cut the crescent in front of the egg. This crescent puncture was not wholly a separate puncture, but, starting in the original opening through the skin, was cut laterally in either direction, partly by the jaws and partly by crowding the beak first one way and then the other. The direction of the beak was but little deflected from the perpendicular, and the cut was made as deep as the length of the beak would allow. The pulp torn away in making the crescent was eaten, just as was done in excavating the egg cavity. The crescent completed, the insect walked away, drew the legs closely under the body, and settled down, apparently to sleep. The time occupied in the process described was distributed as follows:

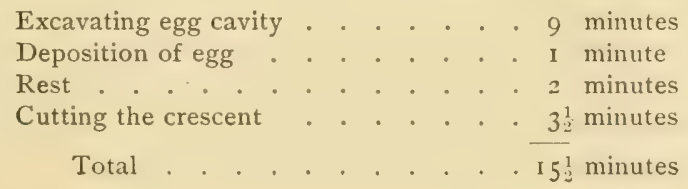


The egrg cavity was cylindrical, with a rounded bottom, and by measurement was found to be 0.04 inch in depth. The egg when deposited very nearly filled the cavity.

The second observation of the complete process was nearly identical with the one described. The insect spent no time in choosing the exact spot, but went to work at once. It worked in a more leisurcly way and did not excavate as deep an egg cavity. Eleven minutes were spent on the cavity, two minutes in depositing the egr, two in rest, and four in cutting the crescent, - a total of nineteen minutes. The egg cavity measured 0.035 inch in depth and was completely filled by the exy. On completion of the process the insect moved a short distance and immediately began a second cavity.

Essential differences from procedure in the two preceding cases were noted in the third complete observation. Excavation of the egg cavity was the same, except that it was deeper in the pulp and of greater extent. After depositing the egs the beetle turned, and with her buk worked the egg back to the bottom of the cavity. Then she began tearing off bits of skin

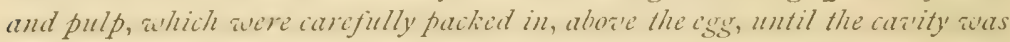
full. ${ }^{1}$ Following this, the crescent was cut in much the same manner as in the preceding cases. Then she appeared to make a final inspection, and added some further packing above the egg. Finally the work appeared to be satisfactory and she walked away and began a second puncture. The time consumed in this process was longer than in the others, and was divided as follows :

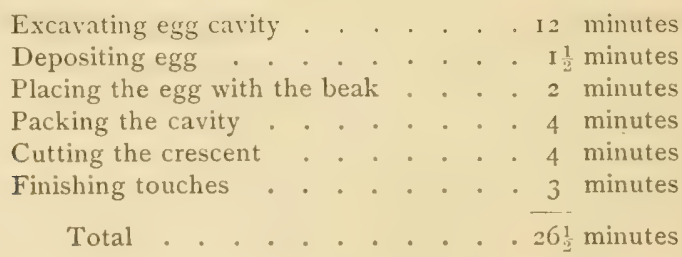

Among the many cases where only part of the process was observed some anomalies were noted. In two cases the insect walked away immediately after depositing the egg and made no crescent cut. In three cases beetles were seen to cut crescents and, moving a short distance, begin other punctures. These crescents had no egg cavities and no eggs were deposited in them. In two cases eggs were found deposited directly in crescent cuts, neither of which had the usual egg cavity. Marked variation in depth of the egg cavity was frequently observed. Not infrequently the cavity is so shallow that the tip) of the egr protrudes, and sometimes its depth is nearly equal to twice the length of the egr. Packing the egrs carity with pieces of pulp is a common, but not unir'sesal, practice; often this is neglected, even where the cavity is deep. . . .

1 Italics are mine. 
When reading of the various processes and acts in insect economy, as observed and recorded in published life histories, it is quite natural to suppose that these processes are fixed, absolute, and unchangeable, while as matter of fact many of them are subject to modifications. Sometimes these variations have apparent reason in surrounding conditions, and again they can be ascribed only to individual peculiarity. . . .

A crescent puncture is usually supposed to represent an egg or an attempt at egg laying, but this does not always hold true, because, as stated above, some crescent cuts are made without the accompaniment of egg laying. On May 27, 1903, fallen apples, twenty-five in number, were picked up at random for examination of the crescent punctures. Nearly all were more or less punctured by the apple curculio, but these punctures are not considered here. Two fruits bore apple-curculio punctures only, so that the number examined for crescent marks was twenty-three. On these twenty-three apples were fifty-eight crescent marks, or 2.52 to each apple. There were also thirty-five feeding punctures made by the plum curculio. Of the fifty-eight crescent cuts, fourteen, or 24.14 per cent, had no egg cavities and contained no eggs. The remaining forty-four crescent cuts had forty-five egg cavities. Some variation in the location of the egg cavities was observed; usually they occupied the center of the crescent, but some of these were not so situated. Of the forty-five egg cavities, thirty-four, or 75.56 per cent, were located at or near the center of the crescent; eleven, or 24.44 per cent, were located near the ends of the crescents. In one case there were two egg cavities within one crescent, one on each side halfway between the center and tip. By another modification one of the egg cavities, instead of being excavated from the surface, was excavated from the bottom at the center of a crescent cut. It was of usual dimensions, extended back obliquely towards the surface, and contained an egg. Evidently in this case the crescent was cut first and the cavity excavated afterwards. ...

The statements we have quoted regarding the details of oviposition of the plum curculio, together with the observations recorded, indicate variation in details sufficient to confuse the layman and even to puzzle the expert if he seek to cover rightly any detail with a general statement that will fit all cases. Two conclusions are open : either some individual insects have faulty instincts or there is more than one acceptable way of performing several of the details of oviposition. The writer accepts the latter conclusion.

From this it is seen that the somewhat complicated process of egg laying varies greatly in detail; that the time consumed varies at least from fifteen and a half to twenty-six and a half minutes; that important details are often omitted; and that in a large proportion of cases even the final object is not attained. Knowing these variations, one is not surprised to learn that of 
the twenty previously recorded observations no two agreed, and not one gave a complete account of what may be called the typically instinctive process. Bearing all these facts in mind, we are not to suppose that still more complicated processes are successfully carried forward in every instance, as we have been led to infer. Instinct is, therefore, not unerring, nor does it insure uniformity of procedure. It looks as if important links in the chain of impulse may be omitted, or, if the series is interrupted for any cause, it may be resumed at almost any point. Certain it is that different series are far from uniform and that very many of them fail of their evident and final object.

Instinctive acts are not always adaptive ; their origin is therefore not in purposeful acts. It is of necessity true that in most corses instinctive acts are for the good of the species and, therefore, by clefinition, adaptive. If they were not for the good of the species they would speedily lead to extinction, which is but another way of saying that instincts not adaptive have long since been blotted out by selection, along with the inclividuals in which they arose.

That such non-adaptive instincts exist, however, is easily shown. For example, when the moth flies into the flame and is killed, by no stretch of the imagination can this be called an adaptive act; nor can it be conceived as having arisen in the purposeful acts of its ancestors. It never could have served any but an evil purpose to the race, and the only element that now stands in the way of the utter extinction of races possessing this instinct is the relative infrequency of the naked flame, so that comparatirely few individuals suffer, - too few to affect either the race or its instincts. We must, therefore, look farther than habit for the origin of instincts.

Instincts originate in reflex action not in habit. If a piece of meat be laid upon the tentacles of an actinian they will immediately contract, infolding the meat and carrying it into the mouth. If a piece of paper be laid upon the tentacles no action follows; but if the paper be covered with the juice of meat the action is the same as if the piece were valuable for food. Tentacles that have arisen from a wound in the side of the body will react to meat and meat juice as do the normal parts, 
infolding and pressing the piece against the body as if endeavoring to tuck it into a mouth, though none is present. ${ }^{1}$ Here it is not intelligence that affords the basis of muscular contraction but chamical action of the meat juices upon the muscle fibers of the tentacles. The same principle governs motion and secretion in insectivorous plants, to which no one would ascribe even the elements of intelligence. Odors excite the salivary glands and make the mouth water, but it is contact that starts the secretion of gastric juice in the stomach.

Light stimulates specific reactions in many forms of protoplasm, and many tissues contract under its influence. It is this contraction that causes the bending of stems toward the light. The iris of the eye contracts, not by nervous impulse but by the action of the light, causing, directly, muscular contraction. Loeb reports ${ }^{2}$ that he has often observed the contraction of the iris of dead sharks under the influence of light "several hours after death, when signs of decomposition had already begun to appear."

Long-bodied insects, if lying with the side to the light, will, because of this, have their bodies bent, with the concave side next to the source of light if positively heliotropic. Whenever they move in this condition they must of necessity move in a curved instead of a straight line, until such time as they are headed directly toward the light. From that time the body is equally illuminated on both sides. It therefore becomes and remains straight, so that future motion must be in a straight line, any deviation being quickly corrected by the uncqual illumination of the body: It is this series of facts, arising from the natural relation between light and protoplasm, and not curiosity, that accounts for the flying of the moth into the candle. Negatively heliotropic animals would of course behave in exactly the opposite manner, but for the same general reasons.

Insects and small worms are said to burrow into dark places for the purpose of hiding. This cannot be true, for under direct experiment small animals often emerge from darkness to light, from hiding to exposure, under the impelling force of an instinct to bring their bodies into contact with as many surfaces as

1 Loeb, Physiology of the Brain, pp. 48-54.

2 Ibid. p. 40. 
possible. In a box they will crawl along the bottom till they come to a side. Here they can touch two surfaces, and motion will then be along the groove where the side meets the bottom until they reach the corner, where a third surface joins, when they are likely not to turn the corner (unless impelled by some stronger instinct) but to come to rest in contact with three surfaces, this affording, apparently, the highest attainable satisfaction. If a tubular opening be founcl, insects of this instinct will crowd into it, if possible, or at least make the attempt. ${ }^{1}$ This instinct to seek greater comfort by getting snugly placed in contact with foreign bodies is present in the higher animals and in man, and it accounts for many of the movements and resting positions so commonly seen.

That this action is not the result of a purpose to hide is eviclenced by the fact that neither light nor darkness has any effect upon it, and that the instinct is not changed even by the removal of the brain. In nature, of course, places that will satisfy this instinct are generally shut away from light, and insects so bestowed are also hidden, - a fact that has given rise to the tradition that the purpose was to secrete themselves from predatory enemies. Of course the tradition itself is not consistent, for intelligent animals seeking food soon learn the favorite haunts of their prey. They therefore know precisely where to look for them and speedily turn them out.

IVe have already seen that protoplasm may be excited to action by a great variety of external agents (light, heat, electricity, chemical substances); that the character of protoplasmic activity may be modified by certain of these external forces, notably light and chemical attraction; that the direction of movement or of growth may also be influenced by the same class of agents (light, chemical substances, heat, gravity); and that in all these ways the activities of living beings are largely dependent upon the nature of the outside forces with which they come into contact. Here lies, to a very large extent, the initial cause of those external acts we ordinarily speak of as instincts, and the remaining elements of causation are to be sought in the internal mechanism of the creature. In all likelihood it is not

${ }^{1}$ Loeb, Physiology of the Brain, p. 93. 
too much to say that in the last analysis instinct is a function of structure; that the ultimate causes of instinctive acts lie in the nature and the surroundings of the protoplasm, - its intcrnal activitics upon the onc hand and its natural reactions to accidental contact with outside forces upon the other.

If this be true, then the causes of instincts lie in the structure of the organism, — using the word "structure" in its broadest sense, chemical and physiological as well as anatomical. Thus if a new creature should suddenly be created, its instincts could be fairly well foretold by any one who knew the morphology of its structure and the nature of its protoplasm. Three fundamental facts should be borne in mind in this connection :

I. Any living being will make use of any organ, part, or faculty with which it is endowed by birth.

2. The impulse to make use of a part may arise either from within (desire) or from without (light, heat, chemical action, gravity, electricity, contact).

3. Manifestly the acts of an organism are limited to its natural organs and faculties. Therefore instincts, like intelligent acts, differ, being restricted to the range of natural endowment, - a restriction which no amount of "willing " will remove or modify.

Intelligence not necessary to the control of even complicated acts. At first thought it scems incredible that a long and complicated series of acts, culminating in a purposeful end, can be directed by any other agency than intelligence. Yet such is the fact, and a mistake at this point has led more than one erolutionist into fatal error.

When we see an insect light upon a particular part of a particular animal, sting it perhaps in such a manner as to paralyze but not to kill, drag it to a cavity wherein eggs have been deposited, store it away as food for the larve, seal all up safely as if with the greatest care, it is difficult not to attribute the highest intelligence and the most careful foresight to so remarkable a series of acts.

Yet we are not to be deceived by the attitude of busy preoccupation or the appearance of intelligent effort. Acts as complicated as these are going on about us every day, with no suggestion of intelligent control. The growth of the embryo in utcro, and the vital processes generally, are even more orderly 
and complicated than those semi-mechanical acts connected with the deposition of eggs and the care of young, which are themselves in their essence not far removed from vital processes.

All motion is reducible to irritability and contractility of protoplesm as its ultimate cause, and any impulse that will produce this effect will lead to action. Furthermore, this action must, from the nature of the case, be characteristic of the organism and its peculiar mechanism.

It has been held that all muscular activity must have its origin in a nerve stimulus of some sort. That this is erroneous is selfevident. Muscle tissue, unsupplied with nerve, is still contractile, and the impulse can still be supplied from a variety of sources (heat, light, electricity, contact).

Nervous impulse is but onc out of many stimuli to muscular contraction, or other activity of living protoplasm, though it is the most rapid and direct, and the one most readily under control of the mind and the will. Restating the proposition, nervous mechanism is not necessary to motion, not even to coördinated motion of a high degree, but it is necessary to the highest coördination of the most complicated organisms; it is necessary as the means by which the will may quickly reach all parts of the machine and direct or set aside mere instinctive motion; it is necessary for the realization of all the possibilities of which a highly organized structure is capable; it is not necessary to action, or even to a high degree of complication in action.

Any effective impulse will serve to stimulate to activity; and, in general with all complicated actions, each act becomes the impulse for the next. If the heart of a frog be cut into several pieces these will all beat rhythmically, "but the number of contractions will vary in the different pieces. The sinus venosus beats most rapidly, and the number of its contractions in a unit of time equals that of the heart before it was clivided. Thus ave sec that the whole heart beats in the rhythm of the part that has the maximum number of contractions per minute. From this ace must assume that the coördination of the huart's actiaity is due to the fact that the part which contracts most frequently forces the other parts to contract in the same rhythm." 1

1 Loeb, Physiology of the Brain, p. 25. 
This shows, not only that a center of coördination exists in the organ itself, independent of nerve centers, but that the action of one part becomes the impulse for crciting action in its neighboring part.

Hydromedusie of different rates of pulsation were united in pairs in Loeb's laboratory by the process of grafting. W'hen the union was complete along nearly all the cut edge the whole beat synchronously, but when the union covered but a small area the two beat separately with a different rhythm. ${ }^{1}$

The heart of the asciclian is an elongated tube, beating so as to send the blood alternately from left to right and from right to left; that is, the impulse seems to originate at one end for a time, and then, after sereral hundred beats, to shift to the other end. It was found that the "area of impulse " was confined to a short section at either end; that each of these sections, if cut away, continued to beat rhythmically, but that the longer middle part seemed incapable of contraction without external stimulus. Commenting on the fact, Loeb says $:^{2}$

These experiments, it seems to me, leave no room for doubt that the change in the direction in the contraction of the ascidian's heart is determined by each of the two ends getting the upper hand alternately and forcing the other center to act in its rhythm for a time. This "getting the upper hand "might possibly mean nothing more than that one end gains the time in which to send off a wave of contraction before the other end begins to contract. For this it is only necessary that a single heart beat of the leading end be delayed or fail entirely, a phenomenon that also appears occasionally in the human heart.

Locomotion in the earthworm is by a series of elongations and contractions of the successive segments of the body, in regular order from the front backward. Noboly ever supposed this serial order to be controlled by conscious intelligence, but it has been assumed to be due to the control of nerve fibers from the ganglia along the dorsal surface of the body. If, however, the worm be cut in two and the parts united by threads, locomotion is entirely successful, even though a considerable space intervenes between the pieces. In this case contraction proceeds backward, segment by segment, as in uninjured worms, suffering no special

1 Loeb, Physiology of the Brain, pp. 26-27.

2 Ibid. pp. 27-29. 
interruption at the point where the nerve connection is severed. The thread serves perfectly to carry the impulse over from the last segment of the anterior piece to the first of the posterior. The unavoidable conclusion is that at least from this point backward each segment derives its impulse not from nore fibcrs but from the segment just ahead; in other words, that the motion of one part becomes a stimulus to appropriate action in a neighboring part.

It is reported that Ribbert transplanted a milk gland to the ear of a guinea pig, and that when the individual became pregnant the gland commenced to secrete milk. Whatever the nature of the stimulus, it was clearly independent of nerve impulse. ${ }^{1}$

It is a well-known fact that if an ant be removed from its nest for a time and then put back, it will be critically examined, but will be received again; if, however, a stranger ant be introduced, it will at once be attacked and killed. How is the difference cletected? This question is largely answered by the fact that, if the ant belonging to the nest be smeared with the juices of a crushed stranger, it will at once be attacked and killed as would the real stranger. ${ }^{2}$ Clearly it is by the odor that the ants detect the difference, much as the dog recognizes his master in daylight or in the darkness, or follows a trail along a crowded street. All this shows that even a slight cause, like odlor, may serve to start the operation of a train of most remarkable reflexes, which, once started, proceeds automatically, each act operating as a stimulus to the next.

This fact is further illustrated in the case of dogs deprived of large portions of the nervous system. Individuals that have lost the spinal cord "almost up to the medulla" may live for years and perform all normal functions. ${ }^{3}$

Goltz entirely removed both hemispheres of the brain from a full-grown dog. The first effects of so violent an experiment are apparently disastrous, but if skillfully done the shock soon subsides and all normal functions of the body proced as boforc. That is, the animal performs the external acts of eating, urination, defecation, etc., the same as when in possession of the brain,

1 Loeb, Physiology of the Brain, p. 206. ${ }^{2}$ Ibid. pp. 220-221. ${ }^{3}$ Ibid. p. 43. 
and apparently according to the same train of reflexes that provide in life generally for such important vital processes as the pulsation of the heart, characteristic action of the various glands of the body, the movements of the intestines, etc. All traces of memory were gone, and the log could not recognize its master. It would avoid objects in walking; but could not recognize food. If, however, the food were brought in contact with thi nose or placed in the mouth, the jaws commenced to work and the food was swallowed and digested as by any other dog. ${ }^{1}$

Dogs in this condition live for months or for years, and perform all the functions of normal animals not requiring intelligent action. All this shows to what extent vital actions are a series of reflexes constituting a train, in which, if one member be started by appropriate stimulus, all the rest follow automatically.

Instinct not founded on habit. The facts that have been cited certainly show that instinctive acts are nothing but the putting to use of parts in possession of the individual and capable of action. They are in that way spontaneous, and whatever meaning or special significance may seem to be involved, it is to be sought, not in the impulse to the act, but farther back in the circumstances and canses that led to the deiclopment of the parts, cach aith its charactoristic capacity. A multitule of causes have taken part in the selective processes by which the several organs and parts of a body have been developed, each capable of performing a special act, either independently or as a part of a complicated series ; but, once assembled, nothing is more natural than that each should perform its proper service, and any stimulus sufficient to start the machinery will of necessity insure the whole train of appropriate results.

Nor are we to be deceived by the affearance of intelligence as the acts proceed. The busy preoccupation of the insect engaged in one of the more complicated processes of egg laying has all the semblance of the highest intelligence, but we must not forget that in many cases the individual must be entirely ignorant of the final result; it will be dead before the eggs hatch; it lives but a single season and, therefore, neither it nor its ancestors ever saw a larva; it is simply playing its rôle in a

${ }^{1}$ Loeb, Physiology of the Brain, pp. $246-248$. 
wonderful series of which it is itself but a part, and of whose beginning and end it is alike ignorant.

The instinct to suck cannot be conceived as founded on habit, because sucking is not a life habit with any individual. It is practiced but a brief season after birth, and then abandoned, leaving no trace or even impulse behind.

It is difficult for us to dissociate these complicated acts from the idea of intelligent control. Yet many of them are performed by plants, in which there is not so much as the beginnings of a nervous system, the impulse traveling from cell to cell, as it is entircly capable of doing in animal tissuc, but at a rate easily overtaken by nerve transmission, whenever the latter is superimposed by the will or otherwise.

Again, we must consicler the exceedingly complicated nature and serial order of the vital processes generally. Most of these processes, it is true, aside from copulation, the laying of the egrer and the care of the young, are carried on inside the body, and therefore out of sight of the observer. If we could by some mental microscope see not only the pulsation of the heart, the movements of the stomach and intestines, and the discharge of accumulated secretions, but also the internal acts of secretion going on within the various glands of the body, with the associated protoplasmic motion and cell division, actively accompanied by the careful division of the chromosomes into exactly equal portions qualitatively as well as quantitatively - if we could sec all this as we see external instinctive acts, we should be led to marvel at the mystery and the complication of vital activity in general. We should involuntarily seck a basis of intelligent control within the organism, whereas we know that the proper place to seek causation is outsicle the creature in the forces that shaped its development and in the higher power that endowed it all with life, whose characteristic act is motion and appropriation of new material. We are, therefore, not to be misled by the complexity of acts having the appearance of intelligence.

We have been led to project intelligence too far down the scale. It may, if present, take hold of and overrule most (not all) instinctive acts, but the vast majority of organic activities go on 
without it. Young things have little or no sense of fear at birth, and at the first consciousness of fear make nothing like intelligent discrimination. The young chick, so Iloyd Morgan tells us, is as much afraid of a flying newspaper as of a hawk, and has no preconceived notion of the danger from bees, which can sting, as compared with that from flies, which are harmless. Its first fears are of "largish things," but experience rapidly" informs it of specific things. It has at first no appreciation of water as water, but when it acciclentally pecks at a shining drop and wets the bill, the presence of the water starts the series of reflexes and the chick holds up its head to swallow, or, if a duck, it "shovels," - each organism reacts in its own characteristic manner, - and both learn rapidly by experience. ${ }^{2}$

Habits follow and are founded upon instincts. The true order seems to be that the earliest attempts are instinctive, but that they are rapidly shaped up and perfected by experience, and in this condition they become habitual. It also appears that no exact line can be drawn between what we call instinct and what is nothing but response to stimuli. Manifestly we hare applied the word "instinct" to those reflex acts that have the appearance of being purposeful. It would apply equally as well to many acts clearly reflex, and when it is shown that these so-called instinctive acts are themselves only reflexes and can be performed perfectly in the absence of all possible application of intelligence, either on the part of the indiviclual or of its ancestors, it appears that our present use of the word is a convenience rather than a fair mark of a scientific distinction.

The theory, therefore, which places habit at the point of origin of instinctive acts clearly takes it out of its proper order in the series. It is a property of the incliviclual rather than of the race, - except as we speak of the habit of a race, meaning thereby the habit developed by all or most of the members of the race. The common development of such a habit is not unnatural since all of the individuals of the race possess the same organs

1 The action of the heart and of the secreting organs is beyond the control of the will, and that of the intestines is largely so; but many parts of the organism are under control either of the will, of impulses intemal to the part, or of causes external to the organism.

2 Morgan, Habit and Instinct, pp. 40-90. 
and are for the most part subjected to the same surrounding conditions.

Carefully examined, this field, interesting as it is of itself, is barren of evidence upon the transmission of habits, though it is the one most often appealed to for proof of the inheritance of acquired characters. The error lies in assuming a causative relation between two acts which are similar. It is true that the habits of the individual and the instincts of the race are similar. They could not be otherwise, seeing that both depend upon the presence of suitable organs, without which the particular act would not be possible, and with which it is certain to appear whenever suitable stimulus is encountered; but habit is founded upon instinct and not instinct upon habit.

The conclusion which seems inevitable is this: that brecders nead haie no foar that the habit of an indiridual, as such, will be inherited by its offspring; but the fact that the habit deacloped at all is sufficiont reason for knowing that its derclopment is aliways possible in the fermily line, whenear sutable conditions arise.

The attention of the student is called to the fact that while we have shown that instinct is not the result of habit, and therefore that its existence is no proof of the transmission of habitual acts, yet this loes not show whether or not the habitual use or non-use of a part will affect the intensity of transmission of that part, or the tendency to make use of it. Having put instinct behind us where it properly belongs, we have now to inquire into the effects of use and disuse.

\section{SECTION VII - EVIDENCE FROM USE AND DISUSE}

The question is not whether use develops and disuse leads to non-development or degeneracy. The facts on that point are already well known. The inquiry is whether the effects of use and disuse are transmitted; whether their influence is direct, not merely indirect by rendering the individuals less or more able to meet the demands of selection; whether specialization of a part is in any way due to use, aside from its effect in developing individuals separately and aside from its connection with increased rigor of selection; whether generalization and 
degeneracy - beyond under-development in individuals, or as associated with cessation of selection - are in any way the result of clisuse; whether an individual is a better parent after a long course in exercise or training, or a worse one after a long life of idleness, than the same individual would have been before; whether a race horse will transmit better speed after he or she has been " dereloped," and has made a record on the track, than he or she would have transmitted if never tracked; whether the later children of a studious or of an athletic man (or woman) will be born with more ability in these clirections than the earlier ones, and whether the younger children of criminals are more inclined to criminality than are those born before the criminal instincts were fully dereloped in the parents. The real question is this: Is transmission augmented or lessened by the degrec of deiclopment to which racial characters have attained in the indiwidual before parentage, and without reference to selection?

In the opinion of the writer there is not yet sufficient evidence on which to base a final decision, and much as we all desire a settlement of the matter, and much as we need to know what the real truth is, nothing is gained by passing premature judgment, and the question must be left for the time unanswered.

For the present the student must content himself with learning to know the field of discussion, and, inasmuch as he must hold his opinions in abeyance, it is important that he know the arguments pro and con. If he do this, and keep his ear to the ground, he will find the question clearing up rapidly in the near future; we may be nearer its solution than the most careful biologists have yet dared to hope.

Not going back of the fact that no somatic variation can possibly become blastogenic, Weismann and his followers deny in toto all possibility of such transmission, although Weismann himself has admitted, as a result of his own experiments with the colors of butterflies as dependent upon temperature, that such all-pervading conditions as heat may penetrate to the germ and affect its character as well as that of the tissues of the body. In the opinion of many recent writers the list of "all-pervading" influences includes much more than temperature alone. 
Weismann's exact words concerning the two varieties of butterflies - the darker Italian and the lighter German - are as follows: "The two varieties may have originated owing to a gradual cumulative influence of the climate, the slight effects of one summer or winter having been transmitted and added to from generation to generation" ; and again, "In many other animals and plants influences of temperature and environment may very possibly produce permanent hereditary variations in a similar manner" ; and still again, " "Many varieties of plants may also be due wholly or in part to the simultaneous variation of corresponding determinants in some part of the soma and in the germ plasm of the reproductive cells, and these variations must be hereditary. Tomperature, and mutrition in its widest scnse, affect the whole body of the plant-the somatic cells as well as the germ cells." 4

In all this it must be noted that Weismann limits action of this kind to such external influences as are able to penetrate the organism and affect the germ plasm directly; whether an influence does this is to be determined in every case by direct experiment, and is not to be assumed from the effect of the same influence upon mere body development.

These citations from Weismann, while not especially bearing upon the topic of use and disuse, are introduced because his position as the leader in opposition to the theory of the transmission of modifications due to external influences is often misunderstood. They are introduced at this point because it is concerning use and disuse that the most vigorous discussions have arisen.

Those believing in the transmitted effects of use and disuse base their belief mainly upon the method of proof by instance, and most of them cite instances that were far better omitted. As long as one side depends upon simple enumeration, and the other mainly upon abstract reasoning, we are not likely to get ahead. As all forms of acquired characters are discussed together, it is practically impossible to cite references dealing exclusively with use and disuse; but in order that the student

1 Weismann, Germ Plasm, p. 400-406.

${ }^{3}$ Ibid. p. 406.

2 Ibid. p. 405.

4 Italics are mine. 
may do his own thinking, some of the principal references relating in part to use and disuse are given in the footnote. ${ }^{1}$

It is important that we know the truth in this matter if possible. If the perfection of development that comes only with use is to any extent transmitted, then we must put our speed horses through a long course of training and develop them fully before we may hope that they will transmit maximum speed. If this theory be correct, then the heifers from a mature cow that has been long in milk and made record, will be capable of developing into better milkers than would be possible if the dam had never made extreme records, or than would be possible with the earlier calves from the same cow (before the extreme records were made). Manifestly the age of the bull will not count, as he is incapable of developing this particular character. All he can do is to transmit, unaugmented and unchanged, the hereditary faculties of milk production exactly as they descended to him. In meat production of course, as in speed, the case would be different, as both sexes may be conceived as capable of adding to (?) or cletracting from (?) the racial intensity by reason of their own development or lack of development.

This subject is now uncler investigation, and while the point would seem to be easy of determination, yet it involves the careful study of all the progeny of many individuals both before and after development. On this point, however, evidence may be expected at no very distant date.

Effect of development upon transmission of speed in horses. In a recent series of articles, ${ }^{2}$ Casper $\mathrm{L}$. Redfield takes the position that the effects of speed development are transmitted, and he cites numerous instances calculated to show that maximum speed is transmitted only from sires with long and

1 Against transmission: Weismann, Germ Plasm, pp. 392-41o. In favor of transmission: Romanes, Darwin and After Darwin, II, 60-287; Cope, Origin of the Fittest, pp. 194-203, 405-42I, and Primary Factors of Organic Evolution, pp. 246-3S4; Eimer, Organic Evolution, pp. I 53-I73, 205-22I. Non-Partisan : Lloyd Morgan, Habit and Instinct, pp. 280-322; Vernon, Variation in Animals and Plants, pp. $35^{2-370 .}$

$2 \mathrm{Mr}$. Redfield's theories are best set forth in a series of articles entitled "Evolution of the Setter," in American Field, LXII, Nos. 25-27, and LXIII, Nos. I-9. They are further set forth in a series entitled, "Breeding the Trotter," published in The Horseman, XXV, Nos, 19-34. 
honorable racing records. The studies would be more conclusive if they included larger numbers of examples, and if these were thrown into two classes, - one gotten after the records were made, the other gotten by the same sires bifore their development.

Mr. Redfield conceives that the sire or dam that is constantly worked up to a safe limit develops thereby a larger stock of what he calis "dynamic force," and that transmission is in proportion to the extent of this force present at the time of procreation. There is no need of involving the subject with new terms. What is in Mr. Redfield's mind is doubtless the same thought that lies at the basis of Cope's theory of growth force, which is one of the strongest of what may be called the dynamic theories of evolution. Everybody recognizes a dynamic basis in transmission, - that which is connected with the intensity of the vital processes. Many forces cause that intensity to vary, and the important question is whether exercise, use, extreme development in the individual, is one of them. Mr. Redfield's articles may be read with profit; whether or not all his conclusions will stand is another matter. The articles are chiefly useful for the large mass of facts presented, which, good as they may be, are not yet sufficient to maintain his theories or to answer the question that horsemen would like to have settled.

Certain outside considerations must be borne in mind in studying this subject:

I. The better the sire as to speed, the better will be his opportunity to get speed, for the more numerous will be his offspring and the better will be the class of mares offered. The same principle holds true as to the dam, for only a good one is worth the fee for a high-class stallion; in other words, the sires and dams with records have better opportunities to produce than do those equally good but without records. Because of this fact the get of one animal must be compared, not with that of another, but with his own of a later or carlier date.

2. Speaking grenerally, the get of the best horses later in life, after they are know'n to be valuable, will be better trained and better developed than the get of the same animals earlier in life and in the hands of more ordinary horsemen. 
3. So much of this exercise, or development, as contributes simply to constitutional vigor and good health will have its effect under quite another principle. Moderate exercise over against absolute inactivity should show some results, but this is entirely outside the present inquiry. What we desire to know is whether the crtreme derelopment of a faculty in an individual will augment its transmission above what would otherwise have been the transmitting power of that individual.

Mr. Redfield's conclusion that this transmission is limited to the same sex - that the development of a sire affects only his mat' offspring - does not rest upon good grounds, and is against what is generally known as to sex transmission. It will be seen later that sires are slightly but not noticeably prepotent over dams in male offspring, and a'ice a'sers; but the difference is slight, and not marked, so far as it has yet been studied by the statistical method, which is the only reliable one for the determination of questions of this character.

\section{Effect of development upon the transmission of milking quality.} It is a widespread belief that a heifer will make a better cow if brought into milk at two years of age than she will make if her milking powers are not developed till later. Will her later calves, dropped say at six years of age, be influenced by the fact that she was a cow at two years of age rather than not until four or five? The question cannot be answered at present, though records are accumulating which will almost certainly afford an answer in the near future. In the meantime it is both fruitless and hazardous to speculate. IVe must turn in another direction for the only known evidence that is raluable, and reason by inference from the behavior of characters under degeneration.

\section{SECTION VIII - EVIDENCE FROM DISAPPEARING ORGANS}

It seems to be true in general that when a part once useful is no longer used, its doom is sealed. It at once begins to degenerate and its final disappearance seems only a question of time. In this way the snake has lost all its limbs; ${ }^{1}$ the whale, its hind

1 The python still has rudiments of the pelvic bones. 
limbs; the wings have gone from the apteryx and appear to be going from the ostrich; eyes of cave insects and fishes are in many cases imperfect or rudimentary; horses, cattle, sheep, and hogs have lost toes that belonged to their ancestors, and parts generally which are functionless are evidently disappearing. How, now, are parts lost, when once they have become useless ?

Economy of Nature not the reason for loss of parts. It is sometimes said that a part no longer used is removed by Nature in the interest of economy. This is bad science. Nature is not economical. She not only supports many expensire and useless parts, such as tremendous horns and tusks, but she often produces necessary products in wanton profusion, such as pollen and fat. Other and deeper causes are at work, - causes more rationally connected with the facts of life, - than any such anthropomorphic reason as economy. Whatever may be true as to economy of growth, it is a fact, not a principle; a result, not a cause.

How parts disappear. IVe are reasonably intelligent upon a part of the process of degeneracy and disappearance, at least in the more active portions of the body. As long as a part is in use, its constant movements increase the flow of blood to that part and it enjoys the extreme development that comes only with maximum nourishment and uniform healthy exercise. When, however, the part is no longer used, the flow of blood is lessened and it suffers from lessened nourishment. In this way the first steps of degeneration are easily accounted for.

If the part has been useful heretofore, it has of course been sustained by selection. Being no longer useful, this influence is withdrawn, and breeding is henceforth totally without reference to this particular part; that is to say, there is absolute "cessation of selection," or panmixia, ${ }^{1}$ as to this part. Under this condition the average parentage would be lower than heretofore, thus accounting for a still forther step in the downward process.

To all this is often added the adverse effect of selection, when for any reason that influence is turned agrinst the part. This "reversal of selection" of course comes only when a part once useful has become not merely useless but detrimental. In

1 Romanes, Darwin and After Darwin, II, 97-100, 29I-306. 
such event there ensues a third stage of degeneracy. Obviously the full effects of selection will depend very much upon the character of the part and its connection with the vital interests of the species.

The influences just mentioned will sufficiently account for extreme degeneracy of a once active part, but they will not account for absolute disafpearance. Any character under such conditions would decrease to a low minimum where its presence becomes insignificant, and there it would remain, but it would not absolutely disappear except through some other agency. That characters, even those which were once important, do entirely disappear, leaving not even rudimentary parts, is evidenced by the disappearance of the legs of snakes, but that the later stages are extremely slow is shown by the rudimentary leg bones of the python and the whale.

Degeneration of eyes in cave-dwelling and deep-sea species. It is a well-known fact that the eyes of cave fishes and insects often exhibit all grades of degeneration, from near the normal down to the merest rudiments. Being entirely useless under the conditions of life, selection is suspended, and Nature is having her way with the remnants of what was once a highly developed organ.

Deep-sea fishes are either in the same condition or else are supplied with enormous eyes of a kind evidently fitted to perceive light rather than to make distinct and clear-cut images.

The gradual failure of parts like the toes that have gone from the horse, or the apparently disappearing vermiform appendix, might be attributed to some failure or imperfection in the germ ; but the instance of disappearing limbs is too clearly connected with disuse, and that of disappearing eyes with lack of what may be called essential conditions of development, to be explained wholly on the ground of imperfection from within as the fundamental cause of degeneracy. ${ }^{1}$

The final disappearance of a useless part is certainly due to some fact other than the withdrawal or even the reversal of selection. There must be morphological units of some kind

1 Light is evidently essential to the origin of the sensitive spot we call the eye, especially in the formation of pigment. 
resident within the germ, from which they slowly disappear when no longer favored by the conditions of life and no longer sustained by selection. These vital units, if ever discovered, will be found to be closely connected with the origin of characters as well as with their preservation.

We shall see later that, wohen a character is undergoing rigorous sclection nea and higher adues than caer before are constantly afpicring. May not the reverse be also true, - namely, that a character on the decline may present its successive decreasing values because of influences entirely internal?

That disappearance of parts is not due entirely to disuse is shown by the fact that the process continues long after the fact of disuse could have the slightest influence. Where a rudimentary tibia further degenerates to a rudimentary pelvic bone, the question of disuse is certainly not involved; neither is use or disuse involved in breeding for high or low oil in corn, for example. Manifestly some biological principle is involved that has not yet been discovered and identified, and, as it is evidently a principle fundamental to transmission and variation, its isolation is exceedingly important.

Characters not dependent upon adaptation. Generally speaking, there is a close correlation between the development of a character and its usefulness to the individual and the species. ${ }^{1}$ This fact has given rise to the impression that all characters are dependent upon teleological principles for their existence. No greater error could be made. It is true that in nature selection operates mostly along utilitarian lines, and in this way after a time it brings most characters into line with the greatest service and the closest adaptation; but selection may operate in any direction, even to the disadvantage of a species. In this case, however, the response is to selection, not to utility.

Neither is development along utilitarian lines necessarily true of those characters that lie outside the field of selection or of those upon which it operates too rarely to impress itself. For example, the instinct to fly toward a source of light would exterminate certain species if naked fire were more generally encountered. That the testicle in mammals should have

1 Known among biologists as teleology. 
descended into an external sac (the scrotum) is in no way useful; on the contrary, it is in many ways unfortunate for the individual. Again, the extreme development of the testes in cattle, and especially in sheep, is most inconvenient, not to say dangerous. Such unusual size is in no way necessary, as we must infer from comparison with other species. It is one of the strange overgrowths of nature, unfortunate, but not sufficiently dangerous to destroy the species. In other words, here are characters upon which selection has never fastened its hold, and consequently they have not been made to square with the highest degree of utility, and have not been brought into the closest "fit." The inference is unavoidable that the existence of a character is not absolutely dependent upon its usefulness. ${ }^{1}$ All this is matter of slight consequence in itself, but it is of fundamental importance when discussing questions touching the disappearance of characters and the transmission of variations.

The origin of characters. Considerations such as here engage the attention, impel the student to raise, in his own mind at least, the ultimate question, What was the origin of racial characters, and how did they come into being?

It may be humiliating, but it is certainly necessary, to say that we do not know, and to freely confess that present information throws little light upon the question. The Lamarckians find a ready answer in asserting that all characters have originated in the necessities of the individual and the race, and in the influence of the conditions of life; but it is both illogical and unscientific to assume that an organ or a part "arose" for no greater reason than that the need for it existed.

The opponents of the Lamarckians, - among whom Weismann is the recognized leader, - depending as they do exclusively upon selection, must assume the preëxistence of the characters on which selection may operate, for selection as such can originate nothing; but this introduces new difficulties, for all higher life is considered to have evolved from lower through the acquisition of characters leading to greater specialization. Now these differentiating characters must have arisen sometime, somewhere, and in some way. Weismann recognizes this difficulty and 1 Of what possible use is the "beard" on the breast of the turkey? 
meets it by assuming that the characters that distinguish the higher races were in some way impressed upon the original protoplasm from without, while the remote ancestors were yet in the singlecelled stare. Thus do the most radical "selectionists" become Lamarckians of the purest kind when driven far enough back. ${ }^{1}$

But is this violent assumption necessary? Life in the singlecelled stage is not fundamentally' different from life in the colony form. A cell is a cell in either case, and its activity - what it can do - is dependent partly upon its ancestry and partly upon the conditions of life, which are its opportunities. To be sure, the single cell is more dependent upon the external world, and reacts more completely to temperature and other external forces, than does the larger colony of highly specialized units (cells), but this is a difference in degree rather than in kind.

In the last analysis we are driven to the conclusion either that all characters were created and implanted in the original protoplasm, - so that living matter, even in its simplest form, has all the potcutialitics of the highest form of life, - or else that the peculiar chemical compounds that constitute living matter are able not only to enter into definite relations with the world at large, but also, perhaps, to effect, from time to time, new combinations among themselves, thus acquiring new or greatly modified characters, sometimes conforming naturally to surrounding conditions, sometimes not. To this latter view the writer strongly inclines.

A chemical element, as iron, acquires no new characters, though it behaves differently under different circumstances. Sulphur is extremely sensitive to surrounding conditions, and many of the organic compounds depend almost entirely for their properties upon the conditions under which their peculiar combinations were effected.

But when life enters the field all the complications are infinitely multiplied, and when we are driven to the last ditch all must agree that the characters possessed by living matter are to a large extent and in some way an expression of the conditions of life. How these conditions impress themselves not only upon the individual but also upon the race is, in some cases at

1. Weismann, Germ Plasm, pp. 4I 5-4I 6. 
least (temperature, food, poisons, and many chemicals not poisons), both evident and easy to understand; in others it is obscure and uncertain to the last degree.

Degeneracy and origin contrasted. Of one thing we are certain: characters are disappearing before our very eyes, and whole races are becoming extinct. What does this mean? Is the world growing poorer in possibilities? Is specialization realized only at the expense of lessened adaptability to new conditions later on? If a part or a character now useless degenerates and disappears will it ever come back, or will a new one arise to take its place if necessity for its presence should return?

These are large questions - questions that we cannot answer, but that we must think about and take into consideration in the studying and answering of easier and smaller questions.

In the meantime we will remember that soles, founders, and the flatfishes generally are developing a new style of living, ${ }^{1}$ and that their eyes are taking a new position with reference to the other body parts. We will not forget that all animals that live in (under) the water, if of much size, are of one general shape, - the shape of least resistance to water. That this is independent of selection is shown in the history of the whale and of the few land mammals that took to the water and whose transformation must have been comparatively recent.

We will remember that, while most "new characters" are but new combinations and different adjustments of old ones, there is, after all, progressive development showing the infusion of something practically new and differcut. Higher life differs from lower in kind as well as in degree. That it springs from the lower is certain, and that something has been added in the process is no less certain.

The world is full of lowly forms of life. The species of singlecelled organisms that are known probably far outnumber existing

1 These fishes, of which there are a number of species, are symmetrical, or nearly so, in the embryo and for a little time afterward, so that at first they swim like any other fish. The swimming bladder is defective, however, and shortly they t irn to one side and lie on the bottom, generally left side down, - though some individuals are reversed. In this position the left (now lower) eye travels upward toward the other side, until the two eyes lie side by side on the right, now the upper, side. 
species of highly developed organisms. Is this the "raw material" out of which new species shall be evolved later on to take the place of what is now so rapidly becoming extinct? It is a moving panorama, and we know neither the beginning nor the end. It is all a part of a vast plan on which the universe is founcled by the Creator. We cannot doubt that the most complex element of it all is life, nor can we cloubt that the most characteristic property of living matter is its progressize development, bringing to light new activities and establishing new relations with the world outside. Whether this property is wholly internal and dynamic, or due, at least to some extent, to outside influences, — this is the chief mystery.

\section{SECTION IX - VARIATIONS DUE TO CAUSES NOT AFFECT-} ING THE GERM ARE NOT TRANSMITTED

This much is a logical conclusion. This much is fundamental and axiomatic. The germinal matter is the only material that passes over from generation to generation. The fertilized germ is the only heritage, and it is the only avenue of transmission. Whatever faculties or endowments the individual may possess, he can transmit only those which can find lodgment and representation in the germ, and whether the causes of germinal changes are internal or external, or both, no variation can be transmitted unless it affects the germ or unless the cause that induced the variation also affected the germ.

Variations due to causes internal to the body but external to the germ are not transmitted. It is entirely possible that certain variations, either of structure or of function, may arise from irregularities in cell division during development due to local rather than to germinal causes. For example, it has already been noted that cells may at any time divide into unequal portions, or into three instead of two parts, giving rise to abnormal if not to pathological conditions.

Almost all portions of the body are subject to those overgrowths called tumors, and while it is not yet definitely known whether the causes of abnormal growth are external or internal to the organism, they certainly are not located in the germ. 
The probability is that they are entirely local, sometimes arising from external injuries, as in galls, and thus outside the present field of inquiry, but more often due to internal clisturbances in the cells themselves at the particular point where the abnormal functioning appears.

Modifications due to external causes sometimes transmitted, oftener not. The effects of such all-pervading influences as nourishment, temperature, chemical action, gravity, etc., are not felt simply by the external parts, but on the contrary they may extend to the innermost parts of the organism, affecting the constitution and the activities of the most highly specialized matter, extending, for all we know, to the very germ, in which case they would certainly be transmitted, whereas there is no ground for belief in the transmission of influences that do not affect the germ.

Summary. When we speak of the transmission of a modification we mean rather the transmission of a claractir as modifict. Strictly speaking, a character will be transmitted in a modified form if the modification affects the gom ; otherwise it will be transmitted in an unmodified form, for the germ is the only hereditary substance, and nothing is transmitted except through the germ.

A modification may arise from causes either internal or external to the germ. If internal it of necessity affects the germ and is transmitted. This is the ordinary cause of hereditary variability. If, on the other hand, the modification arose from external causes, the germ may or may not be affected, and the modification may or may not be transmitted.

There is much reason to believe that many modifications of functional activity are of such a funclamental nature as to influence the germ as well as the soma, and such modifications would be transmitted and inherited by the offspring.

In general, the great effect of the cuironment is to influence diclopment, not to induce new characters. The environment does not decide what characters shall compose the individual, - that is a matter of straight inheritance, - but it does decide to a very large extent what degree of development the various racial characters shall attain in particular individuals. The 
question whether extreme development tends to affect the germ and to become inherited is a question beset with many difficulties. The greatest obstacle to this study is the ever-present fact of selection, which rapidly brings about a close correspondence between organisms and their surroundings. No reliable conclusion can be drawn until this influence is accounted for or eliminated.

In further pursuance of this study we now pass to more definite discriminations as to type and to more exact and critical distinctions concerning variability as expressed not in indivicluals but in numbers sufficiently large to be fairly indicative of the race.

\section{ADDITIONAL REFERENCES}

An Examination of Weismannism. By G. J. Romanes, i vol. Darinin and After Darwin. By G. J. Romanes. 2 vols.

Development and Evolution. By J. M. Baldwin. Science, XVI, $819-821$.

Environment and its Effect on the Transmitting Power of SeEds. By W. W. Tracy. Science, XIX, 738-740.

Experimental Zoölogy. By T. H. Morgan. Chapters IV and V, pp. 43-6r.

Foundations of Zoölogy. By W. K. Brooks. I vol.

Heredity and Instinct. By J. M. Baldwin. Science, III, 439-44I, $55^{8}-559$.

Inheritance of Acquired Characters. By E. D. Cope. Science, V, 633-634; by John McFarland, Ibid., 935-945.

Inheritance of Acquired Characters. (Examples.) By F. H. Herrick. Science, VII, 280 .

Inheritance of Acquired Characters. By D. E. Hutchens (I904). Nature, LXXI, 83 .

Nature of Cancers ani) Abnormal Growths, axi Transmissibility of SAme. By E. B. Bashford. Proceedings of the Royal Society, London, LXXIII, 66-67.

Right- and Left-Eyedness. By G. M. Gould. Science, XIX, 59I-594. The Heredity of Acouren Characters. Boston Medical and Surgical Journal, CXXXVII, 427-428.

Use-Inieritaxce. Direction of Hair in Man and Animals and its Application to Darwinism. By W. Kidd. Science, XV, I42-I43; also in Science, $\mathrm{XX}, 40 \mathrm{I}-407$. 


\section{CHAPTER XII \\ TYPE AND VARIABILITY ${ }^{1}$}

Enough has been shown in earlier chapters to convince the student that variability is an inevitable accompaniment of both reproduction and development, and therefore that variation is to be expected among living beings everywhere.

Before anything like a comprehensive idea of transmission can be developed therefore, it is necessary to study, not indivicluals, but groups, and to establish definite conceptions as to type and variability. In this, as in any other critical study of a race, we proceed character by character, and are careful to include enough individuals to be fairly representative of the race as a whole. ${ }^{2}$

A farmer plants an ear of corn say ten inches in length. What he gets is not a crop of ears all ten inches long, but a group of ears ranging in length all the way from perhaps three or four inches up to eleven or twelve, or even a little more. The same principle will hold if the ear that is planted is nine inches long instead of ten, except that the distribution will be different, lengths running, in general, slightly lower; that is to say, the length of ear of the offspring is not the same as that of the parent, but it constitutes a "distribution" extending both above and below that length. So far as is known, this principle of transmission holds true in all races and for all characters. Stated in more general terms, we may say that the offspring as a whole is not the same as the immediate parents, but that it constitutes a distribution crtending from near the lower to approximately the upper limits of the race. This suggests at once the idea of type and that deviation from type which we call variability.

1 See Bulletin No. Irq, Agricultural Experiment Station, University of Illinois, by the author of this text.

2 Having discovered the type as to several important characters, it would then be possible to select a typical individual. 


\section{SECTION I - TYPE}

What now is our conception of type? If ten-inch ears will not produce ten-inch ears, but something else, and not only something else but a considerable variety of lengths; and if what we get extends both above and below the parent, then we arrive at once at a double conception as to type ; that is to say, the type of the offspring is not the same as that of the parent. The type of the parent is very definite, representing an ideal; but if the offspring is distributed both above and below that ideal, some being better and some not so good, then a close analysis of the real character of that offspring becomes necessary in order to make any just comparison between the two, or to arrive at any adequate conception of type in a mixed population, even in one arising from a selected ancestry.

A concrete case will serve best to illustrate the principle involved. In the year I906 some Leaming corn was raised on good ground from seed ears exactly ten inches in length. A random sample ${ }^{1}$ of this crop, consisting of 327 ears, gave the following distribution as to length :

One ear was 3.0 inches long, one was 4.0 inches, two were 5.0 inches, three were 5.5 inches, nine were 6.0 inches, eight were 6.5 inches, twelve were 7.0 inches, nineteen were 7.5 inches, thirty-two were 8.0 inches, forty were 8.5 inches, sixty-seven were 9.0 inches, sixty-three were 9.5 inches, thirty-eight were Io inches, twenty-one were 10.5 inches, eight were II.O inches, two were II.5 inches, and one was I 2.0 inches long. ${ }^{2}$

1 By a "random sample" is meant a sufficient portion of the whole, taken so much at random as to fairly represent the entire crop, or "population," as the technical phrase goes.

2 Measurements might be taken at quarter inches with a seemingly higher degree of accuracy, but repeated triais show that the same final results follow whether measurements are taken at the quarter inch or at the half inch. The main point is that the numbers shall be sufficient and that the sample shall be representative. Judgment must dictate as to the accuracy of the sample, but the number depends upon the degree of reliability desired. This matter will be fully discussed under the subject of probable error, but experience shows that in studies with corn excellent results can be had with from 200 to 300 ears, and very fair results may generally be had with half that number. 
Put in tabular form as it appears in actual work we have the following: 1

Length of Ears, or Value, $-V$

No. of Ears, or Frequency, $-f$

\begin{tabular}{|c|}
\hline 3.5 \\
\hline $4.0 \ldots 1$ \\
\hline 4.5 \\
\hline $5.0 \ldots$ \\
\hline 5.5 \\
\hline 6.0 NWIIII \\
\hline $6.5 \_$INI III \\
\hline 7.0_ NWNWII \\
\hline 7.5 IN NWI NW IIII \\
\hline 8.0 NWU NWW NWW NWI INW INW II \\
\hline 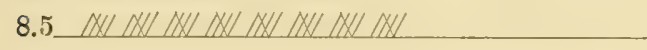 \\
\hline 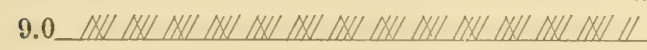 \\
\hline 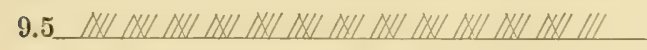 \\
\hline 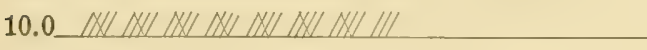 \\
\hline 10.5 NW NWN NN NWL \\
\hline 11.0 NW III \\
\hline 11.5 \\
\hline $12.0 /$ \\
\hline
\end{tabular}

Here we have a "frequency distribution" representing the entire "population," or crop, and as it lies spread out before the eye a glance is sufficient to afford considerable information as to the prevailing type.

It will be noted at once that there are more ears of 9 inches than of any other length, and that the distribution decreases in both directions, but unequally, from this highest frequency.

The mode. This highest frequency, or most common length, shows clearly what is the prevailing type in the crop, as distinct from the selective type of ro inches in the seed ear, and it is held by statisticians and by students generally to be the best obtainable single expression for type. When it is ascertained,

1 This is the most convenient form in which to make the original record. A mark is made for every individual examined, and the additions are readily made. 
therefore, we know at once what is the natural type ${ }^{1}$ of the race or inricty so far as the character in question is concerned, and whon this is determined for a munber of important claracters a'e shall hare a grood knowlidge of the racial type as a whole. Thus we might obtain the mode for circumference, number of rows, weight of ear, color of grain, per cent of cob, or any other desired character, and having done so a typical car of this saricty could be difinitely' described. We thus arrive at an accurate idea of type in a greneral population, and of its definite measurement.

The empirical and the theoretical mode. It is eviclent by inspection of the frequency table that if measurements had been taken at the quarter inch, or some less fraction, the highest frequency would have fallen not at the nine-inch point but slightly above it, for the next frequency above $\left(\sigma_{3}\right)$ is greater than the next one below (40); that is to say, the mode is to some slight extent dependent upon the scheme of measurements adopted.

Any scheme expressed in numbers, either whole or fractional, is of course by nature discontinuous, and the mode arising from such a scheme is at best only an approximation. It is therefore called the empirical mode. If all possible values were represented, however, as is done whenever the theoretical curve is plotted corresponding to the frequency distribution, such a continuous curve will find the true or, as it is called, the " theoretical " mode. It is necessary to recognize this clistinction, although in practical breeding operations the empirical mole arising from convenient measurements is sufficiently accurate.

The coefficient of mode, or modal coefficient. ${ }^{2}$ It is not enough simply to determine which value has the highest frequency, even though this gives us the type; we desire to know also what proportion of all the individuals tends to drop into the type

1 It is evident that the frequency distribution and, therefore, the type of an adult population is something different from that which was born into the race. What that may have been we can never know. Many individuals did not survive, and the development of all was influenced by environment. The final result as represented in adult individuals is all we can consider.

2 The writer has never seen this expression used. It is of little consequence in general evolution, but is of much significance in thremmatology, where the breeder desires to know what proportion of his animals or plants conform to type. I have, therefore, taken the liberty of using it, as here, - " the coefficient of mode, or modal coefficient." 
of the race. This is easily determined in the form of a rate per cent by dividing the highest frequency by the total number of variates. ${ }^{1}$ The highest frequency in this case is 67 , which is over 20 per cent $(20.4+)$ of the total number of ears measured. This we call its modal coefficient because it indicates the percentage of the total population that conforms to type in respect to this character. The modal coefficient of some other variety might be quite different, showing that a higher proportion of one variety may conform to type than of another; or, what is the same thing, that one variety may be more constant and truer to type. The modal coefficient, therefore, is an inder of relatice conformity to typi, a valuable bit of knowledge for purposes of selection.

Modal coefficient partly dependent upon the scheme of measurements adopted. If these measurements had been taken to the quarter inch there would have been twice as many frequencies and each would have been represented by correspondingly fewer ears. The highest frequency, therefore, would have been not 67 but approximately half that number, -33 or thereabouts, and the modal coefficient would have been not 20 per cent but near ro per cent. This being the case, modal coefficients are not directly comparable except when arising from the some systcm of masurments, or after the coefficient has been dirided by the width of the class; thus, $20 \div \frac{1}{2}$ equals $10 \div \frac{1}{4}$.

For the purpose of comparing the variability of races we use the "coefficient of variability," to be described later. The modal coefficient is chiefly valuable for comparing one type with another within the rece, which is all that is required in ordinary breeding.

Practical value of the frequency distribution, the mode, and the modal coefficient. The practical importance of the information afforded by these values must be apparent. By means of the frequency clistribution the breeder is enabled at any time, when he can secure sufficient numbers, to spread out before his eyes a good and fair representation of the whole population of the variety or race he is breecling, with respect to any character which he can measure or accurately estimate.

I By variates is meant the individuals measured (in this case 327 ears). 
When he has ascertained its mode he knows what is the natural type, for mode indicates type; and he then knows by how much, if any, it differs from the type ${ }^{1}$ which he has chosen as the standard for selection. By this he may judge whether and to what extent he is operating at variance with nature.

The mean. There is still another conception of type as to this distribution, and that is the average, or "mean" as it is technically called. It will be noted that the distribution does not decline uniformly both above and below the mode; that is to say, there are six values below and only three above, - from which we conclude that the average length of ear is somewhat different from the most usual length. By multiplying each separate length by the number of ears of that length and adding the products (or, what is the same thing, adding together the lengths of all the ears) and then dividing by the total number of ears, we find the average, or mean length, to be 8.83 - inches.

Accordingly we have the following for the determination of the mean ${ }^{2}$ : Multiply each value by its frequency, add the results, and divide the sum by the number of individuals ${ }^{3}$ or variates.

Applying this principle to the case in hand we have the result seen in the accompanying table: ${ }^{4}$

* It is customary to drop off extremely outlying values in the distribution, but evidently in this case if very large num-

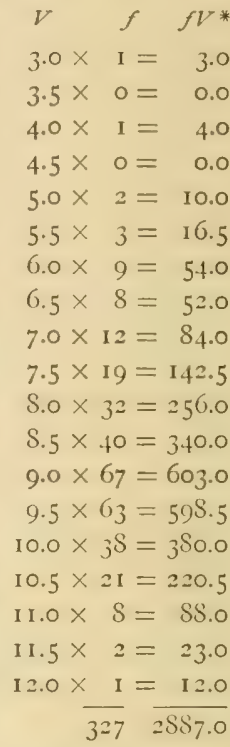

$2887.0 \div 327=8.83-$, the mean length of ear in inches bers had been taken these blanks would have been filled; that is, ears of 3.5 inches and 4.5 inches would have been found; hence all values are included here.

1 Here is another conception of type. The ideal of the breeder, which he accepts as his standard, is a kind of economic or business type, quite distinct from the biological type indicated by the mode. The purpose of all breeding is to bring the two as close together as possible.

2 By "mean" is here meant the arithmetical average, which is the average most commonly accepted and the symbol of which is $M I$. For a discussion of different

averages, see Appendix.
$\quad 3$ The algebraic formula would be $. I=\left(V_{1} f_{1}\right)+\left(V_{2} f_{2}\right)+\cdots\left(V_{n}, f_{n}\right)$
$u$ $f_{1}, f_{2} \cdots f_{r}$ are the frequencies, $V_{1}, V_{2} \cdots V^{r}$ the respective values, and $n$ the number of individuals measured.

4 In this table $V$ stands for values, or magnitudes, - in this case length, and $f$ stands for frequency, or the number of varieties (ears) of each separate measurement. The whole column under $f$ is technically known as a "frequency 
Here we have a third valuation for type $\left(8_{8} 8_{3}-\right)$, representing the average, as distinct from 9 of the highest frequency, or most usual length, and both distinct from the 10 inches of the ear planted.

Practical use of the mean. The mean gives a gond average value of the character, and establishes the practical or commercial value of a race or variety, for it shows what it will do on the avcrage. It is not always, however, a good index of the prevailing type, for, as often happens, the variety with the higher mean may have the lower mode. Neither is the mean always a good index of conditions; for example, in a population of one thousand paupers and one millionaire the mean wealth is fair, but the type is clearly that of the pauper.

Here are three separate and very definite conceptions of type: (I) the icleal, which is used in selecting the parentage; (2) the prevailing type as represented by the highest frequency or most usual length (the mode); and (3) the average length as represented by the mean.

These three conceptions of type - the ideal type of the parent, the prevailing type of the offspring, and the general average of the offspring - have distinct applications to the practical affairs of breeding. ${ }^{1}$ The breeder of pedigreed stock is interested primarily in the ideal and in the mode, or highest frequency, while the general farmer who multiplies or raises it for the open market is most interested in its mean, or average production.

\section{SECTION II - VARIABILITY, OR DEVIATION FROM TYPE}

Having established definite distinctions as to type, the student of transmission should next form equally clear conceptions as to deviation from type, commonly known as variability. ${ }^{2}$

distribution," representing an entire race, spoken of as the "population." The heading $\mathrm{fl}^{\circ}$ means the products of the values (lengths) multiplied by the corresponding frequencies.

${ }^{1}$ It is to be noted that the generation to which the selected parent belonged had also its own mode and mean, which may have been quite different from those of the offspring.

2 The term "variability" should not be understood as expressing departure in the sense of wandering from a fixed standard. Students sometimes gain the 
In the study of variability it is worse than useless to study a few scattered individuals here and there. What we seek is a measure of what may be called the average tendency to deviate from type. Some individuals deviate but little, others more, and still others very much, and we seek a measure of this nonconformity to type. To find this we must study groups of individuals sufficiently large to be representative of their race. This brings us back to the frequency distribution and what it can teach as to variability. ${ }^{1}$

impression that if the law of heredity were infallible all offspring would be of a common type, and that any departure from the type of the race, variety, or breed is to be regarded as by so much a failure of heredity and a concession to variation.

The truth is that all transmission is heterogeneous in the sense that the individuals of any race, whether parents or offspring, belong not to a fixed type but to a frequency distribution similar to the one now under discussion. The itita of type thus arises out of the distribution, and it constitutes a convenient base from which to reckon deviation.

The chief conception to rest in the mind of the student at the present stage of matters is that, whatever the parentage, the offspring will constitute a distribution extending through a considerable range, and that the parent itself also belonged and was drawn from some portion of a frequency distribution not very different from that of the race in general.

Variability is, therefore, not the opponent of heredity but its inevitable accompaniment in transmission, and our problem is to devise methods of accurately measuring and expressing its range and extent in any particular instance.

1 No apology is made for introducing the so-called statistical method of study at this point ; first, because it is the only reliable method of attacking problems in transmission, and second, because this method is everywhere coming into use among careful students. The reader is urged, and the student should be required, not to evade this portion of the subject because the method of treatment may happen to be unfamiliar. On the other hand, he is urged to familiarize himself not only with the method of work but with the point of view involved. If he will do this, both variability and later on correlation and heredity in general will come to have a new meaning, and one far more rational and comprehensive than the hazy notions evolved from the unsystematic study of isolated individuals. The principles involved are for the most part simple, and in this elementary treatise every effort will be made to treat the subject from the standpoint of the nonmathematical reader.

For the convenience of those who may care to pursue a little further some of the more strictly mathematical conceptions involved, an Appendix has been prepared by Dr. H. L. Riet $z$, of the mathematical department of the University of Illinois.

The statistical method of study of problems in heredity, as distinct from the strictly biological, was introduced by Dr. Francis Galton of England (see Natural Inheritance, ISS9), and afterward much extended by Karl Pearson and others (see especially Grammar of Science and I'hilosophical Transactions of the Royal Society). It is now coming into such common use that a quarterly journal 
Again the concrete serves well as a medium for teaching a principle. In this connection we refer once more to our distribution of 327 ears, and note that every ear in the lot deviates somewhat from the mean of $\$ .83$ inches. The range and extent of this deviation are shown in the following table, column $D$ ).

The practical question now is to reduce this column of deviations to a single expression denoting the variability of the population of which this distribution is representative. Manifestly, when this is done, the variability of this distribution can be compared directly and exactly with that of any other, and at the present or any future time. Two methods of procedure are possible in thus securing a kind of general expression for the average amount of deviation, giving rise to two similar but slightly different values, namely, the average deviation and the standard deviation.

The average deviation. If each deviation (column $D$ ) represented an equal number of ears, this single expression could be readily derived by adding the deviations and dividing by the total number. But these deviations do not represent equal numbers of ears. The

Deviation of 327 Ears OF CORN FROM THEIR MEAN LENGTH OF 8.83 INCHES

$\begin{array}{rrr}V & f & D^{*} \\ 3.0 & I & -5.83 \\ 3.5 & 0 & -5.33 \\ 4.0 & I & -4.83 \\ 4.5 & 0 & -4.33 \\ 5.0 & 2 & -3.83 \\ 5.5 & 3 & -3.33 \\ 6.0 & 9 & -2.83 \\ 6.5 & S & -2.33 \\ 7.0 & 12 & -1.83 \\ 7.5 & 19 & -1.33 \\ 8.0 & 32 & -0.83 \\ 8.5 & 40 & -0.33 \\ 9.0 & 67 & 0.17 \\ 9.5 & 63 & 0.67 \\ \text { I0.0 } & 38 & 1.17 \\ \text { I0.5 } & 21 & 1.67 \\ \text { II.0 } & S & 2.17 \\ \text { II.5 } & 2 & 2.67 \\ \text { I } 2.0 & \text { I } & 3.17 \\ & 327 & \\ 110 & & 11\end{array}$

deviation $-5.8_{3}$, for example, represents but one ear, while no less than twelve ears deriated I. $S_{3}$ inches below the mean and two deviated 2.67 inches above, with others unevenly distributed.

Manifestly each deviation should first be multiplied by the number of ears involved, as in the succeeding table $:^{1}$

(Biometrika) is devoted to the reports of statistical studies in evolution, technically known as "biometry."

1 When the deviation is to be obtained in this way the minus sign is disregarded.

* $D$ indicates the deviation of the several groups from the common mean of the race, $8.8_{3}$ inches. Thus, for example, the first ear deviates the difference between 3 inches and $8.8_{3}$ inches, or $5.8_{3}$, which, being below the mean, is written with the negative sign; also the 2 I ears 10.5 inches long deviate $10.5-8.8_{3}$, or 1.67 inches from the mean, and being above the mean, we write it positive. 
$f \quad D \quad D f$

I $\times 5.8_{3}=5.8_{3}$

$0 \times 5.33=0.00$

I $\times 4.83=4.83$

$0 \times 4.33=0.00$

$2 \times 3 . \$_{3}=7.66$

$3 \times 3.33=9.99$

$9 \times 2 . S_{3}=25.47$

$8 \times 2.33=18.64$

$12 \times 1.83=21.96$

$19 \times 1.33=25.27$

$32 \times 0.8_{3}=26.56$

$40 \times 0.33=13.20$

$67 \times 0.17=11.39$

$63 \times 0.67=42.21$

$3_{3} S \times 1.17=44.46$

$21 \times 1.67=35.07$

$S \times 2.17=17.36$

$2 \times 2.67=5.34$

$1 \times 3.17=\frac{3.17}{318.41}$
The result of this calculation is that the total deviation of 327 ears from their average length is $318.4 \mathrm{I}$ inches, some above and some below the mean. If now we divide 3 I $8.4 \mathrm{I}$ by 327 , the number of ears involved, we have $0.97+$ inches, which is a good expression of the arouge deviation of this particular population. If another variety should give a larger quotient, we should conclude it to be more variable. In this manner we may reduce the variability of a whole population to a single expression.

Standard deviation. Mathematicians have another method of calculating variability. It differs from the one just discussed in only one detail; namely, the deviations are squared before multiplication by their respective frequencies, as in the table which follows:

\begin{tabular}{|c|c|c|c|c|}
\hline$V$ & $f$ & $=D$ & $D^{2 *}$ & $D^{2} f t$ \\
\hline 3.0 & I & $-5.8_{3}$ & 33.9889 & 33.9889 \\
\hline 3.5 & 0 & -5.33 & 28.4089 & 00.0000 \\
\hline 4.0 & I & -4.83 & $23 \cdot 3289$ & $23 \cdot 3289$ \\
\hline $4 \cdot 5$ & $\circ$ & -4.33 & 18.7489 & 00.0000 \\
\hline 5.0 & 2 & -3.83 & 14.6689 & 29.3378 \\
\hline $5 \cdot 5$ & 3 & -3.33 & 11.0889 & 33.2667 \\
\hline 6.0 & 9 & -2.83 & 8.0089 & 72.0801 \\
\hline 6.5 & 8 & -2.33 & $5 \cdot 4=89$ & 43.4312 \\
\hline 7.0 & 12 & $-1 . S_{3}$ & 3.3489 & 40.1868 \\
\hline $7 \cdot 5$ & 19 & -1.33 & I. 7689 & 33.6091 \\
\hline 8.0 & 32 & $-0.8_{3}$ & 0.6889 & $22.044^{\mathrm{S}}$ \\
\hline 8.5 & 40 & -0.33 & 0.1089 & 4.3560 \\
\hline 9.0 & 67 & 0.17 & 0.0289 & 1.9363 \\
\hline $9 \cdot 5$ & 63 & 0.67 & 0.4489 & 28.2807 \\
\hline 10.0 & $3^{8}$ & I.I 7 & I.3689 & 52.0182 \\
\hline 10.5 & $2 \mathrm{I}$ & 1. 67 & 2.7889 & 58.5669 \\
\hline 11.0 & 8 & 2.17 & 4.7089 & 37.6712 \\
\hline II. 5 & 2 & 2.67 & 7.1289 & $14.257^{8}$ \\
\hline \multirow[t]{2}{*}{12.0} & I & 3.17 & 10.0489 & 10.0489 \\
\hline & 327 & & & $\Sigma 538.4103 \hbar$ \\
\hline
\end{tabular}

* The column marked $D^{2}$ is secured by squaring the various deviations, thus eliminating the minus sign. For example, $-5.8_{3} \times-5 . S_{3}=33.9889$.

t The column marked $D^{2} f$ is obtained by multiplying the squared deviations each by its respective frequency. For example, $8.0089 \times 9=72.080 \mathrm{I}$.

t The (ireek capital sigma $(\Sigma)$ is the usual sign of summation in mathematics. 
Dividing 538.4103 by 327 after the manner of fincling the average deviation, we have the quotient 1.6465 ; but as the deviations have all been squared during the operation it is necessary to extract the square root of this number in order to arrive at the correct value. The square root of 1.6465 is $1.28+$, and this is the so-called standard deriation of the mathematician, the universal sign for which is the Greek letter, small sigma $(\sigma)$.

Hence, to find the standard cleviation, we have the rule: Find the deviation of each frequency from the mean; square each deviation, and multiply by its corresponding frequency; add the products, divide by the total number of variates, and extract the square root. ${ }^{I}$

Shortening the method. The large decimais can be avoided, and the process of finding both the mean and the standard deviation can be very much shortenerl, by assuming as a mean the nearest probable measurement as determined by inspection of the frequency distribution, and afterward making a suitable correction. For example, in the present instance, we should judge by inspection that the mean cannot be far from 9.0.** This we infer from the fact that the distribution reduces both ways from this point and quite evenly. Proceeding with this assumption, denoting our "gutess" by $G$ and reckoning deriation provisionally from this point, we have the result as seen in the table on the following page.

Considering first the mean: In column $f(V-G)$ we find that after multiplying the deviations from our assumed mean (9.0) by their respective frequencies, the sum of the negative products ( - I 8 I.O) exceeds the sum of the positive products (I 25.0 ) by 56.0 ; that is, the algebraic sum of the products is -56.0 . Our assumed mean is therefore too high by the amount of $-56.0 \div 327+=-$ O. I 7 I. We then reduce our assumed mean

1 Expressed in symbols the formula is $\sigma=\sqrt{\frac{\sum D^{2} f}{n}}$.

* The advantage of assuming this value from which to reckon deviation lies in the fact that it is exact and contains but one decimal, while the true mean has at least two decimal places, making relatively large numbers.

$\dagger$ We divide by the total number (327) because we are dealing with a column of products arising from the introduction of the frequencies. 
by this amount $(9.0-0.17 \mathrm{I}=8.829)$ and arrive at the true mean 8.83 - **

Considering next the standard deviation: In column $f(Y-G)^{2}$ we have 548.00 as the sum of the products of the several frequencies into their respective deviations from the assumed mean, clerived on the same plan as when working from the true mean

\begin{tabular}{|c|c|c|c|c|c|}
\hline$V$ & $f$ & $V-G$ & $f(V-G)$ & $(V-G)^{2}$ & $f(l-G)^{2}$ \\
\hline 3.0 & I & -6.0 & -6.0 & 36.00 & $3^{6.00}$ \\
\hline $3 \cdot 5$ & o & -5.5 & 0.0 & 30.25 & 00.00 \\
\hline 4.0 & I & -5.0 & - 5.0 & 25.00 & 25.00 \\
\hline 4.5 & 0 & -4.5 & 0.0 & 20.25 & 00.00 \\
\hline 5.0 & 2 & -4.0 & -8.0 & I 6.00 & 32.00 \\
\hline $5 \cdot 5$ & 3 & $-3 \cdot 5$ & -10.5 & 12.25 & 36.75 \\
\hline 6.0 & 9 & -3.0 & -27.0 & 9.00 & 81.00 \\
\hline 6.5 & 8 & -2.5 & -20.0 & 6.25 & 50.00 \\
\hline 7.0 & I 2 & -2.0 & -24.0 & 4.00 & 48.00 \\
\hline $7 \cdot 5$ & I9 & $-\mathrm{I} .5$ & -28.5 & 2.25 & 42.75 \\
\hline 8.0 & $3^{2}$ & -1.0 & -32.0 & 1.00 & 32.00 \\
\hline 8.5 & 40 & -0.5 & -20.0 & 0.25 & 10.00 \\
\hline 9.0 & 67 & 0.0 & \multirow{2}{*}{$\begin{array}{r}0.0-181.0 \\
31.5\end{array}$} & 0.00 & 00.00 \\
\hline $9 \cdot 5$ & $6_{3}$ & 0.5 & & 0.25 & 15.75 \\
\hline 10.0 & $3^{8}$ & 1.0 & $3^{8.0}$ & 1.00 & $3^{8.00}$ \\
\hline I0. 5 & 21 & I. 5 & $3^{I} \cdot 5$ & 2.25 & 47.25 \\
\hline I 1.0 & 8 & 2.0 & 16.0 & 4.00 & 32.00 \\
\hline II. 5 & 2 & 2.5 & 5.0 & 6.25 & 12.50 \\
\hline \multirow[t]{2}{*}{12.0} & I & 3.0 & 125.0 & 9.00 & 9.00 \\
\hline & 327 & & \multicolumn{2}{|l|}{ Difference, -56.0} & $\Sigma 548.00$ \\
\hline
\end{tabular}

1). Dividing by the total number (327), we have $548.00 \div 327=$ 1. 6758 , corresponding to the quotient $538.4103 \div 327=1.6465$ of the previous calculation when working from the true mean.

The correction made in the mean was -O.I I ; but as we are now dealing with second powers it seems but natural that this amount should be squared before it can be taken from the quotient 1.6758 . This will be justified by a mathematical proof in the Appendix. The square of $-0.17 \mathrm{I}$ is $0.02924 \mathrm{I}$, or $0.0292+$.

* On the other hand, should the sum of the positive deviations exceed the sum of the negative deviations it would indicate that our assumed value is too small and we should add the correction in order to arrive at the true mean. 
We have therefore as a correction on account of the true mean I. $6758-0.0292+=\mathrm{I} .6466$.

This agrees very nearly with the value 1.6465 previously found, but this shorter method is the more accurate, because fewer decimals have been lost. The square root of I.6466 is $1.28+$, the standard deviation sought, agreeing perfectly with the former value and derived by a very much shorter method.

The student will note that the difference in the two methods is essentially this: in the latter we deal only with deriations, while in the former entire values are involved. It is true that deviations are taken from an assumed mean, but the correction is accurately made, and the whole operation can be carried forward not only with smaller numbers but also without the loss of decimals necessarily involved in the more direct but far more laborious and on the whole less exact method first given. The first method is useful for expounding the principles involved, but the later is far preferable for actual use, not only on account of its brevity, but on account of its increased accuracy as well.

Average deviation and standard deviation contrasted. These two expressions for variability rest upon the same arithmetical principle, but the latter has decided mathematical advantages over the former for many purposes and is the one universally used by mathematicians. The only advantage in the arerage deviation lies in the simpler calculation, as neither squares nor roots are involved. With the shortened method of fincling the standard deviation, however, this advantage is slight.

It makes little difference which is used in practice, provided the same method is always employed. The results obtained differ considerably $(0.97+$ as compared with $1.2 S+)$. The standard deviation is always larger than the average deviation because of the squaring of the several deviations. It thus exaggerates the wider departures from type as compared with the methods employed in finding the average deviation. This being true, results obtained by the two processes can never be compared; that is, when dealing with values clesigned to express variability we must always know whether average deviation or standard deviation is meant. 
The breeder may choose either method, but having once chosen, all his records must be made in the same way. Inasmuch as the standard deviation has distinct mathematical advantages over the simple average deviation, and inasmuch as it is the one commonly employed in mathematical literature, it is the one that will always be employed in this text. When, therefore, a measure of variability is mentioned it will be the standard deviation and not the average deviation that is meant. ${ }^{1}$

Meaning of standard deviation. The standard deviation is a good measure of variability for the character in question and the race involved. It therefore affords a reliable basis for comparing the arriability of one race with that of another as to the charactir under consideration, or of one character with that of another cither in the same or in different races.

In ascertaining the standard deviation the mean, or average, of the race was taken as the basis, and all deviation was reckoned from that. It is manifest, however, that if the modc be taken as a basis, and deviation reckoned from this, following the same methods as for determining the standard deviation, a somewhat different value will result. Whenever, as in most cases, the mode does not coincide with the mean, this will represent the deviation from the prevailing type, which is often of more practical importance to the breeder than the deviation just described, especially to the breeder who proposes to deal with individuals selected with reference not to the mean but to the prevailing type.

In the same manner the breeder may calculate the deviation from his own ideal type or standard and in this way assess the deviation, not from the present mean or type but from the one he hopes to establish. In this way he is able to keep accurately informed from year to year as to the progress he is making and the degree of success that is following his selections.

The writer has never seen either of these determinations used, but they are especially valuable to the breeder who desires to know how a certain variety deviates on the average from its

1 In the section on "Probability Curve" (Appendix) it will be shown that the standard deviation is on the whole preferable from the purely mathematical standpoint. 
oren type or even from his standart of sclection, as well as to know how it deviates from its own mean.

I have therefore recommended their use, calling the one deriation from mode and the other deriation from standart, and earnestly suggest their constant employment by the breeder as a means of acquainting himself with the true nature of the variety he is attempting to improve; for improvement consists often in changing the type as well as in bringing a larger proportion of the population to conform either to the natural or to the accepted standard. ${ }^{1}$ To do this he should make these determinations year by year, and keep the results as a history of the variety or breed as it behaves with him under selection.

Practical meaning of standard deviation. Inasmuch as the standard deviation is an index of variability whether from mean or from type, it expresses accurately the tendency of the variety to wander, so far as the character in question is concerned. It affords, therefore, a basis of comparison of one variety with another, or with itself at some future time. However, one standard deviation ordinarily cannot be compared directly with another for two reasons: first, one mean may be very much larger than the other; and second, the two may be of entirely different units, as inches and pounds, in which case direct comparison is impossible.

Coefficient of variability. We seek, therefore, an abstract expression combining the idea both of standard deviation and of type. Such an expression is known as the "coefficient of variability," and is found as follows: Divicle the standard deviation by the mean as a base, and the result will be an excellent index of variability appearing in the form of a rate per cent. ${ }^{2}$

Thus, for the case in question, we have $1.28 \div 8.83=0.145$, indicating the variability of this population to be over 14.5 per cent of its own mean. Here we have a mathematical expression for comparing variability on a perfectly abstract basis, and by this means we can compare the variability of this population

1 "Standard," as here used, refers to the scale of points, or that which the breeder is trying to establish. It is his standard for selection.

2 The formula is $\mathrm{C}=\frac{\text { standard deviation }}{\text { mean }}$ or $\frac{\sigma}{\mathrm{M}}$. 
with that of any other race, plant or animal, and for any character of which accurate measurements can be taken and a frequency distribution be constructed. ${ }^{1}$

Practical application of the coefficient of variability. The value of this term lies in the fact that it affords an accurate means of comparing directly the variability of one frequency distribution with that of another, no matter what the claractir, whither in the same or different indiaiduals, or bitriecn similar or unlike species. Thus, by this means we may compare the variability of the lingth of an ear of corn with that of its weight; the variability of its circumference with that of its number of rows, or with that of any other measurable character.

IVe can also, in this way, compare the variability of ears of corn with that of their stalks; with that of the length of horse's legs, of what they can pull, or of the rate at which they can travel; with that of the height of men, their weight, length of arms, measurements of the head, - incleed, with that of any object, living or non-living, that possesses variable characters that can be accurately measured, whether by feet, inches, pounds, or by any other unit that can be devised. ${ }^{2}$

By means of this coefficient the breeder may not only ascertain whether one character is more variable than another, but by taking this coefficient frequently, as annually for the same variety or under different conditions, he can know the variability of the same character as influenced by time or circumstances.

In general these determinations enable the breeder to know which way his varieties are drifting, or whether they are standing

1 Clearly, if the mode or the standard of selection has been used as a base in calculating the deviation, then the same value should be used as a base in calculating the coefficient; thus it is possible to secure a coefficient of variability from any desired type as well as from the mean.

2 The coefficient of variability has been worked out for a large number of characters in man, as is shown in the following table (see Vernon, Variation in Animals and Plants, p. 24).

\begin{tabular}{|c|c|c|c|c|c|c|c|}
\hline Nose length & . . & . & . & . & & & 9.49 \\
\hline Nose breadth & . . & . & . & - & & & . 7.57 \\
\hline Nose height . & . & . & . & . & & & .15 .20 \\
\hline Forehead height & . & . & . & . & & & 10.40 \\
\hline Under jaw length & & . & . & . & & & - $4.8 \mathrm{I}$ \\
\hline Mouth breadth & & . & & . & & & 5.18 \\
\hline Head length . & . & . & & . & & & 2.44 \\
\hline
\end{tabular}

Head breadth . . . . . . 2.78

Upper arm length . . . . 6.50

Forearm length . . . . . 3.85

Upper leg length . . . . . . 5.00

Lower leg length . . . . . 5.04

Foot length . . . . . . . . . 5.92 
still in spite of vigorous selection; whether in his selection he is operating with or against nature; whether the type is becoming a little more "fixed" or whether the tendency is more and more to scatter; whether the mean or general average of excellence is approaching or receding from the desired type ; whether, as time passes, variability is lessened or increased; whether, as the result of selection, ncw values are coming in at the upper end. In short, by these calculations he may know whether he is making real progress, or is only dabbling in the whirl of variability that is inevitable with all living things, without influencing at all the trend of the race. It is needless to say that much of this latter sort of ineffective breeding is going on all about us everywhere. The statistical method is the only known method of securing accurate knowledge of type and variability; for type is not simply what we desire, but it is what we actually get, and any breeder is working in the dark who does not know the real nature of the ivhole population of the race he breeds.

\section{SECTION III - PRACTICAL HINTS ON THE TAKING AND GROUPING OF MEASUREMENTS}

It is highly improbable that, in measuring any part of an organism, a perfectly accurate measure is obtained. It may be very easy to measure the length of an object accurately to $\mathrm{I}$ inch, or to O.I inch, or even to O.OI inch, if the ruler is sufficiently accurate; but finally, in trying to measure to as high a degree of accuracy as possible, we come to a point where our ruler fails us. Again, the object measured may be of such a nature that it is futile to try to take measurements beyond a certain degree of accuracy. For instance, it would be useless to try to measure the length of an ear of corn to O.OI inch. Similarly, in weighing substances, it is possible with a good balance to take measurements to ounces or to tenths of milligrams if extreme accuracy were demanded; but finally, in striving after accuracy, the balance fails us, and we must grant that it is highly improbable that the weight which we record is perfectly accurate.

While we thus see that we cannot attain absolute accuracy in any measurements, yet thremmatology is no exceptional field in 
this respect, and experience has shown that measurements can be easily taken of sufficient accuracy to insure very reliable results.

For obtaining the frequency distribution of a population with respect to some measurable character, it is impracticable to lay down specific rules in regard to the accuracy of measurements. This must be settled largely by experience and common sense. It may, however, be said here that in measuring a large population, under the free action of the laws of probability, substantially as many measurements will be taken too large as are taken too small. Hence slight errors in measurements do not appreciably disturb the mean and variability of the population, because they tend to offset each other.

Scheme of measurement. Reverting to the frequency distribution obtained from measurements of corn, it will be noted that this population is distributed in classes or groups which differ by a half inch in length. For the purpose of forming this frequency table there would then be no object in taking the measurements closer than the nearest half inch. This raises the question of the inclusiveness of a class in grouping a population ; that is, Should these measurements have been taken at the quarter inch or perhaps at the even inch? Here, again, no hard and fast rule can be laid down; but it may be said that in general, and for the best results, the class range should be made just large enough for some variates to appear in each class, except, possibly, in a few near the extremes of the range of variability when the total population is not very large.

Sometimes the best unit for measurement and grouping will be evident, but frequently some preliminary work is necessary in order to decide it. For example, in beginning the statistical work with corn we at first took measurements to the quarter inch, with groupings accordingly, but found no results different, either as to mean or variability, from those obtained when measurements were taken to the half inch, and but slightly different from those taken at the even inch. Accordingly we are using the half-inch measurements for extreme accuracy and the even inch for rougher work.

Much judgment must be used in deciding upon the scheme of measurements to be adopted and the groupings to be made. 
If the unit of measurement be large, say two inches for corn, the individual values will not be accurate; the groups will be large, but so far apart that the empirical mode will have but little meaning. If, on the other hand, the units of measure be too minute, say tenths of inches, the work of calculation will be vastly increased, and while the empirical mode will be more accurate, yet the distribution will not be "smooth," as the technical phrase goes; that is, some of the groups near the extremes may not be filled.

The scheme must be so chosen and the groupings so made as to furnish a uniform distribution, with fairly smooth results when the frequency is platted in the form of a curve. The method of platting frequency distributions and dealing with them as "curves of frequency" will be shown in the Appendix."

\section{SECTION IV - PROBABLE ERROR}

Mention has already been made of certain inaccuracies in measurements and groupings of a population. Attention is now called to another source of error which arises from using a limited number to represent the total population. From all these sources slight errors are inevitable. We have no means of determining the exact error, but after the work has been done we may find a measure of accuracy. This measure of accuracy is called the "probable error."

Whatever the source of error, the exact discrepancy in a result which depends upon measurements can never be ascertained. However, the so-called "probable error" does give a measure of accuracy which indicates whether we should expect a large or small error in a determined value. In other words, it indicates the degree of confidence which we should place in results obtained by statistical methods.

1 In general this may be done by laying off the measurements, as inches, half inches, pounds, etc., on a horizontal line, and the numbers of individuals in the distribution as verticals, connecting the points by a continuous curve. As numbers differ greatly in different cases, it is best to reduce them all to the basis of I00, and express all values in percentages. If this is done, then all curves of the same measurement are comparable. 
The real nature of probable error and the methods for deducing the formulas for its calculation will be covered in the Appendix, which is devoted especially to mathematical methods and conceptions, but enough should here be said to give the student an intelligent idea of what is meant by probable error and to acquaint him with the bare formulas for its calculation as to the values here under discussion.

The probable error (denoted by $\pm F$ ) is a pair of divergencies lying one above and the other below the value determincd, and of which we can say with confidence that there is an e'cu chance that the true value lies between these limits. ${ }^{1}$ These numbers are numerically equal, but one is regarded as plus, the other as minus $( \pm E)$, and the two define a range within which, out of a very large number of determinations, at least half the true values would be found. This being the case, we may say of any single determination that the chances are even that any error involved will not fall outside the limits set by $\pm I:$. It is obvious, therefore, that the smaller the probable error the narrower this range, the greater confidence we should place in our determination, and the smaller are the chances of a large error having been made.

The expression "probable crror" may be misleading. It is not, as might be supposed from the words, the most probable cmor. The most probable value is our determination and the most probable crror is sero. Neither does the probable error fix the limits of error, but it is an extremely good measure of accuracy in that it fixes a range above and below the determined value such that the chances are cicn that the true value lies within this range.

Thus, if a series of calculations results in a final number 27.4 , with a probable error of.$\pm \mathrm{I} 2$, it means that out of a great number of cases the true value of one half will lie between $27.52(27.4+.12)$ and $27.28(27.4-.12)$.

If another calculation involving larger numbers or more accurate methods should result in the same value, 27.4 , but a probable error of only \pm .04 , then the true value has an even chance of lying between 27.44 and 27.36 , which is a very

1 There is, of course, also an even chance that the true value lies outside the same limits. 
narrow margin, giving us much confidence in the determination, with only a small chance of being wide of the truth.

Of course the error in a determination has also an even chance of lying outside the limits set by the probable error (E), but the following table will show that it is very unlikely that the error is many times as great as $E$. Thus the chances that the true value lies within the range set by $\pm E, \pm 2 E$, etc, are as follows : ${ }^{1}$

$\pm E$ the chances are even

$\pm 2 E$ the chances are 4.5 to I

$\pm 3 E$ the chances are 21 to 1

$\pm 4 E$ the chances are 142 to I

$\pm 5 E$ the chances are 13 Io to 1

$\pm 6 E$ the chances are 19,200 to 1

$\pm 7 E$ the chances are 420,000 to I

$\pm 8 E$ the chances are $17,000,000$ to 1

$\pm 9 E$ the chances are about $\mathrm{I}, 000,000,000$ to. I

It is extremely improbable, therefore, that an error will be many times as large as the probable error. For instance, it is practically certain that the error is not as large as $9 E$, since the table shows that the chances are about a billion to one in favor of its being smaller than $9 E$.

Thus by giving, along with any result, the calculated probuthle error, the reader may know what degree of confidence is to be placed in the results.

For a graphic illustration of the meaning of $\pm E$, suppose in the following figure the line $A B$ represents our determination, and the lines $a b$ and $a^{\prime} b^{\prime}$ the location of $+E$ and $-E$

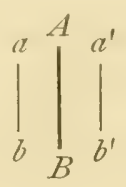

Now this means that if $A B$ is not the true location of the value in question, the chances are even that this location is not outside the limits set by the lines $a b$ and $a^{\prime} b^{\prime}$ representing $\pm E$.

1 C. B. Davenport, Statistical Methods, p. 14. 
If now we set other lines like the following at $\pm 2 E$,

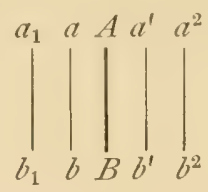

then we know from the table that the chances are 4.5 to I that the true position of $A B$ is not outside the lines $a_{1} b_{1}$ and $a^{2} b^{2}$, each removed twice the probable error from the determination.

Probable error of mean. The probable error of the mean is based upon the standard deviation, as we notice by the following formula : ${ }^{1}$

$$
\begin{aligned}
E_{\text {mean }} & = \pm 0.6745 \frac{\text { standard deviation }}{\sqrt{\text { number of variates }}} \\
& \text { or } E_{M}= \pm 0.6745 \frac{\sigma}{\sqrt{n}} \cdot
\end{aligned}
$$

Substituting for the case in hand, we have

$$
E_{8.83-}= \pm 0.6745 \frac{1.28}{\sqrt{327}}= \pm 0.047+
$$

The student cannot fail to notice the overwhelming influence of numbers in controlling the value of $E$, or to realize that if the number of determinations should become infinite, $E$ would become zero.

Probable error of standard deviation. According to methods of deduction, to be discussed later, the probable error of any determination for standard deviation is found by the following process: divide the standard deviation by the square root of twice the number of variates and multiply the result by $\pm 0.6745 .^{2}$

In the case in point we have $\mathrm{I} .28$ as the standard deviation with 327 variates. Substituting these numbers in the formula, we have

$$
E_{1.28}= \pm 0.6745 \frac{\mathrm{I} .28}{\sqrt{2 \times 327}}= \pm 0.034-\text {. }
$$

1 See C. B. Davenport, Statistical Methods, P. I5. The method by which the constant 0.6745 is obtained will be explained in the Appendix.

2 The formula for probable error of standard deviation is

See C. B. Davenport, Statistical Methods, p. I6.

$$
E \sigma= \pm 0.6745 \frac{\sigma}{\sqrt{2 n}} .
$$


If another distribution should give a smaller $E$, we should conclude that more confidence could be reposed in this second determination than in the first.

Obviously $\pm E$ will decrease as the standard deviation decreases or as the numbers examined increase (see formula). Our numbers are relatively small (327) and our probable error is relatively high, though it constitutes but an insignificant fraction of the determination (1.2S).

Probable error of coefficient of variability. When the coefficient of variability $(C)$ is small (ro per cent or less) its probable error is found by dividing the coefficient of variability by the square root of twice the number of variates and multiplying by \pm 0.6745 ; that is, by the same formula as the one just given, only substituting coefficient of variability for stanclard deviation. ${ }^{1}$ When the coefficient is larger than ro per cent we use a slightly more complicated formula. ${ }^{2}$

Since the variability in question (14.5) is greater than Io per cent we employ the more extended formula and obtain the following :

$$
E_{c}= \pm 0.67+5 \frac{14.5}{\sqrt{2 \times 327}}\left[\mathrm{I}+2\left(\frac{\mathrm{I}+.5}{100}\right)^{2}\right]^{1}= \pm 0.39+
$$

The student will find on trial that for these values the two methods give results but slightly different.

Deviation and probable error illustrated. This whole matter of deviation and probable error is well illustrated in shooting at a mark. Some of the shots will strike the bull's-eye and others will strike at various distances from the center, some yroing wild. Obriously the better the shooting the closer will the shots be clustered about the bull's-eye. The distance of each shot from the center would be its deviation from the mark and the mean of all the deriations of the marksman A would represent the average of his deviations.

1 Formula $E_{C}= \pm 0.6745 \frac{C^{*}}{\sqrt{211}}$.

Methods, p. 16 .

${ }^{2} E_{C}= \pm 0.6745 \frac{C}{\sqrt{2 n}}\left[\mathbf{I}+2\left(\frac{C}{100}\right)^{2}\right]^{\frac{1}{2}}$. See C. B. Davenport, Statistical 
If a circle be drawn about the center with a radius equal to the average deviation of $A$, this circle will fairly well represent his marksmanship; that is to say, the average distance of all his shots from the center is the same as if they all lay on this circle. If the marksmanship of $\mathrm{B}$ is not so good as that of $\mathrm{A}$, his average is greater and the circle correspondingly larger. ${ }^{1}$

Now neither of these circles is an absolutc index of the marksmanship of either $A$ or $B$, unless an infinite number of shots has been made; that is to say, if only a few shots have been fired, the probability of error is great if we assume these circles to be fully representative of the marksmanship, because there is practical certainty that succeeding shots will be either better or worse; indeed, there is always a chance that the next may be a lucky shot and lower the deviation, or a wild one and raise it.

We should therefore conceive of two other circles lying neighbor to each of those representing the calculated deviations. These are represented in the cut by the light-line circles and give a graphic meaning to the probable error. They are drawn so that if the heavy circles do not accurately represent A's and B's marksmanship then the chances are even that the true position of the circle representing A's marksmanship, for example, lies somewhere between the light lines representing the probable error of the computation. The chances are of course also even that it lies beyond these limits, either within or outside. Obviously, the smaller the probable error the greater the confidence to be placed in the calculated deviation. The student is cautioned here that in this illustration the "probable error" refers not to A's or B's failure to hit the target, but to our

1 The question may be raised as to whether there is not a better measure of marksmanship than the average departure of the shots from the bull's-eye. For instance, with the bull's-eye as a center, we may describe circles through each of the shots of $\mathrm{A}$, and construct a circle with the average area of these circles for its area. This circle may then be selected as a measure of A's marksmanship instead of the circle above discussed. The radius of this circle can be obtained by taking the square root of the mean square of the deviations from the bull'seye. It is not important for us to discuss here the relative merits of these two methods of measuring marksmanship, but it is important that we recognize that the method explained in the text is based upon what we may well call "average deviation from an ideal," while that suggested in this footnote may well be called "standard deviation from an ideal." 
calculation in assuming the circles to represent the deviation of each, when only a limited number of shots had been fired.

It is this deviation and not our probable error that is to be taken as expressing crror in marksmanship, for with an infinite number of shots our $E$ would disappear, but the error in marksmanship or the deviation of $\mathrm{A}$ or $\mathrm{B}$ would never disappear. With infinity it would have an exact and fixed value, with $E=0$.

It is obvious that the deviations above alluded to are deviations from an "ideal" (the center of the target) which is comparable to the selection type in practical breeding. It is obvious, too, that these heavy circles represent the means of all the shots fired by the two marksmen, but they do not represent their distribution. That is to say, $\mathrm{A}$, for example, might have put most of his shots, or all of them for that matter, at a uniform distance from the bull's-eye, none hitting the center and none going wild; in other words, his shooting might have been very uniform, but neither very good nor very bad, owing either to bad marksmanship or to a badly sighted gun. Now it is conceivable that

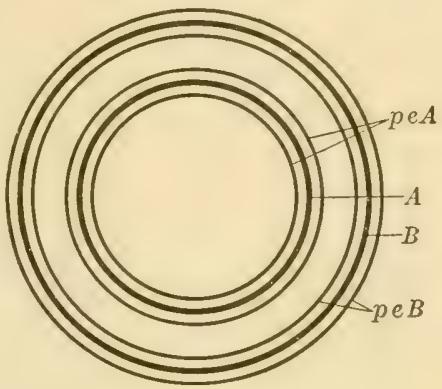

FIG. 44. Let the heavy lines $A$ and $B$ represent the culculatid marks. manship of $A$ and $B$ respectively ; then the light lines $p e A$ and $p e B$ will represent the probable errors in the assumption that lines. 1 and $F$ represent truly the marksmanship of A and B

another marksman, C, should succeed in making an areroge identical with that of $A$, but in a very different manner, - some of the shots hitting the bull's-eye and some going wild. These two men, then, do shooting of an entirely different class the one from the other, that is, make a very different distribution even though they win the same mean. If now, from this mean (represented by the heavy circles), we calculate standard deriation with respect to all the shots fired, we then have a conception of deviation corresponding exactly to that of the ordinary standard deviation; namely, deriation from the mean of all the variates. Thus we illustrate both deviation from mean and deviation from an ideal, together with the probable error involved. 
SECTION V - COMPARATIVE, TYPE AND VARIABILITY FOR DIFFERENT CHARAC'TERS IN THE SAME

\section{POPULATION}

If a variety of characters in the same population be critically studied it will be found that each has its own type and variability. For example, in the population arising from the ten-inch ears already discussed it was found that other characters varied as follows :

Type and Variability for Four Characters of Corn grown from Seed Ears Ten Inches Long

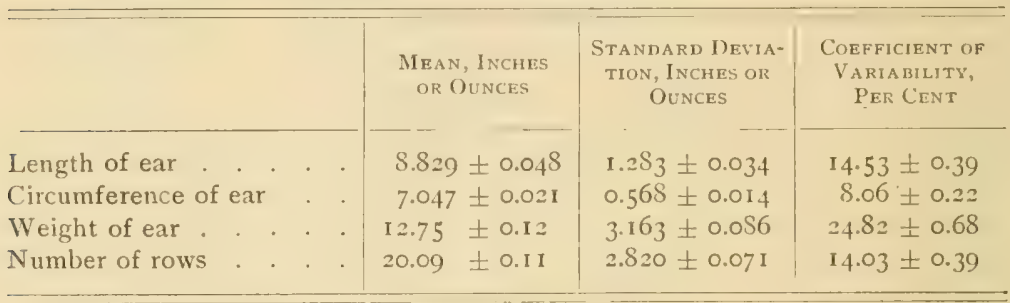

From this we see that each separate character takes its own type and variability. For example, corn is much more variable as to weight (24.82 per cent) than as to any other character measured. This is to be expected, because weight is to some extent the resultant of both length and circumference and would thus partake of the variability of both. But we note also that this corn at least was much more variable as to length than as to circumference ( 4.53 per cent as compared with 8.06 per cent).

Again we note that these ears are much more variable as to number of rows than as to circumference, by which we infer that the width of the liernels is far from uniform, else the two would move together.

This raises the whole question of the relation or bond between clifferent characters, a subject to be discussed in the succeeding chapter on "Correlation." It is sufficient here to remark that the typical ear of this crop of corn is 8.829 inches long, 7.047 inches in circumference, has 20.09 rows of kernels, and weighs I 2.75 ounces. If we should pick such an ear it could stand as 
the actual type of this crop, though the different characters involved would vary differently from this type or mean.

\section{SECTION VI - EFFECT OF SELECTION UPON TYPE AND VARIABILITY}

The whole purpose of selection is to influence type. In the minds of some it is also to reduce variability. The real effect of selection is well brought out in data (see table, page 446) from Dr. Hopkins's experiments ${ }^{1}$ in endeavoring to influence chemical composition of corn by the method of selection. It is to be noted that all four strains sprung from the same original stock (I63 ears), and that each seed selection was made from the highest (or lowest) ears within the several strains; that is, the high-oil stock, for example, originated from those ears showing the highest oil content in the original stock, and was developed by successive selection of the highest oil ears, always within the high-oil stock, and similarly for the other strains.

Discussion of data. A critical study of the columns of means shows a steady rise in the means of both high-protein and highoil strains and a corresponding decline in low-protein and low-oil strains, indicating a prompt response to selection. An inspection of the columns of standard deviations and coefficients of variability, however, reveals the fact that the variability is practically unchanged. This agrees with the mathematical theory, to be developed later, namely, that the effect of selection is to shift the type but not greatly to reduce variability.

The peculiarity of this sort of selection is that it is progressive; that is to say, with each response to selection a new standard is set up still more difficult to meet than was the old one. Under these conditions of continuously advancing: standards and with rapidly advancing types, in only two out of the four cases was the variability apparently reduced, and this whether we regard the standard deviation or the coefficient of variability. This power of response to the demands of an advancing standard of selection is immensely suggrestive and will be considered further under "Heredity."

1 See Bulletin No. IIO, University of Illinois Agricultural Experiment Station. 


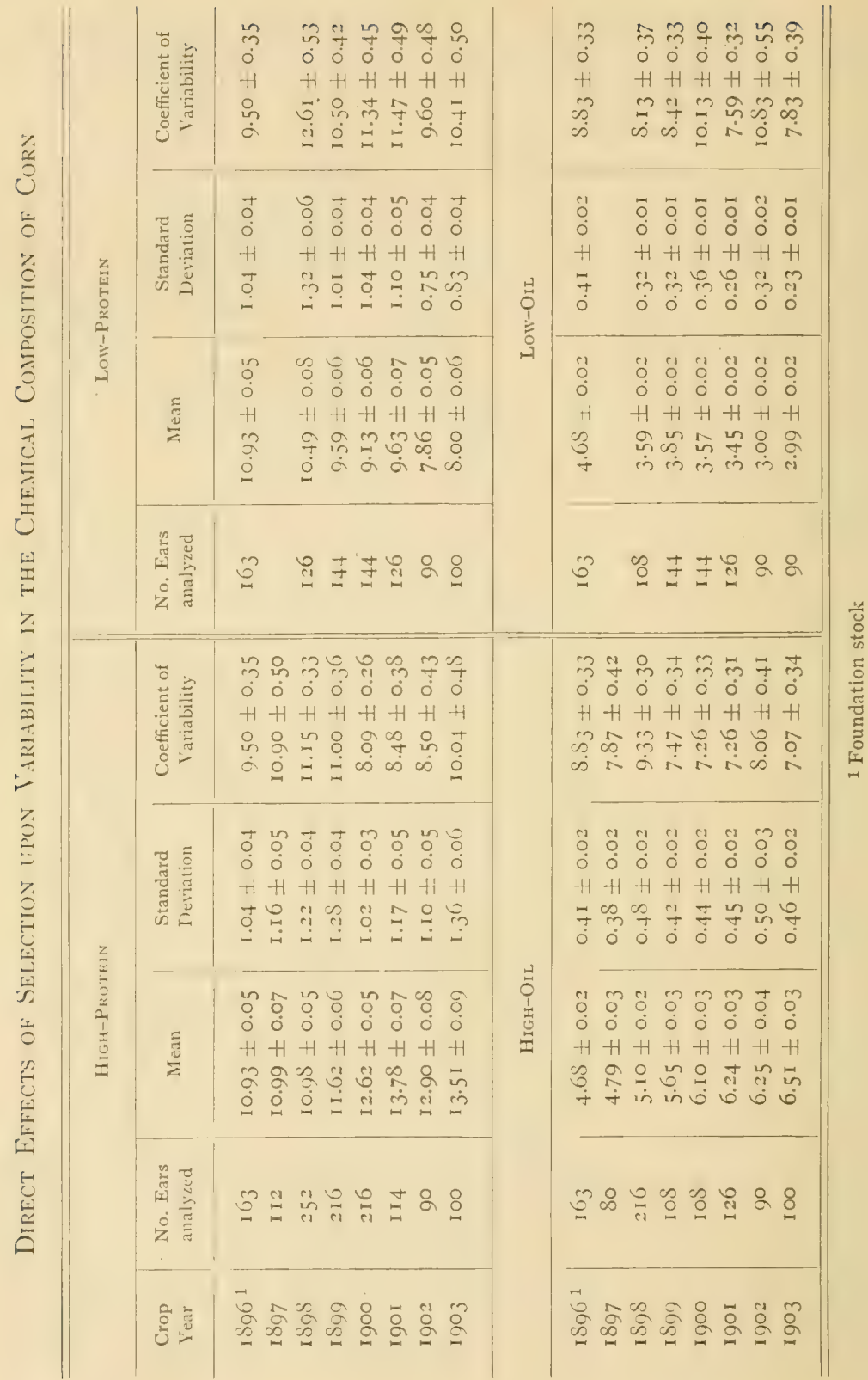




\section{SECTION VII - INDIRECI EFFLCIS OF SELECTION LPON TYPE AND VARIABILITY}

In the table just given we noted only the direct effects upon the character undergoing selection. It now remains to consider how rigid selection of one character may affect other and presumably correlated characters. To this end we construct a table showing the physical characters of the four strains of corn now under discussion, remembering that these strains all developed from the same original stock and that selection was confined to the chemical characters, protein and oil, leaving the physical and physiological characters free to take care of themselves.

Indirect Effects of Selection: Results of Seven Years' Selec'tion for Chemical Composition upon Physical Characters. All Four Strains developed from the Same Original Stock ${ }^{1}$

\begin{tabular}{|c|c|c|c|c|c|c|}
\hline \multirow{2}{*}{ VARIETY } & \multicolumn{3}{|c|}{ LENGTH OF EAR } & \multicolumn{3}{|c|}{ CIRCUMFEIRENCE } \\
\hline & $\frac{1}{\text { Mean }}$ & Stand. Dev. & ${ }^{3}$ Coef. Var. & $\begin{array}{c}4 \\
\text { Mean }\end{array}$ & $\begin{array}{c}5 \\
\text { Stand. Dev. }\end{array}$ & $\begin{array}{c}6 \\
\text { Coef. Var. }\end{array}$ \\
\hline A. High-protein.. & $7.21 \pm 0.04$ & $1.27 \pm 0.03$ & $17.6 \pm 0.4$ & $5.76 \pm 0.01$ & $0.4 t \pm 0.01$ & $7.6 \pm 0.2$ \\
\hline B. Low-protein .. & $7.80 \pm 0.04$ & $1.54 \pm 0.03$ & $19.7 \pm 0.4$ & $6.51 \pm 0.02$ & $0.61 \pm 0.01$ & $9.4 \pm 0.2$ \\
\hline C. High-oil .... & $6.87 \pm 0.04$ & $1.39 \pm 0.03$ & $20.2 \pm 0.4$ & $6.05 \pm c .01$ & $0.53 \pm 0.01$ & $8.8 \pm 0.2$ \\
\hline D. Low-cil ..... & $7 \cdot 48 \pm 0.04$ & $1.30 \pm 0.03$ & $17.4 \pm 0.4$ & $6.65 \pm 0.02$ & $0.59 \pm 0.01$ & $8.9 \pm 0.2$ \\
\hline
\end{tabular}

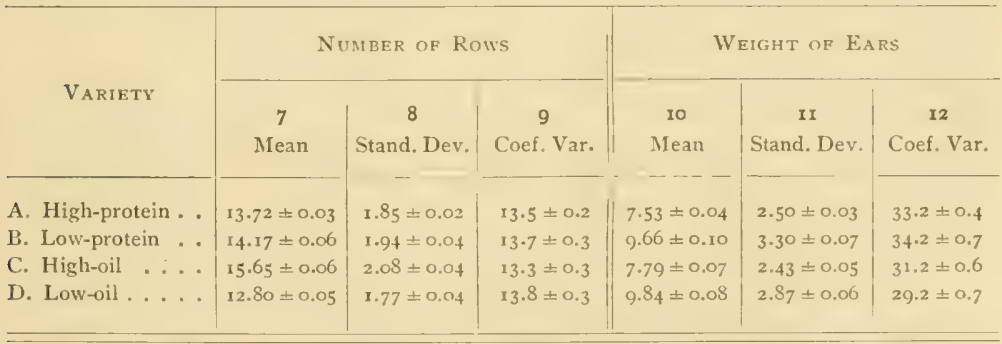

Discussion of data. A critical study of this table reveals some significant facts concerning the indirect effects of selection

${ }^{1}$ See Bulletin No. 119, University of Illinois Agricultural Experiment Station. 
upon characters other than those under special consideration. Such a critical study clevelops the fact that these four strains differing in protein and in oil content have developed also into four distinct strains regarding the purely physical characters, length, circumference, etc.

I. High and low protcin. The ear of the former is the shorter (column I), and the smaller standard deviation and coefficient of variability show it to be less variable as to length (columns 2 and 3). It is also smaller in circumference (column 4), with a less number of rows (column 7), and lighter in weight (column IO). It is also less variable in every respect, both relatively (columns $2,5,8$, and I I) and absolutely (columns 3, 6, 9, and I 2).

2. High and low oil. In the same manner we learn that the high-oil ear is a shorter and a smaller ear than the low-oil, but that it is morc ariable as to length and less variable as to circumference (columns i to 6 ). The low-oil ear is rapidly becoming a twelve-rowed variety, with a lower deviation than any other (column 8 ). It is the heaviest, though not the longest, of the four selected strains.

3. The four sicital strains. Of these four strains developed from the same original stock, the low-protein ear is the longest and the high-oil the shortest. The high-oil is also the most variable as to length (column 3 ).

The low-oil is the largest and the high-protein is the smallest in circumference. The latter is also the least variable and the low-protein is the most variable as to circumference.

The high-oil corn has the largest number of rows, with the lowest variability, and the low-oil the fewest rows, with the least standard deviation but the greatest coefficient of variability.

The low-oil is the heaviest and the high-protein the lightest ear, but the low-protein is the most variable as to weight.

It will be noted that these differences are the nutural results of sclecting, not for these particular characters, but for chemical contcnt. They are therefore the indirect effects of selection, and bring up again the whole subject of correlation, which will be treated further in a later chapter. 


\title{
SECTION VIII - STUDIES IN TYPE AND VARIABILITY
}

\author{
OF THE SAME TARIETY OF CORN RAISED LNIDER
} DIFFERENT CONDITIONS AS TO FERTILITY

The effect of the conditions of life upon variability, as distinct from mere development of the mass, is well illustrated in the behavior of a single varicty of corn (Leaming) grown uncler different conditions as to fertility. This was the crop of 1906 upon land that had been in pasture for eighteen years previous to $\mathrm{I} \$ 95$, in a three-year rotation of corn, oats, and clover from that time till the present, with the fertility treatment indicated in the tables (pages 450 and $45 \mathrm{I}$ ) since the year I9OI.

Discussion of data. As to weight of ear, it will be noted (see tables, pages 450 and $45 \mathrm{I}$ ) that the mode and mean correspond very closely to yield; that is, that increased yield is mainly due to heavier ears. This is inevitable from the uniform method of planting with two stalks to the hill. In respect to variability, however, we can detect little difference except in the last plot, which was planted with three stalks to the hill.

In respect to length and circumference of ear it is noticeable that the higher yields are accompanied by the longer and larger ears for the reasons given above. The most significant fact in the table is that corn is far more variable as to length than as to circumference, but that neither is especially affected by fertility.

A general correspondence between circumference and number of rows is evident, showing a tendency to constancy as to size of kernel, but again variability is not greatly different for the different yields. So far as this instance can be accepted as a safe criterion, we may deduce the following principles:

I. That type is directly and largely dependent upon food supply.

2. That variability is not greatly influenced by specially favorable conditions of life, tending to become less rather than greater as all individuals are afforded ideal opportunities for development. 


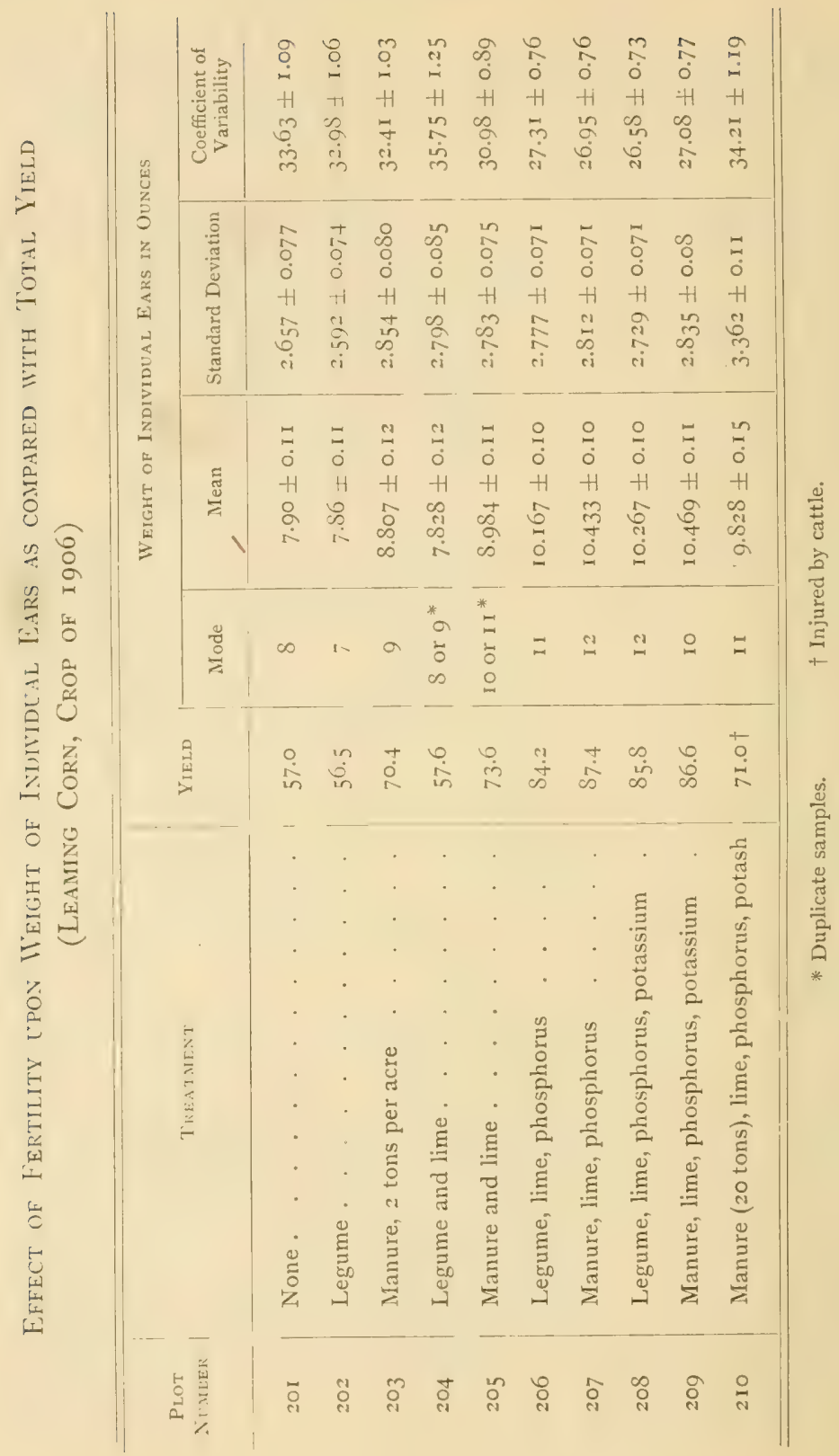




\begin{tabular}{|c|c|c|c|c|c|c|c|c|c|c|c|}
\hline \multirow{4}{*}{ 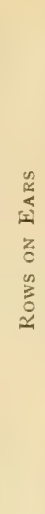 } & 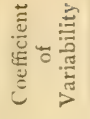 & \begin{tabular}{l}
$\vec{T}$ \\
0 \\
0 \\
+1 \\
0 \\
\hdashline \\
1
\end{tabular} & 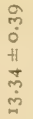 & $\begin{array}{l}\infty \\
0 \\
0 \\
11 \\
0 \\
0 \\
0 \\
1\end{array}$ & $\begin{array}{l}7 \\
0 \\
0 \\
+1 \\
7 \\
7 \\
7\end{array}$ & 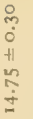 & 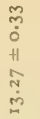 & $\begin{array}{l}0 \\
0 \\
0 \\
0 \\
11 \\
0 \\
0 \\
0 \\
0 \\
0\end{array}$ & $\begin{array}{l}m \\
0 \\
0 \\
+1 \\
m \\
m \\
m\end{array}$ & 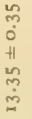 & 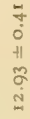 \\
\hline & 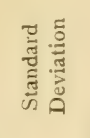 & \begin{tabular}{l}
0 \\
0 \\
0 \\
0 \\
11 \\
$\vdots$ \\
\multirow{2}{*}{} \\
ì
\end{tabular} & $\begin{array}{l}8 \\
\vdots \\
0 \\
\text { il } \\
\text { in } \\
\text { in } \\
\text { i }\end{array}$ & 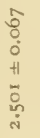 & 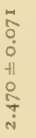 & 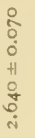 & $\begin{array}{l}0 \\
0 \\
0 \\
0 \\
11 \\
0 \\
0 \\
0 \\
i\end{array}$ & 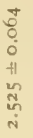 & 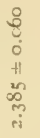 & 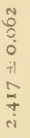 & 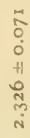 \\
\hline & 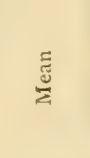 & $\begin{array}{l}\tilde{0} \\
0 \\
0 \\
艹 \\
\hat{N} \\
0 \\
0 \\
=\end{array}$ & 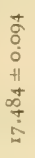 & 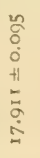 & 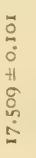 & 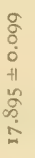 & 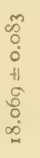 & 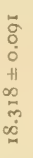 & $\begin{array}{l}0 \\
\infty \\
0 \\
0 \\
11 \\
0 \\
0 \\
0 \\
0 \\
0\end{array}$ & $\begin{array}{l}0 \\
0 \\
0 \\
0 \\
+1 \\
0 \\
0 \\
0 \\
0\end{array}$ & $\begin{array}{l}\stackrel{0}{0} \\
\text { +1 } \\
\ddot{\sigma} \\
\stackrel{0}{4}\end{array}$ \\
\hline & әроIN & 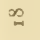 & $\stackrel{0}{-}$ & $\mathscr{\sim}$ & 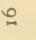 & $\stackrel{\infty}{\infty}$ & $\stackrel{\infty}{\sim}$ & $\stackrel{\infty}{\sim}$ & $\infty$ & $\infty$ & 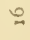 \\
\hline \multirow{4}{*}{ 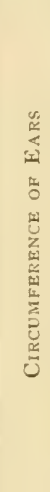 } & 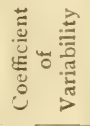 & $\begin{array}{l}\tilde{N} \\
c \\
+1 \\
\text { N } \\
\text { a }\end{array}$ & 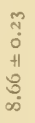 & 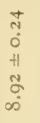 & 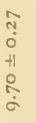 & 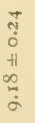 & $\begin{array}{l}0 \\
\vdots \\
0 \\
11 \\
\vdots \\
0\end{array}$ & \begin{tabular}{l}
\multirow{2}{0}{} \\
0 \\
0 \\
11 \\
8 \\
$\infty$ \\
$\infty$
\end{tabular} & $\begin{array}{l}\text { ma } \\
0 \\
\text { t1 } \\
0 \\
0 \\
0\end{array}$ & 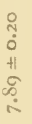 & 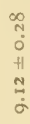 \\
\hline & 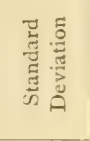 & 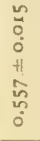 & $\begin{array}{l} \pm \\
\vdots \\
\vdots \\
0 \\
+1 \\
0 \\
0 \\
0 \\
0 \\
0\end{array}$ & $\begin{array}{l}n \\
0 \\
\vdots \\
+1 \\
0 \\
0 \\
0 \\
0 \\
0\end{array}$ & 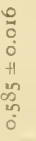 & $\begin{array}{l}2 \\
0 \\
0 \\
0 \\
1 \\
0 \\
\vdots \\
0 \\
0 \\
0\end{array}$ & $\begin{array}{l}m \\
0 \\
\vdots \\
H 1 \\
0 \\
0 \\
0 \\
0 \\
0\end{array}$ & 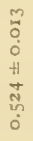 & $\begin{array}{l}0 \\
0 \\
0 \\
0 \\
11 \\
\& \\
\dot{0} \\
0 \\
0\end{array}$ & 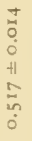 & $\begin{array}{l}\infty \\
\overline{0} \\
0 \\
+1 \\
0 \\
0 \\
0 \\
0\end{array}$ \\
\hline & 节 & 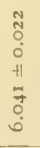 & $\begin{array}{l}0 \\
0 \\
0 \\
+1 \\
\vdots \\
\vdots \\
0\end{array}$ & $\begin{array}{l}\ddot{N} \\
0 \\
0 \\
+1 \\
11 \\
\tilde{n} \\
0 \\
0\end{array}$ & $\begin{array}{l}3 \\
0 \\
0 \\
0 \\
+1 \\
0 \\
0 \\
0 \\
0\end{array}$ & $\begin{array}{l}5 \\
0 \\
0 \\
+1 \\
+1 \\
0 \\
0 \\
0\end{array}$ & \begin{tabular}{l}
$\infty$ \\
0 \\
0 \\
0 \\
+1 \\
0 \\
\multirow{7}{*}{} \\
0
\end{tabular} & 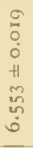 & $\begin{array}{l}\tilde{0} \\
\vdots \\
0 \\
+1 \\
0 \\
0 \\
0 \\
0\end{array}$ & 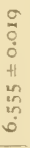 & $\begin{array}{l}\text { N } \\
0 \\
0 \\
+1 \\
\vdots \\
\vdots \\
0 \\
0\end{array}$ \\
\hline & әроIU & ? & ○ & $\dddot{m}$ & $\tilde{6}$ & $\tilde{\omega}$ & oे & $\stackrel{a}{0}$ & $\stackrel{\circ}{\omega}$ & o & 3 \\
\hline \multirow{4}{*}{ 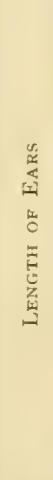 } & 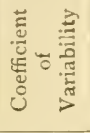 & $\begin{array}{l}8 \\
0 \\
0 \\
+1 \\
\alpha \\
\text { ते } \\
\text { ते }\end{array}$ & 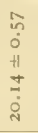 & 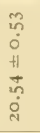 & 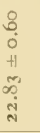 & $\begin{array}{l}0 \\
0 \\
0 \\
11 \\
0 \\
i n \\
0 \\
0\end{array}$ & $\begin{array}{l}7 \\
7 \\
0 \\
+1 \\
01 \\
0 \\
0 \\
0 \\
0\end{array}$ & 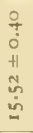 & 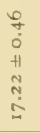 & $\begin{array}{l}\stackrel{0}{0} \\
0 \\
0 \\
+1 \\
0 \\
0 \\
0\end{array}$ & $\begin{array}{l}\text { of } \\
0 \\
\text { in } \\
\text { in } \\
\text { ते } \\
\text { ते }\end{array}$ \\
\hline & 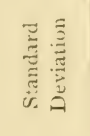 & $\begin{array}{l}0 \\
0 \\
0 \\
0 \\
11 \\
0 \\
0 \\
0 \\
\end{array}$ & 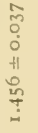 & 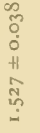 & 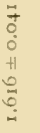 & 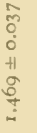 & $\begin{array}{l}m \\
0 \\
0 \\
0 \\
+1 \\
\infty \\
m \\
m \\
\tilde{m}\end{array}$ & 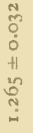 & $\begin{array}{l}0 \\
0 \\
0 \\
0 \\
+1 \\
+ \\
0 \\
3 \\
i\end{array}$ & 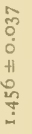 & $\begin{array}{l}0 \\
0 \\
0 \\
0 \\
11 \\
0 \\
\text { In } \\
\text { in }\end{array}$ \\
\hline & $\frac{\Xi}{\bar{J}}$ & $\begin{array}{l}\text { के } \\
0 \\
0 \\
+1 \\
11 \\
0 \\
0 \\
0\end{array}$ & 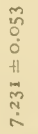 & 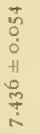 & 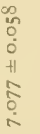 & 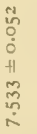 & $\begin{array}{l}0 \\
0 \\
0 \\
0 \\
11 \\
\ddot{0} \\
0 \\
0 \\
0\end{array}$ & 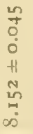 & 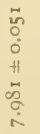 & 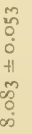 & $\begin{array}{l}\vec{A} \\
0 \\
0 \\
+1 \\
+ \\
0 \\
0 \\
0\end{array}$ \\
\hline & नроा & n & $\cong$ & $\infty$ & n & $\%$ & ? & $\sigma$ & $\infty$ & r. & $\overbrace{}^{n}$ \\
\hline \multicolumn{2}{|c|}{ צสRW.IN LCIT } & $\ddot{\tilde{c}}$ & $\stackrel{8}{4}$ & $\stackrel{n}{e}$ & $\vec{c}^{+}$ & $\sum_{c}^{n}$ & हृँ & ô & క్టి & ¿ & $\stackrel{2}{a}$ \\
\hline
\end{tabular}




\section{TRANSMISSION}

\section{Special Exercises}

The student should have extensive practice in making frequency distributions of different species and in working out standard deviations and coefficients of variability. He will thus not only become familiar with the methods of work, but he will acquire new conceptions of the whole subject of variability and type. The teacher should require the student at this point to make original studies on his own account, and to prosecute the work until he becomes entirely familiar both with the methods and with the conceptions involved.

\section{ADDITIONAL REFERENCES}

The Grammar of Science. By Karl Pearson. Chapter X.

Variation in Animals and Plants. By H. M. Vernon. Chapter I. 


\section{CHAPTER XIII}

\section{CORRELATION}

When studying variability in its simplest form we take the characters separately and determine how each behaves with reference to itself alone; that is, with reference to its own range and type.

\section{SECTION I - MEANING OF CORRELATION}

As the study proceeds, however, and is extended to other particulars, it will be noted that certain characters tend to rise and fall together, as if connected by some causative relation, for example, length and weight of ears, or size and strength of horses; while others appear to vary quite independently of one another, as stature and intellectual power in man, or color and feeding quality in animals.

The whole subject of correlation refers to that interrelation between separate characters by which they tend, in some degree at least, to move together. This relation is expressed in the form of a ratio. Thus, if an increase of one character is always followed by a corresponding and proportional increase in a related character, the correlation is said to be perfect and the ratio is $\mathbf{I}$. On the other hand, if an increase in one character is followed by a corresponding and proportional decrease in a related character, the correlation is said to be negative and the ratio is - I, or perfect negative correlation. Still again, if the characters in question are absolutely indifferent the one to the other, the correlation is said to be zero, indicating mere association under the law of independent probability, without causative relation of any kind.

Examples of perfect correlation are furnished by such obvious relations as those between the power of sight and the presence of eyes; the giving of milk and the presence of an udder; the 
presence of sunlight and the fixing of carbon; and by such other relations as are involved in direct causation.

On the other hand, such a relation as cleafness among telegraphers or blindness among civil engineers or locomotive drivers is unknown, because the conditions are such that the characters in question are mutually exclusive.

In general, however, correlation falls somewhere between - I and unity, and on one side or the other of the zero point ; that is, a degree of relationship exists which is neither absolute, denoting direct causation, nor negative, signifying mutual exclusion. For example, a high degree of correlation exists between length of cob and weight of ear. It does not amount to unity, however, for the circumference also contributes to weight.

Most results in living organisms are the effect of mixed causes, and for this reason correlations are more complicated than may at first appear. For example, many, if not most, good cows have a capacious "barrel" and a roomy udder, and men have been led to assume a perfect correlation between these special characters and milk production; whereas the truth is that the correlation, though high, is something less than unity, because good cows are known with small barrels and with inconspicuous udders. Here is a real need for accurate methods of determining what degree of correlation actually exists. The average man asks whether or not two characters are correlated, and expects a positive answer Yes or No; whereas the question should be, To what extent do the two characters appear together? expecting for an answer a fraction lying somewhere between zero and unity, say perhaps 40 to 60 per cent, as in correlation of length to weight of ear.

The student must distinguish clearly between correlation and mere association. For example, we might ask the question whether black pigs are more subject to cholera than are pigs of other colors. The first step would be to establish a ratio between the number of diseased pigs and pigs in general. This ratio would now express the chances that a particular pig, irrespective of color, will be afflicted with this disease, - that is, by that operation of independent probability which we call chance. If now we find upon inquiry that under the same conditions the ratio 
of cholera subjects to black pigs is higher than the ratio to pigs in general, then we should conclude that an actual positive correlation exists between the black color and this particular disease. On the other hand, if this ratio should be below the ratio of pigs in general, then we should conclude that black pigs are less susceptible to this disease than are pigs of other colors, and that a negative correlation exists, assuming always equal opportunities for infection. This is the only correct method of study, and it would not be safe to conclude that black pigs are peculiarly susceptible simply because most of the pigs that died under our observation happened to be black, for black pigs are more numerous in the cholera belt than all others combined, and under probability alone their absolute mortality must be higher. When expressed in the form of a ratio, however, the truth comes to the surface. ${ }^{1}$

Similarly we may ask the question whether different species of plants or animals tend to attract or repel each other when thrown together in the same territory. Here again the first step is to find the ratio of association under free operation of independent probability, - a ratio based on the relative numbers of individuals of the species in question and the extent of territory, first where no opportunity for association is possible, and second, where such association is possible. If the two ratios differ, then we infer that some degree of correlation exists.

\section{SECTION II - CALCULATION OF COEFFICIENTS OF CORRELATION}

When the presence or absence of the characters in question is absolute, as red or black hair, presence or absence of horns, then the correlation is expressed by a single ratio, as we have seen. But most cases are not of this extreme simplicity; for example, it is said that white cats are deaf. If now all white cats are deaf, then the correlation between albinism and loss of hearing power is absolute, and is expressed by the coefficient $\mathrm{I}$.

I Perhaps it ought to be remarked that this illustration is taken purely at random, as no studies have been made as to the relation between color and this particular disease. 
Suppose, however, that out of 1000 cats taken at random 20 are white, Io are deaf, and 6 are both white and deaf. Is there correlation? Now, according to this assumption, the probability of a cat being deaf without respect to color is $\mathrm{IO} \div \mathrm{IOOO}$, or I to $\mathrm{IOO}$; but the probability of a white cat being also deaf amounts to $5 \div 20$, or $\frac{1}{4}$, showing a high correlation between albinism and deafness.

But to derive an exact expression for this correlation is not so simple as it might seem. According to the conditions which we have already laid down, and in consistency with other phases of the problem of correlation in general, any expression which we may adopt as an efficient measure of this correlation should be such a formula as will become zero when the two characters are indifferent to each other; will become I when the two move together perfectly; and will become - I when they are mutually exclusive.

Yule ${ }^{1}$ has given an elegant measure of this association, or correlation, which satisfies these conditions. To develop this formula he arranges the population as in the accompanying diagram with respect to the characters in question (deafness and color).

Then the measure of associ-

\begin{tabular}{l|c|c}
\hline Cats & White & Not White \\
\hline Deaf & 6 & 4 \\
\hline Not deaf & $\mathbf{I}_{4}$ & 976 \\
\hline \hline
\end{tabular}
ation between deafness and the white color is expressed by

$$
\frac{(6 \times 976)-(4 \times 14)}{(6 \times 976)+(4 \times 14)}=0.9 \mathrm{I}+.
$$

In general, if we have a population arranged with reference to the presence or absence of two characters, $\mathrm{M}$ and $\mathrm{N}$, in numbers $a, b, c, d$, the arrangement would stand the same as above, and the formula would be $\frac{a d-b c}{a d+b c}$. If care be taken to arrange the table so that $b c$ shall be numerically less than $a d$, then the

1 Philosophical Transactions of the Royal Society, CXIV, 257-319. 
correlation is positive. Whenever ad and bc become equal the formula becomes $\frac{0}{a d+b c}=0$, or no correlation; whenever $b$ or $c$ becomes zero, then the formula becomes $\frac{a d}{a d}=1$, or perfect positive correlation; and whenever $a$ or $d$ becomes zero, then the formula becomes $\frac{-b c}{b c}=-\mathrm{I}$, or perfect negative correlation.

This is the simplest formula proposed that will meet the necessary conditions of the case. Pearson ${ }^{1}$ has proposed several others that are much more complicated, and that differ slightly as to results. Strange as it may seem, the problem is a comparatively new one, though the question involved is fundamental and very old. Though other methods are in use for special cases we may safely use Yule's formula for all ordinary cases of association where the question is simply as to presence or absence, without involving considerations of degree; that is to say, when the question is whether or not the cats are deaf, without reference to degrees of deafness; whether or not the patient has smallpox, without reference to the severity of the attack.

When, however, the question is one of possible correlation between characters present in rarying degres, as size, weight, amount of milk, etc., the problem would seem at first thought to be far more difficult; but in truth it has been much more completely worked out than the preceding question.

For example, what is the correlation between length and circumference in ears of corn? In general, long ears are also large ears, but many can be found that are long and slender, many that are short and small, and still others that are short and large. In other words, the two characters, length and circumference, are so related that the two maxima may appear together, the two minima together, the maximum length and the minimum circumference and vice versa, and all grades between. What now is the correlation? To ansiver a question thus complicated we construct what is called a correlation table. 


\section{SECTION III - THE CORRELATION TABLE}

To determine the degree of correlation between any two characters in any race, a so-called "correlation table" is constructed out of the measurements of the two characters as found in a large number of individuals, one character being recorded in columns and the other in rows. Two records are thus made of the same individual, one for each character. Such a table, when finished, consists of a double system of arrays, each dependent on the other, and from whose means and standard deviations the mutual relationships can readily be worked out.

Knowing this relationship and the value of one of the characters we are enabled to calculate the corresponding mean value of the other. The advantages of this for purposes of selection are obvious. The method is best illustrated by an actual example.

For instance, it is evident that the weight of ears in corn depends partly upon their length and partly upon their circumference. To what extent, for example, does it depend upon length?

In order to answer this question definitely a large number of ears taken at random are both weighed and measured, and the data are arranged in tabular form as described above, appearing as follows :

Correlation between Weight and Length of Ear (Leaming CORN)

Weight of Ears in OUNCES

\begin{tabular}{|c|c|c|c|c|c|c|c|c|c|c|c|c|c|c|c|c|c|c|c|c|}
\hline & 2 & & 4 & 5 & 6 & 7 & 8 & 9 & 10 & II & 12 & 13 & 14 & 15 & 16 & 17 & 18 & I9 & 20 & 21 \\
\hline 3. & I & 2 & . & I & . & . & . & . & . & . & . & . & . & . & . & . & . & . & . & . \\
\hline 3.5 & . & 4 & . & I & . & . & . & . & . & . & . & . & . & . & . & . & . & . & . & . \\
\hline 4. & 3 & 5 & 5 & 1 & . & . & . & . & . & . & . & . & . & . & . & . & ${ }^{\circ}$ & . & . & \\
\hline 4.5 & . & 6 & 5 & 4 & . & . & I & . & . & . & . & . & . & . & . & . & . & . & . & . \\
\hline 5. & . & 2 & 4 & 7 & 2 & 4 & . & . & . & . & . & . & . & . & . & . & . & . & . & . \\
\hline 5.5 & . & 2 & 9 & 5 & 14 & 8 & 4 & I & . & . & . & . & . & . & . & . & . & . & . & . \\
\hline 6. & . & I & 2 & 12 & 16 & 13 & I3 & 6 & I & . & . & . & . & . & . & . & . & . & . & . \\
\hline 6.5 & . & . & I & 6 & I I & 26 & I I & 8 & 6 & I & . & . & . & . & . & . & . & . & . & . \\
\hline 7. & . & . & 1 & 2 & 2 & 12 & IS & 12 & 12 & I I & 4 & I & . & . & . & . & . & . & . & . \\
\hline $7 \cdot 5$ & . & . & . & I & 2 & 4 & 20 & 12 & 13 & 2 I & I I & 6 & 6 & I & I & . & . & . & . & . \\
\hline 8. & . & . & . & . & . & 3 & 7 & 19 & 25 & 17 & 22 & I 7 & 3 & I & . & . & . & . & . & . \\
\hline 8.5 & . & . & . & . & . & I & I & 12 & 9 & 23 & 30 & 26 & 26 & 5 & I & . & . & . & . & . \\
\hline 9. & . & . & . & . & . & . & . & I & 7 & 10 & 23 & 35 & 26 & 24 & I 2 & I & 2 & I & . & . \\
\hline $9 \cdot 5$ & - & . & . & . & . & . & . & . & I & 4 & I 4 & I9 & 29 & 17 & IO & I & 3 & . & I & I \\
\hline IO. & . & . & . & . & . & . & . & . & I & I & 3 & 8 & I 8 & 10 & 6 & 4 & 2 & . & . & . \\
\hline 10.5 & . & . & . & . & . & . & . & . & . & . & . & 2 & 3 & 6 & 7 & 2 & 5 & I & . & . \\
\hline I I. & . & . & . & . & . & . & . & . & . & . & . & . & I & I & . & . & . & 2 & I & . \\
\hline I 1.5 & . & , & . & . & . & . & . & . & . & . & . & . & . & . & . & . & I & . & - & . \\
\hline
\end{tabular}


Arrays of a correlation table. In this table each ear is recorded in the proper square to represent both its weight and its length. This being the case, all the ears of the same weight that are also of the same length are recorded together in the same square. This means that the various row's are frequency distributions of weight with respect to length (as I, 6, I I, 26, II, 8, 6, I, the frequency distribution corresponding to the length 6.5 inches), and all the columns are frequency distributions of length with respect to weight. Such frequency distributions with respect to a correlated character are technically known as "arrays." The entire table, therefore, may be looked upon as made up of two systems of parallel arrays with respect to the two characters in question. They are in no respect different from any other frequency distributions; and their means, standard deviations, arriability, and other detcrminations are calculated by the same methods as given in the last chaptcr.

\section{SECTION IV - THE CORRELATION COEFFICIENT}

A mere inspection of the correlation table just given suggests that, in general, short ears are light ears, and that long ears are heavy ears; but what we seek is a statistical constant which will be a measure of this correlation, and which indicates to what extent the weight of ears can be predicted from their lengths. The cofficiont of corrilation is such a constant, and when determined it will be denoted by $r$.

A discussion of the mathematical theory of correlation will be given in the Appendix, but it should be said here, as before, that the coefficient always takes some value between + I and - I. If $r=+$ I there is said to be perfect positive correlation; that is, the two characters are causally connected. If $r=-\mathrm{I}$ there is perfect negative correlation; that is, they are mutually exclusive. If no correlation exists, $r=0$, indicating the two characters as being inclifferent to each other and moving independently. In nearly all cases some actual correlation exists, and, in a general way, we may say that the correlation should be judged by the value which $r$ takes between zero and unity. 
Method of finding correlation coefficient. The method of calculating the correlation coefficient $(r)$ is exhibited in connection with the table on page $46 \mathrm{I}$, showing a representative case, for convenience continuing the same correlation table already constructed. Though the method is somewhat complicated it is given in full.

It is highly important to get at once a general conception of this table and of the method of procedure. All the computations shown except those involved in the column headed $\mathbf{\Sigma} P$ have to do oilly with finding the means and standard doriutions of the population with respect to the two characters in question, according to the method fully treated in the last chapter; that is to say, the columns of figures headed ${ }^{1} f_{l}, f_{l} I_{l}^{\top}, D_{l}, D_{l}{ }^{2}$, $f_{l} D_{I}{ }^{2}, f_{H}, f_{H} I_{H}, D_{H}, D_{H}{ }^{2}, f_{H} D_{H^{\prime}}{ }^{2}$ are all self-explanatory to any one familiar with the meaning of ordinary algebraic symbols, and who knows how to find the variability of a population by the methods already given.

There remains the column of figures headed $\Sigma P$, which it seems worth while to explain in cletail and which is the only special feature in the determination of the correlation coefficient. Each number in this column represents the sum of the products of the corresponding length and weight deviations for every individual in the horizontal array to which the number belongs.

Toshow how these numbers are computed, select, for example, the horizontal array marked Io, and we shall show how to find the number 43 I.O.

In this case

Then

$$
\text { I0 }-7.8 \text {, the mean, }=2.2=D_{L} \text { of row } 10 .
$$

$$
\begin{aligned}
2.2[\mathrm{x}(-0.7) & +\mathrm{I}(0.3)+3(\mathrm{I} .3)+8(2.3)+\mathrm{I} 8(3 \cdot 3) \\
& +\mathrm{I} 0(4 \cdot 3)+6(5 \cdot 3)+4(6.3)+2(7 \cdot 3)]=43 \text { I.o. }
\end{aligned}
$$

All other numbers of the column headed $\Sigma P$ are found in the same way, and the total is written symbolically as $\Sigma D_{L} D_{H} \cdot{ }^{2}$

${ }^{1}$ Read " $f$ sub $\|$;" meaning the frequency of weights; " $f$ sub $W \cdot V$ sub $N$;" meaning the frequency of weights multiplied by the value in weights; " $f$ sub $I$," meaning the frequency of length, etc.

2 The real significance of $\Sigma P$ is best shown by the expression $\Sigma D_{L} D_{W}$, that is, the sum of the products of both deviations of all the individuals in the table. It is written in various ways, but always with the above meaning. 
Correlation of Weight of Ears relative to Length of Ears (Leaming Corn) ${ }^{1}$

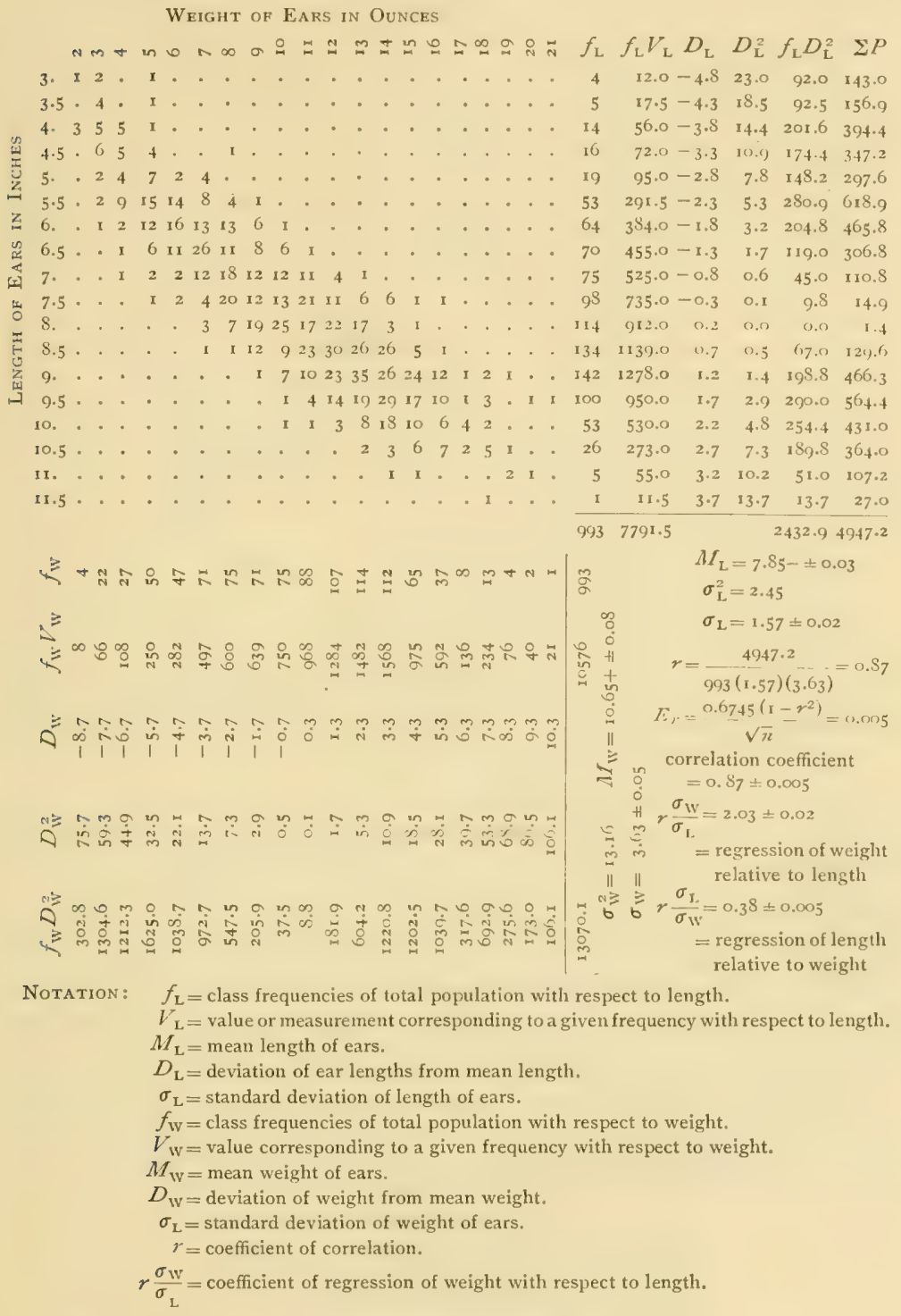

1 We have retained a minimum of decimal places in this table in order to save space. 
The value of $r$ is found by means of the formula

$$
r=\frac{\Sigma D_{L} D_{w}}{n \sigma_{L} \sigma_{\|}} \text {. }
$$

Systematic arrangement of work. The whole process, which seems somewhat complicated, is after all quite simple. To recapitulate, it amounts to multiplying the figures in each square by both their own deviations (that is, by their deviation as to length and their deviation as to weight), and then adding all the results and clividing by the whole number (of ears) multiplied by the product of the two standard deviations. (See formula $r=\frac{\Sigma D_{L} D_{W}}{n \sigma_{L} \sigma_{W}} \cdot$ ) In performing the actual work, however, it is highly important to have a systematic scheme for carrying out the computations in order to avoid confusion in the somewhat complicated details. It has seemed desirable, therefore, to present the matter in the form of a detailed description of the various steps involved.

First stcp. Having given the correlation table of the population, we first add the frequencies in the arrays with respect to both characters; that is, add the numbers in columns and rows of the table. This gives two frequency distributions of the total population, - the one with respect to length of ears $\left(f_{L}\right)$, and the other with respect to weight of ears $\left(f_{W}\right)$.

The one with respect to length has the frequencies $4,5,14$, I6, I 9, 53, 64, 70, 75, 98, I I 4, I 34, I42, I00, 53, 26, 5, I.

The one with respect to weight has the frequencies $4,22,27$, 50, 47, 7 I, 75, 7 I, 75,88, I07, I I 4, I I 2, 65, 37, 8, I 3, 4, 2, I.

Second stcp. For each of these frequency distributions (column $f_{l}$, and row $f_{I I}$ ) the means and the standard deviations must be calculated. The method of making these calculations is the same as the one used for mean and standard deviations in general. It has already been fully explained, and therefore need not be repeated here. A systematic arrangement of the work is shown in connection with the table. The results are:

$$
\text { mean length }=M_{L}=7.85
$$

standard deviation in length $=\sigma_{L}=\mathrm{r} .57$

$$
\text { mean weight }=M_{W}=10.65
$$

standard deviation in weight $=\sigma_{w}=3.63$. 
Third stcp. This is the only part of the work that is really new. In doing the second step we found the deviations of the classes from the mean length and mean weight. These are recorded under $D_{L}$ and $D_{W}$. For example, in row I we find that 4 ears were each 3 inches long; that is to say, they deviated -4.8 inches from the mean length taken at 7.8 instead of 7.85 in orcler to save labor. We next take the number in each square of the correlation table and multiply it by the corresponding deviations both in weight and in length, thereby securing a product which is the result of the full number of variates involved and of their deviation in respect to both characters.

For example, where the column headed 9 ounces and the row labeled 6.5 inches cross each other occurs the number 8 , which indicates that $S$ ears of the population weighed 9 ounces and were 6.5 inches long. In other words, these 8 ears each deviate - I.7 ounces (row $D_{u}$, column 9) from mean weight of ears, and - I.3 inches from the mean length (column $D_{1}$, row 6.5). Ilence for this number 8 we form the product $S(-1.7)(-1.3)=+17.68$. Without regard to labor, we should find such a product for each number in the correlation table. If now all these products be added and the result divided by the product of the two standard deviations into the number of variates in the total population, we shall obtain the correlation coefficient, or the index of correlation which we seek.

The systematic way of carrying out this work is to record the results of this operation for each horizontal array under the heading $\Sigma P$, and then add these results for the arrays to obtain the final result 4947.2 , which is symbolically indicated by $\Sigma D_{L} D_{W}$, or summation $D_{L} D_{W}$.

To illustrate the method of calculation a few of the products recorded in column $\Sigma P$ will be shown.

For example, with 7.8 as the mean length and 10.7 as the mean weight, we have for the array corresponding to length 4 inches,

$$
\left.\begin{array}{r}
-8.7 \times 3 \\
-7.7 \times 5 \\
-3.8 \times 6.7 \times 5 \\
-5.7 \times 1
\end{array}\right\} \text { This gives } 394.4
$$


For the array corresponding to 5 inches,

$$
\left.\begin{array}{r}
-7.7 \times 2 \\
-6.7 \times 4 \\
-2.8 \times-5.7 \times 7 \\
-4.7 \times 2 \\
-3.7 \times 4
\end{array}\right\} \text { This gives } 297.6
$$

Treat all arrays in a similar manner, and, finally, divide the sum of all the products thus obtained (that is 4947.2) by the product of the two standard deviations and the number of variates, bing carcull aliuays to prescrie the full distinction as to plus and mimus signs. This gives

$$
r=\frac{4947.2}{993(1.57)(3.63)}=0.87 \text {, the correlation coefficient. }
$$

The mathematical derivation of this coefficient as a measure of correlation involves too much mathematics to be given here. It may be noticed from the common-sense standpoint, however, that it seems to be a good measure of correlation. To appreciate the meaning of this coefficient, it should be recalled that we take the products of both deviations for every individual in the table, add these products, and divicle the result by the number of individuals. This gives the average of all the products of both deviations. We then divide this arcrage froduct of the individual deviations by the product of the two standard deriations, thus securing an expression whose value is influenced by the deviation of both characters with reference each to the other.

It requires but little mathematical insight to see that if the correlation is positive and considerable, positive values of the two characters correspond and negative values correspond; and further, that all the products of deviations are positive. This makes for a large correlation coefficient. On the other hand, if no correlation exists, for any value of one character we may expect in the long run equal and opposite deviations of the other character, which makes the sum of products of deviations very small. This common-sense examination indicates the real nature of the correlation coefficient.

Fourth stcp. Find the probable errors in the determined values. Those in the means and standard deviations are computed by 
formulas stated in the last chapter. For the probable error in the correlation coefficient use the formula

$$
E r= \pm \frac{0.67+5\left(1-r^{2}\right)}{\sqrt{n}}= \pm 0.6745 \frac{\left[1-(0.87)^{2}\right]}{\sqrt{993}} \pm 0.005
$$

Use of correlation coefficient. The correlation coefficient is a good index of the mutual relation that exists between the characters in question. If it is low, it indicates that they do not depend very much upon each other; if it is high, it indicates that they are in some way closely related; and if it rises to unity, this relation amounts to causation, - that is, one is the cause of the other, or else they are the joint effect of the same causes. The practical advantage of this knowledge for purposes of selection is obvious, especially when one character is easily seen and readily examined and the other is not. An application of the correlation table would correct many popular delusions on this subject, as, for example, the selection of cows by the escutcheon.

Shorter method for calculating $r$, the coefficient of correlation. There is derived in the Appendix a formula which gives the same numerical value for $r$ as $\frac{\Sigma D_{L} D_{\|}}{\| \sigma_{L} \sigma_{\|}}$alreadly used; and while its algebraic expression is a little more complicated, it is much better adapted to numerical calculation, as it aroids the use of decimals until almost the end of the work. In this respect it is similar to the shorter method presented for calculating the standard deviation. If applied to the case of the length and weight of ears of corn the formula is

$$
r=\frac{\Sigma D_{L}^{\prime} D_{\| \prime}^{\prime}}{n \sigma_{L} \sigma_{W}}-\frac{C_{L} C_{\| !}}{\sigma_{L} \sigma_{W}}=\left(\frac{\Sigma D_{1}^{\prime} D_{\| \prime}^{\prime}}{n}-C_{L} C_{\| \prime}\right) \frac{\mathrm{I}}{\sigma_{l} \sigma_{\| !}},
$$

where $D_{\ell}^{\prime}, D_{I}$ ' are deviations from our guess at the means instead of deviations from the mean itself as $D_{l}$ and $D_{\| \prime}$; and $C_{I}$ and $C_{\| \prime}$ are the corrections applied to the guesses at the mean length and weight respectively as used in the shorter method of finding standard deviation.

In other words, we find the standard deviation by the shorter method explained on page 429. Then, in forming the sum of products of deviations, we measure the deriations from the guess instead of measuring them from the means, and divile as 
before by the product of the number of variates and the two standard deviations. Finally we subtract from this result the products of the two corrections to our guesses in finding means, after dividing that product by the product of the standard deviations of the two systems of variates.

We shall present on page 467 an illustration of this shorter method, using for the purpose the correlation between length and circumference of ears of Leaming corn. In this $G_{I_{f}}$ and $G_{c}$ are the guesses at the class mark nearest to the mean of the population as to length and circumference respectively.

Let $I_{L}$ and $I_{l}$, be the mean length and circumference respectively, and $C_{L}$ and $C_{C}$, the corrections to $G_{L}$ and $G_{C}$. which give $M_{L}$ and $M_{C}$, so that $M_{L}=G_{L}+C_{L}$ and $M_{C}=G_{C}+C_{C}$

Also let $D_{L}^{\prime}$ and $D_{\ell}^{\prime}$ represent deviations of class marks from the guesses $G_{i}$ and $G_{C}$ respectively.

Unless one carries through a large number of decimal places the method previously discussed is not only very laborious but it is much less accurate than the shorter method here described.

\section{SECTION V - THE REGRESSION COEFFICIENT}

From the correlation coefficient and the standard deviations with respect to two characters it is easy to obtain what is known as the regression coefficient. To obtain the regression coefficient of the weight of ears relative to their lengths, multiply the coefficient of correlation by the standard deviation of weight, and divide the product by the standard deviation of length.

This gives, for the regression of weight relative to length,

$$
r \frac{\sigma_{H}}{\sigma_{L}}=2.03
$$

Similarly, the regression of length relative to weight is

$$
{ }^{\sigma_{l .}} .
$$

Use of the regression coefficient. The regression coefficient is useful for prediction; that is to say, if we know the deviation of one character from its mean, this coefficient will enable us to 


\section{Correlation Study (Leaming Corn Crop, i905)}

Circumference. $\quad G_{C}=6.3$

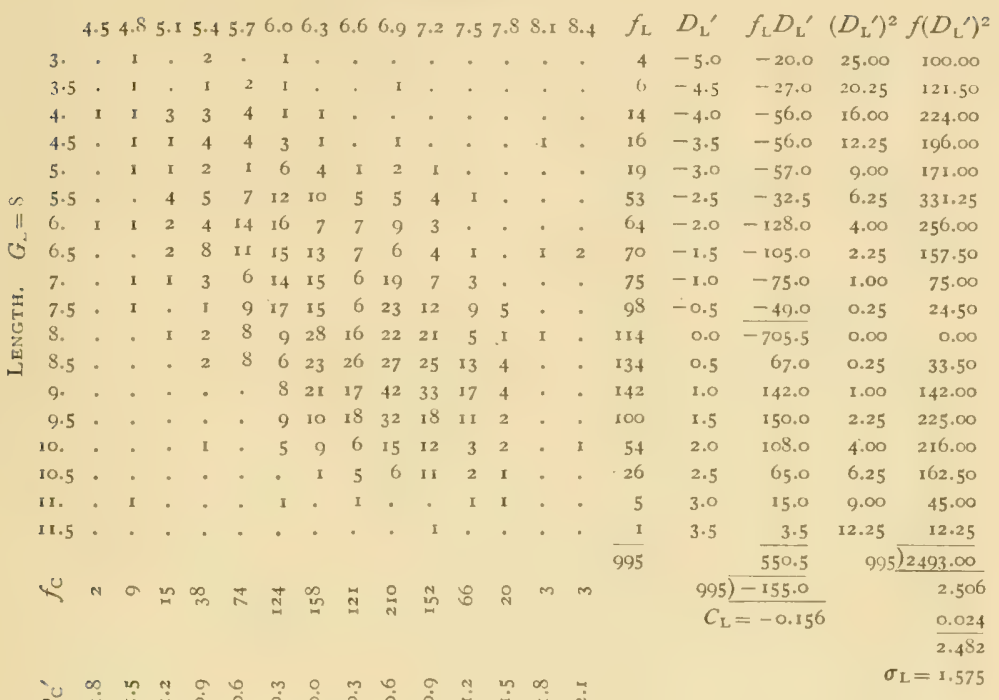

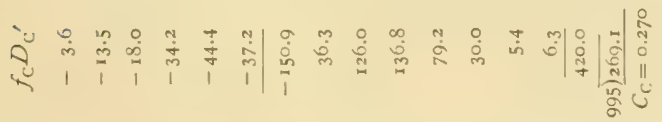

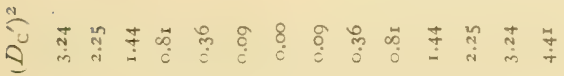

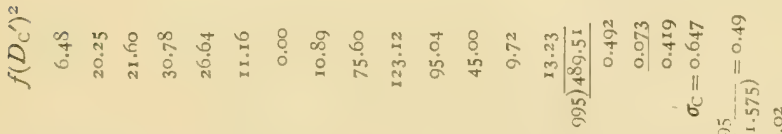



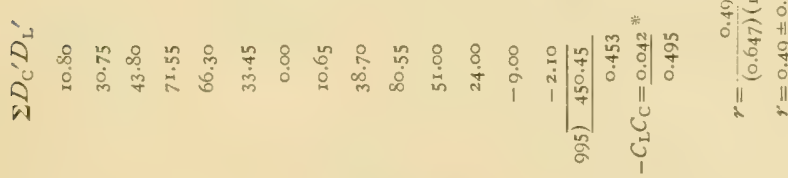

$M K_{\mathrm{L}}=7.844$

$M I_{\mathrm{C}}=6.570$ 
predict what will probably be the deviation of the correlated character from its mean. Thus, suppose we select ears which cleviate, say two inches, from the mean length of ears; that is, which are two inches above the average: the regression coefficient (2.03) of avight relativ'e to lingth indicates that w'e should crpect such cars to be about 4.06 ounces from the mean, that is $2 \times 2.03$. To be more general, if we select ears which have any deviation $x$ from the mean length, we should expect their deviations in weight to center about a value $2.03 x$ from the mean weight of ears for the whole population.

The regression coefficient is thus a fixed ratio between deviations of correlated characters, so that, knowing how much one of the characters differs from its mean in any unit of measurement, say inches, we are enabled to predict how much the associated character departs from its mean in its unit of measurement, say in pounds. Thus if a regression coefficient of weight upon stature is, say 2.I7, we know that any departure from the mean stature will be followed by a departure 2.17 times as great in respect to weight, using in both cases the same units as were used in calculating the coefficient ; for example, feet and pounds, inches and ounces, or even inches and pounds, if these were the units actually used in computing the regression coefficient.

\section{SECTION VI S STUDIES IN SPEED RECORDS OF TROTTERS}

Studies were made of 13,879 trotters possessing records of $2: 30$ or better, in order to learn their distribution as to speed, and the possible correlation of speed with color, and more particularly with sex (see tables, pages 469 and 470).

The data were taken for each quarter second, and the record made a scroll over forty feet long. The matter is here condensed to differences of one second in order to bring it into suitable space.

The original record showed two strange peculiarities. Almost invariably the largest number of records was found on the first quarter second after the even minute, as $2 \mathrm{O}_{4}^{1}, 2 \mathrm{I} \frac{1}{4}$, etc.; that is to say, the records were not evenly spread, or, as mathematicians 


\begin{tabular}{|c|c|c|c|c|c|c|c|c|c|c|c|c|c|}
\hline STV.LOI & & $\stackrel{m}{+}$ & 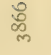 & in & $\begin{array}{l}0 \\
\text { in } \\
\text { in }\end{array}$ & $\begin{array}{c}\sigma \\
0 \\
0 \\
0\end{array}$ & 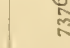 & 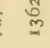 & 岕 & สู้ & 8 & 袩 & लू \\
\hline o. & & $\underline{\ddot{n}}$ & के & क् & 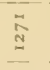 & $\equiv$ & ஜூ & $\stackrel{2}{m}$ & $\stackrel{m}{7}$ & $\stackrel{n}{q}$ & 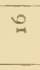 & $\stackrel{\vec{\sigma}}{\sim}$ & in \\
\hline $\begin{array}{l}\text { ô } \\
\text { in } \\
\text { ñ. }\end{array}$ & & है & i & స్ & $\stackrel{\circ}{\circ}$ & $\stackrel{\Xi}{\Xi}$ & in & $\cong$ & के & 8 & + & ; & \pm \\
\hline 交 & & 20 & $\frac{\pi}{n}$ & $\stackrel{\circ}{\circ}$ & in & 今ે & है & $\cong$ & 8 & $\frac{4}{4}$ & 0 & $\approx$ & तี \\
\hline $\begin{array}{l}\hat{n} \\
b \\
\text { in }\end{array}$ & & $\stackrel{N}{7}$ & क्ल & $\stackrel{2}{2}$ & $\stackrel{+}{\sharp}$ & $\stackrel{\substack{n \\
\cong}}{\cong}$ & हु & $\Xi$ & $\stackrel{\infty}{=}$ & $\stackrel{2}{2}$ & a & J & $\tilde{N}$ \\
\hline $\begin{array}{c}0 \\
\text { N } \\
\text { N } \\
\text { n }\end{array}$ & & क् ल & $\underset{N}{n}$ & in & م⿸户ల & $\begin{array}{l}+ \\
\text { D. } \\
\text { D. }\end{array}$ & तี & œ & $\stackrel{\circ}{=}$ & $\stackrel{0}{\square}$ & m & 8 & $\sigma$ \\
\hline $\begin{array}{r}n \\
\text { and } \\
1 \\
\end{array}$ & & 号 & స్ల్ & 5 & $\stackrel{0}{7}$ & : & $\Xi$ & ㅁ & $\stackrel{n}{\Xi}$ & : & N & $\stackrel{\infty}{+}$ & 9 \\
\hline $\begin{array}{c}T \\
i \\
i \\
\text { in }\end{array}$ & & $\frac{4}{4}$ & สั & $\stackrel{\infty}{?}$ & $\overline{8}$ & 总 & बू & $\stackrel{\infty}{n}$ & $\stackrel{+}{\circ}$ & $\approx$ & n & in & 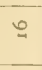 \\
\hline $\begin{array}{l}m \\
1 \\
1 \\
\text { d. }\end{array}$ & & 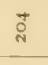 & $\stackrel{m}{\circ}$ & 品 & $\stackrel{\text { iे }}{4}$ & $\hat{8}$ & $\stackrel{n}{m}$ & 8 & $\infty$ & $\stackrel{\sim}{0}$ & - & $\hat{m}$ & 0 \\
\hline$\frac{\pi}{2}$ & & ્ֻণ & હ્ & $\stackrel{N}{\infty}$ & $\underset{N}{n}$ & $\tilde{g}$ & $\stackrel{8}{8}$ & 2 & $\infty$ & a & in & 广 & 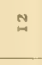 \\
\hline $\begin{array}{l}\text { N } \\
\text { dे } \\
\text { N }\end{array}$ & & $\approx$ & 志 & م્م & $\stackrel{2}{\approx}$ & $\stackrel{8}{+}$ & $\frac{4}{4}$ & के & ๓n & $\approx$ & $N$ & $\overrightarrow{\text { ते }}$ & $\cong$ \\
\hline ஸे & & $\widehat{\text { से }}$ & $\stackrel{8}{0}$ & क् & के & छ & $\stackrel{0}{m}$ & 品 & q & $\mathscr{m}$ & - & $\stackrel{m}{7}$ & I \\
\hline $\begin{array}{r}a \\
\frac{1}{\infty} \\
-\infty \\
-\infty \\
\end{array}$ & & 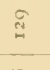 & $\Xi$ & సี & 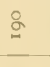 & $\%$ & $\bar{\alpha}$ & in & 5 & J & 1 & $\overline{\text { i }}$ & $\infty$ \\
\hline$\frac{1}{5}$ & & $\stackrel{\infty}{్}$ & \%ू & สె & $\stackrel{5}{ \pm}$ & : & $\stackrel{ \pm}{I}$ & 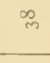 & in & 8 & H & $\cong$ & in \\
\hline$\vec{b}$ & & 8 & $\tilde{o}$ & $\ddot{\Delta}$ & क) & से & $\approx$ & $\stackrel{\circ}{\circ}$ & స్ & $m$ & - & \& & N \\
\hline$\stackrel{0}{i}$ & & $\overline{1}$ & m & ᄋ & 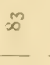 & 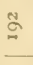 & $\stackrel{\varrho}{0}$ & $=$ & m & 요 & m & $a$ & "t \\
\hline$\underline{i}$ & & t & $\vec{m}$ & $\stackrel{\infty}{=}$ & $\approx$ & $\cong$ & $\Xi$ & $\overline{\text { ते }}$ & $\pi$ & g & - & 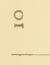 & + \\
\hline $\begin{array}{r}5 \\
0 \\
0\end{array}$ & & $\dddot{7}$ & ले & क & in & 怘 & ㅁ & $=$ & $=$ & $\hat{त}$ & 1 & in & 1 \\
\hline 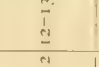 & & is: & $\hat{n}=$ & $\because$ & in & $\cong$ & $\vec{x}$ & $\simeq$ & $\because$ & $\because$ & . & $\%$ & ' \\
\hline$\underline{1}$ & & $\because$ & $\overline{\bar{i}}$ & in & $m$ & 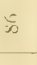 & $\hat{\text { in }}$ & 2 & $\check{\check{c}}$ & $=$ & 1 & n & 1 \\
\hline$\underline{I}$ & & i & $\subseteq$ & $\dot{m}$ & $=$ & 7 & $n$ & क & 0 & + & 1 & $m$ & - \\
\hline$\frac{5}{5}$ & & $n$ & $\bar{c}$ & $\overline{7}$ & 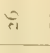 & $\because 8$ & $\bar{m}$ & $=$ & $\Xi$ & $\because$ & 1 & + & $"$ \\
\hline $\begin{array}{c}0 \\
1 \\
0 \\
0\end{array}$ & & $=$ & $T$ & $=$ & $\simeq$ & $\because$ & $=$ & 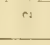 & $m$ & $m$ & 1 & 1 & 1 \\
\hline i. & & $=$ & + & $=$ & i. & $\approx$ & 2 & - & " & n. & 1 & 1 & 1 \\
\hline$\frac{1}{2}$ & & $m$ & - & \pm & - & in & $\cdots$ & 1 & $a$ & 1 & 1 & 1 & 1 \\
\hline in & & - & 1 & - & - & $"$ & - & - & 1 & 1 & 1 & 1 & 1 \\
\hline 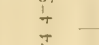 & & 1 & - & - & 1 & $=$ & - & 1 & 1 & 1 & 1 & 1 & 1 \\
\hline$\frac{1}{0}$ & & - & $\bar{r}$ & 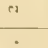 & (1) & + & " & 1 & $=$ & $\pi$ & $\frac{1}{.}$ & 1 & $\frac{1}{.}$ \\
\hline 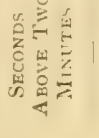 & 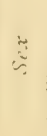 & : & 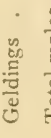 & : & 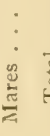 & : & 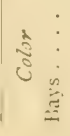 & $\begin{array}{l}\vdots \\
\dot{0} \\
\stackrel{0}{\Xi}\end{array}$ & $\vdots$ & 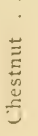 & $\begin{array}{l}\vdots \\
\vdots \\
\vdots\end{array}$ & $\begin{array}{l}\vdots \\
\vdots \\
\text { हैं }\end{array}$ & $\begin{array}{l}\vdots \\
\vdots \\
\check{c} \\
\check{L} \\
\check{L}\end{array}$ \\
\hline
\end{tabular}


say, they were not "smoth," and if the curve were platted there would be an unaceountable "hump " at each quarter second.

The second peculiarity had reference to the last record, $2: 30$. By the principle just stated this number should have been about 70 per cent of the number recorded at $2: 29 \frac{1}{4}$. On the contrary, it was very much greater. (On the principle ramning through the rest of the records we should predict the number at 2:30 to be about 727, whereats the number actually recorded is 1097 , showing conclusively that some 370 horses had been admitted to the 2:30 list that really belongel at $2: 30$ or slower; all of which shows how statistical studies bring to the surface as nothing else will the natural irregularities of observations or whatever abnormal facts connect themselves with our investigations. The table on page 469 is suggestive in many ways.

\section{Reiation of Sex and Color to Speled as expregsed in RATE PER CENT}

\begin{tabular}{|c|c|c|c|c|c|c|}
\hline \multirow{2}{*}{ DFECRUTION } & \multicolumn{2}{|c|}{$2: 30$ AND BRLOW } & \multicolumn{2}{|c|}{$2: 15^{-2: 16}$ AND BEI.OW } & \multicolumn{2}{|c|}{$2: 10-2:$ II AND BeLOW } \\
\hline & Number & Per Cent & Number & Per Cent & Number & Per Cent \\
\hline \multicolumn{7}{|l|}{ Sc $x$} \\
\hline Stallions .... & 4,493 & 32.0 & 330 & 36.0 & $6 \mathrm{~S}$ & 39.0 \\
\hline Geldings.... & 3,866 & $2 \mathrm{~S} . \mathrm{O}$ & 224 & 24.0 & 42 & 24.0 \\
\hline 'Total Males.. & 8,359 & 60.0 & 554 & 60.0 & 110 & 63.0 \\
\hline Mares ..... & 5,520 & 40.0 & 361 & 39.0 & 66 & $37 \cdot 0$ \\
\hline Total .... & ${ }_{13}, 879$ & & 915 & & 176 & \\
\hline \multicolumn{7}{|l|}{ ciolor } \\
\hline Bay .... & 7,376 & 53.0 & 502 & 55.0 & 92 & 52.0 \\
\hline Black .... & 1,362 & 10.0 & 101 & II.O & 25 & 1.4 .0 \\
\hline Brown .... & $\mathrm{I}, \mathrm{SS}_{5}$ & 13.0 & 129 & 14.0 & 24 & 14.0 \\
\hline Chestnut... & 2,220 & 16.0 & 126 & 1.1 .0 & 25 & 14.0 \\
\hline 1)un ..... & 60 & 0.4 & 2 & 0.0 & 0 & 0.0 \\
\hline Gray .... & $75^{2}$ & 6.0 & 46 & 5.0 & 7 & 4.0 \\
\hline Roan ..... & 224 & 1.5 & 13 & I. 5 & 3 & 2.0 \\
\hline Total ... & $13,8,9$ & & 915 & & 176 & \\
\hline
\end{tabular}

From the table above it appears that there is little relation between speed and either sex or color. It is true that, as we 
read across the table, we see that the pereentage of lemales talls slightly as we get into high speeds, hut the ball is very slight. The pereentages as to color vary but slighty, execpe in hlate, which decidedly increases with high speed, and in grat, which as decidedly falls off.

Applying Vule's formulit to the sturly of these ligures, let us, for example, take the bay color and inquire as to the measure of correlation between this color and high speed :

Total mumber of haly.

Number of bays at or below 2:15-2:16 . . . . . 502

Total number of performers . . . . . . . . . 13,879

Total number of performers at or below 2:15-2:16 . . 915

Number not bays at or below $2: 15-2: 16$ is $915-502=413$

Number of bays above 2:15-2:16 is $7376-502=6874$

Number not bays above $2: 15-2: 16$ is $13,879-(7376+413)=6000$

Arranging these values aceording lo Vule's formula, we hatre

\begin{tabular}{|c|c|c|}
\hline & lixy & I NoT lisy \\
\hline 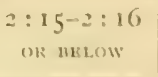 & 502 & 413 \\
\hline Anov' $2: 15$ & $6 S_{7}+4$ & 6090 \\
\hline
\end{tabular}

This gives as a measure of the association of the bay color and speed,

$$
\begin{aligned}
& 6090 \times 502-687+\times+13 \\
& 6090 \times 502+687+\times+13
\end{aligned}=+0.038 .
$$

While this result, 0.038 , shows that the bays have furnished slightly more than their share of the high-speed trotters, it is cloubtful whether this cocflicient is latge enough on enable us to assert any decided correlation.

In much the same way exhatustive studies should be made in all lines of breedinge, and at any expense of time and laber, in oreler that we may possess ourselves of reliable information in reatrol to ats many details ats possible concerning the relations of notable characters in our most valuable races. 
Summary. Correlation is generally a relative matter, and impressions are exceedingly deceptive. Nothing but actual calculation will show the extent to which characters really move together, and the importance of this knowledge is ample recompense for all the labor involved. As will be seen later, the same methods give us the only reliable measure of heredity.

\section{Special Exercises}

Again let the student actually do the work of finding correlation coefficients until he acquires facility in operation and a distinct conception of what is involved.

\section{ADDITIONAL REFERENCES}

Correlation in Rye. Experiment Station Record, XIII, 24I, 641.

Correlation in the Parts of Corn. By A. A. Brigham, Göttingen. Experiment Station Record, VIII, 486.

Correlation in Wheat. Experiment Station Record, IX, 553.

Correlation Mathematics. Science, XXII, 309-3I2.

Correlation of Characters in Corn. (German.) Experiment Station Record, XVI, 46I.

Correlation of Seeds and Color of Fruit. Experiment Station Record, XI, 932-936.

Correlation of the Mental and Physical Characters in Man. By Alice Lee and Karl P'earson. Proceedings Royal Society, LXXI, IO6-II4.

Correlation Theory. Science, XXI, 32-35. 


\section{CHAPTER XIV}

\section{HEREDITY}

"Heredity" refers to the distribution of racial characters among individuals of successive generations. On the principle of heredity all successful breeding operations depend, and the practical breeder needs to know all that is to be known concerning the manner in which succeeding generations are built up out of those characters which constitute the heritage of the race.

To define "heredity" as the direct and personal relation between the individual parent and the individual offspring is not only to restrict its meaning within too narrow limits but to destroy its significance to the breeder and deceive him as to the actual facts of transmission during descent. "Heredity" properly refers to the group that constitutes the parentage and the related group that constitutes the offspring.

All investigations show that both groups vary greatly among themselves, and to predict about where, within the racial range, an indiviclual will fall as compared with its personal parent, this is the object of a critical study of heredity, and the constant aim of the practical breeder. There is no hope that the offspring will be like the parent, except in a very general sense, but to predict how near it is likely to approach the parent, - this is something that requires not only the widest knowledge of the ancestry but the most accurate understanding possible of the facts and principles of heredity. It is the purpose now to inquire somewhat specifically into some of these general facts and principles.

\section{SECTION I - HOW CHARACTERS BEHAVE IN TRANSMISSION}

The particular characters that associate themselves together, constituting a race, variety, or breed, have separate histories as the generations come and go. Each has an identity and a history 
of its own, and each establishes and maintains, apparently, fairly definite relations to certain of its associates (correlation), while with reference to others it seems indifferent if not independent.

Individuals inherit differently. All individuals of the same race possess the same characters, but in different proportions, and no two individuals, even from the same parents, are alike. Some portion of this difference is of course due to development according to the conditions of life, yet all evidence goes to show that, after full allowance is made for this factor, natural differences exist that can be due only to inheritance.

That each individual is in possession of all the characters of the race is evidenced by the fact that his descendants possess them and that he transmits far more characters than are developed sufficiently to be noticeable in his own personality.

Latent characters. Thus characters may be present, but undeveloped, or "latent." Galton asserts that latent characters are "not very numerous "; 1 but it is certain that many characters may remain undevelopel through life and yet be transmitted perfectly. Familiar examples are the occasional secretion of milk by the male sex, already alluded to, and the transmission of the milking quality by bulls as well as by cows. All things considered, it is safe to say that the visible and fully developed characters of an individual constitute but a small proportion of his real possessions. Especially may this be said of a highly differentiated race.

Inheritance not limited to sex. It has been a favorite saying that certain characters are transmitted to one sex but not to the other. There is no evidence of any such limitations to inheritance. The limitations of sex may and do prevent the dciclopment of many characters that we know to be potentially present, so far as inheritance is concerned, because they can be transmitted. In this respect the relation of the male mammal to milk secretion is not different from that of the female that has never yet borne young. The faculty is latent, ${ }^{2}$ or undeveloped, in both

1 Galton, Natural Inheritance, p. I87.

2 The term "latent" is unfortunate. It conveys too strongly the sense of lurking. "Undeveloped" is the sense that ought to attach to this unfortunate term that has now been used too long to be dislodged. 
cases. They differ only in the fact that with the female the development is easily brought about, while in the male it is difficult and in most cases impossible. ${ }^{1}$

Belated inheritance. It is well known that all characters do not develop contemporaneously. Thus the sexual characters become developed just before full stature is attained, and with the failure of the primary sexual characters with advancing age comes the development of many of the peculiarities of the other sex. Then it is that the hen crows, the human female grows more hair, and the voice of the male becomes effeminate. The term "belated inheritance," though fixed in our literature, is unfortunate. It is belated dcrclopmont that is meant. Inheritance comes only at, or rather before, birth; but development is conditioned upon many factors, - among which age and sex are important, but not the principal, considerations.

Blended and exclusive inheritance. Perhaps the first and most noticeable fact is that some characters blend when brought together by transmission, while others remain distinct, being apparently mutually exclusive. Thus skin color in man blends readily, the cross between white and negro being nearly always of some shade intermediate between those of the parents, almost never spotted. ${ }^{2}$ In Shorthorn cattle and in Jerseys the colors frequently, if not generally, blend, while in the HolsteinFriesian they always remain distinct. In horses the blend is common, but in hogs it is practically unknown, so that in a litter of pigs from a black and a white parent the colors will remain distinct; some may be black, some white, and others spotted, but none will be roans or grays.

The same distinction holds as to characters generally. Sometimes the offspring will be intermediate between the parents, showing a blend; and again it will resemble one or the other, or else exhibit traces of both, each distinct and separate.

For example, so far as the matter has been studied, the blend is most perfect as to stature, ${ }^{3}$ and probably as to size in general, but eye color does not readily blend, ${ }^{4}$ nor do "tempers" 5 or

1 The student is reminded that milk secretion among males is not unknown.

2 The spotted skin is not absolutely unknown among humans, however.

${ }^{3}$ Galton, Natural Inheritance, p. 89 .

4 Ibid. p. 145 .

5 Ibid. p. 233. 
"tastes." This being true, we are often disappointed in trying to modify or tone down a vicious disposition by mating with one of milder temper, the progeny tending to follow the average of the race, or else to be as vicious as the objectionable parent.

Particulate inheritance, - inheritance by type, or bit by bit. Characters are often so closely associated (correlated) as to move in company, so that whole groups of characters appear and disappear together, even when there is little or no known causative relation between them. Whether this is merely accidental association of characters not mutually exclusive, and certain to happen occasionally under the law of chance, or whether it is due, rather, to some deeper-lying principle, is perhaps uncertain; but it is surely true that man, for example, runs in types, and whoever has traveled much, or has enjoyed a fairly extensive acquaintance, has met many people of no blood relationship, in places widely separated, who yet were clearly of the same type, and whose similarity became more evident upon closer acquaintance. ${ }^{1}$

Clearly, characters are not altogether independent one of another, and often the greatest difficulty is encountered in breaking up a group, some members of which are desirable and others objectionable. So inheritance is often "bit by bit," as if the unit of transmission were larger and more complex than the single character; as if a kind of permanent partnership were in force. The biological basis of all this, if it really exists, will probably remain for a long time hidden, but coefficients of correlation afford at least a method for determining the degree and the persistence of this copartnership.

Polymorphism and sexual dimorphism. Many races, instead of showing all intermediate gradations from one extreme to the other, in respect to size for example, or color, or any other character or association of characters, will exhibit two, three, or more forms or types, so different and distinct as often to be

1 In practical breeding operations the greatest need exists for exact knowledge of correlated characters. The methods given in the preceding chapter enable the student to determine quantitatively the real extent of correlation, and breeders should prosecute most industriously the study of this subject, until they are well informed as to the real relations of all valuable characters of domesticated animals and plants. 
mistaken for distinct species. In other words, their variations are not continuous but discontinuous.

Thus the earwig is of two distinct types as to size (dimorphism), and many insects exist in three different forms, - larva, pupa, and imago, - the crawling or worm form, the resting stage, and the winged form. ${ }^{1}$

Sex in general means dimorphism, for, almost invariably, marked differences exist between males and females of all species. Sometimes, as in most mammals, the males are the larger, but often the opposite is true, as in the case of many birds and insects. External differences other than size, however, are certain to distinguish the sex by a number of non-sexual characters.

Dimorphism in improved breeds. Most of our improved breeds exhibit more than one type entirely aside from considerations of sex. For example, the Hereford is remarkably constant in color, but there are two distinct types as to form. One is heavily built and long-bodied, with deep flanks and straight thighs; the other is smaller and shorter, with less depth behind and a tendency to rounded buttocks. The fore quarters are not different in the two types, but the differences behind are marked and the types do not readily blend. The breed appears to be almost, if not entirely, dimorphic.

Among the Shorthorns we have no less than half a dozen types that do not readily mix. The pure white is distinct in conformation, as is the Duchess roan, the Cruickshank roan, the cherry red, the dark mahogany red, and the Cruickshank red.

The Percheron horse is dimorphic both as to color and as to form. Whether it will always remain so, or will finally blend into a common type as to color and conformation, time only will tell. The same is true of the Jersey and the HolsteinFriesian cattle, the Berlishire hogs, and the Shropshire sheep.

All widespread and most newly developed breeds are polymorphic, - the first from the external influences and different standards of selection, the second from recently associated dissimilar

1 Excellent material on seasonal dimorphism of butterflies may be found in Weismann, Studies on the Theory of Descent, I, I-IOO; and on polymorphism in insects, Ibid. II, $40 \mathrm{I}-48 \mathrm{I}$. 
characters (see Mendel's law). Whether, in good time, they will blend, or will remain distinst, giving rise to polymorphic forms within the breed, is an important question in which the breeder is always deeply interested. If the polymorphism can be removed, he of course clesires to do it; if not, he must make the best of it and cease wasting time over the unattainable.

In all such cases the breeder is to satisfy himself as quickly as possible whether the polymorphism is temporary or permanent; and if it be permanent, he will do well to choose the type he is to breed, and abandon the effort to blend it with another, - in other words, he must be content to secure his results gradually, by selection.

\section{SECTION II - STATISTICAI, METHOIS OF STUDY OF HEREDITY}

Until recently no phase of evolution has been so badly studied as heredity. The common mistake has been to note a few remarkable individuals and exceptional instances, and from these attempt to deduce the "laws of descent." In this way popular conceptions of heredity have grown up, many of which are exceedingly erroneous, not to say fantastic.

We have only recently learned that studies and conclusions based upon individual instances are worse than useless because of the extreme range of variability, and that to determine the facts of heredity with any degree of reliability, we must studly the race as a ahole, and not simply the separate individuals that compose it.

All this means that the laws of descent are to be discovered by a critical study, not of inclividuals, but of cutire populertions, or at least of proportions of populations sufficiently large to be safely representative.

Unfortunately, the application of the statistical method to the study of this subject is comparatively new, and as it is extremely laborious, the accumulation of a large mass of material will of necessity be a somewhat slow process. ${ }^{1}$

1 Galton was the first to apply present-day methods to the study of heredity, but Pearson and others followed, and a considerable literature is accumulating, to which important additions are being rapidly made. The quarterly journal Biometrika is devoted to the study of this subject by the statistical method. 
Unfortunately again, the first and most exhaustive studies were made outside of our field, and mostly in that of human characters, so that the best material for the study of heredity lies in this field. But later studies, in wider fields, lead us confidently to believe that the same general principles control transmissions everywhere, in all races and with all characters. Accordingly the writer will employ any studies that have been made, in whatever fields, that offer valuable material either for elucidating principles or for illustrating methods of study. The fullest data of all are those collected by Galton, dealing principally with stature, and they will be freely employed for both purposes.

\section{SECTION III - THE REGRESSION TABLE}

As already seen (chap. xii), when a representative population of any race is arranged in the form of a frequency distribution we are able to deduce exceedingly accurate expressions for variability.

If now this distribution be separated and assorted acouding to parentage, we shall have a series of distributions, each of similar parentage, the whole presenting the best facilities possible for the study of heredity. Such a tabular arrangement constitutes what is called a "regression table," and inasmuch as all regression tables present the same general features, we look upon them with confidence as affording reliable data for the study of this most important but otherwise most difficult and apparently self-contradictory subject.

In all regression tables a scale of values (measurements, weights, numerals, or other valuation) is provided at one sicle for the parents, and a corresponding scale along the top for the offspring, or vice versa. ${ }^{1}$

The offspring, considered as adults, is then distributed, each individual being recorded opposite the value representing his

1 Obviously the parental measurements may be arranged along the top and those of the offspring at the side. Every observer follows his fancy in this respect, and as a matter of fact the tables are made in both ways. The writer has become more accustomed to the one described in the text, and for no other reason prefers to arrange the parental values at the side. 
*

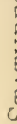

is

它

!

in

동

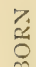

in

点

t

落

1

낭

$\leftarrow$

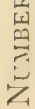

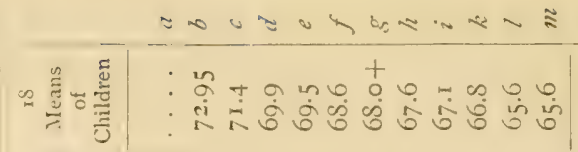

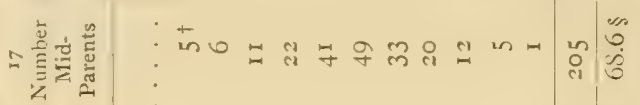

竞考焉:

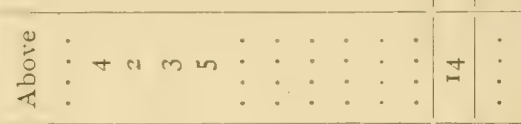

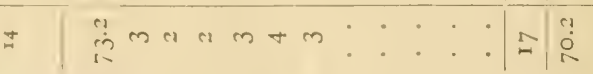

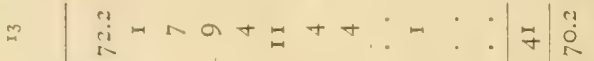

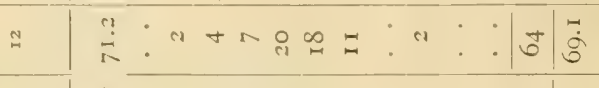

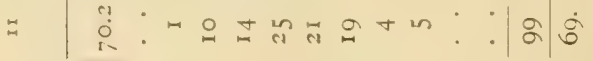

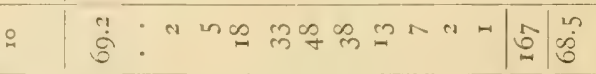

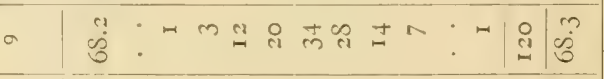

$\infty$

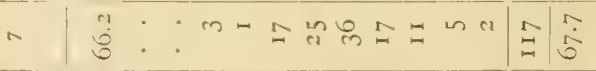

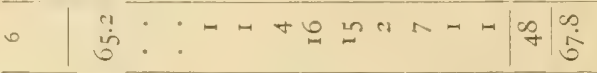

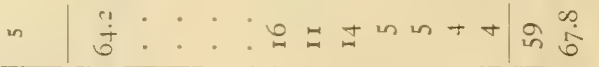

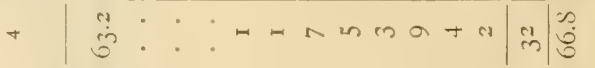

i....... mm $n$

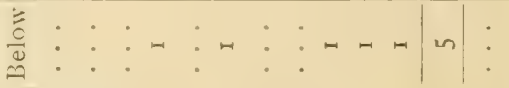

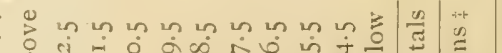

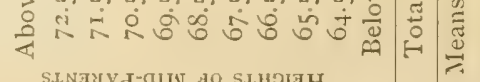
SLNAYVd-AIIN AO SLHDIAH

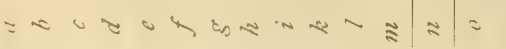

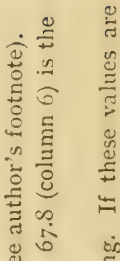

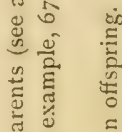

กับ

a

这

다 का

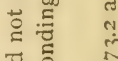

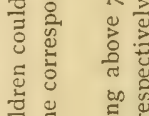

을 号

范

כ)

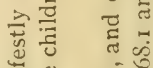

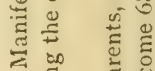

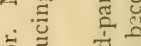

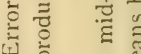

A 2 .

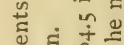

政

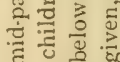

ن.

०े पूल थै

ถ่ ตี ถูก

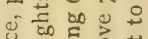

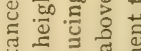

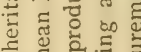

ह

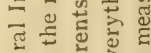

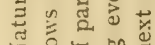

궁

है के है

हैं

의

* tr o.

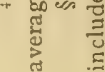


parent and in the column representing his personal value as to the character under question. When completed, such a table will show the number of offspring of each particular value, and also the kind of parents from which they sprung.

In whatever direction its parts are read, such a table consists of frequency distributions whose means and standard deriations may be detemined by the methods alrcady' gia'cu. The horizontals show the distribution of offspring of like parents, the verticals show the range of parents capable of producing like offspring, and the totals represent the respective generations.

One of the first tables of this kind published, and one of the best for our present purposes, is the one on the preceding page, from Galton, based on his studies of the stature of English people. ${ }^{1}$

1 See Galton, Natural Inheritance, p. 208.

In this table the heights were taken in small fractions, but recorded in I-inch groups. For instance, all measurements falling between 66 and 67 inches he recorded as 66.5. In attempting to do this for the sons, however, he noticed "a strong bias in favor of the integral inches." Hence he adopted for these measurements 66.2, 67.2, etc., instead of 66.5, 67.5, etc. As a matter of fact, it makes little difference what scale is adopted, provided the same plan is always observed in the matter of discarding or of recording fractions.

One slight inaccuracy for the individual in the long run offsets another, and as a whole such adjustments do not interfere with results. Trial calculations, too, will show that measurements taken an inch apart give substantially the same results as when taken a half inch or a quarter inch apart.

In this table the heights of the adult children are compared with the heights of the mid-parents; that is, with the average height of the father and the mother after multiplying the mother's height by $\mathbf{I . 0 8}$, because women are, on the average, one twelfth shorter than men. All female heights are, therefore, "transformed" and recorded as male heights. This custom is observed in all statistical studies involving sex; that is, the female values are reduced to their "male equivalents," so that sex differences are eliminated from the mid-parent, or, more properly speaking, everything is reckoned in terms of males.

Early in his studies the question arose whether the mid-parental height is a safe basis; that is to say, whether the child of one tall and one short parent is, in general, the same as the child of two parents whose heights are equal, but whose average height is the same as the average height of the tall and the short parent; in other words, would the children of a 70 - and a 64 -inch parent (average 67 inches) be the same as one of two parents each of whom is 67 inches in height?

After the study of many cases Galton found no. difference. He therefore concluded that a perfect blend takes place in respect to stature, and that the midparental height, after making due allowance for sex differences, may be safely taken as the true height of the mid-parent for purposes of heredity studies (see Natural Inheritance, pp. SS-90). We have since leamed that for extreme accuracy 
It is not likely that all characters behave precisely as does stature, - indeed, it is known that they do not; but all studies go to show that characters of every kind obey the same general laws in descent, and all regression tables that have ever been prepared exhibit the same general features. ${ }^{1}$ This table, therefore, while primarily for the study of stature, may be consiclered as typical of regression tables, and deductions made from it, agreeing as they do with those made from all other similar tables, whatever the race or the character, may be safely accepted as exhibiting fundamental laws in heredity. Because all regression tables afford the same deductions, they may be stated in the form of general principles as outlined in the following sections.

\section{SECTION IV - IIKE PARENTS BEGET UNIIKE OFFSPRING AND, CONVERSELY, LIKE OFFSPRING MAY BE BEGOTTEN BY UNLIKE PARENTS}

In this table the heights of the parents are in the rows $b$ to $m$, and those of the children (as adults) in the columns 2 to 15 . It will be seen at once that the offspring of parents of any given

slight modifications are necessary on account of the parental ancestry, but such corrections are not necessary for present purposes.

Galton calls attention to an error in the first row of children and mid-parents, saying that an error was introduced somewhere in the original tables, which cannot now be corrected. "It is obvious that four children cannot have five mid-parents," he says, but the numbers are so sniall as to be generally discarded, and hence the table is reproduced, error and all. He adds: "The bottom line (fourteen children with one mid-parent), which looks suspicious, is correct" (Natural Inheritance, p. 208).

In calculations generally the extremes (above 72.5 or 73.2 , or below 64.5 or 63.2) are discarded because the numbers are small and because exact measurements are not given. In calculating the general mean, however, two values have been determined, one without the extremes, the other by including the extremes, assuming that measurements above 73.2 averaged 74.2 , above 72.5 averaged 73.5 , below 64.5 averaged 63.5 , and below 62.2 averaged 61.2. The assumption is entirely gratuitous, but it affords a basis for using the extremes, although, as is noticed, it makes but slight difference in the results.

1 Regression tables may be prepared for any character that can be measured, weighed, counted, or in any way accurately determined. It only happens that studies in human stature have been the most complete of any, and are, therefore, used here. 
height are not the same, but, on the other hand, that they constitute a distribution beginning below' the parentage, and extending to a considerable distance abo' ' $c$ it, with the largest number of individuals near the middle of the distribution, close to but not identical with parental height.

Thus the 68 children of 22 parents 70.5 inches high (row $c$ ) are distributed from below 62.2 to above 73.2 inches, a range of I I inches, with the greatest number (I 8 ) slightly below the parental height (70.5). Any other row taken at random will show the same distribution in the stature of the offspring. Heredity, therefore, involves something besides the influence of the immediate parent, which, according to all studies, seldom exceeds 50 per cent of the total influence of the ancestry, leaving the other half to be accounted for by ancestors farther back. ${ }^{1}$

Not only is it true that like parents produce unlike offspring, but the converse is also true, - that like offspring may result from unlike parents. Take any column of the table at random, as column IO, containing the distribution of the 167 children of the uniform height, 69.2 inches. These men of even height were produced by parents ranging in stature all the way from 72.5 inches down to less than 64.5 inches, - a range of 8 inches. To be sure, the parental height that produced the greatest number $(48)$ was 68.5 inches, not far from the common height of the offspring (row 0 , column 16 ) and almost exactly the average height of all the parents (row o, column I7), but the critical study of this and all the other columns will clearly show that the same kind of offspring may be produced by greatly different parents.

These facts show clearly that two sires or dams of equally favorable appearance may have sprung from very different ancestry. They both belong to a distribution covering a considerable range, and if our selection is to be effective we need to know everything possible of the entire group to which the prospective parent belongs, or at least be intelligent as to the portion of the distribution from which he is drawn.

1 Every individual of bisexual parentage has a total of 2046 ancestors within ten generations. Whether these ancestors represent that many different individuals, or whether some are oft-repeated, depends upon the closeness of breeding. 
SECTION V - REGRESSION. IN GENERAL, THE OFFSPRING IS MORE MEDIOCRE THAN THE PARENTS; THAT IS, WHATEVER THE PARENTAGE, THE OFFSPRING EXHIBITS A STRONG TENDENCY TO REGRESS TOWARD THE MEAN OF THE RACE

A glance at any regression table shows an uneven distribution of the population, with the largest numbers near the middle of the table, exhibiting a strong tendency to cluster about the center. Not only is this so, but if any parental row (c to $m$ ) be carefully studied, the following points will be noted:

I. The mean or average heights of the children (column I8) are in no case the same as the heights of the parents. Compare column I 8 with column I.

2. When the parental height is abor' the mean of the race, that is to say 68.5 inches and upward $(c$ to $g$ ), then the mean height of the children is something less than the height of the parent (see any row from $c$ to $g$ ).

3. But when the parental height is below the mean of the race, -67.5 inches and less $(h$ to $m)$, - then the mean of the children is greater than the height of the parent (see any row from $h$ to $m$ ).

To illustrate: in row $\mathcal{e}$ are recorded the heights of the 68 children of 22 mid-parents 70.5 inches high. In column i 8 we see that the mian height of these 68 children was 69.5 inches, or a height one inch bclow the parentage, and by that much nearer the general mean of the race. Again, in row $k$ are recorded the various heights of the 66 children of 12 mid-parents 65.5 inches tall. In column i 8 we see that the mean or average height of these 66 children was not 65.5 inches, as in the case of the parents, but 66.8 inches, or 1.3 inches greatir, and by that much nearer the general mean of the race than were the heights of their parents.

This principle of regression, or, as it is sometimes called, the "drag of the race," represents the "pull" of the ancestors beyond the immediate parents. By this we see that, on the whole, offspring are less exceptional than their parents; or, 
stated in general terms, that the tendency is toward mediocrity, and that offspring are, on the whole, more mediocre than their parents. This is so because, in the absence of sclction, the two thousand or more near-by ancestors, all exercising some influence, were, altogether likely, about an average lot, and their pull is strong toward mediocrity. With rigid selection the average could be greatly raised, making the pull higher; but this results simply in raising the les'el of mediocrity, and the principle would still hold; for it is beyond hope or expectation that these two thousand or more ancestors could all be held at a high level. This is why breeders generally find many disappointments in breeding from exceptional individuals, - their offspring cannot, on the average, be equal to themselves.

On the other hand, the offspring of the inferior parent is helped by the principle of regression, which in this case acts as a "boost" instead of a "drag," "and we hear of such a parent that he "breeds better than himself," - all of which is a credit to the ancestors if not to the individual. However, the children of tall parents, while not so tall as their parents, are yet taller than the children of short parents, giving rise to the peculiar form of the regression table known as its "skew."

This principle of regression through the influence of the ancestry beyond the immediate parent, and the essential mediocrity of the offspring as compared with the parent, are then well established. This is not from any inherent superiority in parents or inferiority in offspring, but from the fact that mediocrity is the common condition of the bulk of the race.

No remedy for regression. Nothing but long-continued selection can ease the race from the clrag of regression, and even then, and always, the offspring are still subject to the pull of a new but higher mediocrity.

1 This principle is the salvation of the "submerged fraction" of humanity, and it is the principal reason why so many successful, even self-made men, spring from unpromising parents. It is entirely possible when the ancestry has been only recently submerged; it is hardly possible when there is a long line of criminal or defective ancestors.

A distinction is to be made, on the one hand, between the children of poor and honest parents who lack advantages but whose blood lines may be excellent, and, on the other hand, those whose ancestors have been submerged for generations; very few of these rise to prominence, or, indeed, can rise. 
This mediocrity is, therefore, a thing always to be reckoned with by the breeder who hopes to attain uniform success with improved strains. He cannot free himself from its influence. We shall see that its pull is not less than 50 per cent. The failure to know this fact, and the willingness to rest the case with the immediate parents and to assume that "like father like son" is the way of heredity, or to accept purity" of blood (pedigree) as synonymous with uniformity of type, - this is the one fertile cause of the greatest failures in stock breeding. The only sure basis of uniform success lies in a uniformly excellent ancestry for at least five or six generations back." Then the "drag of the race" will become a friend and not an enemy of improvement; but, no matter how excellent the ancestry, it can never equal the cricptional parent. In order to make the most of him, therefore, this drag should be reduced to a minimum.

\section{SECTION VI - THE MEASURE OF HEREDITY}

Now this regression is the pull of the ancestry back of the parent, and it is the best argument for the fact that inheritance is partly from the race and not exclusively from the immediate parent. Clearly, we need a measure of the degree of resemblance between the offspring and the immediate parent, so that we may know how much to credit to the parent and how much to credit to the back ancestry through regression. Such a measure of resemblance between mid-parent and offspring will be a good measure of heredity, and it is called the cocfficicnt of hircdity'.

The coefficient of heredity. Fortunately this involves no new conceptions and no new methods. The regression table is nothing more nor less than a special form of correlation table in which, instead of involving two characters in the same set of inclivicluals, we seek the correlation between two sets of related individuals with respect to the same character.

Thus, in the table of statures, we have in fact a correlation table between mid-parents and sons with respect to stature, and its correlation coefficient $(r)^{2}$ is for them a cocfficicut of horedity.

1 See Law of Ancestral Heredity, sect. xiv of this chapter.

2 The coefficient of correlation is everywhere denoted by the letter $r$. 
The coefficient of heredity is therefore nothing more nor less than the correlation coefficient $(r)$ obtained from a regression table in which two sets of individuals related by descent are tabulated with respect to the same character. The methods of finding the coefficient of heredity are precisely the same as those already described for finding the coefficient of correlation; indeed, the correlation coefficient of a regression table is the cocfficicut of heredity.

It is manifest that this correlation table may be constructed not only between mid-parents and offspring, but between fathers and sons, between grandfathers and grandsons, between mothers and sons (or daughters), between uncles and nephews, between brothers and sisters, between brothers and brothers, and, indeed, between persons connected by any' ties of consanguinity whatever, direct or inclirect. In each case the correlation coefficient becomes a good measure of hereditary resemblance.

If a regression table be constructed between fathers and mothers, a correlation would still be found, though the two are united by no blood lines except those common to the race in general. Such correlation comes entirely through selection, and its coefficient $(r)$ is commonly called the coefficient of cross heredity or "assortative mating." It is a good measure of the degree of selection involved in mating.

The table on the next page gives some of the coefficients of heredity that have been determined for different relatives.

Pearson remarks: "Wre see that on the average the intensity" of parental correlation is about 0.3 to 0.5 ; of grand parental, about O.I 5 to 0.3 ; and of fraternal, about 0.4 to 0.6 , - the latter correlation being somewhat reduced when the fraternity consists of members of opposite sexes."

Regression coefficient. The regression coefficient here is computed exactly the same as the regression coefficient from any other correlation table, and it has the same uses, namely for prediction; that is to say, for example, knowing the deviation of a group of mid-parents from their mean, what deviation shall we expect on the part of their offspring?

The use of the word "regression" in the term "regression coefficient" is likely to lead to confusion. We must not assume 
Coefficients of Heredity FOR Different Relationships ${ }^{1}$

\begin{tabular}{|c|c|c|c|}
\hline RELATIONSHIP & Material & Character & Correlation \\
\hline Father and son ..... & English ........ & Stature .. & .396 \\
\hline Father and daughter .. & English ......... & Stature .. & $\cdot 360$ \\
\hline Mother and son .... & English . . . . . . & Stature .. & .302 \\
\hline Mother and daughter. . & English ......... & Stature .. & .284 \\
\hline Mother and son .... & North American Indians & Head index & .370 \\
\hline Mother and daughter. . & North American Indians & Head index & .300 \\
\hline Sire and foal ....... & Thoroughbred horses .. & Coat color & .517 \\
\hline Dam and foal ..... & Thoroughbred horses . . & Coat color & .527 \\
\hline Grandsire and offspring & Thoroughbred horses.. & Coat color & .335 \\
\hline Grandsire and offspring & Basset hound .... & Coat color & .134 \\
\hline Brother and brother .. & English $\ldots \ldots \ldots$ & Stature .. & .391 \\
\hline Brother and brother .. & North American Indians & Head index & $\cdot 379$ \\
\hline Colt and colt ..... & Thoroughbred horses . . & Coat color & .623 \\
\hline Sister and sister .... & English ......... & Stature .. & .444 \\
\hline Sister and sister.... & North American Indians & Head index & .489 \\
\hline Filly and filly $\ldots \ldots$ & Thoroughbred horses .. & Coat color & .693 \\
\hline Brother and sister.... & English ........ & Stature .. & .375 \\
\hline Brother and sister.... & North American Indians & Head index & .340 \\
\hline Colt and filly $\ldots .$. & Thoroughbred horses .. & Coat color & .583 \\
\hline Whole brethren .... & Basset hounds ..... & Coat color & .508 \\
\hline
\end{tabular}

that the coefficient of regression is a dirct measure of the pull of the back ancestry, or that it directly measures the dissimilarity between parent and offspring as shown in the regression table. In fact, we note that when regression is perfect, then the coefficient of regression is zero; and when there is no regression (perfect correlation), then the regression coefficient is large. This is brought out in the cliagram on the following page.

This diagram is a geometrical exhibit of the regression table of statures (page 480 ) It is simply a reproduction of that table, omitting the frequencies and putting in crosses $(X)$ to represent the means of the horizontal arrays ${ }^{2}$ (column IS).

1 Pearson, Grammar of Science, pp. $458-460$.

" The mean of offspring in this table is $6 S$ inches, and we have assumed this value to be, for the present purpose, near enough to the mean of mid-parents and of the race to take the horizontal and vertical lines marked $6 S$ as passing through the mean of the table. 
If there were no regression, that is, if the offspring followed fully the lead of the parent, then parents above the mean would have offspring cqually above the mean; that is to say, parents 69 inches high (one inch above the racial mean) would have children also one inch above the mean, and parents two inches above the mean would have children also two inches above the mean, or 70 inches in height. In this way, were there no

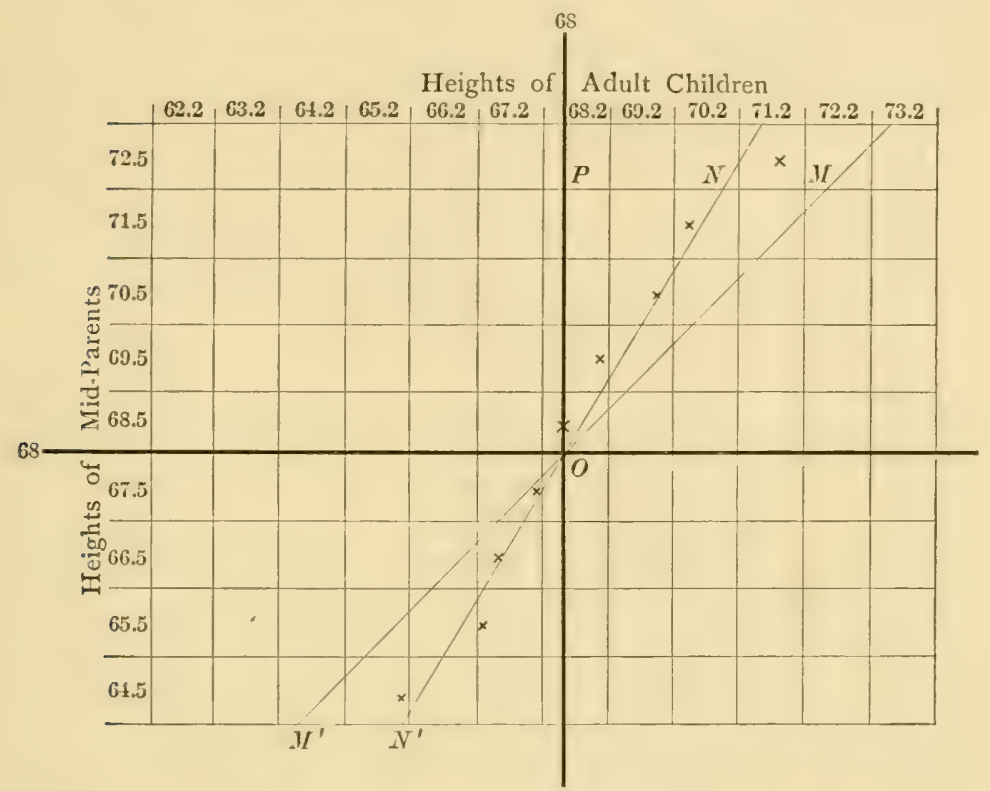

FIG. 45. Diagram representing regression

regression, these points would lie in a line $I I^{\prime} O H /$, with a deviation of $45^{\circ}$ from the vertical.

But regression is a fact, and the children of 70 -inch parents are not 70 inches tall but something less, and so on for other values; so that, when the true means are platted as calculated from the horizontal arrays of the regression table, and then connected by the best-fitting straight line, this line $N^{\prime \prime} \mathrm{O} N$ does not coincide with $M^{\prime} \mathrm{O} I I$, as it would were there no regression. This is known as the regression line, and its slope is the measure of the pull of parental heredity. The measure of this 
slope is our coefficient of regression, and its value is expressed by the ratio of $P N$ to $P M$.

Galton ${ }^{1}$ finds this ratio, when dealing with mid-parents and mid-filial statures, to be approximately as 2 to 3 ; "that is to say, the filial deviation from $P$ (the common mean) is on the average only two thirds as wide as the mid-parental deviation. I call this ratio of 2 to 3 [he says] the ratio of 'filial regression.' It is the proportion in which the son is, on the average, less exceptional than his mid-parent." That is, the deviation of the stature of children from the mean of the race is only about two thirds as wide as that of their mid-parents.

SECTION VII - THE MEAN OF THE OFFSPRING NOT NECESSARILY THE SAME AS THE MEAN OF THE PAREN'TAGE

At first thought it would seem axiomatic that, on the average, the offspring as a whole would be the same as the parents, unless the race is undergoing change. In the table of statures, however, by comparing columns 16 and 17 , row 0 , we see that the mean of all the parents was 68.6 inches (not counting extremes, or 68.7 inches including extremes), while the mean of the adult children was but 68.0 inches (68. I inches including extremes). This indicates a loss in stature of over a half inch in a single generation, unless some other influence is at work to counteract this discrepancy. That counteracting influences are at work we shall shortly discover, but the fact remains that the mean of the offspring is scldom idcutical with the mean of the parcutage. This fact is to be construed as meaning one (or both) of two things, - either that the race is undergoing transformation, or clse that all grades are not equally productive.

In this connection it is to be noted, first, that 68.6 is not to be taken as the mean of the gencration to which these mid-parents belong; that mean might have been either less or more, because not all members of a generation become parents, nor is the parent population a random draft from the generations of the mid-parents.

1 Galton, Natural Inheritance, p. 97. 
No fact is better known among statisticians than that wives differ from daughters and mothers differ from wives, - which means that all women (daughters) do not marry, and that not all wives are mothers (selection); that is to say, parents are a selected draft from the entire population, and we should not expect to find their mean the same as that of their offspring, which closely approaches that of the general population.

\section{SECTION VIII - EXTREMES OF A RACE RELATIVELY LESS} PRODUCTIVE THAN THE MEANS

In the table of statures a strong tendency is evident toward increased height and a still stronger one toward decreased stature (see sect. ix, "Progression"). This being true, the racial distribution would rapidly spread, if not entirely divide, into two races, giants and divarfs, unless prevented by some principle such as natural selection. This principle in this particular instance is evidently relative infertility, a principle easily deduced in at least two ways:

I. One hundred and three children are recorded at or below 64.2 , and only one parent below 64.5 (see table of statures). Clearly most short children do not become parents, else the race would rapidly degenerate as to size. This agrees with common observation, which is that dwarfs do not marry. When, however, the principle is applied to degeneracy and crime, the case is different, for criminals of ten produce more than their normal ratio, and many of their offspring, by the principle of progression (see sect. ix), are frightful degenerates.

To what extent giants marry is not so clearly indicated by this table, but that they are less fertile than the average when they do marry is clearly shown by comparing columns I 6 and 17 .

2. The average of fertility in this table as a whole is almost exactly $4 !(928 \div 205)$ per mid-parent. This average is well sustained in the lower and middle statures, rising in many cases to 5 per mid-parent; but it rapidly lessens in the higher statures, for from 70.5 up it is approximately 3. This, too, agrees with common experience, namely, that crtremes of a race are generally' less fortile than the moans. Indeed, it is commonly 
fertility that fixes type, unless its effects happen to be overcome by some other form of selection; that is to say, the type of highest fertility will become most numerous, and will therefore naturally determine the type of the race.

It is evidently the lessened fertility on the part of extremes, and the higher breeding powers of the mediocre individuals, that in nature assist selection in holding the principle of progression in check, thus generally, but not always, preventing the splitting up of races into smaller varieties; indeed, type is mainly the resultant of relative fertility and selection, - very largely of the former. This is why breeders of highly improved races must needs look well to fertility, for at that point their first troubles will arise, all regression tables indicating that they will never suffer from lack of variability, as seen in the following section.

SICTION IA-PROGRESSION. PARENTS IN GENERAL PRODUCE A FEW. INDIVIDUALS MORE EXTREME THAN THE RACE

What is true of averages is not necessarily true of individuals or of selected groups. Regression applies to the mass, not to the separate individuals that compose it. ${ }^{1}$

Parents in general produce individuals both inferior and superior to themselves, forming a frequency distribution whose mean lies somewhere between that of the parent and the general mean of the race. But the indiriduals extend both ways from this mean and some of them lie well beyond the range represented by the parentage. On this point see any row in any regression table, as row $c$ in the table of statures (page 480 ).

For example, in the case at hand, but 5 mid-parents, and possibly only 4 (Io or 8 parents), are above the height 72.5 inches (see row b), but 3 I children are recorded at 73.2 or above (see columns I4 and I5). The number of extreme children is thus more than three times as great as the number of extreme

1 For example, we have shown that, on the average, offspring are more mediocre than their parents; but for a highly selected offspring (72-inch stature) we should find the parents to be nearer mediocrity than the offspring. 
parents, even with the handicap of 0.7 inch, and only 3 of them were born of extreme parentage (row b, column i 4). Not only that, but the upper limits of height in this table are decidedly with the children rather than with the parents; in other words, children can be found taller than any parent.

At the other extreme of the table, but 6 mid-parents are recorded at or below 64.5 , but no less than 103 children are recorded at 64.2 , or below.

All this goes to show that, notwithstanding the principle of regression, the child population extends over a much wider range than does the parental, - a fact made clearly evident by comparing the offspring (row $n$ ), extending from below 62.2 to above 73.2 , with the parents (column I 7 ), extending only from below 64.5 to above 72.5. While neither of these ranges fixe: the limit, yet the range of the offspring is clearly the greater.

Viewed from any standpoint, offspring cover a wider range of variability than their parents, and some of them lie quite beyond the limits of parentage. This is progression, and its effect is, especially in the case of extreme parents, to send some individuals not only beyond the limits of parents but a'cll biyond the former limits of the race. The practical effect is that the population of any race can be moved either up or down through a large range, and either limit extended at will by the use of highly selected parents.

The behavior of a race uncler rigid selection and the operation of the principle of progression are well illustrated by the table on the next page, the data for which came from the records of ten years of com breeding by Dr. C. G. Hopkins, of the Agricultural Experiment Station of the University of Illinois. In these experiments the purpose was to influence the protein and the oil content of corn by selection. It is notable in this connection that all four strains, - high-protein, low-protein, high-oil, and low-oil, were developed from the same foundation stock.

This table is taken from the experiments in breeding for increased protein in corn. ${ }^{1}$ It will be noted that the foundation stock of corn (IS96), mixed ancestry, showed an average of

1 Bulletin No.116, Agricultural Experiment Station, University of Illinois. 


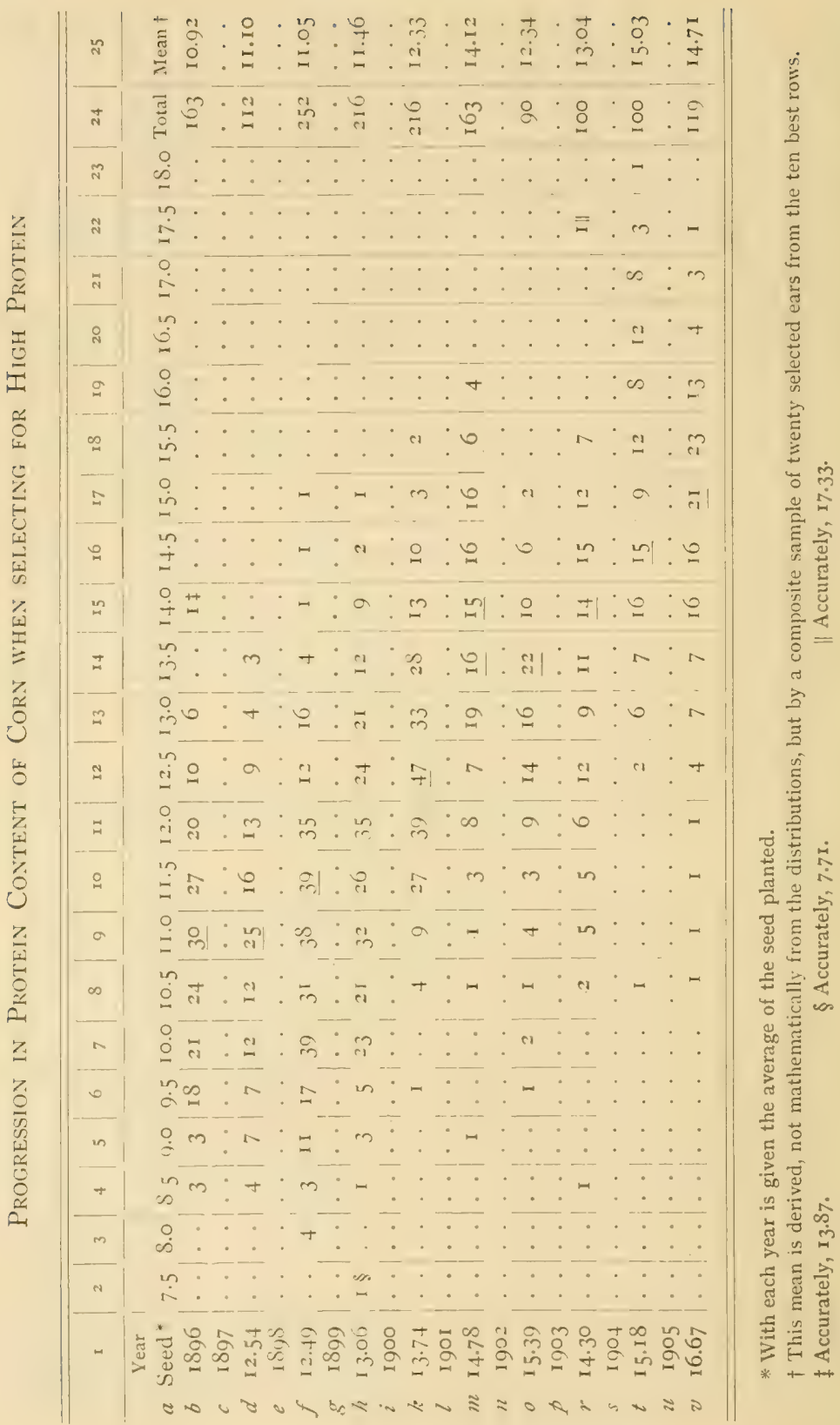


I0.92 per cent, with a mode of I I, and an upper limit (one ear) of 14 per cent, - more accurately I 3.87 .

The result of one year's selection made little evident impression. The second year's crop was not much better. The mode was the same, the average slightly lower, but the distribution became somewhat extended, with the appearance of two higher values represented by one ear each.

In the third year, with still better seed (I 3.06), the distribution extends still farther, but the new value is dow'n, not up. We note, however, a general increase of the higher values, and the mode has moved up a notch. ${ }^{1}$

In the fourth year (1900), with still better seed (I3.74), the lower values lessen and some drop off entirely. One new value appears. All the upper frequencies are increased; the mode has gone up two notches, and we now have no less than 28 ears as good or better than the extreme ear of 1896 .

The same principle continues in I90I, which was an exceptionally good year for protein, and the theoretical mode goes up nearly three points, reaching a par with the single exceptional ear of the foundation stock. It so happens that this year the number of ears examined was the same as that of the foundation stock ( 163 ), and of these ears no less than 77 , or 28 per cent, were the cqual of the cucctional first car. The effects of selection settle back slightly the next year, but in the succeeding season lost ground is recovered and there is produced the exceedingly exceptional ear, I7.33, which has since proved a remarkable parent.

The principle of progression is still further illustrated by the next table, showing the effect of selection both a'dy's. This is taken from Dr. Hopkins's original data in breeding for high oil and for low oil from the same foundation stock. The student should note the decisive manner in which these generations separate themselves from the foundation stock and from each other as selection goes on. Nothing could show more conclusively that under intense and persistent selection new values appear freely as the race becomes liberated from the heavy drag of

1 It can be seen by inspection that the theoretical mode is not so high as the empirical mode, 12. 


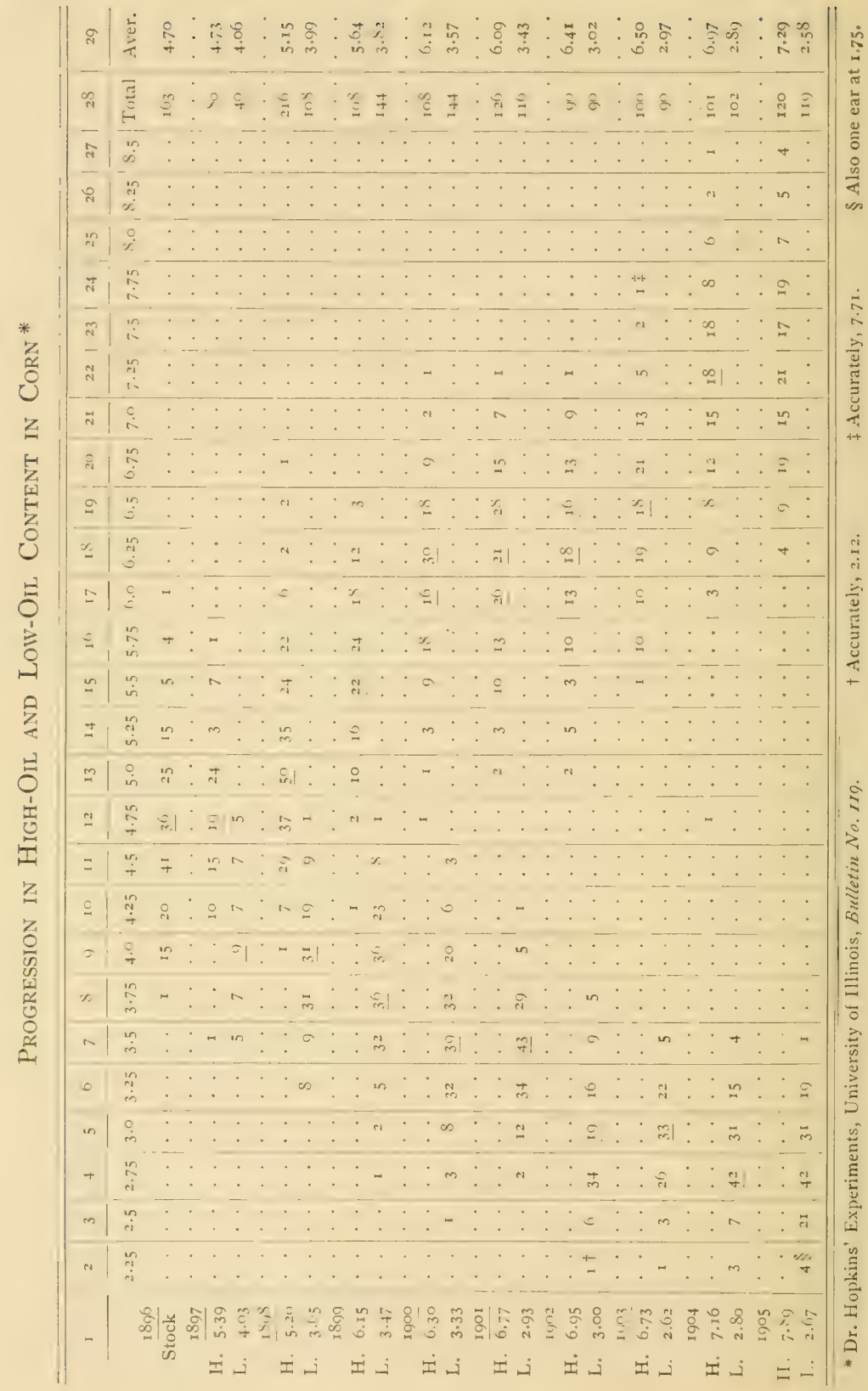


mediocrity, - all of which requires several years, and accounts for the comparatively small effects of the first one or two years' selections.

The same principle is evidently present and at work in stature (see table, page 480 ), for we note that the children cover a wider range than do the parents. It might seem that under the principle of assortative mating these exceptional children would break away and establish a race of giants and one of dwarfs. We are acquainted with some of the causes that prevent this; namely, relatively small fertility in giants (see table) and lack of marriage among dwarfs. As it is, however, the mean of stature is somewhat above the highest fertility (see table).

It used to be said that the offspring is a kind of mean of the parentage, and that the most that could be accomplished by selection was the production of fewer mediocre and inferior and a larger proportion of superior individuals. ${ }^{1}$ We know now, however, that the great bulk of the population will always be mediocre, but that by extreme selection we may secure new upper values quite beyond former limits, not only of the parents but of the race, and that at the same time the cutire population will respond to an upward trend, thereby raising the lea'cl of mediocrity.

All experience in breeding agrees with the principle here set forth. At the University of Illinois, when experiments in corn breeding were first undertaken, the question arose whether the results of the first year or two were anything more than assortative. The effects of selection were not at first pronounced, owing, of course, to the "drag" of previous ancestry. But selection was extremely rigicl, - a fact which rapidly freed the back ancestry from this drag, - - and with this came a decided rise in the mean of the crop; that is to say, the standard of mediocrity was raised, and along with this there appearcel from time to time occasional cars with ralues far aboi'e anything cver found in the foundation stock. These were new values due to the principle of progression, and the fact that the coefficient of variability

1 This position was always untenable, because, given the two best parents in a race, if the offspring is a mean between the two then no offspring can ever equal its better parent. How then was the superior parent produced? Such a doctrine has but one outcome, the bringing of the total population to a dead level of mediocrity. 
is not now growing less (see chap. xii) leads to the conclusion that the upper limits in this breeding experiment will be set by some factor other than the failure of variability; indeed, it is the opinion of the writer that the principle of progression is able always to afford all the material the breeder will need, and that the limits of improvement will be set, when they are set, by some biological, mechanical, or other considerations entirely aside from the failure of variability to present new upper values on which to base selection. ${ }^{1}$ The remarkable fact about progression is that the distributions are not distorted but are all typical of the race (see table, page 496).

This fact of progression betrays a unique principle in heredity, or rather in variability, because progression is over against and in spite of the "drag of the race." Those inclividuals that have overleaped the limits of the race have not only exceeded their own parents, but by much more have they exceeded the comparative mediocrity of their other ancestors." Progression cannot, therefore, be explained by any principle of "mean parentage." It rests upon a principle fundamentally distinct, and is to be regarded as the result of those fortuitous combinations of physiological units which we may expect to occur from time to time in the complicated processes attending reproduction and differentiation, and on which more will be said in Section XII of this chapter.

This suggests what will be later found to be a fact, namely, that in a large sense heredity follows the laws of probability, and in process of time we may expect all possible combinations of the elements that make up characters, and of the characters that make up the race, the largest proportion of which will cluster about a common point, which we call the type, but a few of which will inevitably appear at the extreme limits of the range of possibilities.

1 The greatest menace to extreme improvement is, as has already been said, lessened fertility. According to Pearson all evidence points to the fact that variability will never be reduced by more than about II per cent. See Grammar of Science, p. $48_{3}$.

2 The student will not fail to note that the ancestors of the exceptional parent are of necessity more mediocre than that parent. They will then exert their influence against, not in favor of, progression. 
SECTION X-THE EXCEPTIONAI, INDIVIDLAI, ARISES

EITHER FROM MEDIOCRITY OR FROM THE

EXCEPTIONAL PARENT

Further inspection of the table of statures shows that of the 72 children recorded above six feet in height (see columns 13 , I4, I5), 42, or over one half, were produced by mid-parents 70.5 inches or under; that approximately 22 were produced by micl-parents less than one inch above the mean of the race ${ }^{1}$ (68.6 inches, see row o, column I 7 ); that no less than I 2 , or one sixth of all, were produced by mid-parents recorded at or below the mean of the race; and that I was produced by a 65.5 -inch mid-parent.

From this we see that exceptional indivicluals may arise either from exceptional or from mediocre parents. The probability, however, is greatly in favor of the former. Six 72.5-inch miclparents produced I 3 exceptional children out of a total of 19 , considering everything abore six feet as exceptional. Of this number 6 , or over 30 per cent, exceed their own parents in height. Though the 69.5-inch mid-parents produced a higher number of exceptionally tall chilcren (20), 4 I parents were involved instead of 6 . This is less than 3 per cent of the total children (183), instead of 68 per cent, as in the case of the progeny of the taller parents.

It is at this point that the political scientist and the thremmatologist recognize different principles. Both are interested in exceptional individuals. As we have seen, they may be had either from the general population or from a highly selected parentage. The breeder chooses the latter because he cannot afford to support so large a population for so few exceptional individuals. He takes the highly selected parentage because the proportion of extreme excellence is higher, and because its "drag" is less. He is desirous of using minimum numbers for economic reasons.

The political scientist is limited by no such considerations. If he resort to selection (election) each time a ruler is to be 69.5.

1 Including only one half of the offspring produced by parents recorded at 
chosen, he will always have good material at hand, and, as he is not specially interested in progeny, he is not concerned with the "drag." What he wants is individual service.

If, on the other hand, he adopts the plan of hereditary sovereignty, he will deal with few families at a time; and while, if they are extremely well-bred to begin with, a large proportion will be exceptional, yet a glance at the upper lines of this table will be enough to indicate that he will be confronted by a good many hereditary rulers who are far from exceptional (see especially row $c$ ). He has taken a useless hazard, and this is the inevitable handicap of an hereditary monarchy. From the standpoint of evolution the principle is wrong.

The above ought to make it clear why the breeder and the politician should adopt opposite methods. If service alone is wanted, it is better to find it than to breed it; and that is why it is often better to buy a particular type of animal than to attempt to produce it, especially if the type is at all unusual, as in the case of the "fire horse."

\section{SECTION YI - FRATERNAI, VARIABILITY, - OFFSPRING OF SAME PARENTS NOT IDENTICAL}

The offspring of like parents are not only unlike, but the successive offspring of the same parents vary widely. The only data compiled on this important fact are contained in the table on the following page, from Galton's studies in stature.

This table presents all the characteristics of the ordinary regression table but in a degree slightly less pronounced. This shows that the same laws of regression and progression apply within the family as apply between families.

Alluding to this significant fact, Galton remarks : ${ }^{1}$

It appears that there is no direct hereditary relation between the personal parents and the personal child, except perhaps through little-known channels of secondary importance, but that the main line of hereditary connection unites the sets of elements out of which the personal parents had been evolved with the set out of which the personal child was evolved... This is why it is so important in hereditary inquiry to deal with fraternities

1 Galton, Natural Inheritance, pp. 19-20. Italics are mine. 


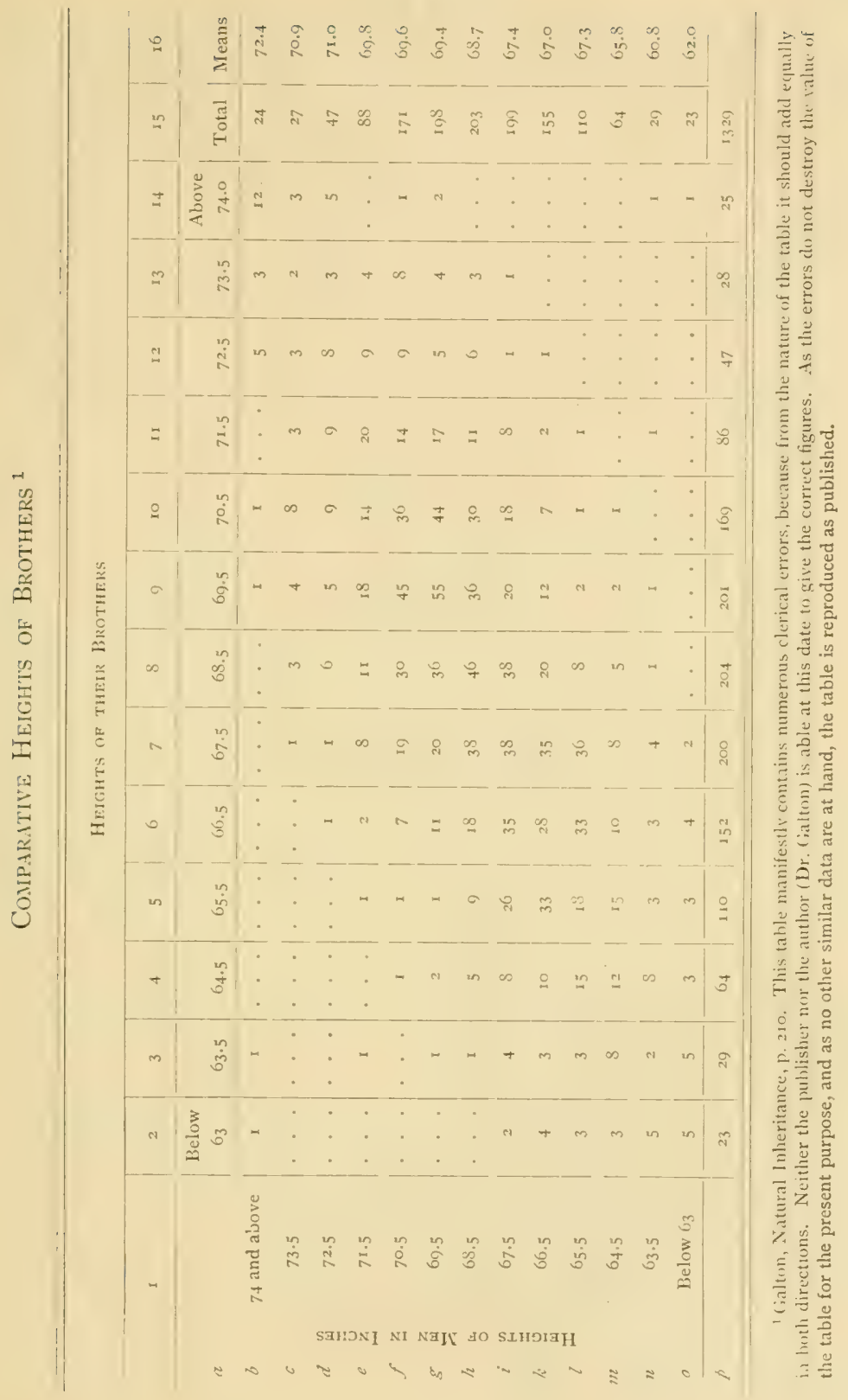


rather than with individuals, and with large fraternities rather than with small ones. IVe ought, for example, to compare the group containing both parents and all the uncles and aunts with that containing all the children.

IIere is the very gist of the whole matter, showing the folly of dealing with individuals in questions of breeding. All the best evidence shows that selection based upon the individual, without regard to the group to which he belongs, will never result in concentration of excellence. It is only by persistent selection, based on the groups as a whole (purity of pedigree in the sense of uniformity of type), that we shall ever free even the family from the drag of the race, and make real progress in improvement.

Harold and Miss Russel, the sire and dam of Maud S., were owned at Woodburn for many years, but of all their get only one, Maud S., developed high speed. Why? The question cannot be answered any further than it can be inferred from the principle just stated and from the well-known methods of cleavage of the nucleus in cell clivision and in maturation; but with these facts in mind, we should hardly expect that two idcntical individuals would ever arise, cven from the same parents.

The earlier offspring live longer than do the younger children of the same parents. Having established the fact that successive offspring of the same parents are different, there remains the task of determining to what extent this fraternal variability is heterogeneous, and to what extent it may be correlated with age or with some similar circumstance that tends to throw the offspring into a regularly graded series of some sort.

On this point we know but little. To the eye this variability appears quite heterogeneous, but studies in longevity, for example, have fully established the fact that in man the older children, on the average, live longer - that is, have longer lives than do the younger ones of the same family. This difference, as between the oldest and the youngest, amounts to no less than four years. ${ }^{1}$ Whether the same or a similar decline takes place in other characters and faculties only exhaustive studies could determine. There is no reason to doubt that general principles apply equally to man and to other animals, excepting in so far

1 See article on "Inheritance of the Duration of Life," by Beeton and Pearson, Biometrika, Vol. I, I'art I, pp. 50-76. 
as social and other somewhat artificial conditions of life may intervene, and we shall anxiously await the results of further investigation into the character and range of difference between offspring of the same parents.

Individuality. Whatever the results of investigation in this direction, and whatever gradual advance or decline may be established among the members of the same family on the arer$a g c$, the fact remains that variability is largely heterogeneous as between indiziduals, and that a marked individuality pervades all offspring, either of the same or of different parents.

This lessened deviation between members of the same family as compared with descendants in general is due to the fact that, among brothers, not only both immediate parents but all ancestors are identical. The differences that do exist within the same family serve to show the aide dia'rgencies possible with the same hercditary' elcments, although, in studying adults, some allowances must always be made for differences in development due to external causes. While members of the same family are in general reared more nearly alike than are members of different families, yet in a large sense every individual has a life history of his own quite distinct and in many senses different from that of any other individual of his own or of any other generation. Now this life history affects derelopment and accounts for some of the differences between adult individuals. Not all of the variability within a family can, therefore, be assigned to hereditary influences, but that a large share of it is due to such influences is rendered extremely likely by the well-known fact that successive ova, spermatozoa, or pollen grains from the same individuals are not alike either in their genesis or in their behavior afterward. The very mechanism of maturation strongly suggests profound qualitative differences, and tends to modify our assumption that all children of the same parents possess identical hereditary elements. The experience of breeders everywhere is that offspring of the same inclividuals are not slightly different but in general they are widely different. Whether, and to what extent, these differences can be lessened by selection and by relative purity of ancestral gametes are questions on which light is sorely needed. 


\section{SECTION XII - CHARACTERS TEND TO COMBINE IN}

\section{DEFINITE MATHEMATICAL PROPOR'TIONS ${ }^{1}$}

The student working with large populations must be struck by the remarkable similarity of the general features of all frequency distributions and arrays. Broadly interpreted, this suggests a strong mathematical basis in reproduction.

In all forms of life halving and doubling are basic processes. The number "two," therefore, as a mathematical conception, lies at the bottom of a large share of our biological problems, especially those of variability, and a little consideration will show that the usual form of the frequency distribution is the natural result of the reproductive processes, - indeed, that the facts of variability largely, though not exclusively, follow the ordinary mathematical laws of combinations and probabilities.

The mixing of pure forms. To illustrate this fundamental fact let us undertake to follow the history of two characters brought together for the first time, and the manner in which they will naturally appear in the offspring.

To put the matter in its simplest form, let us suppose a herd of pure blacks to meet and mingle with a herd (of equal numbers) of pure reds, and that they breed together without restraint, - that is, without sclection. They will then mate incliscriminately; that is to say, a black female will mate indifferently with a black or a red male, - sometimes with one and sometimes with the other.

This being true, one half the offspring of the black females will be pure black (designated by $B^{2}$ ) and one half will be mixed, black and red (designated by $B R$ ).

The same principle applies to the red females, whose progeny will, in like manner, be equally divided between the mixed offspring and the pure reds.

Expressed in tabular form we should then have:

For every 200 offspring of black females

For every 200 offspring of red females

Total distribution, 400 offspring

In proportion of

$$
\begin{array}{r}
100 B^{2}+100 B R \\
100 B R+100 R^{2} \\
100 B^{2}+200 B R+100 R^{2} \\
B^{2}+2 B R+\quad R^{2}
\end{array}
$$

1 This principle was first announced by Quételet, 1846 , in Lettres sur la théorie des probabilités. See Vernon, Variation in Animals and Plants, p. 12. 
It is evident that, whatever the numbers involved, the above is the proportion in which the pure and the mixed forms will naturally appear in the first generation of admixture between two pure forms.

From this we see that indiscriminate breeding of distinct characters results in both "pure" and "crossed," or mixed, forms in their descendants, and this in the proportion of $1: 2: I$. Now this is a short "frequency distribution," in which the middle term represents the individuals of mixed breeding and is equal to the sum of the two extremes.

The second generation, or second remove from pure forms. What now will be the character of the next generation, as bred from the individuals $B^{2}$ (pure black), $B K$ and $R K$ (mixed), and $R^{2}$ (pure red)?

Continuing the assumption of indiscriminate mating and uniform fertility, we shall have the following, remembering what are the relative numbers involved, and that in the long nun ea'ery kind of female will mate with er'ory kind of mali, producing offspring of the following character:

Character of Offospring produced by Females of Different Kinds when mating with Males of Different Kinds Without Selection

\begin{tabular}{|c|c|c|c|c|c|c|}
\hline \multirow{2}{*}{\multicolumn{2}{|c|}{$\begin{array}{c}\text { Females of Different Kindes in } \\
\text { Relative Frequency }\end{array}$}} & \multirow{2}{*}{\multicolumn{2}{|c|}{ THEIK }} & \multicolumn{3}{|c|}{$\begin{array}{l}\text { DifFereNt Kinis OF SiRES iN THEIR } \\
\text { RELATIVE FrEQUENCY }\end{array}$} \\
\hline & & & & $B^{2}$ & $2 B R$ & $R^{2}$ \\
\hline $\begin{array}{l}\text { Offspring of } B^{2} \text { mating with } \\
\text { Offspring of } 2 B R \text { mating with } \\
\text { Offspring of } R^{2} \text { mating with }\end{array}$ & . & $\begin{array}{l}\cdot \\
\cdot \\
\cdot\end{array}$ & $\begin{array}{l}\cdot \\
\cdot \\
\cdot\end{array}$ & $\begin{aligned} & B^{4} \\
2 & B^{3} R \\
& B^{2} R^{22}\end{aligned}$ & $\begin{array}{l}2 B^{3} R^{3} \\
4 B^{2} h^{2} \\
=B R^{3}\end{array}$ & $\begin{array}{rl} & b^{2} R^{2} \\
2 & B R^{3} \\
& R^{4}\end{array}$ \\
\hline 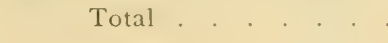 & . & . & .. & \multicolumn{3}{|c|}{$B^{2}+4 B^{3} R+6 B^{2} K^{2}+4 h^{\prime \prime}+K^{4}$} \\
\hline
\end{tabular}

This, then, expressed in its simplest form and the lowest terms, is the population resulting from two generations of indiscriminate breeding between forms originally pure.

Two things are noticeable about this total. First, it has all the characteristics of the ordinary frequency distribution (I, $4,6,4,1)$; and, second, it is a complete expression of the 
binomial $B+R$ expanded to the fourth power according to the binomial theorem.

Succeeding populations follow the law of the binomial theorem except as interrupted by selection or differences in fertility. In the breeding of this third generation intor se we find the numbers becoming rapidly complicated, but from the fact that it always follows the binomial theorem we can write the normal distribution for the fourth generation of descendants of any pair of characters as follows: ${ }^{1}$

$$
\begin{aligned}
(B+R)^{8}= & B^{8}+8 B^{7} R+28 B^{6} R^{2}+56 B^{5} R^{3} \\
& +70 B^{4} R^{4}+56 B^{3} R^{5}+28 B^{2} R^{6}+8 B R^{7}+8 R^{8} .
\end{aligned}
$$

Analyzing this "fourth-generation population," we find :

I. That no less than nine color combinations are represented, ranging all the way from pure black to pure red.

2. That the frequency numbers representing the various combinations, I, $8,28,56,70,56,28,8$, I, form a symmetrical frequency distribution whose total is 256 , only two individuals of which are pure.

3. That future breedings would become rapidly complicated, but that we should alcurys have one pure black and one pure red, with all possible combinations between the two.

4. That the actual color combination of the individual cannot in most cases be inferred from appearances. For example, there is but one real black and but one real red in the whole population. However, the $28 B^{6} R^{2}$ will look like blacks, because there have been six infusions of black to but two of red, while the reverse is true of another equal number, $28 B^{2} R^{6}$. The $70 B^{4} R^{4}$, which is the largest number of all, constituting nearly one third of the entire population, is equally balanced as to color tendencies, but will appar to be of the color that is most noticeable, - in this case probably a dark red.

Combinations of three characters. Though the numbers become more rapidly complicated, the same principles apply when dealing with three or more characters. For example, suppose we introduce a third color, white. We shall then have as the result of the first mating the following:

1 The student can easily verify these figures by the plan already outlined. 


\begin{tabular}{|c|c|c|c|c|c|c|}
\hline & & & & \multicolumn{3}{|c|}{ MaLes } \\
\hline & & & & $b$ & $R$ & $w$ \\
\hline Offspring, female $B$. & . & . & . . & $B^{2}$ & $B R$ & $B W$ \\
\hline Offspring, female $k$. & · & $\cdot$ & $\cdot \cdot$ & $B R$ & $R^{2}$ & $R I V$ \\
\hline Offspring, female $\mathrm{W}$. & . & . & $\cdot \cdot$ & $B W$ & $R U$ & $W^{2}$ \\
\hline Total . . . & . & . & . . & \multicolumn{3}{|c|}{$B^{2}+2 B R+R^{2}+2 B W+2 h I V+W^{2}$} \\
\hline
\end{tabular}

Here we have, after one indiscriminate mating all around, a total of nine animals, of which one is pure black, one pure red, and one pure white, with the other six divided into three groups, each of two colors combined, - that is, all the possible combinations.

If now this generation be bred into, itself, new and strange combinations are inevitable, giving rise to a complicated population, as follows :

Table of Offspring showing the Fourth Generation in the Attempt to Combine Three Characters

\begin{tabular}{|c|c|c|c|c|c|c|}
\hline \multirow{2}{*}{ Dans } & \multicolumn{6}{|c|}{ Stres of Same Breeving as Dams } \\
\hline & $B^{2}$ & $2 B R$ & $R^{2}$ & $2 B W$ & $2 W R$ & $w^{2}$ \\
\hline$B^{2}$ & $B^{4}$ & $2 B^{3} R$ & $B^{22} R^{22}$ & $2 B^{3} \mathrm{IV}$ & $2 B^{\prime 2} R I V$ & $B^{2} V^{2}$ \\
\hline $2 B R$ & $2 B^{3} R$ & $4 B^{2} R^{2}$ & $2 B K^{3}$ & $4 B^{2} R^{3} W$ & ${ }_{4} B R^{2} I V$ & $2 B R^{\prime} W^{2}$ \\
\hline$R^{2}$ & $B^{2} R^{22}$ & $2 B K^{3}$ & $R^{4}$ & $2 B K^{2} I V$ & $2 R^{3} H$ & $K^{22} W^{2}$ \\
\hline $2 B I V$ & $2 B^{3} W$ & ${ }_{4} B^{2} R I V$ & $2 B R^{2} I V$ & $4 B^{2} W^{2}$ & $4 B K^{2} H^{2}$ & $2 b^{3} W^{3}$ \\
\hline $2 R W$ & $2 B^{2} R I V$ & $4 B R^{2} I V$ & $2 R^{3} \mathrm{IV}$ & $4 B R W^{2}$ & $4 R^{2} / V^{2}$ & $2 R^{3} W^{3}$ \\
\hline$W^{2}$ & $B^{2} W^{2}$ & $2 B R W^{2}$ & $K^{22} \mathrm{IV}^{2}$ & $2 B W^{3}$ & $2 R W^{3}$ & $I^{4}$ \\
\hline \multicolumn{7}{|c|}{$\begin{array}{l}B^{4}+4 B^{3} R+6 B^{2} R^{2}+4 B^{3} W+12 B^{2} R W+6 B^{2} W^{2}+4 B R^{3}+\mathrm{I} 2 B R^{2} W \\
+12 B R W^{2}+R^{4}+4 R^{3} W+6 R^{2} W^{2}+4 B W^{3}+4 R^{3}+W^{4}, \ldots \text { total, } \mathrm{I}\end{array}$} \\
\hline
\end{tabular}

Here are eighty-one individuals of no less than fifteen different color combinations, all effected within two generations from purity. Out of the eighty-one individuals, three, and three only, are as pure as if no admixture had been attempted, suggesting that a cortain small proportion will aliog's rumain unmixed in heterogeneons breeding, no matter how long continued. 
All the rest are mixed in color, no matter what their appearance. Of these the $4 B^{3} R$ and the $4 B^{3} \mathrm{IV}$ will most likely appear black, as will also, in all probability, the I $2 B^{2} R I I^{\text {; }}$ because the $B$ elements are clearly in the majority. Similarly, equal numbers will appear red, and other equal numbers will secm to be white, - unless both colors appear, as in roans and piebalds.

There are three sets of six each $\left(6 B^{2} R^{2}, 6 B^{2} I^{2}\right.$, and $\left.6 R^{2} I V^{\circ 2}\right)$ in which but two color elements are present, but in which the appearance will probably be fixed by the color that is most pronounced and which, therefore, tends to dominate the other; thus the $6 B^{2} R^{2}$ will appear as black or very dark red.

Thus it is that appearances are often deceiving, and that which looks like a heterogeneous jumble is, after all, an orderly collection of mathematically exact combinations. A scheme like the above serves to show the exceedingly complicated, yet orderly, systems that necessarily arise in bisexual reproduction, whaterer the characters involicd, - complexity that increases rapidly, indeed almost inconceivably, as generations multiply.

Because of these facts reproduction would be reduced to a problem in probabilities, and we should have all possible combinations presented, were it not for the fact that selection is always at work to eliminate certain unfavored forms, and that differences in fertility serve to give certain combinations still further advantage over others. However, we are not to overlook the fact that, even though certain values be withdrawn from such a distribution, the laws of probability continue to apply to the remaining values, whose combinations will take place as before, and in the end give rise to a distribution not very different in form from that which would have arisen if no values had been removed.

An ultimate confirmation of this statement is found in the fact that most frequincy' distributions are forirly" symmetrical, and that one large cnough to be fairly" "smooth," whateier the numbir of its terms or the siac of its frequcncies, can be closcly' reproduced by cxpanding a binomial. If the distribution be symmetrical, the terms of the binomial should be numerically equal $(B+R$, or $\stackrel{1}{2}+\underset{2}{1})$; but if its mode is not near the middle 
but nearer one extreme, then the terms of the binomial should be numerically unequal ${ }^{1}\left(B+2 R ; \frac{1}{3}+\frac{2}{3}\right.$; etc. $)$, - a case which would fit our illustration had the number of $R$ females been twice the number of $B$ females.

The ease with which all distributions can be fairly well "fitted" shows beyond a doubt that, even with selection and infertility at work, the final result is largely such as would arise from independent probability, - a fact which goes to show that problems in heredity are essentially statistical problems.

The hopeless tangle in which characters soon become involved through bisexual reproduction shows the utter futility of attempting to infer anything whatever from indivicluals, and the almost mathematical certainty of being able to detect almost any principle or law of descent by careful study of entire populations.

1 For the convenience of the student the formula for expanding a binomial to any power is given here. It is

$$
\begin{aligned}
(A+B)^{n}=A^{n} & +n A^{n-1} B+\frac{n(n-\mathrm{I})}{\mathrm{I} \cdot 2} A^{n-2} B^{2}+\frac{n(n-\mathrm{I})(n-2)}{\mathrm{I} \cdot 2 \cdot 3} A^{n-3} B^{3} \\
& +\frac{n(n-\mathrm{I})(n-2)(n-3)}{\mathrm{I} \cdot 2 \cdot 3 \cdot 4} A^{n-4} B^{4}+\ldots+n \cdot B^{n-1}+B^{n} .
\end{aligned}
$$

This formula gives

$$
\begin{aligned}
&(A+B)^{2}= A^{2}+2 A B+B^{2} \\
&(A+B)^{3}= A^{3}+3 A^{2} B+3 A B^{2}+B^{3} \\
&(A+B)^{4}=A^{4}+4 A^{3} B+6 A^{2} B^{2}+4 A B^{3}+B^{4} \\
&(A+B)^{6}=A^{6}+6 A^{5} B+15 A^{4} B^{2}+20 A^{3} B^{3}+15 A^{2} B^{4}+6 A B^{5}+B^{6} \\
&(A+B)^{8}=A^{8}+8 A^{7} B+28 A^{6} B^{2}+56 A^{5} B^{3}+70 A^{4} B^{4}+56 A^{3} B^{5} \\
&+28 A^{2} B^{6}+S A B^{7}+B^{6}
\end{aligned}
$$

Thus in all cases the coefficients form a series like a symmetrical frequency distribution. If, however, the second term be taken as $2 B$, then the coefficients will be substantially altered, forming a skew.

Karl Pearson has well established the fact that frequency distributions obtained experimentally can often be fitted better by the terms of the binomial $(A+B)^{n}$ when $u$ is not restricted to be a positive integer. In this case the expansion does not terminate, but takes the general form

$$
(A+B)^{n}=A^{n}+n A^{n-1} B+\frac{n(n-\mathrm{I})}{\mathrm{I} \cdot 2} A^{n-2} B^{2}+\frac{n(n-\mathrm{I})(n-2)}{\mathrm{I} \cdot 2 \cdot 3} A^{n-3} B^{3}+\cdots
$$

to infinity, in which the general, or $r$ th, term is

$$
\frac{n(n-\mathrm{I})(n-2) \cdots(n-r+2)}{\mathrm{I} \cdot 2 \cdot 3 \cdots(r-\mathrm{I})} A^{n-2+1} B^{r-1}
$$


Distinctions between inheritance and development. There is still another reason why the true nature of an individual cannot always be detected by appearances, and this is found in the relative development of inherited characters.

For example, suppose that in the illustration given on page $506 B$ and $R$ represent size instead of color. If $B$ represents great size and $R$ extreme smallness, then, by all the principles of inheritance, the $28 B^{6} R^{2}$ of the scheme previously discussed are born for something above the mean size, which would be represented by the $70 B^{4} R^{4}$.

Suppose, however, that through insufficient feed many of these indivicluals fail to deiclop the full size which is their birthright. Such individuals then affear small, like the $B^{2} R^{6}$, or perhaps even the $R^{8}$.

So it comes about that these twenty-eight individuals, though born alike as to tendencies with respect to size, and each represented by formula $B^{6} R^{2}$, are yet very different when examined after diviopment, which of necessity depends upon the conditions of life. And so it is that, owing to differences in development, relative quality as we inf ${ }^{\prime} r$ it from the appearance of the adult is but a rough and often misleading indication of the characters actually present through inheritance. Relative strength of characters as inherited may be known with certainty only through an intimate knowledge of pedigrees. Thus a buyer of an adult animal would have great difficulty in selecting, from appearances only, the individual born with greatest tendency to develop unusual size.

The mathematical nature of descent not due entirely to bisexual reproduction. The truth of this statement we deduce from the fact that offspring asexually produced vary on the same plan as do individuals that are bisexually produced. It is to be inferred also from the further fact, already alluded to, that successite offspring of the same parcnts are not alike, but form a distribution of the same general ontlines as that of the total population.

This shows that the mathematical element in reproduction is to be sought not only in bisexual union, but farther back also in the facts of cell division and the splitting of the chromosomes, - if not indeed in their very constitution. 
What is it that is transmitted? Evolutionary literature abounds in such terms as "tendencies," "reversion," "ancestral bias," and many others which imply an intangible something back of the immediate parent. The common impression of transmission is of something "handed down," or passed on from one generation to the next, and that the visible characters represent the inheritance. It is evident, however, that that which is transmitted is not the character, but rather the elements out of which the character is built up, and that these elements are capable of many and varied combinations.

What these elements may be like, and what the ultimate units of variability may be, - whether chromosomes or some infinitely smaller component, - we do not know. Physiological units have not been discovered, but the laws under which they combine to form characters, and under which the characters combine to form individuals within racial limits, - these have been sufficiently studied to warrant the assumption that they follow essentially the ordinary mathematical principles of permutations and combinations working under the laws of probability. ${ }^{1}$ In other words,

1 By "combinations" is meant the number of different groupings that can be made from a given number of objects without regard to the arrangement of the members. Thus, with $a, b, c, d$, taken three at a time, we may have four combinations, namely, $a b c, a b c l, a c d, b c d$; or, taken two at a time, we have six combinations, namely, $a b, a c, a d, b c, b d, c d$. Each of these combinations may have two or more permutations, depending upon the order in which the numbers stand; thus, the combination $a b c$ is capable of the permutations $a b c, a c b, b a c, b c a$, $c a b, c b a$.

The number of combinations possible with a given number of units depends upon the number taken in each grouping.

The general formula is

$$
{ }^{n} C_{r}=\frac{n(n-1) \cdots(n-r+\mathrm{I})}{\mathrm{I} \cdot 2 \cdot 3 \cdots r},
$$

in which $n$ is the total number and $r$ is the number in each group.

The number of permutations, or different arrangements, possible also depends upon the number in each group. The number of permutations of objects taken two at a time is $n(n-1)$; taken three at a time it is $n(n-1)(n-2)$, and so on; taken $r$ at a time it is therefore $n(n-1)(n-2) \ldots(n-r+1)$.

When all the numbers enter into each permutation, then the formula amounts to the multiplication together of all the natural numbers, from unity up to the number itself; that is, the number of permutations of five letters, $a, b, c, d, e$, is equal to $\mathrm{I} \times 2 \times 3 \times 4 \times 5=120$.

To give an illustration of the probability of an event, if a penny be tossed the odds are even that heads will be up, because there is but one alternative. This 
it is the elements of racial characters that are transmitted, and out of these elements all possible combinations arise. Some combinations are unsuited to the conditions of life and others are relatively or absolutely infertile, making blank spots in the system which otherwise would be mathematically complete and substantially regular.

Even with these omissions, however, the distributions into which characters fall lend themselves to ordinary mathematical methods of study, giving the strongest ground for the confident belief that the laws of heredity will not long continue to be regarded as unexplained mysteries, subject to all sorts of exceptions and reversions, but that characters in descent will be found to observe as well-defined and well-known mathematical principles as do chemical elements in their similar but vastly

probability we express as $\frac{1}{3}$. On the other hand, if a dice be thrown, the chance of any particular side coming up is but $\frac{1}{6}$, for there are six possibilities. If a wager be laid that the number 3 comes up, the odds will be five to one against it, for its chances are one out of six. If two dice are thrown, the chance of a 3 coming up on each at the same time is $\frac{1}{6} \times \frac{1}{6}$, or $\frac{1}{35} ;$ but the chance of two different numbers, as 3 and 4 , coming up together is doubled. This is because the chance of one dice turning up either a 3 or a 4 is not $\frac{1}{6}$, but $\frac{1}{3}$; after which the second dice must supply the proper mate, whose chance is but $\frac{1}{6}$, and $\frac{1}{3} \times \frac{1}{6}=\frac{1}{15}$.

This means that in the long run this event will happen once for every eighteen throws, though it cannot be confidently predicted that it will happen on the eighteenth, the thirty-sixth, or on any other particular throw.

The word "chemistry" has nine different letters. By the principle of permutations just laid down, these nine letters are capable of $1 \times 2 \times 3 \times 4 \times 5 \times 6$ $\times 7 \times 8 \times 9$, or 362,880 , different arrangements, only one of which will spell the word "chemistry." If, therefore, the letters of this word should be tossed into the air and left to fall into a groove and arrange themselves in line by chance, the odds would be 362,879 to $\mathrm{I}$ against the letters taking the proper arrangement to spell the word; but if the tossing should be continued, it is certain that in the long run they would fall into the proper order to spell this particular word. Sooner or later, therefore, if the chance differs from zero, the event is sure to happen; and for this reason nothing is more certain than chance, if only a sufficient number of possibilities be afforded.

The mind is lost in the presence of large numbers, and the judgment is confused when the improbal,le happens, yet that these letters would ultimately spell this word by pure chance is an event certain to take place; not only is this so, but in the long run it will happen once for every 362,880 throws made.

A little careful study of the possible combinations, even of a few elements, and of the certain occurrence of possible, even though improbable, events, will lead the student to work with variables in large numbers with greatly increased confidence. 
simpler combinations. This is only another way of expressing the conviction that before many years - if the present activity in statistical studies continues - the laws of descent will be more accurately known than any other phase of biological science. While the individual will always be an uncertain article, as is bound to be the case where the element of chance is involved, yet this same chance, under the doctrine of probability, becomes one of the best lnown and most dependable principles where sufficiently large numbers are concerned. Because of this, the uncertainty as to individuals gives way to the most definite knowledge as to populations, all of which leads to the inevitable conclusion that the systematic study of groups of individuals is the only reliable way to study heredity, and the only method likely to afford data from which we may safely draw conclusions as to laws of descent.

\section{SECTION XIII - MENDEL'S LAW OF HYBRIDS}

Mendel's law, as it is called from its original discoverer, ${ }^{1}$ arises naturally from the principle outlined in the last section, namely, that characters tend to combine in definite proportions, so that the natural offspring resulting from the mating of two lines of parents with different characters, $B$ and $R$, is of the general form $B^{2}+2 B R+R^{2}$. Mendel's law has special reference to the apparently crossed portion of the population $(B R)$,

1 Gregor Johann Mendel, an Austrian monk, and abbot of Brïnn, was born in $1 S_{22}$ and died in $\mathbf{I} S 8_{4}$. He carried out his breeding experiments - mostly with peas - in the garden of his cloister, publishing the results in the form of a few brief papers in an obscure journal in Brünn, 1853-1865. Partly from the obscurity of the journal, but more from the fact that scientists were interested in totally different lines of study, these papers were practically lost to the scientific world for more than thirty years. U pon the appearance of De Vries' paper announcing the rediscovery and confirmation of Mendel's law and its extension to a great number of cases, two other observers came forward almost simultaneously, and independently described series of experiments fully confirming Mendel's work. Of these papers the first is that of Correns (I900), who repeated Mendel's original experiments with peas having seed of different colors. The second is a long and very valuable memoir of Tschermak, which gives an account of elaborate researches into the results of crossing a number of varieties of Pisum sationm (see Mendel's Principles of Heredity, by Bateson, p. I4). The latter experimenter worked mostly on peas; Correns, on peas and corn (maize); while De Vries worked with many species and a great variety of characters. 
and it aims to predict the character of the offspring when these hybrids are bred together.

Ilow will this hybrid breed? Will it continue "pure," or will it break up into its component parts? This question Mendel's law attempts to answer, and the essence of the law can be stated in two propositions:

I. When crossed forms, or hybrids (BR), are bred together, their offspring will not all resemble the crossed parents, but one fourth, or 25 per cent, will be like the original pure parent $B$, another fourth will be like the other original pure parent $R$, and one half, or 50 per cent, will resemble the crossed forms, so that the offspring of the cross will tend to assume the original general form of $B^{2}+2 B R+R^{2}$. Of these the "pure" individuals will breed as true as to the charactir in question as if their ancestors had never been subjected to crossing; and the 50 per cent of crosses, when bred among themselves, will ayain split up into pure and crossed forms in the proportion of $\mathrm{I}: 2: \mathrm{I}$, so that $B R$ bred with $B R$ will give offspring represented by $B^{2}+2 B R^{2}+R^{2}$ indefinitely. In other words, the offspring of hybrids will not all be hybricls, but they will assume the same general proportions that are assumed when pure forms are allowed to breed together indiscriminately.

If this theory be true, it shows the impossibility of breeding a cross true to its own type, on account of its innate tendency to split up into its origrinal pure or uncrossed forms, - a tendency which has long since been encountered by breeders ambitious to fix a fortunate cross, and which has gone far to convince the popular mind of the difficulty in effecting a real cross.

2. The second fundamental in Menclel's law is the distinction between domincut and recessize characters. If the characters in question were evenly "balanced," and equally discernible, then in a population like $I^{2}+2 B R+R^{2}$ the $I^{2}$ would be clearly defined, say black. The $R^{2}$ would also be clearly defined, say red, and the $2 B R$ would be some kind of blend or mixture of the two; in other words, such a population would be easily assorted into three groups in the proportion of $\mathrm{I}: 2: \mathrm{I}$.

On the other hand, suppose one character to be strong and easily noted, as a red color, or a strong, heavy stem, while the 
other is extremely delicate, as a light shade of blue, easily lost in the red, or a lightness of foliage, easily obscured by a heavy stem. ${ }^{1}$ Now, under circumstances such as these, the less noticeable, or "recessive," characters will be a'sible only' in the indiaiduals that are pure recessia's, all others biing dominated by the more pronounced character. Thus, if $D$ stands for dominant (red petal or strong stem) and $r$ for recessive (light-blue petal or delicate foliage), then the actual distribution would be $D^{2}+$ $2 D r+r^{2}$ as before; but in this distribution three out of four individuals would be distinguished by the dominum character, while the less assertive, the recessive character, would be obscured except in the 25 per cent in which it exists unmixed with the dominant. Hence an individual showing a recessive character may be known at once to be pure, but we cannot tell by looking at individuals showing the dominant character, which are pure and which are mixed. We know, in fact, that we have both forms in the proportion of $\mathrm{I}$ to 2 , but in a case of this kind 75 per cent would afpear to be dominant, while only 25 per cent would appear to be recessives. In reality, 25 per cent are pure dominants, the other 50 per cent being apparently dominants, but actually mixed, - a fact that immediately becomes evident when they are bred among themselves, as they at once give rise to the characteristic distribution, with the proper 25 per cent of pure recessives.

Inasmuch as few pairs of characters are equally balanced and equally able to make themselves evident, it is generally true that any generation from crossed parentage will show 75 per cent dominant (really 25 pure and 50 mixed) and 25 per cent recessire, instead of the typical 25,50 , and 25 that would appear if the characters were equally evident and equally assertive.

Distinction between characters and individuals. The reader will be misled if he takes individuals into consideration here instead of characters. The entire discussion applies to characters taken singly, and when we say of an indizidual arising from hybrid parents that he will "breed pure," we mean only as to

1 It is clear that only characters that are "mutually exclusive" can be used in experimenting on or illustrating this subject. 
the single cluracter in question; for example, hybrids of Jerseys and Shorthorns would not breed pure individuals of either breed, though pure Jersey and pure Shorthorn characters would appear freely.

If we are to consider many characters at once, we may easily satisfy ourselves as to the chances of an absolutely pure individual arising out of a hybrid ancestry.

If one fourth of the population arising from a hybrid ancestry can be said to be pure as to a single character, the question arises as to what proportion of this number can be considered as "pure" with respect to two characters.

From the fact that this second character enjoys the same chances as to purity as did the first, we conclude that one fourth of the number, or one sixteenth of the whole $\left(\begin{array}{cc}1 \\ 4\end{array} \times \begin{array}{l}1 \\ 4\end{array}\right)$, will be pure as to two characters. By the same reasoning we know that $\underset{t}{1} \times \frac{1}{4} \times{ }_{4}^{1}$ carried to any number of terms will express the chance of an individual being pure with respect to the corresponding number of characters. If many characters are involved, therefore, the chances of a pure individual arising out of mixed breeding are exceedingly slight, and our chance of being able to "pick him out" by his appearance is still more remote.

Experimental evidence." It remains now to inquire somewhat carefully into the evidence upon which these propositions are founded.

Mendel's first experiments were conducted with garden peas, and covered the following characters : ${ }^{2}$

I. Differences in form of ripe seeds, - either round and smooth or with shallow wrinkles, or else angular and deeply wrinkled.

2. Differences in the color of seed albumen (endosperm) either pale yellow, bright yellow, orange, or green.

3. Differences in the color of the seed coat, - white, gray, gray b:own, leather brown with or without violet spotting.

4. Differences in form of ripe pod, - whether inflated or constricted between seeds.

${ }^{1}$ For a translation of Mendel's original report, see Bateson, Mendel's Principles of Heredity, pp. 40-103, from which are taken the data herein given.

2 Ibid. pp. 45-46. 
5. Differences in color of unripe pods, - light green, dark green, or vividly yellow.

6. Differences in position of flowers, - whether axial or terminal; that is, whether distributed along the main stem or bunched at the top in a "false umbel."

7. Differences in length of the stcm, - varying from 9 inches to 6 or 7 feet.

It is perfectly easy to see that many of these characters would be dominant over others, the less noticeable being "lost" to view in the hybrid forms. For example, dark green would be dominant over light green and over most shades of yellow; long stems over short ones; and dark colors generally over light ones.

The preponderance of the dominant character over the recessive is so pronounced as to lead Mendel to remark that frequently "one of the parental characters was so preponderant that it was difficult or quite impossible to detect the other in the hybrid." In each of the seven crosses of peas, he adds, "The hybrid character resembles that of one of the parent forms so closely that the other either escapes observation completely or cannot be detected with certainty." 1 Of the characters used, the following were found to be dominant ${ }^{2}$

I. The round or roundish form of seed.

2. The yellow coloring of the endosperm.

3. The gray, gray-brown, or leather-brown color in the seed coat.

4. The inflated over the constricted pod.

5. The green color in the unripe pod.

6. The distribution of flowers along the stem.

7. The greater length of stem. In respect to this point the investigator remarks that stems I foot long crossed with stems 6 feet long gave rise to stems from 6 feet to $7 \frac{1}{2}$ feet long.

The first, or hybrid, generation. Because of the overpowering influence of the dominant characters, the "hybrid," or cross, could not commonly" be distinguished from the "pure" parent possessing the clominant character. This agrees with the experience of breeders generally.

1 Bateson, Mendel's Principles of Heredity, p. 49.

2 Ibid. p. 50. 
The second generation, bred from hybrids. When, however, these hybrids were bred among themselves, the recessive characters came into evidence, constituting in all cases approximately one fourth of the offspring, leaving the other 75 per cent to be apparently dominant, - actually, 25 per cent pure dominant and 50 per cent apparently clominant but really mixed.

Thus, in Experiment I (as to form of seed), from 253 hybrids 7324 seeds were obtained in the second trial year. Among them 5474 were round or roundish and 1850 were angular, - a ratio of 2.96 to $\mathrm{I}$.

In Experiment 2 (as to color of endosperm), 258 crossed plants yielded 6022 yellow and 200 I green, - a ratio of 3.01 to $\mathrm{I}$.

Distribution of characters. In each of these experiments bot/ kinds of sed were usually found in the same pod, showing that the ovule, and not the pod, is the unit. Not only was that the case, but the proportion of three dominants to one recessive held only in the long run, and did not hold for individual plants, as is seen in the following table giving the classification of the offspring of the first ten plants in each experiment. ${ }^{1}$

\begin{tabular}{|c|c|c|c|c|}
\hline \multirow{2}{*}{ Plants } & \multicolumn{2}{|c|}{ EXPERIMENT No. I, Form of SEed } & \multicolumn{2}{|c|}{$\begin{array}{c}\text { EXPERIMENT No. 2, COLOR OF } \\
\text { ENDOSPERM }\end{array}$} \\
\hline & Round & Angular & Yellow & Green \\
\hline I & 45 & 12 & 25 & I I \\
\hline 2 & 27 & 8 & 32 & 7 \\
\hline 3 & 24 & 7 & I 4 & 5 \\
\hline 4 & 19 & 10 & 70 & 27 \\
\hline 5 & 32 & I I & 24 & I3 \\
\hline 6 & 26 & 6 & 20 & 6 \\
\hline 7 & 88 & 24 & 32 & 13 \\
\hline 8 & 22 & I0 & 44 & 9 \\
\hline 9 & 28 & 6 & 50 & 14 \\
\hline 10 & 25 & 7 & 44 & 18 \\
\hline
\end{tabular}

From this it appears that the dominant always exceeds the recessive in number, but that the proportion of 3 to $I$ is

1 Bateson, Mendel's Principles of Heredity, p. 53. 
not maintained in each individual plant. As to whether this comes from difficulties in identifying and classifying doubtful specimens, or from some biological reason, Mendel does not express an opinion, though the point is important.

In other experiments the ratio between the dominants and the recessives was in all cases approximately 3 to I. In Experiment 3 (as to color of seed coats), it was 3.I5 to I ; in Experiment 4 (as to form of pods), it was 2.95 to I ; in Experiment 5 (as to color of unripe pods), it was 2.82 to I ; in Experiment 6 (as to position of flowers), it was 3.14 to I ; and in Experiment 7 (as to length of stem), it was $2.8_{4}$ to $\mathrm{I}$, but the numbers were relatively small $(787$ and 277$)$ as compared with the numbers involved in Experiments $I$ and 2.

The third generation, - second from hybrids. According to Mendel," "those forms which in the first generation maintain the recessive character do not further vary in the second generation as regards this character; they remain constant in their offspring.

"It is otherwise with those which possess the dominant character in the first generation (bred from hybrids). ${ }^{2}$ Of these, two thirds yield offspring which display the dominant and recessive characters in the proportion of 3 to $\mathrm{I}$, and thereby show exactly the same ratio as the hybrid forms, while only one third remains with the dominant character constant." That is to say, of the 75 per cent apparent dominants, one third, or 25 per cent, of the whole breeds pure dominants, showing that this proportion is actually what it appears to be, namely, pure dominants, while two thirds, or 50 per cent of the whole, yield both dominants and recessives in proportion of 3 to $\mathrm{I}$, showing their essentially hybrid or crossed nature, and that their dominance is apparent rather than actual. The separate experiments yielded ratios as follows: ${ }^{3}$

In Experiment I, among 565 plants raised from round seed I93 yielded round seeds only, and remained therefore constant in this character, while 372 gave both round and angular seeds in

1. Bateson, Mendel's Principles of Heredity, p. 55 .

2 That is, with the 75 per cent apparently dominant.

${ }^{3}$ Bateson, Mendel's Principles of Heredity, pp. 55-58. 
the proportion of 3 to $\mathrm{I}$. The number of the hybrids as compared with the constants was therefore 1.93 to) 1 . In Experiment 2 it was 2.13 to 1 ; in Experiments $3-7$ the numbers were small, but approximated the same ratio.

From this Menclel states the following conclusion regarding the offspring of hybrids ${ }^{1}$ (italics his) :

It is now clear that the hybrids form seeds having one or other of the two differentiating characters, and of these one half derelop again the hybrid firm, while the other half yicld plants which remain constant and reciz'e the dominant or recessive characters (respectively) in equal numbers.

This conclusion, which seems to be in accord with his results, is clearly against the possibility of effecting a real cross between characters that behave as do those in question.

Subsequent generations. On this point Mendel says : ${ }^{2}$

The proportion in which the descendants of the hybrids develop and split up in the first and second generations presumably holds good for all subsequent generations. Experiments I and 2 have already been carried through six generations, 3 and 7 through five, and 4,5 , and 6 through four ... and no departure from the rule has been perceptible. The offspring of the hybrids separated in each generation, in the ratio of $2: 1: 1$, into hybrids and constant forms.

That is to say, of the offspring of hybrids one fourth resembled one pure parent and ever afterward bred true with respect to the character in question; one fourth resembled the other and also bred true; and one half still remained hybrid, but its offspring, in its turn, fell apart after the same ratio $I: 2: 1$.

When more than two characters are involved. Menclel conducted investigations with plants differing in a number of characters simultaneously and concludes as follows : ${ }^{3}$

That the offspring of the hylirits in which sereral essentially different characters are combined represent the terms of a series of combinations in whith the de'ielopmental series for each pair of differentiating characters are associated. It is demonstrated at the same time that the relation of each pair of different iharatiss in hybrit union is indipendent of the othir differences in the two original parental stocks.

If $n$ represents the number of the differentiating characters in the true original stocks, $3^{\prime \prime}$ gives the number of timls of the combination series, $4^{\prime \prime}$

1 Bateson, Mendel's Principles of Heredity, p. 57.

${ }^{2}$ Ibid. p. $57 . \quad{ }^{3}$ Ibid. pp. $64-65$. 
the number of individuals which belong to the series, and $2^{\prime \prime}$ the number of unions which remain constant. The series therefore embraces, if the original stocks differ in four characters, $3^{4}=8 \mathrm{I}$ classes, $4^{4}=256$ individuals, and $2^{4}=16$ constant forms; or, which is the same, among each 256 offspring of the hybrids (differing in four characters) there are $\delta_{1}$ different combinations, 16 of which are constant. ${ }^{1}$

All constant combinations which in peas are possible by the combination of the said seven differentiating characters were actually obtained by repeated crossing. Their number is given by $2^{\top}=128$. Thereby is simultaneously given the practical proof that thic constant chataitirs which atpear in the several varieties of a group of plants may be obtained in all the associations which are possible, according to the (muthematical) lav's of comblinations, by means of repeated artificial fertilization.

All this has a distinct bearing upon the question of varieties, and its general trend is that changes effected by crossing tend not to remain constant; that is, that a union of clissimilar characters by this means is practically impossible.

It will be noted that Mendel makes no prediction as to what hybrids or crossed forms will look like, but only as to their "essential constitution" and breeding powers.

Gametic purity. This raises the whole question of gametic purity as the most fundamental question inrolved in Mendel's law. If $B R$, when bred with $B R$, in actual experience produces not more $B R$ 's but rather $B^{2}+2 B R+R^{2}$, then it raises an interesting point as to the real nature of the germs arising from the crossed parents $B R$.

If the characters $B$ and $K$ had made a real union, or blend, in the germ, such a distribution among the offspring would be impossible. The crossed or blended forms would themselves breed true, that is to their own type. If they do not breed true, then we conclude that the real cross, or blend, has not been made, and that in some way the characters $B$ and $R$ must remain distinct in the germinal matter of the mixed parent; that is to say, the distribution of the offspring of hybrid parents into two classes, pure forms and hybrids, instead of one form all hybrids, is possible only upon the assumption that the tiio charactirs remain distinct in the parents and in the germ cells thrown off by them, so that the elements are still capable of uniting under

1 The student may test this formula by making the complete expansion. See Bateson, Mendel's Principles of Heredity, p. 64. 
the law of chance. This means that cach parcnt produces successicely germ cells of both cluracters ( $B$ and $R$ ), so that hybrid forms produce pollen, spermatozoa, ova, etc., of both original kinds, which thereafter combine by the law of chance; that is, $B$ of one parent unites with either $B$ or $R$ of the other, producing either pure $I{ }^{\prime}$ 's or $B R$ 's; and also, in a large number of instances, some $R$ 's unite with $R^{\prime}$ 's, producing hybrids, and others with $R$ 's, producing pure $R$ 's from hybrid parents. This is the theory of gametic ${ }^{1}$ purity, an assumption necessarily involved as a fundamental conception in Mendel's law, which, in the opinion of Mendel himself, applies only to characters that do not blend.

Proving or disproving Mendel's law. A good many investigators are trying, often with numbers inadequately small, to prove or disprove Mendel's law as a general principle of heredity. It may not be out of place to call the attention of the student to the uselessness of this attempt, and to direct his attention to the problems connected with this question which really call for further and much-extended study.

First of all, Mendel's law as a general proposition needs no further proof. The experiments on which it was founded have been carefully repeated by De Vries, by Correns, and by Tschermak, who agree as to the correctness of his results. They have been repeated upon many other species, with uniform results in most cases, and no new evidence is worth noting that is not founded on new species and that does not involve relatively large numbers.

Besides all this, the fundamental conception of Mendel's law rests, like Galton's law of ancestral heredity, upon the inevitable mathematical relations in reproduction as outlined in the previous section. This "law" arises, therefore, as a special case out of

1 The term "gamete" is coming to be used, as synonymous with "germ cell," to mean the unfertilized germ, without regard to sex. When it is fertilized it is spoken of as a zygote.

Thus, in the language of these terms, we should say that each hybrid parent produces two kinds of gametes, $B$ and $R$, or dominant and recessive, or whatever characters have been combined. Now some gametes of the $B$ kind will unite with gametes $B$, producing zygotes $B B$ (pure); others will unite with $R$ gametes, producing zygotes $B R$ (crossed); and still other $R$ 'gametes will unite with $R$ gametes, producing zygotes $R R$ (also pure). Of mathematical necessity, for an entire population these proportions will be as I $B B: 2 B R:$ I $R R$. 
the general principle that the binomial coefficients represent populations in general. If there is no blend, then the coefficients represent the proportions of distinct character elements. If a blend has occurred, then the coefficients still represent the proportions within the blend. There is and can be no uncertainty upon this feature of the case. The dominance of some characters over others is a matter of common observation and may be accepted as a matter of common sense.

The feature of Mendelism on which further light is needed is the matter of gametic purity, a biological element of the problem whose universal truth is not yet established, but which seems necessary to a rational explanation of the law as to separation into distinct forms ; indeed, Mendelism in its present form seems to mean substantially that hybrid individuals do not produce hybrid germs, but rather that they produce successively the pure germs of both lines of parentage.

It may be considered that Mendel's law is well established for certain characters; that is, for characters that blend. This may be, however, only another way of admitting its truth for those species which maintain gametic purity - those in whose germs the different characters do not mix (blend), or in which, if they do mix, the process is very slow. This in turn is another way of saying that Mendel's law is a demonstrated fact, the only unanswered question being, To what species and characters does it apply? and the answer to this depends upon the extent of gametic purity. In the opinion of the writer this is the great unanswered question, and here is the object of inquiry toward which all investigation of Mendel's law should be directed, namely, to discover to what species and to what characters its applications are limited.

Upon this point it is worth while to note that crossed forms fall into one of three classes, so far as afpearances are concerned : (I) the hybrid may resemble one of its "pure" parents so closely as to be indistinguishable from it ; (2) it may be a kind of intermediate between the two (different) parents; (3) it may be quite distinct from either parent.

Of these three classes the first is clearly Mendelian, while the second and third are doubtful, - the second exceedingly so. 
Both the second and third, especially the former, suggest a blend, and there is much in the experience of breeders to indicate that certain characters do blend, making a successful union, entirely against Mendel's law, whose operations indicate the non-formation of stable hybrids.

The writer ventures to urge, therefore, not the attempt to prove or disprove this great principle, but the endeavor to learn and define the limits of its action. That it applies to crosses generally there is the greatest reason to believe, and to its general truth many a breeder can testify, when he has seen some of his favorite productions revert to their original forms before his very eyes.

Experiments in crossing Japanese waltzing mice with albinos. ${ }^{1}$ Darbishire conducted extensive experiments with this cross, producing thousands of individuals, which are fully classified in the original, to which reference is here made. It is more work of this kind that is needed. Space forbids giving more than a brief outline of some of the more characteristic conclusions of the experimenter:

I. When the race of waltzing mice is crossed with albino mice which do not waltz, the waltzing habit disappears in the resulting young, so that waltzing is completely recessive in Mendel's sense; the eye color of the hybrids is always dark, the coat color is variable, generally a mixture of wild gray and white, - the character of the coat being distinctly correlated with characters transmitted both by the albino and by the colored parent. There is thus no proper dominance in Mendel's sense, so far as eye color and coat color are concerned, the hybrids differing always in age, color, and generally in coat color, from both parents.

2. When the hybrids produced from the cross described are paired together, the resultant young exhibit a segregation into three groups so far as eye color and coat color are concerned, into two so far as regards the waltzing habit. The phenomenon of segregation is closely similar to that described by Mendel; and in color, whether of eyes or of fur, the proportions are closely identical with those observed by him, - a quarter of the young resembling their albino grandparents, half representing their hybrid parents, and a quarter resembling their waltzing grandparents in so far that they have pink eyes and the same-colored fur, but differing from any of their immediate ancestors in the range of coat color exhibited. The proportion of individuals which cxhibit the waltzing habit is less than one fifth of the whole number of young, and is not a Mendelian proportion.

${ }^{1}$ Biometrika, Vol. III, Part I, pp. I-51. 
3. When hybrids are paired with albinos, half the young produced resemble their albino parent, half resemble their hybrid parent. This result is in accord with Mendel's theory.

Darbishire adds: "There is no eridence that any individuals which could be properly described as 'pure dominants' or 'pure recessives ' exist among the whole series produced."

The reciprocal cross. In Mendel's experiments generally reciprocal crosses, according to all accounts, gave identical results. We know that as a general principle this does not always hold. For example, the common mule, which is the product of the male ass and the female horse, is said to be quite different from its reciprocal cross, the hinny, which is the product of the male horse and the female ass. The one is valuable, while all horsemen seem to agree that the other is lazy and worthless. How much of this is fact and how much is tradition is doubtless somewhat uncertain.

\section{SECTION XIV - THE IAW OF ANCESTRAL HEREDITY}

We have abundant evidence that in a large sense the offspring is the product of something more than the immediate parents. The fact of resemblance to ancestors more remote than the parents, and the additional fact that successive offspring of the same parents are not alike but form an array not very different from that of offspring in general, - these facts alone show us either that the ancestors beyond the parents contribute something to the offspring, or - which is the same thing - that characters are made up out of elements that are handed down from parent to offspring and from which many and various combinations are possible in succeeding generations and even in the same generation.

The interesting question now arises, How are these hichditary infucues back of an indiridual distributed among his ancestors of iarious degrees of consanguinity, from his immediate parents backward?

Manifestly the answer to this question is beset with many difficulties. We could secure a rough approximation by working out the coefficient of heredity between offspring and parent, 
between offspringr and grandparent, and so on indefinitely; but it would need to be done for each character separately. This involves immense labor, and, moreover, we do not ordinarily possess sufficiently accurate information about ancestors back of the parent to enable us to make such calculations. We seek, therefore, some expression for this relation, and such an expression when generalized would constitute a law of ancestral heredity.

\section{How much influence belongs to each separate ancestor? If} inheritance is from the race, or in a more particular sense from the family group, then, in practical breeding affairs, we need a measure of the influence of each ancestor in order to know how much importance to attach to the parent and how much to attach to the several ancestors back of the parent.

One generation back the total heritage rested in the two immediate parents, and, roughly speaking, is to be regarded as divisible equally between them. Two generations back it rested in four grandparents, and, again waiving considerations of prepotency, one fourth of the heritage came from each. The third generation back the heritage was divided among eight greatgrandparents, presumably, on the average, equally, - that is, one eighth coming from each. Still another remove, and no less than sixteen great-great-grandparents contributed to the stream that made the final heritage, and it is fair to assume that each individual contributed its share, namely one sixteenth.

Now these same sixteen individuals have contributed also to the production of many other lines of descent. If we had all the descendants of these sixteen great-great-grandparents of the particular individual we have in mind, they would constitute a large population, and we have no knowledge as to where, in their frequency distribution with respect to the character in question, our special individual might be found. But of all the descendants of these sixteen great-great-grandparents only eight of the next generation contributed to the production of the individual we have specially in mind; again, in the next generation, only four have so contributed, and, last of all, out of the large population descended from these sixteen ancestors, two only have produced our individual. Now the law of ancestral heredity is designed 
to enable us to predict what, on the average, should be the product of this selected ancestry.

Inheritance complex. The heritage from the parent is not therefore a simple thing, but rather a complex stream, or, more properly, two streams that meet from different directions, each made up of currents contributed from many tributaries. How much now has each tributary (ancestor) contributed to the general and composite mixture that we call the heritage?

Galton ${ }^{1}$ macle the first attempt to answer this question, and announced as the law of ancestral heredity that the two immediate parents contributed between them one half (0.5) of the effective heritage, the grandparents one fourth $(0.5)^{2}$, the greatgrandparents one eighth $(0.5)^{3}$, and so on, so that the effective contributions of the successive generations would be represented by the fractions $\frac{1}{2}, \frac{1}{4}, \frac{1}{8}, \frac{1}{16}$, etc., and the total heritage would be represented by the sum of these fractions, which, extended to infinity, would equal $\mathrm{I}$, thus accounting for the total heritage.

This general law applies to gencrations, not to individual ancestors, and these fractions should be still again divicled by the number of ancestors in each generation in order to determine the fractional share contributed by each individual ancestor. The following table exhibits the fractional contribution of each generation and of each individual ancestor according to the law of ancestral heredity as stated by Galton.

Effective Heritage contributed by Each Generation and by Each Separate Ancestor according to the Law of Ancestral Heredity as stated by Galton

\begin{tabular}{|c|c|c|c|}
\hline $\begin{array}{l}\text { Generation } \\
\text { Backiward }\end{array}$ & $\begin{array}{c}\text { Effective Contribution } \\
\text { of Each Generation }\end{array}$ & $\begin{array}{l}\text { NUMBer of ANCES- } \\
\text { TORS INVOLVED }\end{array}$ & $\begin{array}{c}\text { EFFeCtive Contribution } \\
\text { OF Each AnCESTOR }\end{array}$ \\
\hline I & $\frac{1}{2}$ or 0.5 & 2 & $\frac{1}{4}$ or $25.0 \%$ \\
\hline 2 & $\frac{7}{4}$ or $(0.5)^{2}$ & 4 & $\frac{1}{15}$ or $6.25 \%$ \\
\hline 3 & $\frac{1}{8}$ or $(0.5)^{3}$ & 8 & $\frac{1}{67}$ or $1.56+\%$ \\
\hline 4 & $\frac{1}{15}$ or $(0.5)^{4}$ & 16 & $\frac{1}{5}$ or $0.39+\%$ \\
\hline 5 & $\frac{1}{32}$ or $(0.5)^{5}$ & 32 & 1021 or $0.09+\%$ \\
\hline
\end{tabular}

${ }^{1}$ Galton, Natural Inheritance, pp. 134-137; Procedings of the Royal Society', LXI, 402 . 
This series $\left(\frac{1}{2}, \frac{1}{4}, \frac{1}{4}, \frac{1}{1}\right.$, etc.) carried to infinity would account for the total heritage, and if these fractions are correctly taken the influence of each separate ancestor would, on the arerage, be represented by the fractions in the last column.

This "law," at first somewhat arbitrarily derived, and announced with considerable hesitation, has received general support from later investigations, and all researches, mathematical as well as otherwise, tend to establish its substantial accuracy. The first announcement was based upon studies in stature. Somewhat later opportunity was afforded to make exhaustive studies from a large number of Basset hounds of distinct colors and of several generations of known ancestry. This study is reported in full by Galton, ${ }^{1}$ and the results conform substantially to the "law," which, as Galton observes, is "strictly consonant with the observed binary subdivisions of the grerm cells, and the concomitant extrusion and loss of one half of the several contributions from each of the tivo parents to the germ cell of the offspring. ${ }^{2}$

These Basset hounds were especially favorable for a study of this kind. They are of two colors only, "lemon and white," or they may be marked in addition with a third color (black), in which case they are known as tricolor.

It is said that individuals are distinctly of one or the other class, and that transitional specimens are very rare. The pecligrees and color descriptions had been carefully kept by Sir Everett Millais, who had originated the particular stock.

Some $816^{3}$ hounds of known color were descended from parents of known color; in $567^{3}$ cases the colors of the granclparents also were known, and in $\mathrm{I} \& 8$ cases the colors were known for three generations back. Assigning fractional values to parents, grandparents, etc., according to Galton's law of ancestral heredity, and calculating what the descendants should be under the law, it appeared that, according to theory, there should have been Iso tricolor hounds descended from those whose ancestors were known for three generations. In fact, i 8 I such

1 Proceedings of the Royal Society, LXI, 40I-4I2.

2 Ibid. p. 403 .

${ }^{3}$ Not $8 \mathrm{I} 7$ and 577 , as printed. There seems to be an error of $\mathrm{I}$ in the first row. Proceedings of the Royal Society, LX, 409. 
individuals were found, thus furnishing additional evidence at the remarkable agreement between theory and fact in matters of breeding, and tending strongly to establish this law.

All things considered, the fraction seems to be fairly well established as the ratio of the intensity with which, on the average, characters are transmitted at the several matings in bisexual reproduction; and if this be so, the law as stated by Galton may be accepted as substantially correct, especially when we recall the fact that this $\left(\frac{1}{2}+\frac{1}{4}+\frac{1}{8}+\frac{1}{16} \ldots\right.$ to infinity) is the only infinite series whose ratio is $\frac{1}{2}$ and that will add up to unity, thus exactly accounting for the full heritage.

Pearson's method. ${ }^{1}$ Pearson has treated the same problem by somewhat more complete mathematical methods, and arrives at a somewhat more general result, but a result which may be said to agree substantially with that of Galton. He begins by dealing with the question of biparntal inheritance. Then by extending the results obtained he accounts for the total heritage from each generation of back ancestry, taking into consideration the arriability of the cutire population to which each generation of the ancestry belongs; that is, he considers the arriability of the separate generations of ancestors, - a factor which Galton did not take into account.

Biparental inheritance. Starting out with the question of biparental inheritance, the problem, stated in general terms, is as follows: What, on the ar'erges, is the character of offspring of fathers whose deviation from the mean of fathers in general is $h_{1}$, mated with mothers whose deviation from mothers in general is $h_{2} ?^{2}$

In the discussion of this problem let the deviation of this offspring from offspring in general be $h_{3}$, and its standard deviation (in its own array) be denoted by $\Sigma$.

1 Proceedings of the Royal Society, LII, I898, 386-41 2 ; also Pearson, Grammar of Science, pp. 468-4SI.

2 Stated in concrete terms, What is the height, on the average, of the children from those fathers who are, for example, two inches above the average height of fathers, mated with mothers who are one and a half inches below the average height of mothers? In discussions of this kind the student should remember that males and females differ naturally in character valuations, and also that not all males become fathers nor all females become mothers, so that the race descends not from all but from a portion only of the preceding generations. 
As Pearson says, what now are $h_{3}$ and $\Sigma$; that is, what deviation from the mean of offspring in general $\left(h_{3}\right)$ is to be expected in the offspring of these particular parents, and what is their variability or standard deviation with respect to their own mean ( $\Sigma)$ ? Cast in still more general terms, the questions are these: How will the offspring from selected parents differ from offspring in general, and how will they differ among themselves?

Now these are fundamental questions in breeding, and their answer involves the following additional conceptions, all with reference to the character in question:

I. The standard deviation for fathers in general $\left(\sigma_{1}\right)$.

2. The standard deviation for mothers in general $\left(\sigma_{2}\right)$.

3. The standard deviation for offspring in general $\left(\sigma_{3}\right)$.

4. The coefficient of heredity between fathers and offspring reckoned as sons $\left(r_{1}\right)$.

5. The coefficient of heredity between mothers and offspring, also reckoned as sons $\left(r_{2}\right)$.

6. The coefficient of correlation (cross heredity) between fathers and mothers, due to assortative mating $\left(r_{3}\right)$.

Galton consiclered that inheritance from two parents is substantially equivalent to inheritance from a "micl-parent," which should be the mean of the two after transmuting the female values (measurements, for example) into male equivalcuts by multiplying those values by the ratio of the male to the fomale mean, for the character in question.

Pearson, on the other hand, deals first with deviations, and by the deviations of the parents from parents in general he attempts to predict the deviation of their particular offspring from the mean of offspring in general, which is the same as saying "from the mean of the race."

While Galton thus artificially built up a mid-parent to take the place of the two parents, Pearson developed first the theory of "biparental inheritance," taking into account the means and variabilities of the parents, the coefficient of assortative mating, and the coefficient of correlation between offspring and parents, thus leading to the following formula for biparental inheritance : 


$$
h_{3}=\frac{r_{1} \sigma_{3}}{\left(\mathrm{I}+r_{3}\right) \sigma_{1}}\left(h_{1}+\frac{\sigma_{1}}{\sigma_{2}} h_{2}\right)^{*} \text {. }
$$

Where $h_{1}$ and $h_{2}$ are the deviations of parents from the mean of parents for the character in question, $h_{3} \dagger$ is the deviation of the offspring of these parents from offspring in general, $\sigma_{1}$ is the standard deviation of fathers from the mean of their generation, $\sigma_{2}$ is the standard deviation of mothers from the mean of their generation, $\sigma_{3}$ is the standard deviation of offspring in general as to the character in question, $r_{1}$ is the coefficient of heredity between offspring and parents (parents being taken as equipotent, that is, making $\left.r_{2}=r_{1}\right), r_{3}$ is the coefficient of assortative mating.

Now formula (I) may be written as follows:

$$
\frac{\mathrm{I}}{2}\left(h_{1}+\frac{\sigma_{1}}{\sigma_{2}} h_{2}\right)\left(\frac{2 r_{1} \sigma_{3}}{\left(\mathrm{I}+r_{3}\right) \sigma_{1}}\right) ;
$$

or, again, it may be written

$$
\frac{\mathbf{I}}{2}\left(h_{1}+\frac{\sigma_{1}}{\sigma_{2}} h_{2}\right)\left(\frac{\sqrt{2}}{\sigma_{1} \sqrt{\mathrm{I}+r_{3}}}\right)\left(\frac{r_{1} \sqrt{2}}{\sqrt{\mathrm{I}+r_{3}}}\right) \sigma_{3} .
$$

In this form the formula is made up of four factors. Of these the first represents the mid-parental deviation; the second, the variability of mid-parents; the third, the correlation coefficient between mid-parent and offspring; and the fourth, the standard deviation for this particular character. If, now, each of these factors (except $\sigma_{3}$ ) be represented by a single letter, we have the following:

$$
\begin{aligned}
& H=\frac{\mathrm{I}}{2}\left(h_{1}+\frac{\sigma_{1}}{\sigma_{2}} h_{2}\right)=\text { the mid-parental deviation, } \\
& S_{+}^{+}=\frac{\sigma_{1} \sqrt{\mathrm{I}+r_{3}}}{\sqrt{2}}=\text { the variability of mid-parents, } \\
& R=\frac{r_{1} \sqrt{2}}{\sqrt{\mathrm{I}+r_{3}}}=\begin{array}{c}
\text { correlation coefficient between mid-parent } \\
\text { and offspring. }
\end{array}
\end{aligned}
$$

* For derivation of this formula, see Appendix.

$\dagger$ The offspring will, of course, form an array, and the $h_{3}$ is the deviation of its mean from the mean of offspring in general.

$\ddagger$ This last expression is inverted because we desire to use $S$ in the denominator. 
We are now able to put formula (I) in the form of a regression equation, giving the value $h_{3}$ as follows:

$$
\iota_{3}=R \frac{\sigma_{3}}{S} H
$$

which is the deviation of this special population from the mean of the race. ${ }^{1}$

This form of expression of formula ( $I$ ) has the advantage of simplicity. Instead of the deviations $\left(h_{1}\right.$ and $\left.h_{l_{2}}\right)$ of two parents with variabilities $\sigma_{1}$ and $\sigma_{2}$, we now have the deviation $(H)$ of a single artificial mid-parent made by first transmuting female deviations into male values by multiplying by the ratio of male to female variabilities for the character in question and then taking the mean of the male and the transmuted female values. ${ }^{2}$ This is Pearson's mid-parental deviation $(H)$.

$S$ is the portion of this formula which involves the variability of paruts, for it depends upon $\sigma_{1}$ and upon the coefficient of assortative mating $\left(r_{3}\right)$, and when associated with $H$ as it is in the formula it may be looked upon as expressing the variability of the mid-parent.

Likewise $R$ is the portion of the formula which involves the correlation between parent and offspring, and from the form of equation (4) it may be looked upon as the coefficient of correlation between offspring and mid-parent.

If we neglect the coefficient of assortative mating, making $r_{3}=0$, the following conclusions may be drawn from formulas (3) and (4), and the values of $R$ and $S$ :

I. The variability of the mid-parent $(S)$ is equal to that of fathers divided by $\sqrt{2}$.

I Experimental determinations show that for most characters thus far investigated the regression coefficient of offspring as compared with mid-parents is about 0.6 , so that we may write, in general, $h_{3}=0.6 \mathrm{H}$; or, in other words, if a mid-parent deviates a certain amount the offspring may be expected in general to deviate 0.6 of that amount from the mean of the race.

2 This is the $\frac{\mathrm{I}}{2}\left(h_{1}+\frac{\sigma_{1}}{\sigma_{2}} h_{2}\right)=H$ of the formula.

* That is, in $S=\frac{\sigma_{1} \sqrt{1+r_{3}}}{\sqrt{2}}$, if assortative mating be disregarded, $\gamma_{3}$ becomes zero and the formula becomes $\frac{\sigma_{1} \sqrt{1}}{\sqrt{2}}$; but $\sqrt{\mathrm{I}}=\mathrm{I}$, and we have $\frac{\sigma_{1}}{\sqrt{2}}$. 
2. The correlation of sons with respect to mid-parents $(R)$ is equal to that of sons with respect to fathers multiplied lyy $\sqrt{2}$. *

We have answered the question as to the value of $h_{3}$. It remains to answer the question as to the value of $\Sigma$, the variability (standard deviation) of the array of offspring from the particular parents of deviations $h_{1}$ and $h_{2}$. If we assume as before that $r_{1}=r_{2}$, then

$$
\Sigma=\sigma_{i:} \sqrt{\mathrm{I}-\frac{2 r_{1}^{2}}{\mathrm{I}+r_{3}}} .
$$

The fuller treatment of the meaning of this formula will be taken up in a succeeding section on "Selection."

We could now proceed to form a mid-grandparent in the same way by transmuting female values into their male equivalents by multiplying by the ratio of male to female standard deriations. Having four grandparents, we take the mean of the four values thus obtained for our mid-grandparent. Similarly this could be carried back to any number of generations, and we should thus derive a mid-parent for the first, second, third, etc., generations of ancestry. These can be conveniently referred to as the first, second, third, etc., mid-parents of an offspring.

Formula for ancestral heredity. In terms of these mid-parents and their variabilities Pearson has stated, in modified and generalized form, Galton's law of ancestral heredity as follows :

$$
h=\frac{\mathrm{I}}{2} \frac{\sigma}{\sigma_{1}} H_{1}+\frac{\mathrm{I}}{4} \frac{\sigma}{\sigma_{2}} H_{2}+\frac{\mathrm{I}}{8} \frac{\sigma}{\sigma_{3}} H_{3}+\cdots+\frac{\mathrm{I}}{2^{n}} \frac{\sigma}{\sigma_{n}} H_{n}+\cdots,
$$

in which $h$ is the deviation from the mean of offspring in general to be expected in offspring of mid-parents of successive generations backward whose deviations were $H_{1}, H_{2}, H_{3}, \cdots, H_{n} ; \sigma$ is the standard deriation of offspring in general [the $\sigma_{3}$ of formulas (I) to (4) ]; and $\sigma_{1}, \sigma_{2}, \sigma_{3}, \ldots, \sigma_{n}$, etc., are the standard deviations of the mid-parents of successive generations of ancestry.

It may be noted from this formula that if we take no account of differences of variability in successive generations $\left(\sigma=\sigma_{1}=\sigma_{2}=\ldots\right)$, and make the deviations of successive midparents equal $\left(H_{1}=H_{2}=H_{3}=\cdots\right)$, we obtain Galton's series,

* That is, in $R=\frac{r_{1} \sqrt{2}}{\sqrt{1+r_{3}}}$, if $r_{3}$ be disregarded, the formula becomes $\frac{r_{1} \sqrt{2}}{\sqrt{1}}=r_{1} \sqrt{2}$. 
$\frac{1}{2}, \frac{1}{4}, \frac{1}{4}, \frac{1}{16 ;}, \cdots$, by which he accounts for the total heritage. This fractional influence of the different generations, therefore, may be accepted as the best general statement possible of the law of ancestral heredity. The influence of individual ancestors, waiving all considerations of special prepotency, would be found by dividing these fractions by the number of ancestors of that generation ( ${ }_{0}^{1}$ by $S={ }_{6}^{1}$ for great-grandparents). (See table, page 527 , for an extended statement of the fractional influence of generations and separate ancestors.)

The variability of the offspring of an ancestry selected for an indefinitely large number of generations back is given by a formula which is merely an extension of the formula for the variability of the offspring of two selected parents. If we assume Galton's coefficients in the law of ancestral heredity, the formula for the general case may be written as follows:

$$
\Sigma^{2}=\sigma^{2}\left\{1-\frac{r_{1}}{2 \sqrt{2}}-\frac{r_{2}}{(2 \sqrt{2})^{2}}-\frac{r_{3}}{(2 \sqrt{2})^{3}}-\cdots-\frac{r_{n}}{(2 \sqrt{2})^{n}}-\cdots\right\}^{1},
$$

in which $r_{1}, r_{2}, r_{3}, \cdots, r_{n}$, are the coefficients of correlation between offspring and the first, second, third, ..., $n$th midparents. Use will be made of this formula in treating of the reduction of variability by selection.

\section{SECTION XV-LIMIT TO THE REDUCTION OF VARIABILITY}

We often speak of "fixing" the type by selection, meaning by that the reduction of variability. All recent studies, however, go to show that we do not greatly reduce variability by selection, however much we alter the type.

In the records of corn breeding it will be remembered that, while the protein and oil contents rapidly responded to selection, yet the cocfficionts of arriability changed but little ${ }^{2}$ indeed, it is the experience everywhere that variability is but slightly reduced by selection.

This experience accords with mathematical theory. It will be shown in the Appendix that, in general, the variability of an array is obtained from the standard deviation of offspring in

1 See Pearson, Grammar of Science, p. $482 . \quad 2$ See table, p. 446. 
general by multiplying this standard deviation by $\sqrt{\mathrm{I}-r_{1}^{2}}$; that is, in symbolic language, $\Sigma=\sigma_{3} \sqrt{ }+-r_{1}^{2}$ gives the variability (standard cleviation) of an array of offspring whose correlation with the selected parent is $r_{1}$, and in which the rariability of offspring in general is $\sigma_{3}$.

The numerical value of this variability in a given instance depends upon the value of $r$. Now experimental evidence shows that the correlation between parent and offspring ranges all the way from 0.3 , with little or no assortative mating, up to about 0.5 , with the highest selection of both parents that has yet been achieved (see table of coefficients of heredity, page 488 ,

Now in our formula $\sum=\sigma_{3} \sqrt{I-r_{1}^{2}}$ let us substitute these values :

When $r_{1}=0.3, \sum=\sigma_{3} \sqrt{\mathrm{I}-0.09}=0.9539 \sigma_{3}$; that is, in this case, when one parent is selected we get an offspring only about 5 per cent less variable than the offspring in general.

We have already seen (page 533) that when two parents are selected, assuming them to be equipotent, the formula for the variability of the offspring of selected parents is $\Sigma=\sigma_{3} \sqrt{1-\frac{2 r_{1}^{2}}{I+r_{3}}}$.

Let us now make the same assumption as before; namely, take $r_{1}$ first as 0.3 for pangamic mating, and again as 0.5 for the case of perfect assortative mating.

I. If $r_{1}=0.3$ and $r_{3}=0$, then $\Sigma=\sigma_{3} \sqrt{\mathrm{I}-\frac{2 r_{1}^{2}}{\mathrm{I}+r_{3}}}$ becomes $\sigma_{3} \sqrt{\mathrm{I}-\frac{\mathrm{O}-\mathrm{I} 8}{\mathrm{I}+\mathrm{O}}}=\sigma_{3} \sqrt{\mathrm{O} .82}=0.9055 \sigma_{3}$, which means that the selection of both parents out of a race dereloped by pangamic mating will result in the reduction of variability by only about IO per cent.

2. If $r_{1}=0.5$ and $r_{3}=\mathrm{I}$, - that is, with perfect assortative mating and with the highest correlation found in highly bred races, -

becomes

$$
\Sigma=\sigma_{3} \sqrt{\mathrm{I}-\frac{2 r_{1}^{2}}{\mathrm{I}+r_{3}}}
$$

$$
\Sigma=\sigma_{3} \sqrt{\mathrm{I}-\frac{0.5}{2}}=\sigma_{3} \sqrt{\mathrm{I}-0.25}=0.8662 \sigma_{3}
$$


all of which means that the closest selection of both parents (perfect assortative mating) cannot result in the reduction of variability by more than about I 3 per cent.

Moreover, if the entire back ancestry be selected, the variability will not be much reduced below this point. In connection with the law of ancestral heredity (page 534) we gave a formula for the variability of the offspring of an ancestral line selected back for an indefinitely large number of generations. This formula is

$\Sigma^{-1}=\sigma^{2}\left\{\mathrm{I}-\frac{r_{1}}{2 \sqrt{2}}-\frac{r_{3}}{(2 \sqrt{2})^{2}}-\frac{r_{3}}{(2 \sqrt{2})^{3}}-\cdots-\frac{r_{n}}{(2 \sqrt{2})^{n}}-\cdots\right\},(\mathrm{I})$ in which $\Sigma$ is the variability of the offspring of this selected ancestry, $\sigma$ is the variability of offspring in general for the population from which selection is made, and $r_{1}, r_{2}, r_{3}, \cdots, r_{n}$ are the correlation coefficients of offspring and first, second, third, ...., nth mid-parents.

For pangamic mating, $r_{1}, r_{2}, r_{3}, \cdots, r_{n}$ may be taken as

$$
\frac{0.6}{\sqrt{2}}, \frac{0.6}{(\sqrt{2})^{2}}, \frac{0.6}{(\sqrt{2})^{3}}, \cdots, \frac{0.6}{(\sqrt{2})^{n}}, \cdots
$$

Substituting these values in (I), we get

$$
\sum^{2}=
$$

$$
\begin{aligned}
& \sigma^{2}\left\{1-\frac{0.6}{2^{2}}-\frac{0.6}{2(2 \sqrt{2})^{2}}-\frac{0.6}{(\sqrt{2})^{3}(2 \sqrt{2})^{3}}-\cdots-\frac{0.6}{(\sqrt{2})^{n}(2 \sqrt{2})^{n}}-\cdots\right\} \\
& =\sigma^{2}\left\{I-0.6\left(\frac{1}{4}+\frac{1}{4^{2}}+\frac{1}{4^{3}}+\cdots+\frac{1}{4^{n}}+\cdots\right)\right\}^{*} \\
& =\sigma^{2}\left\{1-0.6\left(\frac{1}{3}\right)\right\} \\
& =0.8 \sigma^{2} . \\
& \Sigma=\sigma \sqrt{0.8}=0.8944 \sigma,
\end{aligned}
$$

which means that in the case of pangamic mating the variability is reduced only about II per cent by selecting the entire ancestry.

Basing his remarks on these facts, Pearson says that the Io to I 3 per cent reduction obtained by the selection of two

* The series $\frac{\mathrm{I}}{4}+\frac{\mathrm{I}}{4^{2}}+\frac{\mathrm{I}}{4^{3}}+\cdots$ to infinity is a geometrical progression whose sum is found in the usual manner by dividing the first term by $x$ minus the ratio. 
parents is "almost the limit of the reduction of variability, even if the whole back ancestry be selected." He remarks, of course, that the new variability is from the new type, not the unselected type; but, he adds, "continnons sclection does not indefinitcly" modify variability, however much it shifts the type." 1

The principal function of selection, therefore, is to alter the type, not to riduce sariability, and the facts here cited show the inherent impossibility of "fixing" the type in the sense that individuals will not depart much from it. But, on the other hand, the same principle assures us that, however much we improve by shifting the type, there always remains sufficient variability for still further selection, and as long as variability remains there is hope and possibility for still further improvement. We may therefore fix the type by unchanging standards of selection, in the sense that it will remain stationary and not shift, but we cannot "fix" it in the sense of reducing to any great extent the proportion of individuals that will deviate from it. ${ }^{2}$

\section{SECTION XVI - POIWER OF SELECTION TO PERMA- NENTLY MODIFY TYPES BY THE ESTABLISH- MENT OF BREEDS}

Though selection cannot greatly reduce variability, it is yet immensely powerful in shifting the type, as has been shown, and, if long continued, in so establishing the new type that it will breed true thereafter without selection, ${ }^{3}$ as will now be shown.

This will necessitate a variety of assumptions as to the ancestry back of the parent, according as our knowledge of its character is much or little, and according as it may be assumed

1 Pearson, Grammar of Science, pp. 458, 472-485. (Italics are mine.)

2 Ibid. pp. $4 \mathrm{SI}-485$.

3 "Without selection" here means absolute freedom from the influence of all laws but those of chance. In practice we never realize these conditions, so that it is always necessary to use enough systematic selection to offset the effects of that degree of natural selection which is found to be always at work in nature everywhere. What is meant is, that by continued selection we soon reach a point at which the inherent variability of the race is powerless of itself to alter the type. 
to be mediocre on the one hand or something more than mediocre on the other.

Assuming mediocrity beyond some definite point in the back ancestry. Galton's form of the law of ancestral heredity may be written

$$
h=\frac{1}{2} H_{1}+\frac{1}{4} H_{2}+\frac{1}{8} H_{3}+\cdots
$$

to infinity, in which $/ 2$ has the meaning defined on page 533 .

If the variabilities of successive generations be taken as equal, the fuller statement of this law as given on page 533 reduces at once to this simple form.

As the simpler form of statement gives a good approximate value, we shall, for the sake of simplicity and elegance of results, be content here to investigate what grows out of this law in the way of establishing a character for which selection and breeding are being carried on.

I. If we assume mediocrity back of the immediate parents, we must make

$$
H_{2}=H_{3}=H_{4}=\cdots=\mathrm{o} .
$$

Then

$$
h=0.5 H_{1}
$$

that is, one half the desired character is present in the offspring.

2. If we assume mediocrity back of the grandparents, we must make

$$
H_{3}=H_{4}=\cdots=\mathrm{o} .
$$

Then

$$
h=0.5 H_{1}+0.25 H_{2} \text {. }
$$

If we have a fixed standard of selection, $H_{1}=H_{2}$, and

$$
h=0.75 H_{1} \text {. }
$$

3. If we assume mediocrity back of the great-grandparents, we must make

$$
H_{4}=H_{5}=0 \text {. }
$$

Then

$$
h=0.5 H_{1}+0.25 H_{2}+0.125 H_{3} ;
$$

and with a fixed standard of selection

$$
H_{1}=H_{2}=H_{3} ;
$$

from which

$$
h=0.875 H_{1} \text {. }
$$


If this same line of argument be carried on, so that the fourth generation of back ancestry is selected,

$$
\iota=0.9375 H_{1} \text {. }
$$

Likewise, if the fifth generation be selected,

$$
h=0.9687 H_{1} \text {. }
$$

And if a sixth generation be selected,

$$
h=0.9844 H_{1} \text {. }
$$

The significant point in all this is that six generations of selection, even on a mediocre stock, establish the selected character to within about I.5 per cent. The full significance of this point will appear later.

Finally, if selection of the character of deviation $H_{1}$ be made for $n$ generations, and if we may assume mediocrity in the ancestry beyond the $n$th generation, the amount of the character established is given by

$$
h=\left(\frac{\mathrm{I}}{2}+\frac{\mathrm{I}}{4}+\frac{\mathrm{I}}{8}+\cdots+\frac{\mathrm{I}}{2^{n}}\right) H_{1}=\left(\mathrm{I}-\frac{\mathrm{I}}{2^{n}}\right)^{*} H_{1} .
$$

Making no assumptions as to mediocrity in back ancestry. It has been shown both by experimental and by theoretical methods that if mid-parents with character $H_{1}$ are selected, the offspring will, on the average, exhibit about $0.6 H_{1} \dagger$ of the character in question. The inquiring reader will ask here why this differs from the $0.5 H_{1}$ obtained from Galton's law. It should be remembered that $0.5 H_{1}$ is what we obtained by assuming mediocrity back of the first mid-parents. In general, if we select parents of character $H_{1}$, their special ancestry will exhibit this character to a greater degree than ancestry in general from which the selection is made. It is therefore only common sense to expect a higher value than $0.5 H_{1}$ under the present assumption.

Granting, then, if we can-make no assertion about back ancestry, that an offspring will exhibit 0.6 of the deviation of

$*\left(\mathrm{I}-\frac{\mathrm{I}}{2^{n}}\right)$ is the sum of the geometrical progression $\left(\frac{\mathrm{I}}{2}+\frac{\mathrm{I}}{4}+\frac{\mathrm{I}}{\mathrm{S}}+\cdots+\frac{\mathrm{I}}{2^{n}}\right)$.

† See p. 533; also Proceedings of the Royal Society, LXII, 396. 
selected mid-parents, Pearson has established, ${ }^{1}$ by the theory of multiple correlation, the following results :

1. If selection be made of first and second generations of ancestry, with no knowledge as to back ancestry,

$$
h=0.5122 H_{1}+0.2927 H_{2} \text {. }
$$

If we have a fixed standard of selection, $H_{1}=H_{2}$;

then

$$
h=0.8049 H_{1} \text {. }
$$

2. If selection be made of three generations of ancestry under similar conditions as to back ancestry,

$$
h=0.5015 H_{1}+0.2553 H_{2}+0.1459 H_{3} ;
$$

with a fixed standard $H_{1}$,

$$
h=0.9027 H_{1} \text {. }
$$

3. If selection be made of four generations of ancestry,

$$
h=0.5002 H_{1}+0.2507 H_{2}+0.1276 H_{3}+0.0729 H_{4} ;
$$

with a fixed standard $H_{1}$,

$$
\iota=0.95{ }^{1} 4 H_{1} \text {. }
$$

4. Similarly, for a selection of five generations,

$h=0.5000 H_{1}+0.250$ I $H_{2}+0.1253 H_{3}+0.0638 H_{4}+0.0365 H_{5}$; and with a fixed standard $H_{1}$,

$$
h=0.9717 H_{1} \text {. }
$$

5. Finally, for a selection of six generations,

$$
\begin{gathered}
h=0.5000 H_{1}+0.2500 H_{2}+0.1250 H_{3}+0.0627 H_{4} \\
+0.0319 H_{5}+0.0182 H_{6} ;
\end{gathered}
$$

and with our fixed standard $H_{1}$,

$$
h=0.9878 H_{1} \text {. }
$$

It should be noted that the coefficients which we obtain are approaching more and more Galton's coefficients in the law of ancestral heredity, which means that if selection be carried on for a very large number of generations it does not matter whether the back ancestry of our selection be mediocre or above mediocrity. 
SECTION XVII - BREEDING TRUE, OR STABILITY OF A CHARACTER ESTABLISHED BY SELECTION

It is the object of this section to show that if an improvement has been made in a population, or if a breed has been developed by selection, the offspring will not degenerate if allowed to breed among themselves without selection; that is to say, if by selection a certain per cent of a character has been established on the average, the offspring will breed true to that amount of the character which has been established.

For instance, if selection has been made for six generations of a character $H_{1}$, the amount of this character appearing in the offspring, after this selection, is giren by $6: H_{1}$, if we assume mediocrity back of the six generations of selected ancestry. Now if these offspring with 63.3 of the desired character be allowed to breed together without further selection, their offspring will exhibit the character $H_{1}$ in the amount given by

$$
\frac{1}{2}\left(\frac{63}{64}\right) H_{1}+\frac{1}{4} H_{1}+\frac{1}{8} H_{1}+\cdots+\frac{1}{2^{7}} H_{1}=\frac{63}{64} H_{1}
$$

so that the first generation of offspring after selection has ceased will exhibit the character to exactly the extent that their parents exhibited it.

Let us carry this forward another generation. Then the character will be exhibited in the amount given by

$$
\frac{\mathrm{I}}{2}\left(\frac{6_{3}}{6_{4}}\right) H_{1}+\frac{\mathrm{I}}{4}\left(\frac{6_{3}}{6_{4}}\right) H_{1}+\frac{\mathrm{I}}{2^{3}} I_{1}+\frac{\mathrm{I}}{2^{4}} H_{1}+\cdots+\frac{\mathrm{I}}{2^{n}} H_{1}=\frac{6_{3}^{3}}{64} H_{1},
$$

which again shows the offspring unchanged so far as the amount of the character is concerned; and it is easily seen that this would be true if we should allow breeding to go on for any number of generations without further selection as to the character in question. ${ }^{1}$

For the sake of completeness and generality let us consider the case where selection for a deviation $H_{1}$ has been made for $n$ generations, and where the offspring so produced are allowed

1 It is of course assumed that all forms of natural selection are also excluded. 
to mate without selection. In this treatment we shall assume mediocrity in this back ancestry of the $n$ selected generations of ancestry.

As was seen on page 539 , the character is established in the amount giren by $\left(1-\frac{1}{2^{\prime \prime}}\right) / I_{1}$, and we shall now show that if this offspring be allowed to breed without further selection it will breed true to $\mathrm{I}-\frac{\mathrm{I}}{2^{\prime \prime}}$ of the selected character.

In the first generation of offspring after no selection we should have

$$
\frac{I}{2}\left(I-\frac{I}{2^{n}}\right)+\frac{I}{2^{2}}+\frac{I}{2^{3}}+\cdots+\frac{I}{2^{n+1}}
$$

of the character $H_{1}$ in question.

This series may be written as

$\frac{1}{2}\left(1-\frac{1}{2^{n}}\right)+\frac{1}{2}\left(\frac{1}{2}+\frac{1}{2^{2}}+\cdots+\frac{1}{2^{n}}\right)=\frac{1}{2}\left(1-\frac{1}{2^{n}}\right)+\frac{1}{2}\left(1-\frac{1}{2^{n}}\right)=1-\frac{1}{2^{n}}$.

The amount of the character present is therefore unchanged.

In the second generation of offspring after no selection we should have

$$
\begin{aligned}
& \frac{1}{2}\left(1-\frac{1}{2^{n}}\right)+\frac{1}{4}\left(1-\frac{1}{2^{n}}\right)+\frac{1}{2^{3}}+\frac{1}{2^{4}}+\cdots+\frac{\mathrm{I}}{2^{n+}} \\
= & \frac{1}{2}\left(1-\frac{\mathrm{I}}{2^{n}}\right)+{ }_{4}^{1}\left(1-\frac{\mathrm{I}}{2^{n}}\right)+\frac{\mathrm{I}}{2^{2}}\left(\frac{\mathrm{I}}{2}+\frac{\mathrm{I}}{2^{2}}+\frac{\mathrm{I}}{2^{3}}+\cdots+\frac{\mathrm{I}}{2^{n}}\right) \\
= & \frac{1}{2}\left(1-\frac{\mathrm{I}}{2^{n}}\right)+{ }_{4}\left(1-\frac{\mathrm{I}}{2^{n}}\right)+\frac{\mathrm{I}}{4}\left(1-\frac{\mathrm{I}}{2^{n}}\right)=\mathrm{I}-\frac{\mathrm{I}}{2^{n}}
\end{aligned}
$$

so that the amount of the character present is again unchanged.

The methed here used can be extended to any number of generations. We may show that if it be true for the $r$ th generation of offspring bred without selection, it will be true for the $(r+1)$ th generation. If $\mathrm{I}-\frac{\mathrm{I}}{2^{\prime \prime}}$ of the character has appeared in $r$ generations, then in the next gencration the amount of the character should be given by 


$$
\begin{aligned}
& \frac{I}{2}\left(I-\frac{I}{2^{\prime \prime}}\right)+\frac{1}{2^{2}}\left(1-\frac{I}{2^{\prime \prime}}\right)+\frac{1}{2^{3}}\left(1-\frac{1}{2^{\prime \prime}}\right)+\cdots+\frac{I}{2^{\prime \prime}}\left(1-\frac{I}{2^{\prime \prime}}\right) \\
& +\frac{1}{2^{n+1}}+\frac{1}{2^{r}+2}+\cdots+\frac{1}{2^{\prime}+} \\
& =\frac{\mathrm{I}}{2}\left(\mathrm{I}-\frac{\mathrm{I}}{2^{\prime \prime}}\right)+\frac{\mathrm{I}}{2^{\prime 2}}\left(\mathrm{I}-\frac{\mathrm{I}}{2^{\prime \prime}}\right)+\frac{\mathrm{I}}{2^{3}}\left(1-\frac{\mathrm{I}}{2^{\prime \prime}}\right)+\cdots+\frac{\mathrm{I}}{2^{\prime}}\left(\mathrm{I}-\frac{\mathrm{I}}{2^{\prime \prime}}\right) \\
& +\frac{\mathrm{I}}{2^{\prime}}\left(\frac{\mathrm{I}}{2}+\frac{\mathrm{I}}{2^{2}}+\cdots+\frac{\mathrm{I}}{2^{\prime \prime}}\right) \\
& =\frac{I}{2}\left(\mathrm{I}-\frac{\mathrm{I}}{2^{\prime \prime}}\right)+\frac{\mathrm{I}}{2^{2}}\left(\mathrm{I}-\frac{\mathrm{I}}{2^{\prime \prime}}\right)+\frac{\mathrm{I}}{2^{3}}\left(\mathrm{I}-\frac{\mathrm{I}}{2^{\prime \prime}}\right)+\cdots+\frac{\mathrm{I}}{2^{\prime \prime}}\left(\mathrm{I}-\frac{\mathrm{I}}{2^{\prime \prime}}\right) \\
& +\frac{I}{2^{n}}\left(I-\frac{I}{2^{n}}\right) \\
& =\left(\frac{\mathrm{I}}{2}+\frac{\mathrm{I}}{2^{2}}+\frac{\mathrm{I}}{2^{3}}+\cdots+\frac{\mathrm{I}}{2^{r}}+\frac{\mathrm{I}}{2^{\prime}}\right)\left(\mathrm{I}-\frac{\mathrm{I}}{2^{\prime \prime}}\right) \\
& =\mathrm{I}-\frac{\mathrm{I}}{2^{n}} \text {, since } \frac{\mathrm{I}}{2}+\frac{\mathrm{I}}{2^{2}}+\frac{\mathrm{I}}{2^{3}}+\cdots+\frac{\mathrm{I}}{2^{y}}+\frac{\mathrm{I}}{2^{\prime \prime}}=\mathrm{I} \text {; }
\end{aligned}
$$

which shows that $\mathrm{I}-\frac{\mathrm{I}}{2^{\prime \prime}}$ of the desired character will be present in any generation. Hence the stock will always breed true to the per cent of the character established.

It may be remembered in the above that mediocrity has been assumed in the ancestry back of the $n$ selected generations of offspring; but if this were not assumed, we have seen that after a few generations we obtain approximately Galton's coefficients. Hence, without assuming mediocrity back of $n$ generations, we may safely say that the offspring will breed true to the amount of the character established by selection.

The following table presents the amount of a character established by selection of $1,2,3,4,5$, and 6 generations of ancestry. To illustrate how these breed true we may take the simplest case where 0.6 of the character is established by selecting one generation. Suppose, then, that a generation is produced without selection. The amount of the character present will be given by

$$
\text { (0.6) }(0.5 \text { I 22) }+0.2927=0.6 \text {, }
$$

which illustrates that stability is established. 


\section{Fiffect of Continued Selection upon Variability and Type ${ }^{1}$}

\begin{tabular}{|c|c|c|c|c|c|c|c|c|}
\hline \multirow{2}{*}{$\begin{array}{l}\text { Number of } \\
\text { Genera- } \\
\text { tions of } \\
\text { Selection }\end{array}$} & \multicolumn{6}{|c|}{$\begin{array}{c}\text { FRACTIONAL, CONTRIBUTION OF ANCESTORS, } \\
\text { VARIOUS GENERATIONS }\end{array}$} & \multirow{2}{*}{$\begin{array}{l}\text { Ratio of } \\
\text { Finar to } \\
\text { Setected } \\
\text { Tyeis }\end{array}$} & \multirow{2}{*}{$\begin{array}{c}\text { RATIS OF } \\
\text { FINAL TO } \\
\text { INITIAL. } \\
\text { VARIABILITI }\end{array}$} \\
\hline & I & 2 & 3 & 4 & 5 & 6 & & \\
\hline I & .6000 & $\ldots$ & $\cdots$ & $\cdots$ & $\cdots$ & $\cdots$ & .6000 & .9055 \\
\hline 2 & $.5^{122}$ & .2927 & $\cdots$ & $\cdots$ & $\ldots$ & $\cdots$ & $.80+9$ & .8946 \\
\hline 3 & .5015 & .2553 & .1459 & $\cdots$ & $\ldots$ & $\cdots$ & .9027 & .8945 \\
\hline 4 & .5002 & .2507 & .1276 & .0729 & $\ldots$ & $\cdots$ & .9514 & .89445 \\
\hline 5 & .5000 & .2501 & .1253 & $.063^{8}$ & .0365 & $\ldots$ & .9717 & .8944 \\
\hline 6 & .5000 & .2500 & .1250 & .0627 & .0319 & $.01 S z$ & .9878 & .8944 \\
\hline To infinity & .5000 & .2500 & .1250 & .0625 & .03125 & .015625 & I & .8944 \\
\hline
\end{tabular}

\section{SECTION XVIII - DURATION OF TARIETIES, BREEISS, AND FAMILY STRAINS}

How long can a desired breed or family be retained? There is a popular belief that varieties wear out, and that breeds must of necessity be constantly reënforced by new material or by new combinations to take the place of worn-out stock.

The facts just presented, however, clearly indicate that if a type does not remain true indefinitely either it is the fault of adverse selection, accidental or otherwise, or else it is clue to some physical or biological cause, for the type, once obtained, naturally breeds true.

Again, from the fact that variability is not greatly reducible, we are safe in assuming that types once established by selection will not only remain true but are capable of still further development if we bestow additional attention and selection, and that the upper limits of improvement are fixed, if fixed at all, by some circumstance other than variability. It may be biological, - like loss of fertility or reversal of selection, - or it may be mechanical, but the cause, whatever it may be, that sets a limit to improvement is not connected with variability.

In the last analysis, however, we are bound to raise the question whether all types can be indefinitely maintained, even by the

\footnotetext{
1 Pearson, Grammar of Science, p. 485.
} 
most skillful methods. So far as ordinary law's of erolution gor there is no doubt about it, and we can with confidence assert our ability to maintain a desirable type indefinitely; but are there biological considerations outside of mere variability that tend to extinction? Do species "wear out," or do they come to an untimely end by accident only?

In the opinion of the writer we do not possess sufficient reliable data on this point to warrant confident assertion. It is probably true that species have clisappeared off the earth at a rate not equaled by the production of new species. It is true, too, that among domestic animals some of the most valuable lines have disappeared in spite of the most energetic efforts to preserve them. ${ }^{1}$ In the instance given below the extinction is to be definitely ascribed to barrenness, - a defect perfectly well known to breeders, and considered by them at the time as fortunate, in the interest of high prices, they evidently not appreciating the inevitably fatal consequences of racial barrenness.

On the other hand, many species have persisted from remote times practically unchanged in type (oaks and tulip trees), and as we are fully informed as to some of the causes that resulted in the extinction of favorites, like the unfortunate family of Shorthorns just mentioned, we are warranted in hoping that species in general may be maintained indefinitely.

The conclusion is forced upon us that reliable information is wanting as to whether all types can be indefinitely maintained. No proper attempt was made to save the Duchess family. Its inherent weakness was counted its chief virtue, and there could be but one conclusion. But was its fertility a waning character, which no amount of selection could have strengthened? Again we say that our knowledge is insufficient for the answer.

Summary. Heredity is not the relation between the offspring and his parent simply, but the relation between him and the whole back ancestry. The characters of the individual are the characters of the race. Some are well developed, others are undeveloped or latent, but all are there in some degree.

1 For example, the Duke and Duchess Shorthorns, the most famous family of any breed, - so famous that a heifer brought $\$ 40,600$ at the New York Mills sale in 1873 . 
Different individuals of the same ancestry inherit differently, and in general the behavior of characters in transmission suggests that they are in some way made up of combinations, so that a high degree of variability is inevitable, even with the same elements; as, for instance, a great variety of color effects can be produced with the same three primaries, - red, blue, and yellow.

Some characters blend and others are mutually exclusive, each tending to preserve its identity. On this account, as well as from other causes, such as relative fertility, races often exhibit distinct polymorphism. Inheritance is not so much connected with sex as is popularly supposed. Characters often do not develop until late in life. This is not to be regarded as belated inheritance but as belated development.

The only proper way to study the principles of heredity is by statistical methods, using groups instead of single individuals, from which no general conclusions can be safely drawn.

The regression table brings out clearly the fact that like parents beget unlike offspring; that, in general, the offspring is more mediocre than the parent, but that for silected offspring the ancestry is comparatively mediocre; that the coefficient of heredity between the nearest relatives is seldom above 0.50 ; that the mean of the offspring is not necessarily the same as the mean of the parent; that the means of a race are its most fertile portions; that, in general, a few offspring exceed the previous limits of the race, - that is, progress away from the type if favored by selection; that exceptional individuals may arise either from exceptional or from mediocre parentage; and that successive offspring from the same parents are not identical.

Nothing is clearer than that the inevitable consequences of bisexual reproduction and of the manner of growth by the halving of the cell contents is to insure that character combinations effected in this manner are brought together in definite mathematical proportions not far from those expressed in the expansion of a binomial. This is the real foundation of Mendel's law, for characters that do not blend, and it also expresses the relative proportions of characters that do blend.

The statistical methods of study enable us to derelop the law of ancestral heredity, which agrees closely with experimental 
evidence, and which shows the degree to which the various generations have contributed to the results.

Continued selection will shift the type in any' desired direction, and after a few generations it will "bred true" in its new form. As variability is not greatly reduced by selection, there is alivay's opportunity for improzcment so far as variability is concerned.

\section{Special Exercises}

I. Exercises in great variety in forming regression tables and in deducing the conclusions.

2. Investigations into the operations of Mendel's law, by the examination and identification of laboratory material and if possible by the actual raising of crossed forms for the purpose.

3. Special and definite applications of the law of ancestral heredity to the problems of the breeder, especially in crossing, grading, and line breeding.

\section{ADDITIONAL REFERENCES}

Alternative Inheritance. By Karl Pearson. Proceedings of the Royal Society, LXXII, 505-5IO.

American Trotting Records as Data for Heredity Studies. By Francis Galton. Proceedings of the Royal Society, LXII, 310-315.

Bateson on Pearson's Conception of Heredity. Proceedings of the Royal Society, LXIX, 193-205; Pearson's answer, LXIX, 450.

Chances of Death. By Karl Pearson. Science, VI, 328-330.

Contribution of Several Ancestors to Offspring. By Francis Galton. Proceedings of the Royal Society, LXI, 40I-4I3.

Correlation between Longevity and Fertility. By Karl Pearson. Proceedings of the Royal Society, LXVII, I 59-179, 333-337.

Criterion to Test Tieories of Heredity. By Karl Pearson (1904). Proceedings of the Royal Society, LXXIII, 262-280.

Do Varieties Run Out? By J. Craig. Gardening, 1899, pp. 278-279; also in Experiment Station Record, XI, I 52.

Experimental Evidence upon Mendel's Law. By L. H. Lock. Nature, LXX, 60I-602; by Karl Pearson, 626-627.

Experimental Studies in Heredity. Corn Report of the Royal Society, I902, p. I60; also in Experiment Station Record, XVII, 634. Experimental Zoölogy. By T. H. Morgan. Chapters VI and ViI, pp. 66-166.

Experiments in Crossing White and Black Oats. By J. H. Wilson. Nature, 1904, p. 413; Experiment Station Record, XVI, 462. 
EyE COLOR IN MAN. Philosophical Transactions of the Royal Society, CXCV, A, 79-150.

Formula FOR REgRession. By Pearson and Yule. Proceedings of the Royal Society, LX, 477-489.

Heredity of Coat Characters in Pigs and Rabits. By W. E. Castle. Science, XXI, 737-738, 986.

History of the Development of the Quantitative Study of VARIATION. By C. B. Davenport. Science, VIII, 864 ; Proceedings of the American Association for the Advancement of Science, 1900, XLIX, 197-200.

Hybrid Oranges. By Webber and Swingle. Science, XVII, 262263.

Hybrid Wheats. By W. J. Spillman. Bulletin No. II 5, Office of Experiment Stations; also in Science, XX, 68.

Inheritance in CoAT Color, Thoroughbred Horses. By Blanchard. Biometrika, I, 36I-364; by Karl Pearson, Philosophical Transactions of the Royal Society, CXCV, A, I-49.

Inheritance of Fertilitx. (Race horses and the human race.) By Karl Pearson. Science, 1X, 283-286.

Inheritance of Mental, Characters in Max. By Karl Pearson. Proceedings of the Royal Society, LXIX, 153-155.

Latent Characters and Reversion. By W. E. Castle. Science, XXI, 378-379.

LAw of Ancestral Heredity. By Karl Pearson. Biometrika, II, 2 I I-229, 23 I-236.

Law of Heredity. By C. B. Davenport. Science, VII, I58-16I.

LAw OF REversion. By Karl Pearson. Proceedings of the Royal Society, LXVI, 140-164, 241-244, 316-323, 324-327.

Laws of Ancestral Heredity. By Karl Pearson. Science, ViI, $337-339,551-554$.

Laws of Heredity of Galton and Mendel, and Some Laws Governing Improvement by Selection. By W. E. Castle. Proceedings of the American Academy of Arts and Sciences, XXXIX, $221-242$.

Limits of VARIATION IN PLANTS. (Author says variation is in mathematical ratio.) By J. W. Harshberger. Science, XIII, 251.

Longevity and the Selective Death Rate. Pearson and Beeton. Proceedings of the Royal Society, LXV, 290-305.

Mathematical Contribution to the Theory of Heredity. By Karl Pearson. Proceedings of the Royal Society, LXXI, 288-314.

Matiematical Evolution. By Karl Pearson. Proceedings of the Royal Society, LIV, 329.

Mathematical Evolution. By Karl Pearson. Proceedings of the Royal Society, LXIV: Genetic Selection, 163-165; Inheritance of Fertility, 165-166; Inheritance of Fecundity, 166-167. 
Mathematical Evolution ANo MeNiel's Law. By Karl Pearson (I904). Philosophical Transactions of the Royal Society, CCIII, A, 53-86.

Mathematical Evolution - Correlation. By Lee and Pearson. Proceedings of the Royal Society, LXI, 3+3-356; LXII, I 73-I 75, $386-417$; LXIII, 417-419.

Mathematical Evolution-Some Errors to Be Avoined. By Karl Pearson. Proceedings of the Royal Society, LX, 489-498; On Spurious Correlation, 498-502.

MEASURING VARIATIONS IN ANimals. (Report of a committee of Galton and others.) Proceedings of the Royal Society, LVII, $360-382$.

Mendelax INHeritance of THReECharacters. By William Bateson. Proceedings of the Cambridge Philosophical Society, XII, 153-I 54.

MENDELism. (Experiments with guinea-chicken hybrids.) By M. L. Snyder. Science, XXI, 854-855.

MENDEL'S LAW. (Angora goats.) By W. E. Castle. Science, XVIII, $760-761$.

MENDEL'S LAW. BY A. D. Darbishire. Experiment Station Record, XVI, 232.

Mendel's Law (Exceptions to). By W. J. Spillman. Science, XVI, 709-710, 794-796.

MENDEL'S LAw. (Experiments with mice.) By C. B. Davenport. Science, XIX, IIO-II4.

Mendel's Law And Cytological Investigation. By C. B. Wilson, Science, XVI, 991-993.

Mendel's Law ANd Negro Albinism. By William C. Larrabee. Science, XVII, 75-76.

Mendel's Law. Defense BY Bateson. Cambridge University Press 1902, P. 212 ; Experiment Station Record, XIV, 634.

Mendel's Law, - Discussion, Defense, And Criticism. Biometrika, 1902, No. 2, pp. 228-254; Journal of the Royal Horticultural Society, 1902, pp. 688-695; Experiment Station Record, XIV, 446-447.

Mental and Moral Heredity in Royalty. By Dr. F. A. Woods, Harvard University. Popular Science Monthly, LXI, three articles; LXII, six articles.

New Evidence for Indididulity of Chromosomes. By W. J. Baumgartner. Biological Bulletin, VIII, I-23.

ON the Influence of Selection in Variability. By Karl Pearson. Proceedings of the Royal Society, LXIX, 330-332.

Origin of Black SheEp in a Flock (Mendelian). By C. B. Davenport. Science, XXII, 674-675.

Purity of Gerar Cells. By T. H. Morgan. Science, XXII, 877-879. Regression and Inheritance in the Case of Two Parents. Proceedings of the Royal Society, LVIII, 240-242. 
Rrakission, Hermity, Panmixia. By Karl Pearson. Proceedings of the Royal Society, LIX, 69-70.

Reprodictive, Selection. By Karl Pearson. Proceedings of the Royal Society, LIX, 301-304.

Second-Generation Hybids. By Halstead and Kelsey. New Jersey Experiment Station Report, I902, pp. 377-395; Experiment Station Record, XV, I 52.

SkEW VARIATox. By Karl Pearson. Proceedings of the Royal Society, LVII, $257-260$.

STATEMENT OF MENIEL'S LAW. (With bibliography.) By WV. E. Castle.

Science, XVIII, 396-405; also by L. H. Bailey, XVII, 44I-454

Telegony in MaN. By Karl Pearson. Proceedings of the Royal Society, LX, 273-283.

The Statistical Study of Evolution. By C. B. Davenport. Popular Science Monthly, LIX, 447-460.

VARiability of Individual and Race. By Karl Pearson. Proceedings of the Royal Society, LXVIII, I-5, 372-373.

VARIATION AND CORRElation in MAN - Civilized as CoMpared with Primitive Races.' By Karl Pearson. Science, VI, 49-50.

WONDER HORSES AND MENDELISM. (Several generations of horses with very long manes and tails.) By C. B. Davenport. Science, XIX, 15 I-1 53 . 


\section{CHAPTER XV}

\section{PREPOTENCY}

That all parents are not equally powerful in impressing: racial characters is a fact well known to the merest novice in breeding. It is distinctly shown in all regression tables, and the reason for it is clearly seen in the mathematical nature of reproduction, by which individuals are differently endowed, and by which some few are exceptionally rich in the elements out of which racial characters are developed. When to these facts is added the difficulty of selecting animals by outward appearance, on account of the relation of dominant and recessive characters, we need feel no surprise at the relatively small number of highly prepotent individuals and the large number of reversions encountered in actual breeding.

\section{SECTION I - IATA FRON THE TROTTING RECORDS ILLUSTRATING PREPOTENCY}

Seeking material which would illustrate accurately, and with sufficiently large numbers, the differences in the breeding powers of different individuals, the writer made some studies in the records of trotting-bred horses. These studies covered all animals registered and that had made track or breeding records from the opening of the Register and the Yearbook down to and including the year Igor. ${ }^{1}$

In the consideration of this material, and in the comparison of individuals, four facts must be borne in mind: first, some individuals were too young for their full breeding record to be all in; second, some had enjoyed less opportunity than others, owing to their racing engagements; third, some stallions had access to better mares, and more of them, than had others;

1 It is needless to say that this proved a laborious task, covering many weeks, with two calculators. 
fourth, fashion has much to do, even among race horses, in influencing selection, especially after an individual or a family has acquired a reputation.

Allowing as fully as possible for these facts, the records are worth study for the light they throw upon the question of inherent differences between individuals in respect to breeding powers - differences so great that as we proceed it will be perfectly evident that the line of descent runs through few individuals and quite independent of the mass.

The total number of performers listed - that is, that had made track records good enough to admit them to the 2:30 list at this date (I9OI) - was 26,327 , of which 17,625 , or almost exactly two thirds, were trotters, and 8702 were pacers.

The Register showed that, in all, 34,299 stallions had been recorded at this time, but the breeding record showed that only 6278 , or less than one in five, had produced anything in the list; ${ }^{1}$ that is to say, roughly speaking, 627 sires had produced 26,327 performers, or an average of $4 . \mathrm{I}+$ each.

Great sires. Of these $627 \mathrm{~S}$ sires only 207 had produced $t c n$ or more sires or dams of spced; that is, only 207 had bred well enough to produce either ten stallions each, or ten mares each, capable themselves of producing speed. ${ }^{2}$ In other words, of the whole 34,299 stallions and 6278 sires, only 207 bred speed well enough to send it into the second generation to the extent of either ten sires or ten dams producing speed.

Now these 207 grreat sires themsele's produced diretly 5377 performers (4226-I I5I p.), which is more than one fifth of the cutire list of pirformers, and an average of 26 apiece, or six times the breeding record of the average stallion.

Again, these 207 great sires produced 3 I 55 sires of performers, and they in turn produced 16,536 trotters and pacers (1 $1,737-$ 4799 p.). This is over half of all the sires and over 62 per cent of all the performers of the breed.

1 This was partly, as has been already noted, because some individuals were too young to have made a full breeding record.

2 This, of course, does not include those sires whose produce in sires and dams together equaled ten.

${ }^{3}$ Note that $4226-$ II 5 I p. means 4226 trotters and II 5 I pacers. 
Besides this, these same 207 sires produced 4507 dams of speed, and they produced 669I performers (5I20-I57 I p.); so that about 3 per cent of the sires have produced the sires and dams of somewhere between two thirds and three quarters of the total speed of the race. If we should add the produce of the sires and the dams, we should have $16,536+669 \mathrm{I}=23,227$ apparent grandchildren of those 207 sires. This we cannot do because many of those recorded as offspring of dams are also recorded among the offspring of sires; that is to say, they are duplicatis clue to the fact that many of the 4507 dams were matcd with some of the 3 I 55 sires. We cannot tell, therefore, from these figures what exact proportion of the total number registered may have descended from the 207 great sires.

Distinction between sires of sires and sires of dams. Analyzing these 207 great sires, it was found that they were unequally divided between sires of sires and sires of dams of speed as follows:

Class I. Sires of ten or more sircs of speed, but of less than ten dams of speed, -9 .

Class 2. Sires of ten or more dams of speed, but of less than ten sires of speed, - I I 3 .

Class 3. Sires of ten or more sires of speed and of ten or more dams of speed, -85 .

Of these three classe's, I may be considered as distinctly sires of sires, 2 as sires of dams, and 3 as sires of both sires and dams.

From the table on the following page it appears that:

I. The poorest breeding record was made in every case but one by Class 2, - the sires of ten or more dams but not of ten or more sires. Note the ratios, lines $3,5,7,8$, IO, I 2 , I 3, I 5 . The only case in which they outdid Class I was in the ratio of dams produced per stallion (I 5), which was clearly in excess of Class I (6), which are distinctly stallion breeders (line IO).

2. The great breeding record was made by Class 3 , - the sires that produced both sires and dams freely. In sicry case the ratios are higher than for any other class, whether performers, sires, dams, or produce of sires or dams.

3. Class I, sires of sires, was clearly superior to Class 2, sires of dams, but in all cases inferior to Class 3 , sires of both. 
Breeding Record of Threk Classes of Stallions: i, Sires of Sires; 2, Sires of Dams; 3 , Sires of Both Sires AND Dams of SPEed

\begin{tabular}{|c|c|c|c|c|}
\hline & & Class I & Class 2 & Crass 3 \\
\hline I & Number of sires . . . . . . . . . . & 9 & II 3 & $8_{5}$ \\
\hline 2 & Total performers gotten directly by these sires & 274 & I 357 & 3,746 \\
\hline 3 & Ratio per sire . . . . . . . . . . . . & 30 & 12 & 44 \\
\hline 4 & Sires of performers gotten by each class & 113 & 461 & $2,5^{8} \mathrm{I}$ \\
\hline 5 & Ratio per original sire . . . . . . . . & 12 & 4 & 30 \\
\hline 6 & Performers gotten by these sires (line 4) & 332 & 1396 & $I_{4}, \mathrm{SOS}$ \\
\hline 7 & Ratio per sire (line 4 ) . . . . . . . . & $3+$ & $3+$ & $6-$ \\
\hline 8 & Ratio to original sires(line I) . . . . . & 37 & $12+$ & 174 \\
\hline 9 & Dams of performers gotten by original sires . & 57 & 1677 & 2,773 \\
\hline 10 & Ratio to original sires . . . . . . . & 6 & 15 & 32 \\
\hline I I & Performers produced by these dams (line 9) & 60 & $23+2$ & $4,2 S 9$ \\
\hline 12 & Ratio per dam. . . . . . . . . . . & $\mathbf{I}+$ & $1.5-$ & $1.5+$ \\
\hline 13 & Ratio to original sires (line $\mathrm{I}$ ) . . . . & $7-$ & $20+$ & $50+$ \\
\hline I4 & Performers (line 4) that were also sires of speed & 40 & I 45 & 888 \\
\hline 15 & Ratio to original sires (line 1) . . . . . & $4+$ & $\mathbf{I}+$ & $10+$ \\
\hline
\end{tabular}

4. One outside circumstance helps to relieve the burden of inferiority resting on Class 2. A stallion belonging to an unfashionable line would be used but little or not at all in the stud, while a mare belonging to an equally unfashionable strain would not be equally barred. The result of this discrimination in the long run, and under our methods of study, would appear in the form of sires of dams. To some extent it means, not that these sires did not produce males, but that, being unfashionable, these males had little opportunity. This probably does not account for all differences, even though the turns and caprices of fashion are harder on sires than on dams.

It must not be forgotten in this connection that these I I 3 sires constitute more than one half of the 207 greatest sires of the race. They could not, therefore, have been so very unfashionable.

5. Class I must be largely what it seems to be, - breeders of sires rather than of dams, because there is no reason why its female offspring should have been suppressed. It is clearly superior to Class 2, but inferior to Class 3 . 
6. Class 3 evidently represents the cream of the race, exceedingly prolific and highly fashionable, - the most successful getters both of speed and of breeders.

7. These 85 sires themselves produced directly 258 I sires of performers (30 apiece), this number being over 40 per cent of all the sires of the breed. They produced directly 3746 performers, or 14 per cent of all in the list. The 258 I sires directly gotten by them produced $\mathrm{I} 4, \mathrm{SOS}$ performers, or over 56 per cent of all in the list. This means that a little oecr one por cent of the sires are grandsires to ower half the breed.

The big ten. But the highest relative excellence is well within these $\delta_{5}$ great breelers. Their average get of performers was 44 , or over ten times the average of the race, but out of the 34,299 registered stallions $t c m$, and ten only, produced directly as many as a hundred or more performers each. ${ }^{1}$ The tabulation of the breeding record of these ten greatest stallions is good reading for the student of prepotency, as it shows a breeding power which fully justified their fame as great producers not only of speed but of sires and dams of speed.

Table showing the Record of the Ten Greatest Producers OF SPEED ${ }^{2}$ UP TO AND INCLUding I $90 \mathrm{I}$

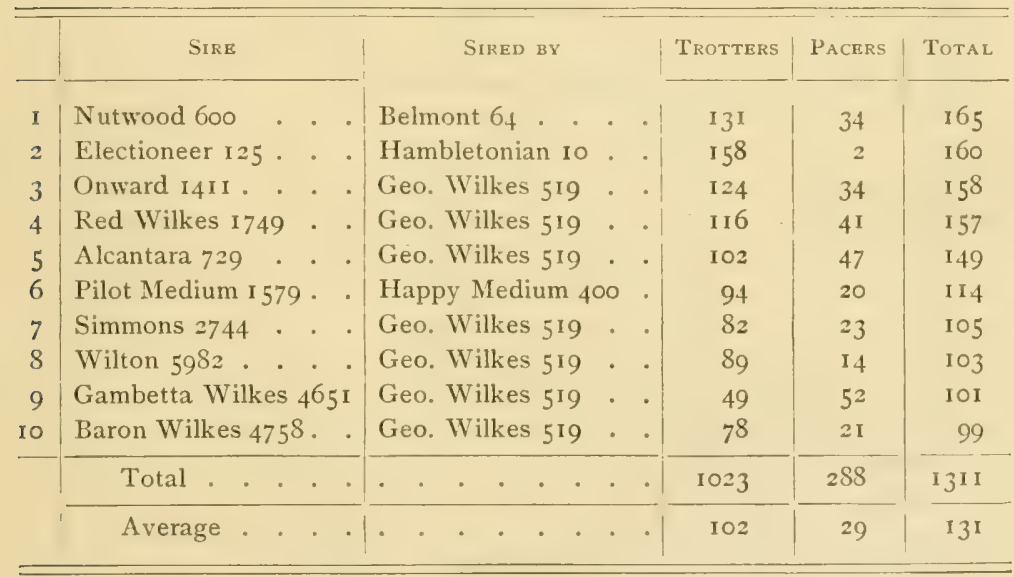

1 One of these goes in at 99.

2 Trotters and pacers. 
This is 32 times the breeding record of the average sire, and nearly five times the record of the 207 great sires, themselves included, or over six times their record exclusive of these ten.

It is worth while to note the sires of these great breeders of speed. Number 2 is by I Iambletonian 10 ; No. 6 is by Happy Medium, he by Hambletonian Io; No. I is by Belmont, by Abdallah, he by Hambletonian Io; and the remaining seven, Nos. 3, 4, 5, 7, 8, -9, I0, are by Geo. Wilkes, by Hambletonian 10. Thus, of this remarkable list of ten stallions, all except one are but two removes from I Iambletonian 10. Of this number, those that were sired by Geo. Wilkes produced 640 trotters and 232 pacers, - in all $\$ 72$ performers, or more than 66 per cent of the whole.

The famous grandsires. Eight stallions of this list have the distinction of being grandsire to over 500 performers, as follows :

Table of Famous Grandsires having 500 or more Performers in the Second Generation ${ }^{1}$

\begin{tabular}{|c|c|c|c|c|c|c|c|}
\hline I & 2 & 3 & 4 & 5 & 6 & 7 & 8 \\
\hline Name & $\begin{array}{c}\text { Per- } \\
\text { formers }\end{array}$ & Sires & $\left|\begin{array}{c}\text { Per- } \\
\text { formers }\end{array}\right|$ & Dams & $\left|\begin{array}{c}\text { Per- } \\
\text { formers }\end{array}\right|$ & $\begin{array}{c}\text { Total Per- } \\
\text { formers }\end{array}$ & $\begin{array}{l}\text { Periorming } \\
\text { Sires and Get }\end{array}$ \\
\hline Geo. Wilkes ('56-'82) . & $\mathrm{S}_{3}$ & 102 & 2410 & 96 & 163 & 2573 & $40-1501$ \\
\hline I Iambletonian 10 ('49-'76) & 40 & 150 & 1694 & So & I 17 & $18 I 1$ & $8-174$ \\
\hline Electioneer $(' 68-90) \ldots$ & 160 & 97 & 942 & 79 & 103 & 1045 & $60-723$ \\
\hline Nutwood ('7o- )..... & 165 & 132 & 693 & 113 & I 84 & 877 & $55-291$ \\
\hline Belmont ('64-'s9) . . . . . & 59 & 74 & 615 & 66 & I 10 & 725 & $25-342$ \\
\hline Almont $\left(' 6_{4}-{ }^{\prime} s_{4}\right) \ldots .$. & 37 & 96 & 569 & 81 & 130 & 699 & $14-212$ \\
\hline Red Wilkes ('74- ) ... & I 57 & 93 & 471 & 79 & I 6 & $5^{87}$ & $5^{6-374}$ \\
\hline Onward ('75- ) ..... & I 58 & 106 & 454 & 57 & $9 \mathbf{I}$ & 545 & $34-228$ \\
\hline
\end{tabular}

1 Column I, name of grandsire; column 2, number of performers of his oron get; columns 3 and 4 , number of sires he got, with their get in the list; columns 5 and 6 , number of dams he got, with the performers dropped by them ; column 7 , total performers gotten by sires and dams, - the second generation; column 8 , number of performers (column 2) that were also sires, and their performing get.

N.B. Many of the sires (column 3) were not performers and many of the performers (column 2) were not sires, Numbers in parentheses refer to year of birth and death. 
Breeders of speed and breeders of breeders. Nothing more forcibly strikes the student working with records of this kind than the fact that some sires are notably sires of speed which ends in that generation, while others, not specially noteworthy for getting performers themselves, yet produce sires and dams of extreme breeding power. See the following table, which is a table of famous producers of speed, and compare with the last table, which is a table of famous producers of produccrs. Especial attention is called in this connection to Wilton, Simmons, and Pilot Medium, - famous getters of speed, - as compared with Almont, Belmont, Hambletonian, and Geo. Wilkes, - none of them famous as direct producers of speed, but all phenomenal breeders of sires and dams of specd.

Table of Fanous Sires and their Descendants; being All that Sired too Performers or Over ${ }^{1}$

\begin{tabular}{|c|c|c|c|c|c|c|c|}
\hline I & 2 & 3 & 4 & 5 & 6 & 7 & 8 \\
\hline Name & $\begin{array}{c}\text { Per- } \\
\text { formers }\end{array} \mid$ & Sires & $\begin{array}{c}\text { Per- } \\
\text { formers }\end{array}$ & Dams & $\left|\begin{array}{c}\text { Per- } \\
\text { formers }\end{array}\right|$ & $\begin{array}{c}\text { Total Per- } \\
\text { formers }\end{array}$ & $\begin{array}{l}\text { Performing } \\
\text { Sires and Get }\end{array}$ \\
\hline Baron Wilkes ('82- ) .. & 99 & 26 & 94 & 21 & 23 & 117 & $2 \mathrm{I}-85$ \\
\hline Gambetta Wilkes ('8I- ) & roI & 30 & III & 17 & 23 & I 34 & I I- 43 \\
\hline Wilton ('so- ) ..... & 103 & 13 & 30 & 9 & 13 & 43 & II -28 \\
\hline Simmons $(179-) \ldots$ & 105 & 26 & $6_{3}$ & 14 & 18 & $S_{I}$ & $3-54$ \\
\hline Pilot Medium ('79- ) .. & I I 4 & I 7 & 32 & 23 & 33 & 65 & $12-23$ \\
\hline Alcantara $(76-) \ldots$ & 149 & 43 & 200 & 27 & 45 & 315 & $19-111$ \\
\hline Red Wilkes ('74- ) ... & 157 & 93 & $47 \mathrm{r}$ & 79 & 116 & $5^{8} 7$ & $5^{6-374}$ \\
\hline Onward $\left(' 75^{-}\right) \ldots$ & 158 & 106 & 454 & 57 & 91 & 545 & $34^{-228}$ \\
\hline Electioneer ('6S-'9o) ... & 160 & 97 & 942 & 79 & 103 & 1045 & $60-723$ \\
\hline Nutwood ('70- ).... & 165 & 132 & 693 & I I 3 & 184 & 887 & $55^{-29 I}$ \\
\hline
\end{tabular}

Attention is especially called to column 7, recording the second generation of performers, as compared with column 2, - those gotten directly. It will be noted of three famous stallions that they were represented by fewer performers in the second

1 This table is made up on exactly the same plan as the previous table; it is designed to be studied in connection with the former, to show the difference between breeders of speed and breeders of breeders. Years are denoted by figures in parentheses. 
generation than in the first, but also that their age is against their second-generation record.

Relation between performance and breeding powers. An at(empt was made to learn whether performers are better breeders than nom-performers. There were at that time 49 stallions in the 2:10 list. Only 2 I of these had get in the 2:30 list, and only four had produced sires of speed.

The breceling record of this class of stallions looks pitifully slim as compared with that of the great breeders. The best breeding record made by a horse in the 2: IO list, up to the time these studies were made, was that of Nelson 4209, who hated produced $28-12 \mathrm{p}$., eight sires $(5-7 \mathrm{p}$.), and three dams $(1-2$ p.). The whole 49 in the 2 : Io list had produced only 194-65 p., and only 13 sires of speed, 8 of which have just been credited to Nelson.

We might conclude that performance is not a very good index of breeding power, but it would be a hasty conclusion if made on this basis. Two circumstances conspire to keep down the breeding record of stallions of extreme speed. One of these is the fact that many of them are young, and the other is the fact that a horse capalule of malinger low records is a'orth more for revcius. than for loveding purposes, and while racing engagements do not absolutely prevent breeding among stallions, as it does amoner mares until their racing days are over, ${ }^{1}$ yet it operates to greatly reduce it. Evidently we shall get little light on our question from this source.

Turning to individuals, we find that Nutwood 6oo, the greatest sire of speed (sec table on page 555), had a record of $2: 18$, but that Electioneer, the next greatest sire of speed, had no record. Of the "big ten," but one has a record as good as $2: I 8$, and his breeding record is the lowest of the lot.

Turning to the greatest grandsires of speed, Geo. Wilkes heads the list with a record of $2: 22$, but IIambletonian ro comes next, having produced more sires of speed than any horse

1 There were also 49 mares in the 2:ro list, - a strange coincidence, — not one of whom had produced anything in the list. This fact is, of course, not to be construed fo mean that they could not produce speed, but rather that they have not, as a class, had the opportunity. What kind of brood mares they would make when tried is another question. 
living or dead, and he has no record; ${ }^{1}$ Electioneer 125 comes next, also with no record; then Nutwood, 2:I $S_{4}^{3}$; Belmont, no record; followed by Almont, 2:39; ; Red Willes, 2:40; and Onward, 2:25 $\frac{1}{4}$.

From this showing of individuals we can arege either that the great breeders were too busy to make racing records of that breeding power is independent of the ability to perform.

Neither conclusion is warranted. In the first place, the breeding record of a stallion with a track record is injured by the fact that he has little opportunity until his racing days are over; but it is helped by the fact that when he is sent to the stud he has a superior class of mares.

Again, it does not follow, if a horse has not made a track record, that he should be considered as unuthe to do it. It may be from some slight defeet or from lack of proper training, from some minor injury, or from one or more of a hundred other causes having no connection whatever with his inherited ability to go.

Evidently, if we are to get any light upon this question from this source, - and it ought to be one of the best of sources for information of this class, - then we must obtain it from large numbers, in which the breeding record of those known to be performers is compared directly with that of those that have no performance record.

Accordingly a table was prepared exhibiting the breeding record of 165 of the principal stallions. They were chosen from the list of 207 that had produced ten or more sires of speed or ten or more dams of speed, and included all inditiducts thert had produced both porforming and non-porformings sires, ${ }^{2}$ cxcept a very few, the data for which were incomplete. The table shows, first (column I), the total number of performers produced by the

1 It was popularly believed that Hambletonian io could go in about 2:40, but he was dead long before anybody knew his value as a breeder of speed.

$213 y$ "performers" is meant those that have a track record of $2: 30$, or better. The term "non-performers" covers all without a record; it manifestly includes two classes, - those that might have made a record under suitable circumstances and those that could not have done so under any circumstances. As there is no way of distinguishing between these two, they are all called non-performers, and the table makes a comparison between those that have made a record and all others, able or unable, that have not done so. 
various stallions without regarel to their breeding powers; second (columns 2 and 3 ), the number of performing sires (that is, sires with speed records $2: 30$ or better), with their get; third (columms 4 and 5 ), the number of non-performing sires, with their get. For convenience there is added (column 6) the track record of those stallions (of the 165 ) which were themselves "performers" ; for example, line 6 : this sire made a record of $2: 23$ on the track. He produced 149 performers, I 9 performing sires that got I I I performers, and 24 sires that never made a record on the track but that produced 89 offspring that were performers. The entire table, covering $\sigma_{5}$ individuals, is given in order that the reader may have at hand the means of making individual comparisons, many of which are, to say the least, remarkable. Names have been omitted, but the reader may be interested to know that line 65 is Geo. Wilkes, 70 is Hambletonian IO, I2O is Nutwood, and I 2 I is Onward.

Breedin: Recori) of i65 Leaind; Stallions to show the Relation between "Performance and Breeding" Powers

\begin{tabular}{|c|c|c|c|c|c|c|}
\hline & 1 & 2 & 3 & 4 & 5 & 6 \\
\hline & $\begin{array}{l}\text { Performing } \\
\text { Get }\end{array}$ & $\begin{array}{l}\text { Sires also } \\
\text { Performers }\end{array}$ & $\begin{array}{l}\text { Performing } \\
\quad \text { Get }\end{array}$ & $\begin{array}{l}\text { Sires not } \\
\text { Performers }\end{array}$ & $\begin{array}{l}\text { Performing } \\
\text { Get }\end{array}$ & Record \\
\hline I & 5 & 2 & I 2 & 12 & I 44 & \\
\hline 2 & 4 & I & 3 & 4 & 6 & $2: 30$ \\
\hline 3 & 53 & 9 & 29 & 23 & 70 & \\
\hline 4 & 16 & 3 & I 2 & 17 & 37 & $2: 291 / 2$ \\
\hline 5 & 55 & 3 & 26 & 5 & I I & \\
\hline 6 & 149 & 19 & I I I & 24 & S9 & $2: 23$ \\
\hline 7 & 59 & 27 & 208 & 21 & 105 & $2: 27$ \\
\hline 8 & 8 & 3 & 9 & 5 & II & $2: 2.4^{1 / 4}$ \\
\hline 9 & 92 & I & 2 & I & 1 & $2: 091 / 4$ \\
\hline IO & 6 & I & 3 & 3 & 3 & $2: 25$ \\
\hline II & 37 & 14 & 212 & 82 & 357 & $2: 293 / 4$ \\
\hline 12 & 20 & 4 & 19 & 9 & 26 & $2: 29$ \\
\hline I3 & 47 & I & I & 6 & 9 & $2: 26$ \\
\hline 14 & 5 & I & I & 3 & 3 & \\
\hline I 5 & 47 & 6 & 12 & 4 & 8 & $2: 263 / 4$ \\
\hline 16 & 31 & 9 & $3^{I}$ & 12 & 17 & $2: 21 \frac{1 / 4}{4}$ \\
\hline 17 & 30 & 7 & 25 & 5 & 12 & $2: 273 / 4$ \\
\hline IS & 46 & 6 & 40 & 3 & 5 & $2: 16 \frac{1 / 4}{4}$ \\
\hline
\end{tabular}




\begin{tabular}{|c|c|c|c|c|c|c|}
\hline & I & 2 & 3 & 4 & 5 & 6 \\
\hline & $\begin{array}{l}\text { Performing } \\
\text { Get }\end{array}$ & $\begin{array}{l}\text { Sires also } \\
\text { Performers }\end{array}$ & $\begin{array}{c}\text { Performing } \\
\text { Get }\end{array}$ & $\begin{array}{c}\text { Sires not } \\
\text { Performers }\end{array}$ & $\begin{array}{l}\text { Performing } \\
\text { Get }\end{array}$ & Record \\
\hline 19 & 32 & I & 1 & 4 & 4 & \\
\hline 20 & 3 & I & 7 & I & I & \\
\hline 21 & 66 & 6 & 20 & 2 & 5 & $2: 171 / 4$ \\
\hline 22 & 19 & 2 & 8 & 7 & 10 & \\
\hline 23 & 65 & 5 & S & 2 & 2 & $2: 12$ \\
\hline 24 & 99 & 21 & $8_{5}$ & 5 & 9 & $2: 18$ \\
\hline 25 & 17 & 10 & $3^{2}$ & 13 & $2 S$ & \\
\hline 26 & 15 & 3 & 3 & 10 & I6 & \\
\hline 27 & 59 & 25 & 342 & 49 & 273 & \\
\hline 28 & $3^{6}$ & 5 & 9 & 5 & 17 & $2: 29$ \\
\hline 29 & $\delta$ & I & I & I & I & \\
\hline 30 & 9 & 4 & I I & 9 & I3 & \\
\hline $3^{I}$ & 6 & 2 & 6 & 4 & 7 & $2: 22 \mathrm{r} / 2$ \\
\hline 32 & 60 & 5 & 9 & 42 & I I 9 & \\
\hline 33 & 92 & I 5 & $s_{3}$ & 10 & 20 & \\
\hline 34 & 25 & 2 & 6 & I & I & $2: 191 / 4$ \\
\hline 35 & 45 & 10 & $5^{2}$ & 4 & I3 & $2: 121 / 2$ \\
\hline $3^{6}$ & 9 & I & I & 3 & 3 & $2: 28$ \\
\hline 37 & 4 & 3 & 22 & ${ }^{13}$ & 33 & \\
\hline $3^{S}$ & 55 & 2 & 10 & 4 & 10 & $2: 18$ \\
\hline 39 & I 7 & I & 7 & I 8 & 29 & $2: 22$ \\
\hline 40 & 14 & 2 & S & 4 & 6 & \\
\hline $4 I$ & $3^{8}$ & 7 & $8 S$ & $2 S$ & $6_{5}$ & \\
\hline 42 & 35 & 2 & 3 & 6 & 8 & \\
\hline 43 & $5+$ & 3 & 5 & I & 6 & \\
\hline 44 & 57 & 21 & $7 \mathrm{I}$ & 34 & I 89 & \\
\hline 45 & $5^{1}$ & 10 & 67 & 16 & 32 & \\
\hline 46 & 18 & 4 & II & 3 & 8 & $2: 23^{3 / 4}$ \\
\hline 47 & 16 & 4 & 13 & 7 & 9 & \\
\hline 48 & I3 & 2 & 66 & 10 & 45 & \\
\hline 49 & $\mathrm{~S}_{5}$ & 23 & 93 & 15 & 16 & \\
\hline 50 & 160 & 60 & 723 & 37 & 219 & \\
\hline $5^{I}$ & 33 & I & I & 4 & 7 & \\
\hline $5^{2}$ & 65 & I & I & I & 2 & $2: 251 / 4$ \\
\hline 53 & 4 & I & I & 4 & 5 & $2: 29$ \\
\hline 54 & 24 & I & I & I & 2 & $2: 28 \% / 4$ \\
\hline 55 & 6 & I & I & 3 & 8 & \\
\hline $5^{6}$ & 25 & 2 & 3 & I & 2 & $2: 15 \frac{3}{4}$ \\
\hline 57 & 22 & 3 & 7 & 3 & 4 & $2: 30$ \\
\hline $5^{S}$ & I 5 & 3 & 5 & II & 14 & \\
\hline
\end{tabular}




\begin{tabular}{|c|c|c|c|c|c|c|}
\hline & $x$ & 2 & 3 & 4 & 5 & 6 \\
\hline & $\begin{array}{l}\text { Performing } \\
\text { Get }\end{array}$ & $\begin{array}{l}\text { Sires also } \\
\text { Performers }\end{array}$ & $\begin{array}{l}\text { Performing } \\
\text { Get }\end{array}$ & $\begin{array}{c}\text { Sires not } \\
\text { Performers }\end{array}$ & $\begin{array}{l}\text { Performing } \\
\text { Get }\end{array}$ & Record \\
\hline 59 & IOI & I I & 43 & 19 & 68 & $2: 22 \mathrm{~T} / 2$ \\
\hline 60 & 20 & 7 & 19 & 10 & 15 & \\
\hline 61 & 15 & 6 & 14 & 26 & $8 I$ & \\
\hline 62 & 15 & 5 & 21 & 3 & $3 I$ & \\
\hline 63 & 4 & 2 & I I & 12 & $5^{2}$ & $2: 231 / 2$ \\
\hline 64 & 10 & I & 9 & Io & 19 & \\
\hline 65 & 83 & 40 & 1501 & 62 & 909 & $2: 22$ \\
\hline 66 & 4 & I & 2 & 17 & 33 & \\
\hline 67 & $3^{8}$ & 8 & 43 & 15 & 47 & $2: 201 / 2$ \\
\hline $6 S$ & 12 & 6 & 17 & 3 & 5 & $2: 271 / 4$ \\
\hline 69 & 75 & II & 85 & 16 & 33 & $2: 15 \%$ \\
\hline 70 & 40 & 8 & 174 & 142 & 2520 & \\
\hline 71 & 24 & 3 & 8 & 9 & 32 & \\
\hline 72 & I 5 & 2 & 4 & 9 & 19 & \\
\hline 73 & $2 S$ & 6 & $3^{2}$ & 2 & 3 & $2: 261 / 2$ \\
\hline 74 & 23 & I & 4 & 3 & 7 & \\
\hline 75 & I 5 & I & 14 & 4 & II & \\
\hline 76 & 23 & I & 5 & 4 & 6 & \\
\hline 77 & 46 & 8 & 74 & 9 & 40 & $2: 2 \times 1 / 4$ \\
\hline $7^{8}$ & 30 & 2 & 5 & 4 & 4 & \\
\hline 79 & 94 & 34 & I 55 & $3^{I}$ & 205 & \\
\hline So & 9 & I 5 & 96 & 29 & I 54 & \\
\hline Si & 4 & I & 6 & 14 & $3^{6}$ & $2: 29$ \\
\hline 82 & 5 & I & 22 & I & 8 & \\
\hline $\mathrm{s}_{3}$ & IS & 7 & 20 & 4 & 6 & \\
\hline $8_{4}$ & 10 & 4 & 9 & 2 & 2 & $2: 21$ \\
\hline$S_{5}$ & 20 & I & I & I & 4 & $2: 28$ \\
\hline 86 & 7 & 3 & 12 & 7 & 25 & \\
\hline 87 & $8_{5}$ & 14 & 172 & II & 12 & \\
\hline SS & 29 & 8 & 19 & 7 & 29 & $2: 211 / 2$ \\
\hline 89 & $3^{8}$ & I & 2 & 7 & 12 & $2: 161 / 2$ \\
\hline 90 & 8 & I & 12 & 6 & $S$ & \\
\hline 91 & 2 & I & 4 & 4 & 5 & \\
\hline 92 & $4^{I}$ & 5 & 43 & $2 I$ & 106 & \\
\hline 93 & 40 & 13 & 32 & 10 & IS & \\
\hline 94 & I I & I & 7 & 10 & I9 & \\
\hline 95 & 4 & I & 10 & 5 & 6 & \\
\hline 90 & 4 & I & 21 & 3 & 7 & \\
\hline $\begin{array}{l}97 \\
08\end{array}$ & 21 & 2 & $\begin{array}{r}7 \\
-S\end{array}$ & $\begin{array}{r}3 \\
12\end{array}$ & 4 & \\
\hline 80 & 31 & 5 & 20 & 12 & 42 & \\
\hline
\end{tabular}


PREPO'LENCY

503

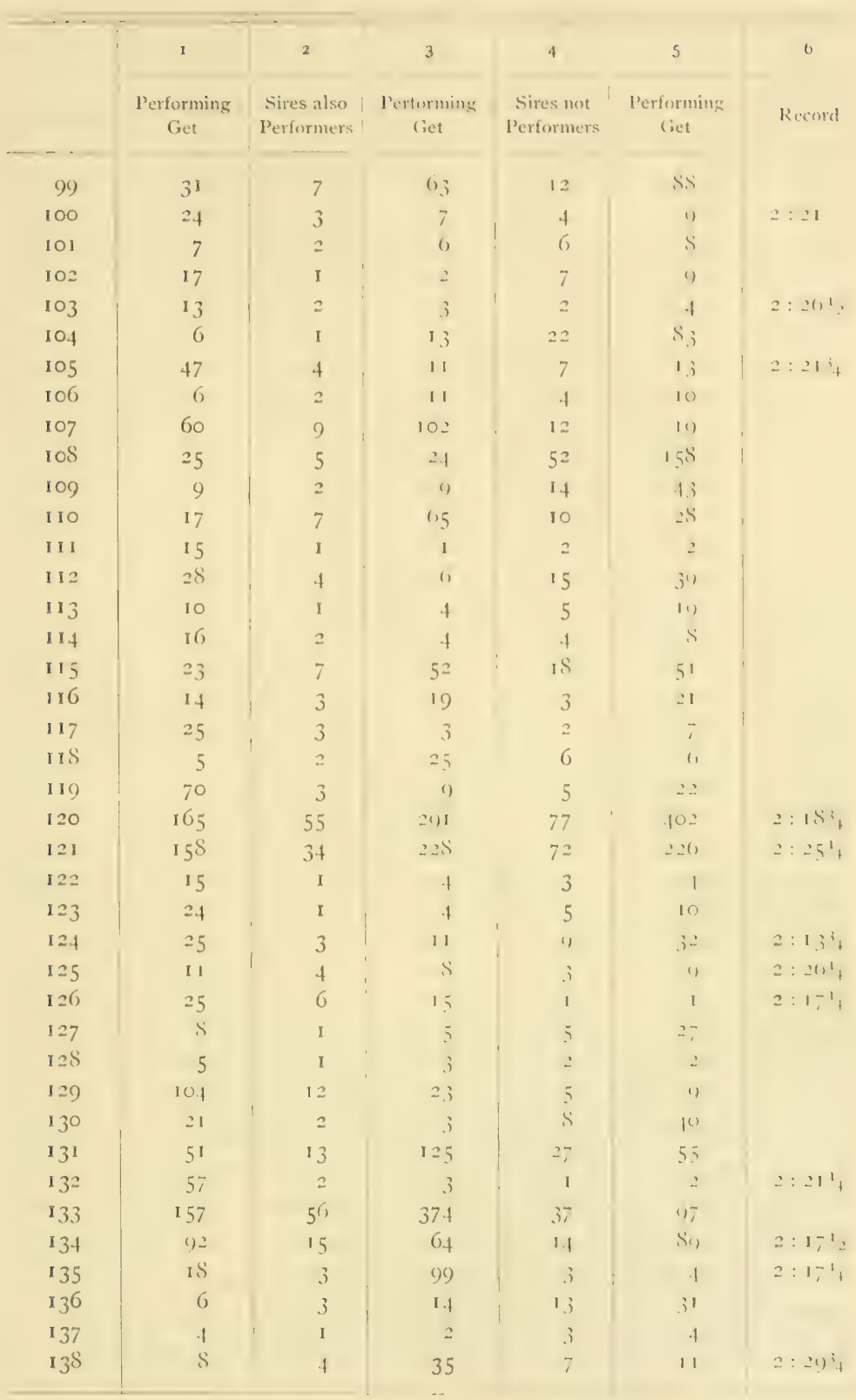




\begin{tabular}{|c|c|c|c|c|c|c|}
\hline & I & 2 & 3 & 4 & 5 & 6 \\
\hline & $\begin{array}{l}\text { Performing } \\
\text { Get }\end{array}$ & $\begin{array}{l}\text { Sires also } \\
\text { Performers }\end{array}$ & $\begin{array}{l}\text { Performing } \\
\text { Get }\end{array}$ & $\begin{array}{l}\text { Sires not } \\
\text { Performers }\end{array}$ & $\begin{array}{l}\text { Performing } \\
\text { Get }\end{array}$ & Record \\
\hline r39 & 96 & 9 & 32 & 6 & 8 & $2: 193 / 4$ \\
\hline 140 & 12 & 2 & 6 & 10 & 17 & $2: 15 \frac{1}{4}$ \\
\hline I 4 I & 4 & I & 2 & I & I & \\
\hline 142 & $8_{5}$ & 4 & 4 & 5 & 6 & \\
\hline 143 & 24 & 6 & 16 & 4 & 6 & \\
\hline 144 & 13 & 2 & 7 & I & 3 & $2: 26 \pi / 2$ \\
\hline I 45 & 34 & 3 & 28 & I & I & $2: 253 / 4$ \\
\hline 146 & $8 S$ & I 3 & 85 & 25 & 54 & \\
\hline 147 & $5^{2}$ & II & 78 & 10 & 20 & $2: 24$ \\
\hline I 48 & 42 & I & I & 4 & II & \\
\hline I 49 & 48 & II & $3^{S}$ & 27 & 55 & \\
\hline I 50 & 22 & 2 & 13 & 2 & 2 & $2: 27$ \\
\hline 151 & IO & I & I & 8 & 24 & $2: 221 / 4$ \\
\hline 152 & 15 & 3 & 5 & 9 & 73 & \\
\hline I 53 & I 5 & I & 2 & 7 & II & \\
\hline I 54 & $3^{I}$ & 7 & $4^{8}$ & 12 & 33 & \\
\hline I 55 & 34 & 5 & I 4 & 36 & 142 & \\
\hline I 56 & II & 3 & IO & I & 4 & \\
\hline 157 & 35 & 7 & 42 & 9 & 16 & $2: 19$ \\
\hline 158 & I & I & I & I & I & \\
\hline I 59 & 65 & 7 & 39 & 5 & 12 & $2: 241 / 2$ \\
\hline 160 & 10 & 3 & 82 & 9 & 21 & \\
\hline $16 I$ & 103 & I I & 28 & 2 & 2 & $2: 191 / 4$ \\
\hline 162 & 13 & 5 & $6 I$ & 20 & 105 & $2: 211 / 2$ \\
\hline 163 & $3^{8}$ & 4 & 10 & 2 & 3 & \\
\hline 164 & 45 & 7 & 27 & I I & 29 & \\
\hline \multirow[t]{2}{*}{ I 65} & $3^{2}$ & 2 & 3 & 4 & 6 & $2: 281 / 4$ \\
\hline & $5^{688}$ & 1062 & 7843 & I94I & 9186 & \\
\hline
\end{tabular}

What light, now, does this throw upon our query? We remember the disadvantage occasioned to the performing sire by the interference of track engagements during his racing years, and we remember, on the other hand, the advantage that comes from his enhanced reputation and the superior class of mares offered.

This table, taken as a whole, shows that these 165 leading stallions (being the ones of highest note that produced both performins and non-porformins sires) produced 5688 offspring and I7,029 grand offspring with track records of $2: 30$ or better. 
This is an average of 34.5 direct get, and the grand-get $(\mathrm{I} 7,029)$ covers two thirds of all in the list $(26,327)$. It is, therefore, the very cream of the breed. What, now, is the breeding record of the performing sires of this list as compared with that of the non-performing?

The non-performing sires (I94 I, column 4) are almost double the number of the performing sires (I062, column 2). These I94 I non-performing sires produced a total of 9 I 86 performers, - a ratio of 4.7 each; while the 1062 performing sires produced in all 7843 performers, - a ratio of 7.4 each.

If any difference in breeding powers is correlated with high speed, it would be reduced rather than exaggerated in this table, for the list of what are called non-performers clearly includes a good many potential performers that had the inherent ability to "go" if all conditions had been favorable.

At the same time it must not be forgotten that the non-performing sires on this list are of the same blood lines as are the performing sires, bingr in ciery casc at lact half-brothers ont of the same sire. ${ }^{1}$ To the writer the conclusion seems inevitable that the heavy difference of 7.4 against 4.7 apiece is in a large sense correlated with the individual ability to "perform."

Turning to indiridual cases, we find that the performing sires got by Geo. Wilkes (line 65) produced on an average 37.5 performers apiece ( $1501 \div 40$ ), while his non-performing sires produced an average of only 14.6 (909 $\div 62$ ), although the popularity of Wilkes' blood was enough to assure almost any son of his a "good chance."

Nutwood (line I 20), the greatest sire of speed, produced 55 performing sires and $77 n 0 n$-performing sires. The first produced at the rate of $5.3(29 \mathrm{I} \div 55)$ and the second at the rate of 5.2 $(402 \div 77)$, - almost exactly the same. Onward (line I 2 I) produced 34 performing sires and 72 non-performing. The first produced performers at the rate of 6.7 each, the second at the rate of $3 . \mathrm{I}$.

Hambletonian IO (line 70), the most successful producer of racing blood and the foundation of almost all modern blood lines,

1 The table is confined to those stallions that produced both performing and non-performing sires. 
produced only 40 performers and 8 performing sires, but Geo. Wilkes was one of these, and the average of $21.7(174 \div 8)$ tells but a small part of the story of these $\$$ performing sires of this remarkable progenitor of speed. His $I \neq 2$ non-performing sires produced speed at an average of 17.7 each. It would be an interesting study to determine how the descendants of these I 42 non-performing sires compared with those of the 8 performing sires, - a study that the writer has left to others ${ }^{1}$ or to a future time.

A conservative conclusion from these clata would be that performance is not an invariable inclex of breeding powers but that on the average the performers are much more likely to get speed than are non-performers of the same breeding.

This difference, if it really exists, is without doubt inherent; indeed, it is not difficult to find instances in which that which seems to be the greneral principle is reversed, so that the nonperfomors are the bitter brecters (see lines 1, 44, 62, 79, I 30 , and I 55); all of which shows that while good negative testimony may be found in a single instance, positive statements must be based upon a comprehensive study of large numbers. And so we need to go through our records and our experience carefully, hunting for the things that constitute ground on which prepotency may safely be predicated. Without doubt purity of blood, in the sense of the highest possible perecnterge of characters farorable to the purpose disired, unalloyed by disturbing factors, will be found to constitute the real basis of prepotency. When discussing the mathematics of breeding it was found that, no matter what the combinations, a fe' individuals will always remain pure. By the same process of reasoning, when we mix the elements of clesirable characters, diluting them as little as possible with "wild blood," we shall, by the same law of probabilities, once in a while effect a phenomenal combination. Such a one is produced by methods not under our control, except as we incrase the probatility by incrasing the intensity of brecing. This is the very heart and soul of "line breeding," and means that the best-bred animal is the most likely to be prepotent. In

1 Work of this kind runs into days, weeks, and months, at a surprising rate. The data given here represent many months of laborious work. 
the meantime it will be well to remember that, to the best of our present knowledge, some individuals seem to be preëminently breeders of porformers; others, breeders of sires; and still others, breeders of dams; while a fiz' are breeders of all three classes.

Importance of the actual test. The student cannot fail to note that the bulk of the business of real improvement is done by a very few really great animals, and that the work of most of the so-called breeding stock is merely that of reproduction in the sense of increase of numbers.

It is perfectly clear that he who is bent upon accomplishing real results will seek for the occasional phenomenal breeder, and, having found him, will make the most of him while he is able to reproduce. It is matter of deep regret that so many of our phenomenal animals, like Hambletonian Io, were never recognized as such until long after they were dead and the opportunity to utilize them to the best advantage had passed forever, leaving us to do the best we can and make the most of the "accidents" that are left behind.

In seeking these phenomenal breeders too much cannot be said concerning the importance of the actual breeding test as the last and final criterion of breeding powers, - a subject on which more will be said later.

\section{SECTION II - PREPOTENCY IN SEX}

There is a traditional belief that in general the sire is prepotent over the dam. In actual practice this is likely to be the case, for the sire is, in most cases, the better bred of the two parents. If a breeder starts out to breed half bloods, and to give his stock the most benefit possible of good blood at the least expense, he will of course provide it through the male side; for with one male he can influence the blood of many offspring, while with the female he can influence but one in horses or cattle, and but few in swine. So it comes about, for purely economic reasons alone, that in general sires are better bred than dams, and on this account should be prepotent.

But, the question of breeding aside, are they prepotent because of sex? On this point speculation has long been at work, 
resulting in a choice collection of "beliefs," covering about all the combinations possible. It is held:

I. That the male is prepotent on greneral principles, because males are stronger and more virile than females.

2. That the female is prepotent, especially among mammals, because her associations with the offspring are so much more intimate, both physiologically and socially.

3. That that parent is prepotent which has the stronger nervous and sexual organization, - whatever that may mean.

4. That the male is prepotent over the forward and upper parts of the body and the mental qualities.

5. That the exact reverse of the last statement is the truth.

6. That the male governs the external and the female the internal organs and parts.

Instances are not wanting to "prove" any of these beliefs; indeed, proof by the method of instance is the favorite form of argument for or against any one of them, and it is not too much to say that by this method these or any other assumptions may be readily substantiated.

We have learned long ago the unreliability of conclusions of this kind, and it is worth while to distinguish clearly, so far as we are able, between what is known and what has not yet been learned touching this important matter.

In general the sexes are equipotent. So far as is now known, no part of the germ cell is naturally predestined to provide any particular part of the body. The germ cells from both parents are bearers of the hereditary substance in the proportion in which they possess it, and either sex may and does transmit any and all the characters of the race to its offspring of either sex. We may say then, in general, that that parent will be prepotent whose hereditary substance is least mixed and, therefore, most intensitied along the line of established characters. The only way we can go farther than the general principle just stated is by extensive studies solving the cocfficiont of horedity between each parent and its offspring of both sexes for different characters separately.

This has been done for a number of characters, both in men and in animals, though the list is too small to do more than to 
indicate the direction, without showing the limits, of prepotency.

The following list from Pearson ${ }^{1}$ includes the most accessible data covering this point. Unfortunately again, much of the material is drawn from studies in man, but fortunately also horses and dogs have been included to some extent. It is all useful in showing the mixed nature of prepotency.

\section{'Table illustrating Prepotency of Sex}

\begin{tabular}{|c|c|c|c|c|}
\hline & ReLationship & MATERIAL & Сharacter & $\begin{array}{c}\text { COEFFICIENT } \\
\text { OF } \\
\text { HEREDITY }\end{array}$ \\
\hline I & Father and son .... & English ....... & Stature ... & .396 \\
\hline 2 & Father and daughter. & English ....... & Stature ... & .360 \\
\hline 3 & Mother and son .... & English ...... & Stature ... & .302 \\
\hline 4 & Mother and daughter. & English ...... & Stature ... & .284 \\
\hline 5 & Mother and son .... & American Indians . . & Head index. & .370 \\
\hline 6 & Mother and daughter. & American Indians .. & Head index. & .300 \\
\hline 7 & Sire and foal ...... & Thoroughbred horses & Coat color. . & .517 \\
\hline 8 & Dam and foal .... & Thoroughbred horses & Coat color. . & .527 \\
\hline 9 & Sire and offspring. . . & Basset hound.... & Coat color. . & .177 \\
\hline ro & Dam and offspring ... & Basset hound.... & Coat color. . & .52 .4 \\
\hline II & Brother and brother. . & English ....... & Stature ... & $39 \mathbf{I}$ \\
\hline 12 & Colt ${ }^{2}$ and colt .... & Thoroughbred horses & Coat color. . & .623 \\
\hline 13 & Sister and sister . . . & English ....... & Stature ... & 444 \\
\hline 14 & Filly ${ }^{2}$ and filly .... & Thoroughbred horses & Coat color. . & .693 \\
\hline I 5 & Brother and sister... & English ......... & Stature ... & .375 \\
\hline 16 & Colt and filly..... & Thoroughbred horses & Coat color. . & .583 \\
\hline
\end{tabular}

Here, in small compass, are results of studies sufficiently extensive to justify careful consideration. The following conclusions are certainly warranted :

I. The English father is prepotent over the mother in respect to stature in both sexes (see lines $\mathbf{I}, 2,3,4$ ); but the reverse is true as to coat color in thoroughbred horses and in Basset hounds, especially in the latter (see lines $7,8,9,10$ ). The conclusion of all this is that sometimes one sex is prepotent and sometimes the other, and, accordingly, that each character must be worked out by itself and for each race separately.

1 Pearson, Grammar of Science, pp. 458, $46 \mathrm{r}$.

$2 \mathrm{By}$ "colt" is of course meant a male foal, and by "filly" a female foal. 
2. To quote Pearson, the male secms to "inherit more" than the female because his coefficient of heredity is higher, with whichever parent the comparison is made (compare lines I, 3, 5, with lines 2, 4, 6). This conclusion Pearson declares is confirmed by data in eye color, as well as in stature, coat color, and head index. ${ }^{1}$

It is a significant fact that for the races and characters here involved the correlation between brother and brother is less than the correlation or similarity between sister and sister (compare lines I I and I 2 with lines I 3 and I4), which means also that sisters resemble each other more closely than do brothers.

3. The resemblance between members of the same sex is closer than that between members of opposite sexes (compare lines I I and I 3 with line I 5 ; also I 2 and I 4 with I6). Pearson also declares that the same principle holds for eye color and head index, and he is inclined to believe it general. ${ }^{2}$

This author points out that this principle, if general, means that "inheritance in a line through one sex is prepotent over inheritance in the same degree with a change of sex" ; that is, that inheritance tends to run in sex lines, which means, to quote Pearson (italics and parentheses mine), "that a man in eye color (for example) more clearly resembles his patcrnal than his matcrul grandfather (or other male ancestors); a woman more closely resembles her maternal grandmother than her paternal grandmother. Again, a nephew is more like his paternal uncle than his paternal aunt; a niece, like her maternal aunt than her maternal uncle." "3

Future investigations will add to our knowledge in these matters, and perhaps modify some general statements now considered safe, but the matter as stated above represents the best conclusions of those who have given most careful attention to the subject up to the present time.

Comparative variability of the sexes. There has been a general tendency to assert that males are more variable than females. ${ }^{+}$This assertion has not been based on actual studies

1 Pearson, Grammar of Science, p. 459.

2 Ibid. p. 459 .

3 Ibid. p. 460.

4 See Geddes and Thomson, Evolution of Sex, pp. 12-13; Darwin, Animals and Plants under Domestication, I, 457 ; Pearson, Chances of Death, pp. 256-260. 
but upon the theoretical ground that males lead a more active life and take the lead in sexual selection. The data just cited seem to substantiate this assertion. For the species and characters involved it appears that male offspring follow more closely the parental type than do the female, and (which amounts to the same thing) female offspring, or sisters, are more nearly alike than are male offspring, or brothers, - tending to the conclusion that males are more variable than females.

Pearson, ${ }^{1}$ however, records data of an exceedingly exhaustive series of investigations of variability in men and women, not absolutely but relatively, as expressed in the coefficient of variability. ${ }^{2}$ While he finds men more variable at certain ages and in certain characters, yet he does not find pronounced and decided differences, nor are these the same for different races of men. He concludes, on the whole, that for all races studied, ancient and modern, and for all characters covered by the studies, there is "no eridence of greater male variability, but rather of a slightly greater female variability."

He finds, for example, that English men are slightly more variable as to height than are English women (4.07:4.03), but that, among Germans, women are considerably more variable than men $(4.26: 4.02)$, as they are also among the French $(4.35: 3.88)$.

In the long bones sometimes one is more variable, sometimes the other; thus, as to the femur, men are more variable: Libyan (5.05:4.46), French (5.05:5.04), Aino (4.65:4.I8), and Neolithic man $(4.73: 4.5 \mathrm{I})$; but with the ancient inhabitants of the Canary Islands the reverse is true (men, 4.64; women, 4.7 I). In all cases examined, except the French (men, 4.975 ; women, 5.365 ), the tibia is more variable in men; but in most cases the humerus and radius are more variable in women.

1 Pearson, Chances of Death, I, 256-377.

2 Manifestly the coefficient of variability is the only correct estimate of comparative variability, because in its calculation each instance is compared with its own type as a base. This is necessary, because the stature of women, for example, is different from that of men; hence the two cannot be compared on any common basis. This is the only way, for instance, as Pearson points out, in which we can compare the variability of man with that of the elephant; in any other way the elephant would appear more variable, because he is bigger. 
The averages of coefficients of variability for all "long-bone" determinations are as follows : ${ }^{1}$

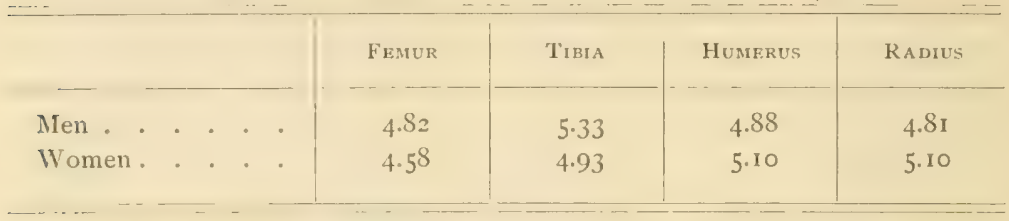

Though these are human, not animal data, yet they involve skeletal measurements which are among the most fundamental of all organic parts, and they scarcely warrant the sweeping assertion that males are decidedly more variable than females. Pearson is entirely justified in his protest against what he calls this "pseudo-scientific superstition" and the sweeping conclusions involving "social and practical consequences" affecting "the whole of our civilization." 2

In body weight, both among English (men, I0.37; women, I 3.37) and among Germans (men, 20.67 ; women, 25.07), women are decidedly more variable than men. ${ }^{3}$

In weight at birth, both among English (boys, I 5.65 ; girls, I 4.44) and among Germans (boys, I 3.567; girls, I 3.278), the males are more variable; but among the Belgians the reverse is true (boys, I4.66; girls, 17.62).

Data of this sort are full, but unfortunately confined mostly to humans. From all sources it seems that men are more variable in "height when sitting" and in "swiftness of blow," but that women are more variable in "stature" (height when stending), "span," "body weight," "breathing capacity," "strength of pull," "squeeze of hand," and "keenness of sight."

As Pearson points out, some of these variants would disappear if women were subjected to the same conditions of life as are men, and we need to be cautious when applying these data to races in general, - for which future researches are sorely needed. We certainly are not warranted in assuming sweeping and fundamental differences in variability between the sexes. Here again

1 Pearson, Chances of Death, I, 305.

2 Ibid. I, 256 and 376 .

${ }^{3}$ Incidentally, the same data warrant our conclusion that the German race, both men and women, are more variable than the English as to body weight. 
is fertile territory for careful and exhaustive statistical studies, which alone will yield reliable results on which principles of selection and breeding may be based.

\section{SECTION III - INFLLENCE OF AGE ON PRLPOTENCY}

Is one parent prepotent over the other merely by reason of age? The question is exceelingly important, but the writer is not aware of reliable data bearing upon the subject. The matter could be determined by sufficient investigation into the offspring from parents with some considerable discrepancy as to age, and by comparing the coefficients of heredity between the offspring of young and those of old parents, not only with each other but with the normal of the race. Helpful as it would be to know the facts upon this point, they have not yet been discovered and it is idle to speculate. We have no choice but to wait until the research is made in what will one day constitute a prolific field for study.

\section{SECTION IV - INFLUENCE OF CONSTITUTIONAL VIGOR UPON PREPOTENCY}

This is an important question, upon which we lack reliable information. Common sense seems to inclicate that the more weakly parent would not be equally influential in impressing his or her characteristics, but we cannot yet say to what extent the character of the reproductive cells is dependent on vigor. Here again speculation can easily run riot; but from the fact that, for other reasons, we should reject the non-vigorous parent, the question loses most of its point except in human affairs, which do not concern us here.

That one stalk in a hill of corn often resists the effects of frost when neighboring stalks are killed is a fact that has long been noted, but whether such plants are prepotent in transmitting resistance to frost is not known. It is a significant fact, however, that Dr. Hopkins, of the University of Illinois, when conducting experiments with soils containing an excess of magnesium, noticed one year a single wheat plant that flourished well where all others succumbed. Saring seed from this plant, 
he found its descendants highly resistant, flourishing well where ordinary wheat totally failed. It was, apparently, a true mutant, with extra strong resistance to magnesium.

Influence of development upon prepotency. Many biologists contend that transmission depends to a large extent upon the derclopment of the parent; that a stallion trotting bred, for example, would get speed much more successfully if he himself were "developed" or "worked" upon the track than would the same stallion kept equally healthful and vigorous but not developed as to actual speed. A natural conclusion of this contention is, of course, that the same sire will get more speed in his middle and later years than would be possible before he was developed.

This is the very point of Casper L. Redfield's recent articles ${ }^{1}$ setting forth what he calls his "dynamic theory of heredity." He brings many instances and much argument in support of the assumption, but in the opinion of the writer the method of proof adopted is not competent to settle the question, nor is any method able to do so that is based upon the simple enumeration of instances.

As with any other question involving great variability, the only way we can settle this is by employing large numbers on both sides of the proposition; in other words, by comparing the speed of all the horses gotten by performing sires late in life with the records of the get of the same sires before development, or at least before long service on the track. Even then we must learn what deductions to make, if any, on account of differences in age; after which, we may hope to learn the real effect of development upon prepotency.

As the matter stands now, the fact of prepotency is patent to both the casual observer and to the careful student; but the reasons for this difference in breeding powers are not yet at all well understood. Here, as in many other directions, we await future studies.

Summary. Individuals of the same ancestry differ marvelously in their breeding powers. Some can produce excellence directly in their own descendants, and others indirectly through

1 See The Horseman, XXV, Nos. 19-41, on "Breeding the Trotter"; also The American Field, LXII, No. 25, and LXIII, No. 9, on "Evolution of the Setter." 
sires and dams they are able to get. The line of descent runs only through the few that can produce brecders of brecders, not simply performers.

Individual excellence is not a certain guide to breeding powers, and many ordinary individuals are among the greatest breeders. This is neither a mystery nor a fault in heredity; it arises from the fact that indiviclual excellence is partly a matter of individual deielopment and not a sure index of real ancestral possessions. The specimen may be only fairly well born, though faultlessly developed, - in which case he will probably be a disappointment as a breeder; or he may be excellently born, but only fairly well developed, - in which case he will breed "better than he is himself" ; still again, he may be well born and perfectly dereloped, which is best of all.

All studies yet made show that, on the average, performers (those individuals possessing high individual merit) are better breeders than non-performers; that is, than those which do not show in their personal development a high degree of excellence, though, as we should at once surmise, there are numerous exceptions, largely arising from our inability to accurately judge individuals by external appearances.

\section{Special Exercises}

Work out special cases of prepotency in the breeding and speed records of trotting and running horses, in the advanced registry of cows, and in the famous families of beef cattle and of swine. Pay special attention to relative prepotency of own brothers and to the correlation between individual performance and breeding powers.

\section{ADDITIONAL REFERENCES}

A Measure of Intensity of Transmission. By Francis Galton, I 899. Nature, LX, 29.

Distribution of Prepotency. (Trotting-horse records.) By Francis Galton. Nature, LVIII, 246-247.

Influence of Sex on Size of Offspring. By F. B. Mumford. Experiment Station Record, XV, 542.

Prepotency and Xenia. By C. Correns. Experiment Station Record, $\mathrm{XI}, 1016$.

Prepotency of Different Plants. By W. W. Tracy: Experiment Station Record, XIII, 324. 



\section{Part IV - Practical Problems}

\section{CHAPTER XVI}

\section{SELECTION}

We have just seen the power of selection to fix type, providing it continue unchanged for five or six generations. By this we discover that selection is the most direct and powerful means of improvement at the disposal of the breeder; incleed, it woukd not be too much to say that it is the only means of permanent improvement that is under his direct control.

In most phases of the breeding problem the stockman or the plant improver is an on-looker merely; but in the matter of selection he becomes an active agent, and his decisions and his acts are powerful either for good or evil in controlling the destinies of the breed or the variety he handles.

In this, to a very large extent, he supplants natural selection, and if he is to succeed he must be well grounded in four fundamentals when he thus takes a hand in the course of nature:

I. He must have a clear idea of what he desires to accomplish, and he must adhere persistently to one standard.

2. He must be well informed as to the history of the breed or the variety he handles and of the variations, both new and old, which it is likely to afford.

3. He must know the general principles involved in selection in order to know the forces with which he deals and what is likely to happen when he interferes.

4. He must have judgment as to when and how far he may depart from sound practice on account of economic or other considerations.

When we reach this phase of breeding operations we begin to touch financial as well as biological principles, and all this must 
be dome with reference both to what is desirable and to what will pay; hence the necessity of considering all phases of the selection problem from a clouble standpoint. Each consideration outlined is self-evident, yet each is of sufficient importance to merit further consideration.

\section{SECTION I - IDEALS IN SELECTION}

Among the multitude of variations which every breed and every variety will present, the breeder must know which are useful. The great mass must be discarded, from the mere point of numbers, and no one cause of failure is more common than a vacillating policy regarding standards of selection.

This uncertainty is due to no other fact than that the breeder does not know quite what he wants. He is "in the market" for "any good thing" that may turn up. In the course of his breeding operations a great many new and more or less promising things will appear. Unless he has unlimited means and boundless space for his operations, these must be discarded with seeming ruthlessness, or he will speedily have an assortment of novelties which if bred among themselves will overrun his premises, and if bred into his permanent stock will produce a reritable jumble, out of which no good thing can come. In this way ancestry and pedigree can become so hopelessly mixed as to be worthless. This may happen with any breed, and even within the limits of purity of blood; indeed it has happened over and over again, in all breeds, through the misguided enthusiasm of breeders working without well-defined standards.

Standards wisely fixed. Standards must not be left to chance. They must not be warped or altered by novelties, no matter how curious or attractive. They must be fixed in advance, like building plans and specifications, and should be fixed in the light of what is ncided and what the bred is likcly to affort. Indeed, the standards should be roughly fixed before the breed is chosen.

Once chosen, standards should be preserved unchanged. As the artist sees his picture before he mixes his colors, and as the sculptor chips away at his marble to bring out the particular figure that stands in his mind, undisturbed and undissuaded 
from his purpose by the many oflurexcellent figures that might be cut from the same material, so the breeder should adhere to his standards doggedly. They should be wisely chosen, it is true, but, once sure of that fact, and with the law of ancestral heredity in mind, nothing should warp the judgment as to change. Everything that helps to secure the ideal should be accepted, and everything else, no matter how attractive in itself, should be pushed aside, - unless, indeed, the breeder have unlimited means and is minded to do not one thing but many things.

Keep blood lines pure. But if the breeder is minded to indulge in experiments outside the chosen standard, these experiments must be carried on separately. Blood lines must be kept pure, not pure within bred lines simply; but, remembering the law of ancestral heredity and the pull of the ancestors back of the immediate parent, they should be kept as pure as selection can make them.

Objects of selection. Indeed, while one object of selection is to reduce numbers, by far the larger object is to purify the ancestry', to the end that inheritance from all the ancestors shall be alike, so that the "pull of the race" shall not be different from the transmission of the immediate parent. This being so, selection according to vacillating standards is no selection at all, and he who returns from each state fair or exposition with n'a rather than improicel standards cannot hope to meet the highest success as a breeder or contribute real excellence to the breed he has chosen.

SECTION II - HISTORICAI, KNOWIEDGE OF THE BREED NECESSARY

This is almost self-evident, and yet the number of breeders who do not possess it, and the readiness with which large money is invested and plans made which will require a lifetime to carry out, - all with the most meager knowledge of the breed that is chosen, - show how lightly this matter rests in the minds of many otherwise intelligent and cautious business men.

These men proceed as if the material they were to work with were new material, ready to be molded for the first time into 
any desired form, while in truth it is very old material, with which many men have worked before, - sometimes for profit, often for amusement merely.

And the breed has inherited the results of all these experiments, both bad and good, so that this material is in some respects the better and in some the worse for what others have tried to do with it. The most ordinary business sense and the commonest biological principles indicate that before the breeder begins serious operations he should know all that can be known of the breed or the variety he proposes to work with. In no other way can he make intelligent selection.

A few crude examples will suffice to illustrate. Many breeders of English strains of cattle will not only destroy a white calf, but will consider its appearance an evidence of impurity of blood, not knowing that these breeds in general have descended from the wild white cattle of Great Britain. It is only recently that white has been restored to favor as a good Shorthorn color.

The Berkshire swine are the result of a cross of the large English hog with the small, thin-haired, plum-colored Neapolitan, and more than one Berkshire herd has been ruined by selecting for breeding purposes the plump, quick-maturing, fine-haired, and most attractive pigs.

A famous breeder of Kentucky was remarkably successful with Shorthorns, yet in his efforts to secure a high head and a low brisket he forgot the natural wild type, and speedily his breeding came to be known by its sloping rump and "split quarters."

The breeder should know the peculiarities of his blood. The first information needed by the prospective breeder is a good knowledge of the inherent fanlts of the breed. Ile needs to know, for example, that the Berkshires are naturally deficient in the hams, and the Poland-Chinas in the shoulders; that the Duroc Jerseys are uneven in type, and the Chester Whites a bit coarse in the bone. Ile needs to realize that the Jersey is sometimes extremely delicate; that many of the Holstein-Friesians are rough, and that the breed is preeminently short-tailed, - hence the provision in the scale of points that the bone of the tail should reach the hock, which was but rarely the case in the foundation stock of the breed. 
The breeder of Shorthorns should know in advance that it is a breed not of one type, but of many types, of varying degrees of excellence. He who expects to breed Herefords should know at once that the breed is one of two types, so distinct as to be almost dimorphic. The Angus breeder should not be surprised at some failures, or at a red specimen, nor the Galloway breeder at considerable roughness, or at the occasional appearance, without warning, of more or less white.

Breeders of the Percheron should know that, of all modern breeds, this retains the most of the Arabian infusion due to the crusades, and that until recently he was a small not a heavy horse, hence his "bone" is to be carefully looked after.

These and a mass of other breed peculiarities, both good and bad, should be fully in the mind of the breeder. Of course most of the advocates of any breed will stoutly resent the slightest implication of faults in their favorites, yet the fact remains that the really able and successful breeders know very well that they must be constantly upon their guard against certain happenings which may be called "faults" or "peculiarities" according to the definition of terms.

\section{SECTION III - GENERAL PRINCIPLES INVOLVED IN SELECTION}

When the breeder determines which individuals shall and which shall not reproduce, he must do it with all the intelligence possible, and with a full knowledge of all that is involved in his decision, which is most far-reaching and irrevocable in its consequences. It is the purpose here to outline some of the principal considerations that should be in mind when these decisions are made.

The purpose of selection. Primarily the purpose of selection is to reduce numbers, or to influence type (whichever way we please to put it), but in the last analysis it is to prevent the birth of unwelcome individuals not suited to the purposes of man; and by as much as the breeder is able to forecast offspring, by so much is he able to surround himself with good and serviceable individuals without resorting to wholesale slaughter 
after birth, with its attendant losses. In all theory we would prevent the birth of umprofitable individuals, and we succeed nearly in proportion as we are skillful in selection.

Selection results in absolute increase in quality, not merely in an elevation of the average by eliminating the lower values. We have been told that selection results only in raising the average by cutting off the lower values, but that the upper values are not influenced thereby. This is clearly an error, as will be seen by a reference to any systematic breeding experiments and especially to the tables giving the results of selecting corn for high or low protein or high or low oil. ${ }^{1}$ Here it is seen that, in the progress of selection, by the use of successively incrasing standards, wie and highthr adues constantly' afpearad. Not only that, but the principle is still operative after ten years of selection, and the cocfficicut of icrriblitity is not, in most corses, growing less. ${ }^{2}$ In general it may be said that the result of systematic selection is to shift the type but not greatly to reduce variability, and when applied to a number of characters at the same time it very clearly and very rapidly defines the type of the strain or breed.

In breeding the beet for sugar, the cow for milk, the horse for speed, or any animal or plant for any definite quality, there is every reason for believing that we have succeeded in producing a higher order of excellence than ever arose spontaneously in the race while in a state of nature; that is to say, we have done more than to raise the avcragc, we have elevated the upper limits.

The upper limits of improvement. Manifestly this increase of quality cannot go on indefinitely. We cannot breed the horse to be as large as the elephant; or, if we could, there will be an upper limit somewhere. What will set these limits is an interesting question. In some cases, no doubt, the limit would be fixed by purcly mechanical principles," in others by physiological restrictions, such as the size of the heart and the labor of

1 See pages 494 and 496 .

2 Ibid.

${ }^{3}$ For example, there is a mechanical limit to the length of leg, or to the size of udder. 
circulating the blood; but apparently we have not yet, in any line, approached a limit so high that variation is not abundantly able to present still higher values. How long this may go on is a question both of scientific and of utilitarian interest, but it will be remembered that variation is supposed not to be reducible below some 85 or 89 per cent of its original amount. ${ }^{1}$

Selection for definite purposes often against valuable qualities, especially fertility, ${ }^{2}$ vigor, and longevity. So intent are we upon securing some coveted character, as early maturity, size, milking or feeding quality, that we orerlook other less visible but none the less essential qualities. This is best seen among our meatproducing animals. For example, it is the heary-fleshed, earlymaturing sow pig that finds her way to the prize ring and ultimately to the fashionable breeding pen. Now this is not the most prolific type of swine, and under this policy of selection, primarily for flesh and fat, it is not surprising that, of all our animals, those bred for meat production are lowest in fertility. We know of no fundamental reason why it must be so ; it simply is so because fertility has been so generally neglected in the exclusive standards and methods of selection employed.

"Fertility," "vigor," and "longevity" are all relative terms. All animals and plants have some degree of vigor, and nearly" all are able to reproduce, at least to some extent. The evils on this score arise not from the non-breeder, or the individual that succumbs in early life, but from those individuals which, though not entirely wanting, are yet deficient in those fundamental qualities that are of necessity correlated with propagation of a rigorous, lasting, and prosperous race. It is the "shy breeder" that comes to nothing, and that is the root of many of the evils of the breeding herd.

The relative values of prolific and of shy breeders may be brought out by comparing three cows, for example, one of which will produce two calves before she stops breeding, another four, and another six. After five generations the fertile femalc descendants of each would be as follows, assuming that one half

1 Pearson, Grammar of Science, p. $4 S_{3}$.

"The word "fertility" is used in preference to "fecundity" because the latter term refers especially to females. 
the calves are males and one half females, and that all descendants are prolific in the same proportion as the originals.

Number of Living and Producing Females at the End of Various Generations, from Cows of Different DEgrees OF Fertility

\begin{tabular}{|c|c|c|c|c|c|c|}
\hline \multirow[b]{2}{*}{ Cow No. } & \multirow{2}{*}{$\begin{array}{l}\text { Total } \\
\text { Calves }\end{array}$} & \multicolumn{5}{|c|}{ Fentales } \\
\hline & & $\begin{array}{c}\text { First } \\
\text { Generation }\end{array}$ & $\begin{array}{c}\text { Second } \\
\text { Generation }\end{array}$ & $\begin{array}{c}\text { Third } \\
\text { Generation }\end{array}$ & $\begin{array}{c}\text { Fourth } \\
\text { Generation }\end{array}$ & $\begin{array}{c}\text { Fifth } \\
\text { Generation }\end{array}$ \\
\hline I & 2 & I & I & I & I & I \\
\hline 2 & 4 & 2 & 4 & 8 & 16 & 32 \\
\hline 3 & 6 & 3 & 9 & 27 & $8 \mathrm{I}$ & 243 \\
\hline
\end{tabular}

It is easy to see that no matter what the individual excellence of cow No. I and her descendants, they could neier build up a herd. Their rate of reproduction is so low as only to keep good the original number. Careful search will cliscover a surprising number of females of this class in the herds of otherwise successful stockmen, - useless from any standpoint except the show ring.

On the other hand, cow No. 2 and her descendants produce at a rate that will not only keep their numbers good but will admit of selection, and this is the case to a greater extent with No. 3, whose descendants in the fifth generation would be no fewer than 243 as compared with 32 for No. 2 and I for No. I. It is easy to see that one such cow as No. 3 in a herd of 20 like No. I would in a few years, by very breeding powers, clominate the herd, at the same time affording generous numbers for selection, whereas the descendants of No. I would afford no opportunity whatever for selection. It is clear to the most castual student that which our stunderds are docidcdly agrainst the highest fortility they are dangerous, if not fortal, to the race.

Need of comparatively large numbers in breeding operations. Obviously, comparatively larege numbers are necessary in order to provide selection with material sufficient for securing uniformity 
in type. Enthusiastic amateurs have of ten attempted to maintain a "small herd of exceptional excellence." Such attempts have always failed, and must fail, for the reason that such a herd affords too little material for selection, and therefore the impossibility of maintaining its type is a bar not only to progress, but even to the bare maintenance of the initial excellence. Suppose, for example, that a small herd of exceptional animals be brought together, - say, three cows and a bull, the choice from many of the best herds. What are the mathematical probabilities of their being able to reproduce their own number of equal excellence before the original herd disappears? It is very slight indeed, unless one of the number proves to be a phenomenal individual breeder, which, in truth, occasionally happens.

Value of the exceptional breeder. The more the matter is studied the more it will be found that the crecllone of any herd or of any breed is sustained and advanced, not by the general mass, but by a faw exceptional, not to say phanomenal, brciders.

The trotting blood of to-day owes its high development to a very few foundation animals, coming to us through Hambletonian IO, and sustained and developed by an insignificantly small percentage of the general mass of stallions. ${ }^{1}$

The excellence of the Shorthorn is maintained in the same way, and it is not too much to say that in all probability there are never living at any one time in any breed more than a score or so of animals that produce anything like a real and positive advance in the breed,, - "The line of descent runs not through their veins."

The exceptional breeder not necessarily the exceptional individual. Neither Hambletonian 10 nor Geo. Wilkes was the best trotter in the breed; indeed, the highest performers have contributed little. They were the offputs, but acciclentally, or of necessity, they were not of the main line of descent. In the corn-breeding experiments already cited, the ear to which the present high-protein stock all traces a'crs not one of the originally' highest protion cars. The great sires and the great dams that

1 See chapter on "Heredity," table of the Big Ten, p. 555.

2 This number is too high. The Shorthorn breed probably never saw twenty such bulls as Champion of England. 
have contributed most to their breeds have often been inconspicuous as individuals and, unfortunately, often have been dead long before their real service to the breed was known and recognized.

Need of the actual breeding test. So valuable is the exceptional breeder, and so impossible is it to know him (or her) in advance by the ordinary methods of judgment, that only the actual breeding test is reliable. The only safe method is to select the herd of females of high fertility and uniformly excellent breeding record, and then, knowing the female side by long and intimate experience, ${ }^{1}$ sclect the sire for his performance record in getting young.

The tests should first be made with a few well-known, and therefore fairly aged, females. Too much cannot be said against the practice of putting a new joung sire at once into full scricice in the herd, no matter ahat his indiaduality or his pedigrec. However promising, he must be subjected to the actual test, and after having proved his breeding powers with known females he shonld be uscet to the utmost as long as he aill bred successfully, and not discarded because of loss of bloom, decline in form, or even for the acquirement of an evil disposition. It is from the proved patriarchs and from the grandmothers of the herd that real excellence will come, and the real value of proved breeders, male or female, is beyond computation. ${ }^{2}$

Install the successor early. It is never too early to seek a new head to an established herd. Proved sires are seldom for sale, and the only recourse for the breeder is to prove his own; indeed, what he needs is a sire that will produce well with his females.

It takes much time and often many trials to find a worthy successor to the head of the herd. Putting it off too long, and a feeling of fancied security, are the two causes of leaving a

I A complete breeding record should be kept of each female separately. See chapter on "Animal Breeding."

2 The author has a mass of data collected from hundreds of breeders, from which it appears that young bulls are commonly preferred because they are cheaper, because their period of service is longer, and because they are more manageable. It appears too that when a "test" is made it is commonly not upon old and known cows, but upon heifers. 
herd without a head, and of the enforced evil practice of using an untried sire.

Comparative value of male and female. In the matter of prepotency, as we have already seen, neither parent has any particular advantage over the other. But this refers to a single offspring, and is only a part of the question. The real difference is one of numbers. Among animals the sire may produce perhaps a hundred in a season, while the dam is limited to one individual or at most (among hogs) to two litters. The trotting records show that certain sires produced literally hundreds of offspring in the list, but the greatest record ever made by any mare was that of Green Mountain Maid, who produced nine living foals.

For purely mathematical reasons, therefore, the female is of vastly less consequence in herd or breed improvement, - indeed, wherever polygamous mating occurs. It is here a question of numbers and opportunity. As regards these, the upper limit of the male is very high and of the female very low, which fact teaches the necessity of extreme care in the selection of the sire, not so much for biological as for numerical reasons. The single female is, therefore, comparaticicly insignificant. Unless she be one of the few phenomenal breeders her individual power for good is exceedingly low, and the readiness of many buyers to pay extreme prices for females, especially of cattle, is wholly unaccountable.

The sire more than half the herd. It has become a proverb that the sire is half the herd. He is far more than that. He is half of the first generation, three quarters of the next, seven eighths of the third, and so on until, if judicions scliction be maintained for a few generations, the character of the herd will be fuict by the sire alonc. This being true, the folly of maintaining a sire with but two or three high-class females is evident; he should have larger opportunity. All this means that, as a beginning, numbers are of more consequence relatively than quality on the side of the dam, and that if the breeder must choose between the two it is better to put a given amount of money into a good number of plain females than into a smaller number of high quality, but that in all cases the sire should have quality and plenty of it, because of the principle here stated. 
Size in the dam; quality in the sire. In many lines of breeding, sia in the sire is considered by many lowecelers as of first importance. This is aganst reatson and biological principles. IVe need in the sire all the desiablet chataters prosible, and these: are: most realily foumel in animals of medimm, not extreme, size. It is complatationly easy to sed size alome, and this can be gotten on the sicle of the diam. The herel must depend for aniformity laredy upon the sire, and he should be freed as moch as possible from the requirement of size. ${ }^{1}$

Natural selection always at work. Natural selection is always at work in field and flock and herd. Of this we may be well assureel. No matter what we desire fo atcomplish, our success or our failure will turn, in the last analysis, upon the fitness of the product to live and to reproduce and the conditions by which it must be surrounded.

Some of the best things amomg both plants and animals are weak of companatively umproblite. Nattual selection is decieledly agamst the stovival, mo matter how valuable they maly be to us. Lack of constitution or vigor is easily seen, but lack of

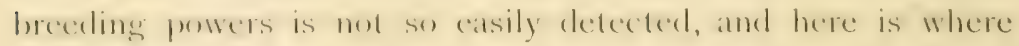
the greatest amount of trouble arises.

We have already seen that in nature the population that is

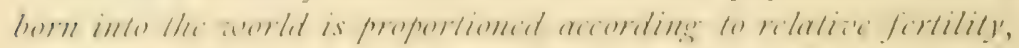
white the penplation that is permitled to remerin is eomelitioned

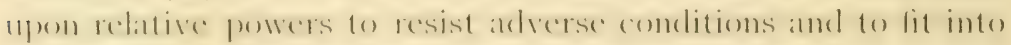
the conclitions of life.

This same: relation obtatus in our herds, with this difference, that ate, whth ont selection, decide athitraty what shall live. That is right and according to economic necessity, - only in doing so we must mot assume that all individuals and lypes are: equally fertile and equally able to propagate themselves.

It may be in certain instances that in order to secure what we desire we shall of necessity procedel temporatrily with some

$1 \Lambda$ manifest exception to this general principle is in the brecling of ciraft horses from farm matres. Ilere size is an oljection on the dam's side, besides being dificult to get. Weight is the chicf desiderattum just now (it will not always be so) in draft-horse breeding, and under present circumstances it must be sought especially in the sire. 
handicap as to fertility, and perhays as to vigor, in which rase these two essential qualities must be borne in mind and the deficiency remedied in future selections. The breeler is merer to forget that natural selection is at work side by side, or perhaps oacr, his best endeavors. In nature the prevailing type is a kind of resultant of the highest fertility and the best "fit."

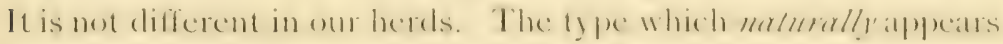
in our herds will be decided not only by our selections but by the relative fertility and vigor of everything present.

Examples are not wanting in which herds, and even whole families, have gene down becaluse of this unequal battle agamst the persistent influence of matural setertion. The nost motalle: instance is the "I Dule and Duehess" family amone Shomthoms, - most excellent inclividuals and true to 1yje, but mol sulficienty prolific to maintain themselves. So they went down and out, submeresel under the inevitable secree of natural selection that the unprolific shall die. The writer does not believe that this most exerellent family meed have heen lost to the heed had the breeders of the day been sufficiently alive to the situation.

This response to inequality in natural fertility of different strains is technically known as "genetic selection," and it is everywhere at work. It must be reckoned with in some form.

Physiological selection. (ieltain individuals are sterile: to carch other. It is a difficulty seldom encountered, but when it does occur it constitutes an effective bar to those particular blood

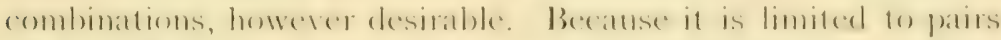
of individuals, its interference is occasional rather than com-

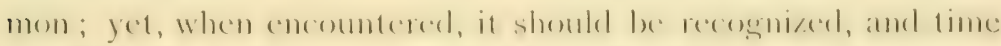
and expense avoided in attempts to overcome it.

Influence of age. Statisties show that at surprisingly latge proportion of sires are so young as to be clearly immature. The effect of this has been moch discussect, and the general opinion seems to be that breeding from immature animals is bad.

1 This family was always known to be "shy breeders." The writer well remembers hearing breeders say, "How fortunate that this is so, clse prices would net be maindainet." Happy would it have been could these same breeders have read their doom in time to save their pockets! They had ample warning, had they known how to read the handwriting on the wall. 
In truth we have little exact information on which to rely, but the writer seriously questions the correctness of this conclusion from the sterndpoint of the offspring. That breeding at an immature age checks the growth of females is next to certain, but it is also true that the heifer will make a better milker and a more certain breeder if bred before maturity and before functions other than milk production have become the prevailing habit of life.

That the progeny of immature animals is necessarily faulty is doubtful. In nature everywhere reproduction bigins bifore maturity, and in man at least it has been shown that the length of life of first children is, on the arerage, four years more than that of the latest born.

Considerations already adranced in connection with testing breeders necessitate the breeding at a comparatively advanced agre, and all things point to the conclusion that in practice breeding may begin early and continue as long as possible. Merely to gain time, if for no other reason, early breeding is to be advocated.

Blemishes and accidental injuries. Notwithstanding popular opinion, the breeding animal is none the worse for accidental injury; that is, so far as his or her breding value is concerned. The question is not whithre the mare is sparined, but what kind of a hock had she naturally, and had she sufficient occasion to be sparined. It is easy to make a bad showing and to say bitter things about the practice of breeding injured animals, but the evidence on inheritance all shows that injuries as such are not transmitted. This should not free the mind from the obligation to judge accurately as to whether the part was moturally perfect or naturally defective.

Difficulties in selection increase rapidly with the number of points on which selection is to be based. This purely mathematical consideration secms not to arrest the attention of breeders as it should. If we select for one point only we get ahead rapidly. That has been the advantage of the trotter. Speed was the only requirement, and while it involved many subordinate conditions, such as a perfect body, rigor, endurance, mental courage, and determination, yet no other requirement has been added. Color, size, style, action, conformation, - all have been disregarded for the one object, speed, 
Over against this the Dutch Belted Cattle, for example, have an absolute color requirement. Every cow must first have a white belt around her body. This certainly has nothing to do with her milking abilities, yet the absurd specification has gone into the very name of the breed, - a fact that will keep the breed materially behind its competitors in matters for which we breed cows. ${ }^{1}$

The more clearly to show the extent of the handicap of striving after many points in selection, let the student work it out mathematically. If but one point is required, and it can be satisfied, say in one tenth of the inclividuals, then the chances of getting it are one in ten, and one tenth of the breed is available.

Now to this let us add a second requirement that can be found in but one third of the individuals. The probability of finding these ta'o points in the same individual will then become not $\frac{1}{10}$ or $\frac{1}{3}$, but $\frac{1}{10} \times \frac{1}{3}$, or $\frac{1}{30}$, and only about three animals in one hundred will meet the requirements.

Need of reducing the requirements to the utility basis. In fashionable animal breeding we have so multiplied our points that we are no longer able to find any very large proportion of them in any one individual, and we are often obliged to tolerate positive evils in order to get the requirements even within the limits of the herd. This is virtually mixed breeding.

What is needed is a return to first principles, - to select a very limited number of the points most important from the utility standpoint. Let these be so few and so pronounced that they may all be found in 'i'ry individual of the breeding herd. Then later, as numbers multiply, other points can be added, a few at a time, upon a practically pure ancestry so for as proizons points are concemed. "This one thing I do" should be worn in the hat of every breeder. A little courage here would soon work wonders ; but " points" have become so multiplied in some of our breeds that all possibility of finding any individuals that possess them all has long ago been passed, leaving us in a

1 The writer hesitates to use as forceful language as the above regarding any breed, because in general all breed's are good, but this is a step so clearly adverse to live-stock interests that no language is too strong in condemnation. 
wellnigh hopeless jumble, with pedigrees meaning next to nothing so far as definite information goes.

Importance of pedigree. Enough has been shown to point clearly to the fact that the simply "good individual" is worthless as a breeder. ${ }^{1}$ He must be the product of a good ancestry, and moreover of the right hind of good ancestry. It is not enough that the animal or plant is not mixed in its blood. We ought to know, and our pedigrees ought to show, what were the special characters of the ancestors. There is yet so much variability in all our breeds that a simple guaranty of noninfusion of outside blood is not enough. Something positive is needed, and great success awaits the breed whose breeders will take a few points at a time and establish a double registry, one of which shall be a record of the destec in which the indiatual actually posscssid the dominant characters of the brod. If the "advanced registry" of some of the dairy breeds can be safegruarded against abuses, and then be used as a basis for selection, it will be of untold benefit to the breeds and to the country at large.

\section{SECTION IV - RATIONAL SELECTION}

When and to what extent to depart from safe general principles on account of economic or other considerations is a matter calling for the most discriminating judgment.

Fancy points. It is perfectly easy to show that if the breeder succeeds in fixing really useful characters he will have his hands more than full; and yet, despite all this, fashion constantly sets certain fancy points, and insists upon their observance. The trouble is not only that most of these fancy points have little or no utility, but also that, like any other caprice of fashion, they are likely to change frequently and without warning, whereas all considerations of selection require constancy and simplicity.

What, then, shall the breeder do? He is bent upon building up a herd of the highest practical value, and he has carefully weighed the relative value of all utilitarian characters. All of a

1 The regression table clearly shows that an inferior individual from a good ancestry is in every way superior to a perfect individual from a heterogeneous ancestry. Both are evils, but of the two the latter is by far the worse. 
sudden, however, fashion thrusts to the front some absurd requirement, and insists that it be met, or the stock will remain unsold. The breeder is in business not for amusement, but for gain, as well as for satisfaction. He must sell his product, or very soon go out of business. He cannot afford to go on producing what nobody will buy, and he is often brought face to face with the alternative, - financial ruin, or the destruction of the herd from the standpoint of the best breeding.

For example, a few years ago all really good horsemen were amazed at the demands of the market for exceedingly high knee and hock action. It was a gait not only awkward to look upon (except to men who were not horsemen), but it was exceedingly hard on the horse, and entirely impractical except for park purposes. Yet this was the demand, for the time, on the part of the buyers who spent their money freely, and it was met by the breeders, for such demands are powerful intluences in setting standards.

Yet no one knew better than these same breeders that the fashion was a passing one. To what extent, then, should studs be disturbed, and standards regarding free, easy, and useful action be upset by a passing whim? Requirements of fashion such as these - and they are many and frequent in the breeding business - call for all the judgment of the breeder, and all his knowledge and skill in meeting issues and in freeing himself and his herd or stud quickly from the evil consequences of illadvised standards.

The whole situation presents a case of steering between difficulties and accepting the least of two evils, - injury to the breeding stock upon the one hand and loss of sales upon the other, and it rivals international diplomacy in the fineness of distinctions to be observed.

There are two ways of meeting situations of this kind with a minimum of danger. One is to meet the demand as far as possible by training instead of breeding; ${ }^{1}$ the other is to introduce whatever is to be introduced at once in the person of the sire,

1 This plan was actually used to its limits in the days of high gaits, when by proper shoeing, driving over rough ground, etc., much was "trained into" the roadster being fitted for market. 
hoping the crate may pass before the old stock of lemales shall pass away. If it does not, then at all hazards some remnants at least must be preserved pure and unalloyed as a nucleus against the day when the pendulum will swing back to the normal, or perhaps to the other extreme.

Breeders' fads. The above has reference to requirements imposed by the buyer. But the fact is, breeders themselves have multiplied their natural difficulties enormously and uselessly by fads of their own invention, the tyranny of which is even worse than that exercised by the alien buyer. Against all this the strongest protest is far too weak.

Why, for example, shoukl a few curly hairs on the back of a hog disculalify him as a breeder? Why are cows and bulls selected by the size or shape of the escutcheon? Why must a Jersey have a black tongue? Why must the tail bone of a IIolstein-firesian cow reach to the hock? Why did the Shorthorn breeders twenty-five years ago carefully kill every roan or white calf, and so yield themselve's to the color craze that for a decade or more the breed made progress backward? Why such frantic horror at the "seventeens," I requiring that a book be written blacklisting literally thousands of the best animals of the breed?

Absurd standards of this character should be resisted to the utmost by every reputable breeder and by every real friend of the breed, whether arising ignorantly or from a malicious desire to natrow the range of possible sales, to discredit the animals of competitors, or to destroy their herds. The writer is well aware that breeders as a cless are second to none, either in intelligenee or in honor. Ite is aware, too, that many of these foolish requirements or objections, like the "querl " on the pig, get started no one knows how, and wain strength by repetition; but the wholesale destruction of reputations, and even of herds and fortumes, by the war on the "seventeens" is convincing proof that individuals, even in this homorable company, are not above the most dastarlly methods of reducing competition. Individlails of this sort are not benu fode breeders; they are commercial pirates who use peeliereed live stock as material for speculation. They have adeled nothing to the excellence of any breed, or to

1 Reference is here made to the Shorthorn importation of 1817 . 
the honor of breeders, who, as a class, are the soul of honor, and would no more falsify a pedigree than they would rob a bank.

Such methods must be met, and breeders' fads generally should receive their everlasting quietus within the confines of our breeders' associations. If this may be, then the inclividual will be reasonably safe; if not, then he must take his chances with the rest, but against this insidious enemy of all grood breeding his voice and his pen should be instant and active, - this in the interest not only of his own business but of the breed he loves.

Fashionable pedigrees. All agree, and the law of ancestral heredity proves, that the ancestry back of the inclividual is extremely potent; yet this potency largely resides in the near-by members, and to see a breeder poring over a pedigree rumning ten or twelve generations back to an "approved" incliviclual on the female side, and then gravely nodeling approbation of a "good foundation," - this is indeed both humorous and pathetic.

According to the law of ancestral heredity as stated by Galton and fully noted in a previous chapter, each generation and ealch individual of the various generations has an influence represented by the following fractions, waiving all questions of prepotency :

Relative Intensity of Blood Lines and Approximately Reiative Influence of Different Generations and Individuats for Ten Generations Backiward

\begin{tabular}{|c|c|c|c|}
\hline $\begin{array}{l}\text { GENFRATION } \\
\text { BACKWARI) }\end{array}$ & $\begin{array}{l}\text { Number of } \\
\text { Ancestors }\end{array}$ & $\begin{array}{l}\text { INFLUence of Geneli- } \\
\text { TION - Ptir Cent }\end{array}$ & $\begin{array}{l}\text { INFHENCE of Each } \\
\text { INDTHUAL - PER CENT }\end{array}$ \\
\hline I & 2 & 50.00 & 25.00 \\
\hline 2 & 4 & 25.00 & 6.25 \\
\hline 3 & 8 & 12.5 & $1.56+$ \\
\hline 4 & 16 & 6.25 & $0.39+$ \\
\hline 5 & 32 & 3.125 & $0.10-$ \\
\hline 6 & 64 & 1.5625 & $0.024 t$ \\
\hline 7 & 128 & 0.78125 & $0.006+$ \\
\hline 8 & 256 & 0.390625 & $0.001+$ \\
\hline 9 & 512 & 0.1953125 & $0.0004-$ \\
\hline 10 & 1024 & 0.09765625 & $0.0001-$ \\
\hline Total & 20,46 & $99.90234375^{1}$ & \\
\hline
\end{tabular}

1 This will be 100 if carried to infinity. 
By this we see that the individual inherits from no less than 2046 individuals within ten generations of ancestry, and that, on the arerege, characters possessed by a single individual of the tenth generation back have an influence amounting to not over one ten thousandth of one per cent of the total heritage, representing a probability of about one in a million - certain to be heard from but of little consequence as a foundation.

Ve must remember that besides the "founclation" there are I023 other ancestors of the tenth generation, and some 1022 intervening ancestors, each more powerful by far than the so-called foundation. Six generations back the influence is but 1.5 per cent for the sixty-four individuals involved, or about 40 of one per cent for each. This is an amount of influence which for practical purposes may be considered as a negligible quantity, and it is for this reason that in many lines the dictum has gone out, "The sixth cross is pure," this meaning that nearly 99 per cent is covered by the "top." We have seen already that this agrees perfectly with mathematical theory.

The so-called foundation is therefore not a foundation, but only a beginning, and it is the top, and not the bottom, that gives character to the pedigree.

This is not intended to disparage purity of pedigree even to the tenth generation and beyond, but it is intended as a protest agrainst the blind following of certain pedigrees because of the "foundation."

Nor is it to be construed as a criticism clirected against breeding along approved lines; far from it; but it is a plea for the careful study and rational valuation of pedigrees.

What deceives the breeder is the fact that the "short form pedigree," as it is often presented, runs only on the female side, so that, of the 2046 ancestors of the first ten generations, only eight or ten females and their sires would appear - the other 2026 not being noted. They exist, however, and their influence is to be reckoned with. Of course it is true that in close breeding the same individual appears many times in a pedigree, and thus his or her infuence is multiplied; but the point here made is that a single individual ten or eren six generations back counts for little so far as its personal influence is concerned. 
Rational standards. In the interest of rational breeding, let ideals be made up of essentials, - a few strong lines, which, like the bold strokes of a great painting, make the picture stand strongly out, unimpaired by a multitude of unimportant details.

Summary. The whole purpose of selection is to modify the type to better suit our purposes, to prevent so far as possible the production of undesirable individuals, and to reduce the population as near as may be to those that are useful in the highest attainable degree.

If the last item is to be accomplished, then the "pull" of the ancestry must be in line with the immediate parentage, which means that there must be a constant, not a fluctuating, standard of selection.

The probability of finding all clesirable qualities in a single individual reduces rapidly as the number of characters multiplies. It is represented by the product of the chances of each, and if many characters are involved it becomes practically impossible to find them all in the same individual. This leads inevitably to heterogeneous breeding within the breed, and to confusion of ancestry with respect to separate characters.

The practical way to "fix" a large number of characters is to do it with one or two at a time, or at most a few at a time, adding others as it becomes comparatively easy to secure them all in the same individual. Common sense dictates that we should begin with the most important from the utility standpoint. In all breeds there are too many animals that do not conform to type, even approximately, and most standards of selection call for too many points.

Fads and fashion, confined for the most part to minor matters, are the bane of good breeding. They must be reckoned with for economic reasons; but in the effort to meet market demands it is sometimes difficult to aroid the fixing of decidedly objectionable characters.

It is the "top," rather than the "foundation," that gives character to the pedigree, and in all cases the individual should conform to the standard of selection. This calls for a degree of detailed information about individuals for at least fire or six generations back, which we do not ordinarily possess, and which 
the records do not undertake to supply, but which breed histories should afford for the enlightenment of young breeders and to the end that unfortunate combinations may not be unwittingly made. The student will recall the difference between brothers, which shows the need for selection even within the pedigree.

When a family becomes famous it is bred with less ability and care than before, whereas it is deserving of greater and more careful attention. But conditions are against it : the individuals have a high commercial value, and everything goes at a strong price, indifferent and bad as well as good. As long as men will pay for breeding, regardless of quality, breeders are likely to sell everything that will find a buyer. A large number of breeders have reported to the writer that they sell Ioo per cent of their animals for breeding purposes. Under conditions such as these all selection is practically abandoned, and we should not expect longer to maintain excellence. Thus it is that the very success of a popular strain is likely to prove its undoing.

This state of affairs sufficiently accounts for most disasters that have orertaken fashionable families in the past, and we are not yet warranted in assuming that a farorite strain is bound quickly to wear out or otherwise come to an end, making it necessary that we should be forever effecting new combinations. Indeed, there is the best of ground for the confident belief that if a tithe of the labor were bestowed upon the preservation of a useful strain that was expended to originate it we might have it with us indefinitely, to the infinite good of the breed and the lasting service of man. It is not a continually recurring strain of new creations that is most needed, but rather well-protected and solidly bred lines of long-established excellence and unquestioned ancestry. Here, in the opinion of the writer, lies the field for future effort of American breeders. 


\section{CHAPTER XVII \\ SYSTEMS OF BREEDING}

Of the various possible systems of breeding some are better adapted to one purpose, others to another; some again are peculiarly adapted to animals, and others to plants. The practical breeder should first of all have a clear idea of what he is trying to do, and then an accurate knowledge of the various systems that can be employed to achieve his purpose.

\section{SECTION I-PURPOSES IN BREEDING}

The general principle that should decide the system to be chosen and adhered to depends upon the answer to the following question: Is the purpose of the breeding to improve the harl, that is, the bome stock of the farmer; is it to improve the breed or variety as a whole; or is it to originate nezu varieties?

The answer to this question should determine the system of breeding to be adopted. These purposes are separate and distinct. The first is herd improvement, the acknowledged object of which is to build up the home stoch until it approaches in excellence the approved breeds or strains. This is purely commercial and purely selfish, in the best sense of the terms, in that the breeder is not operating for the good of anybody or anything but himself and his own, and is not aiming to outdo anybody else; his purpose is, rather, to secure for himself the improvement that others have originated. It is the cheapest and casiest of all forms of breeding and productive of the most rapid results.

The second purpose is, on the other hand, chiefly the improvement of a recognized breed or variety, - an improvement intended to endow the race more richly than ever before. This is the very highest style of finished breeding, and calls for the most intelligent and expensive methods, because in this case the breeder is a leader, not a follower and an imitator. 
The third purpose in breeding is not to improve anything, but to secure something entirely new, different from, and presumably better than, any previously existing strains. In carrying out this purpose the breeder proceeds upon the assumption that our varietics are too few ; that gaps exist which may be filled up; and that it is better to produce something new than to depend upon improving the old. This is the most ambitious of all forms of breeding, and appeals to creative genius rather than to conservative business instincts, which incline to improvement of existing races rather than to the production of new ones. Naturally it is most common in plant breeding, in which numbers are not so serious a matter, and in which breeding operations are less expensive.

As has been remarked, these three purposes in breeding are entirely distinct. These distinctions should be clearly in the mind of the sturlent when situdying systems of breeding, and the breeder himself must be in no sense uncertain as to which one is really in his mind when he begins his breeding operations. If his purpose is to improve his own, let him frankly admit it to himself and proceed accordingly, leaving high prices and hazardous enterprises to others. If, on the other hand, he hopes to do something distinctive for his breed, and is satisfied that he has the money and the patience to do it, then again his purpose is clear cut and his methods are well indicated.

All breeding expensive except herd improvement. ${ }^{1}$ All forms of breeding are costly whometer the purpose is to produce something bettir thern eate bifore. If the purpose is only to multifly cucllence, then it is comparatively cheap, but the original production of excellence, which is breeding in the highest sense of the term, is relatively expensive, because so few individuals, plant or animal, excel either their predecessors or their contemporaries, and so few of these can propagate their own excellence.

With these considerations in mind it is worth while to get a clear iclea of the different systems of breeding available for the various purposes.

1 "Iferd improvement" is an expression used in reference to the home stock, whether plant or animal. 


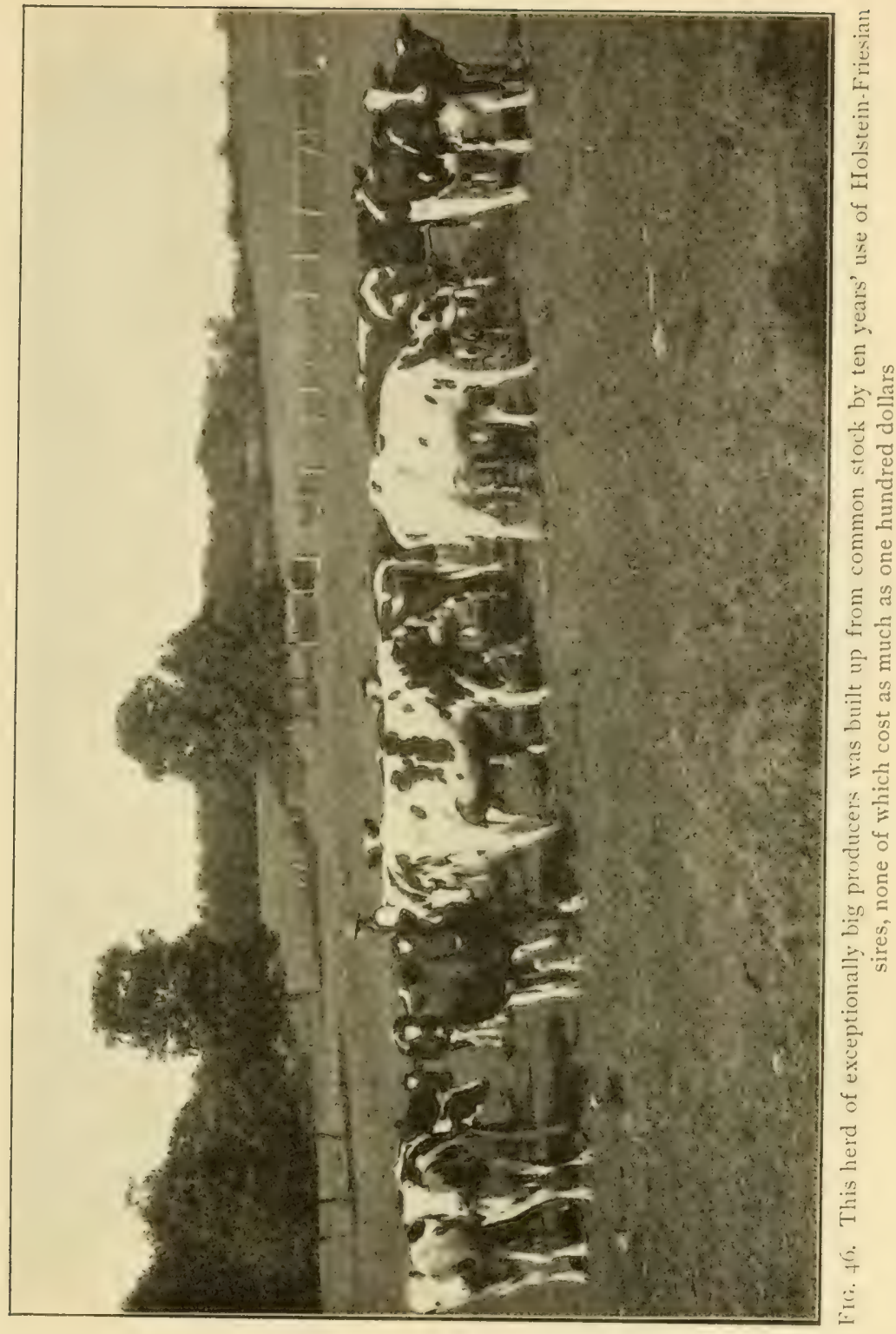




\section{SECTION II - GRADING}

By "grading " is meant the mating of a common or relatively unimproved parent with one that is more highly improved, that is, a "pure bred." The mating might be made either way, but in practice the male is taken for the pure-bred parent for economic reasons. One pure-bred bull with a herd of twenty cows can give all the calves in the herd a pure-bred sire (that is, make them half bloods), whereas if the making of half bloods were attempted in the other way it would require twenty purebred individuals, and the crop of calves would have no more improvement; besicles which, the improvement made would be not in one but in twenty lines, each with its shade of difference.

Expressed in terms of money, it is possible to give all the calves in a herd a pure-bred sire - that is, make them all half bloods - at a total cost of approximately two clollars per calf, assuming, of course, a reasonable number of cows in the herd and a bull at a moderate price but good enough for grading. If the making of half-blood calves were accomplished in the other way, however, - that is, by providing the pure-bred parent on the dam's side, - it would cost, at the same relative rate, close to forty dollars as a minimum. This shows the necessarily extreme cost of pure breds as compared with grades.

Disappearance of Unimproved Blood by the Continuous Use of Pure-Bred Sires

\begin{tabular}{|c|c|c|c|c|}
\hline \multirow{2}{*}{ GENERATIONS } & \multirow{2}{*}{$\begin{array}{c}\text { SiRes } \\
\begin{array}{c}\text { Per Cent of } \\
\text { Purity }\end{array}\end{array}$} & \multirow{2}{*}{$\begin{array}{c}\text { Dams } \\
\begin{array}{c}\text { Per Cent of } \\
\text { Purity }\end{array}\end{array}$} & \multicolumn{2}{|c|}{ OFFSPRING } \\
\hline & & & $\begin{array}{l}\text { Per Cent of } \\
\text { Purity }\end{array}$ & $\begin{array}{l}\text { Per Cent of Un- } \\
\text { improved }\end{array}$ \\
\hline I & 100 & 0 & $50 \quad\left(\frac{1}{2}\right)$ & $50 \quad\left(\frac{1}{2}\right)$ \\
\hline 2 & 100 & $5^{\circ}$ & $75 \quad\left(\frac{3}{4}\right)$ & $25 \quad\left(\frac{1}{4}\right)$ \\
\hline 3 & 100 & 75 & $87.5 \quad\left(\frac{7}{8}\right)$ & $12.5 \quad\left(\frac{1}{8}\right)$ \\
\hline 4 & 100 & 87.5 & $93.75\left(\begin{array}{l}15 \\
1 \\
6\end{array}\right)$ & $6.25 \quad\left(\frac{1}{16}\right)$ \\
\hline 5 & 100 & 93.75 & $96.87\left(\frac{3}{3} \frac{1}{2}\right)$ & $3.12+\left(\frac{1}{32}\right)$ \\
\hline 6 & 100 & 96.87 & $98.44\left(\frac{63}{6} \frac{3}{4}\right)$ & $1.5+\left(\frac{1}{64}\right)$ \\
\hline
\end{tabular}

Improvement by grading is of course limited to herd improvement. It adds nothing to the breed, but it distributes breed 


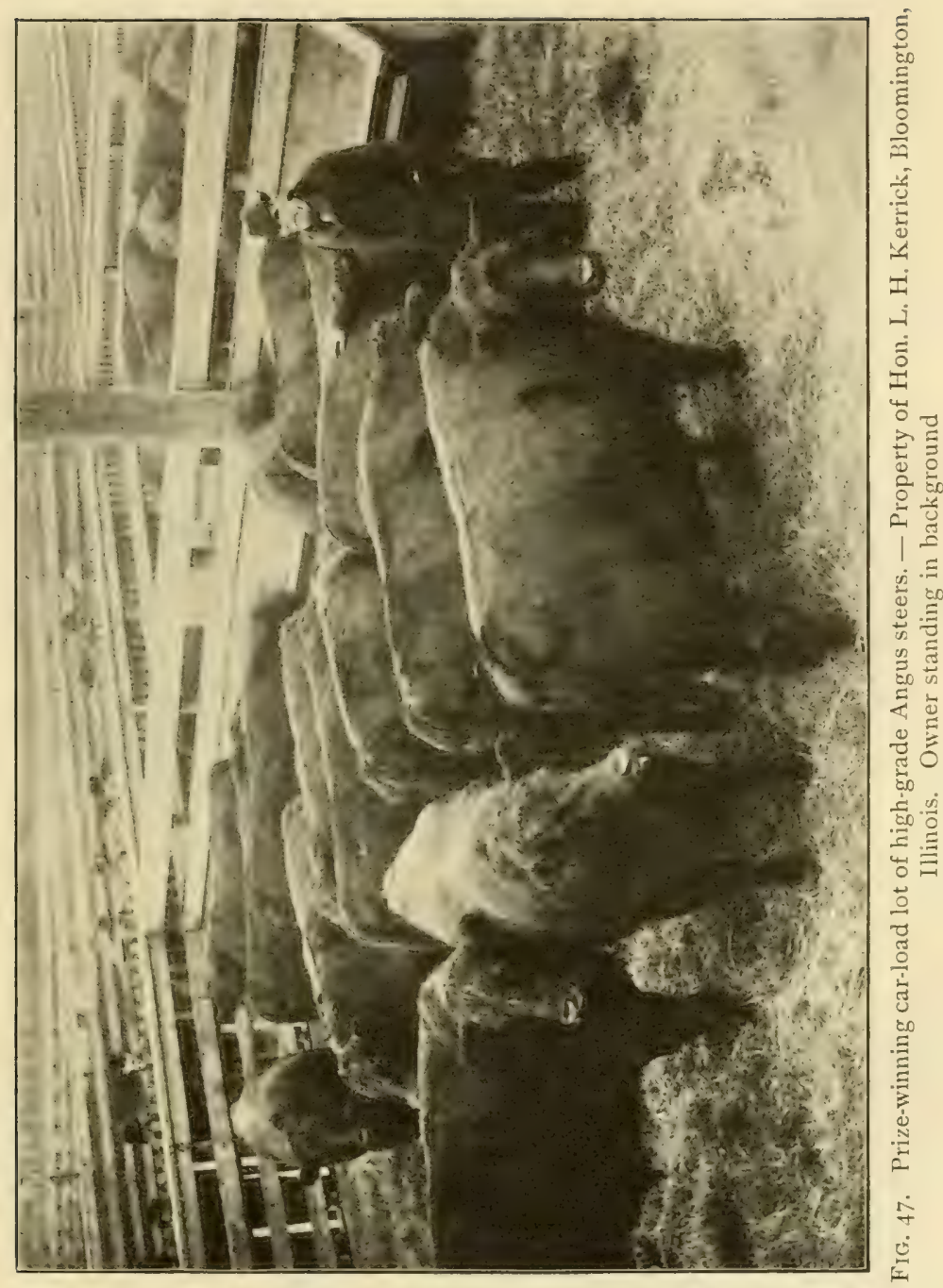

$60 i$ 
excellence rapidly and with extreme certainty. Such a sire is almost surely prepotent over the dams, whatever they may be, and the mathematics of mating shows that if the practice is continued for six generations, but one and a half per cent of the original unimproved blood will remain, as is shown in the table at the bottom of page 602 .

By this we see that the unimproved blood soon becomes insignificant and rapidly disappears. This is why it is that in the early days of a breed the sixth or seventh cross is declared eligible to record.

It should be noted that if any one of these generations be bred with itself (grades with grades) no progress is made. Thus individuals of the second generation are one fourth unimproved, and, bred to a generation of their own kind, they will still remain one fourth unimproved. By the same principle, half bloods bred to half bloods will produce half bloods indefinitely. The effects of grading cease the moment we discontinue the pure-bred sire.

Abuse of grading. The chief drawback in grading is that it is likely not to be followed up. The breeder is almost certain to choose some promising half or three-quarter blood for a sire because he "looks as good" as a pure bred, and then by the law of ancestral heredity all improsement stops except the little that can be accomplished by the slow process of selection.

Advantages of grading. For ccomomic purposes grades mary bc cqual to pure breds, but they are worthless for brecting purposes; this is the plain conclusion of what is well known of the principles of breeding. Grading is cheap. By the use of a single individual it secures at once something more than half of the total excellence of the breed, and if followed up it will secure in time, through sires alone, practically all of $i t$.

This is the system of breeding to be recommended to the great mass of stockmen, and if it could be generally adopted and folloned up it would add millions to American agriculture. Every stockman knows that the great bulk of the best cattle in the markets are high-grade Shorthorns and Herefords. The accompanying figures surely show that the less-known Angus and its close relative, the Galloway, are equally successful for 
grading purposes. The failure to make the most of grading is the largest single mistake of American farmers and the most conclusive evidence of shortsighted business policy on the part both of the general farmer and of the breeder of pure-bred stock.

Breeders of pure-bred stock largely to blame. When breeders themselves stop trying to set up amateurs, who have little money and less experience, with small herds of two or three females, then the longest step will have been taken toward reform in this

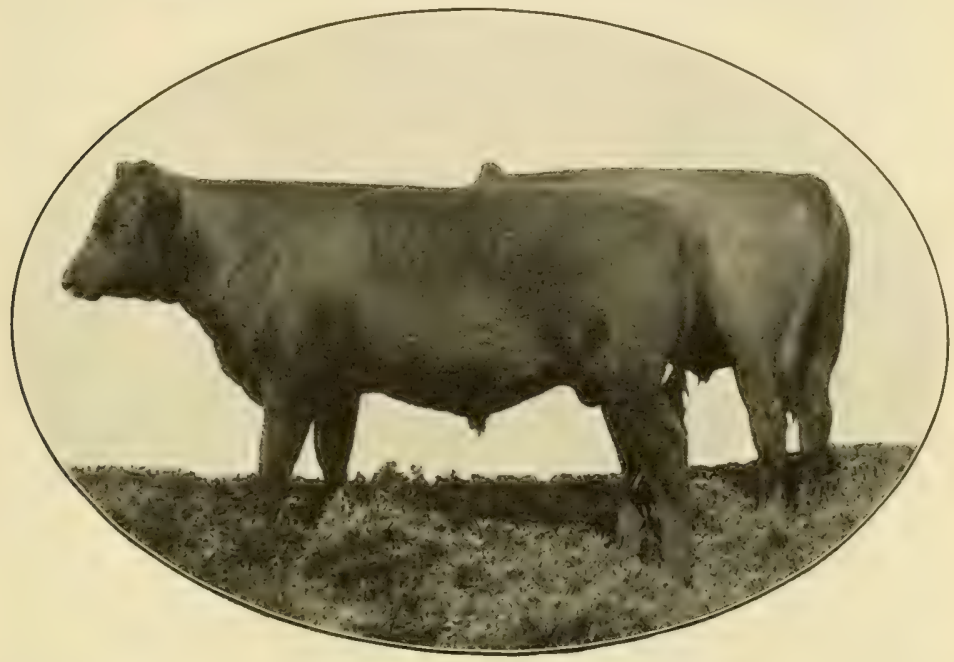

FIG. 48. Seven-eights blood Angus steers, six months old. - Property of Hon. A. P. Grout, Winchester, Illinois

particular. These pitifully inadequate efforts at breeding are foredoomed to failure, after which the unfortunate farmer, smarting under the punishment he suffered by reason of his spasm of enthusiasm for better stock, forthwith and forever curses not only the breed that "let him down," but blooded stock generally and breeders in particular.

The breeder's business is the production of sires. The professional breeder is a producer of sires, and he should sell males, not females. He should take the amateur kindly into his confidence and explain that while he himself is in the business for profit, and his animals are for sale, yet he fully realizes that 
grading is the breeding for beginners. He can easily show the novice that if he will keep his old females, or, if not, get plenty of such as are easily available, he can hare as many grades within a year as he can provide females now, and that speedily he will own a herd that for all practical purposes except breeding will be as good as anybody's, all at a cost of only two or three dollars per calf, and correspondingly less or more for other animals. Such a course will demonstrate at once the excellence of the breed, and make friends, not enemies, of the man and his neighbors.

The burden is upon the breeders and owners of pure-bred flocks and herds to lead in a crusade for grading. They need the market for their excess of males, and if this market were fully developed, and the mass of stockmen fully alive to the advantages of grading, this market alone would absorb at good prices all the male output from our breeding herds, - a consummation they stand sorely in need of attaining.

The female output of our breeding herds should be used, first, to reënforce the home herds, and after that to supply deficiencies in other reputable herds. Any further surplus animals should go to the open market, except in some rare cases in which they are needed for the real founding of new herds.

The main difficulty is that the breeders, as a rule, are too intent upon selling females and setting up a multitude of little breeders in a small business; whereas they should be not only intent, but persistent, in selling males for grading purposes. This is their great market, their natural outlet, and its exploitation is their opportunity. The author has replies from hundreds of breeders on this point. A large share of them profess to expend as much effort to sell females as to sell males, and a few even more. Associations have much to do along this line.

Begin animal breeding by grading. Grading is the safest beginning, even for the prospective breeder of pure-bred stock. Not only is it cheap and safe, but it will bring out clear and strong in the grades the main breed points, and a few generations of grades from low to high will spread out before the eyes of the breeder such a panorama of breed characters as he would not see in years of pure breeding on a small scale; indeed, there is no 


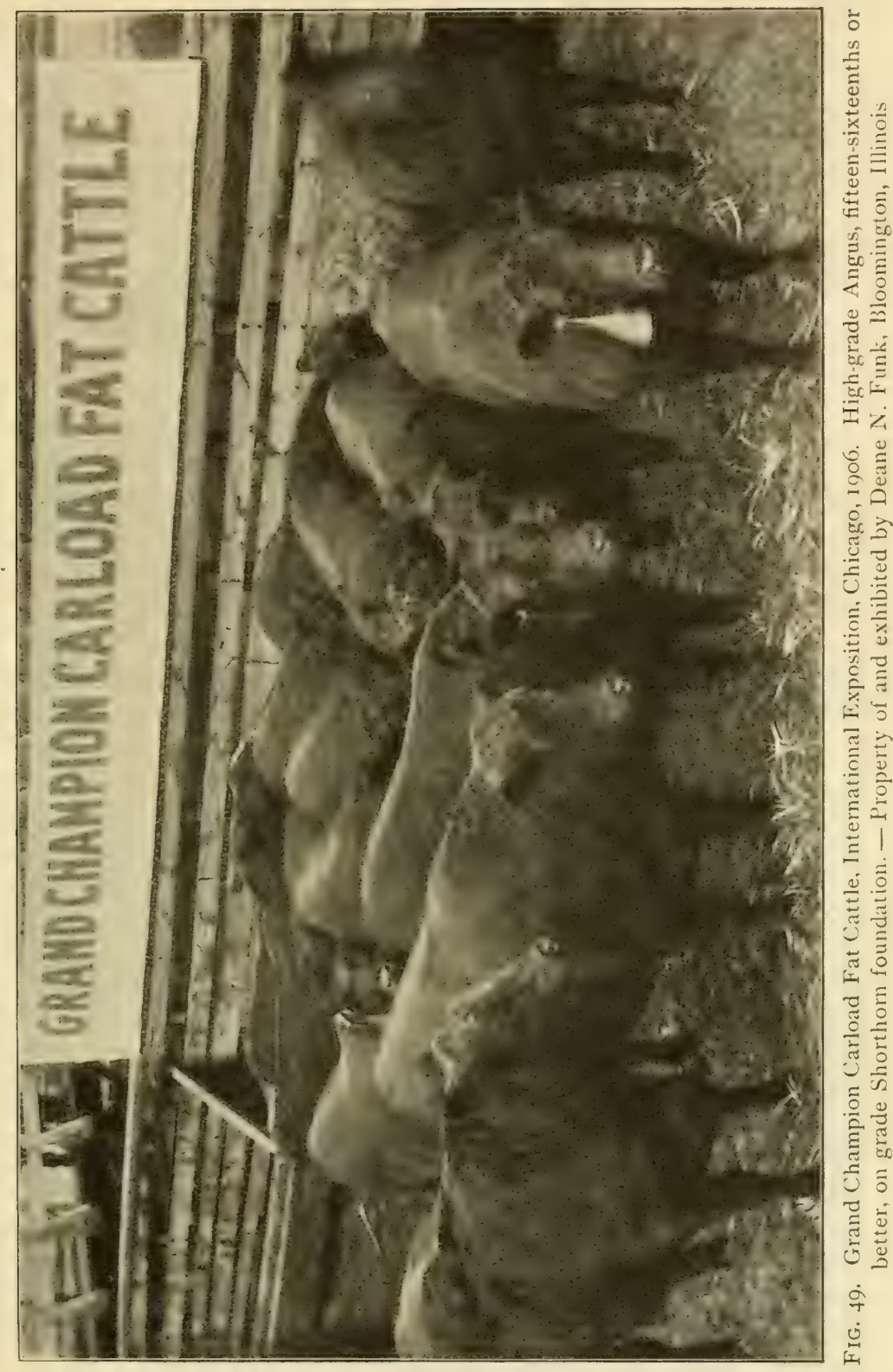


quicker, cheaper, or more thorough way of becoming acquainted with a breed than through its grades.

Disadvantage of grading. The only disadvantage that can be mentioned is this, - that the first results are so eminently satisfactory that some promising grade is likely to be selected as a sire, regardless of the law of ancestral heredity, whereupon all further improvement stops. This is so likely to be the case that it may be said in general that the very success of grading is the greatest guaranty of its failure.

\section{SECTION III - CROSSING OR HYBRIDIZING}

Almost the exact opposite of grading, crossing combines ancestral lines of two distinct races, breeds, or varieties, in the hope either of securing a blend or else of getting a fortuitous combination of characters.

This form of breeding is adapted only to the production of new strains, in which it excels. Of course it so mixes blood lines as to effectually destroy the influence of the ancestry and all meaning and value of pedigree. Its hope is in starting a new strain, which may perchance breed pure.

The operation of Mendel's law teaches how small is this chance. If this law always held with all races and characters, it would of course be impossible to secure permanent strains by crossing, but the fact remains that permanent hybrids lucic frequently becu sccured by this method, especially among plants, which is a noteworthy fact in breeding.

Advantages of crossing. Notwithstanding the operation of Mendel's law as a general principle, crossing is a fruitful source of new strains. Hybridization is better adapted to plants than to animals because of the need of vigorous selection afterward and, therefore, of relatively large numbers. It was a favorite method of plant improvement twenty years ago, but it has fallen largely into disuse because of the inconstancy of Mendel's middle term (the 50 per cent affarent hybrids) and because as good or better results can often be secured by selection alone, withont destruction of the pedigree and the influcuce of the ancestry. 
Disadvantages of crossing (hybridizing). The difficulty of securing a blend out of a violent cross, or indeed anything that will breed pure, and the great mass of long-continued and disappointing reversions experienced, have turned the attention largely away from this system of breeding, to one which, if less spectacular, is eminently safer, and, so far as we now know, fully as fruitful of results.

It is the opinion of the writer, however, that as we learn by experience it will be found that certain races of plants will lend themselves well to this means of producing new rarieties, and that the old-time enthusiasm for hybriclization will return in these exceptional cases.

Crossing is a powerful means of inducing variability, - indeed, it is the most powerful method known to breeders. It is altogether too fruitful of variants to be manageable in animal breeding, and only sheer necessity, after all other methods have failed, would warrant its trial among these slow-breeding races.

If animals are to be hybridized it can probably best be accomplished by combining, not two races simply, but three or more, leaving the one nearest that which is wanted untouched until a fairly favorable cross between two others has been secured. Then the pure form, if bred with the cross, might be influenced thereby, but would of course remain prepotent. Such a plan of action aims rather at the modification of a breed than at the creation of a new one.

Hybrids often sterile. All degrees of productivity are found in hybrids, from extreme fertility to absolute sterility. Some crosses are more fertile than either parent. Such a cross would be made readily in nature. Others are absolutely or nearly sterile. It is safe to assume that about all the possible fertile hybricls were long ago produced in nature, and either went down under natural selection, or became good species before they came into our hands. However, modified strains may yet be hybridized, and sterile hybrids may often be propagated asexually.

The classic hybricl is the mule or hinny, the cross between the horse and the ass, and is nearly always sterile. The lion and the tiger mate freely, in captivity at least, but the mating is in most cases fruitless. Eren here, however, hybricls have been born. 
The reciprocal cross. Strange as it may at first appear, the two possible crosses by interchange of the sexes often, though not always, differ substantially. It is said that the common mule more nearly resembles the ass, and the hinny the horse. Other instances have been noted, and the point has been urged that reciprocal crosses are in general clissimilar. It is the writer's opinion that the rule applies only to those particular characters in which the one parent (either male or female) is prepotent over the other because of sex. However, statistical evidence on reciprocal crosses is almost totally lacking.

The whole subject of hybridization seems at present to promise little of interest to animal breeders beyond the production of the common mule, but if we may place a shrewd guess, it will yet be found a fruitful source of new varieties in certain races of plants, in which propagation is so easily effected by budding, grafting, or other form of asexual multiplication, thus avoiding the effects of Mendel's law in a way quite impossible with animals.

\section{SECTION IV - LINE BREEDING}

By "line breeding." is meant the restriction of selection and mating to the individuals of a single line of descent. The purpose of this system of breeding is real breed improvement, to get the best that can be gotten out of the race, and better than ever before if possible.

Experience has shown that if the purpose be breed improvement, or even herd improvement carried to its limits, it is not enough to confine selection to the limits of the breed. All breeds are exceedingly variable, and real results aiming at anything more than mere multiplication can follow only closely drawn lines within the breed, - breeding in line, or line breeding.

Line breeding excludes everything outside the approved and chosen line of breeding. It not only combines animals very similar in their characters, but it narrows the pecligree to few and closely related lines of descent. This "purifies" the pedigree rapidly and gives the ancestry the largest possible opportunity. The system is eminently conservative. It discourages 
variability, and rapidly reduces it to a minimum. Moreover, whatever variations do occur will be in line aith the prominent characters of the chosen branch of the brece.

Advantages of line breeding. The nature of results secured by this system can almost certainly be predicted; and when they do appear, and improvement is at hand, it is backed up by the most powerful hereditary influence obtainable, because of the simplicity and strength of the an-

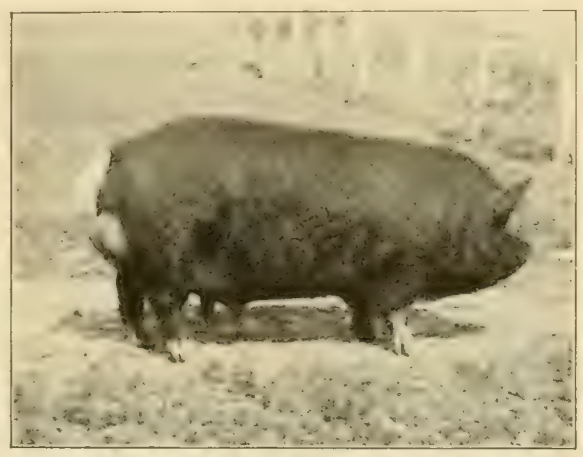

FIG. 50. Baron Duke 63d, a line-bred Berkshire. Property of A. J. Lovejoy, Rosco, Illinois. Figures $5^{1}$ and $5^{2}$ show get of this boar cestry, which, if the selection has been good, all "pulls" in the same direction. The records of all breeds will show the pronounced results that have followed judicious line breeding. A volume could be filled with pictures of famous animals so pro-

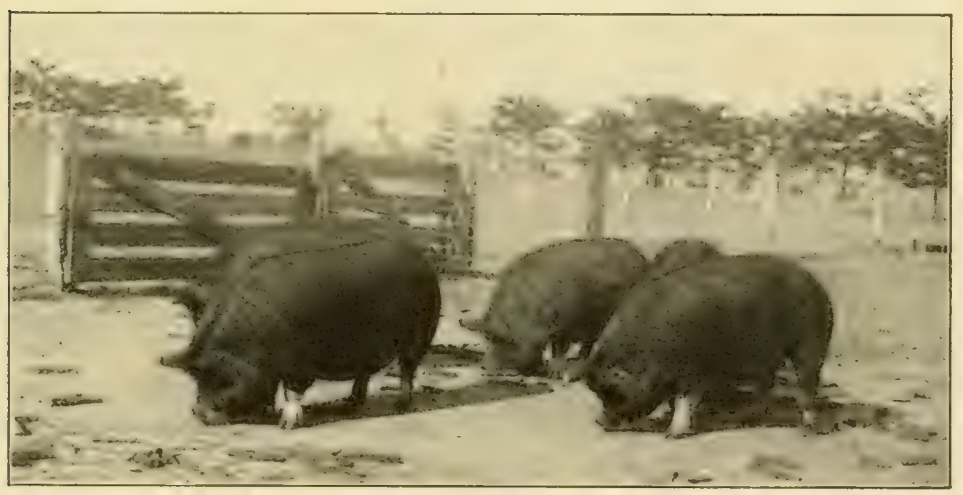

FIG. 51. Line-bred Berkshire pigs. Get of Baron Duke 63d

duced. Those shown are of swine, for the reason that the pig is popularly supposed to be the most sensitive to close breeding:

Disadvantages of line breeding. The chief danger in line breeding is that the breeder will select by pedigree, abandoning 
real individual selection. A line-bred pedigree is valuable or dangerous in exact proportion as the individuals have been kept up to grade. It will not replace selection, but, on the contrary, calls for the most discriminating care within the line.

If the breeder selects by paper, and not in the yards, and a few generations of inferior animals creep in, then line breeding will consign the whole bunch to the limbos quicker and more certainly than will any other known system of breeding, - a

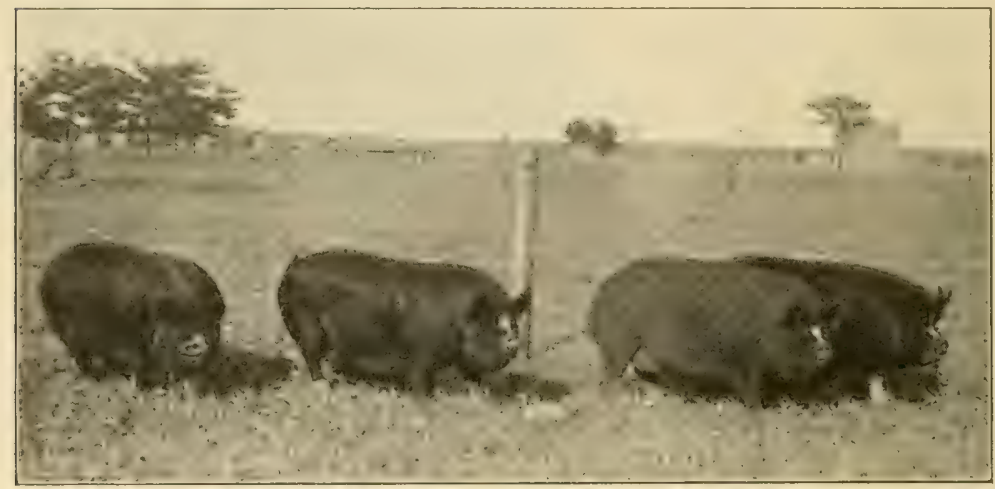

FIG. 52. Line-bred yearling Berkshires. Get of Baron Duke $63 \mathrm{~d}$

fate that has overtaken more than one line that unfortunately became prematurely fashionable.

Line breeding the best system for improvement. No other system of breeding has ever secured the results that line breeding has secured, and if the present state of knowledge is reasonably sound, no other system will ever be so powerful in getting the most possible out of a given breed or variety, especially of animals, and this with the greatest certainty as we go along. The only requirement is, not to abondon indiridual sclection. A pedigree is not a crutch on which incompetence can lean; it is a guaranty of blood lines, - a field inside of which breeding operations and selection may with confidence be confined.

The word "confined" is used advisedly, for, after line breeding has been practiced for a few generations, the ancestry becomes a kind of pure breed of its own, - a breed within a breed, so to speak, - and any attempt to introduce blood from 
other lines is likely to be followed by the pains and penalties of hybridization; for a departure from line breeding is a kind of crossing in a small degree, and so rapidly do blood lines become intensified that line-bred animals assume all the attributes of distinct strains, as they in truth are, and they will be likely to behave as such ever after.

In saying that line-bred animals tend to behave like pure strains, and that their progeny from union with other strains behave like hybrids, it is not meant that such unions should never be made, or that such behavior is as persistent as with real crosses. In truth, many lines are so stubborn as never to blend with others afterward (behaving like the most strongly established races), but, on the other hand, most of them will yield to well-directed and persistent effort; that is to say, a line-bred herd can be modified, and in time made to assume the characters of another family, but the process is attended with a struggle and not a few failures. It has been fashionable at times to clecry line breeding, but the fact remains that a few generations of good breeding soon bring the herd and its career to a point where line breeding must be practiced or a worse alternative must be accepted, for with well-selected strains all outbreeding is mixed breeding.

\section{SECTION V - INBREEDING}

Line breeding carried to its limits involves the breeding together of individuals closely related. When it involves the breeding together of sire and offspring or of dam and offspring or of brother and sister, it becomes inbreeding, or " breeding in and in." It is line breeding carried to its limits, and of course possesses all the advantages and disadvantages of that form of breeding carried to their utmost attainable degree.

Forms of inbreeding. Three forms of inbreeding are possible among animals, namely:

I. Breeding the sire upon his daughter, giving rise to offspring three fourths of whose blood lines are those of the sire, - a practice which, if followed up, soon results in offspring with but one line of ancestry, thus practically eliminating the blood 
of the dam. This form of breeding is prateticed when it is desired to secure all that is possible of the blood of the sire.

2. Breeding the dam to her own son or sons successively, thus increasing the blocel lines of the female side. This form is practiced when it is the dam's blood lines that are to be preserved and condensed. Both systems are necessarily limited to the lifetime of the individuals involved. Either system can of course be approximated by the use of granddaughter or standson, which would by common consent be called inbreedins, but relationship) more remote would generally be regarded merely as line breeding.

3. Breeding together of brother and sister, - a form of inbreeding which preserves the blosed lines from both sire and dam in ignal proportions. It is inferior to either of the others as a means of strengthening previously existing blosed lines, but it is freely employed when the combination has proved caccptionally suciessul, virtually establishing a new type. It has all the dangers of the other two, and in a larger desree, because we have practically no acquaintance with the n'a crombination, whereas in strengthenines the proportion of one line of ancestry ever another, whether it be that of the sire or that of the rlam, we are dealing with previously existing blosed lines known to be harmenious.

Amoms plants there are two forms of inbrecelines, namely:

I. That in which the fertilization is with prollen from another flower on the same plant.

2. That in which fertilization is by pollen of the same flower. This, being hermaphrorlitic, is the closest imaginable inbreerliner, and exceeds anything that is possible with animals.

Advantages of inbreeding. Nobnrly claims arlvantages in inbreeline for sc. but it is the ar me of line breerlings, and when superior individuals are at hand it is the most powerful methorl known of making the mest of their excellence. It is the methror by whic is the highes possible percentage of the blood of an execptional indiyidual or of a particularly fortunate " nirk, " can be pre-creed, fured inter and ultimately made to charar terize an entire line of descent on both sides.

If pelwisted in, the nit-ide blowd di-appears by the same law that governs srading, and the pedigroe is sleedily criched to 
an almost unlimited extent by the blosel of a single animal, in practice, gencrally that of the sire. It is a method mot sos much of originating excellence as of making the most of it when it does appear, and it is not too moch to saly that a largre pros. portion of the really great sires have becen strongly inbred.

An inbred animal is of course conomousily prequencent oves everything else. Its half of the ancestry, lecing largely of irlen. tical blood, is almost certain to deminate thes offspring. Inbresed. ing is, therefore, recognized as the strongest of all boeselinfs,

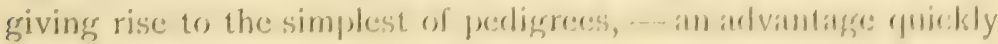
recognized when we recall the law of ancestral hereelity. In this respect it is all that linc lorecrling is and mene:

A second advantage is that successful asserejations of cilouracters are preserved intact and not shattered by the infusion of new strains. If the breceler were dealing with holl at singles chate.

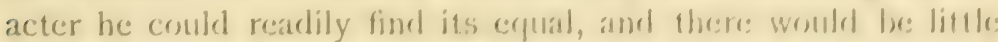
need for inbrecedings; but ceven if brecerlings for loul as singrle: ratili. tarian character, he always has at least. (wo nthers, vigure and fertility, which must be inclueled in selecetion. In practice: lov has many more, and a single individual that contaims all or most of them in a high degree is a veritalde: locomanat; maturally the: temptation is to make the most of an opporemenily which is moms too frequent in the breceding business.

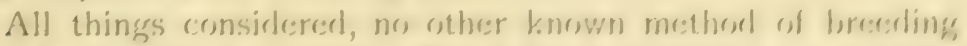

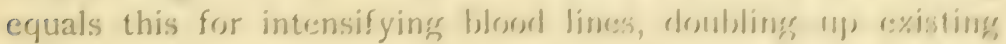
combinatjons, and makingr the most. of r:\%cepoliomal individuals or of unusually valuahle strains.

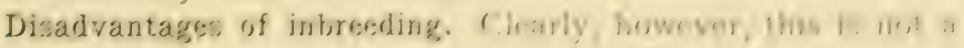
gun to "hit the brear and miss the calf." "This "domblimer, app"

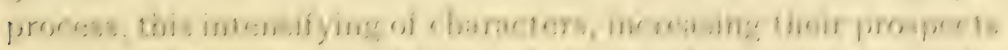

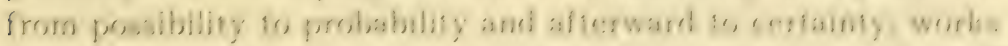

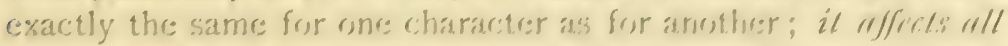

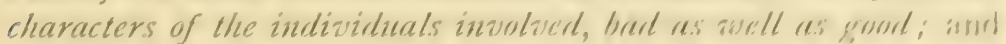

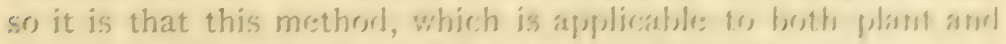

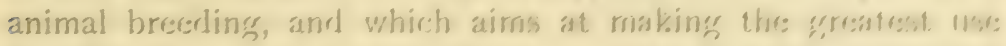

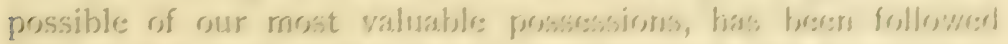

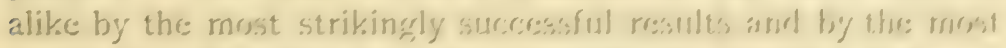

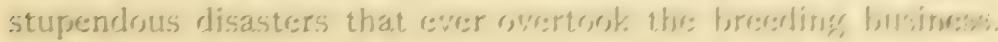


Plenty of examples of successes can be instanced, and every breeder is familiar with them. The failures have been many, but they are not to be counted here, for the blood lines involved are long since extinct.

special dangers from inbreeding. Tradition everywhere has it that inbreeding, if long continued, is practically certain to end in loss of vigor and of fertility, and plenty of instances are given to "prove" it.

Now a rational consideration of the principles of transmission has already led us to expect that bad characters as well as good will be intensified. We could not expect so powerful a method to work only to our advantage and to grant immunity from disadvantage in all cases.

What we want to know is whether, in respect to trouble, we are to look out for likclihood or for cortainty; whether disaster is inevitable, or only extremely probable. This question has been much befogged by certain catchy statements such as, "Nature abhors incestuous breeding," all of which confuse an ethical and social question with the biological one in which only we are interested.

Inbreeding not necessarily disastrous. Our attention is constantly called to " nature's provisions for preventing inbreeding," and to "ingenious derices for inducing cross pollination by insect aid"; but we are not reminded that many species of plants are self-pollinated, nor is our attention called to the many famous sires that were strongly inbrel, nor to the fact that in nature among gregarious animals the head of the herd is sire of practically all the young (so long as he remains master), many of of whom are thus doubly his. Nor do we have it called to our attention that, while corn seems peculiarly sensitive to inbreeding, wheat is self-fertilizing to the closest possible degree, and that it is perhaps the most vigorous, prolific, and all-round cosmopolitan success among our domestic plants.

Lack of vigor and low fertility the two most common defects. If what has been said and shown has any meaning, it is that any character can be bred up or down, strengthened or weakened by this method of breeding. Why then its evil reputation with respect to rigor and fertility? Is there some inherent injury 
from close breeding, or is it merely that vigor and fertility are commonly defective characters and frequently find themselves on the losing side? Undoubtedly it is the latter. There are cases enough of the greatest vigor and fertility of inbred individuals, and of family lines and even of whole species, to set asicle all fear of inevitable injury from close breeding, but a little study will convince us that there is lurking weakness and infertility everywhere. It is said that one third of our children die in infancy. A large proportion of animals and an apparently larger proportion of plants are relatively weak and easily succumb to disease or to the encroachments of their neighbors.

Few individuals are fully fertile, — that is, free and regular breeders, - and fewer yet are both fertile and vigorous. Shortcomings in these two respects may be called the distinguishing defects of both plants and animals under domestication. In nature they constitute the chief points of attack of natural selection, but in domesticated animals and plants we commonly select for other points, even color, trusting to luck for vigor and fertility. Is it any wonder that these lurking evils have crept upon us until they often constitute an insurmountable bar to inbreeding, and have invaded even our most carefully outbred herds?

As inbreeding is the supreme test of a race, so it is of a character ; if a character suffers by inbreeding it is a sign of natural defectiveness and should be accepted as such, and not laid up as an additional instance and a weapon with which to abuse a system with a history of laudable achievement in the past and rich with possibilities for the future.

When we select for vigor and fertility we shall hear less of the evils of inbreeding. In the meantime we shall hear most about it where vitality and fertility are naturally lowest. Both are cardinal requisites, - one for life, the other for reproduction, - and both must be possessed in a high degree by any individual or family line that is to figure much in descent.

Noting, then, the remarkable instances of successful inbreeding, as well as its unexampled capacity for trouble, we arrive at the conclusion that the disaster from inbreeding is probable, but not inevitable. With that much gained, it is worth while to examine further into this disputed territory. 
Darwin's experiments. ${ }^{1}$ Fortunately so far as plants are concerned we are not without some accurate data tending to show the actual effect of inbreeding upon the two most important characters here under discussion, - namely, vigor and fertility, - and for a great variety of species. The experiments are too extensive to fully discuss even by abstract, covering as they do some fifty-seven species, belonging to fifty-two genera; ${ }^{2}$ but their results may be briefly-stated.

The careful study of these experiments shows the following facts: (I) that in gencral, and without a cloubt, crossed forms (both they and their offspring) are, on the arerage, much more fertile and far more vigorous than are the self-fertilized; (2) but that this is not tmic of all spcicies, nor is it truc of all individuals, even within those species most sensitive to inbreeding.

Thus, of the $\delta_{3}$ species tested for height, 26 , or nearly one third, were either within 5 per cent of the height of their crossbred companions, or else exceeded them in height. Of these 26 cases, however, he concludes that 14 were actually inferior, - if not in height, at least in other respects, - leaving 12 , or one seventh of all, that quite clearly were not inferior when inbred, and in some cases were decidedly the better for it. ${ }^{3}$ Concluding, Darwin remarks :

Therefore if we exclude the species which are approximately equal, there are thirty-seven species in which the mean of the mean heights of the

1 Charles Darwin, Cross and Self Fertilization in the Vegetable Kingdom, p. 482 [D. Appleton \& Company]. It is unfortunate that we do not possess equally full and exact data as to inbreeding among animals, but at this point our knowledge is limited to general results and to individual experiences. The marked success of close breeding and even inbreeding in our herds is attributed to the special skill of a "master breeder." That this is not the full explanation is shown by the experience of the United States Bureau of Animal Industry. Some years ago it became necessary to remove the stock of guinea pigs to new quarters a considerable distance away. $\Lambda$ severe storm was encountered en route and only a few pigs were saved. From these few, and with no infusion of outside blood, the present stock is descended, and the writer is credibly informed that the stock is exceptionally vigorous and fertile.

2 The student desiring the data upon the effects of cross-or self-fertilization in general should read chap. vii, pp. $23 S_{-2} S_{4}$, of Darwin's Cross and Self Fertilization, etc.; for data concerning the effect upon seed production he should read chap. ix, pl). 3I2-355; and for data concerning other effects, chap. viii, pp. $2 S_{5}-3$ I I for detailed reports of different species see chaps. ii-vi, especially ii.

${ }^{3}$ Darwin, Cross and Self Fertilization, etc., pp. $279-28_{3}$. 
crossed plants exceeds that of the self-fertilized by 22 per cent, whereas there are only five species in which the mean of the mean heights of the self-fertilized plants exceeds that of the crossed, and this only by 9 per cent. ${ }^{1}$

The writer again calls attention to the fact that while arerages are of prime consequence in commercial transactions, they do not decide principles of breeding, and it is extremely suggestive that even five species were decidedly more vigorous when inbred. It determines definitely that there is nothing inhorently and necessarily cail in inbrecting, por sc, for if such were the case it would make itself evident in every instance.

Speaking of the fertility of self-fertilized flowers, Darwin says, ${ }^{2}$ "Their fertility ranges from zero to fertility equaling that of the crossed flowers ; and of this fact no explanation can be offered." Not only was this true, but the self-fertilized forms were sometimes actually more fertile than the crossed. ${ }^{3}$

This mystery, for which "no explanation can be offered," is largely cleared up by our modern knowledge of heredity, as is shown by what follows.

The total effects of inbreeding. All characters, both good and bad, exist in various degrees in different individuals. The problem in breeding is to secure the strongest combinations of desirable characters, and it is easy to show that this is accomplished by inbreeding. Not only that, but it is also easy to show that the same methods will secure the lowest attainable intensity, a consummation desirable with unwelcome characters, and good to know about as a general possibility.

Take, for example, three intensities of any single character, disregarding for the moment all questions of correlation. Let these three intensities be represented by 3,2, and I, respectively, 2 being the mean.

If, now, we exclude inbreeding, we find three unions possible, - namely, $3+2,3+\mathrm{I}$, and $2+\mathrm{I}$; but if we resort to inbreeding, we make also the matings $3+3,2+2, \mathrm{I}+\mathrm{I}$. Which unions are richest in results? In which have blood lines been most intensified?

1 Darwin, Cross and Self Fertilization, etc., p. $28_{3}$.

2 Ibid. p. 326.

3 Ibid. pp. 322-325. 
Relative Effect of Outbreeding and of Inbreeding

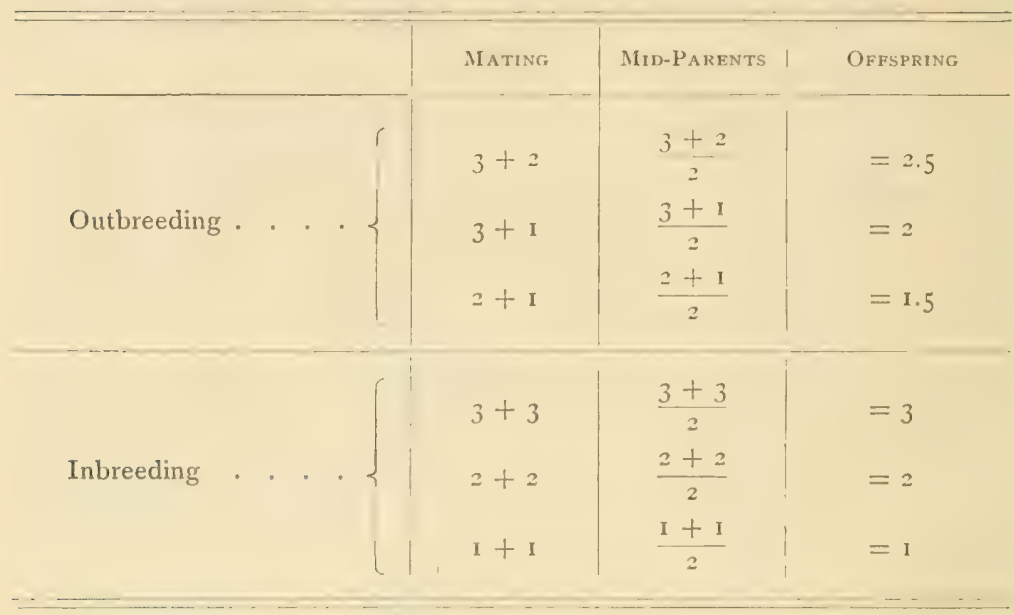

From this we see that both systems produce the same mean, but that inbreeding produces the wider extremes (3 and I). I Hence the greatest ranse of possibilities lies with inbreeding, so far as immediate parmitese alone is concerned, and the advantage is of course still greater in the ancestry farther back.

Again, the table shows what will happen on the averagre under the law of regression, but in exceptional cases the law of progression will apply, from which we see that the advantage for inbreeding is still greater; in other words, it is by inbreeding that the highest and the lowest attainable results can be produced, and this is because no other system can produce so high (or low) a mid-parent, or in the end so "pure" an ancestry. All of this indicates a principle that is abundantly powerful for intensifying good characters or for breeding out evil ones. The fact that it is thus powerful argues against its use with any but superior individuals. Furthermore, inbreeding is a supreme test of excellence, and if a family line or an individual endures it, its characters are above reproach.

Not all inbred individuals inferior to the cross-bred, even in species especially sensitive to inbreeding. One of Darwin's most extensive series of experiments was carried on with the common 
morning-glory (Ifomacr purfurer). ${ }^{1}$ This species was bred both crossed and self-fertilized for ten generations. In every generation the crossed forms were larger than the self-fertilized, the average being as IOO is to 77. Not only that, but they were clearly the more productive. The species, therefore, is one that on the whole is extremely sensitive to inbreeding. Let us, however, analyze the details of the experiment and observe how it fares with individual plants.

Darwin's plan was to put the cross and the inbred seeds into moist sand at the same time, and then to pair them off in the order of their germination. That is, the first cross-bred seed up would be paired with the first inbred seed up as a competitor, 2 the two being planted on opposite sides of the same pot; the second would be paired with the second, the third with the third, and so on. ${ }^{3}$

The following table, reporting the first generation, shows how the results appeared at first.

The average of these six pairs is 86 inches for the cross-bred and 65.6 for the self-fertilized, an initial difference of approximately 20 inches, which on the whole did not greatly change during the ten generations of the experiment.

It will be noted that in this table every inbred plant is inferior to its cross-bred mate; not only that, but no inbred Heights of Cross-Bred and Inbred Stock, - First Generation ${ }^{4}$

\begin{tabular}{|c|c|c|}
\hline $\begin{array}{c}\text { Number of } \\
\text { Pot }\end{array}$ & Crossed & INBRED \\
\hline In pairs & $\begin{array}{l}87.5 \text { in. } \\
87.5 \\
89\end{array}$ & $\begin{array}{l}69 \text { in. } \\
66 \\
73\end{array}$ \\
\hline $\begin{array}{c}2 \\
\text { In pairs }\end{array}$ & $\begin{array}{l}S 8 \text { in. } \\
S_{7}\end{array}$ & $\begin{array}{l}68.5 \text { in. } \\
60.5\end{array}$ \\
\hline $\begin{array}{c}3 \\
\text { Plants crowded }\end{array}$ & $77 \mathrm{in.}$ & 57 in. \\
\hline
\end{tabular}
individual of the series is as good as the poorest cross-bred reported.

1 Reported in full in Darwin's Cross and Self Fertilization, etc., chap. ii, pp. $28-62$.

2 If, however, a seed germinated long before a corresponding mate appeared, it was thrown away, the aim being to mate seedlings that germinated exactly together, giving an even start.

${ }^{3}$ Darwin, Cross and Self Fertilization, etc., pp. II, 12.

4 Ibid. p. 29. 
On this point compare the facts reported in the following table for the fourth generation. ${ }^{1}$

Here again each inbred plant is inferior to its particular mate, but only' threc of the cross-breds equaled the best inbred plant

\begin{tabular}{|c|c|c|}
\hline $\begin{array}{l}\text { Number of } \\
\text { Pot }\end{array}$ & Crossed & INBRED \\
\hline I & $\begin{array}{l}84 \text { in. } \\
47\end{array}$ & $\begin{array}{l}80 \text { in. } \\
44.5\end{array}$ \\
\hline 2 & $\begin{array}{l}8_{3} \text { in. } \\
59\end{array}$ & $\begin{array}{l}73.5 \text { in. } \\
51.5\end{array}$ \\
\hline 3 & $\begin{array}{l}82 \text { in. } \\
65.5 \\
68\end{array}$ & $\begin{array}{l}56.5 \text { in. } \\
63 \\
5^{2}\end{array}$ \\
\hline
\end{tabular}
of the series (8o inches), and all but one of the inbreds were more vigorous than the poorest cross-bred.

The same general fact is noticeable in the next (fifth) generation, though not quite so pronounced, except that in one case the inbred plant equaled its own mate. Evidently something was preparing to happen.

The appearance of "Hero." In the next (sixth) generation there appeared a specially vigorous plant that overtopped its own competitor by half an inch and exceeded in height all but three of the series. Darwin named this plant "Hero," and remarks, "I was so much surprised at this fact that I resolved to ascertain whether this plant would transmit its powers of growth to its seedlings."

Accordingly he fertilized a number of flowers of Hero with their own pollen, and planted the seedlings in competition with other inbred plants and with cross-bred as well. The two tables on the next page show how the descendants of Hero acquitted themselves.

Here, then, out of a species sensitive to inbreeding, has arisen a plant that is strong, vigorous, and prolific, and its own inbred seedlings at once demonstrate their superiority not only to other inbred stock but also to their crossed competitors. As Darwin remarks, ${ }^{2}$ "Hero transmitted to its offspring a peculiar constitution adapted for self-fertilization" ; and again, "It appears, therefore, that Hero and its descendants have varied from the common type not only in acquiring great power of growth and

1 Darwin, Cross and Self Fertilization, etc., p. 34.

2 Ibid. p. 50. 
increased fertility when subjected to self-fertilization, but in not profiting from a cross with a distinct stock."

Here is excellence through inbreeding under what may be called the hardest conditions, and it gives great encouragement to the belief that if it is necessary to secure a strain of plant or animal that will prosper under inbreeding, that strain can be produced, and that its production is a question only of time, patience, and expense. Hero will undoubtedly be called a mutant in these days, but mutants are welcome. It must be borne in mind that Hero was not the only individual that demonstrated its superiority to crossbred plants, but that this was a common circumstance throughout the experiments.

Nor was the morning-glory the only case of the kind. Concerning his experiments with Mimulus (the monkey-flower, of no consequence to us except as showing a principle) he says : ${ }^{1}$

In the third and fourth generations a tall variety, often alluded to, having large white flowers blotched with crimson, appeared amongst both the intercrossed and the self-fertilized plants. It prevailed in all the later selffertilized generations, to the exclusion of every other variety, and transmitted its characters faithfully, but disappeared from the intercrossed plants. ... The self-fertilized plants belonging to this variety were not only taller but more fertile than the intercrossed plants, though these latter in the earlier generations, were much taller and more fertile than the selffertilized plants.

${ }^{1}$ Darwin, Cross and Self Fertilization, etc., p. 348. (Italics are mine.) 
He adds, "This a'aricty secms to har'e become specially adapted to profit in ciery a'ay by solf-fortitiantion, althonght this process "ars so injurions to the parcut plents during the first fout genorations." Darwin's whole discussion of "highly self-fertile varieties" 2 is exceedingly valuable, not only because the author seems to consider the phenomena inexplicable, but more especially because it establishes the fact that the closest inbreeding is not necessarily fatal.

It should be noted, however, that these are exceptional instances, constituting no argument for indiscriminate inbreeding; but they do show that inbreeding is not necessarily headed straight for disaster and with a full head of steam.

The breeding business deals not with averages but with possibilities, and it is high time that the foolish horror of inbreeding be dissipated. If breeders had been as careful in certain other respects as they have been to avoid the slightest form of inbreeding, our flocks and herds would have progressed farther along the road of improvement.

Experience in animal breeding. Any one who will take the trouble to study the pedigrees of famous families in almost any line of stock breeding will find that the foundation blood is most intensely bred. Indeed, the practical breeder working with material that is really of distinctive and peculiar merit comes soon to the point at which close breeding is inevitable, and he must face the issue sooner or later if he is to make any real use of his valuable creations. To breed them out is but to dissipate their excellence, and the only practical course is close breeding.

Among cattle breeders this practice is too well known to need more than a passing mention, but the following extracts from personal letters recently received will show how it works upon the highly organized horse and the quick-breeding, heavyfleshed swine.

The veteran breeder of Arab horses, Randolph Huntington, of Rochester, New York, writes as follows :

With me close breeding has proved a sure test for purity, and my best, most uniform results have been in breeding the dam to her son and to her

1 Darwin, Cross and Self Fertilization, etc., p. 348. (Italics are mine.)

2 Ibid. pp. 347-352. 
grandson, and then breeding the produce together, intensifying such breeding by going back to the grand-dam with the grandchildren, until I had a family.

In this connection we do not forget that Messenger was three times inbred to Godolphin.

The following from A. J. Lorejoy, of Roscoe, Illinois, gives his experience in breeding Berkshires. The quality of his stock is indicated by Figs. 50-52, and his reputation as a successful breeder is fairly won by many years of uniform success. He writes as follows:

We are believers in quite close, even inbreeding. We find the greatest show animals are closely inbred. Sires to half-sisters is the most common form of close breeding, though cousins, nephews, and nieces, and even brothers and sisters are bred together with great success. It of course requires good judgment in mating animals that are particularly strong in individual merit. Should each have a bad defect in any way, we should expect that to be more manifest in the offspring than in the parents, and likewise the good points would be better; so if one mates equally good specimens the produce will be an improvement. There is no sire of any breed so prepotent as an inbred sire. When we get to the point where we feel the need of outside blood we mate an imported sow with our best boar, and from this litter we select a boar to use on the get of his own sire from other sows in the herd; that is, we breed this boar on his own half-sisters.

No man has bred Berkshires more successfully than N. H. Gentry, of Sedalia, Missouri, and no American breeder has been credited with a freer use of inbreeding. This reteran breeder writes as follows :

My experience in inbreeding is that you do good, or fail, in proportion to the quality in the strain of blood; that is, that you intensify what you have, let it be good or bad, let it be weak or strong in constitution. The theory advanced by the mass of people, to the effect that you degenerate size and weaken constitution, is all wrong unless the strain you are inbreeding lacks size as a rule, or lacks constitution. Animals that have plenty of size and a vigorous constitution can have these traits intensified as certainly as you can lessen these traits by inbreeding with strains lacking these essential traits. If you can intensify the one it seems to me as reasonable that you can the other; so a man's success in inbreeding will depend upon what he has to inbreed with. Rightly and intelligently done I have never been able to detect any bad results whatever from inbreeding. I inclose you my prize list of two World's Fairs, and it is especially true of my St. Louis winners that every animal was closely inbred. It has always been strange to me that most every person who has never given the subject 
any study whatever has a decickel notion that inbreeding is dangerous. I presume nur fathers iell us this simply because their fathers told them so and their grandfathers before them, and not one in many thousands has ever given the matter any trial or serious thought. Even with a trial it does not follow that every case will be a success, any more than the mating of animals not related will be a success in every case. The animals mated, whether kin or not, must be suited to produce good results; that is, have no weakness in common, and as much good as possible.

How to practice inbreeding. There are two situations especially indicating this method of breeding. One is grading, in which it may ordinarily be practiced with impunity. The other arises in the very best herds when the breeder finds himself in possession of a small amount of very superior blood and is debating how to handle it. If he insists upon breeding "out" he will lose it by dissipation. He has gone to the limits of line breeding; what shall he do?

In a case of this kind the only course that promises anything is inbreeding. It puts the line to the severest possible test, of course, and the hazard is great, but the possible results are phenomenal. The really good breeder should always be ready to accept whatever hazard is involved.

If it is to be done at all, the best way is to "do it and be done with it," and know the worst at once. Many breeders, fearing the consequences, go at the job gingerly, breeding a little more closely with each successive trial, as if to test the situation before making the bold and final stroke. This, if not successful, is to undermine the situation and accumulate numbers of undesirable indivicluals; in any event it consumes time that is valuable, for animals grow old quickly.

The proper way is to make the boldest stroke at once, so that, if the worst happens, the original stock is left for other trials and the breeder is not possessed of a herd that is destroyed by unsuccessful, half-hearted attempts at inbreeding.

\section{SECTION VI - BREEDING FROM THE BEST}

This has reference to the practice of selecting and breeding from the best individuals but without reference to blood lines. It is probable, incleed it is certain, that in process of time 
cxceedingly valuable races could be established in this way, especially on restricted areas and more particularly with field crops.

But in actual practice the breeder following this method among animals succeeds in getting together a confused jumble, out of which nothing of note can be established. It is the practice followed by primitive races and by careless farmers, and as soon as some attention is paid to strains, to families, and to blood lines, it passes at once into some one of the other forms of breeding already discussed.

In plant breeding the principle operates differently. Here numbers may be employed so extensively that after having chosen the stock we can literally hunt through thousands for the thing we want. This when found is, strictly speaking, a mutant, and having found it the plant breeder may proceed at once to multiply it by cuttings or to breed it pure, possibly by inbreeding, certainly with as little crossing as possible. This is the system followed by Luther Burbank, and by plant breeders generally who are looking for new things, though it is often combined with crossing.

Neither in animal nor in plant breeding, however, are we to expect much success except by regarding ancestral lines and living and working in full realization that the law of ancestral heredity is a fact.

Summary. The system of breeding to be followed depends upon the purpose to be accomplished. Grading is the practical method of improving common stock and of quickly and cheaply getting acquainted with the essential characters of a breed.

If the purpose is breed improvement through the perfection of family lines, then line breeding and even inbreeding will be the systems found most effective.

If new types, new strains, and new creations generally are sought, two courses are open, - either to watch for accidental mutations, or to hasten their appearance by crossing, a form of breeding that produces individuals which are good, but which, under the common law of ancestral heredity, are too bad mixtures to produce a uniform type, and under Mendel's law are too unstable to produce a constant type of any kind. The system 
of crossing is, therefore, best adapted to plants, which can be propagated asexually, and therefore free from the limitations just mentioned.

\section{Special Exercises}

Make calculations showing the relative expense of grading as compared with breeding pure for different classes of animals.

Also make critical study of many pedigrees of famous animals in order to trace the systems of breeding actually employed, especially as to line breeding and inbreeding.

\section{ADDITIONAL REFERENCES}

Loss of Vigor from Inbreeding. By H. J. Webber. Science, I9oI, No. 320, p. 257.

Pollination of Apples and Peas. Experiment Station Record, XIII, 620.

Reciprocal Crosses (with extended bibliography). Maine Station Report, 1904, pp. 81-89. 


\section{CHAPTER XVIII \\ THE DETERMINATION OF SEX}

\section{SECTION I - THEORIES}

The desire to control the sex, or at least to preclict what it will be, is a very old and a very common one. There are apparently about as many theories purporting to cover the case as human ingenuity has bean able to devise (more than five hundred are now known), ${ }^{1}$ and as there is but one alternative in the case, any' theory, no matter how absurd, is certain, uncler the law of probabilities, to come true half the time. Some of the principal theories that have gained popular credence, and which, so far as present knowledge goes, contain no basis of truth, are the following:

I. That one testicle is naturally male, the other female, and that the sex will depend upon the source of the particular spermatozoon taking part in fertilization. - Disproved by the fact that males with but one testicle are yet able to sire both sexes.

2. That successive ova are alternately male and female, so that naturally the sexes would be evenly distributed, and all that is needed to produce sex at will is to choose the proper heat for service; that is to say, if the last young were a female, then service at the first, third, fifth, etc., heats thereafter would produce males, and at the second, fourth, sixth, etc., heats would result in females. - Disproved in the same way as is the first theory; that is to say, females with but one ovary produce both sexes, and the same sex is repeated indefinitely, with no alternation in heats.

3. That the stronger personality, especially in a sexual sense, will impress its sex upon the offspring. - Disproved by the fact that parents of both sorts produce both sexes freely, and by the further fact that in general sires are better bred and stronger

1 Geddes and Thomson, Evolution of Sex, p. 35 . 
specimens than are dams, which shoukl sive a heary preponderance of males, - a fact not substantiated.

4. That service early in heat will probluce a male (some say a female), and that service late in heat (with ovem stale) will poduce the opposite sex. - Disproved by the fact that in nature females, especially in herds, are served early in heat, a fict that should make the offspring practically all of one sex.

5. That the older patrent will determine the sex, - some say the parent nearest the prime of life; not substantiated.

6. That extreme sexulal excitement on the part of the female is almost certain to result in male (some say female) offspring. This is a difficult assumption to prove or to disprove, because everything turns upon what would be called extreme excitement, and the singular fact is that the believers in this theory themselves appear quite mable to decicle which sex is indicated by the violent disturbances of the female; some say one, some say the other, until it looks like a case of the indige test over again.

It is inconceivable that the seneral disturbance of the body attending heat should have the slightest influence upon the chatacter of the union of the nuclear matter of two germ cells, which is all that we now know to be involved in fertilization.

It is noticeable that nearly every theory on determination of sex contains some trate of " male superiority," many going so far as to state that females are undereloped males. This conceit is evideneed wherever an advantage is supposed to exist, as by excess of fertilization, - such advantage being always given to the male.

Any theory not involving obscure distinctions - as does this one caln be casily proved or disproved by the statistical method, alwatys remembering that a correlation up to 50 per cent is inevitable, inclicating no cause at work but chance. It is far more profitable to leare speculation and inquire what is actually known about the causes that determine sex.

Sex differences slight. First of all, the idea of fundamental sex differences is steatly exagererated. About the only attribute that can be ascribed to "maleness" in ereneral throughout the whole range of life is a little higher state of activity, usually but net alwatys acompanied by somewhat decreased size. Typically 
the orum is large, well supplied with nourishment, and not given to activity; while the sperm plasm, spermatozoön, or pollen grain, is small, and poor in forel matcrial, but chatacterized by great activity.

Aside from this, males and females cliffer far less than is popularly șpposed. The artificial comentiomalities and the established divisions of labor exaggerate differences of sex in man, and over-enthusiastic writers have formed out of these exatererattions conclusions as far-reaching as they are grotesque.

Sex differences are few and slight, and mostly comnerded with the serious business of reproduction. We need not, therefore, in secking causes for their determination, look for such as strike at the very foundation of racial characters. Sex is something superimposed division halving the poptulation into one section that may, and another that may not, enter into the full possession of all the endowments of the race.

\section{SECTION II - INFIUENCE OF NUTRITION}

In tadpoles. According to P'fliger, ${ }^{1}$ three forms develop): (a) distinct males, (b) distinct females, and (i) hermaphrodites. In the last case the male organs "develop round primitive ovaries, and if the tadpoles are to become males the inclesed female organs are absorbed."

According to Young, sex in tadpoles remains a longe time indeterminate, and during this time the amount of food exerts a controlling influence upon the sex. He had three broxds of tadpoles.

Brood I, under natural conditions, developerl 54 per cent females, but when fed freely with beef it developed females in the proportion of 78 per cent; the proportion of females form brood 2 was increased by a generous diet of fish, from fo l per cent 10 8 I per cent; and in the same way a diet of froges' flesh raised the proportion in brood 3 from 56 per cent when "left alone" to 92 per cent when feel, - all of which looks as though nutrition has some influence upon sex in frogs at least.

1 Geddes and Thomson, The Evolution of Sex, p. 45.

2 Ibid. 
In plant lice. ${ }^{1}$ In general it may be said that in summer, when favorable conditions of life are at the maximum, these creatures produce parthenogenetically generation after generation, and only females, but with the cool of autumn and its lessened food supply, males appear, and sexual reproduction is resumed; indeed, to quote from Geddes and Thomson, " "in the artificial environment of a greenhouse, equivalent to a perpetual summer of warmth and abundant food, the parthenogenetic succession of females has been experimentally observed for four years. It seems in fact to continue until lowering of the temperature and diminution of food reintroduce males and sexual reproduction." Others have stated that males may be produced at any time merely by letting the plants on which the lice are feeding become somewhat "dried up."

\section{SECTION III - INFLUENCE OF FERTILIZATION}

In bees. ${ }^{3}$ As is now well known, bees are of three forms as regards sex, - drones (males), produced from unfertilized eggs ; workers (females, generally but not always sterile), produced from fertilized eggs; and queens (fertile females), also produced from fertilized eggs but quartered in special cells and given large amounts of special food. As remarked by Geddes and Thomson, "royal diet and plenty of it develops the reproductive organs of the future queens," and at any time "within the first eight days of larval life the addition of a little food will determine the striking structural and functional difference between worker and queen." 4 This fact is often taken advantage of by the nurse bees when disaster threatens through the loss of the queen cells. Hastily extracting some worker larvæ from their ordinary cells, they deposit them in the queen cells, giving them royal food, and speedily they acquire all the characters of any other fertile queen, - a result plainly due either to the character or to the quantity of the food, or to both.

In the case of bees, therefore, fertilization seems to be the deciding factor as to differences between male and female, and

1 Geddes and Thomson, The Evolution of Sex, p. 49.

${ }^{2}$ Ibid. p. 50. $\quad{ }^{3}$ Ibid. pp. $46-48 . \quad{ }_{4}^{4}$ Ibid. p. 47. 
food the element that determines whether or not the female is to be fertile. This is a sex clistinction that cannot hold in the higher forms, where fertilization is necessary to development, whatever the sex, showing that one species may differ from another, even in seeming fundamentals, and teaching us caution in making sweeping generalizations.

In wasps. Von Siebold ${ }^{1}$ conducted investigations with the fertilized and with the unfertilized ova of the wasp, Nematus icntricosus, each kind of which produces both sexes undcr certain conditions.

\section{Developaent of Fertilized Ova}

\begin{tabular}{|c|c|c|c|}
\hline $\begin{array}{l}\text { END of Larval } \\
\text { PERIOD }\end{array}$ & $\begin{array}{l}\text { Number of Fratales } \\
\text { to too Males }\end{array}$ & $\begin{array}{c}\text { END of LaRval } \\
\text { Period }\end{array}$ & $\begin{array}{c}\text { Number of Females } \\
\text { to too Males }\end{array}$ \\
\hline Fifteenth of June. & 14 & August .... & 340 \\
\hline July ...... & 77 & End of August. & 500 \\
\hline July . . . . & 269 & September... & 100 \\
\hline
\end{tabular}

From this we conclude that in general fertilized ova produce females, but not exclusively, the proportion of females being in some accord with temperature or food, or both.

Here the unfertilized ova produced males except when the conditions of development were so favorable as to shorten the larval period to the utmost, leading Geddes and Thomson to remark that even "where Developient of Unfertilized Ova the production of

\begin{tabular}{c|c|c}
\hline \hline $\begin{array}{c}\text { Number of } \\
\text { Experiment }\end{array}$ & $\begin{array}{c}\text { Duration of } \\
\text { Larval State }\end{array}$ & \multicolumn{2}{c}{ Sex } \\
\cline { 1 - 1 } II & 2 I days & All males \\
I2 & 19 days & All males \\
I3 & I8 days & 493 males, 2 females \\
I4 & I7 days & 265 males, 2 females \\
I5 & I7 days & 374 males, 8 females \\
I6 & I8 days & I68 males, I female \\
I7 & 24 days & I male \\
\hline
\end{tabular}
males is the normal condition, favorable environmental influences appear to introduce females."

1 Geddes and Thomson, The Evolution of Sex, pp. 48, 49. 


\section{SECTION IV - SEX IN MAMMALS}

Temperature and nutrition seem to be controlling factors in many of the lower forms, and when such is the case it is remarkable that in every instance the production of females seems to accompany the more favorable conditions, and that of males the harder or less favorable.

Among mammals there is little experimental evidence, but that little points to the same general fact as found among lower animals, though the larger animals are manifestly less directly influenced by surrounding conditions. Girou divided a flock of three hundred ewes into two equal lots, one of which was extremely well fed. This lot was served by two young rams; the other, scantily fed, by two mature ones. The well-fed lot (served by young rams) produced 60 per cent females, the other lot only 40 per cent females. ${ }^{1}$ The difference in age of the rams introduces a second element, but the facts, if true, are significant.

This is about all that is known of this phase of the question. Experimental evidence seems to indicate that abundant food, optimum temperatures, and generally favorable conditions tend $\mathrm{to}$ the production of females; but when we come to predict the limits to which the rule will apply we must proceed with great caution, confessing that our exact knowledge is exceedingly limited.

\section{SECTION Y - THE "ACCESSORY CHROMOSOME" AND SEX DETERMINATION ${ }^{2}$}

Certain inequalities between germ cells in respect to the distribution of chromatin matter have long been known. ${ }^{3}$

1 Geddes and Thomson, The Evolution of Sex, p. 5 I.

2 Wilson (1906), "Studies on Chromosomes, III," Journal of Experimental Zichosy, III, No. I, PP. I-39. Also the following: Beard, The I)etermination of Sex; Castle (1903), "The Heredity of Sex," Bulletin of the Nuseum of Comparative Zoölogy, XL, 4; McClung (1902), "The Accessory Chromosome. SexDeterminant?" Riologriol fulletin, III, I, 2 ; Morgan (I (904), "Self-Fertilization Induced by Artificial Means," Journal of Exporimental Zoïlogr", I, I ; Studies in Spermatogenesis with Special Reference to the Accessory Chromosome, Publication No. 36, Carnegie Institute, Washington, D.C., September, 1905; Wilson, "The Chromosomes in Relation to the Determination of Sex in Insects," Sicnic, XXII. 564 , October, 1905 .

3 Wilson, The Cell, pp. 27I-272. 
For example, Wilson reports that as long ago as I S9I Henking "discovered that in the second spermatocyte division of Pyrrhocoris one of the chromosomes passes undivided into one of the daughter cells (spermatids), which receives twelve chromatin elements, while its sister receives but eleren" ; so that, of the four resulting spermatozoa, two possess an additional chromosome as compared with the other tivo. ${ }^{1}$

Other discoveries were reported, and Paulmier (1898, I S99), working with Anasa in IVilson's laboratory, found that in the first spermatocyte division eleven tetrads appeared, one of which was "much smaller than the others" and seemed "to arise from a single nucleolus-like body . . . and by a process differing considerably from the others." I Ie adds : "In the second (and last) spermatocyte division the (ten) larger dyads divide to form chromosomes in the usual manner; the small dyad, hoacerer, fails to diaide, passing oa'r bodily into one of the spormatids. In this case, therefore, half the spermatids receive ten single chromosomes, while the remainder receive in addition a small dyad." 2

The fact was gradually established that, at least in Hemiptera and in certain other insects, one of the chromatin masses of the male maturation cell differs from its fellows, and undergoes one less division than they, so that, of the group of four spermatozoa arising out of the double division of the spermatocyte, two will possess an additional chromosome as compared with the other two. This additional member has been variously named by different experimenters, the terms "accessory chromosome" and "heterotropic chromosome" being the most common.

Here is about where the matter rested till McClung (I902) advanced the theory that the accessory chromosome is the sc $x$ determinant, assuming (erroneously as we now believe) that if the ovum should be fertilized by one of the spermatozoa containing the accessory chromosome the offspring would then be provided with the accessory and its sex would therefore be male; while if the fertilization should be by one of the spermatozoa destitute of the accessory, the offspring would of necessity be of the opposite sex.

$$
1 \text { Wilson, The Cell, p. } 271 .
$$

2 Ibid. p. 272 . 
This view of the case made it appear that the female cells correspond most closely with those spermatozoa which are destitute of the accessory, and here the matter stood until Montgomery (I9O4), Gross (I904), and Wallace (I905) discovered that, in certain species at least, the ovum has the same number of chromosomes as the spermatozoa with the acce'ssory'. These experimenters came to the conclusion that "only one of the two classes of spermatozoa is functional, namely, that in which the heterotropic (accessory) chromosome is present. Those of the other class were assumed to degenerate, after the fashion of polar bodies." 1

Wilson (I906), shows that "the sexes in hemipters of this type do in fact show a constant difference in the number of chromosomes." 2 Ile has determined, in at least four genera, that the number of chromosomes in the fomale cell corresponds with the larger number in the male cell; in other words, that the "accessory chromosome," though present in but half the spermatozoa, is found in all fimalc germ cells. This being true, the "remarkable" spermatozoa are not those with the accessory, but, on the contrary, those without it, and they are to be regarded as in some sense deficient.

Wilson shows conclusively the opposite of Gross' and Wallace's hypothesis, namely, that when a female cell unites with a spermatozoön destitute of the accessory chromosome, then the accessory of the ovum finds no mate and a male develops; and that, on the other hand, if the ovum happens to be fertilized by one of the spermatozoa provided with an accessory, then each accessory finds its mate, there is then no solitary accessory, and a female results.

Extending his experiments, Wilson finds two kinds of accessory chromosomes, - the one already described, which is smaller than the ordinary chromosomes, and another which is larger. In this connection it should be remarked that his investigations show great differences in size among the chromosomes generally, but that the "accessory" can readily be detected, whether larger or smaller than the others, and that all chromosomes, large or small, - except the accessory, _ can readily be assigned in pairs under the microscope.

1 Joumal of Experimental Zoölogy, III, No. I, p. 2.

2 Ibid. 
It should be said in this connection, too, that in certain species the accessory seems always present, - in both spermatozoa as well as in all female cells, - but that when this is the case the accessories are distinguished by some kind of qualitative difference not understood, but that gives to two of the spermatozoa of each group of four a different character from the other two.

If, therefore, it shall appear that all female cells, after extrusion of the polar bodies, are in possession of this accessory chromosome, whatever its peculiar quality, and if, out of each group of four spermatozoa arising from a single spermatocyte, two are in possession and two are destitute of this accessory, then we have in the spermatozoa themselves a very evident fundamental cause of sex determination, and, as the numbers are equal, under the law of chance the sexes should be equal, as in fact they practically are.

Here in truth would seem to be a fundamental cause of sex determination. Whether it is operative in all forms of life or only in certain species, it is yet too early to even speculate. A fertile field of inquiry is here opened up, and the near future may be expected to afford important additional clata on this most difficult subject.

Summary. There are various circumstances that appear to influence the sex of offspring. These seem, in some cases, to be connected with nutrition, and, in others, with the inherent nature of the germ. The present state of knowledge is insufficient to solve the problem of sex differentiation, but it is safe to say that none of the traditional beliefs are warranted by the known facts.

\section{ADDITIONAL REFERENCES}

Changing Sex in Plants. Tropical Agriculture, I903, pp. 789-79o.

Chromosomes in Relation to Sex Determination in Insects. By C. B. Wilson. Science, XXII, 500-502.

Do Seedless Fruits Require Pollination? Experiment Station Record, XV, I080.

Expermental Zoölogy. By T. H. Morgan. Chapters XXIV-XXVII. Experiments in Heredity and Sex Determination in Moths. Report of the British Association for the Advancement of Science, 1904 , p. 594 . 
Influexce of Nutrition on Sex. Experiment Station Record, XVI, 228.

Parthenogexetic Fertilization in the Honeybee. Experiment Station Record, XV, 792.

Recent Theories in Regard to Determination of Sex. By T. H. Morgan. Popular Science Monthly, LXIV, 97-116.

Sex Control. By Professor Schenck of Austria. Science, VII, $736-$ 738.

Sex Determination in Bees. (A discussion of the Dzierzon-Dickel controversy.) By B. Sporrer. Experiment Station Record, XI, 56I, $657,956$.

Sex Determination, - whether Bud shall be Leaf or Flower. By E. S. Goff. American Garden, I90 I, pp. 330-333, 346-347.

Sex in Mice. By Parsons and Copeman. Proceedings of the Royal Society, London, LXXIII, 32-48.

Sex in Plants a Matter of Nutrition. By T. Mehan. Report, Department of Agriculture, 1898 , pp. 536-548; Experiment Station Record, XI, 910.

Wisconsin Experiment Station Report, 1900, pp. 266-285; igor, pp. 304-316.

Ziegler's Theory of Sex Determination. By T. H. Morgan. Science, XXII, 839-84I. 


\section{CHAPTER XIX}

\section{PLANT BREEDING}

When the principles of breeding are once understood, their application to special cases, either in plant or animal breeding, is largely a matter of common sense, and no extended discussion of particular operations is necessary.

It has already been remarked that the breeder needs the utmost possible familiarity with the particular line he hopes to improve. This familiarity he will get largely through experience, but he cannot afford to neglect any source of information that will enlarge his acquaintance with the breed or the variety, for every item of knowledge will constitute a valuable asset in his business when the time comes, as it surely will, for weighing slight differences in the balance in order to determine questions of selection. This involves detail which only the practical breeder can acquire, and upon which attempted instruction amounts to little more than academic dissertation. Certain special facts and principles, however, run through plant breeding, as distinct from animal breeding, and these it is well to clearly understand in advance of actual operations.

\section{SECTION I - ADVANTAGES AND LIMITATIONS}

Advantages in plant breeding. The plant breeder possesses no less than six distinct advantages as compared with the breeder of animals :

I. Large numbers, giving excellent opportunity for selection.

2. Rapid reproducing powers, resulting in a marvelous saving of time as compared with that necessarily consumed in the slower process of animal breeding.

3. The relative cheapness of individuals, making wholesale destruction economically possible. 
4. The greater likelihood of mutations arising from mere point of numbers, if from no other cause, and the greater ease with which these may be detected if they do arise.

5. The greater chance of preserving mutations, owing to rapid powers of reproduction.

6. The possibility of reproducing asexually by budding, cutting, etc., which overcomes to a large extent the disasters of sterility and avoids the operation of Mendel's law in the propagation of hybrids.

The plant breeder, therefore, not only enjoys superior advantages in selection, but he is free to make full use of mutations and the principle of crossing, - two forms of improvenent all but closed to the animal breeder. Naturally, therefore, his operations assume one of three well-defined forms or systems of breeding:

I. "Straight selection," or breeding from the best, the purpose being the improvement of existing varieties rather than the production of new strains.

2. Maintaining extensive plantings in the hope of detecting spontaneous mutants, the object being the production of new varieties.

3. Crossing, or hybridizing, with the purpose of producing new strains.

New strains produced by either the second or the third method are of course variable and capable of improvement by straight selection. Each system of breeding requires its own methods, suited to the material in hand and the character of the improvement sought. Some species do best with one system, others with another, and only experience can decide which is most prolific of results in a particular case. The first, straight selection, is the safest, and is alacey's certain of results; but most species respond well to the second and the third, which, with suitable material, are capable of the richest results known to all breeding, and are the systems per crellence for the production of new strains.

Crossing has latterly fallen into some clisrepute because of the emphasis laid on Mendel's law, and the principle of mutation is but recently recognized; but the prediction is ventured that the former will be restored to favor in plant breeding, and that 
mutation will yield unexampled results in certain species that are "in the mutable state."

Limitations and disadvantages of the plant breeder. It is not all clear sailing for the plant breeder. He has no less than six distinct limitations which he must recognize in advance:

I. His varieties are subject to the effects of soil and climate in a way quite unknown to the animal breeder. His operations are, therefore, in a large measure local rather than general in their results.

2. The rapid rate of reproduction necessitates wholesale destruction, with the almost certain loss of "good things."

3. It is impossible to prevent accidental crossing of many varieties by insects and by the wind.

4. It is difficult to keep accurate records.

5. The product is cheap and it can be readily and rapidly reproduced by every novice the moment it is on the market. This necessitates that the breeder shall operate as a seedsman in order to secure financial returns for his labors, or else that he shall sell his production to those who are seedsmen.

6. The extravagant claims often made for new varieties possessing little or no merit tend to destroy confidence in new creations. This attitude on the part of a portion of the trade leads the public to discount heavily even the moderate statements of reputable seedsmen, reducing by a considerable percentage the profits of the business.

Two of these natural limitations, - the limitations of the soil and the difficulty of keeping accurate records, - call for special attention.

\section{SECTION II - SOIL AND CULTURE CONDITIONS}

The breeder is all but powerless to alter climate conditions, but the fertility of the land is absolutely under his control. Should the soil for breeding operations be rich or poor? at, below, or above the average of that upon which the crop is likely to be grown?

The argument is often advanced that if breeding operations be carried on upon land below the average of fertility, then the 
strain will prosper even better in the hands of the farmer, and thereby stand more chances of pleasing when put to the actual test on the farm or in the orchard. Specious but faulty, is the only correct verdict as to this position, though it may be maintained, perhaps, as to some special strains particularly sensitive to high fertility. Improvement is what is aimed at by the breeder, - the production of better strains than before. On this there are two significant points:

I. The breeder will not know when he has succeded in producing an improvement unless the soil conditions are good enough to permit full and complete development.

2. All experience goes to show that plants are more variable in soils of high fertility than in soils of low fertility. This is the experience of De Vries, of Darwin, and, so far as is known to the writer, of every plant breeder upon record.

The object in all plant breeding is the production of improvement. This is partly dependent upon fertility, and, in general, plant-breeding operations will be most successful on lands of maximum fertility. Sone acclimatization may afterward be necessary as to soil as well as to climate, but the latter is involved in all plant breeding, - and the former too, for that matter, - for no soil can be fairly representative of any very great extent of territory.

The balance of fertility. Much remains to be learned as to the elements that should predominate in a fertile soil. In general, nitrogen favors the growth of leaf and stem, but there is much reason to believe that seed formation is intimately related to the supply of phosphorus. The botanist will tell us that Saprolegnia, for example, grows luxuriantly in beef extract or peptone, but produces no reproductire organs; grown in nutrient solutions containing abundance of phosphorus, however, reproductive organs form readily, especially in the female.

Without cloubt much remains to be learned in the matter of making up of soils most favorable for the production of clesirable variations in different classes of plants. In this matter of soil fertility and cultural requirements let the plant breeder provide maximum conditions, with no fear of evil consequences. If he can succeed in producing a desirable variety by hook or by 
crook, acclimatization will come to his aid and help him to preserve it.

Of one fact we may be well assured, namely, that every plant is at its best under optimum conditions. That is the time when favorable variations may be most confidently expected; in other words, that is the time when it will respond most favorably to selection. The writer believes this to be the general principle from which to work out the conditions under which the particular strain or strains yield best results, but the plant breeder must not deceive himself into thinking that he will get valuable deviations from type while the plant is enduring hard conditions, the effect of which is to bring everything to the dead level of mediocrity, where little improvement is possible by breeding without first improving the conditions of growth.

\section{SECTION III - SYSTEMS OF PLANTING}

In general, three systems of planting are in use among plant breeders :

I. The nursery system, in which the plants are treated as individuals, each being given abundance of room, and each made the basis of selection at the close of the season.

2. The field system, in which individual plants are not given special opportunities. Seed is saved from the best plants, but no attempt is made to identify and isolate particular parentage. This is "improvement" rather than breeding, and is in common use among general farmers.

3. What Webber calls the Burbank method of crowding thousands of seedlings close together on good soil and then selecting the few that are able to endure the battle and survive.

Each system has its advantages, especially the first and third, but the third merges into the first the moment that really close work begins.

All things considered, it is altogether likely that the greater part of our results will be obtained by the so-called nursery method. In any event this is the method that lends itself to the best grade of work and to the most complete records. It is the one therefore that will receive further consideration here. 
Plot or row in the nursery system. Shall the individuals of a single selection be planted together in rectangles or grown in separate rows? Each system has its advocates, and the matter is to be decided first of all by convenience in cultivation and harvesting, and second by convenience in keeping records. The two systems are well illustrated by the methods employed at the two experiment stations of Minnesota and Illinois.

The plot system. This system is best described by its use at the Minnesota station, where it has been fully elaborated and is constantly employed for all breeding work. ${ }^{1}$ Under this system as there employed the procedure is substantially as follows, taking wheat as an illustration. When a new variety is received or a promising plant is selected its history is recorded on a record sheet $5 \frac{1}{2} \times 8 \frac{1}{2}$ inches and punched at the end for filing. It is given a class name and a number of its own ("Nursery Stock No. - "), and if it sprang from a numbered stock, that number is also recorded as "Parent Stock No. - -." This sheet is known as the "Introductory Sheet," and is reproduced here on a reduced scale.

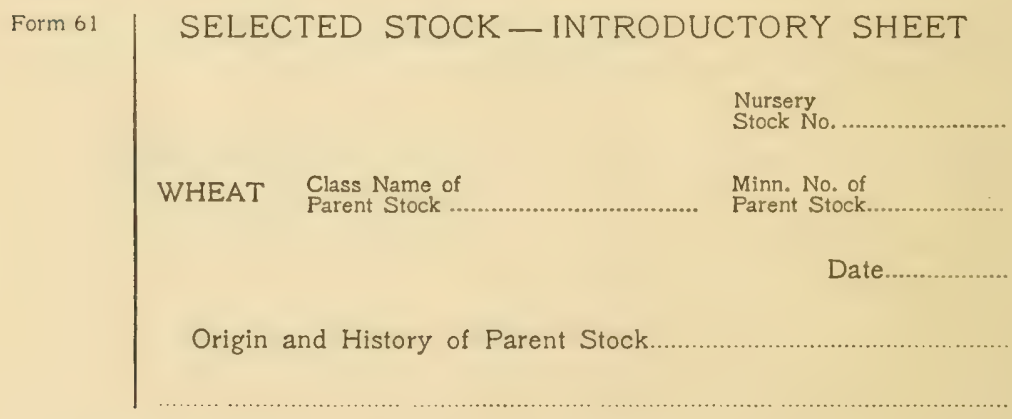

The seed is then planted by itself in a rectangular plot, ten plants square if possible, - hence known as a "centgener plot." Notes are taken both on the centgener plot as a whole and on individual plants, and recorded on sheets (see table on opposite page, reduced size).

1. See. Bulletin 10.62, Minnesota Agricultural Experiment Station, for a full description of the plot system as used at this station. 


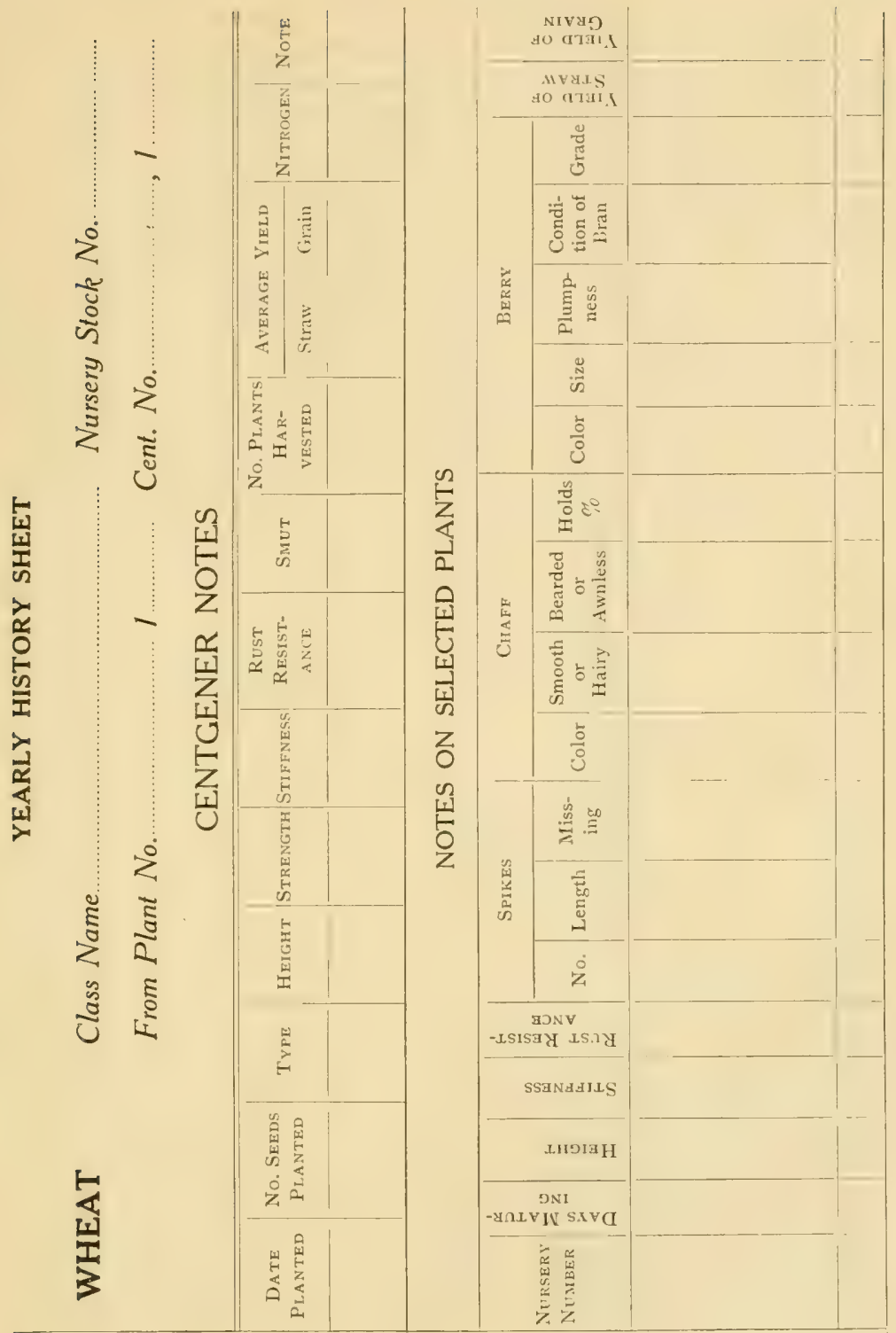

ஜ 
Manifestly all the plants on this centgener plot have the same centgener number, but any promising plant in the plot may be given a "nursery number" at any time, so that after the system is well started every plant has two numbers, its centgener number and its nursery number. From these original records three other sheets are made up as a kind of ledger account with this particular selection, extending perhaps over several years. See forms 65, 67, and 68 .

The system seems somewhat complicated to a novice, but it is easily and quickly mastered by those engaged in the work, and it is applied to all forms of breeding carried on at the Minnesota station.

The row system. This system is best illustrated by its use at the University of Illinois, where it is employed not only for corn breeding but also for wheat, oats, and other plants. The same system has been adopted by the Illinois Seed Corn Breeders' Association, and is in use by all its members.

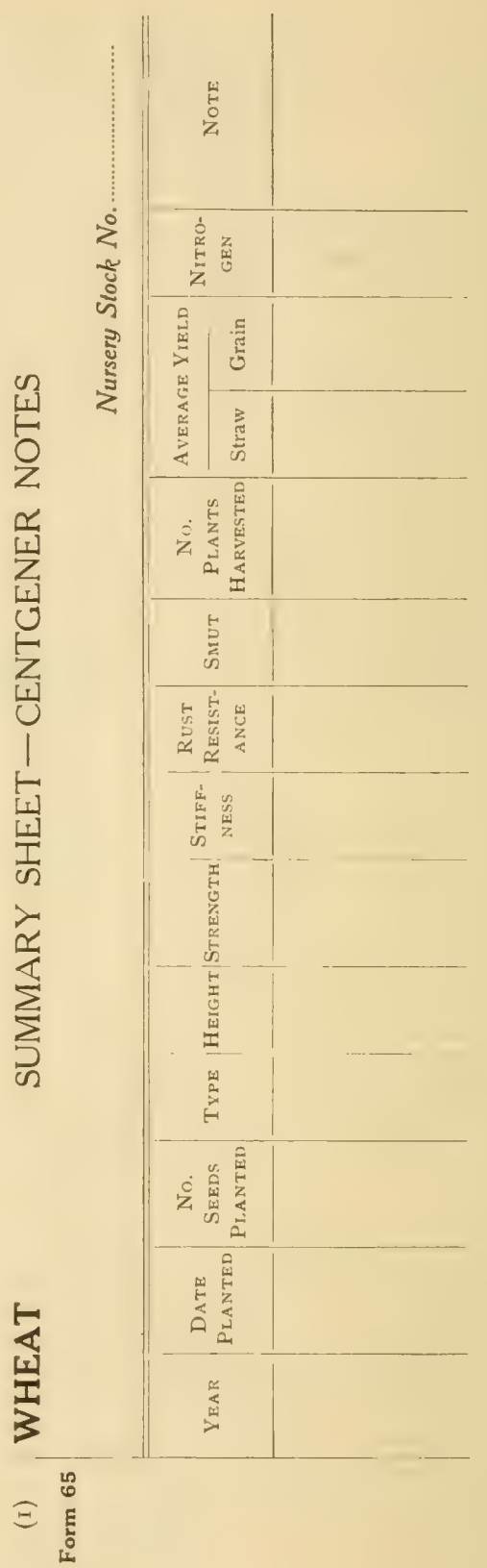



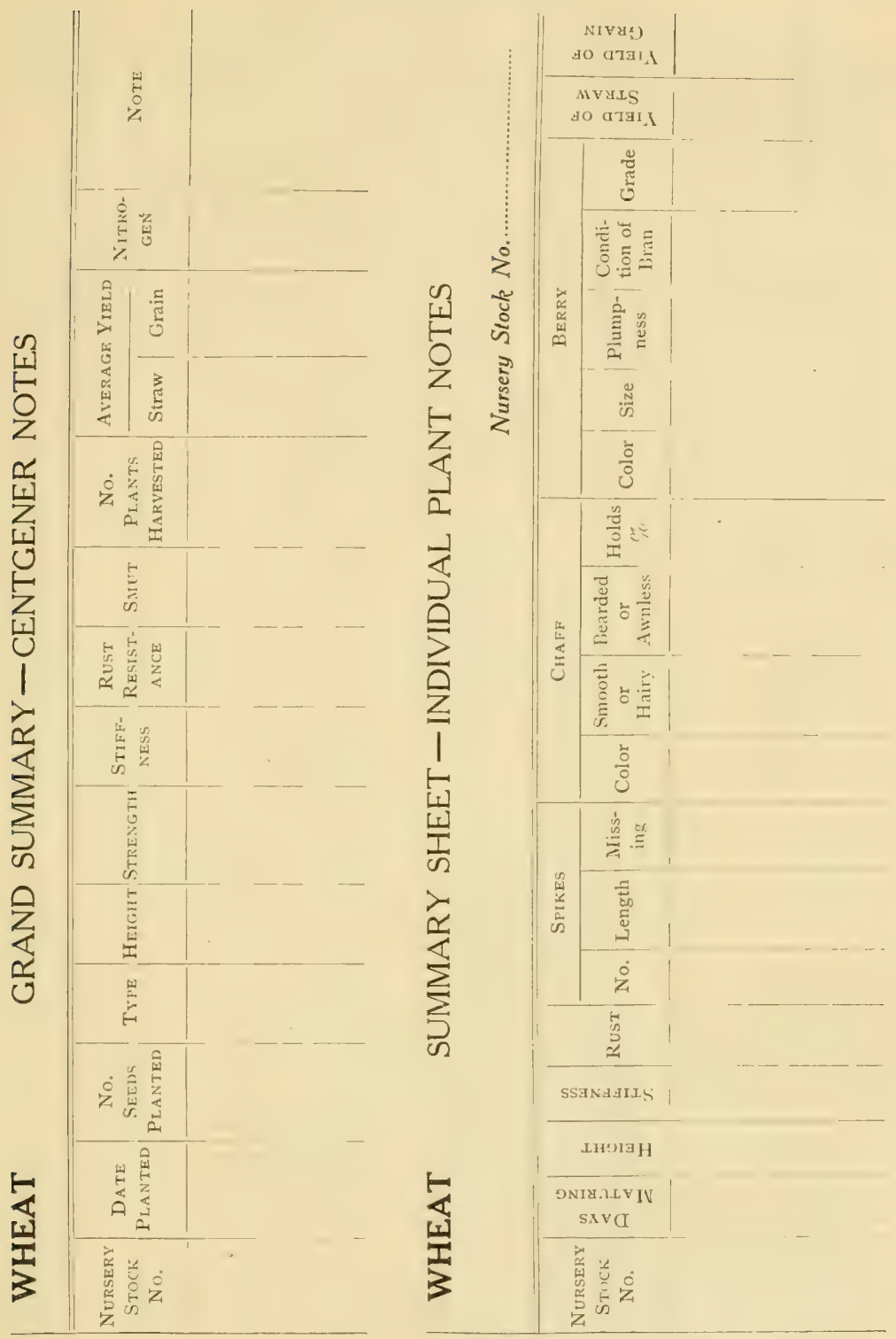

(1)

(2) $\begin{array}{r}0 \\ \vdots \\ 5 \\ 0\end{array}$ 
Under this system each ear of corn, for example, is planted in a separate row, and as many rows will be used as there are ears in the first selection. The ear in the book and the row in the field then have the same number.

Suppose we are starting an experiment on breeding for " high oil." It is the first year, and we have the twenty highest oil ears that could be found out of the seed at hand. These ears will be numbered, not from I to 20 , but from IOI to I 20. Next year they will be numbered 20 I to 220 , or 225 , or to whatever number of ears may be available. The hundreds always show the number of years or generations of improvement, and the rest of the number shows the field number of the ear and of the row in which it is planted. Thus, if we find ear No. 6I2, we know that it is the sixth year of the experiment, or the sixth selection from the original stock, and that it is planted in row No. I 2 of that year's field. This ear, like all others that have been analyzed, has its laboratory number, or "annual ear number," by which its composition may be traced; but its "pedigree number," 6 г 2, for example, is the one from which its breeding is traced. A sample page from such a register book shows the system in full, there being no other records except the chemical analyses. This is the entire record of the high-oil corn for 1902. (See table on opposite page.)

Selecting 607 of this table, for example, we see by the record that its dam of the year before was No. 504; that its number in the chemical laboratory was 3923 ; that the ear was 6.5 inches long, its tip circumference 4.8 inches, and its butt circumference 5.8 inches; that it had 12 rows, and that each row had an average of 48 kernels; that the ear weighed 5.3 ounces, and had 7. I 3 per cent of oil. We learn, too, that it was planted in row No. 7 , which was $7 I_{1}^{6} \bar{t}$ hills long and produced 85 pounds of corn, or at the rate of 63.5 bushels per acre; that there were in all 140 ears of corn in the row, the aicrage oil content of which was $6.65 .^{1}$

As a practical detail in corn breeding, it may be remarked that the best ears are always planted in the middle rows to give them the advantage in the matter of pollination.

1 As determined by a composite sample of twenty average ears. 
— క

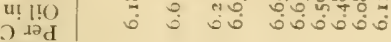

uाix ut ụฺวoมd iाวา $7 a^{d}$

suy 11

s.e. dai

-unN [B? L

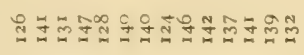

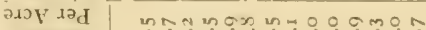

s[aysng

uso.

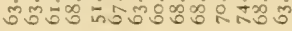

Moy

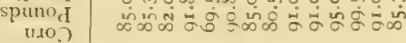

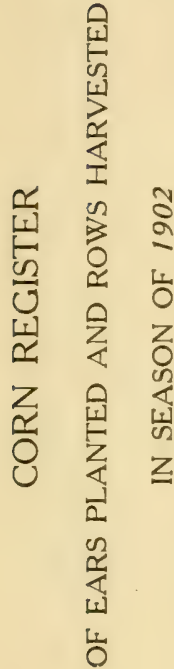

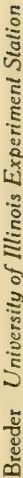

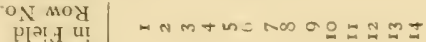
pəicld

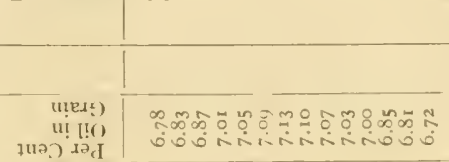

(4)

या แวा०म

วนวว 124

q०o jo

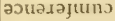

- -

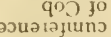

- -II) dI I

qos

รо

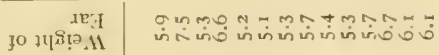

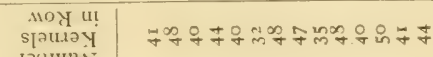

дачนиำ

fo siloy
s[aujay

1جपाuाn

มान jo

วงเวเมูนแกว

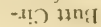

деन jo

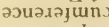

$-315 \mathrm{~d} d i$

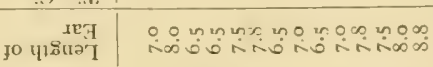

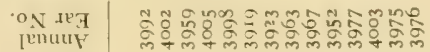

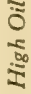

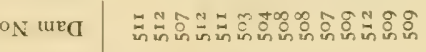

คำ 
As between the two systems the individual must take his choice. The row system seems to have the advantage of simplicity, especially for plants standing in cultivated rows. It is the one most readily understood and most easily managed by the farmer, but either is easy of application.

The performance record. One of the surprises of plant breeding is the very different appearance of the progeny from equally promising individual ears, heads, or other selection. A difference of two to one is not at all uncommon, and not infrequently a row or centgener plot from promising seed proves almost worthless. Hence the necessity of accurate records of entire rows and centgener plots. ${ }^{1}$ There is little use in wasting time on inferior material, for the best individual plant from such a fraternity would be but poor stock for breeding purposes. All individual selections for future planting, then, should be made from rows (or centgener plots) with a high average performance record.

Multiplying plots and fields. After a new strain has proved satisfactory in the nursery row or plot, it must needs be "multiplied" in order to secure a sufficient quantity for sale. It is usually customary to select for at least three generations, as in the production of sugar-beet seed, then multiply by planting out in the open field and selling the total crop for seed. Thus the beet seed of the market is generally two "removes" from the "mother beet," whose selection was made by sugar content.

Seed production a business. It is fashionable to advise the farmer to produce his own seed. From the standpoint of acclimatization it is good advice, but from any other standpoint it is bad counsel, providing, of course, that plant breeders and seedsmen live up to their responsibilities.

It is unthinkable that the farmer who is primarily engaged in production can also be a skilled improver in all that he produces. He may breed one or two lines of animals or plants himself, and if he be a breeder by nature and training, and if he have the leisure, that is well; but by the same token he should

1 The row of the row system corresponds, of course, to the centgener plot of the plot system, and both include the entire progeny of an "individual selection." 
expect some other specialist to provide him with his foundation stock in other lines. No man living, or that will ever be born, will succeed in breeding all lines of animals and plants, except to their confusion and general degradation.

So while it is popular to advise the farmer to produce his own seeds, it is better business policy for him to buy, and spend a little time and patience in acclimating the strains if need be for a year or two, before putting the new stock into common use. This will generally suffice, and if, in a special case, as perhaps in corn, no improved variety will successfully acclimate, then there is nothing to be done but to set about the production of an improved strain of the local variety; but this can be accomplished by a few persons or even by one person as well as it would be done if every neighbor undertook the task.

Improvement, even in plants, is costly business, and when once it is effected a real investment has been made that can be multiplied as often as men and lands are available.

The interests of agriculture demand that some men give their time and genius to the improvement of animals and plants. Their number, however, will never be relatively large, and it need not be. It is expedient that all farmers address themselves to the serious business of learning how to handle and produce on a commercial scale the really excellent creations in plant and animal life that the genius of breeders is able to originate.

\section{ADDITIONAL REFERENCES}

Abstract of Papers read at the New York Conference of Plant And Animal Breeders (September 30-October 2, 1902). Experiment Station Record, XIV, 208-222.

Bibliography. (A reference to forty-eight articles on plant breeding.) Experiment Station Record, $\mathrm{XV}, 770$; also in Experiment Station Record, XVI, 354, thirty-one articles.

Breeding Animals and Plants. By W. M. Hays. Breeders' Gazette, XLI, 892, 944.

Breeding Corn. By C. P. Hartley. Year Book, United States Department of Agriculture, 1902, p. 539.

Breeding Cotton. By H. J. Webber. Year Book, United States Department of Agriculture, 1902, p. 365.

Breeding for Earliness. Experiment Station Record, X, 352. 
Brefding Penxuts. North Carolina Experiment Station, Bulletin No. 168, pp. 421-434; also in Experiment Station Record, XI, 1032.

Breking Wheat. By William Saunders. Agricultural Science, is99, 74-87; also in Experiment Station Record, X1I, 339.

Breeding Work of the Minnesota Experiment Station. By W. M. Hays, Breeders' Gazette, XLIV, 1187.

COFFEe Hybrid. Gardener's Chronicle, 1899 , p. 240.

Coöprerative Breeing. By W. M. Hays. Breeders' Gazette, XLV, 14. Cross, Maize-Teosinte. By J. W. Harshberger. Garden and Forest, I896, p. 522 ; also in Experiment Station Record, VIII, 563.

CROSS-BREEDING OF FRUITS. (Summary of series of experiments covering a number of years.) By J. L. Budd. Iowa Horticultural Society, 1900, pp. 176-178; also in Experiment Station Record, XIII, 454. Crossixg of l'EAs, BEANs, ETC. Experiment Station Record, XVI, 263. Crossing Strawberries. By F. W. Card and G. E. Adams, Rhode Island Experiment Station Report, 1900, pp. 247-267; also in Experiment Station Record, XII, 944.

Crossing Varieties. By B. D. Halsted. New Jersey Experiment Station Report, I901, pp. 389-4II; also in Experiment Station Record, XIV, 568.

Difference in Plant and Animal Breeding. By W. M. Hays. Breeders' Gazette, XLIV, I 132.

ErFect of Sorl on DEvelopuext. Experiment Station Record, XVI, 22. Experiments in PLANT BreEding ON THE DOMINION Experimental. FARus. By William Saunders. Transactions of the Royal Society of Canada, I go2, p. I I 5.

Experiments of LUther BURBaxk. By David Starr Jordan. Popular Science Monthly, LXVI, $20 I-225$.

GERMAN Method OF BREEding SUgar BeETs. Experiment Station Record, XIII, 642-948.

Hybrid, BLACKBERRy-RAspherRy. Experiment Station Record, VII, $36,306$.

Hybrim Corx. By C. P. Hartley. Year Book, United States Department of Agriculture, I902, pp. 539-550.

Hybido Tomatoes. Experiment Station Record, XIII, 348.

HybiridizAtion. (Lists of hybrids and general laws of heredity.) By F. A. Waugh, American Garden, 1899, No. 234, p. 431.

Hybridization. Papers by Bateson, DeVries, Bailey, and Webber. Science, $X, 113-116$.

Hybridization in BeAns, By R. A. Emerson. Nebraska Experiment Station Report, 1903, pp. 33-68; also in Experiment Station Record, XVI, 563-564.

Hyiridization of Barlex, WhEAT, OAts, Axd Fruts. By William Saunders. Transactions of the Royal Society of Canada, I894, pp. I39-142; also in Experiment Station Record, VII, 273-275. 
Hybridization of Cereals. By J. H. Wilson. Report of the British Association for the Advancement of Science, 1904, p. 796.

Hybridization of RYE. By P. Nielson. Experiment Station Record, VII, 204.

Hybridizing Roses and Gooseberries. By J. L. Budd. Iowa Experiment Station Bulletin No. 36, p. 868; also in Experiment Station Report, $X, 47$.

Methods of Planting and Systems of Keeping Records. By W. M. Hays. Breeders' Gazette, XLII, 10, 42, I24, 255.

Philosophy and Practice of Breeding. By Luther Burbank. Popular Science Monthly, I905, pp. 201-225.

Profitable Breeding by Improving Existing Varieties. By L. $\mathrm{H}$. Bailey. Year Book, United States Department of Agriculture, 1896, pp. 297-304.

Reversion and Graft Hybridization. By H. J. Webber. Science, I 896, No. 92, pp. 498-500.

Strawberry Breeding. By N. O. Booth: American Garden, 1900, p. 534 ; also in Experiment Station Record, XII, 246.

Use of Immature Seed gives Inferior Trees. By T. Christy. Gardener's Chronicle, I896, p. I 45; also in Experiment Station Record, VII, 588 . 


\section{CHAPTER XX}

\section{ANIMAL BREEDING}

\section{SECTION I - ADVANTAGES AND DISADVANTAGES}

Advantages. Animal breeding possesses three substantial advantages over plant breeding:

I. More freedom from climatic and other local conditions, so that the product is fitted for a wider range of service.

2. Relatively good prices, because the individual has a high value and is not so easily or so rapidly multiplied.

3. Involving superior beings, animal breeding becomes one of the highest forms of art. In this we are dealing not only with superb physical form, but with mental qualities as well, and here the breeder may come into sympathetic personal relations with the products of his hand and of his genius. The same sympathetic relation will be claimed by the lover of plants, and especially by the lover of flowers; but the fact remains that only with animals do we find consciousness and intelligent response to our moods and passions. What feeling on earth, outside of human affection, can approach the attachment existing between a man and his horse, or between a dog and his master?

Disadvantages. But the animal breeder must face a long line of limitations and disadvantages. Some of these can be easily singled out and stated, others are too subtle for putting into words :

I. Numbers are necessarily few, and reproduction is relatively slow, making selection difficult from mere scarcity of material.

2. Individuals are costly, and those of high breeding powers extremely so. This makes really good breeding not only expensive but in a measure hazardous, for prices have a way of taking a sudden drop, often with little or no warning when nothing better than the open market affords an outlet for surplus stock. 
3. The characters to be selected and bred for are generally not few but many, and difficulties in selection necessarily increase out of all proportion to the number of points to be attained.

4. As if the breeder did not have troubles enough of his own, fashion is continually adding points that demand his attention most imperiously, even at the expense of better things.

5. Animals propagate but slowly, and breeding operations necessarily extend over many years and several generations; the population cannot, therefore, be spread out to riew, and there is more or less uncertainty as to actual family history and individual merit, - all of which makes selection more or less difficult and uncertain.

6. The young of most animals are promising, but selection cannot safely be made at extreme immaturity, for the differences between inferiority and superiority are brought out only in the development that comes with full maturity. The cxccllence of brcading is mainly showe in the capacity for development, and this cannot be foretold except as it may be predicted by a general knowledge of the particular family line and the special blood combination involved. Some of the most promising "young things" are the bitterest disappointment.

7. Animals are difficult of development, and many of the best-bred things are never properly developed.

8. Animals do not reproduce asexually, and their successful production is conditioned upon high sexual fertility. Now, imperfect sexual development is one of the most common, if not the most common, defect in both plants and animals. Plants may be propagated by buds or cuttings, but animals are at the mercy of sexual reproduction. In nature the existing lines are kept at least fairly fertile by natural selection, but in domestication no such controlling influence exists unless supplied by the breeder himself. This lack he must provide for if he hopes to succeed, but it constitutes one of his principal difficulties.

9. Fashion and custom decree that animals shown at the fairs shall be put into extreme condition, and this is a constant menace to the efficiency of a breeding herd.

IO. A strong vein of speculation has entered into many lines of animal breeding, the tendency of which is to unsettle prices 
and conditions generally in what ought to be one of the most steadily conducted of all the industries.

These, in brief, are some of the limitations of the animal breeder. Ile cannot afford to close his eyes to their existence, for they are realities. He will get on best to frankly confess their existence and to meet them to the best of his ability. It remains to examine a little more closely into some of these and other detailed considerations that must engage the breeder's attention.

\section{SFCTION II - FEWER CIIARACTERS FOR SELECTION}

The greatest single improvement possible in present-day animal breeding in most lines would be to free the situation from unimportant characters. At best the breeder must pay regard to a large number of considerations in making selections. Constitutional vigor, high productive powers, and utility for the purpose in mind are fundamental considerations, and the last (utility) is more than likely to cover many points.

Now the difficulties of selection increase at a surprising rate as requirements multiply. If a proper degree of constitutional vigor is found in but one animal out of two, - and it is not higher than that, - then the chances of a particular animal proving satisfactory in this respect are but one out of two, or, as we say, his probability is $\stackrel{1}{2}$. If, again, but one animal out of three is fully fertile, ${ }^{1}$ - and it is doubtful if the proportion is higher, in certain lines at least, - then the chances of a strong constitution and full fertility being found in the same animal are but $\frac{1}{2} \times \frac{1}{3}$, or $\frac{1}{6}$. If, now, we add to this a third requirement found, say, in but one animal out of ten, then our chances have been reduced to $\frac{1}{2} \times \frac{1}{3} \times \frac{1}{10}={ }_{60}^{1}$, which is equivalent to saying that only one animal in 60 taken at random - that is, but one animal in 60 of all that are born into the breed - will fully meet our demands and satisfy our requirements, crect in so for as the characters in question may be related by cansation and to that extcut overlap.

1 By full fertility is not meant the power to reproduce as against absolute barrenness, but rather full and maximum powers of reproduction, - that is, regular breeding throughout a reasonably long life. 
If, now, to this we add, say, three other requirements ("points") represented respectively by $\frac{1}{4}, \frac{1}{15}$, and $\frac{1}{2}$, we have reduced our chances to $\frac{1}{2} \times \frac{1}{3} \times \frac{1}{10} \times \frac{1}{8} \times \frac{1}{1} \times \frac{1}{20}$, or I I 5,200 , - meaning that not one individual in 100,000 will meet our demands. But this is beyond the range of practical selection, and it means that defects will of necessity be accepted. If the same defect were always accepted the damage would be less, but in practice one point is now waived and then another, as we choose the least of two evils, and so defects linger and, behaving according to the principles of Mendel's law, return to plague us long after we supposed ourselves through with them and well freed from their influence. This is really mixed breeding, however pure the pedigree.

Now the principle is this: we should tolerate no more points at any one time than can all be found in the same individuals. When the entire population come to possess these few characters in a high degree, then other requirements can be safely added, because the breding for a fou characters at a time amonnts to practical certainty. The breeds with which this method has been practiced - racing horses and hunting dogs, for example - have outstripped anything known in the rapidity of their improvement, and, moreover, a foundation has been secured on which other requirements may safely be laid; whereas the breeds in which many requirements have been exacted contemporaneously have had a checkered history, full of ups and downs, and the end is not yet,-nor will the end be in sight until the custom is abandoned of requiring at the same time so many points as to put the matter beyond the range of practical selection.

The direct effect of too many points of selection is, first, temptation to overlook, under the stress of circumstances, those fundamental biological requirements, - constitution and high breeding powers; and history shows that this has been repeatedly done, to the extinction of some of our otherwise most promising creations. When this result does not follow, the inevitable consequence of too many points of selection is that we are forced to accept defects, now one and then another, as has been shown, until, under Mendel's law, the breed becomes 
at best a mixture of good and evil, - a mixture, moreover, that will never purify itself, and that can be purified only by a return to first principles.

\section{SECTION III - FASHION}

As fashion decrees the cut of our clothes, so it also decrees the length of the tail of a cow or the shape of her horn, and the height at which a horse should raise his feet from the ground. If fashion would be reasomable, and consistent, and stable, it would not be so bad, for breeders could finally adjust themselves to its demands; but it is not stable, and often it is neither reasonable nor consistent.

Now it is not so easy to change the conformation of an animal as it is to alter the cut of a garment, which means at the most only a turn of the shears this way or that. Every one of these decrees of fashion indicates an additional requirement for selection, and we know what that means to the breeder; not only that, but such decrees are certain to be short-lived, changing for others more or less troublesome. Worst of all, many of these requirements of fashion are to the distinct and permanent disadvantage of the breed - that is, permanent until bred out, which we have learned requires approximately six generations of successful selection.

But the mandates of fashion are to be reckoned with, erratic and troublesome though they may be, for in a very larese measure they ditermine sales and fix prices. Now the breeder is in the business for money, and he must sell stock or abandon all hope of profit, - which in the end means to abandon the enterprise entirely; and no phase of practical breeding calls for more wisdom and shrewdness than this particular problem, - how to meet the changing demands of the market and keep the herd or stud intact and if possible improving.

In so far as these fashions emanate from the open market their control is practically beyond the breeder. I3ut some of the worst of them emanate from among the breeders themselves, who sometimes seem bent on inventing artificial issues on which to make sales. Sometimes there comes a feeling, apparently, that 
there are too many animals of a siven breed available, and that, in self-protection, new standards must be set up.

This is confusing to the buyer and only hurtful to the breed. As a matter of fact, there never were and never can be tow many really excellent animals of any breed. The question of develop)ing the market for pure-bred stuff will be discussed later, but here it is enough to say that no artificial standards should be tolerated in any breed merely to create sales. This matter can be controlled by the breeders themselves in theirown associations, and when it is controlled a large share of the senseless and disturbing "decrees of fashion" will have disappeared and the remaining ones will have been mostly modified into comparative harmlessness. There is tor much homemade law passing from mouth to mouth among breeders, without the sanction of associations, and much of it would never be seriously supported on any floor if the advocates were really required to seriously defend it. Here is a duty that every association owes the breed it advocates and whose interests it maintains.

But when all is said and done, how shall the individual proceed to meet the decrees of a craze that in his jud gment will speedily pass?

There is no better way than by the use of sires that strongly possess the points demanded in the market, always being careful to preserve a goodly number of the best females uncontaminated from the infection. These will form the nucleus of the new herd or stud after the craze has passed and the pendulum has swung back to the normal.

Ifere the breeder must be wise in his judgnent as an whether a new thing is only a passing crowe or is really a permanent improvement in the breed, and here his acemmulaterl knowledge of animals will serve him well; but he should be well atrised that by the proper use of the sire a herd may be marle to furm out a new style of animal for a consiclerable time without in any way affecting the real character of the fonndation, and this can be continued as long as the old streck of females lasts. As the time of their end approaches, however, something must be done to restore their number, or else the new proint must be accepted and bred into the females which really constitute the backbone of every producing herd. 


\section{SECTION IV - SHOW-RING CONSEQUENCES}

Animals that have mate their record in the show ring are none the worse for that fact, and this success adds greatly to their credit as individuals and to the commercial value of their get afterward. Although the excessive fitting required in the ring is often injurious, it is not necessarily so. It is of course true that no animal will remain long in "form," nor can the process of fitting be repeated many times. Show-ring animals are thus often a disappointment to the eye later on, but this is no detriment to their breeding powers. The only danger from excessive fitting is its effect upon fertility, and if this has been impaired it will very soon become evident.

It is a serious question as to whon a breeder can afford to take the risk of putting into the ring a valuable breeding animal. As a matter of fact, if not of necessity, most show animals are young. The writer cloes not share the opinion that show-ring animals have necessarily been injured for breeding purposes, any more than he shares the opinion that show-ring success is a guaranty of breeding powers. Upon this point nothing is reliable but the actual test.

\section{SECTION $\mathrm{V}$-TESTING OF SIRES AND DAMS}

When we remember that variability cannot be reduced below 89 or 90 per cent of the variability of the race, or, in extreme cases, perhaps to $S_{5}$ per cent, and when we appreciate the fact that no matter how much the type (or mean) has been improved the variability remains, then we are no longer surprised at the large number of mediocre individuals that appear even in blood lines the most aristocratic and that have been longest "in the purple."

The necessity for selection, therefore, will always exist, and when we add to this that other fact, that many mediocre-looking animals are after all great breeders and many exceptional individuals bitter disappointments, there is an additional reason for the actual test.

And still again, no one knows positively what will be the result of a new combination of blood lines until it has been 
tried; thus, by every count, we arrive at the conclusion that real progress is assured only with tested animals.

Testing dams. The test of a dam is what she has produced. If the herd has been bred by the owner, - and in general no other course is consistent, - then the records of the herd will show the breeding powers of every female in it, and any one that is not satisfactory should be promptly eliminated. If this course is pursued, then at any given moment the owner of an established herd will be in possession of a tisted herd, so far as the females are concerned, and the only additional testing is of each new sire that is brought into service. ${ }^{1}$

This is a comparatively simple matter if the breeding powers of the female side of the herd are well known. If they are not well known, the breeder is worse off than the ship at sea without rudder or compass; he may multiply animals, but he will never do much real breeding. If a female is brought into the herd, she should on all accounts be brought in on her breeding record if possible; for, of a hundred females, only a few will prove great mothers or even good mothers. If, of necessity, young females are put into the herd, then they must be regarded, like the regular produce of the herd, as under a test until each shall prove herself a breeder entitled to a place in the permanent herd.

Testing young females. This is a job that the breeder has always at hand. His herd is short-lived at the best, and however grood it may be it will become extinct by cleath in a few years unless reënforced from younger stock.

While individuals live many years, it is found in actual practice that the claracter of the entire herd will change in five years with cattle and horses, and in much less time with sheep and swine, unless properly reenforced with young animals. A breeding herd is a moving tide of life, and what the breeder does he must do quickly. He must reënforce the stream while it is at the flood. He must keep the number high by continual reënforcement and not wait till the herd is shrinking on his hands.

1 It is needless to remark that many breeders have not yet learned the principle of maintaining their own stock of females; indeed, a good number confess to buying females to "keep up the herd." 
The testing of young females is a difficult business. There is little use in breeding them to unknown sires, whose own breeding powers are problematical. To do that is to measure one yardstick with another whose standard of accuracy is unknown. The young females should be bred whenever possible to tested sires, and then the breeder will have an accurate measure of what they should be expected to do under herd conditions.

Unity of the herd. The old plan of having represented in the same herd all fashionable families in the person of its females is fortunately passing. Such a herd, no matter how excellent or well bred its individuals, was after all but a motley collection of strongly bred differences, on which no sire ever born could be expected to succeed. Such attempts have involved many breeders in a hopeless tangle of Mendelism, for these violent admixtures of family lines amount to little else than crossing, either in theory or in practice.

The individual breeder succeeds best who attempts to do a distinctive thing, and who preserves one type throughout his collection of females, which is his herd. He will find this diffi. cult enough to accomplish without seeking the multiplication of types; and he will, if he is wise, discard many females in the testing, because, if working with well-known and tested strains of line-bred stock, one or two tests of a particular combination ari as good as a dowen. There is no need that the breeder should waste time and money and live in uncertainty if only he will keep his type distinct, his blood lines pure, and will test every animal that is to have a permanent place in the herd. If he will not do this he will indeed be treading a maze of uncertainty, and will be ready to say at the end of a long life and as the fruit of his experience, "Verily, breeding is a lottery," - an honestly uttered but gross libel on one of the greatest professions on earth.

Testing sires. A well-established herd has always in service a mature and well-tested sire who has proved his breeding power on some of the best-known females of the herd. ${ }^{1}$ Not only that, but the owner of such a herd is always looking for his successor.

1 Among the many answers to questions touching this point a surprisingly large proportion of breeders confess to testing bulls, not on cows of known breeding powers but on heifers. 
If an old and proved sire can be had, that is the sire to buy, but ordinarily sires of this kind are not obtainable, for, if they are really tested sires, they are usually held in the herd that tested them until their period of usefulness is over. If, however, one is available, it is a treasure that should not go begging, as it often does. If the young breeder would make it a rule to buy only old, tested sires, - though there is no virtue in old sires per se, - he would do better breeding than many another with long experience behind him who is constantly accumulating excellence and as constantly dissipating it by the use of untested sires.

The writer has conducted an extensive correspondence with breeders of cattle on this point, and has found that the almost universal practice is to buy a young bull, probably a yearling, and put lim at once into service on the entire herd. This is business suicide, for it constitutes a bar to any rery high degree of success and is, besides, extremely dangerous. It is headed straight for mediocrity - within the breed of course, but it is mediocrity nevertheless.

Testing young sires. This test, to be most valuable, should be made on some of the better females of the herd, whose breeding powers are known. It would be folly to use the very choicest individuals, for they are needed for more certain work with the tested sire at the head; but something must be known about the females on which eren the preliminary test is made, or it is of little value.

As all young things look promising, this test will not be worth much until the young have neared maturity. To be sure, some individuals will be so unpromising as to show it at birth, or soon after, but very many mediocre animals will not make their mediocrity manifest until maturity approaches. They then exhibit their inability to take on the finer finish and the better touches that go with the breed.

On this point experience is full. One of the finest sucking colts ever known to the writer was exceedingly perfect as a yearling, gave good promise as a two-year-old, commenced to fall away as a three-year-old, and before he was five had developed into a veritable "pelter" with ewe neck and sway back. 
If the young that are to show the breeding powers of prospective sires must reach practical maturity much time will be consumed in the process. In cattle, for example, the young must be not less than one, and preferably they should be at least two, years old. Practically a year was consumed in pregnancy, and the bull was a year old at the time of service. This will make the prospective sire at least four years old by the time his breeding powers are actually known. This is the age at which most bulls are sold as "ugly and dangerous," a practice which is deplorable. All bulls are ugly or dangerous, or both, — that is always to be assumed, - but at four years of age they are just ready to enter upon the period of real usefulness, and the records will show that all great sires have done their work not as yearlings but later, after their powers were known.

I Iaving been tested and proved on a portion of the herd, a sire is, of course, placed in full service, and it is but business sense to make the most of him as long as he is able to continue at its head; it is even more business sense to begin at once to look for his successor.

A herd without a head. Herds pass quickly, and a herd without a head is doomed to speedy extinction unless a suitable one can be found. A herd in such a conclition presents a hard problem to the breeder and owner. He has a lot of accumulated excellence, but it is liable to die before he can use it unless he puts a proper sire at the head without delay. If he cannot do that, in all probability dissipation will follow, which is but another form of extinction. In this event the only practical remedy seems to be dispersion, and this is the real reason for more than one of the clispersion sales that come along each year to arouse our wonder.

The dispersion sale affords an exceptional opportunity to secure real excellence in breeders, for animals are there offered that ordinarily no purchaser could buy, but these sales are not necessarily for the best interest of the breed; in many cases it would have been better if the herd could have been kept together.

Tested individuals, male and female, are the backbone of the herd, and these are what the wise breeder will preserve through 
all the ups and downs of his experience. They are his chief stock in trade, and he will cherish them as any other business man would protect a vested interest.

SECTION VI - WEATHERIN( A PERIOD) OF IIEPESSION AND PRESERVING THE HERD

No herd can live without ruining its owner unless sales are made regularly and at good prices. It is a stream that cannot be stopped without damage.

More than once in the history of most breeds a time comes suddenly when for some reason, or for no assignable cause, prices drop and matters collapse generally. This calls for all the ingenuity of the breeder and all his fortitude in dealing with a difficult situation.

One thing is certain, - the herd must be reduced. It is simply business folly to go on multiplying animals in the face of no market. Such a course leads to unmanageable numbers, and when they seem to have lost their value no man has the courage to do for them what a real breeding herd requires. Under conditions such as this the herd is doomed to neglect. It is only a question of time when their hungry eyes will become a positive source of displeasure, if not of disgust, to the owner. No one ever looked upon such a herd without a feeling of sorrow, for its end is extinction, even though the storm pass and the palmy days of the breed return.

Neither is the other extreme to be advocated, - the dumping of everything upon the open market for what it will bring. The writer has seen Shorthorns that cost $\$ 300$ to $\$ 500$ sold to the butcher for $\$ 40$, to be killed and eaten, only because in sudden panic the owners had assumed that the Shorthorns had seen their day.

Now a really excellent breed will never "have its day." If it looks that way it means only that the day will come again, and not so very far in the future. The breed has served us before and it will serve us again, and the man who sells the cream of his herd to the butcher or "shoots his horses to feed to the hogs," - he is the first man on the ground to restock himself 
at long prices from the herds of those who were wise enough to protect themselves and make everything taut while the storm might last, but who continued in business against the day when the market would again want the produce of their herds.

When the herd is reduced at such a time, and it must be reduced, it is the young and unproved stuff that should be sacrificed first, and it is marvelous how much can be sold off withont disturbing the ral muclius or producing part of the herd.

If the herd is not all the breeder could desire, such a time is the most favorable opportunity that will ever appear to perfect the herel by purchase. This is the time to gather in the mothers and the grandmothers of the breed from other herds, keep them out of the butcher's hand, and set them at work; and about the time they have produced another generation, their former owners, or other equally anxious purchasers, will be ready to pay more for a calf than the tested dam and sire both cost. Every one who has lived long among breeders has seen this stampede out of the business followed by an equally insane tumble into it. The solid breeder will hold himself well together at such a time and avail himself of the opportunity both to improve his herd and to reap an assured harvest later on.

\section{SECTION VII - RECORIS}

One of the requirements of all grood breeding operations is an accurate system of records, covering every important detail, leaving nothing to memory. Moreover, the record should be made on the spot.

Herd records. Simple records of all purchases, sales, births, and deaths are matters of ordinary business accounts and inventory, but in addition to these there should be kept what may be known as the performance record of the herd. This consists of three distinctly separate features :

I. An accurate description in writing of every individual animal of the herd, talien not only at maturity but at birth or time of purchase, and as often thereafter as changes in development occur. Such a record should be a descriptive history of the individual from birth to cleath, or at least to disposal, accompanied 
by photographs, if possible, and by any measurements, weights, achierements, or facts of any kind that may later assist the judgment in basing conclusions as to selection. The record of the service males should be especially full and complete.

2. A full description of every individual offspring of every sire of the herd as compared with his own description and with that of the dam, - such a series of descriptions to constitute the breeding record of the sires.

3. A breeding record should be opened with every female of the herd, showing date of service or services, date of birth of all offspring, and some name or number which may serve as an identification mark for every individual produced, whether living or dead.

If these three lines of clata are kept, the breeder will have not only an accurate personal description of every animal constituting the herd and every one produced by the herd, but he will also have a complete breeding record of every animal, both male and female, and the two together will show not only how much each animal has produced but also what was its quality. When breeding operations have been carried on after this plan for a number of years, such records will constitute a mine of information which no memory can supply with sufficient accuracy to be dependable. Trusting to recollection is dangerous, and the fleeting impressions formed of certain individuals become rapidly distorted and unreliable as time passes, - how rapidly no one knows until he has been confronted a few times with his own record made first hand and upon the spot. These records are essential to the best breeding and absolutely indispensable to the man who may succeed to the management of the herd later on.

Breeders too often proceed as if they expected to live forever, or at least as long as the herd exists, whereas we must look forward to the time when an established herd shall outlast the lifetime of its founder, and perhaps two, three, or more generations of men shall be involved in shaping the policies of its breeding, - all of which is possible only when the most perfect records are kept.

In connection with private herd records the following is appended as the plan in actual use by the late Honorable 
L. H. Kerrick, of Bloomington, Illinois, a very successful breeder of the Aberdeen-Angus. These records are kept on cardboard $5 \frac{1}{3} \times 8_{4}^{1}$ inches, and containing no printing whatever. They are kept on edge in alphabetical order in a special box, and it is but a moment's work to note the present condition of any female in the herd; moreover, every time the owner consults a card he is afforded at a glance and involuntarily a picture of the complete breading record of the cow for her cntive lifetime. Two records are presented, one of Fair Lady of Verulam and the other of her first offspring, Fancy Fair.

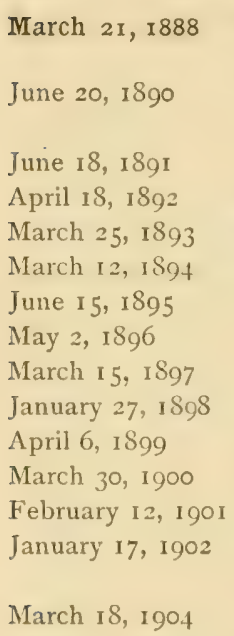

\section{Fair Lady of Verulam}

Fancy Fair

C. 15215

Wapella Boy .

B. 15094

Ebon Lizzie

C. 16988

Bunty . . . . . B. I9I 55

Irene of the Wells .

C. 21243

Lucy M. of the Wells. . C. $2345^{\text {I }}$

Fairie D

C. 24961

Lygia of the Wells

C. 27945

Florence F. of the Wells

C. 32444

Erie of the Wells . . . C. 34196

Senator Hoar of the Wells B. 41949

Statesman of the Wells . B. $47 \mathrm{SI}_{3}$

Bunker of the Wells

B. 57703

Florida of the Wells .

C. $7575^{\mathrm{I}}$

\section{June 23,1890}

\section{Fancy Fair}

I5215

\section{August 20, I 892}

September I0, I 893

July 2, I 894

February 6, I 897

December 28, I 897

January 21, I 899

February 24, 1900

January 15, 1901
Wapella Lady .

C. 16991

Fair Fancy

C. $1924 \mathrm{I}$

Rose $\mathrm{F}$. of the Wells

C. 36968

Little Ben

B. 27944

Operator of the Wells

B. 27962

Graymont of the Wells . B. 32536

Crouje of the Wells.

B. 41945

Miss West of the IVells
Ermine Bearer . I749

Fair Lady of Chil-

licothe . . 2760

Ellenreagh of

Kinnoul Park 10203

C. C. Allen . . I 266

Ebonist . . 5266

Ebonist - . 5266

Ebonist . . 5266

Craigo of Estill. 19518

Craigo of Estill . 19518

Craigo of Estill . 19518

Willis E. Gray - 2475 I

Craft of the Wells $2345^{\circ}$

Craftof the Wells $2345^{\circ}$

Craft of the Wells $2345^{\circ}$

$\mathrm{Painstaker}$ of

Aberlour . 34220

Painstaker of

Aberlour . 34220

Ellenreagh of

Kinnoul Park I0203

Fair Lady of

Verulam . 9122

H. C. Allen . I I 266

Ebonist . . 5266

Ebonist . . 5266

Craigo of Estill . 1951S

Willis E. Gray . 2475I

Willis E. Gray . 2475 I

Royal Judge . . 2037 I

Painstaker of

Aberlour . 34220 
December 16, I90I Andee of the Wells . B. 50342 Painstaker of

Aberlour . . 34220

November 20, 1902 Castro of the Wells . . B. 57747

Painstaker of

Aberlour . 34220

December 2I, I903 Black Heath of the Wells B. 72985

November 27, 1904 Fan-Dan of the Wells B. (Castrated)

Painstaker of

Aberlour . . 34220

Danwessels of

the Wells . $4782 \mathrm{I}$

The plan of the record is simple. It shows that Fair Lady of Verulam, born March 2I, IS8S, was recorded as No. 9122; that her sire was Ermine Bearer I749, and her dam was Fair Lady of Chillicothe 2760 . It shows that her first calf was dropped June 20, I 890 , when she was a little over two years of age. The C. shows that it was a cow calf. It was named Fancy Fair, and recorded as No. I52I5. Her sire was Ellenreagh of Kinnoul Park 10203.

Thus the first name in the first line is that of the cow whose record is to be kept. All that follow in her line are her offspring, and all the names of the second column are their sires, cxcopt the first tavo. Of these, one (the first) is her own sire, the other is her dam. ${ }^{1}$

In transmitting these data Mr. Kerrick observed:

You will see that whenever I am so inclined, I can commence with the letter $\mathrm{A}$ and lift up these cards in order until the right one appears. Instantly, when I lift a card, the life performance of a cow is shown. It does not take long to go through a large herd and see just what each cow is doing. If I find a number of cows not showing a calf reasonably near the date at which I am looking through the cards, we can make a note in each case.

It would be interesting to you to see what this one cow, Fair Lady of Verulam, has done. If a 22 -year-old boy would luckily buy three such cows, he would have a fine big herd at 32 , and after that all the cattle he would want. On the other hand, with a couple of shy-breeding things, and they bringing mostly bulls, he might not have any more at 32 than when he started in the business. A big part of our herd to-day (numbering over 300) are descendants of Fair Lady of Verulam.

These cards might well be extended to cover other details, especially as to whether the offspring is retained in the herd or sold, and, if sold, to whom and at what price.

1 On the back of these cards is an outline for an extended pedigree. 
This latter point is covered in the system in use by A. J. Lovejoy, of Roscoe, Illinois, a well-known breeder of Berkshires, a sample card from whose herd is here reproduced:

Index No. I6. Imported Bessie II 5510I, farrowed April ro to Masterpiece 77000. Farrowed 5, saved 5: boars 4, sow I

Sold boar to J. W. Martin, Gotham, Wisconsin . . \$5 50.00

" " " L. W. Brown, Berlin, Illinois . . . . . . . . . 75.00

" " " J. R. Iogan, Seward, Illinois . . . . . . . . . 50.00

" " " Hibbard \& Brown, Michigan . . . . . . . . I25.00

" sow "Nebraska Insane Hospital, Lincoln, Nebraska . . . 50.00

Total for litter of 1905 .

$\$ 450.00$

In connection with office records of this kind the "breeding book" kept at the barn should record the date of every service, the date, sex, and any distinguishing fact concerning the birth of every individual, living or dead, and any other fact that would prove of the slightest value in estimating what the herd is doing or has done.

If, then, in addition, there were kept an accurate "descriptive record" of every individual that is considered worthy to enter the herd, or to be sold as a breeder, and besides this also as accurate a record of the unadorthy products of the herd, the breeder would have - not only for his own satisfaction and profit when memory fails or becomes confused, but for that of others who may handle his breeding - a record of qualities good and bad on which a skillful breeder can safely base his selection. Without a record such as this all real selection is limited to what is done with the eyes on animals still living, except as a man may be guided by an uncertain memory. How uncertain that is he will realize when he revisits in full prime of life the hills and valleys of his boyhood.

Pedigree records. That which is needed within the herd is equally important within the breed. The facts of heredity go to show that all good breeding requires that the type shall be unchanged for at least six generations, if we hope to get anything like uniformity of offspring. If this be true, we need accurate records covering all important details and all valuable characters for at least the six generations required to produce a stable type. 
Now our pedigree records furnish little information outside of blood lines, and they are totally silent as to what the individuals actually were in their own personalities. This information the breeder needs and must have if he is to succeed. Some slight beginning has been made in the way of track records among racing horses, and in advanced registry among dairy cows. Then, too, breeders aim to supply in their private catalogues some detailed information about particular animals ; but in many cases such description contains so large an element of advertising as to throw doubt upon its accuracy.

As the case stands to-day, there is no way in which an impartial and trustworthy record can be assured even of our most famous and valuable animals. This being the case, the individual breeder coming into the business must devote years of his life to the accumulation of a mass of data more or less reliable, gathered irregularly and often surreptitiously from the undercurrent of side talk in which old and prominent breeders, like other mortals, sometimes indulge.

Now this ought not to be. Such information belongs to the breed and to future breeders, who have a right to know the facts about the animals whose blood lines they are obliged to use; and sometime, in some way, when commercial interests are no longer supreme, - if not before, - an accurate and impartial description of every great animal will be made a matter of permanent record and will find its way into the history of the breed.

It is to the interest of the breed that this should be so, and it is also to the interest of the young breeder, that he may proceed at once and intelligently with his breeding operations and not spend twenty of the best years of his life in collecting information by indirect and of ten devious methods, - information that is by good rights public property, and as such is the rightful heritage of every man from the moment he becomes a breeder of that particular breed.

A brilliant future awaits the breed that will secure and put into its history an accuratc and critical description, at least of every famous animal, said description covering all distinguishing or unusual traits both clesirable and undesirable, and not confined to extravagant praise. 
If this is ever to be done, some feasible plan must be devised. Now in this matter two things are self-evident: first, such truthful and critical description could not be made, or at least made public, during the lifetime of the animal, while large commercial interests were involved; and second, more than one man's judgment should be consulted in making the actual record.

The writer ventures to suggest that while the animal lives, and is in his prime, and while his character and achievements are well known, a full statement of his achievements be made and two critical descriptions be prepared, one by the owner, the other by a committee of the association, - all to find a permanent place in the published records of the breed.

How this object can be achieved is problematical, but until it can be achieved, the best results in animal breeding will never be possible. One thing is certain, - the public at large and the association of breeders in particular have an inherent, if not a vested right, in every animal that comes prominently before the public, and sometime, in some way, this larger right of public ownership will be conceded greatly to the general interests of the breed and to the convenience of future breeders; in other words, not even private commercial interests will always intervene to prevent a record of the facts, until, by the death of all parties possessing actual knowledge, the real personality of a famous animal has become lost beyond the power of restoration.

The blood of not only one famous animal but of many famous animals runs through the pedigrees of all our herds. The fame of some of them rested on real excellence and well-earned merit ; that of others was due chiefly to skillful management, often to shrewd advertising. Animals of both classes possessed points of high excellence, and both classes also possessed defects. The public has a right to the facts, which are no less than a vested interest to every man who owns a breeding herd.

\section{SECTION VIII - DISPOSAL OF SURPLUS FEMALES}

The herd itself must make the first draft upon its female output in order to secure material to reënforce its numbers. Some females will be needed by other breeders of standing to 
replenish or extend their herds. What shall be done with the rest? The answer to this question depends somewhat upon the class of animals and the circumstances of the breeder, but on general principles the destination of surplus females should be the open 'market, and this destination should be reached as soon as possible after unfitness to take a place in the permanent herd is well established.

The one thing that should not be done is to employ this surplus generally as material for the establishment of new herds. There is a feeling among breeders that no animal eligible to registry should be sent to the open market, especially to the shambles. Nothing could be more erroneous. To use surplus females for the establishment of a mulcitude of small, weak herds in the hands of men who have no experience and no genius for breeding, is at first to arouse vain hopes that will not be realized and afterward to bring down curses not only on "blooded stock" and breeders in general but on this special breed in particular.

The safest and the best destination of all surplus females is the open market, where they will sell for what they are worth and be entirely safe and out of the way, with a small but safe balance to their account on the books at home, after having afforcled the best possible practical test of the real commercial value of the type that is being bred in the herd which they represent. In this way all females help to test the herd.

\section{SECTION IX - A MARKET FOR SIRES}

It is quite the opposite with males. The great business of all pure-bred herds is the production of sires, and the country ought to be industriously campaigned in the interest of "placing" sires for grading purposes. If they cannot be sold let them be rented, or in some way gotten at work. Let there be coöperation between breeders, even between breeds, for the placing of sires. Let salesmen cover the country as do agents of machinery, and sell sires on some terms. The practice of grading must be brought into American farming, and nobody is so much interested in this as the breeders themselves. The common 
stock needs the sires for the service, and the breeders need the market.

Breeders are selling too much back and forth among themselves. The breeding business is too much of a mutual benefit association, while there is an undeveloped public with almost unlimited buying powers, that needs to be educated and its buying powers developed. Many a breeder works inclustriously to sell two or three females and a sire to a novice, partly for the money that is in the sale and partly to spread the gospel of better breeding, as he thinks.

It does not work that way. A novice has been started in a small business. The chances are great that he will not succeed. He will either fail and curse the breed, or succeed only indifferently well and make an undesirable competitor who is willing to sell stock of the "same breeding" at prices much below what they must cost when produced by careful breeding.

If the same man had bought a sire he would have been satisfied with the new breed, and he would be on the road to a permanent habit of keeping better live stock. I Ie would then become a customer again and again. From any point of view the breeders must develop the market for sires for grading purposes.

\section{SECTION X-COMMUNITY BREEDING}

Many advantages will follow if an entire community will go into the production of a particular class of animals, as, for example, driving horses. There are a thousand little details in the successful management of any business, and for the best success, mind must react upon mind. If a whole community would go into the production of driving horses and discuss the driver, his breeding, care, development, and education, as communities now discuss corn raising in the corn belt, in a few years every man, woman, and child in that particular locality would "know all about horses." They would soon become skillul drivers, and, as is now the case in the famous blue-grass region of Kentucky, the community would have a reputation that would attract buyers, and a horse would bring more money than he could bring if he were the only one in the neighborhood. 
Let the whole community, as far as possible, breed the serme kind of horse or other animal, so that it may win a reputation for a distinctive product, and it will not only do better breeding than it would if it were to breed many types, but the business will be vastly more profitable. Practically all the canaries of the world are bred in two villages in Germany; and no bird with a false note is ever allowed to live, so skilled have the entire village become in what might be called canary excellence. No individual breeder can ever equal the degree of success which is here evolved, where practically no other interest engages the attention of the community.

\section{SECTION XI - THE YOUNG BREEDER}

There is no reason why the young breeder should not possess himself thoroughly and quickly with the principles of breeding. What he lacks is experience with animals and real information about breeds. He should get his experience either by grading or by association with a good herd, but he must needs pick up his information, much of it at least, from intimate association with men who are in the active business of breeding.

The "sucker" in the sales ring. Above all, the young breeder must keep his head. He does not need and he cannot afford to pay large prices for females. If he sees some old and established breeder bidding high on a young female, he must not assume that he can afford to bid equally high. There are a dozen reasons why the older breeder may want that particular animal, none of which would apply to the young breeder. For example, it may be the only one of that particular breeding outside the herd of the older breeder, and he can perhaps, or thinks he can, afford to pay even more than the animal is worth for the sake of controlling that particular combination; or he may want it to put into the show ring, or to mate with a particular sire. None of these reasons would apply to the young breeder, who, if he buys it, takes it for its merit.

The best way for a young breeder to get his start in pure-bred animals is to get it from a reputable breeder who can be persuaded to sell some really excellent and tested, or partially tested, females. 
It is doing no injustice to any breed to remind the young breeder that the bulk of young things come to very little. He will be safer with old animals - even those of considerable age, providing they are still fertile.

With him much depends upon price. He has no call to pay extreme prices. He cannot sell his stuff at maximum prices till he has been in the business long enough to acquire something of a reputation, and one of his best early reputations is as a careful, juclicious buyer.

If the young breeder ever loses his head in the sales ring, let him not do it on a female, that can at best procluce but few, or upon extremely young things, which stand about as many chances of coming out wrong as they do of coming out right.

\section{ADDITIONAL REFERENCES}

Animal Breeding. By W. M. Hays. Breeders' Gazette, XLV, I99, $252,305,356,461,513,565,608$.

Breeding Bees to increase Lengti of Tongue. By J. M. Rankin. Michigan Experiment Station Report, 1897, p. 127 ; also in Experiment Station Record, XI, 6I, 1062.

BREeding Experinents With Sheep. Missouri Experiment Station, Bulletin No. 53, pp. $167-188$; also in Experiment Station Record, $\mathrm{XIV}, 383$.

Breeding Poultry. Experiment Station Record, XIII, I76.

Breeding Poultry for Egg Production. By G. M. Gowell. Maine

Experiment Station, Bulletin No. 79; No. 93, pp. 69-92; also in

Experiment Station Record, XV, 394.

Breeding Sheep to change Breeding Season. By T. Shaw. Minnesota Experiment Station, Bulletin No. 78, pp. 7I-87.

Cross-Breedixg Chickexs. By E. P. Miles. Virginia Experiment Station, Bulletin No. 96, p. 6 ; also in Experiment Station Report, XI, I074.

Cross-Breeding; Suefr. By F. Winter. Agricultural Gazette, London, 1900 , p. 246.

Cross-Breeding Swine. Experiment Station Record, XI, 1077.

Crossing Cattle. Experiment Station Report, Vili, 720.

Hybrid, Ganecock-Guinea-Fowl. By T. Vilaro. Bulletin of the American Museum of Natural History, 1897, p. 225 ; also in Experiment Station Record, IX, I03I.

Penigree Stock Records. (Report of Committee on Photographic Methods of Preserving.) By Francis Galton. Report of the British Association for the Advancement of Science, 1899, pp. 424-429. 


\section{CHAPTER XXI}

\section{DEVELOPMENT}

Thremmatology is interested in growth as well as in reproduction; in the proper development of valuable characters as well as in their transmission and inheritance.

What an individual comes to be at maturity is a kind of resultant of the characters born into him and the opportunities for their development afforded by the conditions of life. IVhile the surroundings during development cannot in any sense compensate for deficiency in inheritance, the opposite is also true, that the richest heritage is fruitless of results if conditions of life make their development impossible.

External conditions only indirectly causes of variation. Conditions external to the organism thus operate only indivectly as causes of variation. Their good results depend entirely upon the capacity on the part of the inclividual or the breed to avail itself of their advantages, and this capacity is born into the organism or it cloes not possess it, - it cannot be implanted from without. That is to say, no amount of feeding would make draft horses out of those that are racing bred, or beef cattle out of those bred for the clairy, and it would be a great waste of feed to try it. Certain experiments with individual animals, it is true, seem to teach that Jerseys, for example, are successful feeders. Such experiments will deceive no one who realizes the full extent of variability in all breeds, and that indiaiduals can be found to prove almost anything. When some adventurous investigator will take the trouble to feed off three hundred Jerseys against three hundred Shorthorns, and work out the mean and the standard deviation for both, then he will learn that a breed cannot be selected for unknown generations for milk only, and still retain or attain meat-producing powers equal to those of a breed selected almost exclusively for that purpose. If it could, there is little in selection and less in breeding, and the wonder is that 
anybody ever seriously doubted this fact. This is altogether outside the question of the "dual purpose" animal, for no attempt has been made to breed the Jersey for other purposes than milk production. Nor is it a reproach to Jerseys that they do not excel in something for which they have never been bred. As well expect them to take records upon the race track, or to do any other thing for which they have not been fitted by selection.

The influence of the environment is therefore permissive rather than assertive. It affords the material and the opportunity for development of what was born into the individual, and what was not born into it cannot develop, no matter how favorable the environment, - as witness the very different development of two individuals differently born but living under the same conditions of life. If an individual is exceptional, we may say of him with confidence that he was both well born and well conditioned during development. If, on the other hand, he is inferior, we are uncertain whether to attribute it to non-inheritance of valuable characters, or to their failure to develop owing to unfortunate conditions, or to both.

Well-bred individuals should have good conditions. It is manifestly unwise to expend time, money, and thought on the production of individuals highly endowed with the richest possibilities of the race and then fail to provide the necessary conditions for their development. Ordinary business scnse, therefore, dictates that the breeder should secure icleal conditions for the full and proper development of the characters whose improvement he aims to secure through fortunate combinations of blood lines. To see herds of the best-bred animals suffering for feed is at once pathetic from the humanitarian standpoint and unaccountable from a business standpoint.

Having spent time and money for the infusion of the highest possibilities into the herd, certainly business foresight demands that their full realization shall be prevented by no ordinary circumstance; yet what share of the best-bred animals and what proportion of our improved seeds are given full opportunity to show what is really in them?

The breecler is interested for another reason in securing the full development of all that is born into individuals and family 
lines. In no other way can he judge of their real excellence and in no other way is his selection safe. The practice of fitting for the show ring is often deplored, and not without good reason, but of one thing we may be well assured, - we can never be certain of the capacities of an individual until they have been put to the test by development.

One of the hard facts of animal breeding is that the dcaclopment of young things is very often left to men who are not slilled in animal production. They seem to assume that the well-bred animal in some way can get along under less favorable conditions than can the unimproved, - a kind of offsetting of breeding against feeding and care.

Improvement consists in producing animals and plants able to make good returns for good conditions, not merely to exist under hard conditions. This fact ought to be pasted in the hat of every farmer. The purpose of breeders is not to produce strains that can live on next to nothing, and that are able to enclure hard conditions and not dic outright; it is to produce strains of animals and plants that are able to make good roturns for the fuller feed and better care which the civilized and educated farmer can give as compared with nature, which is capricious, or with the unskillful semi-savage, who is improvident.

One of the most serious faults of unimproved strains is that they do not respond to better food or to more of it. They have been selected for generations for their powers of resistance to hard conditions, and that is where their strength lies. Now that we can provide better food, we need animals of higher efficiency ; indeed, that is our argument for better animals. Then, again, having animals of higher efficiency, we need better feed and more of it, and that is our argument for better-bred corn and other crops. So the two - animal breeding and better feeding - react the one upon the other, and both go with better farming and with the greater needs of an advancing civilization.

The well-bred animal is a high-class machine. This fact cannot be too well understood by every man who comes into relations with the well-bred animal, and it is true, whether we consider animals as machines for the producing of milk or meat, of labor, or of body covering; whether we consider that they are to 
minister to our necessities or to cater to our enjoyment by personal service, as with the saddler and the driving horse.

Excellence is not to be measured by the power to withstand deprivation, but rather by efficiency to do work under full feed and under good conditions; and to this end it is but good business sense to secure for each individual the full development of all the useful qualities with which he is naturally endowed.

Development is a study by itself. Here is an entire field almost unexplored. We know, in a general way, that the "energy of embryonic development" is never attained later in life, and that if we would secure full development in growth we must "keep the young thing growing." In some way or other this business of body development, if once checked, is never again fully resumed. It is true that a few experts have learned fairly well how to develop speed in horses, and others how to train saddlers and drivers; but whother we consider the grozeth of the body, the deaclopment of its functions, or the cducation of the mental faculties, we do not yet posscss coen the madincuts of the knowledge of the most successful development. With us only an occasional individual enjoys optimum conditions throughout his life, and only a few exhibit in their ow'n personality the really wonderful capacity of the breed to which they belong. If one were to say that the science and practice of breeding is far better known than that of development afterward, he would be well within the truth, and in the opinion of the writer here will lie some of our greatest improvements of the near future. The excessive fitting of an occasional individual for the show ring, regardless of consequences afterward, is not what is here meant, but rather the orderly and full development, in substantially all individuals, of those qualities which we deem valuable, so that we may fully realize in our animals the qualities we select and breed for in our yards. 


\title{
APPENDIX
}

\section{STATISTICAL METHODS}

\author{
BY H. L. RIETZ, Ph.D. \\ Assistant Professor of Mathematics, University of Illinois
}

\section{SECTION I - IN'TRODUC'TION}

An elementary account of the mathematical theory of statistics in a treatise on Thremmatology needs no justification after the foregoing text. The doctrines of evolution and heredity rest on a statistical basis, ${ }^{1}$ because we are, in general, concerned with groups of individuals, and with occurrences of such a nature that, although we cannot make definite quantitative statements about any one of them taken singly, we can make statements in regard to a large number of them taken together with a degree of certainty which increases as the number increases. For example, a thousand ears of corn may vary in length from three inches to eleven inches and have an average (average to be defined in Section II) length of 8.5 inches. We cannot state with any degree of certainty the length of an ear selected at random out of this group of a thousand ears; but if we select at random five hundred ears out of the thousand we can assert with considerable confidence that the average length of the five hundred ears will differ but little from 8.5 inches.

The important questions in every case are these: In what way can we best describe a population whose variates we have measured? How can we give the meaning and information contained in this mass of figures in a few words or symbols?

A glance at the figures may give a personal impression, but this is not reliable, as is proved by the fact that two persons may each get a very different personal impression, even when examining the same set of figures. We must here resort to more exact methods, and it is the object of this appendix to present in a brief and elementary manner the mathematical methods of dealing with such masses of figures.

\section{SECTION II - AVERAGES}

Meaning and function of an average. The fundamental questions which arise in the discussion of averages are: (I) What is meant by "the average of a system of variates"? (2) Why do we make use of averages at

1 See Karl Pearson, Grammar of Science. 
all? (3) What are the objects of having different kinds of averages? Such questions as these are apt to be overlooked by those who have formed the habit of averaging all kinds of results without careful thought.

In popular language, we speak of the average daily temperature, the average length of ears of corn, the average student, the average citizen; and we should know the exact meaning to be conveyed by these and similar expressions before using them in scientific discourse.

The taking of an average presupposes a population whose variates have a certain measurable character about which we are concerned, and that the measurement of this character differs in different individuals. We attempt to describe this population by putting aside the measurements of individuals and constructing a single intermediate number which shall be descriptive of the total population, in so far as one number can describe a population.

The single intermediate number which answers this purpose is, in the general sense, some kind of an average. We thus use averages for descriptive purposes in the interest of brevity; but, taken alone, an average cannot completely describe a population any more than the motion of the center of gravity of a system of material particles can completely describe the motion of the separate particles.

In stating what an average is we have also stated its function; but, as just indicated, it must not be assumed that an average gives all the information which is to be secured from the measurement of a population. It can only take the place of the mass of figures for certain special purposes. In fact, there has been a tendency, by somewhat careless workers with statistical data, to attach too much importance to averages and not enough to deviations from the average, - a point that will be dealt with in a later section.

There are five different kinds of averages in common use for different purposes. These are (1) the arithmetic mean, (2) the weighted arithmetic mean, (3) the geometric mean, (4) the mode, (5) the median.

While some of these averages have been defined, and used freely in the text, it seems well to restate these definitions together with the others, the better to discuss their respective advantages and disadvantages, and some of the purposes to which each is adapted.

The arithmetic mean. The arithmetic mean of a population of $n$ variates may be defined as follows:

$$
\text { arithmetic mean }=\frac{\text { sum of measurement of } n \text { variates }}{n} .
$$

That is, to find the arithmetic mean of $n$ sidrates, are divide the sum of the measurements of these variates by the number of variates.

Thus, in the case of a thousand ears of corn, the arithmetic mean of the lengths of the ears is the sum of the lengths of 1000 ears divided by 1000 . The use of this kind of an average has always been taken by observers as the best method of combining direct measurements of the same quantity. This is 
the average most commonly employed, and one of the strongest arguments advanced to justify this method is its universal acceptance. It is worth while, however, to call attention to one of the abuses of the arithmetic mean. For instance, if a very few (say four) measurements have been made of a certain character, the arithmetic mean has often been taken as a good index of their meaning; but if these few measurements differ widely, to report their arithmetic mean is to furnish a very misleading and untrustworthy piece of information. This has often been done by those who have given no thought to statistical methods.

There is a sort of commercial point involved in the arithmetic mean which should not be overlooked. For instance, if a real estate dealer sells a hundred lots at various prices, of which the arithmetical average is $\$ \$ 00$, this assures us that if the seller had sold each of the lots for $\$ \$ 00$, instead of selling at different prices, he would have realized precisely the same from the sale of the whole number of lots as he has realized from selling at varying rates, even though we have no information as to what any individual has paid for a lot.

Weighted arithmetic mean. A slight modification of the above method is often used. To illustrate, the thousand measurements of lengths of ears of corn may be arranged, let us say, in half-inch groups as follows:

\begin{tabular}{|c|c|c|c|c|c|c|c|c|c|c|c|c|c|c|c|c|c|}
\hline Inches .... & $|3.0|$ & 3.5 & 14.0 & 4.5 & 5.0 & 5.5 & 6.0 & 6.5 & $7.0 \mid 7.5$ & 8.0 & 8.5 & 9.0 & 9.5 & 10.0 & $|10.5|$ & I1.0 & 111.5 \\
\hline Frequencies. . & 5 & 6 & $\mathrm{I}_{3}$ & 17 & IS & 55 & $6 \mathrm{I}$ & 73 & So $\mid g^{8}$ & II 3 & r34 & 142 & 100 & 53 & 26 & 5 & I \\
\hline
\end{tabular}

where, for instance, the 6-inch group includes all ears whose lengths are between 5.75 and 6.25 . In general, if $v_{1}, v_{2}, \ldots, v_{r}$ represent the class marks, and $f_{1}, f_{2}, \cdots, f_{r}$ represent the corresponding frequencies, then

$$
\text { weighted arithmetic mean }=\frac{f_{1} v_{1}+f_{2} v_{2}+\cdots+f_{r} v_{r}}{f_{1}+f_{2}+\cdots+f_{r}} \text {. }
$$

Stated in words, this mean is obtained by multiplying each mark of a class by the corresponding frequency, and dividing the sum of the products by the total population.

This kind of average is used a great deal in our work and is approximately equal to the ordinary arithmetical average if the groups are fairly narrow. Its advantage over the ordinary arithmetic mean lies in the fact that it is more easily computed. In reporting the mean daily temperature, the average length of ears of corn, the average height of a certain class of men, one of the above kinds of averages is meant. We use these averages so much in this work that we speak of them as "the mean," for brevity, so that when the term "mean" is used without a limiting adjective, it is to be understood that an arithmetic mean is meant.

The geometric mean. The geometric mean of $n$ numbers is found by multiplying the numbers together and extracting the nth root of the product. 
Let us assume that during a decade the attendance at a university increased 100 per cent, and let us propose the problem of finding the average annual rate of increase. Will it do to resort to the arithmetic mean in this case and say that the average rate of increase is 10 per cent? No; an increase of 10 per cent annually would give an attendance $(1.10)^{10}=2.59$ times the attendance at the beginning of the decade. What we really want is $\sqrt[10]{2}=1.07+$; that is, an increase of a little more than 7 per cent each year will double the population in a decade.

The geometrical average is but little used in our work, but it is brought forward here to remind us that an average can, in general, be depended upon only to serve a definite purpose; and, when the purpose is known, we are sometimes confined to one kind of average, or at least able to see the advantage of one kind of average over another. Suppose that we know the protein content of corn to have been increased 50 per cent in ten years' breeding. Can we say that the average annual rate of increase was 5 per cent? Clearly we cannot. What we should do is to take

$$
\sqrt[111]{1.50}-1.00=0.041
$$

and say that the average annual rate of increase is approximately 4 per cent.

The mode. When we speak of the average college student or the average citizen we certainly do not have reference to the arithmetic or geometric average of anything. When we say a man is an average citizen we mean that he represents a type which is met oftener than any other.

If a community has ten millionaires, but all the other citizens are in poverty, an arithmetical average might give the impression that the people of the community are in good financial condition, while really the "average citizen" is in poverty. The averages thus far discussed are in no way suited to describe this population, but the average called the "mode" is useful for this purpose.

If a population be arranged in seriate order with respect to some character, a mode is a value to which there corresponds a greater frequency than to values just preceding and immediately following it in the arrangement. A population may have more than one mode, but the populations with which we shall deal have, in general, only one.

This kind of average seems to be about the same as that of the newspapers when they speak of the average citizen. In a democracy we often hear the cry of "the greatest good for the greatest number," and insist that legislation shall benefit the average man, - the man at the mode.

Reverting again to the thousand ears of corn arranged in half-inch groups, it should be noted that the frequency increases up to the class of mark 9 inches and then decreases. We might conclude that 9 inches is exactly. the mode for this population. It must be remembered that all measurements from 8.75 to 9.25 inches were placed in the 9 -inch group, and that a different grouping might change the frequencies somewhat. Hence 9 is said to be the cmpirical mode, and the theoretical mode is defined as a 
point of greatest frequency of the theoretical distribution, of which the given distribution is a sample. As a disadvantage, it should be mentioned that it is somewhat difficult to determine the theoretical mode accurately. It may also be pointed out that for a very irregular group of figures the mode is practically useless. Its great service is to characterize a type, and with a very irregular group of figures the existence of a type is not manifest ; indeed, a type may not exist.

The median. If all the variates are arranged in serial order, the value corresponding to the middle sariate is called the median of the population. Thus, if we should speak of the wages of a thousand and one laborers, we should mean by the median the wages of the middlemost of these men when they are arranged in serial order with respect to wages; that is, if five hundred received less than $\$ 1.72$, and five hundred received more than $\$ 1.72$, we should say that $\$ 1.72$ is the median wage. The median has the great advantage that it can be easily determined. Very large and very small values do not affect it. It is only a question of being above or below the middle in an arrangement. Its great disadvantages are that it may be totally removed from the type and that it gives no special importance to extreme values.

Averages of whatever kind are designed to exhibit the main features of a population by means of a few well-chosen numbers. We have seen that the particular average selected depends upon the purpose we have in view. Now, if this purpose is merely one of comparison between two similar groups, then almost any kind of an average will do. The ordinary and the weighted means have been used for the most part in this work, but conditions may easily arise where some other average is more suitable. Bowley has well stated the following as the characteristics of a good and suitable average: "If there is a type, it shows it; it gives due influence to extreme cases; it is not easily affected by errors, or much displaced by slight alterations in the system of calculations ; and it is easily calculated."

It is often useful to give more than one average in order to describe a population ; for the relative positions of the mean, the mode, and the median give a good deal more information about the distribution of a population than any single average can give. For a great many distributions Pearson has found an approximate relation to exist between the mean, the mode, and the median. This relation is

$$
\text { theoretical mode }=\text { mean }-3(\text { mean }- \text { median }) \text {. }
$$

It is, of course, possible to form fictitious frequency distributions for which this relation does not hold, but it is important as indicating what nature, in general, provides.

The use of averages for representing what is often spoken of as the "true value" can be better discussed in the section devoted to the probable error. 


\section{SECTION III - GRAPHIC REPRESENTATION OF STATISTICS}

A mere tabulation of any considerable number of figures does not make it possible, in general, for the mind to grasp the main facts which the figures represent; in fact, such a tabulation of, say a thousand figures, may make no impression on the mind which is at all worth mentioning. By the graphic method, however, the chicf characteristics of a mass of figures are presented to the eye by means of a picture or curve. The graph gives, at a crlance, important facts which may be overlooked, or which can be obtained from the figures only by considerable labor.

The use of graphic methods in statistics is very extensive, and has proved to be of great service. In fact, every one who has to deal with complicated groups of figures comes to appreciate the graphic method, as it enables one to perceive relations through the eye.

It is the object of this section to show how graphs are formed from given data.

Frequency curves. Let us consider the graph of the following frequency distribution, in which the first line of the table gives the marks of the classes, and the second gives the number of variates in the classes:

\begin{tabular}{|c|c|c|c|c|c|c|c|c|c|c|c|c|c|c|c|}
\hline 'Values & $\cdots$ & $\cdots$ & 4.0 & $4 \cdot 5$ & 5.0 & 5.5 & 6.0 & 6.5 & 7.0 & $7 \cdot 5$ & S.n & 8.5 & 9.6 & 9.5 & 10.0 \\
\hline Frequen & icies. & ... & I & I & 8 & 33 & 70 & II & 176 & 172 & 124 & fi & 32 & 10 & 2 \\
\hline
\end{tabular}

What we propose to do here is to present a significant picture of this population.

Draw two lines, $O X$ and $O Y$, at right angles to each other (Fig. I). These reference lines are called coördinate axes. The line $O X$ is called the $x$-axis, and the line $O Y$ the $y$-axis. The point $O$, from which we measure, is called the origin, or zero point. Beginning at this point mark off on the $x$-axis equal intervals upon a scale convenient to the problem at hand. From the same zero point lay off equal intervals also on the $y$-axis. These need not be on the same scale as those on the $x$-axis, but should be suited to best bring out the facts to be shown by the graph. Not all graphs are drawn upon the same scale therefore, nor are the two axes of the same graph alike as to spacing or scale.

In the particular case in hand, let each interval along $O X$ represent a half inch. The question as to what each interval shall represent is a matter of the scale used; and the scale must be chosen to suit the particular data in hand. Next, along (). I lay off the class marks. Corresponding to each class mark there is a frequency. From the various points on the $x$-axis

1 This distribution is taken as a representative of any frequency distribution. It is not, however, made up artificially, but actually represents the distribution of eight hundred ears of corn with respect to length. 
which represent class marks lay off lines parallel to the $y$-axis and of lengths corresponding to the various frequencies, according to the scale on the $y$-axis. When this is done there will be a series of parallel lines at equal distances apart, all perpendicular to the $x$-axis and parallel to the $y$-axis, but of lengths corresponding to the various frequencies and therefore unequal. Joining the tops of the lines so constructed by straight lines gives the frequency polygon shown in Fig. I. The tops of the lines thus joined give an orderly arrangement of points, through which it may be possible to draw a smooth curve. If it is impossible to draw a smooth curve through all of them, draw a smooth curve as near as possible to all of them. The curve so drawn is called a frequency curve (not shown in figure).

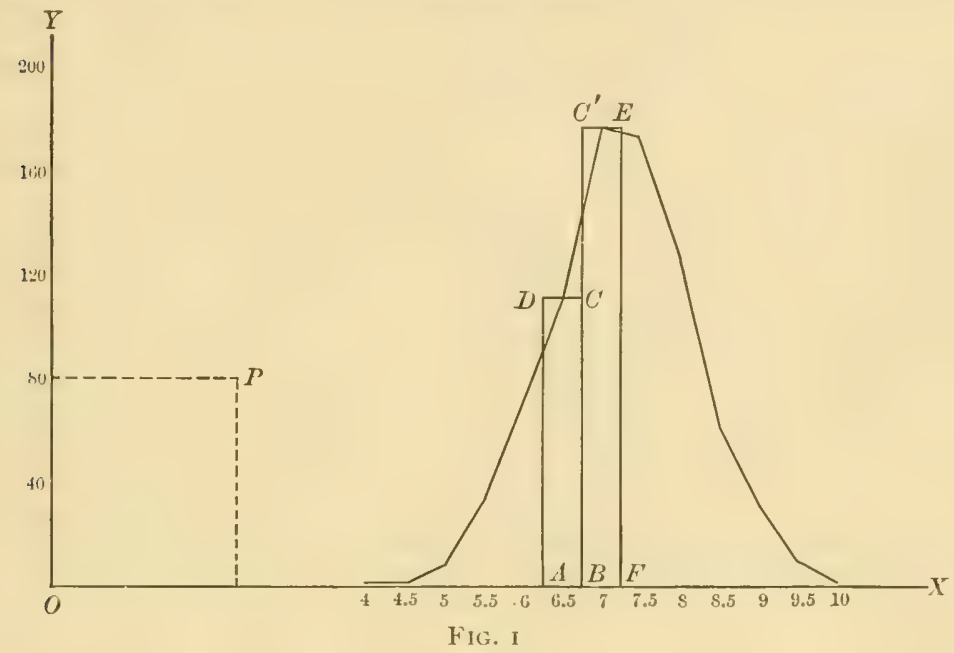

Any point $P$ in the plane represents two numbers: the one number is represented by the distance of the point from the $y$-axis, and the other by its distance from the $x$-axis. The number which gives the distance of $P$ from the $y$-axis is called the abscissa of $P$, and the number which gives its distance from the $x$-axis is called its ordinate. The two numbers together are often spoken of as the coördinates of the point $P$.

Significance of area under curve. Construct rectangles such as $A B C($ ) and $B C^{\prime} E F$ on the ordinates at class marks as mid-lines, making the sides $A D, B C$, etc., bisect the class intervals along the $x$-axis. Suppose, now, that we define unit area as a rectangle bounded by $A B, A D, B C$, and a line parallel to $A B$ and just far enough from it so that the distance between $A B$ and this line represents unit frequency. Then the area of $A B C D$ is $1 \mathrm{IO}$, and the area of all such rectangles taken together is equal numerically to the total population. In drawing the smooth curve mentioned above, we should aim to make the area between the curve the 
$x$-axis, and the two end ordinates (in this case ordinates at 4 and 10 ) equal to the sum of the areas of these rectangles. The area under the curve then represents the total population. This is an important point, because it presents to the eye how much of the population is included between any two measurements. For instance, at a glance you could estimate approximately the portion of the population discussed in Fig. I, whose measurements are between 5 and 8 . The use of the area under the frequency curve will be found helpful in our discussion of "probable error."

Choice of scale. In drawing a graph the question always arises as to what scale shall be used in plotting, but unfortunately no definite rule can be laid down. It may, however, prove useful to call attention to a few points. First, we should choose such a scale that we can plot all the points on one page of the paper used; for it is a great advantage to have the whole graph on one paper, thus making it visible to the eye in its entirety. Second, if the point involved in the investigation is a question of rate of increase or decrease, we should select such a scale as to make the curve reasonably steep. Frequency curves are used a great deal in the study of the social sciences, as well as in natural science. For instance, the sociologist presents the population of a city or country for successive years by using years as the marks of classes, - laying these off along the $x$-axis, - and the population for these years as ordinates.

Negative values easily represented graphically. We often desire to plot negative values as well as positive values, and this is easily accomplished by a slight extension of what has already been done in connection with Fig. I. With the data exhibited in Fig. I it might have been found convenient to use the mean as the origin and to plot the frequency with respect to deviations from the mean. Since the mean is in this case 7.25 , we have the following set of deviations and corresponding frequencies to plot:

\begin{tabular}{l} 
Deviations ..... \\
\hline Frequencies......
\end{tabular}

We should now lay off the positive deviations toward the right from the origin $O$ ) (Fig. 2) and the negative deviations toward the left from $O$. The freguencies should, of course, be plotted upward from.$^{\prime}{ }^{\prime}$, just as in Fig. I. The result of plotting this frequency distribution is shown in Fig. 2. This should bring home to the reader, who is not very familiar with the use of negative numbers, the fact that negative numbers may be just as "real" and useful as positive numbers.

The frequency polygon of Fig. 2 does not differ in form from that of Fig. I. It is only differently related to the lines of reference O.X and $O \mathrm{I}$.

Graphical meaning of median, mean, and mode. If in Fig. I we select on the curve a point whose ordinate divides the area under the curve into two equal parts, the abscissa of this point is the median of the population. The 
abscissa of the center of gravity of the total area under the curve is the mean of the population, and the abscissa of the highest point on the frequency curve is the theoretical mode of the population.

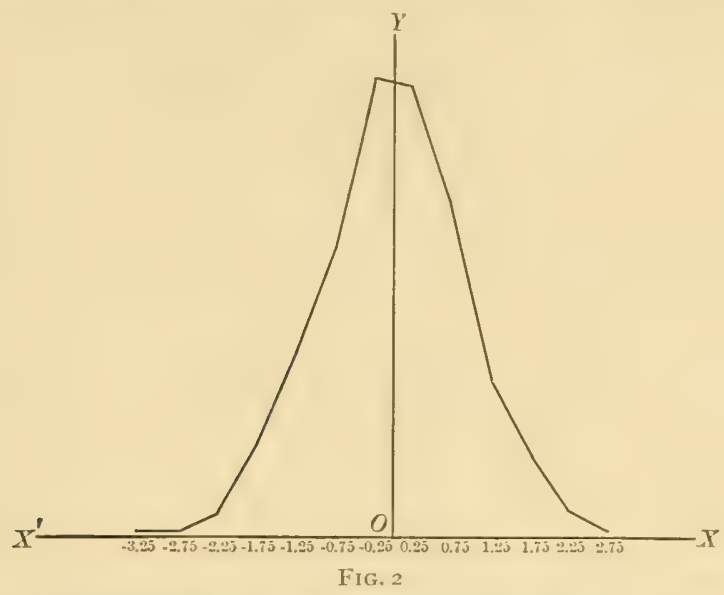

Graph of a mathematical function. A number $y$ is said to be a mathematical function of a number $x$ if to assigned values of $x$ there correspond definite values of $y$.

Thus, if $y=2 x, y$ is a function of $x$, since for any assigned value of $x$ we can compute $y$. In general, if $x$ and $y$ are connected by an equation each is a function of the other. The study of certain functions is of the first rate in importance in the mathematical theory of statistics, and this is much facilitated by the use of the graph of the $x$ function in question. We therefore proceed to show how to form the graph of a few simple functions so as to give the general notion of the graph of functions.

Take coördinate axes, as in Fig. 3, which divide the plane into four quadrants. If the abscissas are positive, they should be laid off to the right

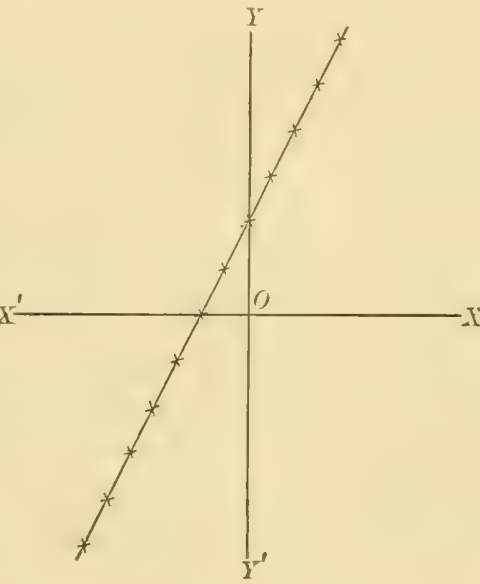

Fig. 3 of $O$; if negative, they should be laid off to the left of $O$. The ordinates, if positive, are to be laid off upward from the $x$-axis : if negative, they are to lx laid off downward. Then whatever two numbers (positive or negative) are given as abscissa and ordinate, the corresponding point can Le located. 
Let us take as an illustration the plotting of the graph of $y=2 x+4$. Here we see from the equation that corresponding to any value assigned to $x$ we get a value of $y$ equal to twice $x$ plus 4 . The corresponding values are as follows:

\begin{tabular}{|c|c|c|c|c|c|c|c|c|c|c|c|}
\hline$x$ & 0 & I & 2 & 3 & 4 & 5 & $-I$ & -2 & -3 & -4 & -5 \\
\hline$y^{\prime}$ & 4 & 6 & S & 10 & 12 & 34 & +2 & 0 & -2 & -4 & -6 \\
\hline
\end{tabular}

Locating, in Fig. 3, the points corresponding to these values, and drawing a smooth curve through them, we have the graph of the function. This graph is a straight line.

We leave as an exercise for the student to find the graph of $y=x^{2}$. For application of graph of function, see "Probability Curve," Section VI.

\section{SECTION IV - "SMOOTHING" OF FIGURES}

Sometimes the frequency distribution of a population arranged with respect to some character has many small irregularities which arise merely from the way in which the measurements were taken and grouped. In such a case a process called "smoothing" can often be employed to obtain regularity. A noteworthy instance of smoothing is to be seen in the adjusting of the population census with respect to age, there being a great many more people who report their ages as 40 than as 39 or $4 \mathrm{I}$. In fact, sometimes the unsmoothed figures show one half more people of age 40 than of age 39 or 41 . It is, then, manifestly desirable to smooth these census returns if they are to give even an approximately correct impression.

In representing such a distribution graphically we have to draw a smooth line in the neighborhood of the points, but not necessarily through any of them. This smooth line is the result of the attempt to present what the distribution would be if the causes of the small irregularities could be removed. Sometimes it is convenient to smooth figures without resorting to a graph. There are some rather refined but complicated algebraic methods ${ }^{1}$ of doing this, but in general a very simple method can be used. To explain this method, take the following frequency distribution (which was obtained by measuring the circumferences of 995 ears of corn), in which the groupings into $\frac{1}{4}$-inch classes are not well selected.

\begin{tabular}{|c|c|c|c|c|c|c|c|c|c|c|c|c|c|c|c|c|c|c|}
\hline Inches & . . & . . & 4.5 & 4.751 & 5.0 & 5.25 & 5.5 & 5.75 & $6.0 \mid 6.25$ & 6.5 & 6.75 & 7.0 & 7.25 & $7 \cdot 5$ & 7.75 & 8.0 & 8.25 & 8.5 \\
\hline Frequer & ncies & . . & 2 & 4 & 13 & 24 & 20 & 74 & 1251 & $18 r$ & 98 & 208 & 55 & 67 & 10 & II & 3 & 2 \\
\hline
\end{tabular}

1 Darwin, Philosophical Magazine and Jourmal, July, x\$77. 
It may be well to explain the chief source of this irregularity. This can be seen by observing two classes, such as the 7-inch class and the 6.75 -inch class. As the measurements were recorded to the nearest tenth inch, the 7 -inch class includes the measurements recorded as $6.9,7$, and 7.I, while the 6.75 -inch class includes only those recorded as 6.7 and 6.8 . This should evidently produce a biased result. Instead of making a new frequency table with a different grouping, we may substitute for each frequency a number derived by smoothing. This smoothing can be accomplished by substituting for each frequency, except the two extreme ones, the mean of the given frequency and the one immediately before and the one immediately after it. Thus, for frequency of ears of length 4.75 inches we should substitute $\frac{2+4+13}{3}=6 !$. But as this is only an approximation, we may as well take the nearest integral value, or 6. For an extreme frequency, we substitute the mean (to nearest integer) of the extreme frequency taken twice and the adjacent frequency taken once. Thus, for the frequency corresponding to length 4.5 inches we substitute $\frac{2+2+4}{3}=2 \frac{2}{3}$, or, in integral numbers, 3. It is sometimes desirable to apply this process more than once to a given distribution in order to give it the desired regularity.

The results of the scheme for the given frequency distribution are as follows :

\begin{tabular}{|c|c|c|c|c|c|c|c|c|c|c|c|c|c|c|c|c|c|}
\hline Inches.... & 4.5 & 4.75 & 5.0 & 5.25 & $5 \cdot 5$ & 5.75 & 6.0 & 6.25 & 6.5 & 6.75 & 7.0 & 7.25 & $7 \cdot 5$ & $7 \cdot 75$ & S.o & 8.25 & 8.5 \\
\hline $\begin{array}{l}\text { Unsmoothed } \\
\text { frequencies }\end{array}$ & 2 & 4 & 13 & 24 & 20 & 74 & I 25 & 98 & $18 I$ & $9^{8}$ & 208 & 55 & 67 & 10 & II & 3 & 2 \\
\hline $\begin{array}{l}\text { ist smoothed } \\
\text { frequencies }\end{array}$ & 3 & 6 & 14 & 19 & 39 & 73 & 99 & 135 & 126 & 162 & 120 & I10 & 44 & 29 & 8 & 5 & 2 \\
\hline $\begin{array}{l}\text { 2d smosthed } \\
\text { frequencies }\end{array}$ & 4 & S & 13 & 24 & 43 & $y^{\prime \prime}$ & 103 & 120 & 141 & ${ }_{13} 9$ & ${ }^{1} 3 \mathrm{I}$ & 1)1 & 61 & 27 & 14 & 5 & 3 \\
\hline
\end{tabular}

In general algebraic terms, if $v_{1}, v_{2}, \cdots, v_{n}$ are the marks of classes and $a_{1}, a_{2}, \cdots, a_{n}$ the corresponding frequencies, in smoothing the $a$ 's we should substitute for them the following values respectively:

$$
\frac{2 a_{1}+a_{2}}{3}, \frac{a_{1}+a_{2}+a_{3}}{3}, \frac{a_{2}+a_{33}+a_{1}}{3}, \ldots, \frac{a_{n-2}+a_{n-1}+a_{n}}{3}, \frac{a_{n-1}+2 a_{n}}{3} .
$$

It can be easily seen from these algebraic expressions that the arithmetic mean of the measurements is scarcely affected at all by smoothing, but that the mode is sometimes considerably changed. In general, the "standard deviation" (to be discussed in Section VII) is but slightly affected by smoothing. 


\section{SECTION V-APIIICATION OF THE 'THEORY OF I'ROIBABILITY}

The reader should understand thorounly that what is commonly known as a "law of nature" is a generalization based upon experience, and that such a law cannot be proved in the strictly logical sense, but only in the sense of establishing a hirh degree of probability in its favor. To illustrate, we may take one of the best-established facts of physical science, namely, that all free bodies are attracted by the earth. The evidence for this statement consists in the fact that the thousands and even millions of bodies which have been observed have, without exception, followed this rule. This has established a very high degree of probability. It is altogether conceivable, however, that some body exists which would be repelled by the earth. Although experience has established an overwhelming probability against such an occurrence, we must not overlook the fact that experience has proved the statement only in the sense that it has established a high degree of probability. It has done this and can do nothing more than this. It is doubtful whether any person living his seen a hundred pennies tossed at random, all of which came heads up, and still it is possible that this might happen. Even if no one has seen them fall with all heads up, we are clearly not justified in concluding that there will always be some heads up and some tails up. If a thousand pennies be tossed at random, the probability that they will all fall heads up is so small that we may safely say, if the whole human race were to devote a generation to the tossing of pennies, a thousand at a time, there would still be a very small probability that any one would toss all heads. All this groes to show that certain possible events have such a slight probability that we should not expect them to happen in the lifetime of a given individual. Just so, as time goes on and observations are made in greater numbers, exceptions may be found to any of the so-called "laws of nature." Such exceptions are, however, in many cases exceedingly unlikely.

It is hoped that the foregoing paves the way for the statement that, while in this subject many results may be stated in terms of probabilities, these results do not differ in reliability on that account from those of any other science based on experience. If a thousand pennies be tossed at random, there is nothing more uncertain than that a given penny will be heads, but it is a matter of common experience that the ratio of the number of heads to the total number of pennies tossed is, in general, nearly one half. We may here recall the statement of Section I, that the theory of probability is needed in this subject because we deal with occurrences and characters of such a nature that we wish to make statements in regard to a large number of them taken together. It is a matter of common experience that results, such as averages and ratios obtained from large numbers of cases, are nearly stationary. We find the average stature of a thousand individuals selected at random from a large population, and are much surprised if, upon taking another random sample of a thousand from the same 
population, their average stature differs materially from that already found. We are not at all surprised if the averages are substantially equal. There are, no doubt, many causes which influence the grow th of each individual differently, but when they are all taken together these small disturbances tend to counterbalance each other. In short, it is regularity in large numbers which we expect. While it may be common sense to expect this, we shall later give a mathematical measure known as the "probable error" to indicate what deviations we should expect in results such as averages derived from a random sample. This discussion leads us to the following definition of probability.

Definition. If, in the long run, out of $n$ possible cases in each of which an event occurs or fails to occur, it occurs $n$ times and fails to occur $n-n_{1}$ times, the probability that the cuent occurs on a given occasion in question is $\frac{n_{1}}{n}$, and the probability that it fails to occur on a given occasion is $\frac{n-n_{1}}{n}$.

In framing this definition we idealize our actual experience. We say the probability of a penny's turning up heads is one half. This may be looked upon as an answer to the following question: What proportion of the pennies tossed should we expect to find with heads turned up if we should continue tossing indefinitely?

This idealization, for purposes of definition, is analogous to the idealization of the crude chalk mark into the straight line of geometry. Since the sum of the probabilities of occurrence and failure is $\frac{n_{1}}{n}+\frac{n-n_{1}}{n}=\mathrm{I}$, the number I is the symbol of certainty. The expression "relative frequency" conveys fairly well the idea of probability.

The following corollary is often easier to apply than the definition.

Corollary. If the entire number of possible cases in which an event is in question can be analyzed into $u^{\prime}$ cases, each of which is equally likely, and $m^{\prime}$ is the number of these cases in ruhich the event occurs, then $\frac{1 \prime^{\prime}}{n^{\prime}}$ is the probability of the event.

Thus, in tossing two pennies, what is the probability that one will be heads and one tails?

There are four different ways in which the pennies may fall: Head and tail, tail and head, head and head, tail and tail. Two of these ways lead to the occurrence of the event. Hence ${ }_{4}^{2}=\frac{1}{3}$ is the desired probability of one head and one tail.

Combination of probabilities. The probuthility that all of at set of indefentent events will occur on any occasion in which all of them are in question is the product of the probabilities of the single events.

Proof. Let $p_{1}, p_{2}, \ldots, p_{r}$ be the separate probabilities of $r$ events. Out of a great number, $n$, of cases, the first will happen on $p_{1} n$ occasions. Out of these the second will happen on $p_{2}\left(p_{1} n\right)$ occasions. Continuing this process, and applying the definition of probability, the theorem is at once established. To illustrate, suppose that among a population of a hundred 
thousand people thirty thousand are vaccinated, and that five hundred persons have smallpox. If vaccination has no influence on the number of cases of smallpox, what is the probability that a person will be both vaccinated and have smallpox?

Since one hundred thousand is a large number, we may give

$$
\begin{aligned}
& \frac{30000}{100000}=\frac{3}{10}=\text { probability that a person is vaccinated; } \\
& \frac{500}{100000}=\frac{1}{200}=\text { probability that a person has smalipox; } \\
& \frac{3}{10} \cdot \frac{1}{200}=\frac{3}{2000}=\begin{array}{c}
\text { probability that a person is both vaccinated } \\
\text { and has smallpox. }
\end{array}
\end{aligned}
$$

Furthermore, $\frac{3}{2000} \times 100000=150$, the number of persons we should expect both to be vaccinated and to have smallpox, if vaccination has no influence on the number of cases of smallpox.

Illustrations of probability. Let us throw ont upon a table at random four pennies; what is the probability that exactly rof them will be heads and the rest tails when $r$ takes values $O, I, 2,3,4$ ?

(I) Probability that 0 will be head and 4 tails is $\left(\begin{array}{l}\frac{1}{2} \\ 2\end{array}\right)^{4}$

(2) Probability that I will be head and 3 tails is $4\left(\frac{1}{2}\right)^{4}$

(3) Probability that 2 will be heads and 2 tails is $6\left(\frac{1}{2}\right)^{4}$

(4) Probability that 3 will be heads and I tail is $4\left(\frac{1}{2}\right)^{4}$

(5) Probability that 4 will be heads and o tail is $\left(\frac{1}{2}\right)^{4}$

In (2) the coefficient 4 appears before $\left(\frac{1}{2}\right)^{4}$ because with four coins there are four different combinations ${ }^{1}$ possible, each consisting of 1 head and 3 tails. Similarly in (3) the coefficient 6 appears because with four coins there are possible six combinations, each consisting of 2 heads and 2 tails.

The above illustration may be generalized and the result put into the following form:

If $n$ coins are thrown upon a table at random, the probability that exactly $r$ of them will be heads and the rest tails is given by the $r+$ Ist term of the binomial expansion $\left(\begin{array}{l}\mathrm{I} \\ 2\end{array}+\frac{1}{2}\right)^{n}$; that is, in other symbols, ${ }^{n} C_{r},\left(\begin{array}{l}1 \\ 2\end{array}\right)^{n}$, where the symbol " $C_{r}$ indicates the number of combinations of $n$ things taken $r$ at a time.

In order that the reader may more fully appreciate the greater probability of getting an almost equal number of heads and tails in tossing pennies than of getting widely different numbers, we present the following table for $n=999$, obtained from Quételet's Sur la théorie des probabilités.

1 For definition of a combination, see text, p. 5 II. 
Columns 1 and 2 give the number of heads and tails whose probability is in question. Column 3 gives the probability of exactly the number of heads and tails indicated in columns $I$ and 2 .

\begin{tabular}{|c|c|c|c|c|c|}
\hline 1 & 2 & 3 & I & 2 & 3 \\
\hline HEAUS & TAILS & Probability & HEADS & TAlLS & Probability \\
\hline 499 & 500 & ${ }^{999} C_{500}\left(\frac{1}{2}\right)^{939}=0.025225$ & $45^{\circ}$ & 549 & 0.0001863 \\
\hline 490 & 509 & ${ }^{999} C_{509}\left(\frac{1}{2}\right)^{999}=0.021069$ & 440 & 559 & 0.0000209 \\
\hline 480 & $5^{19}$ & ${ }^{999} C_{519}\left(\frac{1}{2}\right)^{999}=0.011794$ & 430 & 569 & 0.0000016 \\
\hline 470 & 529 & ${ }^{999} C_{529}\left(\frac{1}{2}\right)^{999}=0.004423$ & 420 & 579 & 0.00000004 \\
\hline 460 & 539 & ${ }^{999} C_{539}\left(\frac{1}{2}\right)^{939}=0.001110$ & & & \\
\hline
\end{tabular}

A glance at the table shows that, in the long run, one should expect 499 heads and 500 tails more than 600,000 times as often as 420 heads and 579 tails. In this connection it is interesting to inquire into a case mentioned on page 692, namely, the probability of getting all heads in a single throw of a thousand pennies. This probability is

$$
\left(\frac{\mathrm{I}}{2}\right)^{1000}=\frac{\mathrm{I}}{(\text { an integer containing } 302 \text { figures })},
$$

and the statement made on page 692 as to the human race (population $\mathrm{I}, 500,000,000)$ devoting itself to tossing pennies is clearly a safe statement. The results in the above table may well be exhibited graphically (Fig. 4 ).

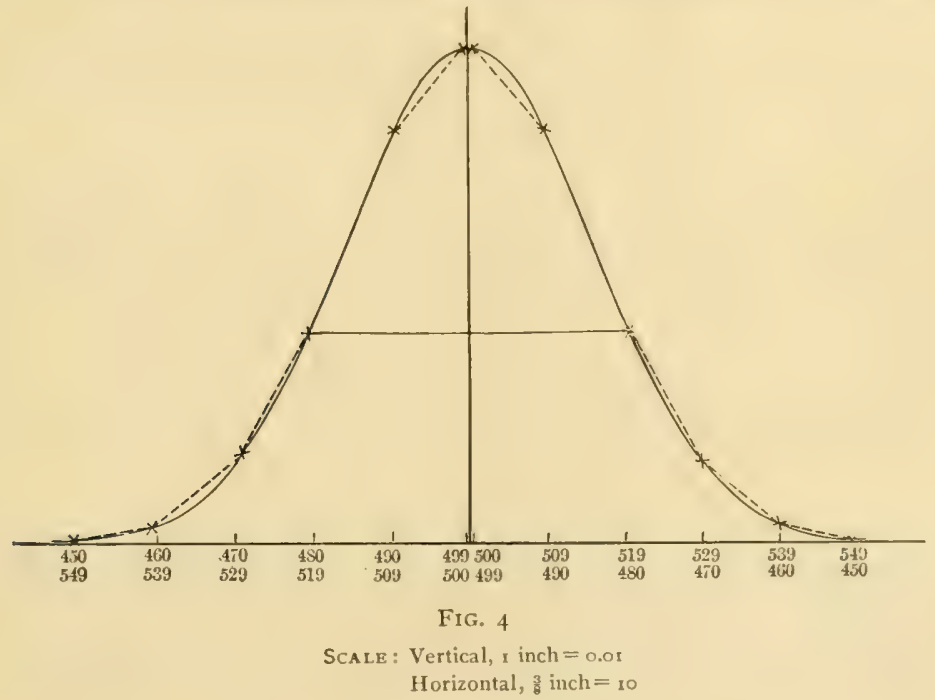


If we had taken all the intermediate integers from 499 with 500 to 440 with 559, we should have had ten times as many points which would arrange themselves along the curve in Fig. 4. By increasing the number of coins and decreasing the horizontal scale, we can gret the points as close together as we please. This curve in Fig. 4 is the so-called probability curve and it approaches very nearly the curve of error, or normal frequency curve, which we are now prepared to discuss.

\section{SECTION VI - NORMAL PROBABILITY CURVE}

It has been found that the frequency curves of a great many biological measurements follow a curve variously known as the "probability curve," "normal probability curve," "curve of error," or "normal frequency curve." In particular it is known as the "curve of error," because if errors which an observer makes in a refined set of direct measurements on a given quantity be plotted as abscissas, the corresponding ordinates of points on this curve represent the frequencies or probabilities of the errors.

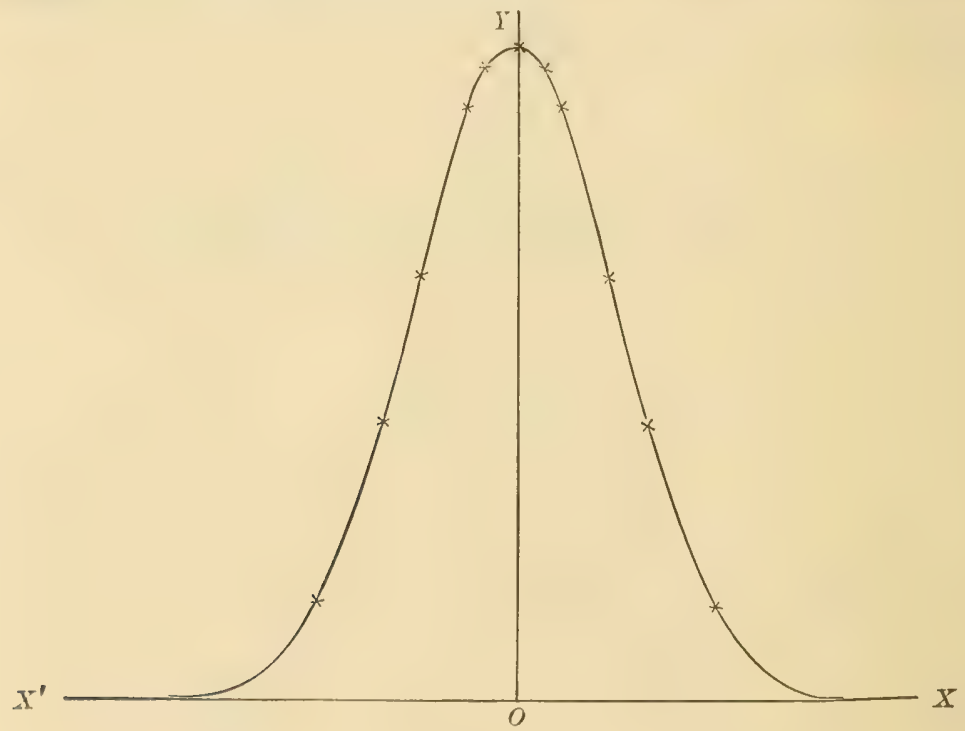

FIG. 5

The general form of the curve is shown in Fig. 5. The origin is taken at the mean. Then, if any mark of a class is above the mean, its deviation is positive, and it would be plotted to the right from the origin $O$, while if the mark of a class is less than the mean, its deviation is negative, and it would be plotted to the left from $O$. For the benefit of those who are 
familiar with the calculus, the derivation of this curve will be treated in a footnote, ${ }^{I}$ but a complete understanding of this footnote is not necessary for reading what follows.

While the equation of this curve has been derived in many different ways by arguments based on a few assumptions as to the nature and causes of deviations from the mean, it must be granted that these assumptions are of such a nature that experiment is an important test as to whether a frequency distribution is of this type. The reader should le on his gruard against the mistake that deviations from the mean, in all classes of measurements, follow closely this law of frequency." In fact, Pearson has found that in many cases frequency distributions obtained in biological study cannot be so well fitted by the normal curve as by what he calls generalized probability curves, which take "skewness" and limit of range into account. These curves lead us in to mathematical complications which cannot be well treated here, but it may be remarked that he obtains these curves from the point binomial $(p+q)^{n}$, where $p+q=\mathrm{I}$, but $p \neq q$, and from a hypergeometric series.

1 While Gauss, Laplace, Quételet, Herschel, and other great mathematicians have derived the equation of the normal curve, and all agree in the result, they differ widely as to hypotheses upon which they base the derivations.

We present here a derivation based upon the hypothesis (see Pearson, Philosophical Transactions, CLXXXVI, A, pp. $343-381$ ) that the normal curve represents a function $y=\phi(x)$ which has a certain slope condition obtained from the point binomial polygon $\left(\frac{1}{2}+\frac{1}{2}\right) m$ (see Fig. 4). This slope condition may be stated as follows:

$$
\frac{\text { slope of side }}{\text { mean ordinate of side }}=-\frac{2 \text { mean abscissa of side }}{2 \sigma^{2}} \text {, }
$$

the $y$-axis being the axis of symmetry and the $\sigma$ being the same for all sides.

In calculus form, this condition would be

Integrating,

$$
\begin{aligned}
\frac{d y}{y d x} & =-\frac{2 x}{2 \sigma^{2}} . \\
y & =k c^{-x^{2}} \sigma^{2 x} .
\end{aligned}
$$

The constant $k$ can be determined by finding the total area under the curve and equating this to the total population $n$ which the area represents. This gives

$$
i={ }_{\sigma}^{\prime \prime} V_{2 \pi}
$$

and the final form of the equation of the normal curve is

$$
y=\frac{n}{\sigma \sqrt{2 \pi}} e^{-2^{2} \sigma^{2}}
$$

in which $\sigma$ will later be shown to be what we shall call the "standard deviation" and $e=2.7 \mathrm{I} S \cdots$, the base of Napierian logarithms.

If equation $(x)$ is to give probabilities instead of frequencies, we must replace $n$ by $\mathrm{I}$ in equation (I).

2 For fulfillment of the normal law in nature, see Edgeworth, Slatistical Journal, Jubilee Number, $188_{5}$, p. 188 . 
However, the normal curves give, in general, at least a valuable first approximation, and we shall follow the usual method of employing statistical constants derived from this curve; for these constants are significant, even if the distribution is not normal. ${ }^{1}$

Area under the probability curve has an important meaning. If we select unit area as explained in Section I I I, the area represents the total population, and the area between the two ordinates, the curve, and $x$-axis represents the number of variates between these ordinates. If we look upon the curve as representing probabilities instead of frequencies our horizontal scale is unchanged but our vertical scale must be multiplied by the total population. Thus, if the population is 800 , as in the case of Fig. I, we should say that what there represented unity should be multiplied ly soo in order that it shall represent unit of probability. Then the entire area under the curve will be unity, and the area between two ordinates, the curve, and $x$-axis is simply the probability that a variate selected at random would lie within this interval.

\section{SECTION VII - PROBABLE ERROR AND STANDARD DEVIATION}

If we have estimated the population of a city at 100,000 and have good reason to think that the chances are even that this is correct within 1000 , we give much more information by stating that the population is $100,000 \pm 1000$ than by giving merely the figures 100,000 and leaving the reader entirely in doubt as to the accuracy of the determination.

In describing a frequency distribution the average gives absolutely no idea as to whether deviations are large or small, - nothing in regard to the spread of the distribution. It is the object of the "standard deviation" to be descriptive of this variability, and it is the object of the so-called "probable error" to indicate what confidence is to be placed in statistical results. The use which has been made of both "standard deviation" and "probable error" makes it unnecessary to dwell longer on this point, but it is our purpose here to show how the formulas used in the text are derived.

Probable error of a single variate. The probalile crror of a single arriate of a population is defined as that departure fiom the mean, on either side, within which cxactly one half the variates are found.

By the use of the probability curve (Fig. 6) the probable error may easily be explained geometrically when we look upon the entire area under the curve as representing the total population. In Fig. 6 we draw two ordinates, $S T$ and $S \% T$, equally distant from the mean, and such that one half of the entire area under the curve lies between them, in other words, is bounded by the curve, the $r$-axis, $S T$, and.$S^{\prime \prime} T^{\prime}$. Then \pm ().Srepresents the probable error of a single variate. If we should use a single variate selected at random to represent the population it is an eren chance that that single variate would be less or more than $O S$ from the best value. 
An approximate value for the probable error of a single variate in any population may be easily obtained in the following manner:

I. Arrange the variates in the order of magnitude.

2. Count one fourth of the variates of least measurements and note the measurement of the upper one of these variates. Let $u$ represent this measurement.

3. Count one fourth of the variates of greatest measurements and note the measurement of the lower of these variates. Let $v$ represent this measurement.

4. Then $\frac{2-" x}{2}$ gives the probable error of a single variate.

The formula for the probable error in a single variate is

$$
E_{s}=0.6745 \sqrt{\sum_{x^{2}}},
$$

where $\Sigma x^{2}$ means the sum of the squares of the deviations from the mean and $n$ is the number of variates. The conception of the probable error of

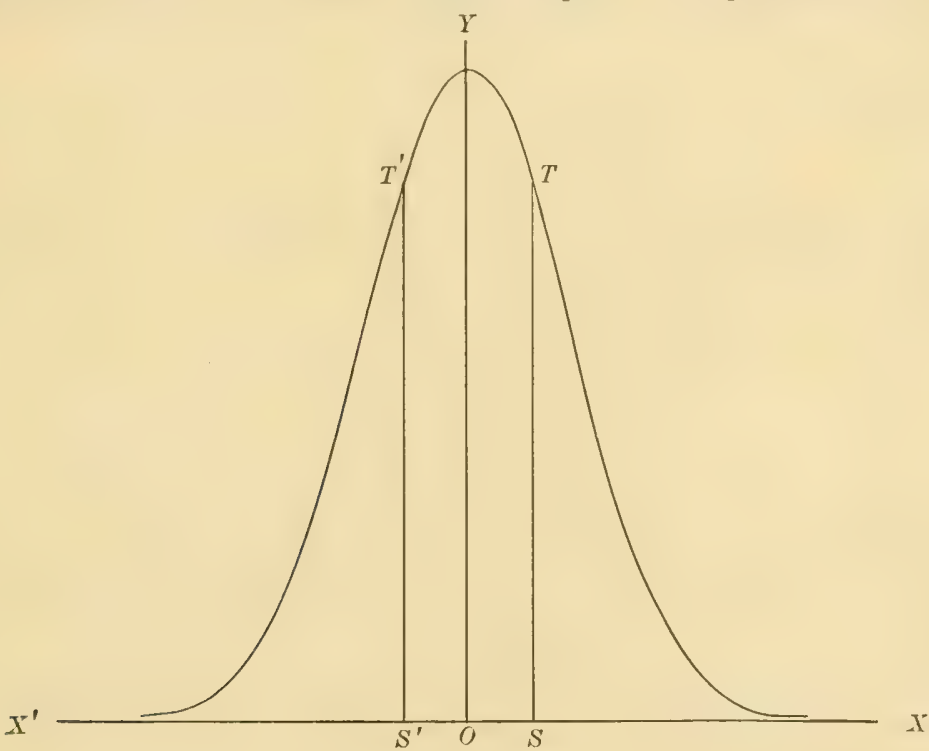

Fig. 6

a single variate is of value because it aids in the derivation of the probable error of other important results. The formula for the standard deviation is (page +29) $\sqrt{\frac{\Sigma^{2}}{n}}$, so that the probable error of a single variate is obtained from the standard deviation of the population by multiplying the standard deviation by 0.6745 . 
As has been pointed out in the text, the standard deviation gives a good idea of the spreat of the distribution. From the accompanying footnote ${ }^{1}$ we are now in a position to appreciate its mathematical significance. It is the $\sigma$ in the equation $y=\frac{1}{\sigma \sqrt{2 \pi}} e^{-\frac{x^{2}}{\sigma^{2}}}$ of the normal probability curve, and bears a similar relation to the probability curve that the radius of a circle bears to the circle. If $\sigma$ is small the probability curve is crowded together so as to resemble curve $A$ in Fig. 7 , while if $\sigma$ is large it is spread out so as to resemble curve $B$ in Fig. 7 .

Hence the standard deviation along with the mean completely describes the distribution when it is normal.2 The expression $\sqrt{\frac{\sum x^{2}}{i t}}$, which is thus a perfect measure of variability for a normal distribution, is a good measure of variability when the distribution is not normal, but it is not completely descriptive of the population.

Another measure of variability is sometimes used which consists simply in taking the arithmetical average of the $n$ deviations, - these deviations being given the positive sign'.

1 This may be written more briefly as

$$
P=\frac{1}{\sigma^{u}(2 \pi)^{2}} l l^{-\sigma_{12}}(\Delta x)^{\prime \prime} .
$$

For a given set of deviations which occur, $\sigma$ should be selected so as to make the

probability $P$ a maximum.
Equating the first derivative $\frac{d P}{d \sigma}$ to zero, we obtain

or

$$
\begin{gathered}
\frac{d P}{d \sigma}=\frac{1}{\sigma n(2 \pi)^{\frac{n}{2}}} l-\frac{\Sigma 12}{2 \sigma^{2}} \cdot \frac{1}{\sigma^{3}} \Sigma x^{2}-\frac{1}{(2 \pi)^{\frac{n}{2}}} \frac{n}{\sigma^{n+1}} l \frac{21}{2 \sigma^{2}}=0, \\
\Sigma x^{2}-n \sigma^{2}=0 .
\end{gathered}
$$$$
\therefore \sigma^{2}=\frac{2 x^{2}}{n} \text {. }
$$

Now, by means of integral calculus, tables are formed of the area included by the curve

$$
y=\frac{1}{\sigma \sqrt{2 \pi}} l=\frac{x^{2}}{2 \sigma^{2}},
$$

the $x$-axis, and any two ordinates at equal distances $\pm a$ from the mean. Such a table with the argument $\frac{x}{\sigma}$ is found in Davenport's Statistical Methods, second edition, pp. 119-125.

This table shows that

$$
\frac{x}{\sigma}=0.6745
$$

when just one half of the area under the curve is included as described above. By definition, the particular value of $x$ given by ( 1 ) is called the probable error in a single variate, and we shall represent it by $E_{s}$.
Hence,

$$
E_{s}=.6745 \sigma \text {. }
$$

2 See Galton, Natural Inheritance, p. 62. 
The formula for this is simply

$$
\frac{\left|x_{1}\right|+\left|x_{2}\right|+\cdots+\left|x_{n}\right|}{n},
$$

where the marks || indicate that the numbers should all be taken with the positive sign.

This measure of variability is usually known as the average deviation.

As to the relative merits of these two measures of variability, the standard deviation is to be preferred. Its relation to the probability curve as indicated above gives it special favor mathematically, although a geometric meaning may also be given to the average deviation.

Probable error of the mean. Since in natural science results are, in general, based on averages, we are more directly interested in the probable error in the mean than in the probable error of a single variate, although

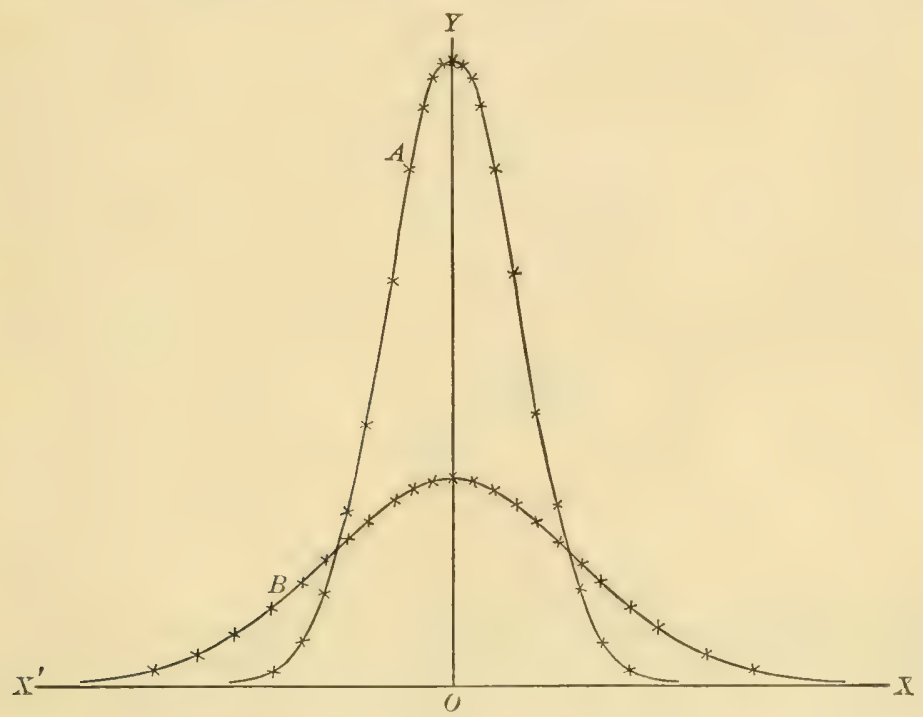

FIG. 7

the latter conception is desirable as a basis for understanding the former. We can best discuss the probable error in the mean by beginning with an illustration.

Suppose that, in determining the average stature of a male population consisting of a million individuals, we select at random groups of a thousand each. We could then, in all, have available a thousand such groups using no individual twice. It is an axiom of statistics, as we have already explained, that the mean statures obtained from each of these groups will 
differ but slightly from each other, but if the measurements are sufficiently accurate there will be some differences. If we should find the mean of these means (we may call it the second mean) we could plot a frequency curve of the distribution of means just as we have for the deviations of the original variates. Of course this curve would be much crowded together, like 1 of Fir. 7. To make this general, with a very large population, and with $n$ in each group instead of 1000 , the following result is obtained :

If $E_{s}$ is the probable error of a single variate, that of the mean of $n$ variates is

$$
E_{M}=\frac{E_{s}}{\sqrt{n}}
$$

that is, to find the probable error of the mean, divide the probable error of a single variate by the square root of the number of variates.

Probable error in standard deviation. Taking up again the million cases of stature divided into a thousand groups as an illustration, supposing that the standard deviation of each of these thousand groups be found, we should see that they differ but slightly. However, if the computations and measurements be very refined there will be deviations. These standard deviations constitute a frequency distribution whose standard deviation can be fourd, and the probable error of the standard deviation can be obtained just as we have shown in the case of a single variate.

Generalizing this so as to have a very large number of groups each containing $n$ variates taken as a sample to represent the population, the probable error of the standard deviation is

$$
E_{\sigma}=\frac{E_{11}}{\sqrt{2}}
$$

that is, to find the probable error in the standard deviation, divide the probable error in the mean by $\sqrt{2}$.

Formulas for probable error in some important statistical constants. Enough has now been said to give the conception of the probable error in any statistical determination and a general notion of the methods by which formulas for the probable error are derived.

It is scarcely necessary to remark that the probable error does not take into account evident mistakes either of observation or computation. We are assuming that these have been eliminated. It has to do with errors (deviations) due to an indefinitely large number of unassignable causes such that the errors are distributed according to the laws of probability.

It seems unnecessary to continue the discussion of probable error in other determinations, but it does seem well to collect together, for purposes of reference, the formulas for the probable error in some of the most important statistical constants. 
In what follows

$\sigma$ is to represent the standard deviation;

$u$ is to represent the number of variates;

$c$ is to represent the coefficient of variability;

$r$ is to represent the coefficient of correlation.

I. $E_{s}=0.6745 \sigma=$ probable error in a single observation.

2. $E_{n f}=\frac{E_{s}}{\sqrt{n}}=\frac{0.6745 \sigma}{\sqrt{n}}=$ probable error in the mean.

3. $E_{\sigma}=\frac{E_{.1}}{\sqrt{2}}=\frac{0.6745 \sigma}{\sqrt{2 n}}=$ probable error in standard deviation.

4. $E_{i}=\frac{0.6745 C}{\sqrt{2 n}}\left[1+2\left(\begin{array}{c}C \\ 100\end{array}\right)^{2}\right]^{\frac{1}{2}}=$ probable error in coefficient of varia$=\frac{0.6745 C}{\sqrt{2 x}}$ approximately, if $C$ is not greater than io per cent.

5. $E_{r}=\frac{0.6745\left(1-r^{2}\right)}{\sqrt{n}}=$ probable error in coefficient of correlation.

6. $E_{R}=\frac{0.6745 \sigma_{1}}{\sigma_{2}} \sqrt{\frac{\mathrm{I}-r^{2}}{u}}=$ probable error in the regression coefficient $r \frac{\sigma_{1}}{\sigma_{2}}$

\section{SECTION VIII - CORRELATION THEORY}

Definition. Two measurable characters of an individual, or of related individuals, are said to be correlated if to a selected series of sizes of the one there correspond sizes of the other whose mean values are finctions of the selected values. The word "sizes," here used, should be taken to mean "numerical measure."

For the sake of concreteness and simplicity, we may think of measuring the correlation of sons with respect to their fathers. To render the above definition in symbolic language and to develop the method of determining the function mentioned in the definition are the first points in the application of mathematics to the theory of correlation. For this purpose, let $x$ and $y$ represent variables such that $y=\phi(x)$ gives the mean value of $y$ corresponding to a selected $x$. Then the problem is to determine $\phi(x)$.

Suppose the following system of corresponding values results from measurement: $\left(x^{\prime}, y^{\prime}\right),\left(x^{\prime \prime}, y^{\prime \prime}\right), \cdots,\left(x^{(n)}, y^{(n)}\right)$, where $n$ is a very large number indicating the population of fathers and corresponding sons. These observations are said to form a total population or universe of observations. As it will be more convenient to deal with the deviations of the observations from their mean value than with the measurements themselves, let $\left(x_{1}, y_{1}\right),\left(x_{2}, y_{2}\right), \ldots,\left(x_{n}, y_{n}\right)$ represent the deviations of 
the observations from their mean value. These deviations may be conveniently represented with respect to coördinate axes (Fig. 8).

By the range along the $x$-axis we shall mean such an interval that ordinates drawn at the extremities of the interval include between them the total population. Thus, in Fig. 8, the range is taken from $a$ to $b$. This range may well be divided into some number, say $s$, of equal parts, each of width $\Delta x$, by ordinates at the points of division. If we let $x_{1}{ }^{\prime}, x_{2}{ }^{\prime}, \cdots$, $x^{\prime}$, be the abscissas of the feet of the ordinates through the middle points of the $s$ classes, we shall call these the marks of the classes of $y$ 's. The values of $y$ which belong to a given class of $x$ are said to form a $y$-array.

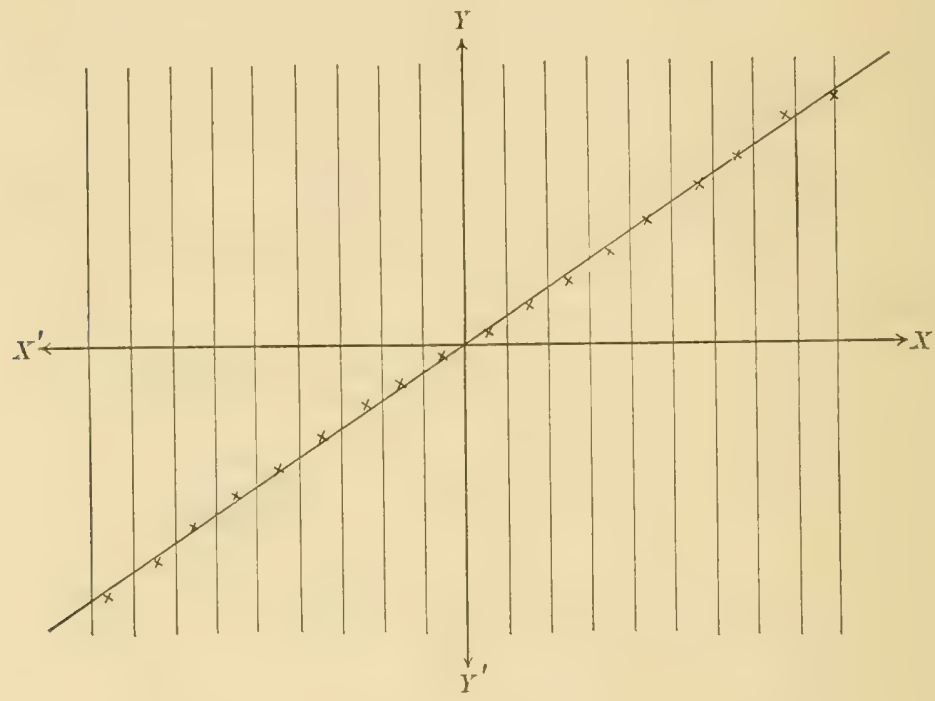

FIG. 8

Let the crosses $(x)$ in Fig. 8 represent the means of the $y$ 's in each of the $s$-arrays. If correlation exists, these means do not lie at random over the field, but arrange themselves more or less in the form of a smooth curve called the "curve of regression." This curve is a crude picture of the function which defines the correlation of the $y$-character relative to the $x$-character. Experience has shown that, in many sets of measurements, this line is approximately a straight line. For this reason, and for simplicity, the line subjected to the condition that the sum of the squares of the deviations (measured parallel to the $y$-axis and weighted with number of points in array) of the means from it shall be a minimum, is called the "line of regression." When the means lie exactly on the line the regression is said to be "truly linear." 
The algebraic details of subjecting a line to this minimal condition are well known to those familiar with the method of least squares. The equatjon of the resulting line is

$$
y^{\prime}=r \frac{\sigma_{1} x}{\sigma_{3}},
$$

where $\sigma_{x}$ is the standard deviation of the population with respect to the $x$-character, $\sigma_{y}$ is the standard deviation with respect to the $y$-character, and $r$ is the correlation coefficient given by

$$
r=\frac{\Sigma}{n \sigma_{x} \sigma_{y}},
$$

where the summation is extended to every two corresponding variates of the population.

Similarly, the regression of the $x$-character with respect to the $y$-character is given by

$$
x=r \frac{\sigma_{x}}{\sigma_{y}} y .
$$

It should be noted that (2) cannot be obtained from (I) by solving for $x$ in equation $(\mathrm{I})$.

Standard deviation of arrays. Suppose that the regression is truly linear, so that the means of the $y$-arrays fall on the line $y=r \frac{\sigma_{y} x}{\sigma_{x}}$, and furthermore that the standard deviations of all parallel arrays are equal. Then the standard deviation of any array must be given by

$$
\frac{\sum\left(y-r \frac{\sigma_{y}}{\sigma_{x}} x\right)^{2}}{n}
$$

where the summation extends to the entire population.

$$
\begin{aligned}
& \frac{\sum\left(y-\begin{array}{c}
r \sigma_{1} x \\
\sigma_{x}
\end{array}\right)^{2}}{n}=\frac{\Sigma y^{2}}{n}-\frac{2 \sigma_{y} r}{\sigma_{1}} \frac{\Sigma x y}{n}+\frac{r \sigma_{y}{ }^{2}}{\sigma_{x}{ }^{2}} \frac{\Sigma x^{2}}{n} \\
& =\sigma_{y}{ }^{2}-2 r^{2} \sigma_{y}{ }^{2}+r \dot{2} \sigma_{y}{ }^{2} \\
& =\sigma_{y}{ }^{2}\left(\mathrm{I}-r^{2}\right) \text {. }
\end{aligned}
$$

Hence the standard deviation of a $y$-array is obtained from the standard deviation $\sigma_{y}$ of the total population with respect to the $y$-character by multiplying $\sigma_{y}$ by $\sqrt{1-r^{2}}$.

Since the first number of (3) is a sum of squares divided by $n$, the sacond number must be positive. Hence

$$
-\mathrm{I}<r<\mathrm{I} \text {. }
$$

This shows that our correlation coefficient must take values between +1 and -1 . 
If $r=+\mathbf{I}$, all the individual points of the population will lie on the line of regression, and we can therefore, when one character is given, tell exactly what the associated character is in magnitude. In this case the correlation is said to be perfect positive correlation. Similarly, if $r=-\mathbf{I}$, the correlation would be perfect negative correlation.

Three variables. The theory of correlation is easily extended to apply to more than two variables. For example, we might investigate the correlation of the statures of sons with respect to the statures of both parents. This is the case of biparental inheritance treated in the text, page 529 , and the formulas there used must be special cases of those which we are about to derive for giving the most probable value of a variable $\approx$ where $z$ is the numerical value of a character correlated with characters of measurements $x$ and $y$.

Suppose that the following system of corresponding deviations from the means have resulted from measurement: $\left(x_{1}, y_{1}, z_{1}\right),\left(x_{2}, y_{2}, z_{2}\right),\left(x_{3}, y_{3}, z_{3}\right)$, $\ldots,\left(x_{n}, y_{n}, z_{n}\right)$. Represent these measurements with respect to coördinate axes in three dimensions. These axes are to be taken at right angles to each other, as is conventional in analytic geometry, and may be referred to as the $x, y$, and $z$ axes. It now requires two letters to mark an array of $z^{\prime}$ s. We shall call $\left(x_{\lambda^{\prime}}, y_{\mu}{ }^{\prime}\right)$ the mark of a class. Now imagine the means plotted for every z-array. If correlation exists, these means will not lie at random in space, but will arrange themselves approximately on a surface called the "surface of regression." The equation of a surface is of the form $z=f(x, y)$. We shall consider only the case where this $f$-function is of the first degree, for the same reasons that we considered only the case of a first degree function in the case of correlation of two variables.

It results that the required function is

$$
z=\frac{r_{x z}-r_{x y} r_{y z}}{\mathrm{I}-r_{x y}{ }^{2}} \frac{\sigma_{z}}{\sigma_{x}} x+\frac{r_{y z}^{*}-r_{x z} r_{x y}}{\mathrm{I}-r_{x y}{ }^{2}} \frac{\sigma_{z}}{\sigma_{y}} y
$$

where $r_{y}$ is the correlation coefficient between the $y$-and $z$-characters, and similar meanings are to be given to the other $r$ 's, as indicated by the subscripts. This equation gives the mean value of the $z$-arrays corresponding to given $x$ and $y$, if they can be given by a linear function. If they cannot be accurately given by a linear function, this equation must merely be looked upon as giving a first approximation.

Standard deviation of arrays. If the equation (I) be used to estimate the value of the $z$-character corresponding to a selected $x$ and $y$, we have the square of standard deviation of each z-array about this estimated value given by the expression

$$
\frac{\mathbf{\Sigma}\left(z_{q}-a x-b y\right)^{2}}{n}
$$

as an average value, where the summation extends to all the observations; and

$$
a=\frac{r_{x z}-r_{x y} r_{y z}}{1-r_{y y}{ }^{2}} \frac{\sigma_{z}}{\sigma_{x}}, \quad \text { and } \quad b=\frac{r_{y z}-r_{x z} r_{x y}}{1-r_{x y}{ }^{2}} \frac{\sigma_{z}}{\sigma_{y}} \text {. }
$$


When expanded and expressed in terms of $r$ 's and $\sigma$ 's, (2) becomes

$$
\sigma_{z}^{2}\left(1-\frac{r_{z x}{ }^{2}+r_{z y}{ }^{2}-2 r_{z x} r_{x y} r_{z y}}{1-r_{x y}{ }^{2}}\right) .
$$

The formulas used in the text in discussing biparental inheritance are special cases of (I) and (3) just derived. This may be verified by making the following substitutions:

$$
\begin{aligned}
& \text { Put } x=h_{1}, y^{\prime}=h_{2}, z=h_{3}, r_{y z}=r_{2}, r_{x z z}=r_{1}, r_{x y}=r_{3}, \sigma_{z}=\sigma_{3}, \sigma_{x}=\sigma_{1} \text {, } \\
& \sigma_{y}=\sigma_{2} .
\end{aligned}
$$

Then in the new notation (I) becomes

$$
h_{3}=\frac{r_{1}-r_{3} r_{2}}{1-r_{3}^{2}} \frac{\sigma_{3}}{\sigma_{1}} h_{1}+\frac{r_{2}-r_{1} r_{3}}{1-r_{3}^{2}} \frac{\sigma_{3}}{\sigma_{2}} h_{2} .
$$

Since in the case discussed in the text the parents were taken equipotent, $r_{1}=r_{2}$, and by making this substitution in (4) we get

$$
h_{3}=\frac{r_{1} \sigma_{3}}{\left(I+r_{3}\right) \sigma_{1}}\left(h_{1}+\frac{\sigma_{1} h_{2}}{\sigma_{2}}\right)
$$

which is the formula used in text. Likewise, if we make these substitutions in (3), we get for the variability of an array of sons

$$
\sigma_{3} \sqrt{1-\frac{2 r_{1}^{2}}{1+r_{3}}}
$$

which is the formula used in the text.

More than three variables. It is easily seen that the methods employed in the case of two and three variables can be extended to any number of variables. However, the complexity of the algebraic expressions becomes so great that it does not seem well to present a more extended discussion here. For the general case of any number of variables, the reader with considerable mathematical training is referred to the treatment by Karl Pearson in the Philosophical Transactions of the Royal Society, A, CLXXXVII, I896, and $\mathrm{A}, \mathrm{CC}, 1903$. In the papers just referred to the general expression is also given for the variability of an array in the case of any number of variables. It is from this general expression that the formula used in the text for the variability of an array of offspring after $u$ generations of selection is derived.

Formula for the correlation coefficient $\mathrm{r}$ which is better adapted to numerical calculation. In the first place, the calculation of the standard deviations of both systems of variates should be done by the shorter method presented on page 465 . 
The value obtained for $r$ on page 705 is

$$
r=\frac{\Sigma_{y} y}{n \sigma_{1} \sigma_{y}},
$$

where $x$ and $y$ represent deviations from the means, and the summation is extended to every pair of corresponding deviations. The calculation of $\frac{\Sigma_{x} x y}{n \sigma_{x} \sigma_{y}}$ can be much shortened by an equivalent formula which we shall now derive. Following the notation on pages let $G_{x}$ and $G_{y}$ represent class marks near the means of the systems of variates indicated by the subscripts, and $C_{i}, C_{y}$ corrections to these class marks which give the correct mean values so that

$$
\begin{aligned}
& M_{z}=G_{x}+C_{i}, \\
& M_{y}=\dot{G}_{y}+C_{y} .
\end{aligned}
$$

Let $x^{\prime}, y^{\prime}$ represent deviations from $G_{x}$ and $G_{y}$ which correspond to deviations $x, y$ from the mean. Then

$$
\begin{aligned}
& x=x^{\prime}-C_{r}, \\
& y=y^{\prime}-C_{y} \text {, } \\
& r=\frac{\sum\left(x^{\prime}-C_{y}\right)\left(y^{\prime}-C_{y}\right)}{n \sigma_{x} \sigma_{y}}, \\
& =\frac{\Sigma x^{\prime} y^{\prime}-C_{y} \Sigma_{x^{\prime}}-C_{x} \Sigma y^{\prime}+\Sigma C_{1} C_{y}}{n \sigma_{x} \sigma_{y}}, \\
& =\frac{\mathbf{\Sigma} x^{\prime} y^{\prime}-C_{y} \mathbf{\Sigma}\left(x+C_{x}\right)-C_{x} \mathbf{\Sigma}\left(y+C_{y}\right)+\mathbf{\Sigma} C_{x} C_{y}}{\| \sigma_{x} \sigma_{y}}, \\
& =\frac{\Sigma x^{\prime} y^{\prime}-\mathbf{\Sigma} C_{1} C_{1}}{n \sigma_{1} \sigma_{1}} \\
& =\left(\frac{\Sigma_{1 y^{\prime} y^{\prime}}}{n}-C_{i} C_{i}\right) \frac{\mathrm{I}}{\sigma_{1} \sigma_{1}} .
\end{aligned}
$$

This is a formula much hetter adapted to computation than the formula

$$
r=\sum_{n \sigma_{x} \sigma_{y}}^{\Sigma_{x y}} .
$$

Its application is shown in the text, page 465 .

\section{SECTION IX-RANDOM SAMPLES}

We know full well that we cannot, in general, measure all the individuals of a race or population whose characteristics we wish to describe. W'e are obliged to get our information and to construct our science by the selection and examination of samples taken at random from a large group) of individuals. To illustrate, it is not practicable to measure the stature nor, in general, any other character of all the adults in the United States. We must be content to deal with a reasonably small number that will make the measurements a feasible undertaking.

An investigator is always concerned about the number of variates which must be measured in order that confidence may be placed in his results. 
For instance, he asks, Of how many ears of corn taken at random must I measure the length in order to obtain, to a certain desired degree of accuracy, the variability of the corn from which selection is made? Must I measure fifty, a hundred, or a thousand ears? Again, how many variates must I take to give a reliable determination of the mean?

Similarly, in any correlation study he will be concerned with the number of variates he must take in order to present a trustworthy determination of the correlation coefficients.

While these questions cannot be answered in advance for all kinds of populations, it is the object of this section to give some assistance to the inquiring investigator in forming a judgment in this matter. The best measure thus far devised upon which to base a judgment is the so-called "probable error."

So far as the mean is concerned, it has been seen that the probable error of a single variate may be obtained approximately by counting, and that the probable error in the mean is obtained from that of a single variate by dividing by the square root of the number of variates. This process can often be applied in a rough way before much labor has been put on a problem, and it becomes a useful guide where the mean alone is in question. It should be remembered that the probable error in any result is, in general, inversely proportional to the number of observations.

A method similar to that just explained for the mean can be used to find the approximate value of the probable error of the standard deviation, since the probable crror of the standard deviation is obtained from that of the mean by dividing by $\sqrt{2}$.

As for the coefficients of variability and correlation, the following tables show the probable errors corresponding to values of the coefficient of variability from I per cent to 25 per cent, with numbers of variates from 25 to I000, and the probable errors of the correlation coefficient for values from o to I, with numbers of variates from 25 to 1000.

If, then, we have an approximate notion as to the value of one of these coefficients, we can find from the table the probable error corresponding to a certain number of variates.

To illustrate the use of these tables, suppose that we know in advance that the coefficient of variability is in the neighborhood of 20 per cent; then with a hundred variates we see from the tables that the probable error would be approximately i per cent, while with five hundred variates it would be only 0.44 per cent. We thus decide upon the number of variates by the magnitude of the probable error and the degree of accuracy desired in our results.

Probable error in estimate of probability from a limited number of observations. While it has been said that, in a general way, the accuracy of a statistical result increases as the square root of the number of observations, this rule is often difficult to apply, and is an inadequate test in many important cases. 


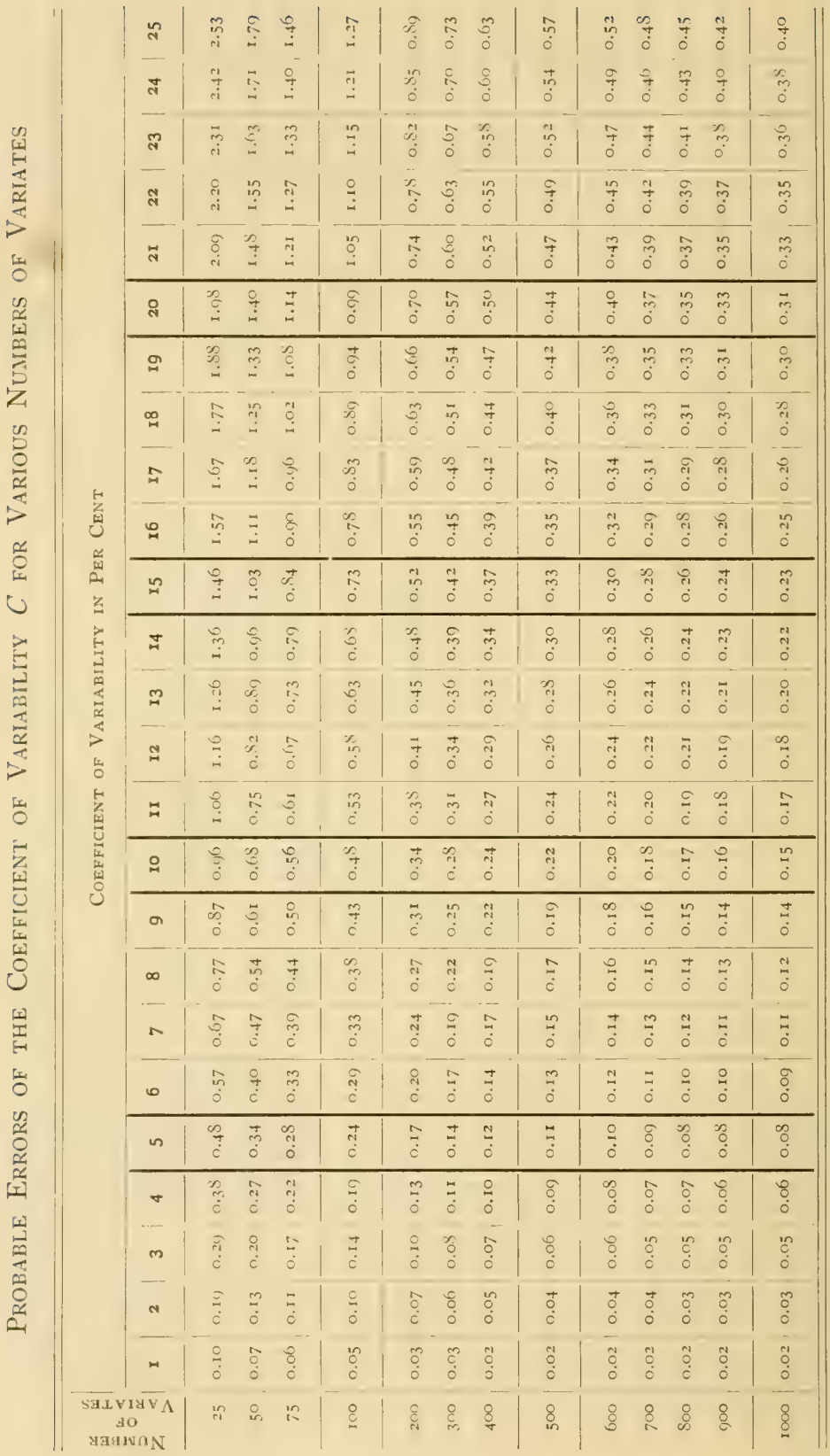




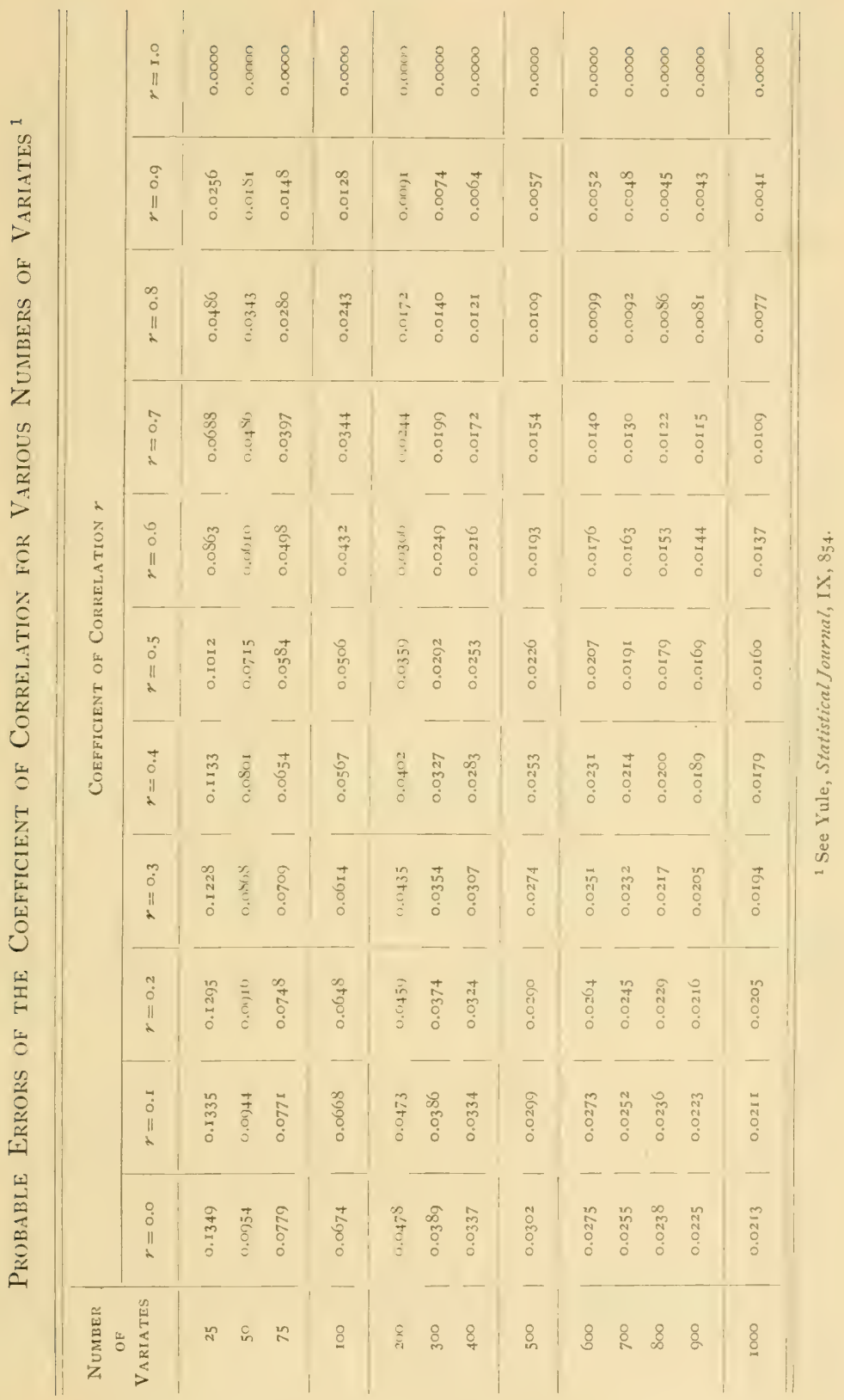


A common and important class of statistical deductions, which should receive very critical examination, may be illustrated as follows:

Suppose that, out of a total of ten years which have been observed, the apple crop in this locality has been injured by frost four years, and has been uninjured six years. If this data for ten years is all the evidence we have bearing on the probability of an apple crop, the best estimate we can give for the probability that the apple crop in this locality will not be injured by frosts in a given year is $\frac{6}{10}$. If, however, our data extend over twenty-five years, in fifteen of which the apple crop has been uninjured by frost, we again give $\frac{6}{10}\left(\frac{1}{2} \frac{5}{5}=\frac{6}{10}\right)$ as the best estimate for the probability that the apple crop in this locality will not be injured by frosts in a given year; and certainly more confidence can be placed in the result than when only ten years were taken.

We might carry our illustration back a hundred years in sixty of which the apple crop in this locality has been uninjured by frosts and we should still give $f_{i} ;$ as the most probable value of the probability that an apple crop in this locality will not be injured by frost in a given year. It should be noted that we are here dealing with the probubility of a probability, or what De Morgan has called the "presumption of a probability."

The critical examination of such probabilities as the above derived from observation should include some criterion which will indicate the accuracy of the approximation when only a limited number of cases can be examined. Such a criterion may be found in the probable error of the probability.

The problem in hand may well be stated in the following general form:

A bag contains an indefinitely large number of white and black balls in unknown ratio; if $m+n$ balls have been drawn as a random sample, and $m$ are white and $n$ are black, we give as the best value of the probability of drawing a white ball $m+n$. What is the probable error in this result? Or, in other words, in $m+n$ trials, an event has happened $m$ times and failed to happen $n$ times; if we deduce from this that $\frac{m+n}{m+n}$ is the probability that the event will happen on a given occasion, what is the probable error in this result?

From the works of Laplace, Poisson, and De Morgan, it follows that the probable error in $m+n$ is given by the formula $\pm \frac{0.67+5}{m+n} \sqrt{\frac{m n}{m+n}}$. Applied to our illustration of the apple crop when the data covered only ten years, this probable error formula gives $t_{10}^{0.67+5} \sqrt{\frac{24}{10}}= \pm 0.104$.

From the magnitude of this probable error, it is at once seen that the result in (derived from ten observations) for the probability that an apple crop will be uninjured by frost can at most be said to be but a crude and 
unreliable approximation. It is more than an even wager that it differs from the true value by as much as $\frac{1}{10}$.

When a hundred years are used, the probable error is $\frac{0.6745}{100} \sqrt{\frac{2400}{100}}$ $=0.033$, which shows that the result $\frac{6}{10}$ derived from a hundred years is much more significant than that which was obtained when only ten years were used.

The following table will show how, with increasing numbers, the probable error in the determination of the probability decreases.

\begin{tabular}{|c|c|c|c|c|c|}
\hline $\begin{array}{l}\text { Numeiz of } \\
\text { OBSERVATIONS } \\
=m+n\end{array}$ & $\begin{array}{l}\text { Number of } \\
\text { Times Event } \\
\text { HAppens }=m\end{array}$ & $\begin{array}{l}\text { Proba HLITY } \\
=\frac{m}{m+n}\end{array}$ & $\begin{array}{c}\text { Probable } \\
\text { ERROR }\end{array}$ & \multicolumn{2}{|c|}{$\begin{array}{l}\text { NUMBERS SUCH THAT WAGER IS } \\
\text { EVEN THAT RANDOM SAMPLE } \\
\text { AGAIN TAKEN LIES BET WEEN } \\
\text { THESE NUMBERS }\end{array}$} \\
\hline IO & 6 & 0.6 & \pm 0.104 & 4.96 and & 7.04 \\
\hline 25 & 15 & 0.6 & \pm 0.066 & 13.35 and & 16.65 \\
\hline 50 & 30 & 0.6 & \pm 0.047 & 27.65 and & 32.35 \\
\hline 100 & 60 & 0.6 & \pm 0.033 & 56.7 and & 63.3 \\
\hline $\mathrm{I}, 000$ & 600 & 0.6 & \pm 0.0104 & 989.6 and & 1010.4 \\
\hline 10,000 & 6000 & 0.6 & \pm 0.0033 & 9967 and & 10033 \\
\hline
\end{tabular}

Remarks. In conclusion, it should probably be said that we have, in the foregoing brief discussion of statistical methods, touched only "the fringe of a great subject." We have for the sake of simplicity confined ourselves to the normal curve of distribution; but it is to be hoped that we have given a general view, in this short space, of the methods by which the formulas are established which are now being commonly used in the quantitative study of evolution, and that the reader may come to see the proper place of statistical methods in solving problems of this character.

Furthermore, it is hoped that the results as here presented will be found practical in the sense that they may be of use to the non-mathematical reader, and pave the way for further investigations along this line. 



\section{N D E X}

Accessory chromosome, as related to sex, 634-637; Gross and Wallace upon, 636; Henking upon, 635; McClung upon, 635; Paulmier upon, 635; Wilson upon, 634,637 .

Acclimatization, bearing of, on instability of living matter, 308-316; by inoculation, 382 ; effect of, upon transmission, 374-386; extent of, 375,376 ; in general, 105, 314-316; of the individual and of the race, 374 ; of races, 384 ; of wood sage on high and low altitudes, $37 \mathrm{~S}$; permanence of, 315 ; to chemicals, $308-3 I I$; to cold, 3I3; to electricity, 3 I4; to high temperature, $3 \mathrm{II}-3 \mathrm{I} 3$; to hot springs, 379 ; to light, $3 \mathbf{I} 3$; to poisons, $3 \mathrm{SI}-$ 382 ; to temperatures, $376,{ }_{3} 81$; transmission of, 374 ; without selection, $379,3^{8}$.

Acid secreted by animals and plants, 267.

Acquired characters, I82, 308-3II ; as distinct from congenital, $354-356$; inheritance of, 349; non-existence of, 358-36o.

Actinian, reaction to chemicals, 273, 274; regeneration in the, 334 .

Adaptations, 350; not universal, 206208, 4 I 2.

Adlum, John, originator of Catawba grape, 134 .

Age, influence of, upon functional activity, 94 ; influence of, upon prepotency, 573.

Albinism common in most species, II4.

Amitosis, I 5 I.

Amœba, response to light, 253,254 .

Amphiaster, 147.

Amphicyon, 50.

Ancestral heredity, formula for, 533, 534 ; law of, 525-534.

Ancestral idioplasm, 173 .

Ancestral units, 173.

Animal breeding, $654-676$; advantages of, 654 ; disadvantages of, 654,655 ; disadvantage of many characters in, 656,657 ; during a depression, 665 ,
666 ; fashion in, $65 \$, 659$; by beginners, 675 ; records in, 666-672; show-ring consequences, 660 ; testing sires and dams, 660-664; testing young females, $66 \mathrm{r}$.

Animals, growth of, influenced by heat, 258, 259; higher regeneration in, 325,326 ; reduction in, compared with plants, 165 .

Ant, polymorphism of, 20 .

Antenna developed as a foot, 43 .

Apes, meristic variation in teeth of, $4 S$, 49.

Apricot, mutant of the plum, 112.

Arrays of the correlation table, 459 .

Artemia, experiments with, $28_{3}$.

Artemia salina, effect on, by degree of salinity, 102.

Artificial parthenogenesis, experiments in, $278-282$.

Assortative mating, 163 .

Aster, 147.

Asymmetry, 70 .

Atavism, 192-194.

Attraction of odors, 275.

Auricular appendages in mammals, 45, 46.

Average deviation, 427; illustrated, $44 \mathrm{I}-443$.

Bacillus tuberculosis excites abnormal growth, 98 .

Bacteria, effect of culture medium upon, 229.

Bailey, experiments in acclimatization, $376-378$; on bud varieties, 181 ; on the gooseberry, I 30 .

Bardeleben, studies in mammx, 47.

Barrenness to certain individuals, $20 \mathrm{I}$.

Bathmic influences, 202-208.

Bear, variation in digits of, 58 .

Bees, effect of food upon, 226 .

Begonia, regeneration of, 238,331 .

Belated inheritance, 475 .

Bert, experiments in grafting, 107.

Biometrika, journal of statistical study, 478 .

Biophors, I4, 208. 
Biparental inheritance, 529-533.

Birds, effect of heat upon development of, 259; variation in digits of, 56 .

Birthmarks, i89-191.

Bisexual reproduction, a cause of variation, 160-163; introduces no new characters, I63.

Blackberry, evolution of, I 3 I-133.

Blemishes on breeders, 590.

Blend in characters, $48 \mathrm{I}$.

Blended and exclusive inheritance, 475 .

Bonnet, experiments in regeneration, 3 I6.

Bonnier, experiments on acclimatization, 378 ; experiments with dandelion, 223 .

Born, experiments in grafting, $108,336$.

Brain not necessary to coördinated motion, 400, 401 .

Breeder's business is the production of sires, 605.

Breeders' fads, 594.

Breeders of speed and breeders of breeders contrasted, 557 .

Breeding, polymorphism in, 476,477 ; problems of, outlined, $3-5$; purposes in, 599, 600; systems of, 599-627; true, or stability of type, 54I-544.

Brown-Séquard, experiments on mutilations, 367 .

Bruce, studies in mammæ, 47.

Bud variation, $18 \mathrm{r}$.

Bud varieties reproduce by seeds, I $8 \mathbf{I}$.

Bull, E. W., originator of Concord grape, I 34.

Bullfinch, effect of food upon, 228 .

Bumblebee, antenna of, developed as a foot, 43 .

Burbank system of planting, 643 .

Burrill, experiments in crossing strawberries, $\mathrm{I} 84$.

Camel, development of foot of, 60 .

Castration, indirect effects of, upon the body functions, Ioo.

Catalytic poisons, 266.

Cats, variation in digits of, 57 .

Cattle, acclimatization of, 375 ; development of foot of, $5^{8}$; meristic variation in digits of, $62,6_{3}$; reversions in, 192 .

Cave animals, 242 .

Cell, the, as a structural unit, I 43, I 44 ; differentiation in, 144 ; effect of gravity upon, 239.

Cell division, as a cause of variation, I 55-18I ; irregularities in, I 50-I 52; mechanism of, $145^{-1} 5^{2}$; outline of, 146,147 ; variation in rate of, 340 ; with and without differentiation, $149-$ 150.

Centgener plots, 644 .

Centrosome, 146.

Cervical fistulæ in mammals, 44 .

Chance, accounts for unusual occurrences, 187; as distinct from cause, 365 ; law of, 365,366 .

Character, defined, I7; meaning of term, I I-I3.

Characters, acquired, 182 ; acquired from the environment, $302-304,308-311$; acquired, inheritance of, $349 ;$ are not "acquired," 358-360; blended, 48r ; combine in definite proportions, 5045 3 ; congenital and acquired, 354356; dependent upon sex, 194-196; development of, how influenced, $36 \mathrm{I}-$ 363 ; dominant and latent, 13,14 ; dominant and recessive, $5 \mathbf{1} 4,515$; elementary, 14 ; how behave in transmission, 473-478; latent, 474; not necessarily adaptive, $4 \mathrm{I} 2$; of adult as influenced by development, 350; of the individual are those of the race, 357 ; origin of, 4 I $3-4$ I 5 ; origin and degeneracy of, contrasted, 4 I 5 ; too many, in animal breeding, 656, 657 ; variability of, in same population, 444 .

Chemical action, acclimatization to, 3083 II ; of secretions, $3_{3}, 3_{8} 8_{4}$.

Chemical effects of light, 2.40 .

Chemical reactions of protoplasm, 264285 .

Chemicals, effect of, upon germination, 27I; rhythmical contraction stimulated by, $276-278$.

Chemotaxis, $27 \mathrm{I}, 272$.

Chemotropism, 271,276 .

Chromatin granules, $\mathbf{1} 45$.

Chromatin matter, $145^{-1} 52$.

Chromomeres, 146.

Chromosomes, $146-152$; but half the normal number of, in parthenogenetic individuals, ISo; composition of, 174; constant in number for the species, 146; number of, even in bisexual reproduction, 146 ; number of, reduced by maturation, restored by fertilization, 170; number of, sometimes halved, I 80 .

Chrysanthemums, experiments with, by De Vries, I I 9-12I.

Cleavage, effect of outside conditions upon, 340; geometrical character of, 339.

Coefficient, of assortative mating, 532 ; of correlation, short method for 
calculation of, $465-468$; of heredity, 486-488; of mode, 422,423 ; of regression, 487-490; of variation, 433 ; of correlation, calculation of, 455 , 464 ; of variability, comparative, 434 ; of variability, meaning of, 434,435 ; of variability, probable error of, $44 \mathrm{I}$.

Cold, acclimatization to, 313 ; effect of, upon color, 264.

Coleoptera, extra eyes in, 51.

Color, correlation with speed in trotters, 468-47I; influenced by temperature, 262-264; not due to presence of light, 242 ; when important, $3 \mathbf{I}$.

Combination of characters, law of, 504$5^{\mathrm{I}} 3$.

Combinations, formula for, $5 \mathrm{II}$; of two characters, 504, 505; of three characters, 506, 507.

Community breeding, 674 .

Comparative value of male and female, 587.

Conditions of life, effect of, upon development, $98-105$; influence of, upon parthenogenesis, 102.

Congenital and acquired characters, $354-356$.

Contact, effect of, upon direction of motion, 235; effect of, upon functional activity, 233-236.

Continuity in variation, $\mathbf{I} 8$.

Cope, on transmission, 354 ; on theory of growth force, $203,204$.

Com, acclimatization of, $375,377,378$; correlation between length and circumference of ears, 461 ; effect of selection upon, 445, 446; functional variation in, $83-86$; influence of locality upon, 222 ; progression in oil and protein content of, 493-498; variability of, 427-43I, 444, 447, 448 ; variability of, as affected by fertility, 449-45I; variation in composition of, $\delta_{3}, s_{4}$.

Corn Breeders' Association, system of planting, 6.6 .

Correlation, 453-47 I ; coefficients of, 455-466; between color, sex, and speed, in trotters, $468-471$; between length and circumference of ear, 461 ; meaning of, 453-455; method of finding coefficient of, $460-468$.

Correlation table, 458 .

Cows, variation in functional activity of, 92, 93; functional variation in, $77-81$; meristic variation in mammæ, 47 ; relative fertility of, 199.
Crab, segments of, influenced by parasite, 100 .

Crandall, observations on curculio, 390-393.

Crayfish, meristic variation in oviducal opening, 44.

Cross, reciprocal, 525 .

Crossing, 608-6ro; advantages of, 608 ; disadvantages of, 609 .

Crystals, growth of, compared with growth of living matter, I 43.

Curculio, egg-laying instincts of, 390393.

Cynips, sting by, produces functional deviation, 98 .

Cytoplasm, I45; in development, I 77.

Dallinger, experiments in acclimatization to heat, 379 .

Dams, testing of, 66 I.

Dandelion, influence of locality upon, 223.

Darbishire, experiments with mice, 524 .

Darwin, experiments in cross and self fertilization, 6r 8-624.

Davenport and Castle, experiments on acclimatization, $3 \mathbf{I} 2$.

Davenport and Neal, experiments on acclimatization of stentor, 3 I O.

Death rate, Weismann on, 202.

De Candolle, experiments on acclimatization, 376 .

Degeneracy contrasted with origin, 4 I 5 , 416.

Degeneration of useful parts, 409412.

Determinants, $152,208,215$.

Determination of sex, 629-637.

Development, 677-680; a study distinct from breeding, 680 ; confusion of, with inheritance, 350 ; dependent upon external conditions, 677 ; does it influence transmission? $36 \mathbf{1}$; effect of, upon transmission, 372,373 , 407-409; from a half ovum, I76; how influenced, $361-363$; influence of food upon, 225-230; influence of gravity upon, 239 ; influence of locality upon, 22I-225; influence of moisture upon, 230-233; influence of use upon, $286-288$; influence of, upon prepotency, 574; limits of, 362 ; mechanism of, $142-154$; not an index of inherited characters, 232 ; originates in geometrical cleavage of ovum, 339; requires good conditions for well-bred individuals, 678 , 679 ; through the cytoplasm, 177. 
Deviation, average, 427 ; nature of, 349, 350; not transmissible as such, 349; standard, 428-431; standard, meaning of, 432,433 .

Deviation and probable error illustrated, 44I-443.

De Vries, experiments of, II4-I29; experience in plant breeding, 642; experiments with chrysanthemum, II9I2I; experiments with primrose, I 2 I-I 29 ; production of new species by mutation, I $2 \mathrm{I}-\mathrm{I} 29$.

Differentiation, by cell division, I 49, I 50; causes of, 343 ; from internal causes, 144; mechanism of, I 42-I 54; polarity and promorphology of ovum, $34 \mathbf{I}-343$

Digitalis, medicinal qualities of, affected by locality, 223 .

Digits, meristic variation in, 53-64.

Dimorphism of earwig, Shorthorns, Herefords, etc., 20.

Disappearance of parts, 306.

Disappearing parts, 288 .

Discontinuity in variation, 19.

Disease, transmission of, 368,384 .

Diseases due to absence of secretions, 269.

Distribution, offspring constitute a, $4 \mathrm{I} 9$.

Dodd, William, originator of plum, 133.

Dog, acclimatization of, 376 ; behavior of, when deprived of brain, 400,401 ; meristic variation in teeth of, 50 ; variation in digits of, 57 .

Dogs, influence of locality upon, 224; telegony in, 186.

Dorfmeister, experiments with butterflies, 262.

Double personality, ro6.

Doubling of parts, as head, 67-69.

Ducks, relative weight of bones of tame and wild, 95 .

Dugong, variation in digits of, 57 .

Dwarfs, reasons for, 27.

Dyads, I66.

Earthworm, meristic variation in generative opening of, 44 .

Earthworms, regeneration in, 317-320.

Earwig, dimorphism of, 20.

Eggs, regeneration in, 324, 325 .

Ehrlich, experiments with mice, 309.

Eimer, on adaptation, 206-208; on theory of orthogenesis, 204-208.

Electricity, acclimatization to, 3 I 4 .

Embryos, regeneration in, 324, 325 .

Endosperm, fertilization of, $\mathrm{I}_{3}$, I $8_{4}$.
Environment, always selective, $35 \mathrm{r}$; bearing of, on instability of living matter, 290-293, 302-304; cause of evolution of the horse, 302-304; direct action of, 307; food, 225-230; general effect of, upon development, $22 \mathrm{I}-225$; how influences type of race, 290-293; influence of, upon cleavage, 340 ; influence of, upon parthenogenesis, 178 ; influence of, upon variability, 220-293.

Epilepsy, transmission of; 367 .

Evidence that is not evidence, 353.

Evolution, knowledge of, needed in breeding, 5 ; not confined to morphol. ogy, 76; Weismann's theory of, 152 .

Ewart, experiments in telegony, 186.

Exercise, effect of, upon functional activity, 95, 96.

Exophthalmia, transmission of, 367 .

External influences as causes of variability, 220-293.

Extra wing, 43 .

Eye, effect of light upon, 243.

Eyes, degeneration of, in cave species, 4II, 4I2; supernumerary, 5 I.

Fads of the breeder, 594.

Fan-top trees, II 2.

Fashion in animal breeding, 658,659 .

Fattening, unsuccessful in darkness, 246.

Fear, not present at birth, 403 .

Female, influence upon, by previous mating, $18_{5}-18_{9}$; maturation and reduction in, $165-169$.

Females, disposal of surplus, 672 .

Féré, experiments of, on chicks, 259.

Fertility, characters correlated with, I 97 ; relative, I96-200; effect of food upon, 226 ; effect of, upon type, r98, I 99; effect of, upon type and variability, 449-45I ; importance of, I99, 584,589 ; less with extremes of a race than with the means, 491 ; often opposed by selection, $5^{8} 3$.

Fertilization, by the polar body, I79, I80; connection of, with mutation, I So ; of endosperm, $183, \mathrm{IS} 4$; manner of, $16 \mathrm{I}$; significance of, 170 ; influence of, upon sex, 632,633 .

Fish, upstream movements, 235 .

Fisher, studies in fistula, 45 .

Flagellata, acclimatization to high temperatures, $379,38 \mathrm{r}$.

Flammarion, experiments with light, $245,246$.

Flatfishes, 415. 
Food, effect of cotton seed on pork, 228; effect of, upon bullfinches, 228 ; effect of, upon body temperature, 230; effect of, upon constitutional vigor, 372,373 ; effect of, upon development, 370-374; effect of, upon fertility, 226; effect of, upon functional activity, 96, 97; energy of, 229 ; excess of, 227 ; how reduced by living beings, 228 ; influence of, upon regeneration, 327,328 ; influence of, upon variability, 225-230; proportion of, used in growth, 225; qualitative effects of, 228-230; quantitative effects of, 225-227; well-bred races require more, 227 .

Foundation not in a remote female, $595,596$.

Fraser on efficiency of cows, 78-8I.

Fraternal variability, 500-50.4.

Free-martins, 176.

Frequency distribution, typical, 421 .

Frequency distribution and the binomial theorem, 509.

Frog, influence of heat upon growth of, 25 .

Functional activity, by suggestion, 243 ; effects of light upon, $241-246$; how dependent upon light, 25I, 254 .

Functional variation, 75-109; between different individuals of the same species, 77-91 ; due to light, 239-254; of same individual from day to day, $9 \mathbf{I -}$ 94 ; induced by castration, 100; induced by contact, 233-236; induced by external influences, $98,101-105$; influenced by food, 96, 97, 225-230; influenced by gravity, 236-239; influenced by hard conditions, 97 ; influenced by the reproductive faculties, I0O.

Functions, discharged only in presence of light, 242, 243; exercised under abnormal conditions, 107-109.

Funk, Deane N., car-load lot of prizering grade cattle belonging to, 607 .

Fürbringer, studies in birds, 42 .

Galls, 98, 270.

Galton, on heredity, 478 ; on law of ancestral heredity, 528 ; on fraternal variability, 500, 501 ; on law of inheritance, I93, I94; on studies of stature, 48 I.

Gametic purity, 5-21.

Gemmules, zo\&.

Genetic selection, 196-200.

Gentry, N. H., on inbreeding, 625 .
Geotaxis, 236.

Geotropism, 236-239; in animals, 238; of sprout and root, II .

Germ, infection of, $\mathrm{IS}_{5}$; influence of age or staleness upon, $\mathrm{I}_{2}$; individuality of, 182 ; variations in, are transmitted, 348 .

Germ plasm and transmission, 355 .

Germinal selection, I62, 213-215.

Germinal vesicle, $\mathrm{s} 66$.

Germination, effect of chemicals upon, 271.

Giants, reason for, 27.

Glands, specific secretions of, 269.

Goltz, experiments on the dog, 400 , 401 .

Gooseberry, evolution of, 130.

Gorilla, extra incisor in, 49.

Grading, 602-608; advantages of, 604 ; abuse of, 604; begin by, to get experience, 606 ; disadvantage of, 608 ; disappearance of unimproved blood in, 602 .

Grafting, frog made up of pieces of two individuals, IoS; illustrating stability of living matter, 335,336 ; mammary gland into ear of guinea pig, 107; spur of cock into comb, 107; two species of animal together, 336 ; two pieces of tadpole, 336 .

Grapes, evolution of, 134 .

Gravity, effect of, upon development, 239 ; effect of, upon living matter, 236-239; effect of, upon protoplasm, 239; effect of, upon regeneration, 329-332; in struggle with polarity, 237.

Great sires, 552 ; the ten greatest, 555 .

Gross on the accessory chromosome, $6_{3} 6$.

Grout, A. P., grade Angus steers belonging to, 605 .

Growth, as influenced by temperature, 254-262; direction of, due to light, 2.49 ; direction of, influenced by heat, 259 ; geometrical character of cleavage in, 339; retarded by light, 245, 246.

Growth force, 203, 204.

Guinea pig, grafting mammary gland into ear of, I07; supposed transmission of mutilations of, 367 .

Habit not the basis of instinct, $40 \mathrm{I}-$ 403.

Habits, are they transmitted? 363 , 386-403; founded on instincts, not the reverse, $401-403$; leamed from 
elders, 353 ; not acquired characters, $35^{\mathrm{S}}-360$.

Harris, 13. F., example of longevity, 89). Heape, experiments on rabbits, 190.

Heart, rhythmic contraction of, 398, 399 .

Heat, acclimatization to, 3II-3I 3, 379$38 \mathrm{r}$; effect of, upon animal activities, 255 ; effect of, upon direction of growth, 259; effect of, upon growth of animals, 258, 259; effect of, upon growth of plants, $255^{-257}$.

Hedgehog, meristic variation in vertebre of, 39 .

Heliotropism, 247 ; conditions that determine, 254; due to the luminous rays, 248 ; general principles governing, 251, 254; of amœba, 253, 254; of insects, 104 .

Henking on the accessory chromosome, 635.

Herbst, experiments in regeneration, 328.

Herd, management of, during depression in prices, 665-666; records of, 666-670; unity of, 662; without a head, 664 .

Heredity, 473-547; coefficient of, 486 , 488 ; coefficients of different relationships, 488 ; famous grandsires, $55^{6}$; law of ancestral, 525-534; manner of, 420-43I ; material basis of, 209; mathematical nature of, 510; mean of offspring not mean of the parents, 490; measure of, 486 ; misconceptions of, 473 ; offspring differ from parents, 482,483 ; offspring more mediocre than the parents, $484-486$; origin of the exceptional individual, 499, 500; progression in, 492-498; proper conceptions of, 473 ; statistical methods in study of, 426 , 478 ; what is transmitted? $5 \mathrm{II}$.

Herefords, dimorphism in, 20.

Hero, the inbred morning-glory, 622.

Herringham, studies on nerves, 43 .

Homœosis, 37; in insects, 43; in vertebra and ribs, 40-42.

Honeybee, eyes united, 65 .

Hopkins, experiments in corn breeding, 83-86, 493-498.

Horns, meristic variation in, $52,53,66$; regeneration of, 326 .

Horse, begging instinct in, 353 ; causes of evolution of, 302-304; defective voice in, 353 ; development of foot of, 58, 59; evolution of, 298-305; extreme age of, 89 ; meristic variation in digits of, 60,61 .
IIorses, acclimatization of, 37.5 ; correlation between color, sex, and speed, 468-47 r; inbreeding in, 624; power of transmission among, 408 ; telegony in, 185 .

Horseshoe kidney, 65.

Hot springs, Infusoria in, $3 \mathbf{I I}$; life in, 379.

Houghton, Abel, originator of gooseberry, 130.

Hunter, experiments in grafting, 107.

Huntington, Randolph, on breeding, 624.

Hybrids, character of descendants of, 514-52I; Mendel's law of, 513-525; sterility of, 607 .

Hypertrophy, 288, 289 .

Idants, I73.

Ideals in selection, 578,579 .

Idioplasm, I 52, 208.

Ids, 146,208 .

Illinois station, system of planting at, 646 .

Immunity, natural and acquired, $3^{82}$.

Improvement, upper limits of, 582 .

Inbreeding, 613-626; advantages of, $61_{4}, 615$; A. J. Lovejoy on, 625; among animals, 624-626; Darwin's experiments on, 6I S-624; disadvantages of, 615; forms of, 613, 614; how to practice, 626 ; often more vigorous than outbreeding, 622-624; lack of vigor and low fertility common defects, 6i 6, 6r 7 ; N. H. Gentry on, 625 ; not all inbred individuals inferior, 620-623; not necessarily disastrous, 616-626; Randolph Huntington on, 624; special dangers in, 616; total effects of, 619, 620 .

Individual, the, $35^{2}$; possesses all the characters of the race, 357,360 .

Individuality in offspring from same parents, 503 .

Infertility a common defect, 616,617 ; effect of, $5^{84}, 5^{89}$.

Inheritance, complex, 527; belated, 475; blended and exclusive, 475 ; from separate ancestors, 527; from the race, I93, 194; not limited to sex, 474; of acquired characters, 292, 349; offspring more mediocre than the parents, $484-486$; particulate, 476 ; progression in, $492-498$.

Inoculation, immunity by, 382 .

Insect poisons, 270.

Instability, of living matter, illustrated by origin of differentiated tissue, 336 338 ; of protoplasm, 398. 
Instinct, 10.4-106; is it founded on habit? $388-390$; not founded on habit, $40 \mathrm{I}-403$; not unerring, 389 .

Instinctive acts, a series of reflexes, 398-40I; not uniformly performed, $390-394$.

Instincts, are they inherited habits?

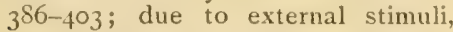
252 ; intelligence not necessary to, 397,398 ; nature of, 387,388 ; originate in reflex action, 394-397; not always adaptive, 394 .

Intelligence not necessary to complicated acts, 397, $39 \mathrm{~S}$.

Intemal influences affecting the race, I 96-2 I 7 .

Intra-uterine influences, IS9-I9I.

Jochemke, variation in functional activity of, 92, 93.

Kangaroo, development of foot of, 60 . Kanthack, experiments with snake poison, 309.

Kerrick, L. II., car-load lot of graded steers, 603 ; on herd records, 668 , 669.

Lamarckians, opposers of, 4I3; views of, 4 I3.

Lancaster, Ray, defines thremmatology, 1 .

Latent characters, 474 .

Law of ancestral heredity, 194, 525-534.

Law of chance, 365,366 .

Life, material basis of, $2 I_{3}$.

Light, acclimatization to, 313 ; chemical effects of, 240; effects of, upon fixation of carbon, 239; effect of, upon functional activity, 24I-246; effect of, upon living matter, $239-254$; effect of, upon regeneration, 328 ; general effects of, 25 1,254 ; influence of, upon direction of growth, 247 ; influence of, upon eyes of dead sharks, 395 ; influence of, upon locomotion, 247; not necessary to development of color, 242 ; not necessary to development of vigor, 244; specific rays of, that exert effect upon growth, 245 , 246 ; vital limits as to, 244 .

Line breeding, 610-613; advantages of, 6 I I ; best system for improvement of, 612 ; disadvantages of, 611,612 .

Living matter, distinguished from nonliving, I 42-143; influence of light upon, 239-254; parallelism with non-living matter, 2IO, 2 II ; relative stability and instability of, 295-346; response to gravity, $236-239$.

Locality a comprehensive term, 224 .

Locomotion, direction of, due to light, 250.

Loeb, experiments in chemotropism, 273-276; experiments in heliotropism, 250-254; experiments in parthenogenesis, $278-282$; experiments in rhythmic contraction, $276-278$; observations upon Infusoria, 308 .

Longevity, 20I, 202; earlier offspring live longest, 502.

Lothelier, moisture experiments, $23 \mathrm{I}$.

Lovejoy, A. J., line-bred swine belonging to, 611, 612; on herd records, 670 ; on inbreeding, 625.

Lubbock, experiments with ants, 273 .

McClung on the accessory chromosome, 635.

Male, maturation and reduction in, 169 , 170.

Male and female, comparative value of, 587.

Mammx, meristic variation in, 46,47 .

Mammary tissue, grafted on ear of guinea pig, 107; in various parts of the body, 46.

Man, auricular appendages on, 45; cervical fistulx in meristic variation in digits of, 54, 55, 69; meristic variation in mamma of, 47 ; meristic variation in ribs of, $40,4 \mathrm{I}$; milk secretion not confined to females, 107 ; telegony in, ISS.

Manatee, variation in digits of, 54 .

Marks due to prenatal influences, I89I 9 I.

Material basis of life, 213.

Maturation in the female, $165-169$.

Maturation and reduction, a cause of variation, $163^{-1} 81$; in animals and plants compared, 165 ; in male and female compared, 164 .

Mean, calculation of, 424 ; of offspring not the mean of the parentage, 490 ; practical use of, 425 ; probable error of, 440 .

Measurements, hints on taking of, 435 ; scheme of, 436 .

Meat production, variation in, $\delta I-\delta 2$.

Mechanism of development and differentiation, I42-I 54 .

Melon, influence of locality upon, $22 \mathrm{I}$.

Men, milk secretion by, 107.

Mendel, Gregor Johann, 5 I 3.

Mendel's experiments, 516-52I. 
Mendel's law, 5I3-525; experimental evidence on, 516-521; experiments with mice, 524 .

Mendel's law and gametic purity, $52 \mathrm{I}$.

Merinos in New Zealand, 223.

Merism, 33.

Meristic variation, $33-74$; cervical fistulx and auricular appendages, 4446; doubling of complicated parts, $64-69$; due to cell division, $72,15^{8}$; homoeosis in, 37; in digits, 53-64; in eyes, $5 \mathbf{I}$; in generative parts, 44 ; in head, 67,68 ; in horns, 52,53 ; in legs, 64 ; in mamme, 46,47 ; in radial series, 70-73; in spinal nerves, 42 ; in teeth, $48-51$; in vertebræ and ribs, $39-42$; in wings, 51 .

Mice, acclimatization of, to ricin, 309; variation in digits of, 58 .

Microsomes, 146.

Mid-parent, Galton's, 481 .

Mid-parent deviation, formula for, 531 .

Mid-parent variability, formula for, $53 \mathrm{I}$.

Milk production, variation in, 78-80, 92,93 ; transmitted by males, 360 .

Milk secretion by males, 105, 107.

Minnesota station, system of planting at, 644 .

Mitosis, as a cause of variation, 155181 ; details of, $145^{-1} 5^{2}$; irregularities in, 150-1 52 ; pathological, I $50-$ I5I.

Mixed breeding, purity in, 507 .

Modal coefficient, 422,423 .

Mode, the, 42I, 422; empirical and theoretical, 422 ; practical value of, 423 .

Modifications due to external influences, transmission of, $34 \mathrm{~S}-4 \mathrm{I} 7$.

Moisture, effect of, upon development, 230-233; effect of, upon spiny growth, 23 I.

Monte Carlo and roulette, 365 .

Morgan, observations on acclimatization to heat, $3 I I$; on fear in chicks, 403 .

Morgan horse, 296.

Morning-glory, Darwin's experiments in inbreeding, 62I-624.

Morphological variation, 25-29; causes of, 27 ; in mulberry leaves, 26.

Moss roses, a bud variety, $\mathrm{I} 8 \mathrm{I}$.

Moths, flight of, determined by light, 250.

Movement induced by contact, 234 .

Mulberry leaves, polymorphism in, 26.

Multiplying plots, 650 .

Multipolar mitosis, $15 \mathrm{I}$.
Mumford, experiments in feeding, 82 ; on pork production, 228 .

Muscle fiber, effect of light upon, 247.

Mutability of species, $298-305$.

Mutants, 2I ; origin of, in toadflax, II 5-1 18 .

Mutation, biological significance of, 136 , 137; distinguished from ordinary variation, IIO; economic significance of, $135^{-1} 36$; in chrysanthemum, I 19$\mathrm{I} 2 \mathrm{I}$; in primrose, I2I-I 29; laws of, $127-129$; in toadflax, II5-1IS; in American native plants, $129-135$; in general, I10-1 38 ; relation of, to reduction and fertilization, I80.

Mutation and elementary species, I28.

Mutation and parthenogenesis, I 79 .

Mutilations, are they transmitted? 363368 ; experiments upon transmission of, 367 ; resemblance of, to natural deformity, 366 .

Nägeli, experiments with copper, 268 .

Narwhal, meristic variation in tusk of, 70.

Natural selection always at work, 588 .

Nectarine, mutant of the peach, I I 2.

Neo-Darwinians, 354.

Neo-Lamarckians, 354 .

Nerves, meristic variation in, 42 .

Neugebauer, studies in mamma, 47 .

Nucleus, $145^{-1} 5^{2}$.

Odors, attraction of, 275 .

Oil content of corn, $83-85$; effect of selection upon, 445,446 ; progression in breeding for, $496-498$.

Old Granny, example of longevity and extreme fertility, $89,90$.

Oöcyte, I65.

Ö̈gonia, 165 .

Orientation, 247.

Origin of characters, $4 \mathrm{I} 3^{-4} \mathrm{I} 5$.

Orthogenesis, 204-208; explained by germinal selection, 2 I 5 .

Osborn, researches on evolution of the horse, 302.

Ossification, 99.

Otocyon, teeth of, 50.

Overfeeding, evil effects of, 227 .

Ovum, 161, I65; polarity of, 341-343; promorphology of, $34 \mathrm{I}$; segmentation of, without fertilization, 177I 80.

Oxygen, effect of, upon protoplasm, 265 .

Panmixia, 288.

Parry, originator of blackberry, 132. 
Parthenogenesis, I6z; but one polar body in, 179; influenced by conditions of life, 178 ; influenced by temperature, $2 S \mathbf{I}$; Loeb's experiments in, $278-282$; but half the normal number of chromosomes in, I 80.

Parthenogenesis and mutation, 179.

Parthenogenetic reproduction, variation in, $177-1$ So.

Particulate inheritance, 476 .

Paulmier on the accessory chromosome, 635.

Pearson, on the bathmic influences, 203; on causes of variability, 220; on fertility, $19^{6}-198$; on law of ancestral heredity, 529; on reduction of variability, 536 ; on telegony, 188.

Pedigree, importance of, 592 ; records of, 670-672.

Pedigrees, fashionable, 595 .

Peloric flowers in toadflax, II 5-I IS.

Performance, as a guide to breeding powers, 558-567; records in plant breeding, 650 .

Pfeffer, experiments on chemotropism, 273.

Photosynthesis, 240.

Phototaxis, 248.

Phototonus, 248 .

Physiological selection, 201, $5^{80}$.

Physiological units, 14, 17, 152, 2082I 3 ; as affected by reduction, I 70 .

Pigs, acclimatization of, 375 ; development of, 58 ; meristic variation in digits of, 63 .

Planarian, regeneration of, 321, 322, 334, 335; regeneration of starving, 327 ; regeneration of, when split, 335 .

Plant breeding, 639-65 I; advantages and limitations of, $639-64 \mathrm{I}$; plot system for, 644-646; soil and culture conditions for, 64I-6.43; systems of planting in, 643-6.49.

Plant lice, effect of conditions upon reproduction, I02; parthenogenetic only at high temperatures, $2 S \mathrm{r}$.

Plants, effect of heat upon growth of, 255-257; regeneration in, 325 .

Plot system of planting in breeding work, 644-646.

Plums, evolution of, 133 .

Poison ivy, immunity to, 308 .

Poisons, acclimatization to, 308-3II; catalytic, 266,267 ; from insects, 270 ; toxic, 268.

Polar bodies, I 64 ; formation of, 167 ; formation of, illustrated, I $6 S$; fertilization by, I 79, I So; formation of second, r67.
Polarity, in struggle with gravity, 237 ; of ovum, 34 I-343.

P'ollination, indirect effect of, $1 \mathrm{~S}_{5}$.

Polymorphism in practical breeding, 476,477 ; with regard to sex, 20.

Poplar, acclimatization of, 376 .

Population, 421 ; type of, 422 .

Pork, effect of cotton seed upon, 228.

Precipitin test, $3 \mathrm{~S}_{2}$.

Preferential mating, $\mathrm{I} 6_{3}$.

Prenatal influences, $189-191$.

Prepotency, 55I, 575; as affected by age, 573; as indicated by perfornance, $55^{8}-567$; as related to constitutional vigor, 573 ; as shown by trotting records, $55 \mathrm{I}-566$; influence of development upon, 574 ; because of sex, 567-570.

l'rimrose, seven mutants in eight generations of, 127.

Probable error, of coefficient of variability, 44I; of mean, 440; of stand. ard deviation, 440; meaning of, 437-440.

Probable error and deviation illustrated, $44 \mathrm{I}-443$.

Progression, a few offspring extreme, $492-498$; in oil content, 496-498; in protein content, 493-495; origin of the exceptional individual, $499,500$.

Proof by method of instance, 187,366 .

Protein content of corn, effect of selection upon, 445,446 ; progression in breeding for, 494,495 .

Protoplasm, a chemical substance with chemical properties, I 44 ; activity of, due to external as well as to internal impulses, 398-40I; as influenced by chemical agents, $264-285$; effect of contact upon, $233-236$; effect of gravity upon, 239; effect of heat and cold upon, 255 ; irritation of, 398 ; effect of external agents upon, 396 . 397 ; physical basis of life, I42, I43 relative stability and mutability of, $295,346$.

Protozoa, but one maturation division in, 165 .

Purity of blood lines essential, 579 .

Python, meristic variation in vertebra and ribs of, 39,40 .

Qualitative effects of food, $228-230$.

Quantitative effects of food, $225^{-228}$.

Race, affected by intermal influences, 196-217; extinction of, 415.

Radial symmetry, 34 
Random sample, 420 .

Rat, tail of, grafted into back, 107.

Rational selection, 592, 593 .

Rats, variation in digits of, $5 \$$.

Réaumur, experiments in regeneration, 3 I6.

Reciprocal cross, 525, 610 .

Records, in animal breeding, 666-672; in plant breeding, $645-649$; of the herd, 666-670; of pedigree, 670-672.

Redfield, Casper L., on prepotency, 574 ; on transmission, 407, 408.

Reduction, a cause of variability, $163-$ ISI, I75, I76; apparent purpose of, I67; connection with mutation, 1 So; end products of, 167,168 ; how accomplished, 169 ; illustrated, 168 ; in the male, 169, 170; in the female, $165-169$; in male and female compared, 164; in plants, 171-173; in animals and plants compared, 165 ; losses sustained by, I70; opportunities for accident during, I7I; Weismann's prediction concerning, I73, I74; results in loss of chromatin matter, 170, 173; significance of, I70.

Reflex action the basis of instinctive acts, 394-397.

Regeneration, bearing of, on stability of living matter, 316-332; by transformation, 232 ; character of restored part in, 326, 327; effect of age upon, 331 ; effect of flowering period upon, 331 ; effect of food upon, 327,328 ; effect of gravity upon, 329-332; effect of light upon, 328 ; effect of temperature upon, 327 ; first in form, afterward in size, 317; growth in, not uniform, 317, 3IS; from oblique surface, 334; heteromorphosis in, 332 ; internal factors in, 332-335; in animals, 316; in earthworms, 3I $7-$ 320 ; in embryos and eggs, 324,325 ; in fish, 318 ; in higher animals, 325 , 326 ; in the planarian, $321,322,334$, 335 ; in plants, 325 ; in salamander, 316 ; in actinian, 334 ; lateral, 333 , 335 ; not always complete, 318-320, 323,325 ; polarity in, $320,329-333$; what determines character of restored part, 333.

Regression, advantage and disadvantage of, 485 ; as to stature, $479-48 \mathrm{r}$; diagram of, 489 ; offspring more mediocre than parents, $484-486$.

Regression coefficient, $466,487-490$.

Regression table, 479-482.

Reversion, 16, 192-194.
Reversion and atavism, 305 .

Rheotaxis, 235 .

Rhinoceros, development of foot of, 60 .

Ribbert, grafting mammary gland on ear of guinea pig, 336 .

Roebuck, meristic variation in horns of, $53,65,66$.

Romanes, on instinct, 389 ; on transmission, 35t; on transmission of mutilations, 367 .

Roots, development of, by external conditions, I04; growth of, in running water, 235 .

Rose, record of, with Nora, 7S-So.

Roulette wheel, how made up, 365 .

Roux, experiments upon segmenting frogs' eggs, $3 \nmid 3$; on preparation of antitoxin, 310 .

Row system of planting in breeding work, 6.46-6.49.

Sachs, experiments on growth of piants, 256.

Salamander, regeneration in, $3 \mathbf{I} 6$.

Saline solutions, effect of, upon development, $282-285$.

Sample, random, 420 .

Sawtly, antenna of, developed into a foot, 43 .

Schmankewitsch, experiments with Artemia, 102,283 .

Sea urchin, Loeb's experiments with, 278,282 .

Seal, variation in digits of, 57.

Secretions, chemical action of, $3_{3} s_{3} s_{4}$.

Seed production a business, 650 .

Seedlings, response of, to gravity, 236 .

Seeds, effect of moisture upon, 232 .

Segmentation, dependence of, upon water content, $17 \mathrm{~S}$; geometrical character of cleavage in, 339; not dependent on fertilization, I 7 S.

Selection, 577-59S; blemishes and accidental injuries bearing on, 590 ; cessation of, 2SS; effect of, upon type and variability, 445,446 ; general principles involved in, $58 \mathbf{I}-592$; historical knowledge of the breed essential in, $579,58 \mathrm{r}$; ideals in, 578,579 ; importance of pedigree in, 592 ; increased number of "points" in, 590 ; indirect effects of, $447-448$; influence of age in, 589 ; fallacy of "foundation" females in, 595, 596; limit of power of, to reduce variability, 534,537 ; natural selection always at work, 588 ; need of large numbers for, 584 ; need of the actual test for, 586 ; 
objects of, 579; often against vigor and fertility, 583 ; physiological, 589 ; power of, to modify type, 29r, 537544; progressive, 197 ; purpose of, 581 ; rational, 592, 593; reduces to utility basis, 591 ; results in absolute increase of quality, $5 S_{2}$; reversal of, 288 ; size in dam, quality in sire, $58 S$; the exceptional breeder not always the exceptional individual, 585 ; upper limits of improvement, $5 s_{3}$; value of the exceptional breeder, $5^{\mathrm{S}} 5$; visible characters deceptive in, 50S, 510.

Selective death rate, $201,202$.

Selective influence of environment, $35 \mathrm{I}$.

Sewall, experiments with snake poison, 309.

Sex, correlation with speed in trotters, 468-470; determination of, 629-637; differences slight, $6_{30}$; in mammals, 634 ; in bees, 632 ; in plant lice, 632 ; in wasps, 633; influence of, upon development of characters, I94-I96; influence of fertilization upon, 632, 633 ; influence of nutrition upon, 631 ; inheritance not limited to, 474 ; related to the accessory chromosome, $634-637$.

Sex determination, theories upon, 629, 630.

Sexes, comparative variability of, 570 , 573 ; equipotent, 568 .

Sharks, effect of light on eyes of dead, 395 .

Sheep, development of foot of, 58 ; meristic variation in digits of, 63 .

Shorthorns, polymorphism in, 20 .

Show-ring consequences, 660 .

Shy breeders, I I $9,200$.

Sire, quality in, $5 S S$.

Sire more than half the herd, $5 \$ 7$.

Sires, great, 552; market for, 673; of sires and sires of dams contrasted, 553-555; testing of, 66z-664.

Smith, Kittie, writing with feet, $2 S 6$, 287 .

Snakes, rudimentary legs of, $5^{S}$.

Spallanzani, experiments in regeneration, 316.

Species, supposed conversion of, $2 \delta_{3}$.

Sperm cell, r6 1 .

Spermatocytes, I69.

Spermatozoön, 161; function of, 2SI.

Spinal nerves, meristic variation in, 42 .

Spireme, I 46.

Sports, 2I, III.

Stability, of type, 296, 297; shown by reversion, 305 ; of living matter illustrated by grafting, 335, 336; by regeneration, 316-335.

Stability and instability of living matter, 295-346 ; illustrated by development and differentiation, $33^{8}$.

Standard deviation, 42S-43I; contrasted with average deviation, $43 \mathbf{I}$; illustrated, 441-4+3; meaning of, 432,433 ; probable error of, 440 ; shortened method of, 429 .

Standards should not be changed, 579.

Starvation, effects of, upon regeneration, 327

Statistical methods, 426 ; need of, in heredity studies, 478 .

Stature, transmission of, $4 \mathrm{So}-484,48 S-$ 493, 499.

Steers, functional variation in, $\delta 2$.

Stentor, acclimatization of, to $\mathrm{HgCl}$, 310 ; regeneration in, 323 .

Stereotropism, 250.

Sterility of hybrids, 609 .

Sterling, originator of blackberry, 133 .

Stirp, I4, I 52, 208.

Strasburger, growth below zero, $3 \mathbf{I} 3$.

Strawberry, evolution of, I3I.

Struthers, observations on ribs, 40.

Stunted animals, 225.

Substantive variation, 30-32; importance of, 3I.

Suprarenal glands, $3 S_{3}$.

Swine, development of foot of, $5^{8}$; inbreeding in, 625 .

Symmetry, 34-37; bilateral, 34, 65-68: dorsal and ventral, as distinct from right and left, in variable parts, $6 \mathrm{~S}-$ 70 ; longitudinal, 36 ; radial, 34 .

Syndactylism, 63, 66 .

Systems of breeding, 599-627.

Tapir, development of foot of, 60 .

Teeth, meristic variation in, $4 \mathrm{~S}-5 \mathrm{I}$.

Telegony, $1 \$_{5-1 S 9}$; in dogs, 186 ; in horses, $1 S_{5}$; in man, ISS; proof by method of instance, $\mathrm{IS}_{7}$; scientific objections to, ISS.

Teleology, principle not universal, 207 . Temperature, acclimatization to, $3 \mathrm{r}$ I$3 \mathrm{I} 3,376-3^{8} \mathrm{I}$; all-pervading, 264; effect of, upon color, $262-264$; effect of, upon growth, $25 t^{-262}$; effect of, upon parthenogenesis, $2 S \mathrm{r}$; effect of, upon regeneration, 327 .

Temperature of the body, 230 .

Ten great sires, 555 .

Teratology, 100.

Testing sires and dams, 660-664. 
Testing young females, $66 \mathrm{r}$.

Tetrads, I66.

Thigmotaxis, 235 .

Thremmatology, compared with evolution, 2 ; defined, 1 ; more than a study in morphology, 8 ; problems of, outlined, 3-5.

Thyroid, effect of extirpation of, $38_{3}$.

Toadflax, experiments with, by De Vries, II5-II 8 .

Toxic poisons, 268 .

Transmission, $347-417$; heterogeneous, 426 ; how characters behave in, $473^{-}$ 478 ; manner of, 420-43I ; effect of acclimatization upon, $374-380$; effect of development upon, 407-409; of disease, 368,384 ; of effects of food, 370-374; of effects of use and disuse, 40.4-407; of liabits, 386-403; of immunity, $3 S_{2}$; of mutilations, 364368 ; of stature, 480 ; of variation, $348-417 ;$ not unless germ is affected, 416,417 ; offspring not like'parents, 482,483 ; offspring more mediocre than the parents, $484-486$; origin of the exceptional individual, 499,500 ; progression in, 492-498; what is transmitted, 5 II.

Trembley, experiments on regeneration, 3I6.

Trotters, correlation between color, sex, and speed, 468-471.

Trotting records showing prepotency, $55^{1-566 .}$

Tumors, 99, $27 \mathrm{I}$.

Turtle, double head of, 67 .

Twins, 176 ; identical, 176 ; from a single ovum, 176 .

Type, as distinct from the individual, $35^{2}$; conceptions of, 420-425; effect of environment upon, 290-293; effect of fertility upon, $198,199,449$, $45 \mathrm{I}$; effect of selection upon, 445, 446 ; mutability of, 298-305; natural, 422 ; power of selection to modify, 537-544; selection standard for, 420 , 425 ; stability of, $296,297,541,544$.

Type and variability, 4I-45I.

Use a function of structure, 387 .

Use and disuse, effect of, $2 S_{5-290}$; effect of, upon functional activity, 95, 96 ; effects of, when transmitted, $404^{-}$ 407 ; influence on transmission, 363 .

Variability, among offspring of same parents, 500-504; as affected by fertility, 449-45I ; coefficient of, 433 ; determined from groups, 426 ; deviation from type, 425 ; effect of selection upon, 445, 446; erroneous conceptions of, 425,426 ; highest in fertile soils, 642 ; in heights of brothers, 50I ; in oil and protein, 445,446 ; in physical characters of corn, 447 , $44^{8}$; limitations of, 8,9 ; limit to the reduction of, 534-537; measure of, by average deviation, 427 ; measure of, by standard deviation, 428-43I ; nature of, 10, 11 ; of different characters in same population, 444; of sexes, 570-573; ultimate unit of, 1 5-17; units of, 208-213.

Variation, bud, r8r; causes of, I4I347 ; caused by bisexual reproduction, $160-163$; caused by cell division, I55-I 81 ; caused by external influences, 220-293; caused by reduction process, $163-181$, 175,176 ; causes of, must be studied, I4I ; confined to racial characters, $35^{8}$; continuous and discontinuous, 18-22; correlated, I6; does not extend to non-living matter, 23 ; due to age or staleness of germ, ISz; due to temperature, 262-264; functional, 75Iog; functional, due to age, 94 ; functional, between different individuals of the same species, 77-91; functional, due to use or disuse, 95,96 ; functional, from day to day, same individual, 91-94; functional, induced by external influences, 98, IOI-IO 5 ; functional, influenced by feed, 96,97 ; how far possible, 295-346; induced by food, 225-230; influence of moisture upon, 230-233; influenced by fertility, 196-200; influenced by the reproductive functions, 100 ; influenced by slight chemical changes, 210,211 ; in chemical composition of seeds, - corn, $83-86$; in fertility, 90 ; in general body faculties, 86 ; in meat production, $\mathrm{S}_{\mathbf{I}}-83$; in milk secretion, 77-8I, 9I-93; in parthenogenetic reproduction, 177-180; in sugar production, 86; in race, caused by external influences, 290-293; in rate of cell division, 340 ; in vital functions, S7-89; internal causes of, 155-217; kinds of, $17-23$; morphological, substantive, meristic, functional, 22 ; meristic, 33-74; morphological, 2529; substantive, 30-32; quantitative 
and qualitative, 17 ; nature of, 356 364; not notably less in parthenogenesis, 178 ; through inheritance of modifications, 292 ; units of, 208-213; universality of, 7,8 .

Variations, due to causes internal to the germ, are transmitted, 348 ; due to external influences, transmission of, $348-417$; due to causes not affecting the germ not transmitted, 416,417 ; occur according to the binomial theorem, 508, 509 .

Varieties, duration of, 544, 545.

Vigor, often opposed by selection, $5^{8} 3$.

Vital limits as to light, 244.

Vöchting's experiments on gravity and polarity, 237 .

Wallace on the accessory chromosome, 636.

Water, effect of, upon growth, 230-233.

Wattles, 46.

Wayland, originator of plum, I 33 .

Weeping varieties, II 2 .

Weismann, experiments with butterflies, 262-264; on death point, 202; on germinal selection, 214 ; on origin of characters, 413,414 ; prediction of,
I73, I74; prediction concerning loss of hereditary matter, 173,174 ; on transmission, 354 .

Whale, variation in digits of, 57 .

Wheat, acclimatization of, 376 ; influence of locality upon, 222.

White, Hugh, originator of Clinton grape, 134 .

Whitney, fund for exploration, 302 .

Willow, effect of gravity on growth of, 238.

Wilson, John, originator of blackberry, I 32 .

Wilson on the chromosome, 634 .

Wing, supernumerary, 43 .

Wings, supernumerary, $5 \mathrm{I}$.

Writing with the feet, $2 \$ 6,287$.

Xenia, $\mathrm{IS}_{3}, \mathrm{I} 8_{4}$

Young breeders, 675 .

Yucca moth, 105; instinctive acts of, 388.

Yule's formula, 456,457 .

Zoja, experiments in regeneration of blastomeres, 325 . 




$0_{0}$

(is

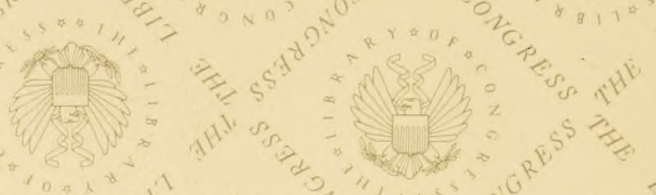

$100 \%$

(c)

100 a

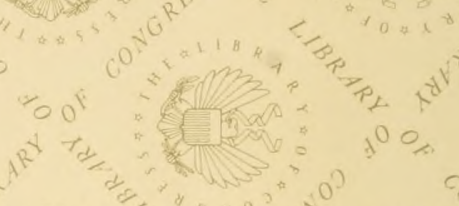

$\cos _{x \rightarrow 1}$

$+3$

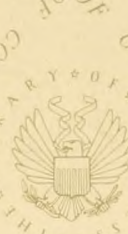

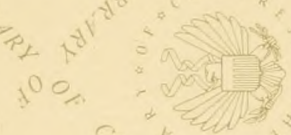
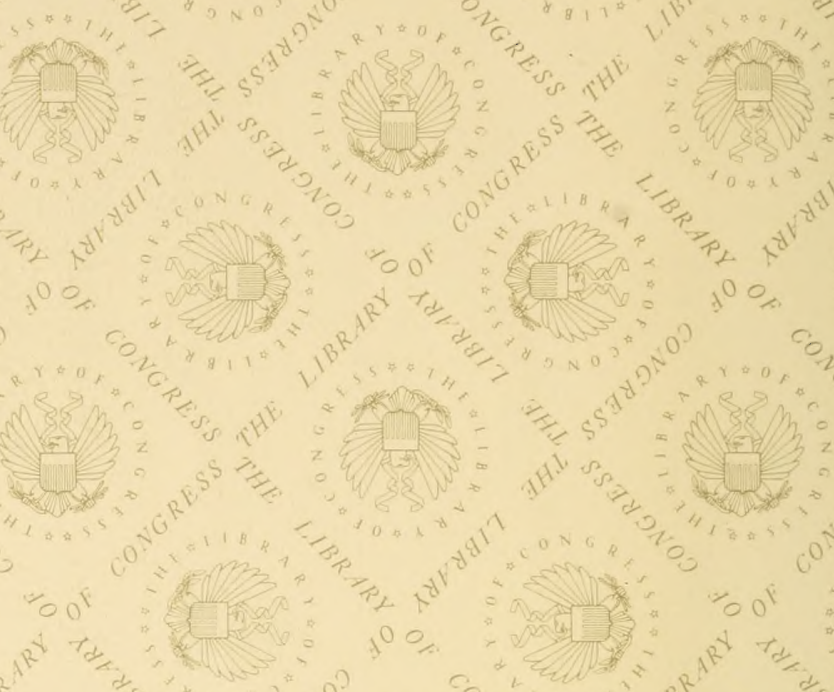

$\operatorname{li}^{+} \frac{1}{2} \frac{1}{2}$

"0 01

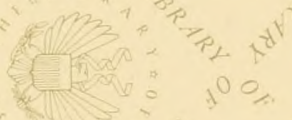

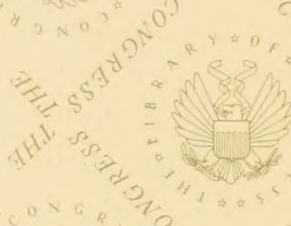

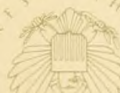

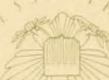

$p_{2}, x^{+t^{2}}=5$

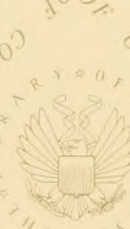

(F) $=$

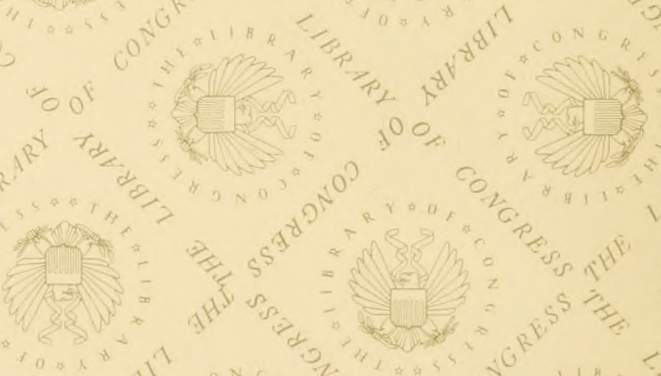

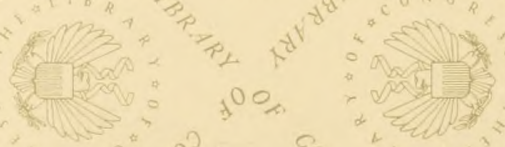

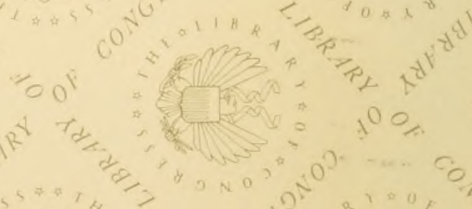





\section{LIBRARY OF CONGRESS \\ |||||||||||||||||||||||||||||||||||||||||||||||| \\ |||||||||||||||||||||||||||||||||||||||||||||||||||||||}

00027466783

ats 